\title{
IntechOpen
}

\section{Theoretical and \\ Methodological Approaches \\ to Social Sciences and \\ Knowledge Management}

Edited by Asunción López-Varela 



\section{THEORETICAL AND \\ METHODOLOGICAL APPROACHES}

TO SOCIAL SCIENCES AND KNOWLEDGE MANAGEMENT

Edited by Asunción López-Varela 
Theoretical and Methodological Approaches to Social Sciences and Knowledge Management http://dx.doi.org/10.5772/2273

Edited by Asunción López-Varela

\section{Contributors}

Yijun Liu, Wenyuan Niu, David Phipps, Krista Jensen, J. Gary Myers, Bayram Tay, Thomas Dan-Olof Marmefelt, Hajime Eto, Pierre Robillard, Okwy Okpala, Christopher Odogwu Chidi, Caleb Kangai, Oliver Mtapuri, Roberts Muriisa, Maria Covadonga De La Iglesia Villasol, Katerina Hlavackova-Schindler, Puvanasvaran A.Perumal, Renan Martins Pombo, Sylvain Cibangu, Nikola Štambuk, Paško Konjevoda, Mohammad Reza Noruzi, José G. Vargas-Hernandez

\section{(c) The Editor(s) and the Author(s) 2012}

The moral rights of the and the author(s) have been asserted.

All rights to the book as a whole are reserved by INTECH. The book as a whole (compilation) cannot be reproduced, distributed or used for commercial or non-commercial purposes without INTECH's written permission.

Enquiries concerning the use of the book should be directed to INTECH rights and permissions department (permissions@intechopen.com).

Violations are liable to prosecution under the governing Copyright Law.

\section{(cc) BY}

Individual chapters of this publication are distributed under the terms of the Creative Commons Attribution 3.0 Unported License which permits commercial use, distribution and reproduction of the individual chapters, provided the original author(s) and source publication are appropriately acknowledged. If so indicated, certain images may not be included under the Creative Commons license. In such cases users will need to obtain permission from the license holder to reproduce the material. More details and guidelines concerning content reuse and adaptation can be foundat http://www.intechopen.com/copyright-policy.html.

\section{Notice}

Statements and opinions expressed in the chapters are these of the individual contributors and not necessarily those of the editors or publisher. No responsibility is accepted for the accuracy of information contained in the published chapters. The publisher assumes no responsibility for any damage or injury to persons or property arising out of the use of any materials, instructions, methods or ideas contained in the book.

First published in Croatia, 2012 by INTECH d.o.o.

eBook (PDF) Published by IN TECH d.o.o.

Place and year of publication of eBook (PDF): Rijeka, 2019.

IntechOpen is the global imprint of IN TECH d.o.o.

Printed in Croatia

Legal deposit, Croatia: National and University Library in Zagreb

Additional hard and PDF copies can be obtained from orders@intechopen.com

Theoretical and Methodological Approaches to Social Sciences and Knowledge Management Edited by Asunción López-Varela

p. cm.

ISBN 978-953-51-0687-6

eBook (PDF) ISBN 978-953-51-5117-3 


\section{We are IntechOpen, \\ the world's leading publisher of Open Access books}

Built by scientists, for scientists

\section{$4,000+$ \\ Open access books available \\ $116,000+$ \\ International authors and editors

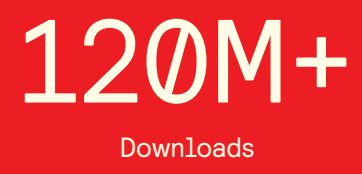

Our authors are among the

151

Countries delivered to

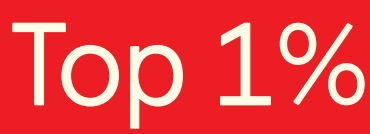

most cited scientists

Contributors from top 500 universities

$12.2 \%$

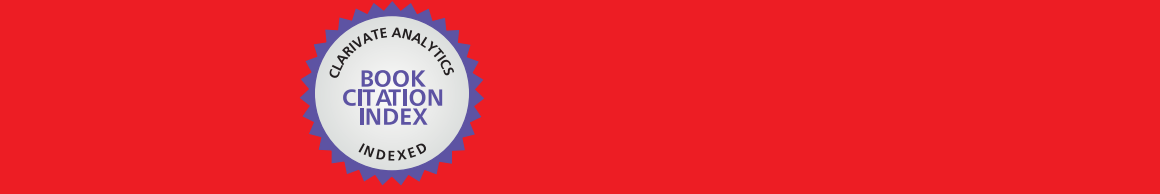

WEB OF SCIENCE ${ }^{\mathrm{M}}$

Selection of our books indexed in the Book Citation Index in Web of Science ${ }^{\mathrm{TM}}$ Core Collection (BKCI)

\section{Interested in publishing with us? \\ Contact book.department@intechopen.com}





\section{Meet the editor}

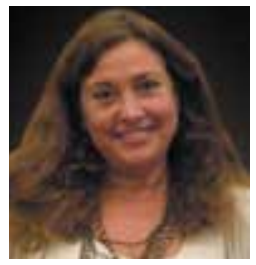

Professor Dr Asunción López-Varela BA, MA (Hons.), $\mathrm{PhD}$ (Hons.) teaches at Universidad Complutense Madrid, Spain. Her areas of research of include socio-semiotics, comparative cultural studies and intermediality. She is a member of the Executive Committee of the European Network for Comparative Literary Studies and the Harvard Institute for World Literature, and coordinates the research program: Studies on Intermediality and Intercultural Mediation. Dr López-Varela participates in the advisory boards of Cultura - International Journal of Philosophy of Culture and Axiology, Journal of Comparative Literature and Aesthetics (JCLA), CLCWeb - Comparative Literature and Culture, and Cypriot Journal of Educational Sciences (CJES). 



\section{Contents}

\section{Preface XIII}

Section 1 Social Research Methods 1

Chapter 1 Social Research Methods in Higher Education: A Critical Analysis of Methodological Issues and Emerging Trends at the Zimbabwe Open University 3

Caleb Kangai

Chapter 2 Methodology Transfers Between Social Sciences and Humanities in Relation to Natural Sciences, Technology and Government Policy 33

Hajime Eto and Shinichi Yamamoto

Section 2 Quantitative and Qualitative Approaches 53

Chapter 3 Causality in Social Studies Education $\mathbf{5 5}$ Bayram Tay

Chapter 4 The Assumption of Non-Gaussianity in Natural and Social Sciences and Its Influence on Detection of Causal Relationships 77

Kateřina Hlaváčková-Schindler

Chapter 5 Qualitative Research:

The Toolkit of Theories in the Social Sciences

Sylvain K. Cibangu

Section 3 Coding and Cartographic Models 127

Chapter 6 A Simulation Approach to Validate Models Derived from Observational Studies 129 Pierre N. Robillard and Simon Labelle

\section{Chapter 7 Cartographic Generalization Applied} to Social Networks Maps in the City of Curitiba in Brazil 147 Renan M. Pombo, Luciene S. Delazari and Claudia R. Sluter 
Chapter 8 Open-Source Tools for Data Mining in Social Science 163

Paško Konjevoda and Nikola Štambuk

Section 4 Knowledge Management 177

Chapter 9 Applying Social Sciences Research for Public Benefit Using Knowledge Mobilization and Social Media 179

David J. Phipps, Krista E. Jensen and J. Gary Myers

Chapter 10 Re-Visiting Ethnographic and Orthodox

Research Methodologies: Field Research

Experiences from an African Perspective 209

Oliver Mtapuri

Section 5 Public Opinion and Citizenship 225

Chapter 11 Social Physics: An Interdisciplinary

Way to Explore the Mechanism of Public Opinion 227

Yijun Liu and Wenyuan Niu

Section 6 Enterpreneurship, Employment and Industrial Relations 245

Chapter 12 The Methodology of Formulating Iranian National Policy of Entrepreneurship: A Conceptual Framework 247

Hassan Danaeefard and Mohammad Reza Noruzi

Chapter 13 Theoretical Approaches to Employment and Industrial Relations: A Comparison of Subsisting Orthodoxies 263

Christopher Odogwu Chidi and Okwy Peter Okpala

Chapter 14 Human Capital Resourcing Practices

and Organisational Performance: A Study

of Selected Organisations in Lagos State, Nigeria 279

Christopher Odogwu Chidi and Okwy Peter Okpala

Section 7 Lean Behaviour and Sustainability 293

Chapter 15 Enhancing Productivity Through Lean Behavior 295

A. Perumal Puvanasvaran

Chapter 16 Organizational Sustainability:

The Case of Handcrafts Micro-Business

in Southern San Sebastian, Jalisco, México 319

José G. Vargas-Hernández

Chapter 17 Strengthening Institutional Capacity

for Science, Technology and Innovation in Uganda 331

Muriisa R. Kabeba 
Chapter 18 A Didactic and Methodological Lesson of the Study of Economics and the Skill Development of Students

Maria Covadonga De la Iglesia Villasol

Section 8 Reforms and Responses to the Global Crisis 365

Chapter 19 New Public Management and Reforms in Iran:

Analysis of Government Downsizing 367

Hassan Danaee fard, Tayebeh Abbasi and Mohammad Reza Noruzi

Chapter 20 Media of Exchange and Liquid Assets of Political and Market Enterprises: A New Monetary Perspective on Medieval French Monetary Mutations 387

Thomas Marmefelt 



\section{Preface}

The umbrella term Social Sciences and Humanities refers to a plurality of fields outside the Natural or Physical Sciences. Disciplines as different as anthropology, archeology, architecture, art, cultural studies, economics, education, geography and environmental studies, history, law, languages and linguistics, political science, philosophy, psychology, sociology or translation studies, all share the concern for human relations and socio-cultural practices.

In the ancient world, the alliance between political and religious power had guaranteed the interdisciplinary dialogue between the Natural Sciences, the Social Sciences and the Humanities, all closely associated to institutional control. The move towards anthropocentric approaches took place at different times in different regions of the world, alongside the socio-cultural, political, economical and technological forces that shaped each territory, from the establishment of the first learning centers and universities, to the discovery of new ways of looking at worlds beyond our own by means of the telescope. But our world not only changed at the pace humans marked through their changing practices and innovations. Environmental issues, such as the fact that papyrus could not be used in the wet climates of Northern Europe, shaped the way human technologies were used and where. From Chinese paper and the printing press, to contemporary digital communication and networked society, the complexity of human life is such that knowledge divisions are there to set the foundations for groundbreaking innovations across all fields. To say that the growth of the Social Sciences took place mostly in the $18^{\text {th }}$-century, coinciding with political and economic reforms, sometimes in the form of violent revolutions, that sought national and territorial cohesion in Europe, would be to cast aside similar changes in other parts of the world.

There are many difficulties involved in writing an introduction to a series of volumes that seek to provide an overall picture of human society and cultural habits across differing disciplines, various nations, distinct methodologies and, in some cases, diverse time spans. The volumes oscillate between 'positive' approaches to knowledge, based sense experience and statistical analysis, focused on deduction and description, and interpretative positions, more inductive and prescriptive. Specialization and interdisciplinarity walk hand in hand in a dialogue that seeks to speak across the bio-physical, the socio-cultural and the artistic, under the common 
desire to understand humanity more clearly and direct our actions in more effective ways by means of theoretical discussions, innovative ideas, the encouragement of public debates, and interaction across societal structures.

InTech collection on Social Sciences and Humanities is unique and groundbreaking in its combination of questions and answers from very diverse fields and disciplines. It is also a point of connection between East and West, North and South, developed and less developed countries. It includes chapters compiled by institutional research agencies, established scholars, alongside work by younger researches, all of which point to fruitful alternative routes for further exploration and good practice, seeking to identify current impasses in times of crisis, and to create opportunities and avenues for future change. Key underlying principles are the support innovation and sustainability across the world, the fostering of collaboration amidst the Sciences (both Natural and Social) and the Humanities, and the private and the public sectors of society. The chapters collected address societal challenges across diverse cultures and environments.

Oftentimes the pathways to discovery are laid on shaky ground in order to open up possibilities despite the risks involved. InTech collection Social Sciences and Humanities: Theories and Application is doing just that.

The first volume of InTech collection is entitled Theoretical and Methodological Approaches to Social Sciences and Knowledge Management and includes chapters that move from theoretical and methodological issues and the analysis of quantitative and qualitative tools, to more concrete applications of these.

Prof. Caleb Kangai's (Zimbabwe Open University) contribution "Social Research Methods: a critical analysis of methodological issues and emerging trends" opens the volume because it offers an excellent introduction which helps identify current issues and trends in the application of methods for social science research. His discussion provides a forum for social scientists to reflect on the contemporary issues affecting the Social Sciences. This is followed by a review of the three most widely used theoretical approaches in the Social Sciences. Prof. Hajime Eto (Professor emeritus, University of Tsukuba, Japan) and Prof. Shinichi Yamamoto (Director Research Institute for Higher Education, Hiroshima University) draw the connection between "Methodology transfers between Social Sciences and Humanities in relation to Natural Sciences, Technology and Government policy". Their chapter describes the paradigms that disciplines in the Humanities and Social Sciences have followed in the West, and their influence on Eastern countries, particularly in the case of Japan. The authors make a claim for the need to incorporate knowledge from the East in the collective memory of a global world. Japanese cultural input in this article remains relatively low, almost a symptom of the need for greater participation of Eastern and Southern cultures in the construction of global paradigms. 
The study of causality is of great relevance to understand the differences between the Natural Sciences and Social Sciences and Humanities. The processes of deduction and induction play a role in both theory and application and depend on the various notions of causation, probabilistic in the Physical Sciences, and contingent in Social Studies. Prof. Bayram Tay (Ahi Evran University, Turkey) revises the development of logical thinking, which includes both induction (generalizations and observations) and deduction (results obtained through the process of reaching a conclusion by comparing). Prof. Katerina Hlavácková-Schindler (Commission for Scientific Visualization, Austrian Academy of Sciences) takes the issue one step forward by studying causality from non-deterministic and Non-Gaussian perspectives and formal mathematical approaches to detect cause-effect relationships, namely the Granger causality, transfer entropy, directionality index and other information-theoretical measures. Departing from quantitative analysis, Prof. Sylvain K. Cibangu (University of Washington) offers a toolkit of theories on qualitative research. The author claims that despite the rise in qualitative research methods in published textbooks and journals, reflections on social science theory remain scant, and that the assumption that the Social Sciences deal with subjective non-measurable phenomena may have reduced the potential role of theory construction. The chapter maps the broader history of qualitative research and outlines some practical consequences for theory building in the Social Sciences.

Continuing with papers that focus on general theoretical and methodological issues, the volume moves on to coding, a strategy used in protocol analysis to extract values for quantitative variables from qualitative data collected from observations. Human behavior is characterized by coding schemes, and statistical tools are used to derive models of the behavioral patterns emerging from the tasks under study. In "Validation of Information Models derived from Observational Studies" Prof. Pierre N. Robillard (Computer Engineering, Ecole Polytechnique de Montréal, Canada) provides a simulation approach to validate models derived from observational studies, enabling analysis of the impact of individual qualitative variability on coding transcripts from protocol analysis. This study constitutes one of the few approaches that enable the modeling of real activities. It is of general interest and can be applied to any observational study. Quantitative cartographic generalizations applied to the mapping of social networks is the object of Prof. Renan M. Pombo, Luciene S. Delazari and Claudia R. Sluter (Federal University of Parana, Brazil) study on the implementation of a software to preserve spatial reference in the graphs and matrices of social networks by means of aggregation and displacement operators which render readable representations on a smaller scale such as mobile phone screens. The paper includes graphics obtained by means of this cartographic software. Open-source tools for data mining in the Social Sciences are revised by Paško Konjevoda and Nikola Štambuk (Ruđer Bošković Institute, Croatia) and their advantages and disadvantages are described. The paper offers the web addresses where these tools are available as well as textbooks and manuals describing them. 
Moving onto knowledge mobilization, Prof. David J. Phipps, Prof. Krista E. Jensen, and Prof. Gary Myers (York University, Canada and KMbeing.com,) discuss activities that encourage relationships between researchers and decision makers by means of social media, Twitter in particular. Their examples come from Canada, the US and the UK. Prof. Oliver Mtapuri (University of Limpopo South Africa) focuses on the particular case of Africa, and introduces researchers to the problems of doing research there. The multiplicity of languages and dialects, literacy levels, particular communal traditions and sensibilities, are among the challenges to be overcome in circumstances of data collection. In many cases, translation from vernacular to English and vice versa does not capture the essence of discussions and offer contradictory and ambiguous results.

Public opinion reflects social realities, the integration of mass consciousness, ideas and emotions. The subject of opinion is the general public, the object is a particular focus of the community, and the ontology is the tendentious comments or remarks of this focus. Prof. Yijun Liu, Wenyuan Niu (Institute of Policy and Management, Chinese Academy of Sciences Beijing) explores the mechanism of public opinion within the perspective of social physics. Three main theories, social combustion theory, social shock wave theory and social behavior entropy theory, are involved in social physics. When large-scale individuals or groups discuss some incident, it enters an active period from latent period of opinion. This indicates that opinion is built step by step and formed at last by integration of local viewpoints with key points from opinion leaders. The level of opinion formation during the different stages can be quantitatively decided by number, scale and intensity. The study of the formation of public opinion is useful in order to build a quantitative method to predict behavior.

Another area of interest in the Social Sciences is entrepreneurship, employment and industrial relations. This volume offers several chapters dedicated to explore different aspects such as policymaking, object of study for Prof. Mohammad Reza Noruzi (Tarbiat Modarres University, Tehran, Iran). His approach involves problem identification, instruction compiling, formulation, performance and evaluation, as well as counting alternatives related to problem solving applied to the Iranian case. Similarly, Prof. Christopher Odogwu Chidi and Prof. Okwy P. Okpala (University of Lagos, Nigeria) examine employment and industrial relations theories, presenting extensively five traditions which show differences and commonalities. The five theories under study (unitary, systems, conflict, Marxist and social action theories) recognize three actors/participants/social partners involved in industrial relations. However, with the emergence of transnational organizations emphasis is shifting to the concept of "tripartism-plus", where none of these theories have a comprehensive coverage. Furthermore, in the following chapter, the authors investigate human capital resourcing practices and organizational performance in selected organizations in the food, beverage and tobacco industry in Lagos State, Nigeria. In testing the first hypothesis that there is a positive relationship between recruitment practices and organizational performance, it was found that there is a positive relationship between 
them $(\mathrm{r}=0.45 ; \mathrm{p}<.05)$. In testing the second hypothesis that there is a positive relationship between selection practices and organizational performance, it was found that there is a positive relationship between them $(\mathrm{r}=0.49 ; \mathrm{p}<.05)$. These results are statistically significant for they mean that as recruitment and selection practices improve, so does organizational performance.

Another issue in industrial relations is lean behavior and its relation to productivity, the focus of Prof. Perumal Puvanasvaran's chapter which examines the particular case of a manufacturer of aerospace composite. Results for this research are collected and analyzed by means of statistical software, and the analysis, in the form of index values, percentages, supported by comparing the results of surveys and monitoring of real life data of the case study presented, is revealing. José Vargas-Hernández (Univ. Guadalajara, Mexico) also explores sustainable and efficient organizations, this time in Mexico. His paper analyzes sustainability and efficiency of organizations committed to handcrafts micro-businesses and their environmental impact in the municipalities of Gómez Farías and Zapotlán el Grande. The initial hypothesis departs from the consideration of the scarce social capital of organizations that limits development's sustainability. The research method employed is ethnography, complemented with fieldwork in the form of informal interviews, and documental and bibliographic research. The outcomes demonstrate that the drama of economic efficiency and sustainable development of micro-business is tied to constraints of social capital, findings that have implications for the design and implementation of economic and social policies. The case of Uganda is debated by Prof. Muriisa R. Kabeba (Dean Mbarara University of Science \& Technology, Uganda), who writes on the need to strengthen institutional capacity for science innovation and technology in this country and discusses institutions and institutional challenges. Points of special relevance for the author are motivation for scientists, guaranteed protection of their research rights, and recognition of their scientific work. The paper also discusses challenges in the coordination of the different research organizations in the country. Finally, Maria Covadonga De La Iglesia Villasol (Univ. Complutense Madrid, Spain) defends the importance of university training in aspects of innovation and creativity, which are particularly lacking in the Spanish context, and need to be encouraged in order to converge with the European Space of Higher Education framework. Her paper offers an interesting discussion of the current trends on university education in Europe.

Several chapters focus on different responses to the global crisis. Mohammad Reza Noruzi and Tayebeh Abbasi (Tarbiat Modarres University, Tehran Iran) examine the Iranian system and downsizing as a strategy of public management. Various analyses show complex set of conclusions. In some cases, downsizing plans are performed on the basis of regulations and management professionalization. At times it is an unnecessary decision intended to reduce public costs. According to the authors, there is also an unsuitable context for privatization and many contradictions in the exchanges between public and non-public sectors in Iran, where the decrease in the number of ministries and offices does not seem to have affected the costs incurred by 
the government. Therefore, the role of government should be investigated, public costs should be controlled seriously, and some activities of low productivity and high energy consuming should be identified and revised. Downsizing should be performed without any significant harm in welfare affairs. A different approach is taken by Prof. Thomas Marmefelt (University of Södertörn, Huddinge, Sweden) who examines liquidity as a crucial link between the financial system and the real economy. The historical experience of liquidity with partial monetary separation in pre-modern economies provides valuable lessons that shed some light on contemporary systems. In the pre-modern European monetary system, such as medieval France where monetary mutations were based on metal-content reductions, the medium of account was independent. Coins had an intrinsic value, in the sense of being a commodity, but also an extrinsic value, in that sense of being an institution. Pre-modern money could be seen above all as a clearing device that later with the gold standard turned into a commodity. Units of account in pre-modern Europe were based on social convention and could therefore be adjusted to control liquidity. Along similar lines, Thomas Marmefelt explains that market prices possess a language-like symbolizing function, and that language is required to establish a shared meaning of money, property, exchange, and price, in order to give market prices that function. The chapter explores how sustainable growth requires that financial system finds the correct liquidity based on a balanced between desired innovation and demands by consumers.

Asunción López-Varela

Universidad Complutense Madrid

Spain 


\section{Section 1}

Social Research Methods 



\title{
Social Research Methods in Higher Education: A Critical Analysis of Methodological Issues and Emerging Trends at the Zimbabwe Open University
}

\author{
Caleb Kangai \\ Zimbabwe Open University, \\ Zimbabwe
}

\section{Introduction}

The world today is witnessing an explosion of social science research. Unlike other disciplines in the natural sciences, social science research has attracted much attention due to its dynamism and complexity (Dube and Pare, 2003). As modern societies become more complex in their structure, needs and demands, social problems have also become more complex. As a result, several social theories and social research methods have been developed and continue to develop in an effort to understand diverse social problems. Presently, social research is in the process of rapid change. This chapter focuses on current methodological issues and emerging trends in social science research. It argues that the challenge for social scientists and researchers worldwide is to continue in the search for a unified consensus on what methodology might have the power and refinement to connect a proposed "grand theory" with the various midrange theories which, with considerable success, would continue to provide usable frameworks for increasing the overall knowledge of society. Social research is experiencing a paradigm shift that calls for the re-examination of current methodologies. The present review helps identify current methodological issues and trends in social science research. It is important that as social researchers, we should understand the philosophical, theoretical, and methodological debates influencing our work and careers, their implications for research and our place within them.

This discussion provides an academic forum for social scientists and researchers to reflect on the contemporary issues and emerging trends affecting their careers. This is important as this will help to shape the orientation of both old and young social researchers to be innovative and go beyond conventional boundaries. This chapter also puts young social scientists - who have not yet become 'attached' to certain sets of theories, concepts and methodology - in advantageous position to understand the current issues and emerging trends in social sciences research.

\section{Aim/purpose of the chapter}

This chapter serves two purposes. First, the chapter examines the historical, philosophical, and theoretical developments that have influenced social research methods worldwide. 
Second, the chapter reports on current issues and emerging trends in social research with particular reference to social research at the Zimbabwe Open University.

\section{Organization of the chapter}

After defining social research, the chapter traces the historical and controversial development of social research covering the deductive - inductive debate, the positivist constructivist debate, and the quantitative - qualitative debate. In order to help readers understand major issues and emerging trends in contemporary social research methods, this chapter examines the experiences of social researchers at the Zimbabwe Open University.

\section{Types of sciences}

Sciences are broadly divided into natural (or physical) sciences and social (or human) sciences. Natural sciences include disciplines such as geology, biology, meteorology. Social Sciences include various disciplines dealing with human life, human behaviour, social groups and social institutions. They consist of Anthropology, Behaviour Science, Commerce, Demography, Economics, Education, Geography, History, Law, Linguistics, Management, Political Science, Psychology, Public Administration, Sociology, and Social Work.

\section{What is social science?}

Literature defines social research as research conducted by social scientists (Ragin, 1994; Firebaugh, 2008). By the late 19th century, the academic social sciences were constituted of five fields: jurisprudence and amendment of the law, education, health, economy, trade and art. More specifically, social research examines a society's attitudes, assumptions, beliefs, trends, stratifications and rules. Popular topics of social research include poverty, racism, class issues, sexuality, voting behaviour, education, gender constructs, policing and criminal behaviour. Social research determines the relationship between one or more variables. Even though social research is most often conducted by social scientists or sociologists, it is an interdisciplinary study crossing into subjects like criminology the study of crime; politics, the study of power; economics the study of money and business; psychology study of the mind; philosophy study of beliefs and morals; and anthropology and the study of culture. Social Science research is a systematic method of exploring, analysing and conceptualising human life in order to extend, correct or verify knowledge of human behaviour and social life. In other words, Social science research seeks to find explanations to unexplained social phenomena, to clarify the doubtful, and correct the misconceived facts of social life. In a broader sense social research is the scientific study of society.

Social Sciences are not exact sciences like physical sciences, as they, unlike the latter, deal with human beings. Human nature and man's environment are so complex that it is more difficult to comprehend and predict human behaviour than physical phenomena. No two persons are alike in feelings, drives or emotions. No one person is consistent from one moment to another. The behaviour of human beings is influenced by biological, psychological, socio-cultural, temporal and environmental factors. It is difficult to see the underlying uniformities in the diversity of complex human behaviour. 
Early German hermeneuticians, such as Wilhelm Dilthey, pioneered the distinction between natural and social science. Since the 1960s, a general weakening of deductivist accounts of science has grown side-by-side with critiques of scientism or 'science as ideology'. Writing in his book "Constructing Social Research", Charles C. Ragin (1994) says social research involves the interaction between ideas and evidence. He further argues that ideas help social researchers make sense of evidence, and researchers use evidence to extend, revise and test ideas. Social research thus attempts to create or validate theories through data collection and data analysis, and its goal is exploration, description and explanation. Social research seeks to find social patterns of regularity in social life and usually deals with social groups (aggregates of individuals), not individuals themselves.

\section{What is research?}

Research is the collection of information to test new ideas or to disprove old ones. Scientists become famous for discovering new things that change how we think about nature, whether the discovery is a new species of dinosaur or a new way in which atoms bond. Many scientists find their greatest joy in a previously unknown fact (a discovery) that explains some problem previously not explained, or that overturns some previously accepted idea. This is the essence of research. Natural/physical science research is the search for knowledge in order understand the physical world. On the other hand, social research is the search for knowledge about individual human beings and their societies.

\section{Why do social research?}

One might still wonder why societies and nations pay people who do social research. Why does a society devote some of its resources to this business of developing new knowledge about the social world, or what has motivated social researchers to devote their lives to developing this new knowledge? One realm of answers lies in the desire to improve people's lives. For example, geneticists try to understand how certain genes are passed from generation to generation; biologists trace the pathways by which diseases are transmitted; earth scientists try to develop better models to understand physical disasters such earth quakes floods.

The second realm of answers lies in a society's desires for economic development. Many earth scientists devote their work to finding more efficient or more effective ways to discover or recover natural resources like petroleum and ores. Plant scientists seeking strains or species of fruiting plants for crops are ultimately working to increase the agricultural output that nutritionally and literally enriches nations. Chemists developing new chemical substances with potential technological applications and physicists developing new phenomena like superconductivity are likewise developing knowledge that may spur economic development. In a world where nations increasingly view themselves as caught up in economic competition, support for social science research is nothing less than an investment in the economic future.

The third realm of answers lies in humanity's increasing control over our planet and its environment. Much science is done to understand how the toxins and wastes of our society pass through our water, soil, and air, potentially to our own detriment. Much science is also done to understand how changes that we cause in our atmosphere and oceans may change 
the climate in which we live and that controls our sources of food and water. In a sense, such science seeks to develop the owner's manual that human beings will need as they increasingly, if unwittingly, take control of the global ecosystem and a host of local ecosystems. Lastly, societies support science because of simple curiosity and because of the satisfaction and enlightenment that come from knowledge of the world around us.

\section{Philosophical assumptions of social research}

In any discipline, there will always be a number of underlying philosophical predispositions in the projects of scientists. Some of these predispositions involve the nature of social knowledge itself, the nature of social reality, and the locus of human control in action. As social scientists, we must understand the three philosophical assumptions that influence social research. These are epistemology, ontology, and methodology. Epistemology means knowledge and how it is acquired (Bryman, 2001). It concerns clarification of the researcher's beliefs about how knowledge is created. There are two contrasting views: normative epistemology and interpretative epistemology. The normative view holds that research creates knowledge by building on the foundations of accepted and rationally defensible theory (positivism). The interpretative view is that research must set aside existing knowledge and discover new knowledge from internal coherence (constructivism).

\section{Epistemology}

Epistemology in the positivist paradigm supports the idea that the social world can be investigated through natural science methodologies. Hypotheses have to be tested by empirical approaches. Data need to be objectively analysed through scientific methods. In contrast, epistemology in the constructivist paradigm supports the idea that knowledge can be acquired by investigating the phenomena in many ways, because the social context is different from natural science.

\section{Ontology}

Ontological assumptions concern the nature of the world and human beings in social contexts (Bryman, 2001). Ontology in positivism assumes that social phenomenon is independent from other factors. It assumes that social phenomena can be defined and studied objectively apart from the people who make it. This means that different researchers can have different conclusions from one study.

\section{Methodology}

Methodological assumptions focus on analysis of the methods used for gathering research data (Louis Kohen, Lawrence Manion, Keith Morrison, 2001). In positivist paradigms, the scientific method (quantitative) is used to observe the phenomena. It uses mathematics calculations to generalize the finding and test the theory. In contrast, the constructivist paradigm uses qualitative methods (observations, fieldwork notes, interviews) to investigate the phenomena.

Social science scholars and researchers are moving away from the idea that social science should reflect the aims and methods of natural science through a critique of these methods 
as inapplicable to social research. These assumptions are very important as they help the social scientist to have an appropriate philosophical orientation to his/her work.

\section{Social research as a science: Issues to consider}

One of the challenges social researchers have had to fight against is the negative perception that social research is not scientific in the same sense as natural sciences. This view raises a number of questions. What constitutes a 'science'? What are the nature of its methods? What is the type of data that it should collect? In order to resolve these questions, we need to understand the main ideas and debates about what constitutes scientific research.

A science is often thought of as being a coherent body of thought about a topic over which there is a broad consensus among its practitioners. Chalmers (1999) argues that science is based on hard facts, the facts are presumed to be claims about the world that can be directly established by a careful, unprejudiced use of the senses. Scientific study is based on what we see, hear and touch rather than on personal opinions or speculative imaginings. If observation of the world is carried out in a careful, unprejudiced way then the facts established in this way will constitute a secure, objective basis for science (Chalmers 1999). Scientific research consists of two primary functions (1) the development of theory and (2) the testing of substantive hypotheses that are deduced from theory. The scientist, therefore, is engaged in the use, modification, and or creation of theory. To this end when traditional theorists talked about science they often meant the "hard" or natural sciences. In the 19th century, any studies concerning societies where not considered as sciences in the true sense of the word. However, today social research is considered as scientific in every aspect.

Contemporary intellectuals have often disagreed about the extent to which the social sciences should mimic the methods used in the natural sciences. The founding positivists of the social sciences (Dodds, Lawrence, \& Guiton, 1984); Kember \& Dekers, 1987); Osman \& Wagner, 1987) have argued that social phenomena can and should be studied through conventional scientific methods. On the other hand, proponents of social sciences (Campbell and Stanley 1963, 1966; Cook and Campbell, 1979) supported the idea that there is a need for an interpretive approach to the study of human action, a technique radically different from natural science. The fundamental task for the philosophy of social science has thus been to question the extent to which social research may be characterized as 'scientific' in relation to fundamental epistemological foundations. These debates rage within contemporary social sciences with regard to objectivity, subjectivity, inter-subjectivity, role of the social science researcher, the complexity of the matter, and practicality in the conduct of social research.

\section{Objectivity}

Objectivity is sine qua non of the scientific - method. It means the willingness and ability to examine evidence dispassionately. It is the first condition of research. Objectivity means basing conclusion on facts without any bias and value judgement. The conclusion should be independent of one's personal beliefs, likes, dislikes and hopes. Both the data and the inference drawn from their analysis must be free from bias and prejudices. Objectivity, along with generalization and explanation, are considered as fundamental characteristics of a science. It is often assumed that if our values do not enter into our research, the findings are objective and above criticism. Objectivity is, therefore, defined as the basic conviction 
that there is or must be some permanent, ahistorical matrix or framework to which we can ultimately appeal in determining the nature of rationality, knowledge, truth, reality, goodness, or rightness (Bernstein ,1983). According to Durkheim (1964), the social scientist must study social phenomena in the same state of mind as the physicist, chemist or physiologist when he probes into a still unexplored region of the scientific domain. In order to achieve objectivity, the researcher must be detached from the topic under investigation. The challenge for social researchers is how they can be detached from their studies.

In the early days objectivity was associated with 'hard', experimental, standardizing and quantifying research methods whilst 'soft', interpretative, open and qualitative-descriptive methods have been considered as subjective. Positivist (quantitative) researchers speak of not becoming 'over involved' with participants. Over-identifying with the 'subject' of the research was said to prevent 'good' research. The researcher should be detached and hence objective. This idea that in order to achieve objectivity, a social researcher must be detached from the study was first challenged in American sociology in the 1960s and the critique was taken up in German discussions in the 1970s. In the middle of the 1980s, the debate between positivists and constructivists centred on the problems of validity and generalization of findings obtained with qualitative methods. The fact that a social researcher is part of the human society which he studies is believed to give rise to certain limitations. According to positivists, qualitative social research findings suffer from lack of validity and generalization. However, according to constructivist (qualitative) researchers the idea that 'rigorous research' involves the separation of researchers from the subject of their research was not only a mythical aim, but also an undesirable one which disguises the myriad of ways in which the researcher is affected by the context of the research or the people who are a part of it. If the aim of positivism is to collect and assemble data on the social world from which we can generalize and explain human behaviour through the use of our theories, then it shares with empiricism the belief that there are 'facts' which we can gather on the social world, independently of how people interpret them. Objectivity brings us to the understanding that there is a world out there that we can record and can analyse independently of people's and even our own interpretations of it. Thus objectivity is defined in terms of researchers' detachment from the social world, as well as the accuracy of their data collection instruments. Social researchers, simply need to refine their instruments of data collection in order that they are neutral recording instruments much as the ruler measures distance and the clock, time. Instead of seeing people in the research process as simply sources of data, social science researchers argue that research is a two way process that must employ both quantitative and qualitative methods and is influenced by both objectivity and subjectivity.

\section{Subjectivity}

Contrary to the contentions of positivists that social researchers must be detached from their investigations, Qualitative researchers such as Moustakas (1994) have argued that social researchers, cannot know this world independently of people's interpretations of it. The only thing that we can know with certainty is how people interpret the world around them. The central interest of social researchers, is focused upon people's understandings and interpretations of their social environments, part of which has been termed a 'phenomenological' approach to researching the social world (Moustakas 1994). Social 
research thus becomes more than a reflection of our opinions and prejudices: it substantiates, refutes, organizes or generates our theories and produces evidence which may challenge not only our own beliefs, but also those of society in general.

\section{Social scientist as part of what is studied}

The qualitative researcher does not stand outside or above the study, but is situated within the very processes being studied (Denzin, 2001). Social research reflects, despite the researcher's best intentions, the values and viewpoints of the inquirer and is theory-laden. In social research one cannot escape the reality that the researcher is an instrument that filters data through own paradigms. The researcher will always be subjective and the research intuitive and value laden. In reality, the social researcher is in actual fact a part of the study.

\section{Complexity of the subject matter}

The subject matter of research in social sciences, viz., human society and human behaviour is too complex, varied and changing to yield to the scientific categorization, measurement, analysis and prediction. Yet the actual practice of science shows that there are not only different perspectives on a given phenomenon, but also alternative methods of gathering information and of analysing the resultant data (Williams, 2000). The use of different perspectives in the study of science has led to the development of natural sciences and social sciences. Social sciences, however, does differ from natural sciences, in that people being studied may also actively participate in the study. Unlike objects in nature, humans are selfaware beings who confer sense and purposes on what they do. We can't even describe social life accurately unless we first grasp the concepts that people apply in their behaviour (Giddens, 1997).

\section{Human problems}

A social scientist faces certain human problems, which the natural scientist is spared. These problems are varied and include refusal of respondents, improper understanding of questions by them, their loss of memory, their reluctance to furnish certain information, etc. All these problems cause biases and invalidate the research findings and conclusions. Subjects and clients, as well as investigators, have personal values that are apt to become involved in the research process. One should not assume that these are freely exploitable. The investigator must have respect for the client's values. The quality of research findings depends upon the soundness of decisions made by the social researcher on such crucial stages of his research process as definition of the unit of study, operationalization of concepts, selection of sampling techniques and statistical techniques. Any mistake in any of these decisions will vitiate the validity of his findings.

\section{The historical development of social research theory}

Historically, social research has had to fight a continuous battle for recognition as an empirical process in the study of human activities. In the 19th century, Greek philosophers and theorists advocated for the scientific study of human society. The French philosopher Auguste Comte (19th century) was an important early figure in the development of social 
science theories. He believed society could be studied scientifically and objectively at a time when most societal changes were explained in religious terms. It was during that time that many thinkers developed theories about society, followed later by methodologies for testing theories and developing new ones. Theory helps social scientists make sense of patterns observed in everyday society. It also helps keep researchers from being taken in by patterns that could just be flukes. Theory also helps shape social research and gives it direction. In this way, theory acts as a guide, pointing researchers to the most interesting issues of society, including its politics, economics and other interactions. Finally, theory helps researchers understand social phenomena in such a way that can suggest actions.

\section{Inductive vs. deductive methods}

Deductive reasoning was first described by the ancient Greek philosophers such as Aristotle. The deductive approach refers to the process of "drawing conclusions by applying rules or principles; logically moving from a general rule or principle to a specific solution" (Woolfolk, 2001, p. 286). Deductive methods involve beginning with a general concept or given rule and moving on to a more specific conclusion. Deductive reasoning is the process of reaching a conclusion that is guaranteed to follow, if the evidence provided is true and the reasoning used to reach the conclusion is correct. The conclusion must also be based on the evidence previously provided.

Not everyone from 300 B.C. to 1600 A.D. was willing to bow to the authority of Aristotle. Many scientists believed Aristotle's arguments were faulty. However, after 1600 A.D., it became apparent to several people - Galileo Galilei in Italy, Francis Bacon in England, Tycho Brahe in Denmark, and others - that there were no subtle logical errors in Aristotle's use of the deductive method. The problem was that the deductive method, while wildly successful in mathematics, did not fit well with scientific investigations of nature.

In order to use the deductive method, you need to start with axioms - simple true statements about the way the world works. Then you use these axioms to build your logical system of nature. If your axioms are true, everything that follows will be true, but Galileo and his contemporaries realized that the problem was that it was enormously difficult to determine "simple true statements about the way the world works". Although, the deductive approach allowed researchers to move from the general assumption to the specific application, it was not fruitful in arriving at new truth.

However, during the Middle Ages, the quest for knowledge led critical thinkers such as Francis Bacon, to challenge the authority of the religious dogma that dominated the search for truth. The acceptance of incomplete or false major premises that were based on old dogmas or unreliable authority was critically challenged. Bacon also recognized the obstacle that the deductive process placed in the way of discovering new truths and advocated direct observation of phenomena, arriving at conclusions or generalisations through the evidence of many individual observations. This was a reverse approach from the deductive process. Thus it was referred to as the inductive process. Since 1600, the inductive method has been incredibly successful in investigating nature - surely far more successful than its originators could have imagined. The inductive method of investigation has become so entrenched in science that it is often referred to as the "scientific method". The inductive method (usually called the scientific method) is the deductive method "turned upside down". 
The deductive method starts with a few true statements (axioms) with the goal of proving many true statements (theorems) that logically follow from them. The inductive method starts with many observations of nature, with the goal of finding a few, powerful statements about how nature works (laws and theories). In the deductive method, logic is the authority. If a statement follows logically from the axioms of the system, it must be true. In the inductive (scientific method), observation of nature is the authority. If an idea conflicts with what happens in nature, the idea must be changed or abandoned.

This reliance on empirical evidence or personal experience was an important step in the direction of scientific inquiry. The deductive method of Aristotle and the inductive method of Bacon gave birth to two research paradigms - positivism and constructivism. Mertens (2005) argues that Aristotle's logical deductive approach to research heavily influenced the positivist (quantitative) paradigm and Bacon's inductive approach has been associated with the constructivist (qualitative) paradigm. Charles Darwin's idea of the integrated deductive - inductive process has led to the support for the pragmatic paradigm (the quantitative qualitative approach). The earliest attempt to integrate the deductive - inductive methods was made by Charles Darwin in the nineteenth century. However since the time of Charles Darwin social researchers and scholars have been engaged in a polemic debate about the methodological appropriateness of each of these three approaches to social research.

\section{The research paradigm debate}

A research paradigm is a way of looking at the world. It is an assemblage usually comprising the researcher's ontology, epistemology, methodology and axiology (Moore, 2007). It is composed of certain philosophical assumptions that guide and direct thinking and action of the researcher. Researchers such as Lather (1992) and Lincoln and Guba (2000), have identified three major paradigms that have influenced social research: Positivism, Constructivist, and Pragmatism. The present discussion focuses on how these three paradigms have influenced the development of social research theory and social research methods. According to Guba (1978), most research work is guided by either positivism (quantitative) research paradigm or constructivism (qualitative) research paradigm or a mixture of the two paradigms (pragmatism). Each approach represents a fundamentally different inquiry, and researcher actions are based on the underlying assumptions of each paradigm (Patton, 1990).

\section{Positivism}

This was the dominant paradigm that guided early social research. It is commonly referred to as the quantitative paradigm. Positivism is based on the rationalistic, empiricist philosophy that originated with the deductive approach of Aristotle, John Lock, August Compte, and Emmanuel Kant (Mertens, 2005). The deductive method argues that knowledge must proceed from the general to the specific. Under a deductive methodology, a researcher begins with a hypothesis, then makes observations or collects data to test that hypothesis. Based on empirical evidence from the study, the researcher then decides whether to accept or reject the hypothesis. The deductive methodology, in short, tests theories and hypotheses. Positivism assumes that the social world can be studied in the same way as the natural world and there is a method for studying the social world that is 
value free and that explanations of a causal nature can be provided and events or observations are influenced by a single theory or paradigm. The adoption of a positivist perspective is accompanied by a broad commitment to the idea that the social sciences should emulate the natural sciences (Lee 1989). Epistemologically, positivist studies are premised on the existence of a priori fixed relationships within phenomena capable of being identified and tested via hypothetic-deductive logic and analysis. Causal relationships, which are the basis for generalized knowledge, can predict patterns of behaviour across situations. Ontologically, positivist research assumes an objective physical and social world that exists independently of humans. The researcher is seen to play a passive, neutral role, and does not intervene in the phenomenon of interest. Keeping in spirit with this set of beliefs, a theoretically grounded positivist study is likely to be conducted with the ideas of establishing appropriate measures (qualitative and/or quantitative) for the constructs being studied; establishing or testing causal relationships; determining the domain to which the study's findings can be generalized; and demonstrating that the inquiry is value free. The criteria for judging the quality of such positivist studies, in opposition to interpretive and critical case studies, are related to the traditional validity and reliability tests used in the natural sciences (Yin 1994). Specifically, the primary criteria for classifying a theoreticallygrounded article as positivist include the following:

- $\quad$ adoption of a positivist perspective clearly stated in the study,

- $\quad$ evidence of formal research hypotheses or propositions

- evidence of qualitative and/or quantitative measures of variables or constructs

- $\quad$ explicit purpose of theory testing or theory building

- $\quad$ concern for validity and reliability issues as used in the natural sciences

The positivist perspective has long been associated with "scientism" the view that the methods of the natural sciences may be applied to all areas of investigation, be it philosophical, social scientific, or otherwise. Positivist (quantitative) research reflects the traditional scientific approach to problem solving. It uses experimental methods and numerical data to test hypothetical generalizations. It assumes that there is a single reality that can be broken down into variables. The purpose of this type of research is to test hypotheses that have been developed before the research project started and to form conclusions that can be generalized to other situations. The emphasis in this approach is upon measurement, comparison, and objectivity. Proponents of the quantitative paradigm include Dodds, Lawrence, \& Guiton, 1984); Kember \& Dekers, 1987); Osman \& Wagner, 1987). These researchers maintain that quantitative methods are inherently preferable to non-quantitative methods. However, among most social scientists and historians, orthodox positivism has long since fallen out of favour. Today, practitioners of both social and physical sciences recognize the distorting effect of observer bias and structural limitations.

\section{Constructivism}

This paradigm can be traced to the inductive approach propounded by Charles Bacon. The inductive method, in contrast with the deductive method, goes from the specific to the general. Under this methodology, social scientists observe social phenomena, identify patterns and then analyze them to reach broad conclusions and develop new theories, based on research findings. The proponents of constructivist paradigm were Campbell and Stanley $(1963,1966)$, 
Cook and Campbell (1979). According to Cook and Campbell (1979), constructivist paradigm rejects the position that social events or observations are influenced by a single theory or paradigm. The basic assumptions guiding the constructivist paradigm are that knowledge is socially constructed by people active in the research process and that researchers should attempt to understand the complex world of lived experiences from the point of view of those who live it (Schwandt 2000). Smith and Manning (1982) suggest that qualitative research is better for initial exploratory research into unknown or unfamiliar phenomena. It enables the researcher to develop concepts, conjectures, interpretations and theories empirically grounded in the investigated systems. In the real world, events cannot be teased out from the context in which they are inextricably embedded, and understanding involves the relationships among all of the many parts of the whole. Thus qualitative methods - which emphasize both inner and outer knowledge of man in his world- are preferable (Filstead, 1970).

The constructivist paradigm emphasizes that research is the product of the researchers and cannot be independent of them. (Mertens, 2005). The most obvious distinction between quantitative and qualitative research is the form of data collection and presentation. The fundamental difference between constructivism and positivism, however, lies in the realm of theory. Data within positivism is theory-driven and designed to test the accuracy of the theory. Constructivism, on the other hand, is a method of research which has not referred explicitly to the theory guiding its data collection procedures. Theory is grounded in the study and develops as the study is conducted. Lincoln and Guba (2000) identify qualitative methods as the preferred methods for researchers working in the constructivist paradigm.

\section{Pragmatism}

So far we have discussed two main research paradigms: positivism (quantitative) and constructivism (qualitative) paradigms. Pragmatism is a paradigm that provides the underlying philosophical framework for mixed methods research (Maxcy, 2003; Patton, 2002; Tashakkori and Teddlie, 2003). This approach was first proposed by Charles Darwin. This research design involves methods that use mixed data (numbers and text) and additional means (statistics and text analysis). A mixed method uses both deductive and inductive scientific methods, has multiple forms of data collecting and produces eclectic and pragmatic reports. Pragmatic philosophers (Maxcy, 2003; Patton, 2002; Tashakkori and Teddlie, 2003) have rejected the scientific notion that social science enquiry was able to access the truth about the real world solely by virtue of a single scientific method. To this end, the most appropriate paradigm is the mixed research approach in which the researcher is not confined to one research method. A mixed research design is a general type of research that includes quantitative and qualitative research data, techniques and methods. All these paradigm characteristics are mixed in one case study. According to Mertens (2005) mixed methods can be applied at four different levels: problem identification, data collection, data analysis, and discussion of research findings. Greene and Caracelli (2002) argue that social researchers mix methods to a varying degree at various points in their research and still call their work mixed method research. However ZOU undergraduate researchers called their research work qualitative.

Two main types of a mixed method paradigm are: (1) mixed research method, and (2) mixed model design. A mixed research method is a research in which you use quantitative data for one stage of a research study and qualitative data for a second stage of a research. A mixed 
model design is a research in which you use both quantitative and qualitative data in one or two stages of the research process. The mixing of quantitative and qualitative approaches may happen in every stage of the research. The advantages of a mixed methods paradigm are:

1. The strength of the research;

2. Use of multiple methods in a research helps to research a process or a problem from all sides;

3. Usage of different approaches helps to focus on a single process and confirms the data accuracy. A mixed research complements a result from one type of research with another one. This research does not miss any available data.

A quantitative component of a mixed research assumes the usage of deductive scientific method while qualitative component assumes inductive scientific method. Moreover, a quantitative approach collects quantitative data based on exact measurement applying structured as well as validated information collection. For instance, rating scales, closedended items and responses. This approach produces statistical reports with correlations.

A qualitative component uses qualitative information from interviews, field notes, openended questions etc. This approach considers a researcher to be the major means of information collection. At the end of a research this approach supposes a narrative report with context description, quotations taken from research material. The aim of a mixed method design is to summarize positive aspects of two approaches and produce a highly accurate data. Bryman (2001) argues for genuine integration, where qualitative and quantitative findings are mutually informative and talk to each other - a conversation - to construct a negotiated account. The triangulation metaphor has been a hindering factor; it should not be just a matter of testing data against each other, but about forging an overall or negotiated account of the findings that brings together both components of the conversation or debate.

\section{Issues and emerging trends in social research: The case for Zimbabwe Open University}

The present discussion makes reference to social research work by lecturers and students at the Zimbabwe Open University.

\section{The Zimbabwe Open University: Background information}

The Zimbabwe Open University (ZOU) is the largest state funded university, in Zimbabwe, established on $1^{\text {st }}$ March 1999, through an Act of Parliament (Chapter 25:20), to cater for a substantial component of people who, by design or unintentionally, could not be accommodated in conventional universities, by offering them the opportunity to study in their homes and at their workplaces through distance education. During the time of this study (2005 - 2011, ZOU had approximately 18700 students, 1795 academic staff and 395 non - academic staff, four faculties, (1) the faculty of Arts and Education, (2) the faculty of Science, (3) the faculty of Commerce and Law and (4) the faculty of Applied Social Sciences, offering approximately 50 undergraduate degree programmes, 5 diploma courses, 10 masters' degrees and 5 doctoral degrees. 


\section{The research dissertation: Definition and structure}

A dissertation is a research project undertaken by post graduate students in partial fulfilment of their master programmes. A research project is a purposeful collection of student work that exhibits to the student and others, the student's efforts, progress, or achievement in a given area (Reckase, 1995). The major goal of the research project is to assess the ability of students to apply knowledge to solve real-life problems. The dissertation is designed by the student in consultation with a tutor (project supervisor) under the overall guidance of the Regional Programme Coordinator (co-supervisor). Basically, there are two types of research projects structured and unstructured. For a structured project, students are given topics to work on, what to look for and guidelines to follow. All Master programmes at the ZOU, require students to undertake unstructured research projects. The student identifies a research topic and works through the project with minimum guidance from the research supervisor. Unstructured research projects are self-directed and should be organized into five chapters involving a number of activities as illustrated in Table 1.

\begin{tabular}{|c|ll|}
\hline Chapter & Activities \\
\hline 1 & - & specifying the research problem \\
& - & formulating the research topic or title \\
& - & presenting background of the study \\
& - & presenting statement of the problem \\
& - & formulating hypotheses or research questions \\
& - & discussing the significance of the study \\
& - & stating delimitations and limitations \\
& - & defining key terms \\
\hline 2 & - & outlining organization of the study \\
& - & Reviewing literature \\
& - & Explaining constructs or key concepts \\
\hline 3 & - & Analysing previous research studies \\
\hline 4 & - & designing the research: creating, adopting or adapting a research design \\
& - & describing the population, sample and sampling techniques \\
\hline 5 & - & collecting data \\
\hline & - & analyzing and presenting data \\
& - & discussing research findings \\
\hline & - & drawmarizing major findings of the study \\
& - & making recommendusions \\
& - & compiling references \\
\hline
\end{tabular}

Table 1. The Dissertation Process, Chapters and Activities.

The dissertation is undertaken in the third and final year of the masters programme. Students are expected to work with their supervisors throughout the research project and to submit a typed dissertation report of approximately $15000-20000$ words to their 
Department one month before the end of their programme. Before embarking on their dissertation, the research topic must be approved by the project supervisor.

\section{Research design and methodology}

The present study adopted the mixed methodology research paradigm involving quantitative techniques in the conceptual analysis and qualitative techniques in the relational analysis of the dissertations. We employed the model developed by Caleb Kangai, Richard Bukalia, Farirai Musika and Barbra Mapuranga in an earlier study in 2011 that focused on research projects submitted by B. Ed students.

There are two general categories of content analysis: conceptual analysis and relational analysis. Both types of content analysis were applied. Conceptual analysis focused on establishing the existence and frequency of certain concepts in the dissertations. In this study conceptual analysis was undertaken by using open coding. Open coding is the part of analysis that pertains specifically to naming and categorizing phenomena through close examination of data (Mertens, 2005). In order to come up with a checklist of concepts and codes, the four researchers initially analysed 10 dissertations. Our analysis produced a checklist of six subcomponents of the dissertation: research questions, data form, research paradigm, research design, data collection instruments, procedures for data analysis and procedures for presentation and discussion of research findings. These concepts were categorized and coded as presented in Table 2.

\begin{tabular}{|l|l|l|}
\hline Concept & Category & Code \\
\hline Research Questions & Descriptive & D \\
& Explanatory & E1 \\
& Exploratory & E2 \\
\hline Data Form & Numbers & N \\
& Words & W \\
\hline Literature Review & Concepts/constructs & CS \\
& Context of the study & CX \\
& Theory & T \\
& Previous Research Studies & \\
\hline Research Paradigm & Positivist (Quantitative) & P \\
& Constructivist (Qualitative) & C \\
& Pragmatic (Quantitative - Qualitative) & P(Q-Q) \\
\hline Research Design & Experimental & E \\
& Non-Experimental & NE \\
\hline $\begin{array}{l}\text { Data collection } \\
\text { instruments }\end{array}$ & Questionnaires & Q \\
& Interviews & I \\
& Observations & O \\
& Tests & T \\
& Documents / records & D/R \\
\hline Presentation and \\
discussion of findings
\end{tabular}

Table 2. Checklist of Concepts, Categories and Codes. 


\section{Reliability of the methods}

We checked the reliability of the coding a ( $90 \%$ agreement is suggested; .8 for Cohen's kappa). To establish the dependability of the categorization decisions, we worked in pairs. First one researcher coded all the items and the second researcher independently coded a $10 \%$ random sample of those items. The proportion of simple agreement between the coders was 0.95 .

To conduct relational analysis of the dissertations we adopted and adapted the relational model developed by Kangai, Bukalia, Mapuranga and Musika (2011) in an earlier study (see figure 1).

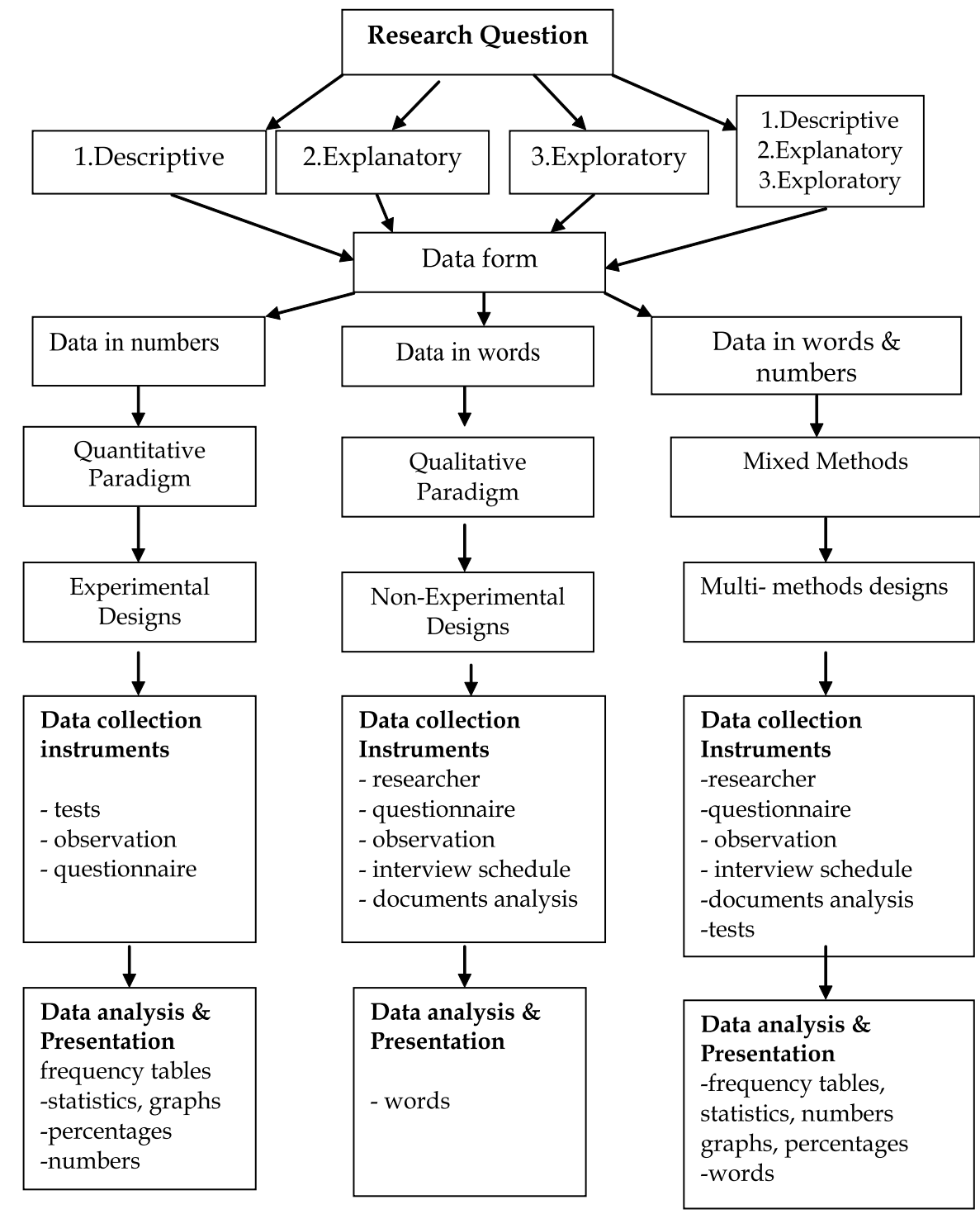

Fig. 1. Relational Analysis Model for Post - Graduate Dissertations Adapted from Kangai, Bukaliya, Musika and Mapuranga (2011). 
The primary purpose of this analysis was to establish whether the dissertations submitted by post-graduate students met and satisfied the scientific enquiry research process. Although there is no universally accepted format for the research process, most studies adhere to the general sequence of scientific enquiry. Scientific enquiry is a sequential research process that is based on recognized methods in data collection, analysis and interpretation. The typical steps in the scientific enquiry are (1) Define a problem (2) state the hypotheses to be tested. (3) collect and analyse data (4) interpret the results and draw conclusions about the problem (McMillan and Schumacher, 1993). Different universities and even faculties in the same university may adopt adapt this process to suit their local conditions and needs. Students' dissertations at the ZOU are structured following eight stages. We analysed each of these subcomponents of the dissertation in order to establish the extent to which each was related to the other parts.

1. Define the research problem

2. State research questions/ Hypotheses

3. Review related literature

4. Describe and explain the research paradigm and design

5. Describe data collection instruments and procedures

6. Describe data analysis techniques and procedures

7. Data presentation and discussion of findings

8. References

\section{Sample}

Data for the current study was collected from a stratified random sample of 100 dissertations, which were submitted by ZOU postgraduate students between 2005-2010. The 100 dissertations were distributed as follows (See table 3).

\begin{tabular}{|c|c|c|c|c|c|c|c|}
\hline Degree Programme & Dissertation & $\mathbf{2 0 0 5}$ & $\mathbf{2 0 0 6}$ & $\mathbf{2 0 0 7}$ & $\mathbf{2 0 0 8}$ & $\mathbf{2 0 0 9}$ & $\mathbf{2 0 1 0}$ \\
\hline $\begin{array}{c}\text { Master of Business } \\
\text { Administration (MBA) }\end{array}$ & $\mathbf{5 0}$ & 10 & 10 & 10 & 10 & 10 & 10 \\
\hline Master of Education (M.Ed) & $\mathbf{5 0}$ & 10 & 10 & 10 & 10 & 10 & 10 \\
\hline $\begin{array}{c}\text { Master of Science Special Education } \\
\text { (MSc- SPED) }\end{array}$ & $\mathbf{5 0}$ & 10 & 10 & 10 & 10 & 10 & 10 \\
\hline Master of Philosophy (MPhil) & $\mathbf{5 0}$ & 10 & 10 & 10 & 10 & 10 & 10 \\
\hline Total & $\mathbf{2 0 0}$ & $\mathbf{4 0}$ & $\mathbf{4 0}$ & $\mathbf{4 0}$ & $\mathbf{4 0}$ & $\mathbf{4 0}$ & $\mathbf{4 0}$ \\
\hline
\end{tabular}

Table 3. Stratified Random Sample of Post- graduate Dissertations content analysed for the current study.

\section{Conceptual analysis results}

\subsection{Dominant research questions and hypotheses}

The first concept we analysed was the research questions or hypotheses that guided each study. 


\begin{tabular}{|l|c|c|c|c|c|c|c|c|}
\hline \multirow{2}{*}{$\begin{array}{l}\text { Degree } \\
\text { Programmes }\end{array}$} & \multicolumn{8}{|c|}{ RESEARCH QUESTIONS } \\
\cline { 2 - 9 } & \multicolumn{2}{|c|}{ Descriptive } & \multicolumn{2}{|c|}{ Explanatory } & \multicolumn{2}{c|}{ Exploratory } & \multicolumn{2}{c|}{ Hypothesis } \\
\cline { 2 - 9 } & $\mathbf{N}$ & $\%$ & $\mathbf{N}$ & $\%$ & $\mathbf{N}$ & $\%$ & $\mathbf{N}$ & $\%$ \\
\hline MBA (50) & 15 & $\mathbf{3 0}$ & 6 & $\mathbf{1 2}$ & 23 & $\mathbf{4 6}$ & 6 & $\mathbf{1 2}$ \\
\hline M.ED (50) & 14 & $\mathbf{3 6}$ & 8 & $\mathbf{1 6}$ & 24 & $\mathbf{4 0}$ & 4 & $\mathbf{8}$ \\
\hline MSC.-SPED (50) & 16 & $\mathbf{3 2}$ & 5 & $\mathbf{1 0}$ & 26 & $\mathbf{5 2}$ & 3 & $\mathbf{6}$ \\
\hline MPHIL (50) & 13 & $\mathbf{3 2}$ & 9 & $\mathbf{1 8}$ & 23 & $\mathbf{4 0}$ & 5 & $\mathbf{1 0}$ \\
\hline Totals (200) & $\mathbf{5 8}$ & $\mathbf{2 9} \%$ & $\mathbf{2 8}$ & $\mathbf{1 4} \%$ & $\mathbf{9 6}$ & $\mathbf{4 8} \%$ & $\mathbf{1 8}$ & $\mathbf{9 \%}$ \\
\hline
\end{tabular}

Table 4. Research questions most preferred by ZOU Post- graduate students.

Our findings indicate that most of the dissertations 182 (91 percent) employed research questions rather than hypotheses. Defining the research questions represents one of the most important steps to be taken in any empirical study (Benbasat et al. 1987; Eisenhardt, 1989; Mays and Pope, 1995; Miles and Huberman, 1994). Most of the reviewed dissertations (79 percent) specified clear research questions, 12 percent of the questions were not very clear, and 9 percent did not state research questions. The fact that 79 percent of the analysed dissertations were guided by very clear research questions is quite encouraging since a clear research question expresses the essence of an inquiry, allows one to easily link a study to its practical and theoretical contributions, and is the backbone of a solid research design (Mason, 1996). Exploratory questions (48 \%) dominated in the dissertations compared to descriptive (29 percent). Yin (1994) explains that social research is most likely to be appropriate for "how" and "why" questions because these deal with operational links needing to be traced over time, rather than mere frequencies of incidence. In the present study, explorative research questions, what questions were the most frequent followed by how and why questions. Research studies which address a what question are most appropriate when the purpose of the study is to explore a new phenomenon (Yin 1994). Our findings support this contention. Indeed, what questions were most frequently posed in exploratory and descriptive studies. It is interesting to note that whilst descriptive questions dominated in research projects submitted by ZOU undergraduate students, (Kangai, Bukalia, Musika and Mapuranga, 2011), exploratory questions were more dominant in post graduate dissertations, although the shift is not very significant. Amongst post graduate students, descriptive research questions are still very popular. This finding agrees with Benbasat I. and Weber R. (1996) who argue that a descriptive study is less demanding than an exploratory or an explanatory one. For descriptive studies, little theory is said to be needed, causal links do not have to be made, and analysis is minimal. In a dominant research question used by ZOU undergraduate student was the questionnaire.

\section{Context of the study}

The second concept we examined was the context of the study. Placing the study in its theoretical context can be bewildering especially to beginning researchers. Beginning researchers often have the perplexing task of building a convincing argument in support of their current research. This is done by presenting background of the study. The background of the study starts by identifying the research problem and relating it to the prevailing theory. The background highlights the importance of the problem and explains why it is a 
research problem in its present context. The context of the problem must identify the knowledge gap the present study aims to fill. The knowledge gap refers to the inadequacies of the existing theories in explaining the current research problem. Failure by findings of previous studies in explaining the present research problem also reflects the knowledge gap. The knowledge gap indicates a mismatch between the general theory and phenomenon under study.

A detailed description of the research context is necessary to assess the credibility of the research results and to determine their generalizability (Benbasat et al. 1987; Yin 1994). Several aspects of the research context are important. The first relates to the setting: a detailed account describing where the research was conducted and the specific period of time under investigation. Other key aspects are related to the moment data was collected in relation to the time the events occurred, whether there were one or more data collection periods, whether the researcher was able to gain sufficient access and spend enough time to develop an intimate understanding of the setting and the phenomenon of interest, and whether the researcher collected data during the course of the events (on-going) or $a$ posteriori.

According to the present study, 26 percent of the dissertations clearly presented the context of the study, 57 percent were not very explicit about the context surrounding their research and 17 percent failed to place their studies in the appropriate conceptual and theoretical framework. First, we found that while most social researchers (98 percent) described to some extent where their research was conducted (site description), only 32 percent stated the specific period of time the study was conducted. Providing the latter information is important since the period defines the frame of reference under which phenomena are investigated (Pettigrew, 1989). Findings from social research are often time-bound and potentially confounded with time (Laudon 1989). The physical and social environment of a phenomenon is dynamic. Social values, attitudes, beliefs, customs, and norms change with time. Thus the time the study was undertaken is very important for the purposes of credibility, validity and generalization of the findings. Finally, we noted that only 19 percent of the articles reported how much time the researcher (s) had spent on the study, while 32 percent of the analysed dissertations provided no information about the nature of the data being collected.

\section{Review of related literature}

The third concept we analysed was the link between the study and prevailing literature. Prior theorizing constitutes an essential input in social research design (Lee, 1989; Yin, 1994). Markus, 1983). Literature review, besides reflecting important theoretical issues, also begin to tell researchers where to look for relevant evidence. Hence, positivist social research presumes that the theory of interest is stated explicitly in the first place and that predictions following from the theory are also explicitly stated (Lee 1989). However, in qualitative social research, the researcher may begin with no theory since the theory will develop from the study. Findings of the present study are encouraging with regard to these two desired attributes. Indeed, almost all of the 200 dissertations (76 percent) reviewed in this study stated explicitly the theory of interest while 87 percent stated clearly the various predictions deducted from the theory itself. As discussed above, 
another valuable approach for social research is the consideration of rival theories (propositions) and the analysis of the evidence in terms of such rivals (Lee 1989; Yin 1994). Our finding on review of rival theories was disappointing as only 24 percent of all dissertations considered rival theories in their literature review. A review of rival theories will enable the researcher to identify the knowledge gap in the theory of interest. The knowledge gap refers to the inadequacies of the existing theory in explaining the current research problem. This means that students failed to present the scholarly debate surrounding their area of study.

The fourth concept for analysis was the research design. The results of our analysis are presented in table.

\begin{tabular}{|l|c|c|}
\hline Research Paradigm & Number & Percentage \\
\hline Quantitative Research & 14 & $7 \%$ \\
Qualitative Research & 40 & $20 \%$ \\
Mixed methods (Quantitative - Qualitative) Research & 126 & $63 \%$ \\
No Mention of Research Paradigm & 10 & $5 \%$ \\
\hline
\end{tabular}

Table 5. The Dominant Research Paradigm. N= 200.

Although Patton (2002) notes that the choice of qualitative methods might be appropriate in many social research studies because they are based on humanistic values. The present study shows that there is a trend towards the use of mixed methods as 63 percent of all the dissertations we reviewed employed quantitative and qualitative methods.

Another interesting finding was that $5 \%$ of the projects did not identify a paradigm. Leaders in the field of research do not all agree as to the need to acknowledge an underlying paradigm, nor do they agree on the role that such paradigms serve in the research process. Whilst Patton (2002) says research paradigms are unnecessary and possibly handicapping, Schwandt, (2000), argues that research paradigms are inescapable. The need for a paradigm is also supported by Mertens (2005), who maintains that a researcher's theoretical orientation (paradigm) has implications for every decision made in the research process, including choice of methods. Skrtic (1991), argues that the absence of a research paradigm, renders the research atheoretical since it rests on an unexamined and unrecognized theory. In an earlier content analysis of research project submitted by ZOU undergraduate students, Kangai, Bukalia, Musika and Mapuranga (2011) found that 22 percent of the students did not mention the research paradigm that influenced their studies. Our results indicate an improvement in post graduate research studies. Our findings agree with Patton (1999) and Lincoln and Guba (2000) who note that many changes have occurred in the status of paradigms and choice of methods over the last decade such that various paradigms are beginning to "interbreed".

\section{The dominant research design}

The fifth concept we analysed was the research design. We based our analysis on Borg and Gall's classification of research questions. According to Borg and Gall (1989) there are two categories of research questions- descriptive and relational. Descriptive questions lead to non- experimental designs and relational questions lead to experimental designs and the use 
of both leads to mixed methods. We, therefore, identified and classified research designs as experimental, non-experimental or mixed methods (see Table 6).

\begin{tabular}{|l|c|c|}
\hline Research Design & Number & Percentage \\
\hline Experimental & 2 & $1 \%$ \\
Non - Experimental & 178 & $89 \%$ \\
Mixed Methods & 20 & $10 \%$ \\
\hline
\end{tabular}

Table 6. The Dominant Research Design in ZOU Postgraduate Dissertations.

Our results show that 178 (89 percent) of post-graduate dissertations employed non experimental research designs. Only 20 (10 percent) of the dissertations used mixed methods and 2 (1 percent) used experimental designs. Our findings agree with McMillan \& Schumacher (1993), Borg and Gall (1996), Mertens (2005)and (Kangai, Bukalia, Musika and Mapuranga, 2011) who have noted that descriptive surveys are the most basic of methods, popular and used pervasively in social research. Descriptive surveys are mainly used to describe peoples' characteristics such as their attitudes, beliefs, habits, values, demographics, behaviours, opinions, desires, ideas, and other types of information and do not help much in developing theory or testing theory. We have argued elsewhere (Kangai, Bukalia, Musika and Mapuranga, 2011) that the confinement of students' research projects to one or two research designs is a function of the lack of theoretical foundations in research methodology.

\section{Data collection methods}

The sixth concept we analysed involved data collection instruments. A major strength of mixed methods data collection is the opportunity to use many different sources of evidence to provide a richer picture of the events and/or issues than would any single method (Sawyer 2001; Yin 1994). A multi-method approach to research involves several data collection techniques, such as interviews and observations organized to provide multiple but dissimilar data sets regarding the same phenomena (Mingers, 2001). As shown in Table 5 , most dissertations (62.5 percent) employed multi-methods followed by the questionnaire (21 percent) and interview (16.5 percent).

\begin{tabular}{|l|c|c|}
\hline Method & Frequency of use & Percentage \\
\hline Questionnaire & 42 & $21 \%$ \\
Interview & 33 & $16.5 \%$ \\
Observation & 0 & $0 \%$ \\
Documentation & 0 & $0 \%$ \\
Tests and Measurements & 0 & $0 \%$ \\
Multi-methods & 125 & $62.5 \%$ \\
\hline Total & $\mathbf{2 0 0}$ & $\mathbf{1 0 0} \%$ \\
\hline
\end{tabular}

Table 7. Data collection instruments mostly preferred by ZOU Post-graduate research students. 
This finding is consistent with the research paradigm most preferred by the students. In addition to using multiple data collection methods, the specific use of a combination of quantitative (e.g., questionnaires) and qualitative (e.g., interviews) data sources is often advocated (Reichardt and Cook, 1978; Kaplan and Duchon, 1988 and Patton, 1999). As Eisenhardt (1989) stressed, quantitative data can keep researchers from being carried away by vivid, but false, impressions in qualitative data, and it can bolster findings when it corroborates those findings from qualitative evidence. The use of both qualitative and quantitative methods of data collection is supported by Kirsch and Cummings (1996) who argue that explanatory and exploratory social research tend to rely more heavily on both multiple data collection methods and a mix of qualitative and quantitative data than do descriptive ones.

Our findings point to an emerging positive trend in the use of mixed methods as compared to other previous content analysis studies. For example, Mingers (2001) observed that about half of the studies included in their sample used multiple data collection approaches. Alan Bryman (2007) also did a content analysis of 232 articles, published between 1994 and 2003, with 'mixed' research methods. He found that only $18 \%$ of the articles succeeded to mix (integrate) qualitative and quantitative methods. He remarks (p. 8):

As a result of the efforts of methodologists and researchers, there is nowadays considerable understanding of a variety of issues, such a various ways in which quantitative and qualitative research can be mixed... However, at the same time, the fundamental issue of the degree to which mixed methods researchers genuinely integrate their findings has not been addressed to a significant extent. In other words, how far do mixed methods researchers analyze, interpret, and write up their research in such a way that the qualitative and quantitative components are mutually illuminating? "genuinely integrate".

The present finding represents an improvement in social research methodology over the past decade since 62.5 of our sample effectively employed mixed methods.

\section{Data triangulation}

The most important advantage of using multiple sources of evidence is the development of converging lines of inquiry (Patton, 1999; Yin 1999). The process of combining multiple data sources is called triangulation (Jick, 1979). Any finding or conclusion in social research is likely to be much more convincing and accurate if it is based on several different sources of information. However, our findings are disappointing in that fewer than one-third (30 percent) of the studies just mentioned using some form of data triangulation but did not elucidate how this was done and how the triangulation contributed to the validity and reliability of the findings. These findings are once again in line with those of Kangai $\mathrm{C}$., Bukalia, R., Musika F. and Mapuranga, B. (2011) who showed that triangulation was rarely used by ZOU undergraduate research students.

\section{Elucidation of the data collection process}

Data Collection Process the seventh concept to be analysed. In addition to mentioning methods of data collection, it was quite encouraging to observe that more than 58 percent of all dissertations in our sample did elucidate how data was collected. This represents a very 
important aspect of social research, since a clear description of the data sources and how data was collected enhances the reliability and validity of the findings (Benbasat, I. Goldstein, D. K. and Mead, M., 1987). However, the fact that 42 percent of the studies did not clearly elucidate how data was collected, is a course for concern. In social research, the reader must be able to tell what sources of information were used and how data was collected in order to judge the reliability of the information. The omission of such information undermines the credibility of social research studies.

\section{Elucidation of the data analysis process}

The eighth concept to be examined was the data analysis process. As Eisenhardt (1989) stressed, analyzing data is "both the most difficult and the least codified part of the process". It was, therefore, important to first assess the extent to which social researchers elucidated the data analysis procedures. In order to do so, we classified each article in the database as providing either "no information," "a brief description," or "a clear and detailed description" of the analytic procedures and/or rules followed.

Our findings show that a large number of dissertations (70 percent) did not explain how data was analysed, while 22 percent provided a brief description of the data analysis process, and 8 percent provided a clear and detailed description of analytic procedures. For example one researcher wrote, "Data collected was quantitatively analysed in tables". This represents another serious shortcoming since a clear description of the analytic procedures allows other readers to better understand the findings. In addition, since positivism considers qualitative data to be vulnerable to subjective interpretation and to surpass human ability to compile, a clear description of the data analysis process allows us to judge whether or not the results are the fruit of a systematic and rigorous process.

Our findings reveal that social researchers at the ZOU did not provide much information about the adoption of preliminary data analysis techniques. This represents another serious deficiency since technical rigor in analysis is a major factor in the credibility of qualitative findings (Patton 1999). Social research data can be analysed as it is being collected. Overlapping data collection with data analysis not only gives the researcher a head start in analysis but, more importantly, allows researchers to take advantage of flexible data collection. Indeed, a key feature of qualitative research, in general, and research, in particular, is the freedom to make adjustments during the data collection process.

\section{Discussion of findings}

The ninth and final concept we analysed was the discussion of research findings. Discussion of research findings must demonstrate a logical chain of evidence. To increase the reliability of the information presented in a case study, a key principle to be followed is the maintenance of a logical chain of evidence (Benbasat et al. 1987; Yin 1994). As Yin (1994) explained, the principle is to allow an external reviewer or observer to follow the derivation of any evidence from initial research questions to ultimate study conclusions. Furthermore, the observer should be able to trace the steps in either direction (from conclusions back to initial research questions or from questions to conclusions). The process should be tight enough so that evidence presented in the project or dissertation report is assuredly the same evidence that was collected during the data collection process. When this is achieved, social 
research has addressed the methodological problem of determining internal validity. In order to assess whether the authors of the dissertations we analysed had maintained a chain of evidence, we used the relational model (see figure ) to evaluated the extent to which we were able to move from one portion of the study to another, with minimal cross-referencing to methodological procedures and to the resulting evidence. Hence, for all of the articles included in our sample, we were able to trace the steps from initial research questions to conclusions in only 44 percent of them.

\section{Comparison with related literature}

When building theories from social research, it is of utmost importance to compare the emergent concepts, theories or hypotheses with the extant literature. Examining literature that conflicts with the emergent theory is likely to enhance confidence in the findings of the study (Eisenhardt, 1989). Literature discussing similar findings is important as well because it ties together underlying similarities in phenomena normally not associated with each other. The result is often a theory with stronger internal validity, wider generalizations and higher conceptual level. Notwithstanding the potential benefits associated with such a tactic, Table 8 reveals that only 11 percent of all exploratory case studies related their findings to conflicting literature while 37 percent of them discussed similar literature. In short, tying the emergent constructs, hypotheses, or theories to extant literature is crucial in theory building since the results usually rest on a small number of cases.

\section{Summary of key findings}

Table presents a synthesis of the key findings and of the present content analysis of social research methodologies applied by ZOU post-graduate students.

\begin{tabular}{|c|c|c|c|c|c|c|}
\hline CONCEPT & \multicolumn{6}{|c|}{ STATUS } \\
\hline $\begin{array}{ll}\text { 1. } & \begin{array}{l}\text { Research } \\
\text { questions }\end{array}\end{array}$ & Clear & $79 \%$ & Not clear & $12 \%$ & Not stated & $9 \%$ \\
\hline $\begin{array}{ll}\text { 2. } & \begin{array}{l}\text { Dominant } \\
\text { research } \\
\text { question }\end{array} \\
\end{array}$ & Exploratory & $48 \%$ & Descriptive & $29 \%$ & Explanatory & $14 \%$ \\
\hline $\begin{array}{ll}\text { 3. } & \text { Literature } \\
\text { review }\end{array}$ & $\begin{array}{c}\text { Analysed } \\
\text { theory of } \\
\text { interest }\end{array}$ & $67 \%$ & $\begin{array}{c}\text { Analysed } \\
\text { rival theories }\end{array}$ & $24 \%$ & $\begin{array}{c}\text { Analysed } \\
\text { unrelated } \\
\text { theories }\end{array}$ & $9 \%$ \\
\hline $\begin{array}{l}\text { 4. Context of } \\
\text { the study }\end{array}$ & $\begin{array}{c}\text { Clearly } \\
\text { presented }\end{array}$ & $26 \%$ & $\begin{array}{c}\text { Poorly } \\
\text { presented }\end{array}$ & $57 \%$ & $\begin{array}{c}\text { Not } \\
\text { presented }\end{array}$ & $17 \%$ \\
\hline $\begin{array}{ll}\text { 5. } & \text { Dominant } \\
\text { research } \\
\text { paradigm }\end{array}$ & Quantitative & $7 \%$ & Qualitative & $20 \%$ & $\begin{array}{l}\text { Mixed } \\
\text { methods }\end{array}$ & $63 \%$ \\
\hline $\begin{array}{ll}\text { 6. } & \text { Dominant } \\
\text { research } \\
\text { design }\end{array}$ & Experimental & $1 \%$ & $\begin{array}{c}\text { Non } \\
\text { Experimental }\end{array}$ & $89 \%$ & $\begin{array}{l}\text { Mixed } \\
\text { methods }\end{array}$ & $10 \%$ \\
\hline $\begin{array}{ll}\text { 7. } & \text { Data } \\
\text { collection } \\
\text { methods }\end{array}$ & Quantitative & $16.5 \%$ & Qualitative & $21 \%$ & $\begin{array}{l}\text { Multi- } \\
\text { methods }\end{array}$ & $62.5 \%$ \\
\hline
\end{tabular}




\begin{tabular}{|c|c|c|c|c|c|c|}
\hline CONCEPT & \multicolumn{6}{|c|}{ STATUS } \\
\hline $\begin{array}{ll}\text { 8. } & \text { Data } \\
& \text { analysis } \\
\text { procedures }\end{array}$ & $\begin{array}{c}\text { Clearly } \\
\text { Explained }\end{array}$ & $8 \%$ & $\begin{array}{c}\text { Poorly } \\
\text { explained }\end{array}$ & $70 \%$ & $\begin{array}{c}\text { Not } \\
\text { explained }\end{array}$ & $22 \%$ \\
\hline 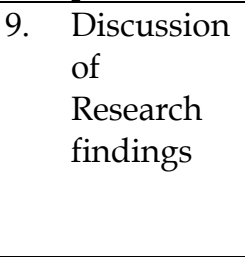 & $\begin{array}{c}\text { Clearly } \\
\text { Related to } \\
\text { research } \\
\text { questions, } \\
\text { theory and } \\
\text { other studies }\end{array}$ & $32 \%$ & $\begin{array}{c}\text { Poorly } \\
\text { Related to } \\
\text { research } \\
\text { questions, } \\
\text { theory and } \\
\text { other studies }\end{array}$ & $66 \%$ & $\begin{array}{c}\text { Did not } \\
\text { relate to } \\
\text { research } \\
\text { questions, } \\
\text { theory and } \\
\text { other studies }\end{array}$ & $2 \%$ \\
\hline $\begin{array}{l}\text { 10. } \text { The } \\
\text { research } \\
\text { process }\end{array}$ & $\begin{array}{l}\text { Logical chain } \\
\text { from research } \\
\text { question to the } \\
\text { conclusions } \\
\text { clearly } \\
\text { established }\end{array}$ & $44 \%$ & $\begin{array}{l}\text { Logical chain } \\
\text { from } \\
\text { research } \\
\text { question to } \\
\text { the } \\
\text { conclusions } \\
\text { poorly } \\
\text { established }\end{array}$ & $48 \%$ & $\begin{array}{l}\text { No Logical } \\
\text { chain from } \\
\text { research } \\
\text { question to } \\
\text { the } \\
\text { conclusions }\end{array}$ & $8 \%$ \\
\hline
\end{tabular}

Table 8. The Status of Social Research by ZOU Post-Graduate Students: Methodological Issues and Emerging Trends.

Table 8 summarises major issues and emerging trends in social research. Our exhaustive assessment reveals that while modest progress has been made with respect to some specific attributes or criteria, there are still significant areas for improvement. In particular, the following 10 key findings should engage further reflection and action among the social research community: Little progress has been made over the years in several areas and our challenge as social researchers is to refine our tools and double our efforts in search of appropriate and quality research methodologies.

\section{Conclusions}

\subsection{Clear research questions}

It is encouraging that 79 percent of the research questions are clearly stated. However, it is a requirement that all research should be guided by clear research questions or hypotheses as 12 percent questions were not clear and 9 percent of the studies did not state research questions.

More attention should be paid to the definition of initial research questions given that, without a research focus, it is easy to become overwhelmed by the volume of qualitative data often collected in case research. Without a clear research question, it is more difficult for the reader to understand the focus of the study and how it relates to a larger domain or area.

\section{Type of research question}

As expected, what questions were mostly adopted in exploratory research. 


\section{Literature review}

It is disturbing that although most studies (76 percent) analysed the theory of interest as well as the various predictions following from the theory itself. However, failure by some dissertations to consider rival theory and in some cases, to review related literature, is a serious deficiency affecting the quality of social research. Exploratory social researchers must continue to define a priori constructs in order to help them make sense of occurrences, ensure that important issues are not overlooked, and guide their interpretation and focus when conducting theory-building research. Social research must consider rival theories in order to increase predictive power.

\section{Context of the study}

Most social researchers (57 percent) were not explicit about the context of their study (time period, time spent at site, nature of data, etc.). More information about the context is needed to increase the credibility of the results and to determine whether they are generalizable. Contextual information also helps the external observer to get a better sense of the "bigger picture."

\section{Data collection}

Qualitative social research offers the opportunity to use many different sources of evidence. Seizing this opportunity represents one of the intrinsic advantages of the mixed methodology. Data collection instruments and procedures must be clearly described and explained. One shortcoming we identified in the present study was that ( 62.5 percent) of the dissertations did not elucidate how data were collected. Most dissertations omitted data collection plans and procedures. Multi-methods represented the most widely used data collection method in post graduate dissertations. However, researchers rarely described how questionnaires, interviews, observations or other methods were conducted (eg. sampling, number of interviews and interviewees, profile of interviewees, transcription, use, and validation of an interview guide, etc.). While reviewing documentation was used in several studies, most authors did not elaborate on how documents were used in the analysis and how they contributed to the findings. A clear description of the data sources and the way they contribute to the findings is an important aspect of the reliability and validity of social research findings.

\section{Data analysis}

Another major deficiency we discovered in the post graduate dissertations we analysed was that 70 percent of all surveyed dissertations failed to provide a clear and detailed description of the data analysis plans, procedures, and rules. Clear descriptions of the analytic procedures used allow the reader to better understand the findings and judge the extent to which they are the fruit of a systematic and rigorous process.

\section{Discussion of research findings}

Any research is guided by either research questions or hypotheses, Literature review is usually conducted in order to identify the knowledge gap to be filled by the present study, and related 
previous research studies are analysed so as to what knowledge other researchers have contributed in the same field of study. When data have been collected and analysed, the findings must be presented and discussed in a logical manner. Readers are interested to know the extent to which the findings address the demands of the research questions. The findings must be discussed in the context of the prevailing theory and the rival theories. This helps readers to assess the contributions of the study to the existing theory (body of knowledge). The findings must also be compared with findings of related previous studies. This places the study and the findings in the relevant field. Our findings from the current analysis of postgraduate dissertations, revealed that most student (66) failed to relate their findings to the research questions, prevailing theory and the previous studies. This deficiency makes it difficulty to assess the significant contribution a particular research has made in a particular discipline. Only $24 \%$ of all dissertations related their findings to conflicting literature while $76 \%$ discussed similar literature. There is need to compare findings to both similar theories and rival theories. Comparison of findings with both conflicting and similar literature increases the confidence in the research findings i.e. generalization and validity. Hence, this practice must be more widely applied in social research.

\section{Conclusions}

Findings from the present case study of social research at the ZOU have a bearing on the current 'quantitative - qualitative' paradigmatic debate. Our findings support proponents of mixed methods (Patton, 1990; Strauss \& Corbin 1990) who believe that qualitative and quantitative research methods can be effectively combined in the same research project. In fact, Patton (1990) advocates for a "paradigm of choices" that seeks "methodological appropriateness as the primary criterion for judging methodological quality. Mixed methods, Patton (1990) argues, will allow for a "situational responsiveness" that strict adherence to one paradigm or another will not. Our findings also show that many changes have occurred in the status of paradigms and choice of methods over the last decade such that various paradigms are beginning to "interbreed". In support of mixed methods, Russek and Weinberg (1993) claim that by using both quantitative and qualitative data, scial research can give insights that neither quantiattive nor qualitative research paradigm could provide alone. However, it is important to note that the field of social research has not yet reached the point of full integration of paradigms.

\section{Recommendations}

For the further maturation of our field, we believe that a careful consideration of the recommendations listed below is likely to enhance the overall rigor of social research. Specifically, for each of the areas considered in the present review, we encourage social researchers to:

- Identify clear research questions

- Clearly explain the context of their research problem including the knowledge gap to be filled by their research

- Take advantage of pilot cases in order to help refine the design and the data collection plans

- $\quad$ Consider both the theory of interest and rival or alternative theories in order to increase the validity and predictive power of social research 
- provide detailed information with respect to the data collection methods (e.g., interviews, questionnaires, direct observation, etc.) and procedures (e.g., sampling strategies, number of interviews interviewees, use of an interview guide, instrument validation, etc.)

- Effectively use tables to summarize information about the data collection process

- Triangulate data in order to increase internal validity of the findings and provide clear explanations on how the triangulation process is achieved

- Provide clear descriptions of the analytic methods and procedures (especially the dominant mode of analysis) and provide external observers with sufficient relevant information so they can follow the derivation of evidence from initial research questions to conclusions and vice-versa.

- a means of reflecting on the data

- compare findings with extant literature (both similar and conflicting) in exploratory so as to increase the confidence in the findings.

Although adhering to all of the rules of social research is encouraged, it does not necessarily make a social research good de facto. The present study has focused on content analysis using the combined conceptual and relational model as just one of many approaches to assess the high quality social research. Other key aspects of quality could be considered in the future. Relevance and contribution of social research to new knowledge could be investigated. Use of computers in social research is also another fertile ground for research. Surveying researchers about their perceptions on social research could also provide valuable information.

In conclusion, it is clear that current research standards have evolved and are more demanding of social researchers than they were in the early 1980s. However, social researchers face the challenges of designing a study in a systematic and manageable yet flexible manner and integrating the results into a coherent document (Marshall and Rossman, 1995). Again, in the future, social researches need to be better organized and documented as a way to help us learn, get meaningful results, and develop a cumulative body of knowledge in our field. This calls for conceited effort by all genuine social researchers, social scientists and social scholars worldwide, to double our efforts towards the development of this field and to share the knowledge, thus created, through publications.

\section{Reference}

Benbasat, I., and Weber, R. (1996) "Research Commentary: Rethinking "Diversity" in Information System Research," Information Systems Research (7:4), pp. 389-399.

Benbasat, I., and Zimud, R.W. (1999) "Empirical Research in Information Systems: The Practice of Relevance," MIS Quarterly (23:1), pp. 3-16. Bonoma, T. V. "Case Research in Marketing: Opportunities, Problems, and a Process," Journal of Marketing Research (22), 1985, pp. 199-208.

Benbasat, I., Goldstein, D. K., and Mead, M. (1987) "The Case Research Strategy in Studies of Information Systems," MIS Quarterly (11:3), , pp. 369-385.

Borg, W. R. and Gall, M. D. (1989). Educational Research. White Plains. N Y. Longman.

Bryman, A. (2001) Social research methods, Oxford: Oxford University Press

Bryman, A. 2007. Barriers to integrating quantitative and qualitative research. Journal of Mixed 
Campbell, D. T., and Stanley, J C. (1963). Experimental and Quasi-Experimental Designs for Research on Teaching. Chicago, Rand McNally.

Campbell, D. T., and Stanley, J. C. (1966). Experimental and Quasi-Experimental Designs for Research. Skokie, I L. Rand McNally.

Charles C. Ragin (1994). Constructing Social Research: The Unity and Diversity of Method', Pine Forge Press,

Cook, T.D., and Campbell, D. T. (1979). Quasi-Experimentation: Design and analysis issues for field settings. Chicago, Rand McNally.

Denzin, N. K., \& Lincoln, Y. S. (2000). Handbook of qualitative research ( 2 nd ed.). Thousand Oaks, CA: Sage Publications.

Denzin, Norman K. \& Lincoln, Yvonna S. (2005). "Introduction: The discipline and practice of qualitative research". In N. K. Denzin \& Y. S. Lincoln (Eds.), The Sage Handbook of Qualitative Research (3rd ed.), pp. 1-33. Thousand Oaks, CA: Sage.

Dodds,A.E., Lawrence, J. A.,\& Guiton, P. (1994). University students' perceptions of influences on external study. Distance Education, 5(2), 174-185.

Dube and Pre (2003). Information Systems Positivist Case Research. Management Information Systems Quarterly Vol. 27 No, \$, pp 597-635. December 2003, 597.

Durkheim, Emile. 1895. Rules of the Sociological Method. Cited in Wacquant (1992).

Eisenhardt, K. M. (1989) "Building Theories from Case Study Research," Academy of Management Review (4:4), , pp. 532-550.

Filstead, W. J. (1970). Qualitative Methodology. Chicago: Markham. Gage, N. L. (1989). The paradigm Wars and their Aftermath. Educational Researcher, 18(7), 4-10.

Gall, M., Borg, W., \& Gall, J. (1999) Educational research: An introduction (6th ed.). New York: Longman.

Giddens, A. (1990). The consequences of modernity. Stanford, CA: Stanford University Press.

Glenn Firebaugh , (2008). Seven Rules for Social Research', Princeton University Press.

Greene, J., and Caracelli, V. J. (2002). Making Paradimatic Sense of Mixed Methods Practice. Thousand Oaks, C A: Sage.

Guba, E. G. (1978). Toward a methodology of naturalistic inquiry in educational evaluation. Monograph 8. Los Angeles: UCLA Center for the Study of Evaluation.

Guba, E. G., \& Lincoln, Y. S. (2005). "Paradigmatic controversies, contradictions, and emerging influences" In N. K. Denzin \& Y. S. Lincoln (Eds.), The Sage Handbook of Qualitative Research (3rd ed.), pp. 191-215. Thousand Oaks, CA: Sage.

Jick, T. "Mixing Qualitative and Quantitative Methods: Triangulation in Action," Administrative Science Quarterly (24), 1979, pp. 602- 611.

Kangai, C., Bukalia, R., Musika., and Mapuranga, B., (2011) Content Analysis of Research Projects submitted by undergraduate Students $(2000$ - 2009) at the Zimbabwe Open University: Implications for Quality Assessment. Turkish Online Journal of Distance Education-TOJDE ISSN 1302-6488 Volume: 12 Number: 1 Article in January

Kaplan, B., and Duchon, D. (1988) "Combining Qualitative and Quantitive Methods in Information Systems Research: A Case Study," MIS Quarterly (12:4), , pp. 571-587.

Kember, D., \& Dekkers J. (1987). The role of study centres for academic support in Distance Education, 8 (1), 4-17.

Lather, P. (1992). Critical frames in Educational Research: Feminist and post structural perspectives: Theory and Practice.

Laudon, K. C. "in J. I. Design Guidelines for Choices Involving Time in Qualitative Research," 
Lee, A. S. (2001) "Challenges to Qualitative Researchers in Information Systems," in E. M. Trauth (ed.), Qualitative Research in IS: Issues and Trends, Idea Group Publishing, Hershey, PA, pp. 240-270.

Lee, A. S. "Integrating Positivist and Interpretive Approaches to Organizational Research," Organization Science (2:4), 1991, pp. 342-365.

Lee, R.M. (1993) Doing Research on Sensitive Topics, London: Sage.

Lincoln Y and Guba EG (1985) Naturalist Inquiry, Sage Publications, Newbury Park, CA.

Lincoln, Y. S., and Guba, E. G. (2000). Paradigmatic controversies, contradictions and emerging confluences. Thousand Oaks C A: Sage.

Markus, M. L. "Case Selection in a Disconfirmatory Case Study," in J. I. Cash and P. R. Lawrence (eds.), The Information Systems Research Challenge: Qualitative Research Methods (Volume 1), Harvard Business School

Marshall, Catherine \& Rossman, Gretchen B. (1998). Designing Qualitative Research. Thousand Oaks, CA: Sage Publications, Newbury Park, CA,

Mason, J. (1996) Qualitative Researching, Sage Publications, Newbury Park, CA,

Maxcy, S. J. (2003). Pragmatic threads in mixed methods research in the Social Sciences: The search for multiple modes of inquiry and the end of philosophy of formalism. Thousand Oaks, C A: Sage.

Mays, N., and Pope, C. (1995) "Rigour and Qualitative Research," British Medical Journal (311), pp. 109-112.

McMillan, J. H., and Schumacher, S. (1993). Research in Education: A Conceptual introduction. 3 th Edition. Happer, Collins College Publishers.

Mertens, D. .M., (2005). Research and Evaluation in Education and Psychology: Integrative diversity with quantitative, qualitative and mixed methods. 2nd Edition London. Sage Publications.

Miles, M. B., and Huberman, A. M. Qualitative Data Analysis: An Expanded Sourcebook. Sage Publications, Beverly Hills, CA, 1994.

Mingers, J. (2001) "Combining IS Research Methods: Towards a Pluralist Methodology," Information Systems Research (12:3), pp. 240-259.

Osman, R., \& Wagner G. A. (1987). New Zealand management students' perceptions of communication technologies in correspondents education. Distance Education, 8(1), 47-63.

Patton, M. Q. (2002). Qualitative Evaluation and Research Methods. Thousand Oaks, C.A: California, Sage.

Patton, M. Q. (1990). Qualitative evaluation and research methods (2nd ed.). Newbury Park, CA: Sage.

Patton, M. Q. (2002). Qualitative research \& evaluation methods (3rd ed.). Thousand Oaks, CA: Sage Publications.

Reckase, M. D. (1995). Portifolio assessment: A theoretical estimate of score reliability.

Reichardt, C. S., and Cook, T. D. (1978) "Beyond Qualitative Versus Quantitative Methods," in T. D. Cook and C. S. Reichardt (eds.) Qualitative and Quantitative Methods in Evaluation Research, Sage Publications, Thousand Oaks, CA, pp. 7-32.

Russek, B. E., \& Weinberg, S. L. (1993). Mixed methods in a study of implementation of technology-based materials in the elementary classroom. Evaluation and Program Planning, 16 (2), 131-142.

Schwandt, T. (2000). Three epistemological stances for qualitative enquiry: Interpretivism, Hermeneutics and Social Constructionism. Beverly Hills, C A: Sage. 
Sheets, R. H.. Diversity pedagogy : examining the role of culture in the teaching-learning process. Boston : Pearson/Allyn and Bacon, 2005.

Smith, R. B., \& Manning, p. K. (1982). A Handbook of social science methods, volume 2: Qualitative methods. Cambridge, MA: Ballinger.

Teddlie, C. and Tashakkori, A. (2003). Major issues and controversies in the use of mixed methods in Social and Behavioural Sciences. Thousand Oaks, C A: Sage.

Lawrence W. Neuman (2006). Social Research Methods: Qualitative and Quantitative Approaches', 6th edition, Allyn \& Bacon

Woolfolk, Anita. (1994). 8th Ed. Educational Psychology. Boston: Allyn and Bacon, 2001.

Yin, R. K. Case Study Research, Design and Methods (2nd. Ed.), Sage Publications, Beverly Hills, CA.

Zikmund, William G. (1991), Business Research Methods, 3rd ed., Chicago, The Dryden Press. 


\title{
Methodology Transfers Between Social Sciences and Humanities in Relation to Natural Sciences, Technology and Government Policy
}

\author{
Hajime Eto ${ }^{1}$ and Shinichi Yamamoto ${ }^{2}$ \\ 1 Professor emeritus, The University of Tsukuba, Tsukuba, \\ 2 Professor, Director of Research Institute for Higher Education, \\ Hiroshima University, Higashi-Hiroshima, Hiroshima, \\ Japan
}

\section{Introduction}

Social sciences may be regarded as derived from humanities such as philosophy and history, but have been developed in different ways (sometimes parallel but often opposite ways) from humanities. In this chapter, social sciences denote the set of social scientific branches (economics, sociology, etc.) while social science denotes the general concept abstracted from each of social sciences. Analogously, natural sciences denote the set of natural scientific branches (physics, chemistry, etc.), while natural science denotes the general concept abstracted from each of natural sciences. An analogy is the difference between behavioral sciences (the set of behavioral psychology, behavioral sociology, etc.) and behavioral science as a general concept. The general concept abstracted from natural and social sciences and humanities (including arts) are referred to as knowledge (the modern version of medieval scientia). This chapter mainly or almost exclusively discusses western knowledge and ignore eastern or southern one because western knowledge is worldwide dominant in the latest centuries. The global transfer of methodologies of knowledge is a challenging theme but too comprehensive for one chapter. Therefore it will be left for future research.

Since the Renaissance, particularly the Galileo's revolution of physics from Aristotelian phusika or scholastic physica, many philosophers have discussed the characteristics and methods of natural sciences. They have also discussed social sciences but very differently in discussing natural sciences. This chapter discusses their differences to clarify the fundamental characteristics of social sciences in relation to humanities, instead of presenting a particular theory of or the particular application of a theory of social sciences or humanities. In this discussion, the separation of social sciences from humanities are considered as a 'crisis' of knowledge, where crisis (krisis) means the decisive turning point in clinics or the radical change by conflict in drama as originally meant in Greek. Later, crisis began to mean the crossing to sharply or crucially change the direction. Hereafter, premodern knowledge will be referred to as scientia. 


\section{Paradigms of social sciences and their shifts}

Humanities was central in scientia and concentrated itself on the study of the classics. Humanities was relatively free from the paradigm shift because it founded itself on the succeeded classics having the eternal values, although the introduction of Aristotelian metaphysics to West affected the paradigm of Bible-based knowledge and the comprehensive recognition of the entire Greek classics affected the belief in Christianity. Despite these influences, humanities retained the exegetic methodology of the classics. This affected the methodology of social sciences, and many social scientists were scholastically engaged in closely reading and faithfully interpreting or commenting on the classical books by Smith or Marx. The rise of social sciences little affected the paradigm of humanities at first. The concept of paradigm has been associated with science revolution [Kuhn, 1962] and used as a general (sometimes too general and ambiguous) term. This chapter uses this word as a general term and replaces this with methodology in more specific discussions.

Before the medieval era like in Greece, without particularly established 'bibles', humanities (particularly, philosophy in Greek) was rather natural historic (if not natural scientific) in embracing what may be called zoology, cosmology, etc. Greek knowledge was transferred to and developed in East, but not transferred to Europe except for Islamic Iberia. The Greek classics were rediscovered and became central in humanities in Europe in the Renaissance almost for the first time although Aristotelian metaphysic was already influential there.

Legal systems and their enforcements have often been called justice. This shows that they have been originally founded on ethics or religions. Pre-modern legal scientia was founded by jurisprudence that was a branch of theology or humanities. This helped political leaders persuade people. Policy-making and the implementations were founded by philosophia including social ethics, religions, and the view of history and historical lessons, all in humanities. This stabilized the paradigm of social scientia and prevented it from the paradigm shift against the social development. In the modern legal system, the constitutions mean the fundamental principles supposedly stable for decades, found other laws, and constrain "the evolution of law". This idea is extended to technology as the standardization, often stipulated by law.

When the close studies of ethical or religious texts were developed, the exegetic method dominated the study of classics. This method sought for the eternal truth uninfluenced by the shift of 'vulgar' life in time, and was developed by scholiasts as a positivism to strictly respect what was really written and to ignore the prevalent practices in contemporary life. This positivism prevented scientia from paradigm shift. While humanities specialized itself in the study of classics, the exegetic method influenced jurisprudence in the interpretation of laws, which was supposed to be eternal.

Ironically, classic-based humanities for the eternal truth drove a radical revolution in entire scientia, called the Renaissance. This revolution was to revolve from the Christian classics (the Bible) to non-Christian Greek classics or to rediscover the latter, and to transform the positivism but not to deny the positivism. The Renaissance physicists succeeded the exegetic positivism and successfully transformed it from reading the given texts like scholastic physica (translated from Aristotelian phusika) into experimenting the earthly movement, as ancient and medieval astronomers observed and predicted the heavenly movement. Motivated by the theological system, Newton unified the earthly and heavenly movements 
into a universal theory by mathematically formulating the observed data. Centuries later, this founded the physics-mimicked economic theories, although the Galilean experimentalism is succeeded in social sciences only by psychology except for the recent experimental or behavioral economic methods. .

The denial of the Aristotelian phusika led to the denial of Aristotelian multi-disciplinary unification of knowledge encompassing oikonomia, politika, ethikos, zoologia, kosmologia, meteorologia, and many others. Modern science proudly distinguishes itself from scientia or metaphysics by the specialization of knowledge into narrow branches. The unification of theories of physics is only within physics, as the unified axiomatic system of geometry is only within geometry. This scientific systemization by narrowing the zone contributed to the success in scientific development.

Many philosophers and historians of natural sciences have almost unanimously regarded the Galilean and Newtonian methodologies as the first great steps of modern scientific paradigm. But they have no consensus about the paradigm of social sciences. Some of them seem to regard social sciences as occupied by para-dogmas or pseudo-dogmas, although paradigm is believed etymologically unrelated to dogma.

Dogmas or not, social sciences have had their paradigms and shifted them several times in the last few centuries. Each shift has been investigated by historians of social sciences, but little has been discussed about the shift in general. Philosophers usually prefer general discussions rather than case studies, but few succeeded in the general discussion of paradigm shifts in social sciences. To avoid the confusion of paradigm with dogma, the discussions below will avoid this term and use the word of methodology.

\section{Views of humans and history in social sciences}

In the ancient era, the studies on law and politics were founded on the ground of theological idea of justice. After theology was replaced with humanities, social scientia borrowed the methodology from humanities, which often explained social phenomena by historical background with the view of human behaviors and acts in the course of historical development or as path-dependent. Upon the view of history of ideas, Hegel founded his Encyklopädie of total knowledge and unified various branches of knowledge via dialectical logic demonstrated in the historical development of various ideas. Introducing the economic or materialistic view into the dialectic logic in historical development, Marx presented the view of history as the unifying core of social sciences. In opposition to founding social sciences upon history, however, the ideas of social engineering and social technology blamed the Marxian view of social sciences as the 'historicism' or the mixture of social sciences with social philosophy. In the meantime, the view of humans as homo economics rather than homo sapiens has been the core of the prevalent neoclassic economics, where sapience means wisdom or intellect and is often synonymous to the humanness or benevolence possibly to selflessly consider others (altruism). Before the homo economics view, the view of humans as homo sapiens was (and is outside economics) almost exclusively prevalent and has been used as the terminology of anthropology in distinction from more primitive humans (e.g., home erectus).

The view of humans as homo economics or the economic rationalism validated the physicsmimicked economic theories. Physics took the economy-mimicked view of nature and 
successfully constructed a set of calculations of variations to explain natural 'behaviors' as minimizing the 'power' or 'energy'. This method led physics to successfully explain the ball shape of the earth as resulting from the 'purposed effort' to minimize the surface area for the given volume. Economics imported this economy-mimicked methodology of physics and successfully modeled the human behavior to minimize the cost and similarly to maximize the profit by multiplying by -1 .

The success of modern science largely owes to that of physics, particularly that of mechanics. The Cartesian view of human body (phusike) as a machine was successfully extended to societies. As machine is mechanical and calculable, the mechanistic view of everything as mechanicals promoted the application of mathematics to everything including society. In legal prudence and political science, the monarchical system and the congressional system were unified as 'the Emperor as Machine' theory that viewed the Emperor as no dictator but as controllable by congress.

Yet another view of humans is to view humans as mankind or humankind, where 'mankind' or 'human-kind' means humans collectively or the genus (kind). With deep knowledge of the Greek classics, Marx followed the traditional ethics to view humans in society like Aristotle, Kant, Hegel and Feuerbach. The Feuerbach's view of humans as mankind affected the Marxian view of humans as beings fostered in history. As history is the product of the collaboration of humans, the view of humans in history is nearly synonymous to the view of humans as collaborative or societal beings. In the first half of the 1800s, before Marx, many intellectuals from the established families accused the capitalism that promoted the competition motivated by individual desires, and they proposed the 'renaissance' of cooperative society or community called the socialism or communism. Workers from fallen but formerly established families in agricultural areas led workers to the socialism movement. This radically changed the intellectuals' movement into the class struggle, or the individuals' movement into unionized movement of similar kind of workers or 'workers-kind'. The mankind view of humans helped workers unions oppose the individual competition principle. The history of borderless collaboration of Jewish businesses also helped the international unionization of Jewish-led communism movement against the pursuit of the wealth of each nation.

The resources within Europe limited its growth. The alternatives were to decrease the population; to revolutionize the social system for the equal distribution of resources to all; or to bring the resources outside to Europe. The progress of transportation technology and its adroit management allowed Europe to choose the last one. This meant the inequality of humans between Europe and elsewhere and resulted in the denial of the Marxian economic and anthropological equalities (i.e., same kind) including his view of humans as mankind. The competition was intensified at the global and domestic levels and led the world to the war. The worldwide prediction of global war may partly be one factor of the Kant renaissance, who discussed the eternal peace in Napoleonic Wars in the entire Europe. World War I replaced the neo-Kantian philosophy with the neo-Hegelian philosophy, the state philosophy of which justified the worldwide domination of the best system for the global and eternal peace. This idea was supported by Heidegger, Jaspers, Tanabe, and other intellectuals besides economists. Traditional ethics and lesson-based view of history failed to prevent the repetition of global war. Traditional humanities proved to be materially powerless. The only lesson of the wars is to replace the armed race with the economic one by extending the homo economics view to the view of the world. 
After the prevalence of the philosophical methodology of history on the basis of the views of history, history in academia resumed the scholasticism to limit itself to the exegetic positivism of historical documents. The progress of information technology helps historians in academia efficiently process the documents in ivory towers. Mass media contributed to the renaissance of the view of history that great leaders or single events determine the course of history. This helped management science stress the decisive power of decisions made by leadership. Outside academia, Marx saw the driving force of history in the economic or materialistic desire as in Smith, and focused on the conflicts between the production system and technological development and that between the ruling and the ruled classes. Although Marx himself failed to discuss technology in detail, philosophers including Heidegger discussed the effects of technology on societies and humans in the 1900s. As its effect on humans, the alienation of humans from technological societies motivated philosophers to develop the existentialism to rediscover the value of spiritually independent individuality. This stimulated the anti-industrialization feeling, affected economic policies, and later, promoted technology assessment.

The Hitler's view of the global conflict as resulting from the division of the world between the have and the have-not nations helped some social scientists and historians to interpret the struggles in Asia and Africa against West. Further, the Toynbee's method to explain history via the responses against the outer stimuli as in the ecological living world helped social scientists interpret the struggles in Asia and Africa against West. After the competition between the views of history was replaced with the quantitative analyses of social sciences, social sciences almost exclusively explained the status quo in precise ways. The range of quantitative social sciences is limited by the data availability as the range of astronomy is limited by the performance of telescopes. Meanwhile, history remains useful as the source of fictions in novels, comics, and other media.

Around 1900, theoretical philosophers (epistemologists and logicians) like Russell viewed humans as rational and logical (maybe termed homo logos). They were optimistic about the human capability to construct symbolic logic, mathematics, and linguistics of artificially constructed symbolic language. This program was to unify all sciences including social sciences and psychology in rigorously unifying ways with the persuasive power over all rational beings beyond national borders and classes. This was called the unity of science or unified science movement. This optimism was broken by Gödel in the early 1930s in the era of the fall of traditional social sciences and the rise of different types of social sciences between Great Panic and World War II. This failure encouraged the idea to base intellect on emotion. The national emotion was proved powerful in decision-making via the development of mass-media as sociologists predicted.

After the failure of formal logic as the power to unify all sciences, logicians and mathematical philosophers with the help of linguists conceptually generalized the strict symbolism to more flexible semiotics to embrace all kinds of language- or symbolexpressible knowledge by following Kant, the epistemology of whom was to consider only the perception itself instead of the thing itself. Semiotics is the first methodology jointly originated in mathematics, physics, linguistics, poetics, aesthetics, etc. and influencing social sciences, particularly sociology. The semiotic methodology is to "poeticize" all knowledge and thereby justifies the deviation of knowledge from the reality. This is acceptable and applicable not only to aesthetics but also to the theories of mass-media, election campaigns, 
social panics, marketing, and the likes. Economics remains uninvolved in semiotics itself because it has already involved itself in money by valuing physical or material commodities symbolically in monetary terms. In this respect, economics is nothing but semiotics. Another origin of semiotics is from medicine, physics and astronomy. The symptom is the most important information to "symbolize" the internal state of body. Astronomers observe images via lens, and modern physicists read the numerals on measurement instruments. Instead of experiencing the reality, economists read the statistical data provided by government, "operate" economic theories in terms of differential or integral calculus, and use computers, as poets create the imaginations of worlds and minds, and painters create worlds and emotions on paper. In this respect, the idea of homo economics is consistent with that of homo logos. As homo logos of logical intelligence is deeply related with homo sapience, homo economics may be considered as related with homo sapience in this respect.

The view of humans is now experimentally examined to provide the evidences of the pros and the cons of viewing humans as homo economics. This view is also called the economic rationalism and is often attributed to Smith, an ethicist in a Catholic city. As the economic rationalism is opposite to the classic rationalism that respects the religious and ethical reasonableness, it fails to persuade non-economists. Using natural or behavioral scientific methods, brain scientists and psychologists test the homo economics view of humans [Gazzaniga, 2005; Camerer and Fehr, 2006; Warmeken and Tomasollo, 2006; Haidt, 2007].

\section{Mathematical, quantitative and physicalistic methodologies in social sciences and humanities}

Many philosophers and historians of natural sciences regard physics (particularly, mechanics) as the core of natural sciences and the mathematics as its core method, which all sciences are supposed to make efforts to use.

Astrology and the calendar based on real observation and calculation provided the foundation of decision-making (divination). Policy making of government and families' (or clans') household (oikonomia) also depended on the calendar and the financial calculation like the calculation of inventory or provision. Under the strong control of myths, religions and historical lessons (all in humanities), social techniques collecting data for taxation and the calculation were undervalued. Despite the economic usefulness, calculation was not respected as knowledge (sophia). Greek philosophers were interested in geographic figures and the properties of natural numbers including their ratio. As the calculation was important in distribution, algebra as the fundamental theory of calculation was developed in the Islam countries, where the studies of complicated figures or patterns were also developed. Owing to the development of transportation, such knowledge was transferred to West, where mathematics was combined with physics (particularly dynamics) during and after the Renaissance. Much later, this provided economics with powerful methods.

Transportation techniques including calculation techniques for road or bridge construction, shipbuilding and navigation accomplished the expansion of territories to obtain more resources and knowledge. This stimulated social technocrats in judiciary and administration into geography including anthropology, and contributed to the development of natural sciences, science-based technologies and humanities. Informed of the customs in various civilizations, for example, Montesquieu presented the comparative philosophy of law. 
Conversely, the method of social sciences owes to humanities, natural sciences and technology. Extending the Arabic and Cartesian view of blood circulation in human body to the circulation of commodities and money, Quesnay, a surgeon and a physiocrat, presented tableau économique to represent economic flows and made the first step towards econometrics and economic planning. But this physiological view of economics failed to win the worldwide support of economists, the majority of whom have almost exclusively regarded physics aided by mathematics as central in science. This may partly be explained as the bias of the mainstream manufacturing- and trade-oriented Anglo-American economists against the land-based French physiocratic economists. Naturally, manufacturing-based economy was rather related to mechanics, while agriculture-based economy was relatively related to biology.

Biology (historically, botany, zoology, entomology, and ichthyology were not unified) seldom affected the methodology of social sciences after the modern civilization succeeded in conquering the nature by using mechanical technology based on physics. Contrarily, the severe competition of home economics after Industrial Revolution induced Darwin to think of the natural selection as the driving force for biological evolution.

Ethics (praktische Philosophie) was influenced by biology like the collaborative behaviors of bees and ants, but theoretical philosophy (epistemology and logic) considered physics and mathematics as central in natural science. Before February Revolution, Comte at École Polytechnique presented positive philosophy to found all knowledge including social study (sociologie) upon natural sciences, particularly physics. Later, Marx claimed his social science (Sozialwissenschaft) to be as rigorous as physics. Around 1900, Frege, Russell and Hilbert attempted to construct the logico-mathematical system upon which all knowledge is supposed to stand. This yielded the logico-mathematical movement toward the unified science (unity of science) in the 1930s in Vienna and then Chicago, where many Jewish scholars fled from Vienna. At the same time, the biological idea of general systems was developed in Vienna.

Today, many social scientists, particularly economists, take the physico-mathematical view of natural sciences, although they have almost no knowledge of the recent states of natural sciences and mathematics. Actually, central in physics is solid-state physics or alternatively called material science, which is located between physics and chemistry (solid-state chemistry). Many papers in this field appear in the journals of the fields called chemical physics, physical chemistry or chemistry itself even if all the authors have the background of physics [Eto, 2003; Whiteside, 2007]. Biology is shifting towards biochemistry and is often renamed life science. Biochemical views date back to the origin of pharmacology, which has paid deep attention to material or chemical processes in living bodies. Deeply connected with pharmacology, medical scientia from China, India, Arabia to Roma had long considered human bodies (and sometimes also the minds) as controlled by the subtle rate between various fluids or moisture (humidity) like humor. Rather than mechanically, Descartes biochemically considered mental and emotional states as determined by the internal liquid secreted from pineal body. In many aspects, chemistry is rather central in natural sciences. As chemistry and biology or life science are rather central in natural sciences today, mathematics is no longer the central method of natural sciences [Eto, 2008a; Kemsley, 2009]. 
Behavioral sciences like psychology have developed and prefer factors analysis, structural equation modeling. LISREL and other information processing type methods or data analysis to mathematically well-founded methods [Kruskal, 1964; Tukey, 1977; Bentler, 1986; Bollen, 1989; Jöreskog, 1997; Hayashi, 2002]. Mathematics including mathematical statistics retains the central position of methods only in economics. Even economics, although slightly, revised mathematical statistics for economic analysis purposes. Many papers in physics are to report the experiment results without using mathematics, although mathematics is still an important subject in high-school and undergraduate education of physics. Today, the most mathematicsprone science is economics rather than physics. Indeed, most papers in economic journals are full of mathematics, and mathematical tools are the most important subjects in undergraduate and graduate training of economics. As the ancient empires stressed calculation for taxation and budgeting, Petty presented the concept of political arithmetic from his experience with finance and is often respected as a forerunner of economics. Mathematics is now the tool for finance rather than physics as will be discussed below.

Social scientists learn some established knowledge of natural sciences from textbook but almost never collected the data through observations and experiments by themselves. Except for sociologists, social scientists (particularly, economists) are usually provided data by governments or sponsors and have almost never trained for data collection. In this respect, economics are in common with mathematics. Therefore it is easy for economists to use undergraduate mathematics without tedious work of data collection. The similarity or dissimilarity in data acquirement may decisively affect the similarity or dissimilarity in the methodologies of sciences.

In regard to data acquirement, mathematics has been distantly divided into abstract mathematics developed in the 1900s and physics-motivated mathematics developed in the $1700 \mathrm{~s}$ ans 1800s. Economic theories almost exclusively use differential/integral mathematics and elementary linear algebra, which are no longer central in modern mathematics. In this regard, economics is actually in no relation with modern mathematics. Statistics is no longer mathematics-centric but rather toward informatics to fully exploit the information carried in data [Benzécri, 1973; Hand, 1998; Hayashi, 2002]. In regard to methodology, economics is isolated in the knowledge world. After the failure of logico-mathematical program of the unified science movement, philosophy seemed fallen into the isolation but succeeded in allying other knowledge by constructing semiotics. Today, only economics is isolated in the knowledge world, although it is in a close relation to economy-centric government.

In the meantime, various fields demand economic knowledge in the economy-dominant society. The healthcare sector is the most severely suffering from the economic pressure [Zweifel and Breyer, 1997; Olsen, 2009] and is required to get more profit possibly at the sacrifice of healthcare quality in the aging society even in the country of socialism tradition [Yan et al., 2011]. Here, economics is requested to come to the real world instead of the comfortable world of government and banks and acquire real data by using own hands in collaboration with medical personnel [Nielsen and Mather, 2011] by designing surveys themselves [Hayashi, 1984]. Also, many other welfare problems including environment and education wait for "true welfare" economics. Besides the history of science, evidence-based humanities such as archaeology, anthropology and history have already collaborated with natural science and technology in essential ways [Balter, 2006]. 


\section{Policy sciences in relation to mathematical/quantitative methods}

As real worlds demand medical and agricultural sciences and engineering to serve human lives, social sciences have been requested to be usefully applicable to real problems. The idea of policy science is as old as that of social sciences themselves but has little been implemented as a new interdisciplinary field except for economic planning, research and development (R\&D) management, and science and technology (S\&T) policy and management. This section discusses why it has been so.

Almost all social scientists have been interested in real societies as persons. But the involvement in real societies often obstructed the free activities because of the gap between contemporary secular worlds and the pursuit of the eternal truth. For the freedom, many social scientists fled from real societies to ivory towers usually protected by the church power. Under the church protection, humanities to study the classics were dominant in universities. Under this influence, social sciences developed the method not to involve themselves deeply in urgent problems. Like the division of labor between natural sciences and engineering and that between Classics scholars and religious practitioners such as priests, labors have also been divided in social sciences between social scientists in academia and practitioners in real society. The former has so distantly separated themselves from the latter that the former has not admitted the latter in the professional societies. Their information exchange channels have been cut off.

As mathematics including mathematical statistics and quantitative data analyses are usually regarded as independent of interest and neutral between the conflict of interest, the development of mathematical theories and statistical analyses were expected to generate a new type of social sciences. This was called policy science. Indeed, the first professional journal in policy sciences was issued by a publisher in mathematical sciences. .

Mathematical approach still dominates policy sciences in academia, but those who deal with policy studies outside academia have increased in number and have had more varieties in their approaches. While academics tend to insist on beautiful models and sophisticated methodologies, administrators and practitioners in government and business prefer more practical approach by observing actual cases or data even when they discuss the same problems with academics. Today, the tension between these two groups becomes stronger; or between academic circles of policy scientists using advanced mathematical models and practitioners in government and business not using mathematics so often except simple calculations and data analysis. Anyway, academics in policy sciences cannot neglect the demands from practitioners in various fields.

In the meantime, several new scientific associations in policy sciences have emerged and developed their activities in Japan. One of their specific characters is that they consist of practitioners in the government and business besides professionals in universities and research institutions. They also accept interdisciplinary approaches of research because their main purpose is to solve real problems of their concern. Nihon Keikaku Gyosei Gakkai (Japan Association for Planning Administration), for example, was established in 1977 by the researchers and practitioners in the fields of public administration/finance, national land development, economic planning, and so on, insisting the need of closer collaboration of academia, government and industry. Kenkyu Gijutu Keikaku Gakkai (The Japan Society for Science Policy and Research Management: JSSPRM) was established in 1985 for the 
promotion of practical interdisciplinary studies on science/technology policy and innovation. They said "So far JSSPRM has been providing researchers and practitioners in this field with unique opportunity of networking or information exchange." More recently, Seisaku Kagaku Gakkai (Association for Policy Sciences) was established in 2009.

In addition to these associations, policy science-oriented research activities are observed in other fields of research. In the educational research field, policy studies are one of the most prevalent approaches rather than traditional pedagogical ways of research. The Japan Society of Educational Sociology, having played the leading role of performing empirical research on educational phenomena, deals with many kinds of policy-oriented research recently. Japanese Association of Higher Education Research also put on the emphasis on practical problem solving at the ages of university reform. Same trends are observed in other academic associations in educational research.

As for the universities in Japan, the number of schools and departments with title "policy" has increased especially in private universities. They need more efforts to recruit more students into their institutes, because the population of 18-year-olds has been decreasing since early 1990s and the decrease may not stop within several decades. Thus, universities need to attract the youth to attend. One of the ways for being attractive is to reform and rename their schools and departments. The new names include "modern," "international," "environment," "information," and "policy," even though the teaching staff remain as same as before the renaming. But social science departments are challenged by the reform of the humanities departments, where English and foreign study professors are trained in USA and learn the American way of humanities to stress the contemporary problems for practical purposes against the traditional stress on the study of classics. Social science departments need the own philosophy to compete with the humanities departments.

Back to the 1970s in Japan, there was a group who intended to establish the graduate school of policy science in Saitama University, one of the national universities. They started planning and succeeded in establishment of Graduate School of Policy Science in 1977, just one year behind the start of Graduate School of Management and Policy Science, the University of Tsukuba, which will be mentioned in the next section.

The policy science group of Saitama University strongly oriented themselves to the policy analysis in real fields. Thus, they invited not only scholars in social sciences, such as in economics and political science, but also bureaucrats who were actively working for the ministries of finance, construction, international trade and commerce, agriculture, science/technology and education. The dean of the school, who later became the first president of Seisaku Kenkyu Daigakuin Daigaku (The National Graduate Institute for Policy Studies: GRIPS), had the strong belief that academics and policy makers should cooperate intensively and thus policy makers and bureaucrats at central and local governments should be educated well; preferably they should have PhDs.

In contrast to the philosophical discussions on natural sciences and technology [e.g., Eto, 2008b], non-Marxian social scientists had avoided the philosophical discussions on social sciences policies. The postwar reform of education and bureaucracies required social science students to study some natural sciences and humanities. Without publishing the design philosophy of social sciences in systematic manners, however, Uehara extended a commercial college to include the departments of sociology and law (now Hitotsubashi 
University), and Yanaihara established a comprehensive research institute for social sciences including history. Gradually, however, universities and government began to "forget" such a cultural training and to stress the specialization. Three decades later, a systematic view of policy sciences with some regard to other fields appeared [Yoshimura, et.al., 1982]. This book was critical of the situation in government office that most of the bureaucrats had only the background of positive law without any scientific manner in their undergraduate programs and that they often lacked the knowledge and the way of thinking in economics, statistics, mathematics and humanities. Further, this book criticized that the bureaucrats in Japan leaned exclusively on legal aspects and didn't or even couldn't care about identifying the real problem and its solving. Moreover, it pointed out that the bureaucrats consumed most of their time for the discussion of legal consistency with the existing legal system. In the meantime, the retired diplomats of Japan serve as the presidents of the International Court of Justice and the International Tribunal for the Law of the Sea, respectively, after the Japanese judges with academic backgrounds of law retired from the court and the tribunal, respectively.

In international organizations such as OECD have many professional staff. In the Department of Science, Technology and Innovation (DSTI), the professional staff produces regulatory reports and policy papers on these fields, while serving for committees consisting of delegates and scientists from member countries. The Center for Educational Research and Innovation (CERI) has led educational thoughts and practices for member countries by their practical research and survey. A couple of decades ago, the idea of "Recurrent Education" was presented and began to affect the life-long learning policy in Japan.

\section{Social sciences and its relations with humanities in the design of universities}

Natural sciences including engineering, medicine and agriculture are relatively borderless. Humanities are either national (the studies of own culture like language, literature, philosophy and history) or foreign (the studies of foreign culture). Social sciences are in between. The globalization limits the national characters of social sciences. When Japan ceased to isolate itself from the world, Japan had to adapt itself to the world, where the western systems dominated. The task of social sciences of Japan was to adopt the western ones possibly with some adaptation to the reality of Japan.

In 1867, the Emperor of Japan restored the power and began the radical modernization by the westernization called Meiji Restoration. The first modern university called The University of Tokyo (now University of Tokyo) was established in 1877. The word "university" was translated into Japanese "daigaku," so named after daigaku, the school for training bureaucrats in ancient Japan. The task of The University of Tokyo was to train bureaucrats with western knowledge in adaptation to the West-dominant world. Professors were all invited from West with high salaries. They gave lectures on the western culture and systems all in their languages. Students merely dictated the lectures without mutual discussion. This university had no department of Japanese or Asian studies, which were left to traditional schools with the original research activities in the fields [Eto, 1993]. This teaching style of lecturing on foreign knowledge and dictating the lectures without mutual discussion was (and is) long succeeded in the discipline system, where young assistant or associate professors follow the senior professors and succeed the positions. 
It had been the only university in Japan until 1897, after The University of Tokyo was renamed The Imperial University and then The Imperial University of Tokyo and set up the departments of Japanese and Asian studies. In 1897, the second imperial university was established in Kyoto, which was mainly for law, science and engineering at first. The third and fourth imperial universities set up in Sendai and Fukuoka, respectively, were mainly for medicine, science and engineering at first.

The Imperial University of Tokyo had two main missions; the first one was to introduce advanced knowledge from overseas especially from West; the second was to train talented personnel for bureaucrats, engineers and various kinds of specialists that were essential for the rapid modernization of Japan. To realize these missions, this university consisted of five colleges, i.e., Colleges of Law, Medicine, Engineering, Literature, and Natural Sciences in this order. The former three colleges dealt with applied studies while the latter two colleges were for basic and academic matters. In this sense, the main concern of the government over higher education was applied matters rather than the promotion of basic sciences that was an important function of universities in West. This application-purposed character of Japanese higher education became clearer when the government added imperial universities and different kinds of higher education institutions later, i.e. technical colleges, commercial colleges, teachers colleges (higher normal schools) and so on, all of which had practical missions of training professionals in their fields.

Whether applied or basic, however, Japan had to quickly introduce western knowledge and technology because she needed the rapid modernization for going along with West, with the aim of being recognized as advanced countries and joining their circle instead of being colonized like neighboring countries in Asia. Thus, the universities heavily depended on "imported knowledge" at the first stage of their development.

The import of established knowledge had characterized every field of sciences in Japan until the early 1900s. However, in the field of natural sciences, things were gradually improved and some distinguished achievements of original research made Japanese scientists being worldwide famous, such as Kitazato and Honda, although such excellent scientists were limited in number. However, in social sciences, the situations had not or even have not improved. Some critics say that Japanese scholars in social sciences have only introduced knowledge and theories from overseas and talk about them among domestic colleagues and to students; they know much about what and how foreign scholars say or do but never think of the contextual difference between the country that foreign scholars work and Japan; they only learn but publish no original article not only in international journals but in domestic ones; they keep their status of scholar by only translating foreign works into Japanese [Kuroki, 2009].

Without disciplinary system, elementary and secondary school teachers attempted the selfinquiry education around 1930. But government oppressed it because it was associated with the democratic movement [Johnston, 2006]. As Army demanded the promotion of science education, the self-inquiry education method was promoted for science education by Hashida, a physiologist, moral philosopher and the Minister of Education. Accused as a war criminal, however, he committed suicide in 1945. In the 1930s and the early 1940s, social scientists made efforts to build social sciences appropriate to Japan. For example, Ono tried to replace the Napoleon Code-based legal system with the Japanese practice-based ones. But Allied Forces arrested them as nationalists or prohibited their publications as nationalistic. 
Regarding the import of social sciences from West, things worsened after World War II. For the purpose of oppressing the nationalism, Allied Forces utilized the energy of west-minded social scientists. They were given the task to lead people toward western ideas and their social activities were encouraged. Social scientists who criticized government in the prewar period were highly praised, while those who kept silence were blamed.

The increase of universities and students worsened the professors' environment for creative research. Far more universities and colleges were established. There were only 47 universities in 1940 but the number increased to 228 in 1955 and 382 in 1970; out of the 382, 75 were national, 33 were local public, and 274 were private universities [Monbu-Kagakusho, 2010]. Along with the increase of institutions, enrollment in universities also rapidly increased. It was about 500 thousands in 1955 but, by 1970, it reached to 1,400 thousands, and the enrollment ratio of 18-year-old student into higher education jumped to over 30 percent. The massification of higher education became one of the problems in keeping quality of faculties as well as students and university education. The massification more seriously affected the departments of social sciences, because it was relatively inexpensive for private institutions to establish new schools and departments, and students tended to prefer studying social sciences and humanities to studying science and engineering. Around the 1970s, more than 40 percent of students enrolled in social sciences departments. In addition, most of the newly established private universities and departments in social science had no graduate program.

As is easily imagined, professors were involved in too heavy duties of teaching to conduct creative research. Teaching subjects of social sciences for undergraduate programs needed little research because teaching was often only to read textbooks or even without use of textbook. Many professors read their notebooks in the classrooms slowly so that students could dictate professors' reading. At national universities, things were a little bit better, but graduate programs in social sciences were only allowed in pre-war universities, such as imperial universities and a few other institutions. In this environment, there were little creative research activities. In most graduate programs in social sciences, faculties tended only to follow research results by foreign scholars and graduate students tended to read books and articles written in English or other foreign languages without critical thinking regarding the contents. There was almost no room for the development of original research in social sciences.

Moreover, the term "university autonomy by faculties" occupied the way of university management after World War II. As a lesson from the governmental control of social sciences, the post-war Constitution of Japan guaranteed academic freedom and the School Education Law put an article saying "universities should have faculty meetings to discuss about important matters for the universities," while declaring in another article that university president should have the power on and the responsibility for institutional management. Faculty positions in national and local public universities were strongly protected by law, thus employment and dismissal could not be done without consent of faculty meeting. By this protection of their positions and little pressure for creative research, some professors in social sciences, especially in economics where Marxian school took the majority, became very politically sensitive and their main activities were to criticize government policies for outwards and, for inwards, they strongly insisted on departmental autonomy against their presidents. This annoyed Ministry of Education and Science responsible for management of national and other universities. 
The economic growth of Japan around the 1960s allowed government to invest in various projects. The campus unrest motivated government to design and to set up a new national university often called the university of new idea (officially, the University of Tsukuba), in which the social science department was designed to be the most radically innovative. For the first (and the last) time among seventy-five national universities (at that time), the University of Tsukuba was set up by the virtually new laws (generally named the law of the establishment of the University of Tsukuba; precisely, the drastic revision of the Law of Establishment of National School and Institutions and other related laws, government orders and the orders of the Minister of Education). After the nationwide hot debates, this law passed the Diet Houses in the early October of 1973 shortly before Oil Embargo in the late October. The sudden depression of economy in the end of 1973 did not obstruct this project.

With no precedent, this "law" stipulates the names of the departments. Among the legally stipulated departments, two are related to social sciences: One is sociology and the second is shakai-kogaku (social engineering or social technology). The governmental order based on the law stipulates the graduate schools and their names of programs including Graduate School of Management and Policy Sciences (simply, policy sciences hereafter).

After World War II, the imprisoned Marxian economists were released and occupied many faculty positions in the economics departments of major universities after the nationalistic professors were all purged by the Allied Forces (virtually the US Forces). A couple of decades after World War II, government regained the power over universities through the budget control, but the paper-and-pen sciences including economics remained nearly out of the control. Government allocated the faculty of shakai-kogaku and the graduate school of policy sciences more budgets than other social science-related ones for the reason that these two use computers.

Against the Marxian theory, Popper presented the idea of social engineering. Shortly after World War I, Russian Revolution, socialism or communism movements in Europe and China, nationalistic self-determination movements in East and Ireland, social engineering denoted the manipulation of the society, close to the socialism or communism. In the late 1930s and the early 1940s, Popper presented the idea of social engineering, the same name but another meaning. With the background of the methodological discussions of quantum physics, he learned the systematic idea (systems engineering in the today's term) from mechanical engineering and presented the idea of piecemeal reform of society by cutting off the long-range view of history from social sciences. But almost all of the faculties of shakaikogaku had never heard of the term of social engineering or the name of Popper. Almost all of the faculties had never heard of the Pound's social engineering view of evolutionary legal system.

In the innovative decades around the 1960s, some staff of RAND Corporation, a most representative think-tank, considered technology as the engine of historical development and as carrying the social meaning. They called this view of technology as social technology. But almost all of the faculties of shakai-kogaku had never heard of the term of social technology or their editing journal "Technological Forecasting and Social Changes" published by a mathematical science publisher. The same publisher was publishing another journal "Policy Sciences" but only a few faculties of policy sciences knew this journal although never opened even a single page. 
Almost all of the faculties of shakai-kogaku and policy sciences were aware only of the disciplinary system in ivory tower and therefore naively believed the disciplinary system as the only possible system or the interdisciplinary system as absurd. Without natural scientific idea, almost none of them knew the success of biochemistry as an interdisciplinary field. When the student organization of the university planned a seminar of Egami, the most representative pioneer of biochemistry in Japan, the university administration ordered the students to cancel it for the reason that he was a leftist. Almost all of the faculties of shakaikogaku and policy sciences naively believed the new social sciences promoted by government merely as the non- or anti-Marxian social sciences or as the US-originated social sciences. Indeed, President and Vice-Presidents of the university repeatedly and proudly pronounced the novelty of the university as the US-oriented university with the USimported system. The first chairman of Graduate School of Policy Sciences and many other faculties repeatedly and proudly pronounced this graduate school as having been established for US-originated economics. Its official publications have never used the term of policy sciences. "The New Idea" of the new university was a novel form of Westimported knowledge. It was really novel as the model of anti-leftist university.

The faculties of shakai-kogaku and policy sciences knew the term of cybernetics and the name of Wiener but only the term and the name. Some regarded cybernetics as a pro-Marxism social science. Almost all of the faculties regarded computers merely as statistical data calculators rather than information processing systems. Allocated an enormous amount of budget by government, some of the faculties put a huge amount of numeric data into computers and simulated every combination of possible cases of economic situations and took pride of this as a new social science.

Originally, the University of Tsukuba was not designed for the innovation of natural and social sciences and humanities. At first, this project was called the relocation of university rather than the design of university with new idea. Actually, it was motivated to promote economy at the cost of universities and R\&D institutes. More specifically, it was mainly motivated for more profitable use of land within Tokyo by relocating "useless" institutes to rural areas. The leading members of the planning board of the university (mainly physicists) ignored the balanced development of natural and social sciences and humanities, and once seriously planned to exclude social sciences and humanities as useless. Their view of physics as the model of all knowledge affected the design of the new university, and viewed social sciences and humanities merely as ideologies, either left or right without scientific base.

Government promised universities and R\&D institutes to increase the budget if they "sell" the expensive land in Tokyo" to more "profitable" sectors. This promise was partly broken. Government including the Ministry of Education and the Science and Technology Agency (now Ministry of Education and Science) failed to recognize the importance of information exchange of universities and R\&D institutes with governmental organizations including budgeting offices. This forced professors and scientists to consume many hours to visit the government offices in Tokyo for budgeting negotiation [Eto, 2005]. Far away from government and business centers, social scientists were engaged in mathematical tools of social sciences with little relevance to real societies.

It has passed nearly forty years since the establishment of the University of Tsukuba. Although Tsukuba is still called the University of New Idea, the environment surrounding higher education has been completely changed. One of the biggest turning points of change 
was around 1990, when the Cold War ended by disintegration of former Soviet Union, and the bubble economy in Japan was collapsed, which later seriously affected Japanese politics and economy. Left-wing opposition parties decreased their seats greatly in the Diet and people who used to oppose the higher education policies by the government lost strong support by such parties. At the same time, the ruling Liberal Democratic Party also decreased their seats in accordance with the growth of centrist parties, which caused the political instability. In this situation, the main concern of universities and faculties shifted from the protection of university autonomy to the acquisition of more financial support from government. Science and engineering faculties were particularly so, and some national universities such as ex-imperial universities succeeded in getting more resources by the "prioritizing of graduate education" policy, which was initiated by the Ministry of Education and Science for the purpose of improvement of research infrastructure of selected universities [Yamamoto, 2007].

The second big turning point was in 2001-2004. In 2001, Prime Minister Koizumi took office and immediately started to implement reform policies in various fields, particularly in the postal services, based on his radical market-oriented mind and neo-liberalism thought. In higher education, "Toyama Plan" was opened in public without pre-negotiation with national universities. This was very unusual in decision-making style in Japan, where the negotiation beforehand is crucially important for consensus formation [Eto, 1984]. The plan aimed at the radical reform of national university system, i.e., merging and reorganizing of existing national universities, the introduction of managerial method developed in private enterprise, and the introduction of competitive environment based on third-party evaluation of universities. The plan said only 30 institutions were allowed to grow as research-intensive universities by the selective resource allocation of the government. By this plan and for related reasons, the university reform in Japan progressed swiftly and reached to the incorporation of national universities in 2004. In the same year, the accreditation system of universities and colleges was also introduced. Every university and college must be evaluated by one of the authorized accreditation agencies every seven years and the failure of being accredited might cause serious problems on the further existence of universities and colleges.

The third and the biggest problem particularly for private institutions continued to grow. It is the decline of 18-year-old population. It was 2 millions in early 1990s but it has continued to decline to 1.2 million in 2010 and will be 0.7 million in the middle of the $21^{\text {st }}$ century. Because of the heavy reliance on young students, it is very serious for universities in Japan. Data provided by the Ministry of Education and Science says that nearly 95 percent of university freshmen are at the age of 18 or 19. The number of adult freshmen over 25 years old is only 2 percent, while the average of this figure at the OECD member countries is 20 percent. By the decline of the population, about one half of private universities did not enroll the enough numbers of students in 2010 and this will cause the decrease of their tuition revenue. This is directly related to the managerial problems. In this situation, student recruitment activities have become serious, and the raise of the attractiveness by university reform is the top priority of their institutional running.

Teaching of social sciences has become more application-oriented, practical and vocational oriented in many institutions in response to the needs of students, while research in social sciences is needed to reform fundamentally. After the Report of Higher Education System in 
the $21^{\text {st }}$ Century issued by the National Council on Education in 2005, the public concern and governmental policy on higher education have shifted to the quality assurance of university teaching, which means the existing way of and contents of teaching must be examined and improved so that Japanese higher education may be more recognized globally. The Science Council of Japan issued a report on quality assurance for university education in each field in 2010 by the request of the Ministry of Education and Science. Although the Council says only the basic idea and does not give details on the contents and levels of each field including social sciences, more concern will be focused on the contents and levels of teaching in details. It is seriously feared for the standardization of university teaching in the field of social sciences in the near future. It may either kill the fundamental character of university education, which should be different from that of secondary education at high schools, or it may improve the quality of teaching and research on social sciences to some extent.

In the 2010s, the relationship between universities and government is expected to be different from that in the 1960s and 70s. Social sciences in Japan are expected to be more than the tools for opposing the governmental policies and rather important measures to educate students for good citizens with rich culture. Social sciences should be more contemporary with society, and students should learn the analytical and critical way of thinking through lectures. As for research in social sciences, the relevance to the actual society in Japan should be stressed for their further development, while the originality and quality should be improved for global recognition. However, the fact shows that the Japanese way of management and the theoretical and empirical studies on it were often ignored in international journals or remained only as a "Galapagos species" [Eto, 2008c]. In this situation, it is feared that the stress to increase original research activities might drive social science professors just to "apply" foreign-made theories to Japan without regard to the reality of Japan.

\section{Conclusion, limitations, remaining problems and future research}

This chapter discussed the mutual relationships between social and natural sciences, humanities, and technology in regard to their methodological influences. The discussion was mainly or nearly exclusively focused on the western knowledge including higher education systems. The non-western ones were mentioned only as a case of West-influence on East. But one can not ignore the historical fact that many of the western ones originally came from East and that East developed its own ones. The eastern ideas and systems have developed in different ways (sometimes analogous but often opposite ways) from the western ones, but are often ignored even within East. The same comment holds for South. This chapter is no exception.

Highly but locally developed knowledge or intellectual systems in East are often regarded as Galapagos species or isolated minorities and are ignored or despised or even hated as enemies in the West-dominant world like non-European languages and non-Christian religions. Many crops were bred and domesticated from wild grasses in central or western Asia in dry climate unfavorable to agriculture and transferred to West, where very few crops were originated [Doebly, 2006; Balter, 2007]. That is, West just imported knowledge from East as well as Africa (e.g., cattle keeping), American Continent (e.g., potato), Pacific Islands (e.g., banana) and, besides food, Siberia (e.g., the domestication of dog). Long after 
that, West began to build the leading civilization. In analogy, Eastern civilization may take time to be recognized worldwide as Greek culture was recognized in Europe nearly two thousands years after. The Buddhists and Hebrews predicted the declines of their knowledge and the revivals again. The same comment may hold for the knowledge of Africans, American natives and Pacific Islanders.

Under the globally dominant western systems in societies and knowledge, non-western countries accept and follow the western knowledge system to avoid the isolation [Eto, 1993]. But the non-western ones survive in the invisible undercurrents. For example, western ideabased laws are radically modified in enforcement by police, prosecutors and judges in nonwestern countries, where law is one thing and the enforcement is another. In such situations, many universities of Japan avoid to offer the lectures of jurisprudence and Constitution. Another example is the so-called the Law of the Establishment of the University of Tsukuba (officially claimed as the university of new idea). This law stipulates to establish the department of shakai-kogaku (social engineering), but actually in its implementation only a few years after the pass of the law in the Diet, the department of shakai-kogaku (social engineering) was semi-officially replaced with the department of economics in accordance with the traditional idea of university.

Related to the view of humans as homo economics and an economic sin, a Buddhist Honen in the suburbs of Kyoto in the 1100s discussed so great power of the Buddha's palm to remedy any evils and cleanse any sins. Here, evils and sins mainly denoted the economic activities for profits that were prohibited in the classic Buddhism. His theory was developed further by his disciple Shinran, and their school called the Jodo School is today the most popular among the Buddhism schools in Japan. Here Jodo literally means the pure or sinless land as the quasi-paradise for general citizens or paradise above the purgatory and below but near Heaven. Honen and Shinran admitted all common people to paradise if not Heaven and thereby approved economic activities as remediable on the great palm of Buddha. Later, however, the economic power overwhelmed the power of the great Buddha's palm and destructed the agriculture-based society.

The classic ideas valued the agriculture and undervalued commerce. But agricultural activities kill noxious insects. Therefore, the Indian Jainists were engaged in commerce to avoid killing, while living in extremely simple manners. The commercial activities were free from $\sin$ if not for profit but for just distribution. The Muslims were engaged in the commerce between the East and the West but avoided the capital gain to lend money at interest. These traditional wisdoms are not known among modern economists. This blind spot must be clarified and the gap between modern western ideas and the classic nonwestern ones must be filled someday.

This chapter did not discuss the ethics of social scientists. The mega-quake and tsunami in 2011 affected the ethics of natural scientists, engineers and medical doctors. After the disaster, they participate in social activities. The public also expects the social scientists' activities, but finds that they remain relatively inactive possibly because of the university reform, which seems to affect the ethics of social scientists in the opposite direction from that of natural scientists.

This chapter merely followed the prevalent idea to regard the Greek culture as the origin of the scientific ideas. But some physicists date back the origin of the relativity and the 
quantum theories to the Indian Vedas, 3000 or more years old. The ideas of evolution and genetics could date back to the origin of breeding grains or cattle from grasses or wild animals in East Africa, and West or Central Asia in the Neolithic Age. Scientific evidences demonstrate that the species of dog (Canis familiaris; family Canidae) was bred from species of wolf (Canis lupus; family Canidae) about or over 10,000 years ago in East Siberia [Savolanien, et al., 2002]. The currently prevalent idea to date back the origin of the modern idea to the Greek classic era might be needed to reconsider on the basis of evidences.

\section{Acknowledgement}

The authors heartily thank Professor H. Matsumoto, Japan Coast Guard Academy, for his providing us with the information of the studies of law, and Professor K. Takemura, Waseda University, for his useful advices on psychometrics.

\section{References}

Balter, M. “Radiocarbon dating's final frontiers”, Science, Vol. 313, pp. 1560 - 1564, 2006.

Balter, M. “Seeking agriculture's ancient roots”, Science, Vol. 316, pp. 1830 - 1835, 2007.

Bentler, P.M. "Structural modeling and Psychometrika: An historical perspective on growth and achievement", Psychometrika, Vol.51, pp.35-51, 1986.

Benzécri, J.-P. L'Analyse des Données. tom 1. La Taxonomie (The Data Analysis, Vol. 1, The Taxonomy), Dunod, Paris, 1973.

Bollen, K.A. Structural Equations with Latent Variables, John Wiley, New York, 1989.

Camerer, C.F. and Fehr, E. "When does 'economic man' dominate social science?", Science, Vol. 311, pp. 47 - 52, 2006.

Doebly, J. “Unfallen grains: How ancient farmers turned weeds into crops", Science, Vol. 312, pp. 1318 - 1319, 2006.

Eto, H. "Behaviour of Japanese R\&D organizations -- Conflict and its resolution by informal mechanism", in Eto, H. and Matsui, K. (Ed.) RED Management Systems in Japanese Industries, pp. 139 - 239, North-Holland, Amsterdam, 1984.

Eto, H. "Prudence of science and technology policies: A historical review", in Eto, H. (ed.) RED Strategies in Japan -- The National, Regional and Corporate Approach, pp. 225 296, Elsevier, Amsterdam, 1993,

Eto, H. "Interdisciplinary information input and output of nano-technology project", Scientometrics, Vol. 58, pp. 3 - 31, 2003.

Eto, H. "Obstacles to emergence of high/new technology parks, ventures and clusters in Japan", Technological Forecasting and Social Change. Vol. 72, pp. 359 - 373. 2005.

Eto, H. "Scientometric definition of science: In what respect is the humanities more scientific than mathematical and social sciences?" Scientometrics Vol. 76, pp. 23 - 42, 2008a.

Eto, H. "The philosophy of science\&technology policy," http://topics.scirus.com/The Philosophy of Science\&Technology Policy.html. Elsevier, Amsterdam, 2008b,

Eto, H. "National management system in global era: Methodological assessment of its possibility", International Journal of Business and Systems Research, Vol. 2, pp. 325 342,2008 c.

Gazzaniga, M.S. The Ethical Brain, Dama Press, Washington DC, 2005.

Haidt, J. “The new synthesis in moral psychology”, Science, Vol. 316, pp.998 - 1002, 2007. 
Hand, D.J. “Data mining: Statistics and more?" American Statistician, Vol. 52, pp. 112-118, 1998.

Hayashi, C. Chosa no Kagaku (Survey Science), Kodan-sha, Tokyo, 1984

Hayashi, C. Data no Kagaku (Data Science), Asakura, Tokyo, 2002

Johnston, J.S. Inquiry and Education: John Dewey and the quest for democracy, State University of New York Press, Albany, NY. 2006.

Jöreskog, K.G. “LISREL”, Behaviormetrika, Vol.24, pp.95-102, 1997.

Kemsley, J. "Philosophizing Chemistry, Philosophers delve into the central science", CEEN (Chemistry and Engineering News), Vol. 87, No. 40, pp. 41 - 42, 2009.

Kruskal, J.B. "Nonmetric multidimensional scaling: A numerical method", Psychometrika, Vol.29, pp.115-129, 1964.

Kuhn, T. The Structure of Science Revolutions (monograph: International Encyclopedia of Unified Science, Vol. 2, No. 2). 1962.

Kuroki, T., Rakkasan Gakucho Funsenki (How I Fought Alone as the University President), Chuokoronsha, Tokyo, 2009.

Monbu-Kagaku-sho (Ministry of Education and Science), Monbu-Kagaku Hakusho (Education and Science Whitebook), Tokyo, 2010.

Nielsen, L., and Mather, M., "Emerging perspectives in social neuroscience and neuroeconomics of aging" Social Cognitive E Affective Neuroscience, Vol. 5, pp. 149 164, 2011.

Olsen, J.A. Principles in Health Economics and Policy: Distributing health care, Oxford University Press, Oxford, 2009.

Savolanien, P., et al., 'Genetic evidence for an East Asian origin of domestic dogs', Science. Vol. 298, pp. 1610 - 1613, 2002.

Tukey, J.W. Explanatory Data Analysis, Addison-Wesley, Cambridge, MA. 1977.

Warmeken, F. and Tomasollo, M. "Altruistic helping in human infants and young chimpanzees", Science, Vol. 311, pp. 1301 - 1303, 2006.

Whiteside, G.M. "Revolutions in chemistry", CEEN (Chemistry E Engineering News), Vol.85, No.13, pp.12-17, 2007.

Yamamoto, S. "Doctoral education in Japan," in Powel, S. and Green, H. (Ed.) The Doctorate Worldwide, pp. 181-193, Open University Press, Berkshire, 2007

Yan, Z. et al. "Patient satisfaction in two Chinese provinces; rural and urban differences", International Journal for Quality in Health Care, Vol. 23, pp. 384 - 389, 2011.

Yoshimura, T., et.al. (Ed.) Policy Science, Center for Multimedia Education, 1982

Zweifel, P. and Breyer, F. Health Economics, Oxford University Press, Oxford, 1997. 


\section{Section 2}

Quantitative and Qualitative Approaches 



\title{
Causality in Social Studies Education
}

\author{
Bayram Tay \\ Ahi Evran University, \\ Turkey
}

\section{Introduction}

Science is a scientific process which uses scientific methods to obtain information, and it studies the facts within their relations, not individually. In this regard, individual facts and objects have no scientific importance. Therefore, science aims at observing and explaining the relations between facts which seem desultory at first (Yildirim, 1998). As a consequence, it can be said that scientific information includes the cause and effect relation. The cause and effect relation can be expressed by causality concept.

\section{Historical and philosophical foundations of causality}

Causality is usually defined as the relation between cause and effect. There are two terms constituting the causality. These are the terms of cause and effect. Cause can be defined as a thing, a condition or a creative agent-a thing making something emerge thus without its existence it is impossible for this something to exist-that has the capability to change a thing and create a phenomenon or an event (Cevizci, 1999; Akarsu, 1998). In Latin, there are two words which mean cause. The first one is "causa" and the second one is "ratio". Although "ratio" means "mind" or "intelligence" it also means "cause" (Ozcinar, 1997). The Latin usage of this word appears to show a relation between intelligence and cause. It cannot be coincidental that "ratio" means both intelligence and cause in Latin because intelligence everyday runs different activities to answer the word cause. Human curiosity and surprise which are first directed to them and then to the nature also revealed the need for them to be able to explain and understand both themselves and the nature. This need brings various "whys (cause)" along with it, and human mind tries to answer (solve) these questions. Answering the whys, i.e. finding out the consequence, is a mental activity which is a typical example of causality itself. Consequently, causality can be expressed as a principle of reasoning.

Effect, which is the answer of cause, can be expressed in philosophy and science through the terms of impression or result. So effect can be defined as any event resulting from a certain cause, any impression stemming indissolubly from a cause, any fact found out based on a factor or any phrase succeeding a condition which expresses the phrase and a certain result (Cevizci, 1999). By this definition, the emergence of effect can emerge depends on a cause or a condition. However, it can be concluded here that effect/impression expression is a mental process since explaining an expression stating a condition requires a mental activity. Thus causality is the term for the relation between cause and effect. That is to say, causality 
is the glue binding entities to each other in time (Denkel, 1996). Causal explanation also entails processes which "accord with reality" to belong to reality, namely; it entails the reality itself to be constituted of process-control (Piaget, 1982).

The fact that no common definition for causality is provided in all scopes of science, and it has been a controversial issue requires the debates to be reviewed only in general terms in order to comprehend it. Aristotle, who classified causality first, distinguished between four types as follows: the material cause (causa materialis), the efficient cause (causa efficiens), the formal cause (causa formalis) and the final cause (causa finalis) (Aristotales, 1985; Yildirim, 1997; Cevizci, 1999). Aristotle's final causality explanation also influenced the Middle Age philosophers and provided the basis for the Middle Age scholastic philosophy. Until the $16^{\text {th }}$ century Aristotle's thoughts were the primary source of reference in solving a scientific problem (Berry, 2003).

Although Aristotle emphasized sense data and founded the sciences of nature by observation, depiction and classification methods, the traditional sciences relying on his thoughts lost their functionality in 17th century. However, human sciences which particularly appeal to imagination became unsatisfactory in the same way as they expressed some contradicting values with uncertain validity (Northrop, 1998). This conception began to disappear during the Renaissance while improvements achieved gave rise to modern philosophy. From the perspective of causality, the idea that the anything which happens in nature depends on a firm causality, is also an output this period (modern ages) (Reichenbach, 1998). In modern philosophy, knowledge came to the forefront, and epistemology was given priority. Arguments about the source of the knowledge introduced two different views, which are the rationalist (intellectual) and the empirical (experientialist) approaches. While rationalism accepts the intelligence as the primary and the only indispensable source of our knowledge, empiricism gives precedence to the senses and the things which can be observed directly (Ozlem, 2003).

Empiricism and rationalism are two key arguments of causality (it is also true in terms of epistemology). Yet those advocating empiricism attribute causality to experiment while those advocating rationalism attribute it to intelligence. Descartes, Leibniz and Spinoza can be remarked as the supporters of rationalism, while Locke, Hume, Hobbes and Berkeley can be remarked as the supporters of empiricism. However, Kant who was a rationalist initially and later influenced by Hume can be remarked as both a rationalist and an empiricist (Ozlem, 2003).

David Hume's and Immanuel's causality explanations constitute significant arguments, and therefore knowledge of the outlines of thereof will be helpful. Hume explained the causality concept and tried to base his explanation upon experimental conscious of causal relationships by utilizing Newton's experimental approach (Cevizci, 1999). He used the words "impressions and ideas" to explain causality, which in modern philosophy appear in the form of sensory experience and concept. He also suggested that all our knowledge derive from impressions (sensory experience) which generate an idea in our minds (Hume, 2000; Denkel, 1996).

According to him, it is not coincidental that our ideas have certain relationships and are connected to each other. He argues that there are some connectives binding our ideas in three ways, i.e. resemblance, contiguity in time or place, and cause or effect (Hume, 2000; Cevizci, 1999). 
Hume also suggests that there is a reasoning not based on experiment but on the cause and effect relation which underlies our knowledge of facts. He emphasizes this knowledge emerges by seeing certain objects are constantly conjoined and experienced, or in other words; it emerges empirically (Hume, 1976). In this respect Hume describes eight criteria for cause and effect relation. These are as follows:

1. Cause and effect must be contiguous in sense of time and place.

2. Cause must occur prior to effect.

3. Cause and effect must be constantly connected with each other.

4. The same causes generate the same effects, i.e., the same effect doesn't occur unless there is the same cause. This principle is gained through experiences and mostly derives from our intellectual causality.

5. Several different facts (things) can have the same effect. This must be by the quality of the event which is common among them. However, similar causes imply similar effects; we always attribute this causality to this environment where we find or discover similarity.

6. This principle is formed on the basis of the previous principle. Several different similar effects can differ from each other at the same level or the same way. We have to consider this difference (in respect to irregularity) in causality since similar effects imply similar causes.

7. If the cause in facts increases or decreases, it is accepted as emerging from an integrated effect, or integration of several effects or different parts of the cause, i.e., the presence or absence of the cause is related with the ratio of presence or absence of effect. This necessary connection proves that a certain part, that is the effect, is the cause of the other part. When warmth and happiness (contentment) are exemplified, happiness increases as warmth increases a piece, and vice versa. Again, a certain amount is intended here.

8. A fact which emerges in any time duration and has no effect is not the only cause of the effect. Nevertheless, it must be supported by the other principles. For instance, similar causes are expected to succeed similar effects. It is must be supported by the principle of contiguity in sense of time and place. Yet, it cannot be the sheer cause.

Although Kant seems to be a supporter of rationalist epistemology, in essence, he is both a rationalist and an empiricist since he doesn't fully or absolutely agree the dogmatic views of rationalists also the views of empiricists who try to explain everything by means experiments. $\mathrm{He}$ is critical of both views. Thus, his thoughts about causality are both empirical and rationalist (Kant, 2004; Kant, 2006; Gunay, 2004). Kant regards the causality as the beginning of the work for classifying the thoughts (knowledge) and the center of philosophical arguments, and he suggests causality evokes particularly experiencing (Ewing, 1969).

Hume and Kant have different points of view about causality principle. In general terms, Kant is considered as rationalist (intellect), Hume as empiricist. Their causality explanations differ due to the views they represent. Causality is briefly explained here with regards to empirical and rationalist views.

Let us consider two facts which are $\mathrm{X}$ and $\mathrm{Y}$. $\mathrm{X}$ stands for cause and $\mathrm{Y}$ stands for effect (influence, consequence). According to this; the judgment that $X$ is the cause of $Y$ must describe a phenomenal connection observed between $X$ and $Y$. It means whenever $X$ emerges, $\mathrm{Y}$ also emerges. This relation which is an observational subject is a companionship. 
Asserting $X$ is the cause of $Y$ means $Y$ always follows $X$, or $X$ and $Y$ always go with together. In this context causality expression has no further meaning. The relation between $X$ and $Y$ does not express a necessary connection or a tie. As for rationalists, while they do not deny the contribution of observation in causality, they assert observation alone is not sufficient to explain causality and they also suggest existence of something beyond observation, i.e. metaphysical explanation, is necessary. In this sense according to rationalists, causality occurs in two stages. The first stage is observation, and the second stage is necessary connection that is beyond observation. Empiricists criticize the rationalists on the grounds of their metaphysical explanations being non-scientific (Yildirim, 1998; Ozlem, 2003).

As it is understood from these explanations causality, according to empiricists, is an outcome of our experience or habits but according to the rationalists, it is a necessary connection and a result of our prior knowledge which often doesn't rely on experience. In this context, causality is a basic principle that is used in both natural sciences and social sciences (history-sociology, cultural sciences, and spiritual sciences) and also these sciences rely on this principle.

\section{Causality in social sciences and social studies}

Human's effort to explain and understand themselves first and then the environment they live in lies on the roots of natural and social sciences. Environment refers to natural and social neighborhood since a human being lives as a social being in the natural environment. Having two living spaces, i.e. natural and social, causes the human being to perceive, understand, set up and organize two different environments. Clarifying the nature-related activities is based on a certain systematic, and easier than those which are related with social surrounding. The order of nature is the main factor for this fact. However, the order is at the moment, and as it involves cosmologically a long period it is more usual to define and explain the events with regards to repetition and continuity which involve shorter periods in social events. Thus, it is well known that human life and social facts in antiquity were different from later times. Both human lifestyle and their scientific activities were affected by the properties of the period they lived in. In this regard the first "cause" which emerged with polytheism in antiquity was different from the "cause" seen in monotheism in later periods. However, improvements in science and technology affecting human life in different ways, even faster today, made it more difficult for society-related sciences to systematize the events and phenomena because repetition and continuity decrease in regard to time.

The philosophy of social sciences deals two forms of dualism, i.e. understanding-explaining and cause-meaning, and the scientific efficiency of social sciences will rise in line with the extent to which these two forms are able to be explained. The definition of the term "general" substantially differs in social and natural sciences, therefore the one used in social sciences should be defined first in order to understand them both. The term "general" used in natural sciences is timeless and universal which means having repetition and continuity at a high percentage (covering cosmologically a long period). However, the situation is quite different in social sciences as in most cases events do not re-occur. For instance, the events such as the Independence War of Turkey or French Revolution are unique events, and they cannot occur twice. However these events happened or remained with their inner and peculiar properties. It is not possible for them to re-occur with different properties and consequences, and they can only be comprehended by the aid of their peculiar properties. 
Understanding them is only possible by knowing or taking into account all the properties of the period in question. Therefore, in social sciences the term "general" cannot be timeless and universal, which by no means rules out the use of term "general" in social sciences. General is peculiar to that period; it is in its mechanism and often occurs once (Ozlem, 2003; Fay, 2005).

Another characteristic of sciences is revealing to which extent a decisiveness occurs between rules-principles and actions. In social sciences, it is known or should be known that between the rules-principles and actions, there is not an absolute consistency but a contingent, consistency with a percentage. Therefore, there is a contingent causality in social sciences and explanations are deductive as in natural sciences. In this respect, explanations provided by social sciences, which have premises comprised of "meaning related" propositions, require understanding the action first. Thus, the explanation will require describing in which period, how and in which way the thing that is to be explained happened. Then, as the last activity "understanding-action" relation will be considered (Ozlem, 2003).

The term "general" used in social sciences and natural sciences can be described as follows (Ozlem, 2003):

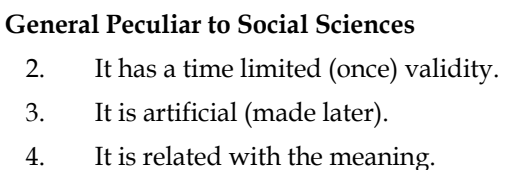

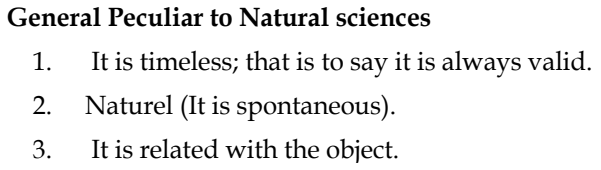

Table 1. General in two science groups (Ozlem, 2003)

When table 1 is reviewed it can be said that social sciences are peculiar to human while natural sciences are peculiar to nature. Keep in mind that the term general related with human is contingent. Since this contingency constitutes the properties of knowledge it means that the causality concept in social sciences will be different from the causality concept in natural sciences. As a matter of fact, natural sciences have been explained through determinist causality for years. This determinist approach ( $a$ is the cause of $b$ ) was faltered with quantum physics and disappeared. Instead, a probabilistic causality understanding became dominant (instead of $a$ is the cause $b, b$ probably follows $a$ ). In this respect causality perception of social sciences and natural sciences seem similar but the properties of timelessness (present), spontaneity, repetition and continuity (with regard to cosmological time) of natural sciences are very limited in social sciences. What made the causality concept differentiate is that natural sciences deal with things unlike social sciences, which deal with human. Therefore, causality is probabilistic in natural sciences but it is contingent in social sciences. It cannot be said that causality concept has been accepted in the same way by the social scientists from past to present. In social sciences, causal explanations focused on the factors which engender cultures and their change, unintended results, functional relations and genealogical origins (Fay, 2005).

Causality relations in social sciences differ from bare coupling principles. According to Fay (2005); "the cause of e is c" is completely different from "c occurred and then e occurred". The fact that c occurred before e doesn't mean c caused e. However, saying that $c$ is the cause of e means c's occurrence is a necessary or sufficient condition for e's occurrence. If e 
can occur without c, or if e doesn't occur despite c's occurrence then c is not the cause of e. As it can be understood from this explanation, causal propositions state condition. It is like if c occurs so will e or if c didn't occur nor would e. But to verify such a relation it is necessary to show why e and c are correlated beyond coincidence and in this respect unlike the bare coupling principles, it should explain all the examples of a causal proposition.

It is seen that in social sciences, causal explanations are provided with genetic explanation. Genetic explanations present the explained e event as the last ring of a string of events which engender this. Therefore, such explanations are based on depiction (description) of successive phases which make e. In genetic explanations, there aren't only phases successively put together, that is to say there is not only chronological ordering. It is necessary to show that all phases produce the next phase to finish in the same way like in the aforementioned event. Here, the explanations are stated as "under the conditions of ...". This is due to the fact that, in social sciences most events are often once and in genetic explanation of the event, it is stated that the event happened under the conditions of that time and the result of this happening; in other words, its consequence emerged under the conditions of that time (Fay, 2005).

There is a significant relation between causal structure of genetic explanations and their efficiency. According to Fay (2005), standard causality evaluation has been made in the context of language of necessary and sufficient conditions since Mill. Most of the social scientists deal with the concept of causality in this way. According to Mill's analysis, if $\mathrm{c}$ is the cause of e, c is the sufficient condition or necessary condition of e. For a causality which is dealt with in the context of necessary and sufficient condition, albeit hidden, the need to refer not only to certain c events but also to a rule which expresses an unchanging functional relation between $\mathrm{C}$ (independent variable), which is a class of events and E (dependent variable), which is another class of events, emerges. When Mill's causality thoughts are reviewed, it is found out that the genetic explanations in which certain causal relations are suggested are implicitly based on some general rules. Most of the social scientists do not approve reaching such general rules as there is singularity in social events and it is not explained by any general rule. However, Fay states that Mill, in his own causality explanations, asserts the existence of an absolute general rule in a causal explanation which covers the events that are dealt with.

Davidson (1970) states that causal explanations are heteronymous or homonymic (intrinsic). Homonymic (intrinsic) explanations have the same vocabulary and structure with the basic rule of the explanation. Heteronymous explanations are the explanations which require using completely different terms from its own terms in order to express the rule on which they are based (Fay, 2005).

In social sciences, causal reality has been used to explain the causality. According to this view, the cause of an e event is a basic mechanism which results in e under appropriate conditions. These underlying mechanisms have natural forces, and these forces are causally responsible for partial results. Thus seeking for the causes is not looking for general rules but for such mechanisms (Fay, 2005).

Although there is not an agreement on the causality concept, it is an indispensable concept in explaining social sciences. Causality concept in social studies, which created a study field by using the information produced by social sciences, will be explained again in the same way that social sciences use it. In this sense causality in social sciences and causality in social 
studies will be in line with each other. When social information is defined as a field of study (Savage and Armstrond, 1996) comprised of humanistic and social sciences aimed at improving civic skills, it is seen that it involves social sciences such as history, geography, economics, sociology, anthropology, psychology, philosophy, politics and law. In this sense, without further detailing, it is beneficial to address the methodology of history and science of geography and their characteristics related to causality. History and science of geography will be addressed as they have respectively a larger part and more subjects in social studies.

History can be defined as a scope of science, which studies and explains the events related with human within the frame of past-present and future considering the cause-effect relations and grounding it to documents. From this point of view, it is seen that history is founded within the frame of the human being's past, grounding to documents and causeeffect relation concepts. This shows history is directly related with causality. It is also known that every science has its own terminology that is its own language. In this respect questioning the existence of causality concept in the language of history can be necessary. Hence, it will be probable to find causality if the language of history is reviewed. On that account applying to classification of the language of history by Husbands will be appropriate. Husbands categorizes the language of history into four. These are as following:

1. Historical Time Language (era, century, period, Middle Age (Middle time), modern),

2. Language of The Historical Period (cause, chronology, similarity, difference, etc.),

3. Language of Historical Depiction and Analysis (revolution, monarchy, triearchy, democracy, etc.),

4. Language of the Past (special terminology) (demesne, kadi, governor etc.) (Husbands, 1996; Dilek, 2002).

Cause, chronology, similarity and difference expressions in the language of the historical period seem to explain the methodology of history, because while explaining the events history will order (chronology) them from past to present, detect the similar and different aspects thereof and eventually will focus on their causes by depicting what kind of a relation exists between them. Explaining the causes is one of the fundamentals of science of history because according to Carr (2006), history reviews the causes. Historians also continuously need to ask "why" questions in order to reach historical truths (Uzun, 2006). Since the answers to "Whys" bring out the cause and effect, it can be necessary to explain historical events considering this fact. In this sense, the need to determine a historical event's relation with the cause and effect can also emerge. A historical event has a characteristic, that is to say; it is the effect of a former event and the cause of a subsequent event. It is explained in the following example.

Sentence 1: The Armistice of Moudros is a cease fire treaty, concluded on 30th October 1918, between the Ottoman Empire and Allies declaring the war was over.

If the sentence is analyzed; The Armistice of Moudros is an effect of World War I.

Sentence 2: After the Armistice of Moudros was signed, Mustafa Kemal and his comrades evaluated the situation and set off to Anatolia on 19th May 1919 and started the War of Independence.

If the sentence is analyzed; The Armistice of Moudros is the cause of starting the War of Independence. 
A historical event appears as an effect of a former event and as a cause of a following event. It can be explained in history by causality concept. So we can say that the cause (reason) which exists in the language of history and its questioning in history paved the way for us to reach the causality concept. Therefore, historians cannot neglect the causality principle in history writing.

Voss (1998) stated the difficulty and complexity of apprehension of causality in history, particularly when it is considered in a historical aspect. This view about the complexity derives from the fact that the causes of historical events can be various, that is to say; they can be expressed in different ways.

Another difficulty of historical causality is that history has no control group like life. Events and actions take place only once. It means in order to make a particular effect, setting or detecting some situations, event or actions necessary and/or sufficient should be formed without using experiential ways (Voss, 1998). Explaining historical events and together with presenting the causality will be possible within the uniqueness of the events. After all history is idiographic with this feature.

In social studies, geography is another discipline, which has one of the largest parts with regards to its subjects. Geography, like history, is an application people have been performing for a very long time and with this feature, it is one of the oldest study fields (Gulbenkian Komisyonu, 2005; Ozguc and Tumertekin, 2000). Geography began to renew itself as a discipline substantially in German universities at the late nineteenth century. In this period, geography aimed at filling the gap between natural sciences and human sciences (Gulbenkian Komisyonu, 2005).

Geography with its simplest and the most common meaning is the introduction to the world (Doganay, 1992). Within the frame of our subject, it is possible to find causality in geography in its purpose. Doganay (1992) states that the essential purpose of geographical view accordingly the purpose of geography is; "to study the formation causes and effects of geographical phenomena...". The abovementioned study of causes and effects will lead us to the causality concept. One of the main aims, probably the most important, of geography is to study and reveal the reciprocal relations between human-environment and scientific effects of these relations on human. While doing this it is seen that the two views, i.e. determinism (environmentalist view) and possibilism (humanistic view), have been supported as the methodic approaches in geography since the late nineteenth century till today (Doganay, 1992; Ozguc and Tumertekin, 2000). They cover the causality concept with their context. According to the determinist view, natural environmental factors are dominant on human (society), therefore, while analyzing human-environment relations in geography primarily environmental factors should be focused on in the first place, but according to the opposite possibilist view human is not under the control and hegemony of environment, but the environment is under control and hegemony of humans thesis is supported. It is well known that determinism constitutes the ontological aspect of causality. In other words, it constitutes the deterministic aspect of the nature (natural environment in geography). As a matter of fact, the supporters of determinism in geography primarily accept the natural environment's influence. In this respect, geography methodologically involves the causality concept of geographical. 
Various methods are used in Geography. These are rationalism (reasoning), experience (experiment), monocausal and multicausal explanations. The monocausal and multicausal explanations among these also indicate the existence of causality in geography. Although monocausal explanation has an extensive range of application in human and economic geography, it is well known that monocausal events are not common in geography. But multicausal explanations emerged on the basis that various and different factors are influential on social events' occurrence (Gungordu, 1997). Both of these explanations can be expressed through causality.

There are three principles referred to when the basic principles of geography are considered: distribution, interrelationship and dependence, and causality. Researches in geography are carried out or should be carried out considering these three basic principles. The distribution principle expresses the distribution or presence of geographical subjects in a certain field. The interrelationship and dependence principle proposes that all facts and phenomena which are subjects of geography are related with another event or phenomenon. The third principle is causality and can be expressed as everything has a cause (Doganay, 1993).

Causality concept which is a requisite for scientific knowledge has a significant place in social sciences, consequently in social studies. As it is explained above, social studies getting information from many sciences, that is to say, benefiting from many disciplines, integrating this information in an interdisciplinary way emerged the necessity that the information, which constitutes the social studies, should be generated scientifically and have scientific consistency. Social studies curricula which will be created in this sense must be prepared considering this feature. Hence, it can be said that in social studies content curricula, especially that was prepared as a product of constructivism in United States of America, New Zealand, Israel, Canada, Switzerland, Australia and Turkey, has this property.

\section{Development of causality in children}

When the studies about the development of causality in children are reviewed (De Avila ve Struthers, 1967; Berzonsky, 1971; Fuson, 1976; Stepans and Kuehn, 1985; Schlottmann, 1999; Spiliotopoulou, 2001; Charles, 2003; Inagaki, Hatano and Inagaki, 2006 et al.) it is seen that they are based on the studies made by Piaget. Piaget, (1969) in his work "The Child's Conception of Physical Causality" under the chapter which he named "Causality and Child" states that causality has 17 types in children. The 17 causality types which were determined by Piaget about the development of children's causality concept take place in three main stages. Two of them are precausality while the third stage is real causality. Those causality types are shown in Table 2 .

1. Psychological Causality or Causality about Motivation/Stimulation (Psychological Causality/Motivation Type): It is the most primitive type of causality and the most continual of other causality types. Psychological causality is in the precausality stage. In his explanations, a child uses psychological expressions and expressions based on motivation. "God or people send us dreams because we did what we should have done." (Piaget, 1969), "The nightmares are sent to us because we are bad." and "Tomorrow it will rains because someone wants us to be ill." (Charles, 2003). These kinds of sentences are examples for children's such explanations. Piaget thinks that this way of thinking continues for a very long time. 


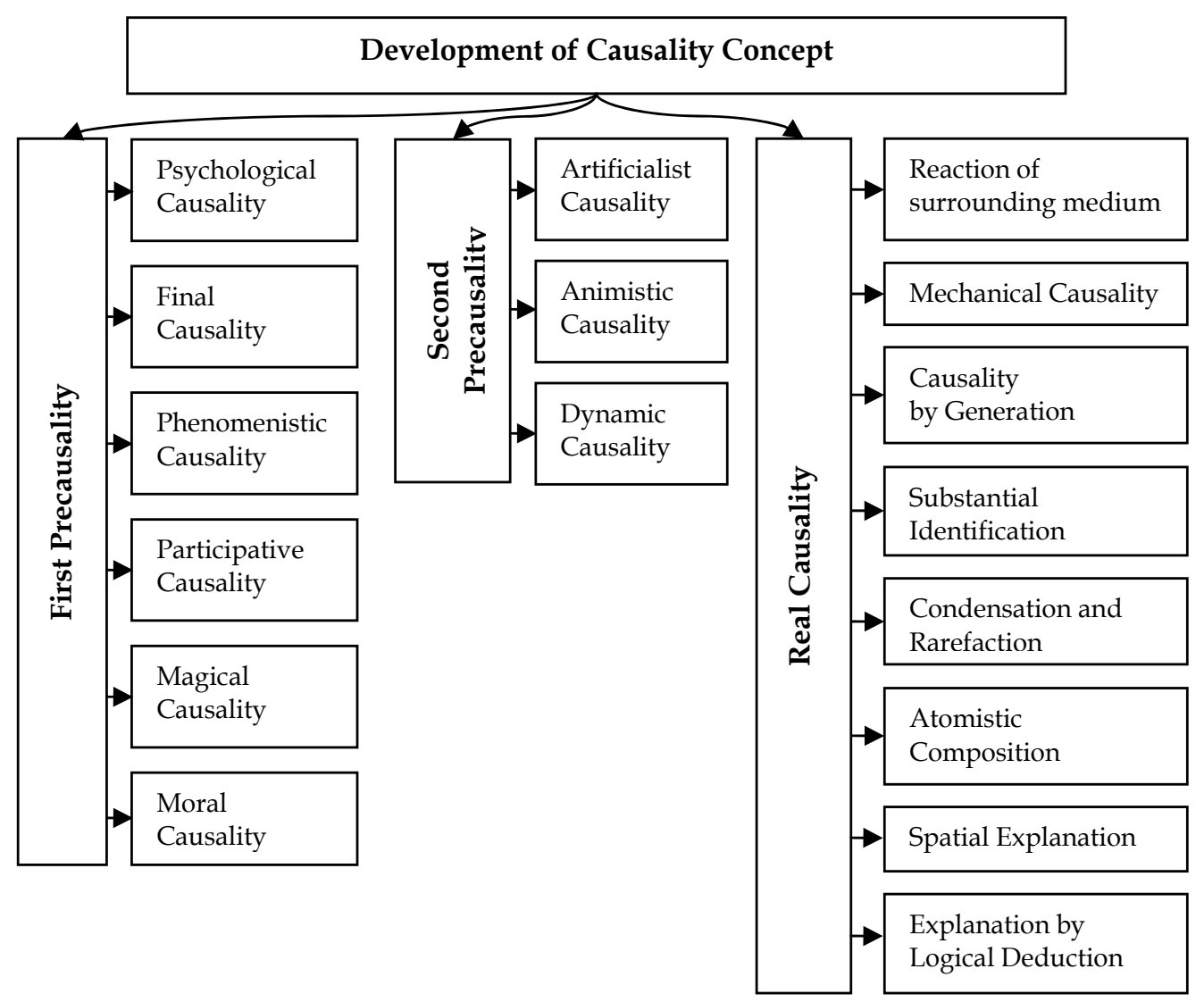

Table 2. Development of causality concept (Piaget, 1969)

2. Consequentalism/Final Causality (Finalism): This type of causality is a successor of the former, and they overlap. However, over time it is separated from the previous one. Children behave thinking that there is an explanation for everything. They make their explanations definite simply by the force of finalism. The sentences "Ducks have web feet to swim better." (Piaget, 1969), "The river flows because it runs to the sea." (Charles, 2003) are examples for children's such explanations.

3. Phenomenisic Causality: This type of causality addresses a relationship between two phenomena to explain events and these phenomena are not related with life at all except their contiguity. In other words, contiguity in time between two phenomena is in such a way that one is mentioned as the cause or the effect of the other (Piaget, 1969).

4. Participative Causality: This type of causality is seen more often than the previous ones, but it disappears after 5-6 years of age. This type's principle is being successive: It is designed between two objects which have similarity or closeness relationship. In this design, the thing which enables them to become above a distance from each other is thought to be common or the source of emerging is accepted as one thing, that is to say, becoming of the first enables the other to become. The following sentence can be mentioned as an example for children's this type of explanations "The air or shadow in a room stem from the air or shadow out of the room" (Piaget, 1969). 
5. Magical Causality: This type of causality is comprised of many features of the participative causality. Gestures, thoughts or touchable objects are assigned or associated with impression, and much participation established between these objects emerges. Thus, some words have influence on some objects. Roots of participative causality are related with some instances of logical structures. But participative and magical causalities are more related with psychological causality. The child's own desires are not influential on himself, but they are seen as conditions in his all reality thoughts. In other words, signs of reality are general and this reality is the result of most objects' first complexity between itself and external world. This implies the primitive psychological causality (Piaget, 1969). The following sentence can be an example for children's this type of explanations "Rude words make a person rude." (Charles, 2003).

6. Moral Causality: This type of causality is closely related with the abovementioned causalities. The child explains the presence of any behavior or attribute referred to for requirements, but these are always related to morality, i.e. the view that "things happen because they have to" comes to the foreground: "The clouds must be moved for the night", "People must go to bed in order to sleep", "The ships have to swim, otherwise they can't use anything", etc. (Piaget, 1969).

7. Artificialist Causality: In this type of causality, psychological causality or pre-causality is described in neither sole moral nor physical terms. The descriptions state that both substance and conscious do not play any role in life. The event and object are described first, and then men's activities are designed as objects. In other terms, the descriptions are made through objects and humans' acts (Piaget, 1969).

8. Animistic Causality: In this type of causality, an existing character or form is described by an inherent biological tendency, which is both alive and conscious. Animistic causality defines artificialist causality (Piaget, 1969).

9. Dynamic Causality: In this type of causality, while the vitality of objects belonging to previous period is appropriately eliminated, inherent strengths of activities and behaviors of objects still remain. Thus, there happens a mandatory primitive intellectual confusion with the life itself. Consequentialism lives longer than pre-causality while dynamic causality lives longer than vitality (Piaget, 1969).

10. Reaction of the Surrounding Medium: This is the type of causality where the child for the first time comes up with realistic physical explanations. The movements of objects are described physically, though in a primitive way. There are traces of dynamic causality but no magic, stimulus, intention or mystery in such descriptions (Piaget, 1969).

11. Mechanical Causality: This type of causality emerges in 7-8 years-old children. For children, who previously thought that any movement was driven by two types of forces (internal and external), the internal (the force of the object itself) and the other force gradually turn into a less needed internal force. At this point, the explanation is a mechanic one. Examples of children's such explanations are as follows: "The wind pushes the clouds, pedals power the bicycle" (Piaget, 1969), "A bicycle moves because it has pedals" (Charles, 2003).

12. Causality by Generation: In this type of causality, explanations for movement demonstrate that mechanic causality loses its significance and that explanations for how objects are actually produced are more easily accepted. Explanations through causality 
by generation are not independent from previous types of causality (Piaget, 1969). Examples of children's such explanations include "The sun was taken out from a cloud; the clouds are made up of smokes" (Charles, 2003).

13. Substantial Identification: This type is similar to the abovementioned generation type of causality. Children think that objects are not made of other things but come into existence through phases such as burning and melting/boiling. Substantial identification is often seen in 8-10 years-old children (Piaget, 1969). Examples of children's such explanations include "There are many small clouds side-by-side in the sky" (Charles, 2003).

14. Condensation and Rarefaction: This type is characterized by condensation and depressurization (rarefaction) schemes. There are explanations over the substances that form the basic attributes of objects. For example, stone is hard due to the properties of the substances that comprise it, i.e. stone was comprised through the condensation and combination of its components and therefore, it is hard. Some examples of children include "Water is light because it is 'thin' or 'fluid/liquid", "Wood and stone are heavy because they are 'big', 'thick' and 'full'" (Piaget, 1969).

15. Atomistic Composition: So far, it was thought that objects were formed through condensation or rarefaction of source substances. This process continues inevitably until they are conceived as sooner or later products of particles connected with loose or tight bounds. The child's conclusion on how stones are formed is as follows: "Stones consist of little, tiny stones which are formed by particles on the earth." In other terms, small pieces are joined together and form objects (Piaget, 1969).

16. Spatial Explanation: This type of causality is seen in children over 9-10 years old. And children use advanced forms in their explanations (Piaget, 1969).

17. Explanation by Logical Deduction: This type of causality contains explanations representing the principle of sufficient reason. Principle of logical deduction includes all mechanic, spatial, atomistic, etc. explanations. Therefore, this period covers 10-11 years and onwards (Piaget, 1969).

The concept of causality develops through overlapped and sequential processes. Piaget determined seventeen types of causality in this development and divided these seventeen types of causality into three main stages. He called the first two as precausality while he called the last one as real causality. He stated that the last stage is reached by 11-12 years-old children. According to Piaget (2007a), there are types of causality as well as types and grades of awareness. In cases where the child acts as if he knows that "one thing is caused by another" or that something caused another; we come across with a primitive type of causal relationship or the functional equivalent of causality even when children are not aware of causality. Later, when the same child becomes aware of the relationship in question, this awareness may emerge in several different types -i.e. spirited, artificialist, finalism, mechanic and dynamic, etc.- as it fits to the needs and interests of that moment. Therefore, the succession of these types never occurs in a definitive stage order. The relationship types used by the adults and the wise are also temporary as the types used before by the child and the primitive.

\section{Instructional elements of causality}

In order to explain the causality term and determine the development levels of students, identifying the context of this term and forming other main terms may be needed. Causality 
can be defined as the cause and effect relation between the event, phenomenon and objects. Cause defines event, phenomenon and object's being, as to effect it defines the result, effect that this being presents (emerges). In this context, when it is instructionally viewed causality falls into two parts: Determining the causes and finding out the effects. Determining the causes may refer to deduction, finding out the effects may refer to induction. This may require the collocation of deduction and induction, which is hypothetical-deduction. However, Aristotales (1989) viewed scientific explanation relying on causality. He formed a connection between causality and scientific explanation, thus there existences a relation between process, scientific process skills and causality. Scientific process skills are, in general terms, discussed in three categories. These categories and sub-categories are as follows:

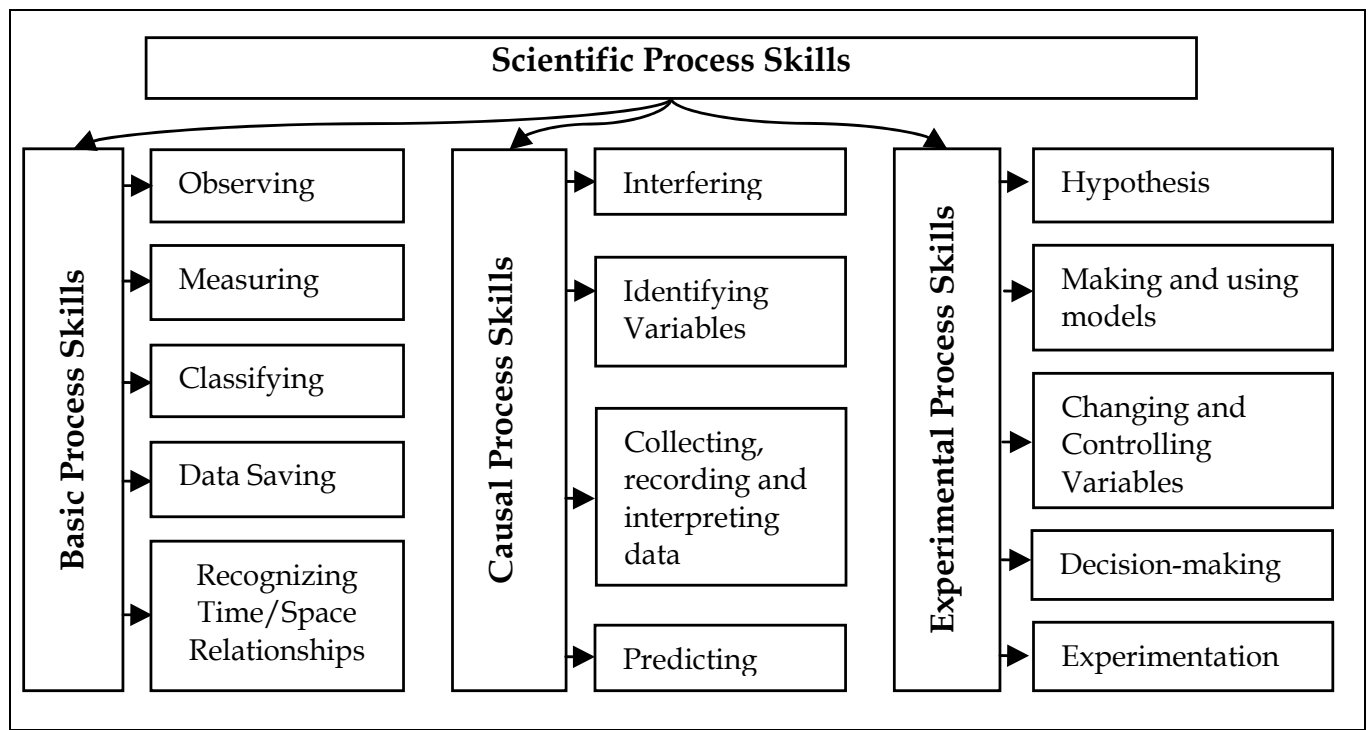

Table 3. Scientific process skills (Cilenti, 1985; Ayas et al., 1997; Turgut et al., 1997; Akdeniz, 2005)

Scientific process skills consist of basic process skills, causal process skills and experimental process skills. Among these skills, the classification skill, relating skill and deduction skill are the skills that can be directly used to determine the education and development of causality concept. However, all scientific process skills are closely related to causality. In this respect, when both scientific process skills and hypothetic-deductive reasoning are considered together, the educational components of the concept of causality can be determined as shown below:

The educational components of the causality concept are as follows:

1. Defining,

2. Relating,

3. Sorting,

4. Classification,

5. Change and Continuity,

6. Determining the Causes (Deduction),

7. Reasoning (Induction). 


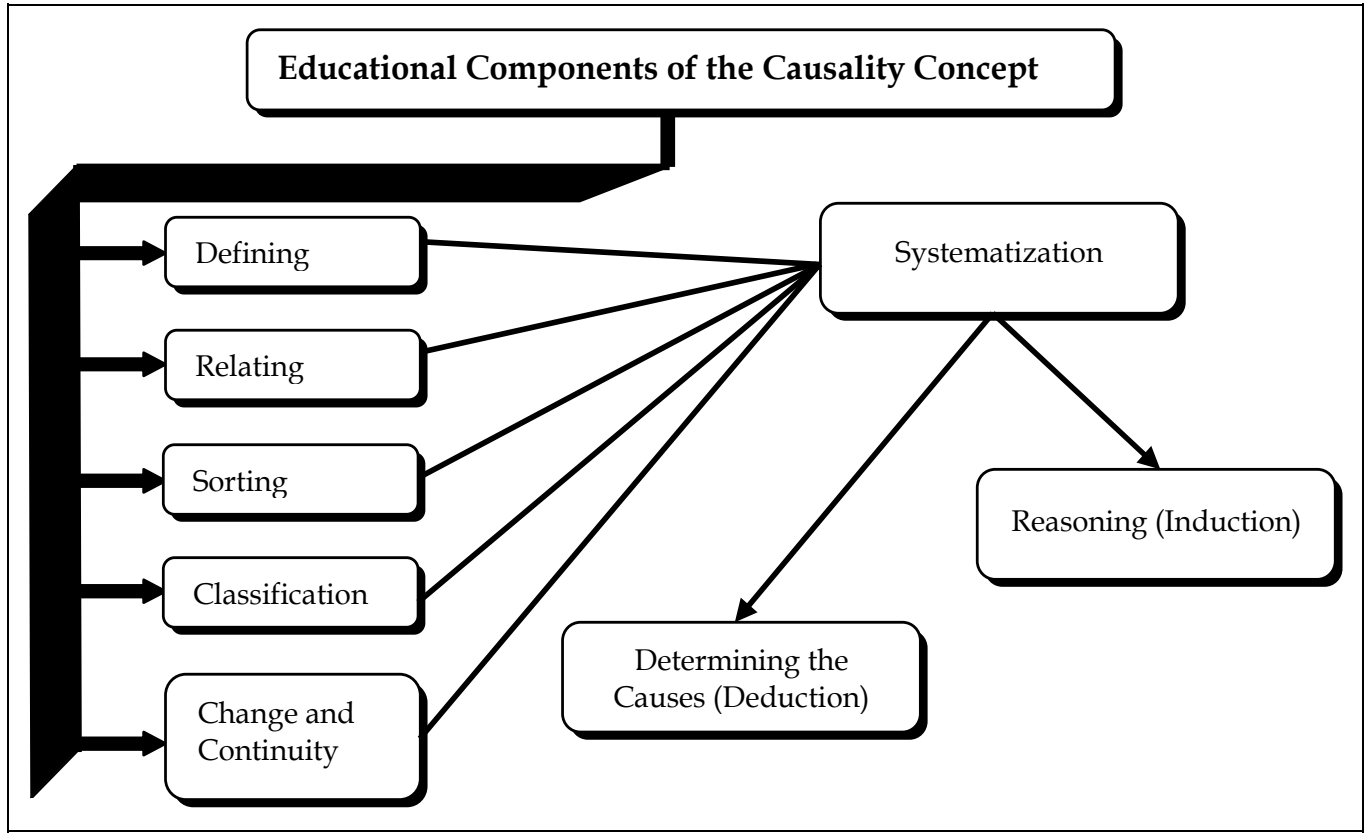

Table 4. Educational components of the causality concept (Tay, 2007; Tay, 2011)

These concepts have their specific properties, yet they complete each other. While the first four of abovementioned skills comprise the main factors in understanding and forming causality, the change and continuity element surrounds these four concepts and thus the concept of causality. A good comprehension of the causality concept could be achieved by systematizing these four elements along with change and continuity, determining causes in some cases through this systematization, deducing the outcomes in some cases and promoting both of them in some cases. In this respect, children should be provided with these skills in order to enable them to develop, improve and utilize the concept of causality. These skills are described briefly in the following sections.

\subsection{Defining}

The scientific method is defined as both an action-based (phenomenal) and mental (descriptive) process which encompasses the ways to describe and explain the universe (Ozlem, 2003). Accordingly, the existence of scientific knowledge is made possible by defining this knowledge. The defining process reveals the existence of knowledge, and once the knowledge is defined, it can be included into the group(s) it belongs to. Defining operationally is important (Cilenti, 1985). As a matter of fact, the ability to draw-up operational definitions, which is one of the scientific process skills, indicates that. In this respect, since the ability to draw-up operational definitions represents the ways to observe and measure any given variable, defining process is the first step in determining the causal relationship between two objects, phenomena and events. Whether the definition is operational or not is an important property as it affects the meaning of that definition. When the defining process is carried out in general terms but not in operational terms, this might 
lead to formation of wrong causal relationships. For instance, if we consider capitulation in general terms, we cannot establish a correct relationship between the capitulations given to the French by the Ottoman Empire and what it intended to achieve. For that reason, definitions should be operational.

Defining start with the children's "to....." words towards the ages of 5 or six. For example, the sentence "a table to write on" can be exemplified. The eye catching point in the sentence is that the definition is operational (Piaget, 2004). Children begin to make first logical defining when they 7-8 years-old and they will not have the ability to make surrounding definitions until they are 11-12 years-old.

\subsection{Relating (resemblance, analogy)}

Relation is the connection which the newly getting formed thought assumes it has found between two new thoughts, two beings or two facts; by this thought these beings or the facts can closely resemble, influence each other, although there is not a perceivable causal participation.

Environment has a significant influence on the development of child's relation thought. Hence at the beginning stage, the baby's life cannot be thought apart from the mother's life. The baby's basic requests and needs end up with the mother's or the indirect environment's response. The extension of the baby's each crying is an activity of the mother-father and even his desires which are never mentioned are always foreseen. Briefly, although the small child cannot distinguish exactly his own activities from the external activities, in this sense, there is a sheer continuity between the mother-father's lives and personal activity. This continuity influences the development of relation thought in child (Piaget, 2005). Children begin to understand many of the concrete and abstract relations at the preschool stage. While concrete relations can be perceived through the senses, the abstract relations should be designed in thought. In other words, connecting will be possible as much as the abstract relations are concretized. In connecting activities of preschool children concretization should be benefited from, since at this stage they are bad at establishing the cause and effect relations (Gander and Gardiner, 2004).

The child begins to understand the relations between the concrete objects and conditions as he begins to see better in which way the people and ideas are similar and different. This fact is the first necessary step for the child to understand and create these concepts, and make generalizations with these concepts (Karadeniz, 2002).

According to Hume, our ideas being in relation and connected to each other in certain ways is not coincidental. Hume states that there are some connective bonds which tie our ideas to each other in some certain aspects. According to this, these ideas connect to each other when they have some certain properties. These properties are three according to Hume: similarity, continuity in time or place and cause-effect relation.

According to Claparède the likening conscious in child (that is understanding the similarity between two objects) emerges long after the differentiating conscious (that is after understanding the differences between two objects). It means that children use similarity without deep thinking. Therefore, development of similarity conscious requires becoming 
conscious. Becoming conscious comes true when there is inconsistency (Piaget, 2007b). In this context and therefore, it may be better to develop the relation skills by inconsistency, in other terms by differences.

According to the child's mental development, the child's relating ability in intuitive stage has a way of sensing which derives from evaluating all perceptional qualities of designed collection without needing to examine the relation between the objects (Piaget, 2004) therefore it is sublevel. In concrete operational stage the child establishes valid relations between the objects he observes, in formal operational stage his relating skill completes its development and becomes able to do the relating without considering whether concrete or abstract (Charles, 2003).

\subsection{Ordering}

The ability of arranging a set of elements according a certain relation is ordering. Piaget called this conceptual ability as "seriation". This ability starts developing at preschool stage, the child's ordering ability of objects according to weight, size or other quantitative properties develops and matures at concrete operational stage and fully develops at formal operational stage (Gander and Gardiner, 2004; Karadeniz, 2002). Ordering ability is one of the scientific thinking abilities according to (Akt. Arslan and Tertemiz, 2004).

Ordering ability includes ordering the events, facts and objects with respect to a property determined among these. When it is viewed in context of causality presenting the causality between two events might be closely related with the order of the events. Here, this ordering is usually related with before event and after event. For example, to present the existence of the causal relationship between two events which took place in history, it is needed to know which happened before and which happened later. In the same way in geographical events, it is also needed.

\subsection{Classification}

It is the process of grouping the objects, facts or events in respect to their relations with each other and properties determined as results of observation. Doing a good classification depends on having observed the properties of objects, facts and events and their relations with each other well (Cilenti, 1985).

Classification that is one of the skills in basic process categories of scientific process skills is an induction (generalization). Our brain gradually organizes the information by grouping, according to similarities or differences, all the living or nonliving; concrete or abstract concepts which we perceive by our organs or have through our mental skills. To distinguish between the concepts by comparing we need to have distinctive information about them. In this sense, it requires specifying the borders of concepts and identifying them considering their basic distinctive properties. Neglecting this property of identification might cause incorrect concepts, in other words, misconceptions (Erbas and others., 2005). Doing the identification properly is the prior condition of doing a proper classification.

There are two elements in an efficient classification process. One of them is the power of learning the figures; it requires considering all related perceptual properties of a group of 
objects, knowing the meanings of the words and keeping them in mind. The other one is the power of organizing the perceptions; it requires using the logical principles in grouping process. According to Piaget, learning the figures gains importance in developing concepts. According to Vygotsky, these two powers are always in an interaction. Knowledge about the meanings of the words reflects the power of organizing the information related with the objects. The information is managed by the classification power. In other words, a person who is deficient in the perceptions related with the objects cannot classify efficiently. An individual whose power of selecting and applying certain principles lacks is unsuccessful in an inefficient classification, even if he has substantial perceptions about the objects (Karadeniz, 2002).

In intuitive stage, classification skill improves from the deficient grouping state towards an efficient grouping skill in accordance with the child's mental development, in concrete and formal operational stage this skill improves with its all aspects and becomes able to test, make explanations conforming to the logical law and make regressions (Charles, 2003). Skill of grouping the objects according to a certain common feature can be stated as classification. According to Ginsburg and Opper (1965), children in concrete operational stage can classify according to many levels of conceptual hierarchy as long as the objects are concrete and not imaginary (Akt. Gander and Gardiner, 2004).

\subsection{Change and continuity}

Positive or negative conditions which the events, facts or objects had over time can be called as change. As for continuity, it means events, facts and objects protecting their existence in the course of time in other words, it means their continuity. Change and continuity can be the condition or continuity of events, facts and objects in the course of time. When it is viewed from the point of causality, establishing the causal relation correctly between the two things can require knowing the conditions and continuity of the two things in the course of time. Thus, children's ability of establishing causal relations is closely related with this change or continuity perception or skills development. According to Sunal (1981), the starting point of change concept is the differences. First, the differences should be introduced to the children and these differences should be observed by the children. According to him, change cannot be identified unless they are observed. Three categories are important for change. These are observation, discussion and recording (Sunal, 1981). Schlotmann (1999), states that there is a natural connection between continuity and children's understanding the causality. This indicates continuity concept is a significant in understanding the causality.

\subsection{Identifying the causes (deduction)}

The Child performs very little reasoning and it is performed especially on rare and special instances because of the difficulties in being aware of own thoughts. He finds all kinds of deductions, as generalization and effect, difficult. He puts successive judgments side by side instead of connecting them. Thus, there is no internal pressure or necessity on his thoughts. Even when the child starts making generalizations and deductions he remains unfamiliar with the formal deduction because of failing in getting out of personal beliefs and reasoning on a hypothesis put forward by others (Piaget, 2007b). 
Before the ages 7-8 even until the ages 11-12 at which deduction completely rises, the whole structure of the child's reasoning is as following: The child reasons on individual or special instances and doesn't look for whether there is a contradiction between these. These individual or special instances constitute the conscious experiences which are not reflectional yet (Piaget, 2007b).

\subsection{Concluding}

The method which goes to the common from the single, special one and reaches general propositions from facts one after another is called induction (Akarsu, 1998). Induction might mean reaching a conclusion, because there is reaching to a whole, in other words, to a conclusion from facts one after another. In this regard, reaching a conclusion includes effect (influence, consequence) which is one of the two main categories of the causality concept.

In children, induction or deduction take place in the stage of "explanations conforming logical law". The explanations in this stage increase in most of the children about at the ages of eleven-twelve. That is to say in children, logical thinking includes induction (generalizations and observations are made) and deduction (results obtained through the process of reaching a conclusion by comparing) (Charles, 2003).

\section{Conclusion}

The relation between cause and effect is the most general definition of causality. Empiricists regard causality as an outcome of our experience or habits, but according to rationalists, it is a necessary connection and a result of our priori knowledge which is often independent of experience. In this context, causality, used in both sciences, is one of the basic principles that nature-related and social sciences are based on.

In social sciences, there is a contingent and percentile consistency between rules, principles and actions, not an absolute consistency. Hence, in social sciences all the descriptions should be made considering that there is a contingent consistency. In an educational curriculum, the information produced by social sciences is instructed through mono disciplinary, multidisciplinary or interdisciplinary approaches. Conceiving a cause and effect relation is one of the skills that the students are supposed to attain in social sciences lesson which is constituted on the basis of multi-disciplinary and interdisciplinary approaches. Having the students to attain this skill may require dealing with causality educationally. In educational point of view, causality comprises the skills of defining, relating, sorting, classifying, change and continuity, determining the causes, and reasoning. Students' development stages and accordingly their mental development level are important in teaching these skills. Properties of children's intuitive, concrete and formal operational stages should be taken into consideration when teaching the causality concept and related skills, until they reach the logical thinking stage.

\section{References}

Akarsu, B. (1998). Felsefe Terimleri Sozlugu [The Dictionary of Philosophy Concepts], Inkilap Yayinlari, Istanbul, Turkey 
Akdeniz, A. R. (2005). Problem Cozme, Bilimsel Surec ve Proje Yonteminin Fen Egitiminde Kullanimi [Using Problem Solving, Scientific Process, and ProjectBased Learning Method in Science Education], In: Fen ve Teknoloji Ogretimi [Teaching Science and Technology]. S. Cepni, (Ed.), 93-114, Pegem A Yayincilik, Ankara, Turkey

Aristotales. (1985). Metafizik [Metaphysics], Cilt.I [vol.I], A. Arslan, Ege Universitesi Yayinlari, Izmir, Turkey

Aristotales. (1989). Orgonon IV. Ikinci Analitikler [Organon IV. Second Analytics], Translated by H. R. Atademir, MEB Yayinlari, Istanbul, Turkey

Arslan, A. (Gursel) \& Tertemiz, N. (2004). Ilkogretimde Bilimsel Surec Becerilerinin Gelistirilmesi [Developing Scientific Process Skills in Elementary Education], Turk Egitim Bilimleri Dergisi, 2, 4, 479-492

Ayas, A. P.; Cepni, S.; Johnson, D. \& Turgut, F. (1997). Kimya Ogretimi [Teaching Chemistry], YOK/Dunya Bankasi Milli Egitimi Gelistirme Projesi Yayinlari, Ankara, Turkey

Berry, A. (2003). Bilimin Arka Yuzu [The Backyard of Science], Translated by R. L. Aysever, TUBİTAK Populer Bilim Kitaplar, Ankara, Turkey

Berzonsky, M.D. (1971). The Role of Familiarity in Children's Explanations of Physical Causality. Child Development, 42, 3, 705-715

Carr, E.H. (2006). Tarih nadir? [What is History], Translated by G. Misket\&G. Gurturk, İletisim Yayinlari, Istanbul, Turkey

Charles, C.M. (2003). Ogretmenler İcin Piaget İlkeleri [Piagetian Principals for Teachers], Translated by G. Ulgen, Nobel Yayin Dagitim, Ankara, Turkey

Cevizci, A. (1999). Felsefe Sozlugu [Philosophy Dictionary], Paradigma Yayinlari, Istanbul, Turkey

Cilenti, K. (1985). Fen Egitimi Teknolojisi [The Technology of Science Education], Kadioglu Matbaasi, Ankara, Turkey

De Avila, E. \& Struthers, J.A. (1967). Development of a Group Measure to Assess the Extent of Pre-Logical and Pre-Causal Thinking In Primary School Age Children, Boulder Valley School District RE-2, Boulder, CO

Denkel, A. (1996). Anlam ve Nedensellik [Meaning and Causality], Kabalci Yayinevi, Istanbul, Turkey

Dilek, D. (2002). Tarih Derslerinde Ogrenme ve Dusunme Gelisimi [The Development of Learning and Thinking in History Course.], Pegem A Yayincilik, Ankara, Turkey

Doganay, H. (1992). Cografyaya Giris 1: Yontem-İlke ve Temel Terminoloji [Introduction to Geography 1: Method, Principal, and Basic Terminology], Ataturk Universitesi Yayinlari, Erzurum, Turkey

Doganay, H. (1993). Cografya'da Metodoloji, Genel Metotlar ve Ozel Ogretim Metodlari[The Methodology in Geography, Main Methods and Specific Teaching Methods], Millî Egitim Bakanligi Yayinlari, Istanbul, Turkey

Erbas, S.; Simsek, N. \& Cinar, Y. (2005). Fen Bilgisi Laboratuari ve Uygulamalari [Science Education Laboratory and Practices] , Nobel Yayin Dagitim, Ankara, Turkey

Ewing, A.C. (1969). Kant's Treatment of Causality, The United States of America: Archon Boks. 
Fay, B. (2005). Cagdas Sosyal Bilimler Felsefesi [The Contemporary Philosophy of Social Science], Translated by İ. Turkmen, Ayrinti Yayinlari, Istanbul, Turkey

Fuson, K. (1976). Piagetian Stages in Causality: Children's Answers to "Why", Elementary School Journal, 77, 2, 150-158

Gander, M. J. \& Gardiner, H.W. (2004).Cocuk ve Ergen Gelisimi [Child and Adolescent Development] , Translated by B. Onur, İmge Kitapevi, Ankara, Turkey

Gulbenkian Komisyonu. (2005). Sosyal Bilimleri Acin [Open the Social Sciences], Translated by S. Tekeli, Metis Yayinlari, Istanbul, Turkey

Gunay, M. (2004). Metinlerle Felsefeye Giris [Introduction to Philosophy with Texts], Karahan Kitabevi, Adana, Turkey

Gungordu, E. (1997). Cografya'da Ogretim Metodu, İlkeler ve Uygulamalar [Teaching Method, Principals, and Practices in Geography], Copy Star Fotokopi ve Dizgi Merkezi, Ankara, Turkey

Hume, D. (1976).Insanin Anlama Yetisi Uzerine Bir Sorusturma [An Enquiry Concerning Human Understanding], Translated by O. Aruoba, Hacettepe Universitesi Yayinlari, Ankara, Turkey

Hume, D. (2000). An Enquiry Concerning Human Understanding, Canada, Ontario, Kitchener: Batoche Boks.

Husbands, C.T. (1996). What is History Teaching? Language, Ideas and Meaning in Learning About the Past, Buckingham: Open University Pres.

Inagaki, K.; Hatano,G. \& Inagaki, K. (2006). Do young children possess dinstinct causalities for the three core domains of thought? , 05.05.2006, Available from http://www.interdisciplines.org/causality/papers/2

Kant, I. (2004). Pratik Usun Elestirisi [Critique of Practical Reason], Translated by I.Z. Eyuboglu, Say Yayinlari, Istanbul, Turkey

Kant, I. (2006). Egitim Uzerine [On Pedagogy], Translated by A. Aydogan, Iz Yayincilik, Istanbul, Turkey

Karadeniz, G. (2002). Cocukta Kavram Gelisimi [The Concept Development of Child], Istanbul: Marmara Universitesi Sosyal Bilimler Enstitusu

http://www.koniks.com/data/doc/KAV_GEL.doc. adresinden 15 Haziran 2006 tarihinde alinmistir.

Northrop, F.S.C. (1998). Bilimsel Dusunmede Ilk Adim [The First Step in Scientific Thought], In: Bilim Felsefesi C. Yildirim (Ed.), 204-211, Remzi Kitapevi, Istanbul, Turkey

Ozcinar, S. (1997).Bilimde Nedensellik İlkesi: Nedensellik İlkesi Acisindan Olgucu Felsefe Uzerine Elestirel Bir Inceleme [The Causality Principal in Science: Critical Perspective on Phenomenological Philosophy In Terms Of Causality], Ankara Universitesi (Master Thesis), Ankara, Turkey

Ozguc, N. \& Tumertekin, E. (2000). Cografya "Gecmis-Kavramlar-Cografyacilar" [Geography "Past-Concepts-Geographers"], Cantay Kitapevi, Istanbul, Turkey

Ozlem, D. (2003). Bilim Felsefesi "Ders Notlari"[ Philosophy of Science "Course Notes" ], Inkilap Kitabevi Sanayi ve Ticaret A.S., Istanbul, Turkey

Piaget, J. (1969). The Child's Conception of Physical Causality, Translated by M. Gabain, Littlefield: Adams \& CO 
Piaget, J. (1982). Yapisalcilik [Structuralism], Translated by F. Akatli, Dost Kitapevi Yayinlari, Istanbul, Turkey

Piaget, J. (2004). Cocukta Zihinsel Gelisim [The Development of Mind in Child], Translated by H. Portakal, Cem Yayinevi, Istanbul, Turkey

Piaget, J. (2005). Cocugun Gozuyle Dunya [The World from Child's Point of View], Translated by I. Yerguz, Dost Kitapevi Yayinlari, Ankara, Turkey

Piaget, J. (2007a). Cocukta Dil ve Dusunce [Language and Thought in Child], Translated by S.E. Siyavusgil, Gunumuz Turkcesi: Y.T. Gunaydin, Palme Yayinlari, Ankara, Turkey

Piaget, J. (2007b). Cocukta Karar Verme ve Akil Yurutme [Making Decision and Reasoning in Child] Translated by S.E. Siyavusgil, Gunumuz Turkcesi: Y.T. Gunaydin, Palme Yayinlari, Ankara, Turkey

Reichenbach, H. (1998). Klasik Fizik'in Empirik ve Rasyonel Yonleri [Empirical and Rational Aspects of Classic Physic], In: Bilim Felsefesi C. Yildirim (Ed.), 212-218, Remzi Kitapevi, Istanbul, Turkey

Savage, T.V. \& Armstrong, D.G. (1996). Effective Teaching in Elementary Social Studies, USA: Prentice Hall

Schlottmann, A. (1999). Seeing It Happen and Knowing How It Works How Children Understand the Relation Between Perceptual Causality and Underlying Mechanism. Developmental Psychology, 35, 1, 303-317

Spiliotopoulou, V. (2001). Entities of the World and Causality in Children's Thinking, Research in Science Education, 113-118

Stepans, J. \& Kuehn, C. (1985). Children's Conceptions of Weather. Science and Children, 23, 1, 44-47

Sunal, C. (1981). The Child and the Concept of Change, Social Education, 45, 6, 438-441

Tay, B. (2007). Sosyal Bilgiler Ogretimi Kapsaminda İlkogretim Ogrencilerinde Nedensellik Kavraminin Gelisimi [The Development of Causality Concept in Primary School Students in The Instruction of Social Studies], PhD. Thesis, Gazi Universitesi, Egitim Bilimleri Enstitusu, İlkogretim Sosyal Bilgiler Ogretmenligi Bilim Dali, Ankara, Turkey

Tay, B. (2011). Sosyal Bilgilerde Nedensellik Kavrami ve Ogretimi [Causality Concept and Teaching Causality in Social Studies] In: Ozel Ogretim Yontemleriyle Sosyal Bilgiler Ogretimi [Teaching Social Studies Through Specific Teaching Methods], Tay, B. \& Ocal A. (Ed.), 437-488, Pegem A Yayincilik, Ankara, Turkey

Turgut, F.; Baker, D.; Cunningham, R. \& Piburn, M. (1997). Ilkogretim Fen Ogretimi [Elementary School Science Education], YOK/Dunya Bankasi Milli Egitimi Gelistirme Projesi Yayinlari, Ankara, Turkey

Uzun, H. (2006). Tarih Bilimi ve Tarihte Nedensellik [Science of History and Causality in History], Kirsehir Egitim Fakultesi Dergisi, Cilt 7, Sayi 1. ss: 1-13

Voss, J.F. (1998). Issues in the Learning of History. Issues in Education, 4 (2), 163-209. http:/ / web.ebscohost.com/ehost/detail?vid=6\&hid=117\&sid=d1b4df3e-306f4a7d-aed2-7ff4862faf99\%40sessionmgr104 adresinden 12 Agustos 2006 tarihinde alinmistir. 
Yildirim, C. (1997). Bilimsel Dusunme Yontemi "Yazilar, Bildiriler, Tartismalar"[Scientific Thinking Method "Writings, Proceedings, and Discussions], Bilgi Yayinevi, Ankara, Turkey

Yildirim, C. (1998). Bilim Felsefesi [Philosophy of Science], Remzi Kitabevi, Istanbul, Turkey 


\title{
The Assumption of Non-Gaussianity in Natural and Social Sciences and Its Influence on Detection of Causal Relationships
}

\author{
Kateřina Hlaváčková-Schindler \\ Commission for Scientific Visualization, Austrian Academy of Sciences \\ Austria
}

\section{Introduction}

The concept of causality is changing as human knowledge changes. Causality as an abstract notion has been traditionally studied in the field of metaphysics in philosophy. The Greek philosophers understood the time causality as explanation in general (Aristotle, 350 B.C.). The search for causes was a search for "first principles", which were meant to be explanatory. In the more recent philosophy introduced by (Newton, 1687) was causality connected with determinism. The current experimental science reveals the non-deterministic notion of cause, which has to be also taken into consideration. The introduction of probability theory into all scientific disciplines allows to formalize and mathematize the wider conceived notion of cause.

This paper does not deal with the philosophical approach to causality, to this we refer the reader for example to the works (Mackie, 1988), (Hume, 1896), (Russo, 2009) or (Pearl, 2000). Here we deal exclusively with the formal mathematical approaches to detect cause-effect relationships, namely with Granger causality and transfer entropy and their application in sciences.

The generally non-deterministic approaches to causality apply various probability distributions to model the real-world phenomena. The selection of an appropriate or inappropriate model to fit the real world data has obviously an important influence on the credibility of the achieved conclusions.

In the present paper we discuss the influence of the selection of a data model for detection of causal relationships between two or more time series. We focus here on cases when the Gaussianity of the investigated process can be assumed and when not. The causality detection methods considered here are Granger causality (Granger, 1969) and transfer entropy (Schreiber, 2000). We investigated time series with a wider class probability distributions than Gaussian, the generalized Gaussian probability distributions. These distributions are given parametrically. We set conditions on their parameters so that one can from their values decide whether the relationships between the involved time series are unidirectional causal or whether no causality is present.

Being aware of outstanding philosophical papers on causality in the sciences, for example (Illari et al., 2011), we are though not aware of any similar publication on mathematically 
conceived causality and their application in natural and social sciences, neither of any analysis of the probabilistic assumptions about the investigated time series and their influence on causality detection by Granger causality or transfer entropy.

The paper is organized as follows. The application of various probabilistic models in natural and social sciences is discussed in Section 2. Granger causality and its application in natural and social sciences is treated in Section 3. Section 4 deals with transfer entropy, directionality index and other information-theoretical measures, inclusive their applications. Section 5 devotes a special attention to causal relationships among Gaussian time series and generalized Gaussian time series. In Section 6 we presented original and simple criteria for phenomena having generalized Gaussian distributions given parametrically which decide about a presence or absence of causality between these phenomena given by concrete time series. Section 7 discussed the criteria deciding about the applied causality detection method and concludes with the importance of the achieved results.

We presented original and simple criteria for phenomena having generalized Gaussian distributions given parametrically which decide about a presence or absence of causality between these phenomena given by concrete time series.

\section{Probabilistic distributions and their application in natural and social sciences}

Gaussian, more frequently called "normal" distribution has a special position among all probability distributions used in data modeling and is the most popular. It has been known for a relatively long time, is simple and analytically tractable. Its symmetry about its mean value is one of the basic principles realized in nature as well as in human culture. The bell shape of its graph makes the normal distribution attractive for modeling of real world data in many scientific or social disciplines.

Indeed, many common natural or social phenomena show to have normal distribution. For example, such phenomena as women's height, Brownian motion of particles, milk production by cows and random deviations from target values in industrial processes fit a normal distribution (Limpert et al., 2001). However, many phenomena which fit normal distribution, have been shown that they fit also log-normal distribution or generalized normal distribution or, more precisely, fit it even better. What is the difference between normal and log-normal distribution? Both forms of variability are based on a variety of forces (causes) acting independently of one another. A major difference is however that the effects can be additive or multiplicative, thus leading to normal or log-normal distributions, respectively (Limpert et al., 2001).

The length of spoken words in phone conversation (Herdan, 1958), the length of sentences (Williams, 1940) have been shown to have log-normal distribution, as well as the age of marriage (Preston, 1981) or income (Statistical yearbook in Switzerland, 1997). Prices, incomes or populations, i.e. phenomena which grow exponentially, are often skewed to the right, and hence may be better modeled by other distributions than by the normal one, such as the log-normal distribution, Pareto distribution or skewed generalized normal distribution. Statistical inference using a normal distribution is not robust to the presence of outliers. When outliers are expected, data may be better described using a heavy-tailed distribution such as the Student's t-distribution. 
Generalized normal distribution (generalized Gaussian distribution), first time mathematically defined in (Nadarajah, 1995) can model for example Brownian motion of particles or fractional Brownian motion with a better precision than the normal distribution (Zinde-Walsh \& Phillips, 2003). Other experiments have shown a better approximation precision of the generalized Gaussian distributions than of the Gaussian distributions, for example (Sharifi \& Leon-Garcia, 1995), (Moulin \& Liu, 1999) for in image processing and video analysis, (Bicego et al., 2008) for EEG time series modeling.

The modeling in linguistics applies mostly Gaussian mixtures. Mixtures of generalized Gaussian distributions have been recently used in text independent speaker identification (Sailaja et al., 2010) and showed that it outperforms the earlier existing text independent speaker identification models. This model was applied for speaker identification like voice dialing, banking by telephone, telephone shopping information services etc.

Exponential distribution has been frequently used in modeling in astrophysics, for example the Weinman exponential distribution has been shown to be a good model for dusty galactic discs (Misiriotis et al., 2000).

To summarize, other probability distributions than the normal one have an important role in modeling both in natural and social sciences. We will call them non-Gaussian distributions in the following. The selection of a correct distributions for modeling natural or social phenomena is of great importance, especially when mutual interactions among these phenomena are investigated. A crucial question is whether there are causal relationships among the studied phenomena. This leads to a formal definition of causality and causal measures.

In the following chapters we define formally two causality detection measures, namely the Granger causality and transfer entropy.

\section{Granger causality}

The introduction of the concept of causality into the experimental science, namely into analyses of data observed in consecutive time instants (time series), is due to C.W.J. Granger in (Granger, 1969), the 2003 Nobel prize winner in economy. In his Nobel lecture (Granger, 2003) he recalled the inspiration by the Wiener's work and identified two components of the statement about causality:

1. The cause occurs before the effect; and

2. The cause contains information about the effect that is unique, and is in no other variable.

As Granger put it, a consequence of these statements is that the causal variable can help to forecast the effect variable after other data has been first used (Granger, 2003). This restricted sense of causality, referred to as Granger causality, G-causality thereafter, characterizes the extent to which a process $X_{t}$ is leading another process $Y_{t}$, and builds upon the notion of incremental predictability. It is said that the process $X_{t}$ Granger causes another process $Y_{t}$ if future values of $Y_{t}$ can be better predicted using the past values of $X_{t}$ and $Y_{t}$ rather then only past values of $Y_{t}$. The standard test of G-causality developed in (Granger, 1969) is based on a linear regression model.

In the following we will define Granger causality by using the notation from (Barnett, 2009). Let $\oplus$ denotes concatenation of vectors, so that for $\mathbf{x}=\left(x_{1}, \ldots, x_{d}\right)$ and $\mathbf{y}=\left(y_{1}, \ldots, y_{m}\right)$ 
$x \oplus y$ is the $1 \times(d+m)$ vector $\left(x_{1}, \ldots, x_{d}, y_{1}, \ldots, y_{m}\right)$. Given jointly distributed multivariate random variables $\mathbf{X}$ and $\mathbf{Y}$ i.e. random vectors in $R^{d}$, we denote by $\Sigma(\mathbf{X})$ the $d \times d$ matrix of covariances $\operatorname{cov}\left(X_{i}, Y_{j}\right)$ and by $\Sigma(\mathbf{X}, \mathbf{Y})$ the $d \times m$ matrix of cross-covariances $\operatorname{cov}\left(X_{i}, Y_{\alpha}\right)$. Let $\Sigma(\mathbf{X} \mid \mathbf{Y})$ denotes the $d \times d$ matrix

$$
\Sigma(\mathbf{X} \mid \mathbf{Y})=\Sigma(\mathbf{X})-\Sigma(\mathbf{X}, \mathbf{Y}) \Sigma(\mathbf{Y})^{-1} \Sigma(\mathbf{X}, \mathbf{Y})^{T}
$$

define when $\Sigma(\mathbf{Y})$ is invertible.

Suppose we have a stationary multivariate stochastic process $\mathbf{X}_{t}$ in discrete time (i.e. marginal distributions are jointly distributed). Denote $\mathbf{X}_{t}^{(p)}=\mathbf{X}_{t} \oplus \mathbf{X}_{t-1} \oplus \cdots \oplus \mathbf{X}_{t-p+1}$ for $\mathbf{X}$ along with $p-1$ lags so that $\mathbf{X}_{t}^{(p)}$ is a $1 \times p d$ random vector for each $t$. Given the lag $p$, we use the shorthand notation $\mathbf{X}_{t}^{-}=\mathbf{X}_{t-1}^{(p)}$ for the lagged variable.

Suppose we have three jointly distributed stationary multivariate stochastic processes $X_{t}, Y_{t}, Z_{t}$. Consider the regression models

$$
\begin{gathered}
\mathbf{X}_{t}=\alpha_{t}+\left(\mathbf{X}_{t-1}^{(p)} \oplus \mathbf{Z}_{t-1}^{r}\right) \cdot A+\epsilon_{t} \\
\mathbf{X}_{t}=\alpha_{t}^{\prime}+\left(\mathbf{X}_{t-1}^{(p)} \oplus \mathbf{Y}_{t-1}^{(q)} \oplus \mathbf{Z}_{t-1}^{(r)}\right) \cdot A^{\prime}+\epsilon_{t}^{\prime}
\end{gathered}
$$

where $A$ and $A^{\prime}$ are the matrices of regression coefficients, $\alpha_{t}$ and $\alpha_{t}^{\prime}$ are the constant terms and the random vectors $\epsilon$ and $\epsilon^{\prime}$ comprise the residuals, so that so that the predictee variable $\mathbf{X}$ is regressed firstly on the previous $p$ lags of itself plus $r$ lags of the conditioning variable $\mathbf{Z}$ and secondly, in addition, on $q$ lags of the predictor variable $\mathbf{Y}$. By stationarity this expression does not depend on time $t$, so we omit $t$ from the notation. The G-causality of $\mathbf{Y}$ to $\mathbf{X}$ given $\mathbf{Z}$ is a measure of the extent to which inclusion of $\mathbf{Y}$ in the second model (3) reduces the prediction error of the first model (2). The standard measure of G-causality in the literature is defined for univariate predictor and predictee variables $\mathbf{Y}$ and $\mathbf{X}$, and is given by the natural logarithm of the ratio of the residual variance in the restricted regression (2) to that of the unrestricted regression (3).

(Barnett, 2009) have shown that G-causality can be expressed as

$$
\mathcal{F}_{\mathbf{Y} \rightarrow \mathbf{X} \mid \mathbf{Z}}=\ln \left(\frac{\Sigma\left(\mathbf{X} \mid \mathbf{X}^{-} \oplus \mathbf{Z}^{-}\right)}{\Sigma\left(\mathbf{X} \mid \mathbf{X}^{-} \oplus \mathbf{Y}^{-} \oplus \mathbf{Z}^{-}\right)}\right)
$$

where ln denotes the natural logarithm.

\subsection{Extensions of Granger causality}

The linear framework of Granger causality given by equations 2 and 3 has been widely applied not only in economy and finance (for a comprehensive survey of the literature see i.e. (Geweke, 1984)), but also in diverse fields of natural sciences, i.e. climatology (see (Triacca, 2005) and references therein) or neurophysiology, where specific problems of multichannel electroencephalogram recordings were solved by generalizing the Granger causality concept to multivariate case (Blinowska et al., 2004; Kamiński et. al., 2001). Nevertheless, the limitation of the present concept to linear relations required further generalizations.

Recent development in nonlinear dynamics (Abarbanel, 1993) evoked lively interactions between statistics and economy (econometrics) on one side, and physics and other natural 
sciences on the other side. In the field of economy, (Baek \& Brock, 1992) and (Hiemstra \& Jones, 1994) proposed a nonlinear extension of the Granger causality concept. Their non-parametric dependence estimator is based on so-called correlation integral, a probability distribution and entropy estimator, developed by physicists Grassberger and Procaccia in the field of nonlinear dynamics and deterministic chaos as a characterization tool of chaotic attractors (Grassberger \& Procaccia, 1983).

Another non-linear extension of Granger causality is so called correntropy (Park \& Principe, 2008).

A non-parametric approach to non-linear causality testing, based on non-parametric regression, was proposed in (Bell et al., 1996). Following (Hiemstra \& Jones, 1994), (Aparicio \& Escribano, 1998) succinctly suggested an information-theoretic definition of causality which include both linear and nonlinear dependence.

Another nonlinear extension of the Granger causality approach was proposed by Chen et al. (Chen et al., 2004) using local linear predictors. An important class of nonlinear predictors are based on so-called radial basis functions (Broomhead \& Lowe, 1988) which were used for nonlinear parametric extension of the Granger causality concept (Ancona et al., 2004; Marinazzo, 2006).

In physics and nonlinear dynamics, a considerable interest recently emerged in studying cooperative behavior of coupled complex systems (Boccaletti et al., 2002; Pikovsky et al., 2001). Synchronization and related phenomena were observed not only in physical, but also in many biological systems, i.e. (Schäfer et al., 1998; 1999) or in (Paluš et al., 2001a;b; Quyen et al., 1999; Schiff et al., 1996; Tass et al., 1998). In such physiological systems it is not only important to detect synchronized states, but also to identify drive-response relationships and thus the causality in evolution of the interacting (sub)systems. (Schiff et al., 1996) and (Quyen et al., 1999) used ideas similar to those of Granger, however, their cross-prediction models utilize zero-order nonlinear predictors based on mutual nearest neighbors. A careful comparison of these two papers (Quyen et al., 1999; Schiff et al., 1996) reveals how complex is the problem of inferring causality in nonlinear systems. While the latter two papers use the method of mutual nearest neighbors for mutual prediction, (Arnhold, 1999) proposed asymmetric dependence measures based on averaged relative distances of the (mutual) nearest neighbors.

(Ge et al, 2009) presented a novel approach which is an extension of Granger causal model and also shares the features of the bilinear approximation of dynamic causal model (David et al., 2006). The authors demonstrated face discrimination learning-induced changes in interand intra-hemispheric connectivity and in the hemispheric predominance of theta and gamma frequency oscillations in sheep infero-temporal cortex. The results provide the first evidence for connectivity changes between and within left and right infero-temporal cortexes as a result of face recognition learning.

\subsection{Application of Granger causality in natural and social sciences}

As already said, the Granger causality was introduced by its author in econometry and applied by him and his followers mainly in econometry, finance and market analysis, for example in (Granger, 1969), (Poon \& Granger, 2003). Other applications in humanities and social sciences are in linguistics and psychology (Gilbert \& Karahalios, 2009) or demography (Feridun, 2007). 
Granger causality has also been extensively applied in natural sciences, for example in medicine, especially to neuroscience (Smith et al., 2011) for the functional magnetic resonance method and (Hesse et al., 2003), analysis of EEG signals or causal interaction in neural populations (Seth \& Edelman, 2007) and in many other papers. Granger causality was applied as well as in climatology (Kufmann \& Stern, 1997), in cognitive and systematical biology, (Kim et al., 2011), (Fujita et al., 2010) etc.

The main drawback of Granger causality and its extensions as a model dependent method are their instability which can cause a high variability in the final estimation of errors in (2) and (3). As an alternative, we will present in the following model-free methods whose formal definitions apply information-theoretic functionals.

\section{Information-theoretical causality measures}

Using distributions of random processes and their definitions, introduce the information-theoretic causality measures determinism into the notion of causality.

(Paluš et al., 2001b) proposed to study synchronization phenomena in experimental time series by using the tools of information theory. Mutual information, an information-theoretic functional of probability distribution functions, is a measure of general statistical dependence. For inferring causal relation, conditional mutual information or so called transfer entropy can be used.

\subsection{Transfer entropy}

Transfer entropy as a non-linear causality measure was introduced in (Schreiber, 2000). It is an information-theoretic measure of time-directed information transfer between jointly dependent processes.

Let us first remind some basic definitions. The differential entropy of a (continuous) random vector $\mathbf{X}$ taking its values in $R^{d}$ with the probability density function $p(\mathbf{x})$ is defined by

$$
h(\mathbf{X})=-\int_{R^{d}} p(\mathbf{x}) \ln p(\mathbf{x}) d \mathbf{x} .
$$

If $\mathbf{X}$ is a discrete (multivariate) random variable given by a set of possible values $\left\{x_{1}, \ldots, x_{n}\right\}$ then the entropy can explicitly be written as

$$
H(\mathbf{X})=-\sum_{i=1}^{n} p\left(\mathbf{x}_{\mathbf{i}}\right) \ln p\left(\mathbf{x}_{\mathbf{i}}\right)
$$

where $p$ denotes the probability mass function of $X$. With $\mathbf{X}_{t}, \mathbf{Y}_{t}, \mathbf{Z}_{t}$ defined as before, the transfer entropy of $\mathbf{Y}$ to $\mathbf{X}$ given $\mathbf{Z}$ is defined as the difference between the entropy of $X$ conditioned on its own past and the past of $\mathbf{Z}$, and its entropy conditioned, in addition, on the past of $\mathbf{Y}$ :

$$
\mathcal{T}_{\mathbf{Y} \rightarrow \mathbf{X} \mid \mathbf{Z}}=H\left(\mathbf{X} \mid \mathbf{X}^{-} \oplus \mathbf{Z}^{-}\right)-H\left(\mathbf{X} \mid \mathbf{X}^{-} \oplus \mathbf{Y}^{-} \oplus \mathbf{Z}^{-}\right)
$$

where $H(. \mid$.$) is the conditional entropy. For stationary variables, similarly as for Granger$ causality, the transfer entropy does not depend on $t$, so we omitted it from labeling. Transfer entropy is a Kullback-Leibler distance of transition probabilities. 
It was shown in (Hlaváčková-Schindler et. al., 2007) that with proper conditioning, the transfer entropy is equivalent to the conditional mutual information (Paluš et al., 2001b). The latter, however, is a standard measure of information theory (Cover \& Thomas, 1991). More details on the information-theoretic methods for causality detection can be found in our review paper (Hlaváčková-Schindler et. al., 2007). Marschinski and Kanz in 2002 suggested so called effective transfer entropy to reduce the bias of transfer entropy on small data sets (Marschinski \& Kantz, 2002).

\subsection{Transfer entropy, other information-theoretical measures and their application in natural and social sciences}

Turning our attention back to econometrics, we can follow further development due to (Diks \& DeGoede, 2001). They applied a nonparametric approach to nonlinear Granger causality using the concept of correlation integrals (Grassberger \& Procaccia, 1983) and pointed out the connection between the correlation integrals and information theory. (Diks \& Panchenko, 2005) critically discussed the previous tests of (Hiemstra \& Jones, 1994). As the most recent development in economics, (Baghli, 2006) proposes information-theoretic statistics for a model-free characterization of causality, based on an evaluation of conditional entropy.

The information-theoretical approaches to causality detection are model free and can detect non-linear causal relationships, which are their advantages with respect to the approach of the linear Granger causality.

The nonlinear extension of the Granger causality based the information-theoretic formulation has found numerous applications in various fields of natural and social sciences. Let us mention just a few examples.

The Schreiber's transfer entropy has been used in climatology, i.e. (Mokhov \& Smirnov, 2006; Verdes, 2005), in physiology, i.e. (Verdes, 2005), in neurophysiology, i.e. (Chávez et al., 2003) and also in analysis of financial data, i.e.(Marschinski \& Kantz, 2002).

(Paluš et al., 2001a;b) applied their conditional mutual information based measures in analysis of electroencephalograms of patients suffering from epilepsy.

Other applications of the conditional mutual information in neurophysiology are due to (Hinrichs et. al., 2006) and (Pflieger \& Greenblatt, 2005).

Causality or coupling directions in multimode laser dynamics is another diverse field where the conditional mutual information was applied (Otsuka et al., 2004). (Paluš \& A. Stefanovska, 2003) adapted the conditional mutual information approach (Paluš et al., 2001b) to analysis of instantaneous phases of interacting oscillators and demonstrated suitability of this approach for analyzing causality in cardio-respiratory interaction (Paluš et al., 2001b). The later approach has also been applied in neurophysiology (Brea et al., 2006).

More recent applications of information-theoretical functionals in natural sciences (medicine) are for example in (Van Dijck et al., 2007), inferring and quantifying causality in neuronal networks (Chicharro et al., 2011), (Vicente et al., 2010), in the computer simulation of human-robot interaction in (Sumioka et al., 1997) or in the relationship of predator-prey in etiology (Bochmann, 2007).

The information-theoretical functionals applied in social sciences are mostly in financial applications: i.e. application of transfer entropy to the information flow between various 
financial time series (Dimpfl et al., 2011) or analysis of the Korean stock market by transfer entropy (Baek et al., 2006). Applications of transfer entropy in linguistics can be found in the book (Baeyer, 2005).

Social media (for example Twitter or Facebook) serves to researches as an important source for studying social interactions. One important problem is the characterization and identification of influentials, which can be defined as users who influence the behavior of large number of other users. To characterize influence (in other words a causal relationship) in Twitter, researchers have suggested number of followers, mentions, and retweets (Cha et al., 2010), and Pagerank of follower network (Kwak at al., 2010). (Ver Steeg et al., 2011) however argue that the purely structural measures of influence (causality) can be misleading (Ghosh \& Lerman, 2010) and high popularity does not necessarily imply high influence (Romero et al., 2010; Ver Steeg et al., 2011).

More recent work has used the size of the cascade trees (Bakshy et al., 2011) and influence-passivity score (Romero et al., 2010). One serious drawback of existing methods is that they are based on explicit causal knowledge (i.e., $A$ responds to $B$ ), whereas for many data sets such knowledge is not available. (Ver Steeg et al., 2011) suggest a model-free transfer entropy approach to detect causal relationships and identifying influential users based on their capacity to predict the behavior of other users.

Having reviewed the relevant literature and also after extensive practical experience, we can state that the information-theoretic approach to the Granger causality plays an important, if not a dominant role in analyses of causal relationships in nonlinear systems.

In the following we define a practical criterium for detection of causal relationships among time series by means of transfer entropy.

\subsection{Directionality index}

To measure causal structure on small data sets and to allow conclusions about the dominant direction of the information flow, the (causal) directionality index was defined for transfer entropy or conditional mutual information by Paluš in (Paluš \& A. Stefanovska, 2003) and analogically in (Rosenblum \& Pikovsky, 2001). It is given by

$$
D I(\mathbf{Y} \rightarrow \mathbf{X} \mid \mathbf{Z})=\frac{\mathcal{T}_{\mathbf{Y} \rightarrow \mathbf{X} \mid \mathbf{Z}}-\mathcal{T}_{\mathbf{X} \rightarrow \mathbf{Y} \mid \mathbf{Z}}}{\mathcal{T}_{\mathbf{Y} \rightarrow \mathbf{X} \mid \mathbf{Z}}+\mathcal{T}_{\mathbf{X} \rightarrow \mathbf{Y} \mid \mathbf{Z}}},
$$

where $X, Y, Z$ are time series. Paluš et al. in Paluš \& A. Stefanovska (2003) consider special cases for $Z$, the so called phase increments of $X$ and $Y$ :

$$
D I(\mathbf{Y} \rightarrow \mathbf{X})=\frac{\mathcal{T}_{\mathbf{Y} \rightarrow \mathbf{X}}-\mathcal{T}_{\mathbf{X} \rightarrow \mathbf{Y}}}{\mathcal{T}_{\mathbf{Y} \rightarrow \mathbf{X}}+\mathcal{T}_{\mathbf{X} \rightarrow \mathbf{Y}}}
$$

where $\mathcal{T}_{\mathbf{Y} \rightarrow \mathbf{X}}=H\left(\mathbf{X} \mid \mathbf{X}^{-} \oplus \boldsymbol{\Delta} \mathbf{X}^{-}\right)-H\left(\mathbf{X} \mid \mathbf{X}^{-} \oplus \mathbf{Y}^{-} \oplus \boldsymbol{\Delta} \mathbf{X}^{-}\right)$and $\Delta X=X(n+k)-X(n)$ and similarly $\mathcal{T}_{\mathbf{X} \rightarrow \mathbf{Y}}=H\left(\mathbf{Y} \mid \mathbf{Y}^{-} \oplus \boldsymbol{\Delta} \mathbf{Y}^{-}\right)-H\left(\mathbf{Y} \mid \mathbf{Y}^{-} \oplus \mathbf{X}^{-} \oplus \boldsymbol{\Delta} \mathbf{Y}^{-}\right)$and $\Delta Y=Y(n+k)-Y(n)$.

The index varies between -1 and 1 , where negative values imply that the information flow from $X$ to $Y$ dominates and positive vales indicate a large information flow from $Y$ to $X$.

The definitions in the literature on the concrete subintervals are unfortunately not united. As well as there exist other modifications of the causal directionality index in the literature. We 
will use the following definitions. The case when the index equals to -1 or 1 respectively we call explicit unidirectional causality from $Y$ to $X$ or $X$ to $Y$ respectively. The case when the directional index is in interval $<-1,0)$ we call it a prevailing unidirectional causality from $X$ to $Y$ (and analogically when the directional index is in interval $(0,1>$ we call it a prevailing unidirectional causality from $Y$ to $X$ ). In case the directionality index equals to 0 , we call it an absence of unidirectional causality.

\section{Gaussianity and causal relationships}

To avoid any misunderstanding, we assume that the investigated random processes are given by time series with a finite number of data (a discrete case). We assume that one knows the probability (density) distribution of the processes which the time series represent, and these we define for a continuous case by explicit analytical formulas. By terms a probability density function or probability distributions which will be used in the text we mean a probability density distribution function.

(Barnett, 2009) recently proved that if all processes (time series) $X, Y, Z$ defined by (4) are jointly Gaussian then Granger causality and transfer entropy are equivalent (up to a multiplication constant of 2). This result provides for the first time a unified framework for data-driven causal inference that bridges information-theoretic and autoregressive methods. For practice it means that in the complexity sense cheaper linear test can be applied for detection of causality, when one knows the time series are Gaussian.

We investigated in our paper (Hlaváčková-Schindler, 2011) the question, to which other multivariate probability distributions of the time series can be the equivalence (up to a multiplicative constant) of the two causality measures extended. In the same paper we extended the equivalence of these two measures to the generalized normal distribution, to the log-normal distribution and Weinman exponential distribution. Since a lot of phenomena in nature and social areas have these distributions, have our results practical implications for studying causal relationships among those phenomena.

In the following we will further investigate generalized Gaussian distributions given parametrically and causal relationships among them. Let us recall their definition.

\subsection{Generalized Gaussian distributions}

Generalized Gaussian distributions which was defined in 1995 by Nadarajah in (Nadarajah, 1995) is a parametrical class of distributions containing all Gaussian and Laplacian distributions as special cases.

Generalized Gaussian density (GGD) is defined as

$$
p(x ; \alpha, \beta)=\frac{\beta}{2 \alpha \Gamma(1 / \beta)} e^{-(|x| / \alpha)^{\beta}}
$$

where $\Gamma($.$) is the Gamma function \Gamma(z)=\int_{0}^{\infty} e^{-t} t^{z-1} d t, z>0$. The parameter $\alpha$, the scale parameter, models the width of the pdf peak (standard deviation) and $\beta$, the shape parameter, is inversely proportional to the decreasing rate of the peak.

The generalized Gaussian distribution for $\beta=2$ is the Gaussian distribution and for $\beta=1$ the Laplacian distribution. 


\subsubsection{Special subclasses of generalized Gaussians and their Kullback-Leibler divergence}

Let us recall that the Kullback -Liebler distance (KLD) of two parametrical (general) probability density functions $p\left(x, \theta_{p}\right)$ and $q\left(x, \theta_{q}\right)$ is defined as

$$
D\left(p\left(x, \theta_{p}\right) \| q\left(x, \theta_{q}\right)\right)=\sum_{x} p\left(x, \theta_{p}\right) \log \frac{p\left(x, \theta_{p}\right)}{q\left(x, \theta_{q}\right)}
$$

where $\theta_{p}$ and $\theta_{q}$ are parameters of probability distributions $p$ and $q$ respectively and $x \in R$.

It can be easily shown that for two probability density functions which are generalized Gaussians $p\left(x, \alpha_{1}, \beta_{1}\right), p\left(x, \alpha_{2}, \beta_{2}\right)$, the Kullback-Leibler divergence can be expressed as

$$
D\left(p\left(x, \alpha_{1}, \beta_{1}\right) \| p\left(x, \alpha_{2}, \beta_{2}\right)\right)=\log \left(\frac{\beta_{1} \alpha_{2} \Gamma\left(1 / \beta_{2}\right)}{\beta_{2} \alpha_{1} \Gamma\left(1 / \beta_{1}\right)}\right)+\left(\frac{\alpha_{1}}{\alpha_{2}}\right)^{\beta_{2}} \frac{\Gamma\left(\left(\beta_{2}+1\right) / \beta_{1}\right)}{\Gamma\left(1 / \beta_{1}\right)}-\frac{1}{\beta_{1}} .
$$

A subclass of generalized Gaussians where the shape parameter $\beta$ is fixed is defined as

$$
\mathcal{P}_{\beta}(\alpha)=\left\{p_{\beta}(x ; \alpha), \alpha \in R^{+} \mid p_{\beta}(x ; \alpha)=\frac{\beta}{2 \alpha \Gamma(1 / \beta)} e^{-(|x| / \alpha)^{\beta}}\right\} .
$$

The Kullback-Leibler divergence between two pdfs from $\mathcal{P}_{\beta}(\alpha)$ is

$$
D\left(p_{\beta}\left(x, \alpha_{1}\right) \| p_{\beta}\left(x, \alpha_{2}\right)\right)=\log \left(\frac{\alpha_{2}}{\alpha_{1}}\right)+\left(\frac{\alpha_{2}}{\alpha_{1}}\right)^{\beta} \frac{1}{\beta}-\frac{1}{\beta} .
$$

For $\beta=1$ it is a Laplacian distribution. In this case is the Kullback-Liebler distance between two Laplacian distributions

$$
D\left(p_{1}\left(x, \alpha_{1}\right) \| p_{1}\left(x, \alpha_{2}\right)\right)=\log \left(\frac{\alpha_{2}}{\alpha_{1}}\right)+\left(\frac{\alpha_{1}}{\alpha_{2}}\right)-1 .
$$

For two Gaussian functions with parameter $\alpha_{1}$ and $\alpha_{2}$ and $\beta_{1}=\beta_{2}=2$ holds

$$
D\left(p_{2}\left(x, \alpha_{1}\right) \| p_{2}\left(x, \alpha_{2}\right)\right)=\log \left(\frac{\alpha_{2}}{\alpha_{1}}\right)+\frac{1}{2}\left(\frac{\alpha_{1}}{\alpha_{2}}\right)-\frac{1}{2} .
$$

\subsection{Transfer entropy as a conditional Kullback-Leibler divergence}

Define $p(X):=\{p(x) \mid x \in X\}$ and similarly $p(X, Y):=\{p(x, y) \mid x \in X, y \in Y\}$ for joint probability distributions. Kullback-Leibler divergence $D$ between two joint probability density functions $p(X, Y)$ and $q(X, Y)$ can be expressed as

$$
D(p(X, Y) \| q(X, Y))=D(p(X) \| q(X))+D(p(Y \mid X) \| q(Y \mid X))
$$

(the chain rule from Cover (Cover \& Thomas, 1991).) Similarly, the conditional Kullback-Leibler divergence can be expressed as

$$
D(p(X, Y \mid Z) \| q(X, Y \mid Z)=D(p(X \mid Z) \| q(X \mid Z))+D(p(Y \mid X, Z) \| q(Y \mid X, Z))
$$

where

$$
D(p(X \mid Z) \| q(X \mid Z))=\sum_{x \in X, z \in Z} p(x, z) \log \frac{p(x \mid z)}{q(x \mid z)}
$$


Transfer entropy can be in terms of Kullback-Leibler divergence rewritten as

$$
\mathcal{T}_{x \rightarrow y \mid z}=D\left(p\left(x_{n+1} \mid x_{n}^{(k)}, y_{n}^{(l)}, z_{n}^{(k)}\right)|| p\left(x_{n+1}^{(k)} \mid z_{n}^{(k)}\right),\right.
$$

where $x_{n}^{(k)}=\left(x_{n}^{1}, \ldots, x_{n}^{k}\right), z_{n}^{(k)}=\left(z_{n}^{1}, \ldots, z_{n}^{k}\right)$, and $y_{n}^{(l)}=\left(y_{n}^{1}, \ldots, y_{n}^{l}\right)$.

We will use the statements from Sections 5.1. and 5.2 in proofs of our results in Section 6.

\section{Causality in time series with generalized Gaussian distributions: our results}

The time series having generalized Gaussian distributions given parametrically allow to study and to express the relationships between them by means of their parameters. In the framework of definition of the directionality index introduced in section 4.3, we investigated the cases of the prevailing unidirectional causality, the explicit unidirectional causality and the absence of causality.

In the following we consider generalized Gaussian distributions given parametrically with the same shape parameter $\beta$. We give conditions on the relationships between the parameters of the involved probability distributions so that causal relationships between them can be detected by the directionality index.

Theorem 1. Assume that times series $X, Y$ have generalized Gaussian distributions given parametrically by $p_{X}\left(x, \alpha_{x}, \beta\right)=\frac{\beta}{2 \alpha_{x} \Gamma(1 / \beta)} e^{-\left(|x| / \alpha_{x}\right)^{\beta}}$ and $p_{Y}\left(y, \alpha_{y}, \beta\right)=\frac{\beta}{2 \alpha_{y} \Gamma(1 / \beta)} e^{-\left(|y| / \alpha_{y}\right)^{\beta}}$, $\alpha_{x}, \alpha_{y}, \beta>0$. Then

(i) there is a prevailing unidirectional causality from $Y$ to $X$, if for the parameters of both distributions $p_{X}\left(x, \alpha_{x}, \beta\right)$ and $p_{Y}\left(y, \alpha_{y}, \beta\right)$ holds $\frac{\beta \log \left(\frac{\alpha_{x}}{\alpha_{y}}\right)^{2}+\Gamma\left(\frac{1}{\beta}\right)\left[\left(\frac{\alpha_{y}}{\alpha_{x}}\right)^{\beta}-\left(\frac{\alpha_{x}}{\alpha_{y}}\right)^{\beta}\right]}{\Gamma\left(\frac{1}{\beta}\right)\left[\left(\frac{\alpha_{y}}{\alpha_{x}}\right)^{\beta}+\left(\frac{\alpha_{x}}{\alpha_{y}}\right)^{\beta}\right]-2} \in(0,1>$.

(ii) there is a prevailing unidirectional causality from $X$ to $Y$, if for the parameters of both distributions $p_{X}\left(x, \alpha_{x}, \beta\right)$ and $p_{Y}\left(y, \alpha_{y}, \beta\right)$ holds $\left.\frac{\beta \log \left(\frac{\alpha_{x}}{\alpha_{y}}\right)^{2}+\Gamma\left(\frac{1}{\beta}\right)\left[\left(\frac{\alpha_{y}}{\alpha_{\alpha}}\right)^{\beta}-\left(\frac{\alpha_{x}}{\alpha_{y}}\right)^{\beta}\right]}{\Gamma\left(\frac{1}{\beta}\right)\left[\left(\frac{\alpha_{y}}{\alpha_{x}}\right)^{\beta}+\left(\frac{\alpha_{x}}{\alpha_{y}}\right)^{\beta}\right]-2} \in<-1,0\right)$.

(iii) there is an explicit unidirectional causality from $Y$ to $X$, if for the parameters of both distributions $p_{X}\left(x, \alpha_{x}, \beta\right)$ and $p_{Y}\left(y, \alpha_{y}, \beta\right)$ holds $\beta \log \left(\frac{\alpha_{x}}{\alpha_{y}}\right)^{2}=\left(\frac{\alpha_{x}}{\alpha_{y}}\right)^{\beta}\left[1+\Gamma\left(\frac{1}{\beta}\right)\right]-2$.

(iv) there is an explicit unidirectional causality from $X$ to $Y$, if for the parameters of both distributions $p_{X}\left(x, \alpha_{x}, \beta\right)$ and $p_{Y}\left(y, \alpha_{y}, \beta\right)$ holds $\beta \log \left(\frac{\alpha_{x}}{\alpha_{y}}\right)^{2}=2-2 \Gamma\left(\frac{1}{\beta}\right)\left(\frac{\alpha_{y}}{\alpha_{x}}\right)^{\beta}$.

(v) there is an absence of unidirectional causality between time series $X$ and $Y$, if for the parameters of both distributions $p_{X}\left(x, \alpha_{x}, \beta\right)$ and $p_{Y}\left(y, \alpha_{y}, \beta\right)$ holds $\beta \log \left(\frac{\alpha_{x}}{\alpha_{y}}\right)=-\frac{1}{2} \Gamma\left(\frac{1}{\beta}\left[\left(\frac{\alpha_{y}}{\alpha_{x}}\right)^{\beta}-\frac{\alpha_{x}}{\alpha_{y}}\right)^{\beta}\right]$ and $\left.\left[\left(\frac{\alpha_{y}}{\alpha_{x}}\right)^{\beta}+\frac{\alpha_{x}}{\alpha_{y}}\right)^{\beta}\right] \neq 2$.

Proof: The items (i) -(v) can be proven by a direct application of the directionality index and the expression of transfer entropy by means of Kullback-Leibler divergence for generalized Gaussian distributions $D(X \| Y)=\frac{2 \beta \alpha_{x} \alpha_{y} \log \frac{\alpha_{y}}{\alpha_{x}}+\alpha_{x}^{2}-\alpha_{y}^{2}}{\left(\alpha_{x}-\alpha_{y}\right)^{2}}$. 


\subsection{Causality detection between two Laplacian distributions}

For the Laplacian distributions holds $\beta=1$, which simplifies the conditions set in Theorem 1 for the remaining parameters. Theorem 1 ist then for the remaining parameters reformulated as follows.

Corollary 1. Assume that times series $X, Y$ have Laplacian distributions given parametrically by $p_{X}\left(x, \alpha_{x}\right)=\frac{1}{2 \alpha_{x}} e^{-\left(|x| / \alpha_{x}\right)}$ and $p_{Y}\left(y, \alpha_{y}\right)=\frac{1}{2 \alpha_{y}} e^{-\left(|y| / \alpha_{y}\right)}, \alpha_{x}, \alpha_{y}>0$. Then

(i) there is a prevailing unidirectional causality from $Y$ to $X$, if for the parameters of both distributions holds $\frac{\alpha_{x}}{\alpha_{y}}-1 \geq \log \left(\frac{\alpha_{x}}{\alpha_{y}}\right)>\frac{\alpha_{x}^{2}-\alpha_{y}^{2}}{2 \alpha_{x} \alpha_{y}}$.

(ii) there is a prevailing unidirectional causality from $X$ to $Y$, if for the parameters of both distributions holds $\left.\frac{2 \log \left(\frac{\alpha_{y}}{\alpha_{y}}\right)+\frac{\alpha_{y}}{\alpha_{x}}-\frac{\alpha_{x}}{\alpha_{y}}}{\frac{\alpha_{y}}{\alpha_{x}}+\frac{\alpha_{x}}{\alpha_{y}}-2} \in<-1,0\right)$.

(iii) there is an explicit unidirectional causality from $Y$ to $X$, if for the parameters of both distributions holds $\log \frac{\alpha_{x}}{\alpha_{y}}=\frac{\alpha_{x}}{\alpha_{y}}-1$.

(iv) there is an explicit unidirectional causality from $X$ to $Y$, if for the parameters of both distributions holds $\log \frac{\alpha_{y}}{\alpha_{x}}=\frac{\alpha_{y}}{\alpha_{x}}-1$.

(v) there is an absence of unidirectional causality between time series $X$ and $Y$, if for the parameters of both distributions $p_{X}\left(x, \alpha_{x}\right)$ and $p_{Y}\left(y, \alpha_{y}\right)$ holds $\log \frac{\alpha_{x}}{\alpha_{y}}=-\frac{1}{2}\left[\frac{\alpha_{y}}{\alpha_{x}}-\frac{\alpha_{x}}{\alpha_{y}}\right]$ and $\frac{\alpha_{y}^{2}+\alpha_{x}^{2}}{\alpha_{x} \alpha_{y}} \neq 2 \alpha_{x} \alpha_{y}$.

Proof: The items (i) -(v) can be proven by a direct application of the directionality index and the expression of transfer entropy by means of Kullback-Leibler divergence for Laplacian distributions.

\subsection{Causality detection between two Gaussian distributions}

For the Gaussian distributions holds $\beta=2$ and Theorem 1 for the remaining parameters simplifies into the following corollary.

Corollary 2. Assume that times series $X, Y$ have Gaussian distributions given parametrically by $p_{X}\left(x, \alpha_{x}\right)=\frac{1}{\alpha_{x} \sqrt{\pi}} e^{-\left(|x| / \alpha_{x}\right)^{2}}$ and $p_{Y}\left(y, \alpha_{y}\right)=\frac{1}{\alpha_{y} \sqrt{\pi}} e^{-\left(|y| / \alpha_{y}\right)^{2}}, \alpha_{x}, \alpha_{y}>0$. Then

(i) there is a prevailing unidirectional causality from $Y$ to $X$, if for the parameters of both distributions $p_{X}\left(x, \alpha_{x}\right)$ and $p_{Y}\left(y, \alpha_{y}\right)$ holds $\frac{4 \log \left(\frac{\alpha_{x}}{\alpha_{y}}\right)+\sqrt{\pi}\left[\left(\frac{\alpha_{y}}{\alpha_{x}}\right)^{2}-\left(\frac{\alpha_{x}}{\alpha_{y}}\right)^{2}\right]}{\sqrt{\pi})\left[\left(\frac{\alpha y}{\alpha_{x}}\right)^{2}+\left(\frac{\alpha_{x}}{\alpha_{y}}\right)^{2}\right]-2} \in(0,1>$.

(ii) there is a prevailing unidirectional causality from $X$ to $Y$, if for the parameters of both distributions holds $\left.\frac{4 \log \left(\frac{\alpha_{x}}{\alpha_{y}}\right)+\sqrt{\pi}\left[\left(\frac{\alpha_{y}}{\alpha_{x}}\right)^{2}-\left(\frac{\alpha_{x}}{\alpha_{y}}\right)^{2}\right]}{\sqrt{\pi})\left[\left(\frac{\alpha_{y}}{\alpha_{x}}\right)^{2}+\left(\frac{\alpha_{x}}{\alpha_{y}}\right)^{2}\right]-2} \in<-1,0\right)$.

(iii) there is an explicit unidirectional causality from $Y$ to $X$, if for the parameters of both distributions holds $\log \left(\frac{\alpha_{x}}{\alpha_{y}}\right)=\frac{\sqrt{\pi}}{4}\left[\left(\frac{\alpha_{x}}{\alpha_{y}}\right)^{2}+\left(\frac{\alpha_{x}}{\alpha_{y}}\right)^{2}\right]-\frac{1}{2}$.

(iv) there is an explicit unidirectional causality from $X$ to $Y$, if for the parameters of both distributions holds $\log \left(\frac{\alpha_{x}}{\alpha_{y}}\right)=1-\sqrt{\pi}\left(\frac{\alpha_{y}}{\alpha_{x}}\right)^{2}$. 
(v) there is an absence of unidirectional causality between time series $X$ and $Y$, if for the parameters of both distributions $p_{X}\left(x, \alpha_{x}\right)$ and $p_{Y}\left(y, \alpha_{y}\right)$ holds $\log \frac{\alpha_{x}}{\alpha_{y}}=-\frac{\sqrt{\pi}}{4}\left[\left(\frac{\alpha_{y}}{\alpha_{x}}\right)^{2}-\left(\frac{\alpha_{x}}{\alpha_{y}}\right)^{2}\right]$ and $\left(\frac{\alpha_{y}}{\alpha_{x}}\right)^{2}+$ $\left(\frac{\alpha_{x}}{\alpha_{y}}\right)^{2} \neq \frac{2}{\sqrt{\pi}}$.

Proof: The items (i) -(v) can be proven by a direct application of the directionality index and the expression of transfer entropy by means of Kullback-Leibler divergence for Gaussian distributions.

In praxis, for time series given by finite number of observations, one can apply methods for finding parameters of corresponding generalized Gaussian distributions, which these data sets interpolate. Knowing these parameters, the statements in our theorem and corollaries provide simple decision criteria about a presence or absence of causality between two time series.

\section{Conclusion}

In this paper we presented linear and non-linear methods on causality detection among time series, namely Granger causality and transfer entropy, respectively. We discussed their applications both in natural and social sciences. For the purpose of selecting a method for causality detection among time series, the approach of using statistical hypothesis testing techniques, applied in G-causality has several difficulties. The issue of multiple testing which is being done sequentially in general does not provide an optimal solution. In addition, there is no objective guideline for the choice of the input of the individual test (i.e. the size of the time series interval) and it is unclear how would such a choice influence the detection of causality. The main drawback of model dependent methods for causality detection is their instability. As expected, with a small or moderate number of observations, models close to each other are often hard to distinguish and the values of the model selection criterion are usually close to each other. A small change on the data may result in a choice of a different hypothesis. As a consequence, the causality detection based on the selected hypothesis may have a high variability (ill-posedness of the problem). The main conceptual advantage of the Granger causality model over the information-theoretical approaches to causality detection is its linearity and its straightforward generalization for multivariate time series testing. The main conceptual drawback of Granger causality is its inability to detect eventual non-linear causal relationships.

For the reasons stated above, in this paper we focused on causality detection by transfer entropy. We dealt with the Gaussian and some non-Gaussian distributions of natural phenomena as well of phenomena occurring in social sciences. We presented original and simple criteria for finite time series having generalized Gaussian distributions given parametrically representing natural or social phenomena which decide about a presence or absence of causality between them.

Our results can considerably simplify current more computationally demanding data processing methods applied in natural and social sciences for detection of causal relationships.

\section{References}

Abarbanel, H.D.I. (1993) Introduction to Nonlinear Dynamics for Physicists. Lecture Notes in Physics, World Scientific, Singapore. 
Ancona, N., Marinazzo, D. \& Stramaglia, S. (2004) Radial basis function approach to nonlinear Granger causality of time series. Physical Review E 70(5): 6221.

Aparicio F.M. \& Escribano, A. (1998) Information-theoretic analysis of serial dependence and cointegration. Studies in Nonlinear Dynamics and Econometrics 3: 119-140.

Aristotle (350 B.C.) Physics. The Internet Classics Archive. Translated by R. P. Hardie and R. K. Gaye, http://classics.mit.edu//Aristotle/physics.html

Arnhold, J., Grassberger, P., Lehnertz, K. \& Elger, C.E. (1999) A robust method for detecting interdependences: application to intracranially recorded EEG. Physica D 134: 419-430.

Baek, E.G. \& Brock, W.A. (1992) A general test for nonlinear Granger causality: Bivariate model, Working paper, Iowa State University and University of Wisconsin, Madison.

Baek, S.K., Jung, W.S., Kwon, O. \& Hie-Tae Moon, H.T. (2006) Transfer entropy analysis of the Korean stock market. Physica A: Statistical Mechanics and its Applications, Elsevier Science.

Baghli, M. (2006) A model-free characterization of causality Economics Letters 91: 380-388.

Bakshy, E., Hofman, J.M., Mason, W.A. \& Watts, D.J. (2011) Everyone's an inluencer: quatifying influence on twitter. In Proc. fourth ACM international conference on Web search and data mining, WSDN'11, pages 65-74, New York, NYm USA, ACM.

Barnett, L., Barett, A.B. \& Seth, A.K. (2009) Granger causality and transfer entropy are equivalent for Gaussian variables. Phys. Rev. Lett. 103, 238701.

Bayer, H.C. (2005) Information: The New Language of Science. Harvard University Press, Harvard.

Bell, D., Kay, J. \& Malley, J. (1996) A non-parametric approach to non-linear causality testing. Economical Letters 51: 7-18.

Bicego, M., Gonzalez-Jimenez, D., Grosso, E. \& Alba Castro, J.L. (2008) Generalized Gaussian distributions for sequential data classification. 978-1-4244-2175-6/08/2008 IEEE.

Blinowska, K.J., Kuś, R., and Kamiński (2004) M. Granger causality and information flow in multivariate processes. Phys.Rev. E 70 050902(R).

Boccaletti, S., Kurths, J., Osipov, G., Valladares, D.L., \& Zhou, C.S. (2002) The synchronization of chaotic systems. Physics Reports 366: 1-101.

Bochmann, O., Lizier, J.T., Mahoney, J., Obernosterer, G. \& Pahle, J. (2007) Computational mechanics and information measures in food webs. Workshop on Computational Biology, University of Santa Fe.

Brea, J., Russell, D.F. \& Neiman A.B. (2006) Measuring direction in the coupling of biological oscillators: A case study for electroreceptors of paddlefish. Chaos 16: 026111.

Broomhead D.S. \& Lowe, D. (1988) Multivariate functional interpolation and adaptive networks Complex Systems 2: 321- 355.

Cha, M., Haddadi, H., Benevenuto, F. \& Gummadi, K.P. (2010) Measuring user influence in twitter: The milion follower fallacy. ICMSM'10: In Proceedings of international AAAI Conference on Weblogs and Social.

Chávez, M., Martinerie, J. \& Le Van Quyen, M. (2003) Statistical assessment of nonlinear causality: Application to epileptic EEG signals. Journal of Neuroscience Methods 124: 113-128.

Chen, Y., Rangarajan, G., Feng J. \& Ding, M. (2004) Analyzing multiple nonlinear time series with extended Granger causality. Phys. Lett. A 324: 26-35.

Chicharro, D., Andrzejak, R.G. \& Anders Ledberg (2011) Inferring and quantifying causality in neuronal networks. BMC Neuroscience 12(Suppl 1): 192. 
Cover T. \& Thomas, J. (1991) Elements of Information Theory. John Wiley and Sons, New York, NY, Chapter 9.

David O., Kiebel S., Harrison L., Mattout J., Kilner J., et al. (2006) Dynamic causal modelling of evoked responses in EEG and MEG. NeuroImage 30: 1255Ü1272.

Diks, C. \& DeGoede, J. (2001) A general nonparametric bootstrap test for Granger causality. Global Analysis of Dynamical Systems. Chapter 16, eds Broer, Krauskopf and Vegter, 391-403.

Diks C. \& Panchenko, V. (2005) A note on the Hiemstra-Jones test for Granger non-causality. Studies in Nonlinear Dynamics and Econometrics 9 (4):1-7.

Dimpfl, T., Huergo, L. \& Peter, F.J. (2011) Using transfer entropy to measure information flows from and to the CDS market. Proc. of the European Economic Association and Econometric Society, 25 - 29 August 2011, Oslo, Norway, 2011.

Feridun, M. (2007) Immigration, income and unemployment: An application of the bounds testing approach to cointegration. Journal of Developing Areas 41 (1): 37-49.

Fujita, A., Severino, P., Sato, J.R. \& Miyano, S. (2010) Granger causality in systems biology: Modeling gene networks in time series microarray data using vector autoregressive models. Advances in Bioinformatics and Computational Biology, LNCS 2010, Volume 6268/2010: 13-24.

Ge T., Kendrick K.M., Feng J. (2009) A Novel Extended Granger Causal Model Approach Demonstrates Brain Hemispheric Differences during Face Recognition Learning. PLoS Comput Biol 5(11):e1000570.

Geweke, J. (1984) Inference and causality in economic time series models. Handbook of Econometrics, eds Z. Griliches and M.D. Intriligator, North-Holland, 2: 1101-1144.

Ghosh, R. \& Lerman, K. (2010) Predicting influential users in online social networks. In Proc. KDD workshop on Social Network Analysis (SNAKDD), May 2010.

Gilbert, E. \& Karahalios, K. (2009) Widespread worry and the stock market. Association for the Advancement of Artificial Intelligence.

Granger, C.W.J. (2003) Time series analysis, cointegration, and applications. Nobel Lecture. In: Les Prix Nobel. The Nobel Prizes 2003, ed. Tore Frängsmyr, Nobel Foundation (Stockholm, 2004): 360-366.

Granger, C.W.J. (1969) Investigating causal relations by econometric and cross spectral methods. Econometrica 37: 424-438.

Grassberger, P. \& Procaccia, I. (1983) Measuring of strangeness of strange attractors. Physica D: $189-208$.

Herdan, G. (1958) The relations between the dictionary distribution and the occurrence distribution of word length and its importance for the study of quantitative linguistics. Biometrika 45: 222-228.

Hesse, W., Moller, E., Arnold, M. \& Schack, B. (2003) The use of time-variant EEG Granger causality for inspecting directed interdependencies of neural assemblies. J Neurosci Methods 124: 27-44.

Hiemstra, C. \& Jones, J.D. (1994) Testing for linear and nonlinear Granger causality in the stock priceÜvolume relation. Journal of Finance 49: 1639-1664.

Hinrichs, H., Heinze, H.J. \& Schoenfeld, M.A. (2006) Causal visual interactions as revealed by an information theoretic measure and fMRI. NeuroImage 31 1051-1060.

Hlaváčková-Schindler, K., Paluš, M. Vejmelka, M. \& Bhattacharya, J. (2007) Causality detection based on information-theoretic approaches in time series analysis. Physics Reports 441: 1-46. 
Hlaváčková-Schindler, K. (2011) Equivalence of Granger causality and transfer entropy: A generalization. Applied Mathematical Sciences 5(73) 3637-3648.

Hume, D. (1896) A Treatise of Human Nature. Selby-Bigge, ed., Clarendon Press.

Illari, P.M., Russo, F. \& Williamson, J. (2011) Causality in the Sciences. Oxford University Press.

Kamiński, M. Ding, M, W.A. Truccolo, W.A. \& Bressler, S.L. (2001) Evaluating causal relations in neural systems: Granger causality, directed transfer function and statistical assessment of significance Biol. Cybern. 85: 145-157.

Kim, S., Putrino, D., Ghosh, S. \& Brown, E.N. (2011) A Granger causality measure for point process models of ensemble neural spiking activity. Computational Biology7 (3) Open Access Publication.

Kufmann, R.K. \& Stern, D. (1997) Evidence for human influence on climate from hemispheric temperature relations. Nature 388, 3 July 1997, p.39.

Kwak, H., Lee, C., Park, H. \& Moon, S. (2010) What is twitter, a socila network or a news media? In Proceedings of the 19th international conference on World wide web, WWW'10, pages 591-600, New York, NY, USA, ACM.

Limpert, E., Stahel, W.A. \& Abbt, M. (2001) Log-normal distributions across the sciences: Keys and clues. BioScience 51 (5) May 2001.

Mackie, J.L. (1988) The Cement of the Universe: A study in Causation. Clarendon Press, Oxford, England.

Marinazzo, D., Pellicoro M. \& Stramaglia, S. (2006) Nonlinear parametric model for Granger causality of time series. Physical Review E 73: 066216.

Marschinski, R. \& Kantz, H. (2002) Analysing the information flow between financial time series - An improved estimator for transfer entropy. European Physical Journal B 30: 275-281.

Misiriotis, A., Kylafis, N.D., Papamastorakis, J. \& Xilouris, E.M. (2000) Is the exponential distribution a good approximation of dusty galactic disks? Astron. Astrophys. 353: 117-123.

Mokhov, I.I. \& Smirnov, D.A. (2006) El Nino-Southern oscillation drives North Atlantic oscillation as revealed with nonlinear techniques from climatic indices. Geophysical Research Letters 33 L03708.

Moulin, P. \& Liu, J. (1999) Analysis of multiresolution image denoising schemes using generalized Gaussian and complexity priors. IEEE Trans. Inform. Theory vol. 45: 909-919.

Nadarajah, S. (1995) A generalized normal distribution. Journal of Applied Statistics 32 (7): 685 Ü694.

Newton, I. , (1687) Philosophiae Naturalis Principia Mathematica A new translation by I.B. Cohen and A. Whitman, University of California press, Berkeley 1999.

Otsuka, K., Miyasaka, Y., \& and Kubota, T. (2004) Formation of an information network in a self-pulsating multimode laser. Phys. Rev. E 69: 046201.

Paluš, M. \& A. Stefanovska (2003) Direction of coupling from phases of interacting oscillators: An information-theoretic approach. Phys. Rev. E 67: 055201(R).

Paluš, M., Komárek, V., Procházka, T., Hrnčíř, Z. \& Štěrbová, K. (2001) Synchronization and information flow in EEG of epileptic patients. IEEE Engineering in Medicine and Biology Magazine 20: 65-71.

Paluš, M., Komárek, V., Hrnčíř, Z. \& Štěrbová, K. (2001) Synchronization as adjustment of information rates: Detection from bivariate time series. Phys. Rev. E 63: 046211. 
Park, I. \& Principe, J.C. (2008) Correntropy based Granger causality. IEEE International Conference on Acoustics, Speech and Signal Processing ICASSP 2008.

Pearl, J. Causality (2000) Models, reasoning and inference. Cambridge University Press.

Pflieger, M.E. \& Greenblatt, R.E. (2005) Using conditional mututal information to approximate causality for mutlivariate physiological time series. Int. J. Bioelectromagnetism 7: 285-288.

Pikovsky, A., Rosenblum M. \& Kurths, J. (2001) Synchronization. A Universal Concept in Nonlinear Sciences. Cambridge University Press, Cambridge.

Poon, S.H. \& Granger, C.W.J. (2003) Forecasting Volatility in Financial Markets: A Review. Journal of Economic Literature 41: 478Ü539.

Preston, F.W. (1981) Pseudo-log-normal distributions. Ecology 62:355-364.

Romero, D.M., Galuba, W., Asur, S. \& Huberman, B.A. (2010) Influence and passivity in social media. Social Science Research Newtor Working Paper Series, August 2010.

Quyen, M.L.V., Martinerie, J., Adam, C. \& Varela, F.J. (1999) Nonlinear analyses of interictal EEG map the brain interdependences in human focal epilepsy. Physica D 127: 250-266.

Rosenblum, M.G. \& Pikovsky, A.S. (2001) Detecting direction of coupling in interacting oscillators. Phys Rev E 64: 045202.

Russo, F. (2009) Causality and causal modelling in the social sciences. Measuring variations. Springer, New York.

Sailaja, V., Srinivasa Rao, K. \& Reddy, K.V.V.S. (2010) Text independent speaker identification with finite multivariate generalized gaussian mixture model and hierarchical clustering algorithm. Int. Journal of Computer Applications 11 (11): 0975-8887.

Schäfer, C., Rosenblum, M.G., Kurths, J. \& Abel, H.H. (1998) Heartbeat synchronized with ventilation. Nature 392: 239-240.

Schreiber, T. (2000) Measuring information transfer. Phys. Rev. Lett. 85: 461-464.

Schäfer, C., Rosenblum, M.G., Kurths, J. \& Abel, H.H. (1999) Synchronization in the human cardiorespiratory system. Phys. Rev. E 60: 857-870.

Seth, A. K., Edelman, G. M. (2007) Distinguishing causal interactions in neural populations. Neural Comput. 19(4): 910-933.

Sharifi, K., Leon-Garcia, A. (1995) Estimation of shape parameter for generalized Gaussian distributions in subband decompositions of video. IEEE Trans Curcuits Syst. Video Technol. 5: 52-56.

Schiff, S.J., So, P., Chang, T., Burke, R.E. \& Sauer, T. (1996) Detecting dynamical interdependence and generalized synchrony through mutual prediction in a neural ensemble. Phys. Rev. E 54: 6708-6724.

Smith, S.M., Miller, K.L., Salimi-Khorshidi, G., Webster, M., Beckmann, C.F., Nichols, T.E., Ramsey, J.D. \& Woolrich, M.W. (2011) Network modelling methods for FMRI. Neuroimage 54 (2): 875-891.

Statistical yearbook in Switzerland (1997).

Sumioka, H., Asada, M. \& Yoshikawa, Y. (2007) Causality detected by transfer entropy leads acquisition of joint attention. IEEE 6. Intern. Conference on Development and Learning, London. 264-269.

Tass, P., Rosenblum, M.G., Weule, J., Kurths, J., Pikovsky, A., Volkmann, J., Schnitzler, A. \& Freund, H.J. (1998) Detection of n:m phase locking from noisy data: Application to Magnetoencephalography. Phys. Rev. Lett. 81: 3291-3294. 
Triacca, U. (2005) Is Granger causality analysis appropriate to investigate the relationships between atmospheric concentration of carbon dioxide and global surface air temperature? Theor. Appl. Climatol. 81: 133-135.

Van Dijck, G., Van Vaerenbergh, J. \& Van Hulle, M.M. (2007) Information theoretic derivations for causality detection: Application to human gait, LNCS, Vol. 4669: 159-168.

Verdes, P.F. (2005) Assessing causality from multivariate time series. Physical Review E 72: 026222.

Ver Steeg, G. \& Galstyan, A. (2011) Information transfer in social media, October 2011, arxiv.org/PS ache/arxiv/pdf/1110/1110.2724v1.pdf.

Vicente R., Wibral M., Lindner M. \& Pipa, G. (2010) Transfer entropy: a model-free measure of effective connectivity for the neurosciences. Journal of Computational Neuroscience doi:10.1007/s10827-010-0262-3: 1-23.

Williams, C.B. (1940) A note on the statistical analysis of sentence length as a criterion of literry style. Biometrika 31:356-361.

Zinde-Walsh, V. \& Phillips, P. (2003) Fractional Brownian motion as a differential generalized Gaussian process. Lecture Notes Monograph Series Vol. 41. 


\title{
Qualitative Research: The Toolkit of Theories in the Social Sciences
}

\author{
Sylvain K. Cibangu \\ Master of Arts in Social Sciences, Regis University, Denver, \\ Master of Communication in Digital Media, University of Washington, Seattle,
}

USA

\section{Introduction}

In recent years, the social sciences ${ }^{1}$ have witnessed an explosive surge in textbooks, disciplines, specialties, publication venues, research centers, etc. However, few books have addressed the theoretical status of the social sciences as a whole. One reason is that theory is simply understood as a purely intellectual exercise withdrawn from reality and human progress. Another reason is that for a long time, qualitative research, the toolkit of theories in the social sciences, has been considered inferior and weak in scientific research. Still another reason is that authors tend to situate qualitative research's beginnings in the 1920s or 1960s, while leaving aside important social theorists. As a result, misunderstandings concerning social science research have increased. For example, social science research has been equated with the endeavor to interpret meaning and/or study subjective and nonmeasurable phenomena. While such assumptions can be true, they represent a distortion of social science research and reduce the potential role of theory construction in the social sciences. This chapter takes stock of the role of theory in the social sciences. To this end, the chapter maps the broader history of qualitative research, diffuses some misunderstandings, appraises the specifics of qualitative research, and outlines some practical consequences for theory building in the social sciences.

The last few decades, the social sciences have seen a burgeoning interest in qualitative research even as textbook materials have become one of the most flourishing markets of our times. A Web tally of the five largest and best known publishing houses of social science materials in the world-Sage Publications, headquartered in Thousand Oaks, CA, US (http://www.sagepub.com); Taylor and Francis Group, headquartered in London, UK (http://www.taylorandfrancis.com/); Pearson Education, headquartered in Upper Saddle River, NJ, US (http://www.pearsoned.com); Cengage Learning, headquartered in Stamford, CT, US (http://www.cengage.com); and Rowman and Littlefield, headquartered in Lanham, MD, US (http://www.rowmanlittlefield.com) - shows that for the year 2010 alone two research methodology textbooks appeared every day. This tally does not involve the

\footnotetext{
${ }^{1}$ For the purposes of this chapter, the social and human sciences are taken to be interchangeable since these groups of sciences had been criticized all together for having a weak scientific character. For interested parties, Cibangu's (2010) article sketches the differences between the social and human sciences.
} 
outputs of university presses, which commonly publish textbooks and references, or of small-scale publishing companies unincorporated by the five publishing companies listed above. Also worth noting is the fact that publishing houses increasingly require textbooks to include both quantitative and qualitative research to ensure a broader marketing and more persuasive academic reach. "Hardly any handbook is published today that does not have a chapter on qualitative research methods" (Flick, 2002, p. 6). However, despite the rise in qualitative research methods in published textbooks and journals, reflections on social science theory remain scant.

The relevance of qualitative research for social science theory owes much to the core of qualitative research. In effect, it is not that quantitative research cannot generate theories (see Shoemaker, Tankard, \& Lasorsa, 2004), but that qualitative research, as apparent below, has a much greater realm of theory creation than does quantitative research. The legendary theory productivity of qualitative research stands on the fact that qualitative researchers deal with the real word in its fullness whereas quantitative researchers intentionally manipulate the world through the lenses of pre-set laboratory and/or laboratory-like questionnaires and samples (Creswell, 2008, 2009). Quantitative research pursues the controllability and manipulability of the independent variable(s) at the exclusion of all external and internal factors (hence the word independent) to allow for the predictability of intended outcomes (dependent variables). The required manipulability of quantitative research reduces, and most often disguises, the already infinite scope of people's lifeworlds and creativity. Quantitative researchers disconnect themselves from the real world in order to manipulate and study the selected phenomenon. Qualitative researchers, however, seek out and immerse themselves into the real, uncontrolled, crude, and non-manipulated world (of humans) to derive and interpret the hidden patterns (theories). It becomes clear that there are more theories to discover in the real world than there are in a laboratory and the like.

On another level, the growing, and often erratic, combination of qualitative and quantitative methods has led many to believe that qualitative research can be best studied only in comparison with and/or in appendix to quantitative research. Such a belief belies the theoretical/practical role of qualitative research. Two good examples are gender and food. Though women can be seen as complimentary to men, should they be studied only in comparison with men? Certainly, women present a nature proper to them that requires no comparison with men. Though they can be mixed with rice to form one protein-filled meal, should beans be studied only as supplementation to rice? Beans present specific characteristics of their own, irrespective of rice. Still, the good news concerning the combination of qualitative and quantitative methods is that it calls for good social science research. Brady, Collier, and Seawright (2006) remarked, "too many qualitative and quantitative studies are simply bad work. We believe that both sides in the qualitativequantitative debate would be more credible if they began by acknowledging how hard it is to do good social science" (pp. 354-355). Familiarity with discussions of qualitative research, through increased exposure and involvement, can help the social sciences to achieve sound research of qualitative methodology needed for theory creation. This chapter is organized into six parts: (1) definitions of basic concepts, (2) theory/theoretical contribution, (3) historical background of qualitative research, (4) misunderstandings around qualitative research, (5) specifics of qualitative research, and (6) practical consequences. 


\section{Definitions of basic concepts}

Before we begin our discussion, the clarification of a few basic research concepts is helpful. There has been an increase in the use of varied-and often confusing-terminology in research textbooks. A typical example is with the concept research process alternatively called research methods (Crowther \& Lancaster, 2008; Graziano \& Raulin, 2010; Patton, 2002; Thomas, Nelson, \& Silverman, 2005), research methodologies (Ackroyd, 2006; Noble \& Bestley, 2005; Yeboah, 2008), research design (Creswell, 2009; Hakim, 2000; de Vaus, 2001; Gschwend \& Schimmelfennig, 2007), social research (Babbie, 2010), or analyzing social settings (Lofland, Snow, Anderson, \& Lofland, 2006), among others. For the sake of simplicity, four concepts central to our debate need precision: (1) methodology, (2) method, (3) qualitative research, and (4) case study.

First, we will begin with the two closely related terms: methodology and method. While it is impossible to put enough stress on the importance of methods and methodology in research design, it is equally difficult to arrive at a fixed and unified definition of both terms. For example, in their landmark works on research, Brady, Collier, and Seawright (2010), Collier, Brady, and Seawright (2010), Denzin (2009, 2010), Denzin and Lincoln (1998, 2003a, 2003b, 2005, 2008a, 2008b, 2008c, 2011), and Lincoln and Denzin (2008a, 2008b) employed methodology and method interchangeably, whereas Mason (2002), Teddlie and Tashakkori (2009), and Tashakkori and Teddlie (2010) described methodology and methods as different. ${ }^{2}$ This chapter regards methodology as a suite of methods governed by chief philosophical underpinnings of research, namely, positivism and interpretivism (details below), and method is understood to be a selected strategy or technique with which to address the questions raised in the research process (e.g., discourse analysis, focus group, observation, interview, etc.). ${ }^{3}$ The third term that needs definition after methodology and method is qualitative research. Carried to its fundamental roots, qualitative research is a research whose means of data analysis is not statistical, and which can involve one participant $(n=1)$, be it a document, event, process, individual, concept, organization, etc. We will discuss the characteristics of qualitative research later; for now this basic definition should suffice. Fourth and last, case study is a systematic investigation of an individual, phenomenon, idea, document, etc. (Ragin, 1987, 1998, 1999, 2004, 2009; Stake, 2008; Yin, 2003, 2009). While it can be quantitative, case study in this chapter is taken to mean qualitative case study. Let us turn now to the concept of theory and theoretical contribution.

\section{Theory/theoretical contribution}

In this section, we will discuss theory and its corollary, theory construction. First, theory figures among the least valued concepts of academia and industry. All too often, theory is understood as a body of concepts withdrawn from reality. More specifically, theory is seen as an anti-thesis to practice. However, theory lies at the core of research practice and human

\footnotetext{
2 Beware that entire chapters have been reproduced verbatim across Denzin's and Lincoln's oeuvre. Two examples, among others, Denzin's \& Lincoln's (2008a) preface and Denzin's \& Lincoln's (2008b) introduction to Landscape of Qualitative Research have been copied and pasted as Denzin's \& Lincoln's (2008d) preface and Denzin's \& Lincoln's (2008e) introduction to Strategies of Qualitative Inquiry, respectively.

${ }^{3}$ This definition is a revised version of Picard's (2007) definition.
} 
existence. Humans have a tendency to theorize their experience into patterns. For example, we see things, love people, and make decisions based on the implicit or explicit theories we hold. The critical theorist Adorno (1951/1974) noted, "since utopia was set aside and the unity of theory and practice demanded, we have all become too practical. Fear of the impotence of theory supplies a pretext for bowing to the almighty production process" (p. 44 ). To a great extent, the mass production of technologies has accentuated this theoryphobia. Authors have also believed that theories do not exist nor do we need them in our existence. This position denies the importance of theory to both the natural and social sciences.

There is no theory. In fact, I don't know of any theory in the social sciences. I don't think the term theory should be applied to fields as intellectually thin as the social sciences. So there is no theory... Theory is very different from understanding... We live our lives often pretty successfully without any theories about other people... There is very few areas of human life where there is anything you might call a theory. Even in biology... Use your sense...you can get as good a sense of the world as anybody does [without theory]. (Chomsky, 1998, n/p)

As shown below, theory is essential to science and human reality. But it should be noted in passing that Chomsky proved to be an influential theorist in the social sciences, in general (detail below), and the field of linguistics, in particular. Without theory, no science and skill can be taught and/or improved over time. It is the case that both the natural and social sciences are made up of a common core of materials used in the fundamental courses of these fields. Sociologist Parsons (1938) wrote, "this common core is not only a body of discrete miscellaneous facts - [but] it is closely integrated with a logically elaborated body of theory - much of which... is stated in a highly generalized form" (p. 13). Theory constitutes the core body of a science's literature, which is criticized, evaluated, and revamped over time in a logically articulated manner. The best way of imagining theory is through the idea of criticism, the substance of theory. Imagine what would become of science and human existence without criticism? One of the goals of repressive regimes is to prevent criticism. The more repression a society is faced with, the less progress and freedom it suffers. "Theory and theorising play key roles in both the natural and the social/behavioural sciences... Both natural and social sciences are empirical in nature, with theory as the primary product" (Venable, 2006, p. 2). Theory plays a vital role in research processes and human existence. Critical discussion allows progress, invention, and creativity. The now widely accepted notion of freedom of speech is an expression of critical discussion.

Another commonly believed idea presents theory as an anti-thesis to reality. As Muirhead, one of Aristotle's best commentators, explained,

Theory is sometimes thought of as concerned with general laws, and therefore as the antithesis of fact and reality. But this, of course, is a misunderstanding. The function of theory is not to carry us away into a region of abstraction and comparative unreality, but to put us into closer touch with fact. $(1900$, p. 21)

Theory constitutes a tool with which to gain a tighter grasp of reality. Theory is a set of propositions that explain specific relationships between the phenomena being studied. "Theories, however, are a large part of our world, framing the way issues are seen, shaping 
perceptions of salience, and thus slanting debate toward certain policies rather than others" (Nussbaum, 2011, p. xi). Theories are the lenses through which we interact with the world.

While there is no unified definition or exhaustive list of theory, it is possible, and in fact important, to derive the commonly featured characteristics of a theory. This chapter explains the four characteristics of a theory outlined by Eisenhardt (1989) and Whetten (1989). Eisenhardt's and Whetten's works have served as the classical materials of theory construction in the social sciences. ${ }^{4}$ In simpler and softer terms, Whetten (1989) defined theory as follows,

A complete theory must contain four essential elements... What. Which factors (variables, constructs, concepts) logically should be considered as part of the explanation of the social or individual phenomenon of interest... How. Having identified a set of factors, the researcher's next question is, how are they related?... Such a step adds order to the conceptualization by explicitly delineating patterns. In addition, it typically introduces causality... Why. What are the underlying psychological, economic, or social dynamics that justify the selection of factors and the proposed causal relationships? This rationale constitutes the theory's assumptions - the theoretical glue that welds the model together...Who, When, and Where. These conditions place limitations on the propositions generated from a theoretical model. These temporal and contextual factors set the boundaries of generalizability, and as such constitute the range of the theory [italics in original]. (pp. 490-492)

The whats and hows describe the concepts, constructs, characteristics, frameworks, and theories gleaned from the bodies of literature concerning the thing being studied. The whys explain the observed patterns and lay out the discrepancies and similarities. The whos, whens, and wheres set the limitations inherent in the researcher's suggested theory and chosen methodology. In essence, theory represents a set of demonstrated relationships between selected variables or constructs. In other words, with a proposed theory, the researcher aims to capture and demonstrate the missing relationship(s) in the observed patterns or regularities. Theory is not just mere speculation about the observed relationships, but a definite contribution to the field and the world. This chapter insists on contribution to the researcher's field (for contribution to the world, see Cibangu, in press).

The second and last point after the definition of theory is the issue of theoretical contribution. Without contribution, science becomes a nuisance or mere verbiage. Like theory, theoretical contribution is seldom discussed, but it provides the substance of scientific work. As should now be clear, by probing the whats, hows, whys, whos, whens, and wheres within a body of work, a sustained discussion of the theories offered in selected social science literature is likely to strengthen the theoretical contributions in the selected area of research. In order to make a contribution, a suggested theory needs to be unique and novel in relation to the literature. Theoretical contribution endeavors to detect and address the gaps, insufficiencies, and weaknesses in relevant literatures to propose newer and tighter relationships about the selected phenomena. Theoretical contribution comes from the

${ }^{4}$ For advanced discussion about theory construction (also called theory development or theory building), de Jong (2010), George and Bennett (2005), Haynes and Carroll (2010), and Jaccard and Jacoby (2010), among others, supplied helpful reflections. 
theory arrived at in a research. Theory construction allows researchers to craft contributions to their fields. Put more clearly, theoretical contribution distinguishes scientific work from other forms of work, such as political speech, newspaper articles, mission statements, art works, diaries, commentary, personal communication, TV shows, etc. One can undertake a research that impacts, and in fact changes, the whole world, but without theory, the research/project is not scientific. It is not the efficient and usually (cost-)effective implementation of the research project and its practical consequences that make the project scientific, but the theory arrived at. Eisenhardt (1989) wrote,

An essential feature of theory building is comparison of the emergent concepts, theory, or hypotheses with the extant literature. This involves asking what is this [observed finding and tendency] similar to, what does it contradict, and why. A key to this process is to consider a broad range of literature. (p. 544)

Such a process requires a broad-based groundwork in the wealth of theories attendant on the key concepts of the selected social science research. The point here is to juxtapose conflicting literatures and findings. Eisenhardt (1989) explained,

Conflicting literature represents an opportunity. The juxtaposition of conflicting results forces researchers into a more creative, framebreaking mode of thinking than they might otherwise be able to achieve. The result can be deeper insight into both the emergent theory and the conflicting literature, as well as sharpening of the limits to generalizability of the focal research. (p. 544)

Selected literature lays bare a number of concepts, hypotheses, and theories. The researcher does not seek to settle the debate, but to raise awareness about the conflicting and solid relationships encountered in the literature. Exposure to and involvement with these and many other relationships are not a solution, but a toolkit needed for firmer and newer research insights. Since qualitative research has grappled with the philosophical underpinnings of social science research, the background of qualitative research is important in order to ensure solid theoretical contributions in the social science literature.

\section{Historical background of qualitative research}

The background of qualitative research comprises a range of thinkers and related theoretical traditions. Qualitative research is deeply rooted in the social sciences' developments. Nevertheless, despite its recent forays in the publishing market, research methodology literature has accorded little attention to the history of qualitative research. As demonstrated below, this methodological restriction has precipitated innumerable misunderstandings about qualitative research and its potentials for theorization. Much of the literature situates the beginnings of qualitative research and its textbooks in the 1960s and 1970s (Flick, 2002, 2009; Gobo, 2005; Knoblauch, Flick, \& Maeder, 2005; Patton, 2002). The most common history of qualitative research is the one succinctly proposed under the rubric of (eight, now nine) historical moments. This history of qualitative research is limited to the US (Denzin, 2010; Denzin \& Lincoln, 2005, 2008b; Lincoln \& Denzin, 2003, $2008 \mathrm{~b}$ ), and is said to have started in the 15th - 16th centuries under the banner of descriptive anthropology or ethnography (Vidich \& Lyman, 1998, p. 46). Originally, the historical moments of qualitative research were five (Denzin \& Lincoln, 1998). As Denzin 
and Lincoln noted, "we employed an arbitrary historical model" (2005, p. xiii). This is not to say that there is a perfect historical model, but that significant portions of qualitative research's tradition have been omitted. However, works of qualitative research trace as far back as Antiquity. In fact, Antiquity brims with examples of qualitative work. For our discussion, I will present three most important ancient writers: the Egyptian Ptahhotep (25th c. BC, Parkinson, 1991), Herodotus (5th c. BC, 1957, 1960), and Aristotle (4th c. BC, 1853).

Despite the disparity between educated and uneducated people in his times, the ancient Egyptian philosopher Ptahhotep valued the necessity of talking to both groups of people. He wrote, "be not proud for being wise. Consult with the ignorant as with the wise" (Parkinson, 1991, p. 66). Textual analysis of this passage reveals interesting consequences for our discussion. The Oxford American Dictionary (1999) provides the following synonyms for the term ignorant: illiterate, uneducated, uninformed, unlearned, unlettered, and untutored. It can be argued that in-person interview of illiterate individuals was found to yield invaluable information. Theorization is taking place around the lived experiences of participants, outside the context of classrooms and laboratories. Because they involve human sensory expressions to their full extent, lived experiences can be used as a basis for theory formation. Such a preferred mode of inquiry represents an accomplished qualitative technique since, per statistical norms, a written questionnaire or survey requires informants to be educated.

The second ancient author is Herodotus. Conducted over three decades using in-person contacts with respondents, as well as open-ended interviews, Herodotus' (5th c. BC, 1957, 1960) work constitutes, to my knowledge, the largest recorded fieldwork material concerning world population. Despite its limitations, Herodotus' study brought to bear highly detailed knowledge about selected respondents, providing details of aspects as diverse as cultural, linguistic, political, geographic, historical, and physical information, all of which is unfeasible with standard quantitative method. For focus purposes, the present chapter does not consider the whole nine-volume collection of Herodotus' histories nor does it intend to supply a specialized qualitative research study focused on Herodotus' work. Interested parties can read Lateiner (1989). Herodotus' work points to the significance of historical methods (Gilderhus, 2009; Howell \& Prevenier, 2001; McCullagh, 1984), which has yet been underutilized in qualitative research. For example, with its probe of reasoning, researchers' biases, fact (re)presentation, and sources/documents, historical criticism is a powerful tool for any research design. Historical methods help look at authors and events in the past, their lifeworlds, works, effects, and the significance of their embedded theories. The idea is to immerse in the real context of participants and interpret the patterns carried over time beneath people's lifeworlds.

The third work is Aristotle's (4th c. BC, 1853) famous posthumous Organon, especially the sections Prior Analytics, On Interpretation, and Posterior Analytics (see also Corcoran, 1974, 2003, 2009). Aristotle propounded the idea of context-bound universal truths. He laid out the procedures of the validity and invalidity of scientific knowledge on the basis of one or a small number of cases (i.e., syllogisms). He understood theories as scientific knowledge or universal truths. It is inexplicable that research methodology theorists have taken the validity of scientific knowledge to be equated with and limited to specific statistical tests of 
experimental research while leaving aside Aristotle's magisterial writing. As Corcoran (2003) stressed, "by setting forth in clear and systematic fashion the basic methods for establishing validity and for establishing invalidity, Aristotle became the founder of logic as formal epistemology" (p. 261). Logic is ideally case-specific and context-bound analysis, the core feature of qualitative research. Common to logic are the following areas: causality, counterfactual analysis, inference, etc. (see Baumgartner, 2008, 2009a, 2009b, 2010; Braham \& van Hees, 2009; George \& Bennett, 2005; Heckman, 2005; Hendrickson, 2010; Morgan \& Winship, 2007). In lieu of and contrast with randomized and representative samples, Aristotle established the validity of scientific knowledge by using (1) a purposefully selected small number of cases, (2) everyday human experience, and (3) real life context. All these criteria, as apparent in this paper, lie at the heart of qualitative research's canon. In the Aristotelian methodology, human sensory expressions were not a threat to scientific knowledge; rather, they enabled it. In this regard, scientific knowledge combined the natural and human sciences. Theorization in Antiquity implied the concepts of people's lived experiences, lifeworlds shared over time, context-bound universal truths, and logical validity.

In the meantime, Antiquity saw authors who rejected human sensory expressions and real context as reliable sources of knowledge. Plato (5-4th c. BC, 1997), for example, defended universal knowledge as one that is unchanging, non-context-specific, stable, and independent from the sensible world. Though beyond the scope of the present chapter, the argument of realism in Plato's work can very well be made, which implies that knowledge is context-specific, see Grabowski (2008) and Plato (4-5th c. BC, 1921, 1925, 1966). For advanced discussion of Aristotle's realism see Fine (2004) and Rickless (2007), among others. This chapter focuses on Aristotle because of his seminal work (4th c. BC, 1853) on the validity of scientific discourse. Notable examples of the rejection against human sensory expressions were also the Milesian (from Miletus, the ancient Greek city) Greek philosophers such as Thales (7th-6th c., BC), Anaximander (7th-6th c., BC), and Anaximines (6th c., BC), among others, who considered supernatural, artistic, religious, mystic, and human expressions as irrelevant to and incompatible with scientific knowledge (Duignan, 2011; Gill \& Pellegrin, 2006; Gottlieb, 2002). Aristotle's seminal work (4th c. BC, 1853) Organon inscribed itself against such anti-human and purerationalistic science, to give just one example. The Aristotelian work left an indelible impact on research methodology from Antiquity onward. The three selected ancient writers Ptahhotep (Parkinson, 1991), Herodotus (5th c. BC, 1957, 1960), and Aristotle (4th c. BC, 1853) show that universal knowledge involves context-limited conditions (i.e., inperson contact, illiterate individuals, small cases, etc.), human sensory expressions, and lifeworlds shared over time. In this sense, knowledge integrates the human and natural sciences since the patterns observed in the natural sciences do not differ from those encountered in people's lifeworlds.

The Middle Ages inherited Antiquity's scientific discourse, whose methodology was based on human sensory expressions, logic (case analysis), people's lifeworlds, and the combination of the natural and human sciences. In the 11th century, in his famous encyclopedia of medical knowledge, the Persian scholar Avicenna (1025/1970) provided a case analysis methodology for the purposes of experimental medicine. Avicenna's work aligns itself with a research practice called casuistry (Jonsen \& Toulmin, 1988), 
widespread in the Middle Ages, which means the study of cases, from the Latin word casus [case]. Casuistry goes back to the Aristotelian case analysis logic. Another characteristic is that the Middle Ages, especially the 1400s and early 1500s, saw a great emphasis placed on human ideals and values to infuse technology. And medieval technology expanded tremendously with the press and the construction of public buildings and cathedrals across Europe. Related exemplars include Leonardo da Vinci (1452-1519), Johannes Gutenberg (1398-1468), and Leon Battista Alberti (1404-1472), among others (Burke, 1999; Copenhaver \& Schmitt, 2002; King, 2003; Kristeller \& Mooney, 1979). Technology was taken to be integral to human ideals and values. To be scientific, educated, or learned during this time was synonymous with acquiring technical skills as well as the humanities. The humanities concerned themselves with human sensory expressions and lifeworlds. Put differently, the human sciences are ones that deal with the inner and/or exterior behavior of humans active or recorded in writings or art works whereas the social sciences consider human interaction to be the central piece in the study of social reality. One can see that scientific discourse does not draw its legitimacy from the rift between the natural and social sciences, nor is it exhausted by the natural sciences. Coupled with case-specific analysis, the Antiquity-inherited mindset of human-natural integrated science grew into modernism, with the view to improve human material and spiritual conditions.

In the 1600s and 1700s, however, the modernist era brought case-specific (logic/philosophy) and context-bounded knowledge and related human values and ideals under considerable attack. In this chapter, modernism is not understood as literary work, art design, and architecture, but as the line of thought, inherited from positivism, which teaches the power of reason to conquer nature and improve human conditions (see Kumar, 2005). The modernist era defended a new idea of science that magnified the power of reason to conquer nature. Several historiographers of science (Burke, 1999; Copenhaver \& Schmitt, 2002; King, 2003; Kristeller \& Mooney, 1979) showed that the modern period started principally with the English philosopher Bacon (1561-1626) and French philosopher Descartes (1596-1650). Bacon (1605/1960) wrote New Organon in an attempt to replace Aristotle's (4th c. BC, 1853) Organon. Bacon's goal was to present scientific knowledge in the manner of natural laws and properties, which Bacon thought to be unchanging, stable, universal, and independent from context and human feelings. Big numbers reflected universality and stability whereas small numbers symbolized variability and particularity. Qualitative research was accorded less value due to its characteristic of small-number samples. The subtitle of Descartes' (1637/1987) work, Discourse on the method, is also suggestive: to guide well the reason and seek truth in sciences. Both the Baconian and Cartesian lines of thought celebrate the absolute power of reason. Since natural laws could be replicated to make machines and tools, they were thought to be the explanation and foundation of human reality. Bacon's (1605/1960) New Organon was based on the famous four idols and accompanying aphorisms that present human nature as a definite deterrent to correct reasoning. Correct reasoning had to detach itself from human inherently misleading tendencies, by using objective tools of measurement and neutral knowledge procedures. For Bacon, human feelings and preconceived ideasthat is, biases - were the sources of error from which to methodically protect the intellect. Relegated to metaphysics or philosophy, theory was viewed as a diversion from natural 
laws and human progress. Therefore, science was intended to allow full, objective, and undisturbed use of reason to conquer nature, enlighten the intellect, and improve human society. This era led to the Enlightenment or Age of Reason. Replication (replicability) of natural laws created an unrestrained pursuit of law-like explanations of reality such that human expressions were seen as alien to and incompatible with natural laws.

Curiously enough, it was within the Age of Reason setting that most universities were formed in North America and Western Europe and that traditional social sciences - such as sociology, anthropology, political science, psychology, and economics-came to be recognized as academic disciplines. Consequently, the Age of Reason influenced the ways in which social science and qualitative research methodology was undertaken for a long period of time. Knowledge acquisition was understood as the endeavor to conquer and master the universe and its objective laws. For example, it was in allegiance to the Baconian (Bacon, 1605/1960) aphorisms that French social thinker Durkheim ${ }^{5}$ (1894/1988) proposed aphorisms that were detached from passions and opinions to define social facts as forces that prevailed upon, and that were independent from and exterior to individuals and their feelings and preconceived ideas. More particularly, Comte (1848/1999) popularized the word positivism by presenting sociology as positive, since it replicated objective principles. Positivistic research methodology seeks knowledge through observation and experiment, in opposition to human feelings, emotions, dreams, ideals, and the like. As is clear above, Bacon's (1605/1960) work has left its marks on social science methodology and has embodied the cornerstone of positivism. It is problematic, then, that so many research methodology discussions bypass Bacon's work.

Nevertheless, since the 1800s and early 1900s, Bacon's (1605/1960) methodology has drawn strong criticism from within the social sciences, the most poignant manifestations of which took place from a variety of fronts under the banner of anti-positivism. The goal of this criticism was to demystify the Enlightenment's claim to absolute powers of reason and the accompanying objective universal methodology. Science's goal shifted from the replication of objective natural laws to the in-depth understanding of nature and individuals. Authors were as diverse as Bachelard (1884-1962), De Tarde (1843-1904), De Tocqueville (1805-1859), Dilthey (1833-1911), Freud (1856-1939), Husserl (1859-1938), Marx (1818-1883), Mill (18061873), Simmel (1858-1918), Weber (1864-1920), and Windelband (1848-1915), among others. With this host of critics, Bacon's science project did not succeed in overthrowing the tenets inherited from Aristotle's Organon (4th c. BC, 1853). For example, Bachelard (1934) outlined the limitations of realism and rationalism defended as absolute in positivism. De Tarde (1890/1993) championed the study of interaction between small numbers of individuals. De Tocqueville (1840/2004) defended the importance of knowledge embedded in the particulars. Dilthey $(1988,2002)$ propounded the scientific status of the human sciences and related lived experiences. Freud (1900/1913) showed that reason is governed by irrationality. Husserl (1913/1962, 1952/1980) presented metaphysical subjective philosophy as a rigorous discipline. Mill (1843/1872) provided a detailed invaluable account of causal

\footnotetext{
${ }^{5}$ An important clarification to make here is that in his other works, Durkheim $(1893 / 2007,1912 / 2008)$ presented us with substantive, yet very often forgotten in research methodology materials, support for case study research, see also Rawls (1996) for further discussion of Durkheim.
} 
inferential knowledge using a small number of cases. Marx (1867/1977) observed that the power of reason engendered increased contradictions in society between the poor and the rich, exploitation and progress, production and dehumanization, etc. Simmel (1908) and Weber (1921/1972) propounded a sociology that looks at individuals' lived experiences. And Windelband (1894/1980) identified scientific knowledge in situated historical circumstances. In one form or another, these authors have expanded on qualitative research's doctrine.

For focus sake, however, three key figures need attention: Simmel (1858-1918), Weber (18641920), and Bachelard (1884-1962). Simmel (1908) and Weber (1921/1972) were credited with applying anti-positivism in the social sciences by propelling the German concept verstehende Soziologie, commonly translated in English as interpretive sociology, to suggest a more effective means of social research beyond the traditional arm-chair research. Interpretive sociology's views have pervaded research methodology across disciplines. It is not possible to chronologically and semantically distinguish Simmel from Weber in their usage of the term interpretive sociology, since they each presented significantly similar and contemporary insights of the term. Chief among these insights was an increased focus on historical context, experience, and meaning in the path to a better understanding of cases and of social phenomena from the perspective of concerned parties. Also similar and contemporary to Simmel and Weber was the notion verstehen, which gained prominence in hermeneutic and phenomenological circles ${ }^{6}$. Although seldom acknowledged in research methodology materials, interpretive sociology embodies the protocol in light of which the concept of interpretivism has been ascribed to the social sciences in general and to qualitative research in particular.

While it can involve - and even be limited to, as is often the case - the study of meaning, text, or human experiences, interpretive sociology, or interpretivism, outstrips a human-, text-, or meaning-bounded conception of qualitative research by inquiring about all aspects of reality, from objects to human experiences to social phenomena7. The widespread meaning-bounded perception, or semanticization, so to speak, of interpretivism falls short of qualitative research's tradition. In fact, the connotation of meaning for the German verb verstehen is overwhelmingly minor. Out of six outlined meanings (Duden, 1996, p. 1667) of verstehen, four are important for our discussion (I changed the enumeration, for ease of use):

1. in Bestimmter Weise auslegen, deuten, aufassen,

2. sich in jemanden, in jemandes Lage hineinversetzen können,

3. eine Verhaltenweise, eine Haltung, eine Reaktion, ein Gefühl eines anderen) vom Standpunkt des Betreffenden gesehen, natürlich, konsequent, richtig, normal finden,

4. gut können, beherrschen... Also interesting is Denzin's (1997) application of the concept interpretive, wherein Denzin went beyond the visual and scriptural dimensions of text and meaning by suggesting the performance of text and people's lives, ethnodrama or ethnoperformance. Interpretivism makes re-live people's lifeworlds.

\footnotetext{
${ }^{6}$ For further discussion about the concept interpretive in general one can read Hiley, Bohman, and Shusterman (1991), Mueller-Vollmer (2006), and Moran and Mooney (2002), among others.

${ }^{7}$ For a broader view of interpretivism, see Denzin and Lincoln (2005), Lincoln and Denzin (2008a), Korte (1999, pp. 86-116), Treibel (1999, pp. 111-132).
} 
The best example of meaning reductionism is with language. One can know, in fact even be a native speaker for, the meaning of the words used by a person, but be unable to understand the person's experiences (i.e., struggles, pains, feelings, views, etc.) behind how the words are being used. Another example is with technology. One can understand what a car is or means and still lack in-depth understanding about the nuts and bolts, so to speak, of the car. Simmel (1908) propelled the importance of not only understanding the Other, but of the Other being understood ${ }^{8}$. Interpretivism serves as the marker of qualitative research (Denzin \& Lincoln, 2008b; Knoblauch, Flick, \& Maeder, 2005) and rebuts the positivistic postulates of methodology. It follows that interpretivism aims to decipher the patterns that compound the clues, puzzles, and forces hidden beneath specific cases and/or human experiences, feelings, ideas, values, beliefs, symbols, etc. When articulated by the researcher, patterns observed beneath lived experiences are turned into theories.

Another seminal, yet often forgotten, contribution to the qualitative tradition is the philosopher of science Bachelard (1884-1962), whose majority of works remains unknown and non-translated in English-speaking academia. Bachelard (1938/2004) was noted for his critique of Comte. Toward this end, Bachelard developed at length the concepts of knowledge shifts and of the discontinuous progress of science, from which Kuhn's (1996) idea of paradigm shift has been drawn. The Russian-born French philosopher of science Koyré (1892-1964), whose (Koyré, 1939) works Kuhn (1996) extensively read and referenced, acknowledged these concepts as having been borrowed from Bachelard. Methodology is thus understood to be a continuous rupture of established and imposed assumptions, truths, and knowledge. Bachelard (1934) was of the opinion that there was no such thing as absolute realism and rationalism, the key tenets of positivism. ${ }^{9}$ As is clear below, this is an important statement of qualitative research. At another level, Bachelard (1938/2004) stated that researchers need to break away from the pride of universal certainties and from the greed for particular certainties.10 It follows that qualitative research does not seek global/universal or local/particular certainties as an end product but, instead, for the purposes of deep-rooted understanding. In their pursuit of theories, researchers need to be aware of this epistemological risk: the pride of universality/globalism and the greed of particularity/localism.

In the 1920s, qualitative research received further impetus from the advent of nontraditional school-driven movements of research. For example, integrating Marxism and Freudianism, the Frankfurt School (Honneth, 2007; Horkheimer \& Adorno, 1947/1989; Martin, 1996; Wiggershaus, 1998) criticized the ways in which reason was not objective and rational, as had been proclaimed in the Age of Reason, but was instrumentalized to create and maintain massive domination and poverty in industrial societies. Armed with in-person small-scale interaction and participant observation, the Chicago School (Bulmer, 1984)

\footnotetext{
8 "durch Verstehen Andrer und Verstandenwerden" (Simmel, 1908, p. 674).

9 "Il n'y a ni réalisme ni rationalisme absolus" (Bachelard, 1934, p. 6). This book has been translated into English by Beacon Press (Bachelard, 1934/1985).

10 "Rompons, ensemble, avec l'orgueil des certitudes générales, avec la cupidité des certitudes particulières" (Bachelard, 1938/2004, p. 290).
} 
undertook dedicated field research in the vastly impoverished outskirts of Chicago. A later version of the Chicago School, symbolic interactionism, emphasized meaning in the processes of everyday interaction.

The second half of the twentieth century moved toward contemporary qualitative research wherein the all-too-devastating effects of the two world wars brought to light both the value and frailty of humans and their societies. In the 1960s and 1970s, postmodernism (Rosenau, 1995; Ward, 2003) began to posit that authority, knowledge, certainty, truth, subjectivity, and so on, are not imposed, essentialized, or absolute from the top down, but are locally and individually constructed. Postmodernism will determine much of contemporary qualitative research, all of which recalls the demystification of absolute reason of the modernist era. As Lincoln and Denzin (2003) stated,

We do not "choose" to be postmodern. The historical moment has chosen us. The implications of this understanding, of this resituating of the argument are enormous... We are not free to "choose" postmodernism. It is the historical moment when the modernist epoch ends. (p. 1060)

Therefore, qualitative work is understood along the lines of postmodernism in its varying forms. With its reversal of the top-down absolute power of reason, postmodernism has led to different variants of locally-driven and empowering movements of thoughts such as postcolonialism, gender studies, cultural studies, etc. One of the most known variants of postmodernism that has been carried in contemporary qualitative research is constructivism. In this chapter, constructivism and constructionism are considered interchangeable (see especially Ratner, 2006 or Berger \& Luckmann, 1967; Burr, 2003; Kukla, 2000; Searle, 1995) since the proposed difference (see Patton, 2002, p. 97) between the two terms is not consequential in the broader context of the social sciences. Much of the literature shows that constructivism has come to be one of the major determinants of contemporary qualitative research (Charmaz, 2009; Lincoln \& Denzin, 2003; Patton, 2002). Constructivism envisages research's results and involved assumptions as culturally, racially, historically, politically, sexually, and ethically constructed. The 1960s and 1970s have also been taken to be the classical beginnings of contemporary qualitative research with authors such as Glaser and Strauss (1967), Garfinkel (1967), and Blumer (1969). In the 1980s, the teaching of qualitative research began to gain mandatory status across curriculums. In the 1990s and 2000s the spectacular advances of information and communication technologies have brought into greater focus the complexity of an increasingly networked world and its challenging dilemmas such as a rise in poverty, natural disasters, ethnic conflicts, and incessant incurable illnesses, to name but a few, making qualitative research all the more valuable to a goal of deepening understanding and making the world a better place.

As should be now clear, qualitative research's rebuttals against positivism have carried different forms at different times since Bacon (1605/1960), although interpretivism has now come to serve as the generic term. After the debates that rocked methodology circles in the 1800s and onward, it is safe to state that the "-ism" terms and related schools employed in qualitative research literature can be best understood in light of or reaction against 
positivism. ${ }^{11}$ Benton and Craib (2001), Kincaid (1996), and Rosenberg (2008) offered some of the most invaluable sources of information about different methodological schools of thoughts in the social sciences. Interpretivism leans toward the idea of solid understanding. Constructivism insists on the fact that understanding is individually and collectively specific. Historicism focuses on socio-historical developments when understanding human actions. Naturalism seeks to understand targeted people and selected phenomena not in experimental and manipulated settings, but in their real-life context. These trends each have a particular flavor with which they formalize and envisage a theory. The awareness about the history behind the foundational assumptions that decisively, and too often, inconspicuously undergird our research processes is important in order to fruitfully engage in any discussion of theory construction. Frequently, research methodology textbooks have propelled fragmentary views of qualitative research's tradition (i.e., figures, schools of thoughts, methods, etc.) to the point that misunderstandings have accrued concerning qualitative research.

\section{Misunderstandings around qualitative research}

The key idea behind the misunderstandings that affect qualitative research resides in the belief that qualitative research is unfit for generalization. Without generalization, one cannot craft theories. "It is exact that the case study is a detailed examination of a solitary exemplar, but it is false to utter that a case study cannot grant unswerving information about the broader class" (Ruddin, 2006, p. 797). More clearly, without generalization, knowledge and its object (i.e., the world or reality) become meaningless. Flyvbjerg (2006) distinguished five most common misunderstandings concerning qualitative analysis.

Misunderstanding 1: General, theoretical (context-independent) knowledge is more valuable than concrete, practical (context-dependent) knowledge.

Misunderstanding 2: One cannot generalize on the basis of an individual case; therefore, the case study cannot contribute to scientific development.

Misunderstanding 3: The case study is most useful for generating hypotheses; that is, in the first stage of a total research process, whereas other methods are more suitable for hypotheses testing and theory building.

Misunderstanding 4: The case study contains a bias toward verification, that is, a tendency to confirm the researcher's preconceived notions.

Misunderstanding 5: It is often difficult to summarize and develop general propositions and theories on the basis of specific case studies. [emphasis in original] (p. 221)

The misunderstandings translate the efforts of different authors that have memorialized qualitative research's traditions, the most predominant of which was Aristotle's concept of context-embedded universal knowledge. We use case-derived generalization more than we think. Another common consequence of these misunderstandings is that qualitative research tends to be seen as synonymous with the social sciences and quantitative

\footnotetext{
${ }^{11}$ Further debate about positivism can be found in Habermas' (1968/1971, pp. 65-186) work. This work, in my view, exposes one of the strongest thrusts of positivism against common misrepresentations. For example, post-positivism should not be considered as identical to or representative of positivism (see Morgan, 2007, p. 61).
} 
research with the natural sciences, respectively. However, this belief is not plausible. Quantitative research textbooks, materials, techniques, methods, software, and procedures come from the hard work of social scientists (Babbie, 2010; Lofland, Snow, Anderson, \& Lofland, 2006). More specifically, social scientists have crafted techniques such as polls, sampling, and surveys, which have been serving as unparalleled authoritative and outstanding tools and guidelines for generalization and scientific rigor. Generalization is not incompatible with qualitative research. There are several reasons why generalization drawn from qualitative research can be useful, of which I have selected three most common.

First, generalization helps make sense of reality. As Flyvbjerg (2001) noted,

Without generalization, we could not interact with our world in a coherent mannerthat is to say, we would need continual repetition of the same mental procedures for each new experience. One can often generalize on the basis of a single case, and the case study may be central to scientific development via generalization as supplement to other methods. (p. 425)

Knowledge comes through generalization. An essential point is that one can live without statistical knowledge, but one cannot function without generalization. Along the same lines, Little (1998) wrote, "a central claim is that social phenomena do not support strong generalizations, akin to laws of nature; rather, we find only weak, phenomenal regularities among social processes and entities" (p. ix). The claim looses ground on a number of levels and reflects a simplistic view of the social world. Popper presented powerful arguments against this claim (see Cibangu, in press). Among the myriad social phenomena that reveal rigid regularities are unemployment, mortality, housing, urbanization, transportation, inflation, group/crowd dynamics, etc. Consequently, "generalization can happen from any research method" (Lucas, 2003, p. 240). Generalization becomes a matter of reality. Particularly pertinent is the historian Gaddis' (2002, pp. 62-66) idea of particular generalization and general particularization. Briefly put, particular generalization helps see generalization within particulars (narratives) whereas general particularization sees particulars as pointers to generalization. The idea shows how generalization without particularization, and vice versa, is impossible (see also Hellström, 2008; Niaz, 2007; Onwuegbuzie \& Leech, 2010). The deeper the knowledge one has about a case, the easier it is to generalize that knowledge for a larger population.12 It is no accident that another word for case in this context is example. And the qualifiers of case or example are concrete, specific, practical, etc. There is no such thing as an abstract and general example. The more concrete the case about a set of propositions is, the stronger the generalization of the propositions will be. This is where the widespread idea of weak generalization for qualitative research or the social sciences is defeated (Little, 1998; Rosenberg, 2008; Smith, 2003; Williams, 2000; Wallerstein et al., 1996). A weak example means superficial, not deep, knowledge. Note that generalization is not uniformization or denial of particularities.

\footnotetext{
${ }^{12}$ For further research on generalization in qualitative research, Brady's and Collier's (2010), Holland's and Herstad's (2000), and Gomm's, Hammersley's, and Foster's (2000) works, to name a few, are recommendable.
} 
A second reason generalization is useful is the research's contribution to the researcher's field. As Marshall and Rossman (2011) clarified,

In examining a specific case or set of individuals, the writer [qualitative researcher] should show how she is studying a case in a larger phenomenon. By linking the specific research questions to larger theoretical constructs or to important policy issues [general applicability], the writer shows that the particulars of the study serve to illuminate larger issues and therefore hold potential significance for that field. (p. 7)

This does not mean that any statement made in qualitative analysis has to be generalized, but that case-specific knowledge, obtained through research, has implications beyond a sampled pool of participants. Contribution makes theory valuable among scientists. A helpful terminology is one of wider resonance or significance. As Mason (2002) maintained,

There is a variety of ways in which generalizations can be made in qualitative research... You may well wish to derive cross-contextual generalities from strategically focused local/contextual studies. You may wish to make claims that have a wider theoretical resonance [emphasis mine]. (p. 39)

Wider resonance implies that applicability can be carried and shared across contextual boundaries and individualities. As was shown in the historical background, from Antiquity to 19th- and 20th-century authors, general theories have been drawn from casespecific knowledge and applied to the larger population. Some notable examples of theories that have been replicated to larger populations are Marx's (1867/1977) theory of class conflicts, Chomsky's (1965) generative grammar, and Piaget's (1926) theory of imitation and innovation, among others. Marx selectively observed a few companies in Germany, and generalized his theory to the rest of the world. Chomsky examined a few English native speakers within his reach in Philadelphia and generalized his generative grammar to all speakers in the world. Piaget observed his own three children and generalized his theory of imitation and innovation to all children of the world. Although these theories are not immune to criticism, they have, however, helped generate other theories, and have each become a momentous pillar of research in sociology, linguistics, and child psychology, respectively. None of these theories-or many others, from Ptahhotep (25th c. BC, Parkinson, 1991) to contemporary qualitative research just discussed above-has used a randomly selected representative sample, but rather a few cases deliberately selected in a naturalistic approach, which is characteristic to qualitative research.

The third and last argument for the use of generalization is that qualitative research has the ability to assess the extent to which controlled quantitative research can be applied in real world situations. Graziano and Raulin (2010) made clear that "case-study and naturalistic research can also enhance the generalizability of research findings, especially in areas in which the research has been conducted in laboratory settings" (p. 116). Statistical tests presuppose a laboratory-like research setting, the outcomes of which qualitative research aims to validate in the real world. Not only is generalizability germane to qualitative research, but it also needs qualitative research to fully unfold the real world. 
A variant appellation of qualitative research's generalization is tied to the concepts of replicability and measurability, which are inherited from the Enlightenment Age (discussed above). It is widely believed that social phenomena are not as replicable/measurable as natural phenomena. Instances of replicable social phenomena, however, are numerous; to name a few: terrorist attacks, ethnic conflicts, murder, bankruptcy, mass protests (such as those in early 2011 in North Africa and the Middle East), etc. Measurability or quantifiability is another concept that is thought to be unfit for qualitative research. Sutton (1998) showed that there are certainly social phenomena that are quantifiable and others that are not. Examples of quantifiable social phenomena include population, democracy (see Freedom House website, http://www.freedomhouse.org), salary, famine, poverty, epidemic, etc. In the same way, there are also natural phenomena that cannot be replicated and/or measured. Astronomy abounds with examples such as movements of the universe, the collision of planets, the formation of Earth, etc.

Misunderstandings around qualitative research are also exacerbated by the tendency to equate qualitative research with just one method. In detail, qualitative research comprises the following methods: logic, ethnography, discourse analysis, case study, open-ended interview, participant observation, counseling, therapy, grounded theory, biography, comparative method, introspection, casuistry, focus group, literary criticism, meditation practice, historical criticism/research, etc. This list is by no means exhaustive, but it is common to limit qualitative research to one of these and similar methods. Furthermore, these methods can be conducted in deference to quantitative tradition. To illustrate, Morgan $(1996,1997)$ referred to quantitative uses of focus groups. However, by identifying the specifics of qualitative research one can ensure a broader view, clearer implementation, and fairer assessment of qualitative methodology's tradition.

\section{Qualitative research's specifics}

While qualitative research has been the object of extensive literature over the last several decades, its specifics have received only passing attention. The specifics of qualitative research provide an efficient tool with which to craft theories. On the one hand, there is a steady consensus among researchers "that the very term qualitative research means different things to many different people [emphasis in original]" (Denzin \& Lincoln, 2005, p. xvii). On the other hand, the diversity behind varied suggested meanings of qualitative research reveals some commonly reflected features. In fact, authors have increasingly acknowledged that definite commonalities traverse the differing implementation of qualitative research (Denzin, 1997; Denzin \& Lincoln, 1998, 2008a, 2008b; Flick, 2002, 2009; Knoblauch, Flick, \& Maeder, 2005; Marshall \& Ross, 2010; Maxwell, 2005; Patton, 2002; Silverman, 2010; Sutton, 1998; Tashakkori \& Teddlie, 2003, 2010; Teddlie \& Tashakkori, 2009). Recognition of commonalities, however, does not warrant the promotion of qualitative research's core principles. As Flick stated, "another result is that qualitative research is in danger of falling into different fields of research and methodological discussions and that in the process core principles and ideas of qualitative research across these different fields could be omitted" (2009, p. xxi). Even more challenging is the fact that, although it is a methodology in and by itself, qualitative research is oftentimes taught as an appendix to or sub-segment of quantitative research methodology. Moreover, while current university curriculums are 
equipped with a whole department devoted to statistics and related positivistic tests, there is no such thing as a department of hermeneutics or casuistry and related methods entirely honed in qualitative research. Most qualitative research experts are either quantitativetrained professionals or ad-hoc specialists, both of which camouflage the specifics of qualitative research. This paper has selected four most important, not mutually exclusive, specifics or components that distinguish qualitative research as: (1) ethnographic, (2) contextual, (3) experiential, and (4) case-analytic.

First, an ethnographic component represents one of the most known, yet forgotten, specifics of qualitative research. The tendency is to relegate ethnographic work to anthropologists for the simple reason that ethnography is thought to require an excessive amount of siteimmersion that an ordinary research timeline cannot afford. Whether it takes a long or short period of time for immersion depends on the researcher's stated priorities, but immersion remains the key defining feature of an ethnographic component-or, as others would argue (Becker, 1996; Silverman, 2010), of fieldwork. Experience shows that we do ethnographic work in our day-to-day lives more than is usually thought. How often do we involve all our senses and body in the setting of what we are talking about or what we want to learn or experience? People live with other people to gain a fuller understanding of other people's needs and lifeworlds. An ethnographic component requires the researcher to be immersed in the spatiality and temporality of the selected participants and their lifeworlds. Ethnographic qualitative research privileges the immersion into the participants' or case's real world and embedded experiences. Peshkin (1988) asserted,

This way [ethnographic research] enables us to bring, first, the complete range of our senses to our investigations; second, sufficient time in which to be attentive; and, third, the breadth of scope, that is, the fullness of what we are willing and able to attend to. (p. 416)

Our whole senses, attentive observation, and the fullness of the phenomenon studied all determine qualitative work. Note that the Baconian (Bacon, 1605/1960) doctrine, discussed earlier, rejects human senses as an impediment to knowledge acquisition. Qualitative research cherishes sense perception as the privileged portal to participants' or case's situated world.

The second component of qualitative research is the contextual component. The contextual component requires the researcher to speak as fully as possible to the particulars of participants' or a case's situated physicality. The term context can be applied to a variety of items such as word, proposition, argument, conservation, idea, book, etc. However, not everything that qualifies as knowledge is contextual or qualitative. Pertinently, contextspecific knowledge lies at the heart of Aristotle's tenets. Aristotle (4th c. BC, 1924) explained,

For it is not man [in general] that the physician cures, except incidentally, but Callias or Socrates or some other person similarly named, who is incidentally a man as well. So if a man has theory without experience, and knows the universal, but does not know the particular contained in it [universal], he will often fail in his treatment; for it is the particular [man] that must be treated. (I, I. 981a)

Qualitative research treats knowledge that is context-dependent. Qualitative research deals with real-life context, present or past. The qualitative context is a context within which the 
selected phenomenon or topic takes place and from which the qualitative researcher draws first-hand data of the study. Even in the case of a context that no longer exists (such as one that occurs with criminology, history, drama, art, text, etc.), qualitative research seeks to rebuild the real-life context and to draw findings as consistent with the original context as possible. This is where statistical data and their numerical outcomes are too insufficient to knit together and reignite the real world of the requisite context. Such an endeavor of reconstruction and enactment of the real-life world, within which participants' or a case's everyday existence burgeons, constitutes the proper domain of qualitative research methodology. In essence, contextual knowledge is one that allows a foreigner to go and live in the place being researched as easily as the natives.

The third qualitative research specific regards an experiential component. The experiential component provides the researcher with the spectrum of feelings, emotions, insights, views, beliefs, and values with which participants live in and interact with the real world. Qualitative research's component of experiential knowledge flows from hermeneutic and phenomenological circles. In a nutshell, hermeneutics concerns itself with the meaning of individuals' actions manifested in documents, texts, events, or social reality, whereas phenomenology studies the Lebenswelt (lifeworld) of human existence. French philosopher Ricoeur (1950, 1969, 1971, 1972, 1986a, 1986b, 1995) presented one of the best integrations of hermeneutics and phenomenology for the social sciences. It is questionable that, despite its immense academic legacy (including its use for linguistics, philosophy, semantics, ethics, theology, law, history, political science, literature, etc.) the Ricoeurian work has received little to no attention in social science and qualitative research literatures. With Ricoeur, social reality is seen as a universe of meaning(s) within and with which individuals share and experience the real world. Therefore, the researcher's immersion into and enactment of participants' lived experiences are central to qualitative research. This implies that qualitative research does not simply seek participants' experiences, but the shared meanings behind those experiences. In the same way, qualitative research does not simply seek participants' meanings, but the essence (such as common ground) or the world behind those meanings.

Pointing to experiential knowledge in slightly different words, Marshall and Rossman (2011) described qualitative research as research in which

analysis proceeds from the central assumption that there is an essence [emphasis in original] to an experience that is shared with others who have also had that experience. The experiences of those participating in the study - those who had a similar experience [who are, then, purposefully sampled] - are analyzed as unique expressions and then compared to identify the essence. (pp. 20-21)

As can now be understood, experiential knowledge obtained through research does not imply mere subjectivism and bias on behalf of the qualitative researcher, as is widely believed, but rich objective regularities systematically garnered from participants' lived worlds. Clearly, regularities are not a reserved seat of the natural sciences. As Aristotle (4th c. BC, 1924) put it so well,

It would seem that for practical purposes experience is in no way inferior to art; indeed we see men of experience succeeding more than those who have theory without 
experience. The reason of this is that experience is [not only] knowledge of particulars, but art of universals [emphasis mine]. (I, I. 981a)

Experiential knowledge is the art that helps learn, discern, and grasp the universals, which in turn make one knowledgeable about life and others. Close textual analysis reveals that

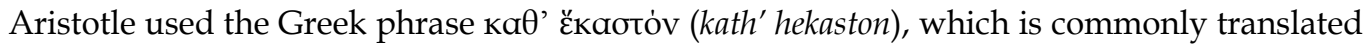
by the English word particular. Two distinct connotations of hekaston are worth-noting for our discussion. Hekaston means a single individual, every single one, each one. Aristotle did not use the word sample or section of a population for individual, much less larger population (for universal). Moreover, according to the long-standing authoritative EnglishGreek lexicon (Liddell \& Scott, 1843/1996), kath' hekaston reflects an accusative usage that implies the sense of standard, norm, or order. The accusative connotation is thus translated as "in accordance with the particular," which implies the standards or patterns embedded in the particular. Aristotle was aware that lived particulars abound with regularities, the forces and patterns of which ought to be relived, brought to light, understood, and articulated as theories. Quite recurrent in qualitative research's tradition, the Aristotelian insight has become an often forgotten pillar of social science methodology: understanding the world and the self to control natural laws better. Social theorist Habermas (1993) wrote,

Enlightenment thinkers...still had the extravagant expectation that the arts and sciences would promote not only the control of natural forces but also the understanding of the world and of the self... and even the happiness of human beings. The twentieth century has shattered this optimism. (p. 103)

The Aristotelian view of science, art, and experiment, for which Enlightenment theorists fought, resists and challenges the modern statistical-fixed understanding of empirical knowledge and the non-objective consideration of human experience. The information age purports the Enlightenment's dream that knowledge is power with which to master the forces of nature and improve humans and their world. It is inexplicable that arts and human experience have been viewed as mere subjectivity, alien to and autonomous from universal empirical knowledge. At the same time, positivistic and statistical tenets have come to be seen as the only valid form of knowledge, devoid of and separate from subjective and artistic expressions.

Note that qualitative research is not a sensualistic, sentimentalist, lyricist, particularistic, or romanticist enterprise, although these elements can be part of the data collection process. The collection process is aimed at reporting the participants' sensations and experiences while employing a disciplined inquiry that is concerned with the patterns, laws, and regularities (hence objective dimensions) that undergird the participants' experiences and lifeworlds. "Qualitative research, however," explained Marshall and Rossman (2011), "is neither subjectivist nor biased (all too common criticisms)" (p. 5). It becomes clear that the dichotomy between objective and subjective is inappropriate for qualitative research's identity. It is often argued that qualitative research is subjective (Myers, 2009), whereas quantitative research is objective. It is also reasoned that qualitative research derives information from the subjective experiences of the participants and quantitative research yields the objective numerical dimensions the phenomenon of interest. As argued above, while these propositions can be true, they lead to undue oversimplification of qualitative research. 
The fourth and last specific aspect of qualitative research is its case-analytic component. Case-based analysis is one of qualitative research's most important features. A case-analytic component requires the researcher to unpack the patterns and forces beneath the essence embedded in the case being studied. Common to case study is the confusion about the term case itself. Despite its merits, for example, renowned methodologist Yin's (2009) recent "definition of a 'case' being... a concrete entity, event, occurrence, action, but not an abstract topic such as a concept, argument, hypothesis, or theory" (p. $x$ ) is one of the many statements that confuses qualitative research's readers. A thorough review of Yin's oeuvre raises significant questions as to the assumptions beneath the definition given above. While Yin (2009) acknowledged that "[his] book does not cover all uses of case studies" (p. 4), his definition of a case pretends to concern all case types. Perhaps more interestingly, Yin (2003) claimed to be dealing only with the genre of case studies related to organizational and institutional phenomena, without denying the importance of other genres of case studies. In the meantime, Yin's proposed definition (2009) remains one of the most commonplace definitions of case study in research methodology curriculums.

For example, one can perform a case study, so to speak, on a hero to resolve a nation's crisis. One can perform a case study on a rock on the seafloor to see how to strengthen concrete to avoid oil spillage. One can perform a case study on the September 11 attacks in the US to enhance hospitals' readiness. One can perform a case study on the concept of how comfort can heal and strengthen the depressed and brokenhearted. One can perform a case study on a criminal's argument to protect the vulnerable in a neighborhood. One can perform a case study of a hypothesis to improve a domestic appliance. One can perform a case study on a theory to improve research in a specific field. In each of these instances, the deeper the knowledge that the case uncovers, the better the result. As has now become clear, case studies have the potential to involve any topic, depending on the questions raised by the research and the methods used.

Having clarified what constitutes a case, we now need to clarify what characterizes a case study in the qualitative research sense of the term. One of the most indicative features of a case study is thick description. Meanwhile, qualitative research has come to mean any descriptive study, and the ways in which the term descriptive, or description, has been implemented and evaluated remains unclear. Geertz (1973/2000), it should be underlined, is the social exemplar who, in importing interpretive sociology's principles (see Simmel, 1908; Weber, 1972) into anthropology, has made description one of the cornerstones of qualitative work. It is no accident that Geertz (1973/2000) spoke of thick description, an important precision that is inexplicably off-sided when it comes to regarding qualitative research as descriptive. In other words, not every description or descriptive study is qualitative or a component of qualitative research. Commonly attributed to Geertz (1973/2000), but originated by 20th-century British philosopher Ryle (1968/1996), thick description aims not to simply articulate the properties of an object, event, or case in and by itself, but to unearth the hidden patterns of essence beneath the object by deriving "the stratified hierarchy of meaningful structures" (Geertz, 1973/2000, p. 7) or by "sorting out the structures of signification" (p. 9). Thin description, however, has nothing to do with the structures and patterns deep-hidden beneath the phenomenon, but provides one easily seen layer of meaning (e.g., size, percentage, frequency, etc.), which "is a surface expression" (p. 17) of 
that which is being studied. Put clearly, the more strata of meanings one pulls out, the thicker the description of the selected case is, and the deeper the obtained knowledge. Thick is another qualifier of deep understanding. To be thick and deep, one has to have as many layers as possible. One layer constitutes a thin or surface understanding (e.g., numbers).

Qualitative research's specifics provide us with a clearer grasp of theory contribution. While researchers diagnose the whats, hows, wheres, whens, whys, and whos within a given literature to capture the relationships leading to a theory, they can use qualitative research's specifics to craft firmer theoretical contributions to their fields. A theory that does not immerse in the real world, re-enact the real context, capture the regularities hidden behind lived experiences, and/or provide a thick description of that which is being studied cannot fully account for the whats, hows, wheres, whens, whys, and whos involved in literature. Numbers alone are only a fraction in this endeavor.

\section{Practical consequences}

This chapter is not proposing a one-size-fits-all formula of theorizing in the social sciences. However, with its largely neglected traditions and theory construction, qualitative research constitutes a tremendous tool of theorization. The belief that theory is an anti-thesis to reality can lead to a notion of theory conceived as incomplete without a specific practice. In addressing the elements of theory: whats, hows, wheres, whens, whys, and whos, researchers achieve a potent grid of not only application, but evaluation. The evaluation of theory should also consider the use of and responsiveness to qualitative research traditions on the one hand, and the specifics of qualitative research on the other hand. Failure to undertake a well-informed account of theory in the social sciences will only perpetuate the misunderstandings outlined above.

The description of the misunderstandings does not seek to assert that qualitative research is the best methodology and the full-fledged generalization toolkit, but rather it advocates for greater awareness about the potentials of qualitative research and its role in contributing to a more informed research process. Equally, the four specifics of qualitative research supplied above are not intended to be comprehensive, but rather to work as a blueprint of how and why to apply, evaluate, and make theoretical contributions in the social sciences. This memo is not an empirical applied study that prescribes and summaries recipes of qualitative research for the social sciences, but it is an invitation to find a tighter contributive grasp of qualitative analysis among the social sciences. No one can make the necessary contributions without a firm grounding in the wealth of various theorists with which qualitative research's history teems.

\section{Conclusion}

Familiarity with qualitative research's tradition takes social scientists to several untapped areas with which to craft theories. Theory is a tool with which to gain a handle of reality. Some of the pitfalls to be aware of are the drive for local particularities, the privileging of the idea of general truths, an enmity between the natural and human sciences, a pursuit of sentimentalism or subjectivism, an over-strict limitation of interpretivism to text or meaning, etc. Practicalities are left to the discretion of researchers as localities vary widely. Qualitative 
research is an art of deeply understanding or enacting ideas, feelings, objects, individuals' experiences, and social phenomena. Qualitative research seeks not only to understand others, but also to allow others to be understood. It can be argued that the deeper the social sciences' knowledge of qualitative research is, the better is the understanding of our lifeworlds and nature.

\section{Acknowledgements}

Many thanks to Carol E. Warrior, Traynor Hansen, and Thomas A. Stuby of the University of Washington for their helpful comments on earlier versions of this chapter.

\section{References}

Ackroyd, J. (2006). Research Methodologies for Drama Education, Trentham, Sterling, VA.

Adorno, W.T. (1974). Minima Moralia: Reflections from Damaged Life (E.F.N. Jephcott, Trans.), New Left Books, London. (Original work published 1951)

Aristotle. (4th c. BC, 1853). The Organon (In two volumes, O. F. Owen, Trans.), Henry G. Bohn London.

Aristotle. (4th c. BC, 1924). Metaphysics (W.D. Ross, Trans.), Clarendon Press, Oxford, UK.

Avicenna. (1970). A Treatise on the Canon of Medicine: Incorporating a Translation of the First Book

(O. C. Gruner, Trans.), A.M. Kelley, New York. (Original work published 1025)

Babbie, R.E. (2010). The Practice of Social Research (12th ed.), Wadsworth, Belmont, CA.

Bachelard, G. (1934). Le Nouvel Esprit Scientifique, PUF, Paris.

Bachelard, G. (1934/1985). The New Scientific Spirit (A. Goldhammer, Trans.), Beacon Press, Boston, MA.

Bachelard, G. (1938/2004). La Formation de l'Esprit Scientifique: Contribution à une Psychanalyse de la Connaissance Objective, Edition de Poche, Paris.

Bacon, F. (1960). The New Organon and Related Writings, Bobbs-Merrill, Indianapolis, IL. (Original work published 1605)

Baumgartner, M. (2008). The Causal Chain Problem. Erkenntnis, Vol.69, No.2, pp. 201-226.

Baumgartner, M. (2009a). Uncovering Deterministic Causal Structures: A Boolean Approach. Synthese, Vol.170, No.1, pp. 71-96.

Baumgartner, M. (2009b). Inferring Causal Complexity. Sociological Methods $\mathcal{E}$ Research, Vol.38, No.1, pp. 71-101.

Baumgartner, M. (2010). Causal Slingshots. Erkenntnis, Vol.72, No.1, pp. 113-133.

Becker, S.H. (1996). The Epistemology of Qualitative Research. In R. Jessor, A. Colby, \& R.A. Shweder (Eds.), Ethnography and Human Development: Context and Meaning in Social Inquiry (pp. 53-71), Chicago University Press, Chicago.

Benton, T., \& Craib, I. (2001). Philosophy of Social Science: The Philosophical Foundations of Social Thought, Palgrave, New York.

Berger, L.P., \& Luckmann, T. (1967). The Social Construction of Reality: A Treatise in the Sociology of Knowledge, Anchor, New York.

Blumer, H. (1969). Symbolic Interactionism: Perspective and Method, Prentice- Hall, Englewood Cliffs, NJ. 
Brady, E.H., Collier, D., \& Seawright, J. (2006). Toward a Pluralistic Vision of Methodology. Political Analysis, Vol.14, No.3, pp. 353-368.

Brady, E.H., Collier, D., \& Seawright, J. (2010). Refocusing the Discussion of Methodology. In H.E. Brady \& D. Collier (Eds.), Rethinking Social inquiry: Diverse Tools, Shared Standards (2nd ed., pp. 15-32), Rowman \& Littlefield, Lanham, MD.

Braham, M., \& van Hees, M. (2009). Degrees of Causation. Erkenntnis, Vol.71, No.3, pp. 323344.

Bulmer, M. (1984). The Chicago School of Sociology: Institutionalization, Diversity, and the Rise of Sociological Research, University of Chicago Press, Chicago.

Burke, P. (1999). The European Renaissance: Centres and Peripheries, Blackwell, New York.

Burr, V. (2003). Social Constructionism (2nd ed.), Routledge, New York.

Charmaz, K. (2009). Shifting the Grounds: Constructivist Grounded Theory Methods. In J.M.

Morse, P.N. Stern, J. Corbin, B. Bowers, K. Charmaz, \& A.E. Clarke (Eds.),

Developing Grounded theory: The Second Generation (pp. 127-154), Left Coast Press,

Walnut Creek, CA.

Chomsky, N. (1965). Aspects of the Theory of Syntax, MIT Press, Cambridge, MA.

Chomsky, N. (1998). The New World Order; On the Social Sciences and Theory, October 18, 2011,

Available from: <http://www.youtube.com/watch?v=pcRZQvU1C9Q>

Cibangu, K.S. (2010). Information Science as a Social Science. Information Research, Vol.15,

No.3, June 27, 2011, Available from:

<http:/ InformationR.net/ir/15-3/paper434.html>

Cibangu, K.S. (in press). Karl Popper and the Social Sciences. In M.A. López-Varela (Ed.), Social Sciences and Humanities: Applications and Theories (Vol. 1), InTech, Rijeka, Croatia, October 25, 2011, Available from: <http://www.intechweb.org/>

Collier, D., Brady, E.H., \& Seawright, J. (2010). Sources of Leverage in Causal Inference: Toward an Alternative View of Methodology. In H.E. Brady \& D. Collier (Eds.), Rethinking Social Inquiry: Diverse Tools, Shared Standards (2nd ed., pp. 161-200), Rowman \& Littlefield, Lanham, MD.

Collier, D., \& Elman, C. (2008). Qualitative and Multi-method Research: Organizations, Publication, and Reflections on Integration. In J.M. Box-Steffensmeier, H.E. Brady, \& D. Collier (Eds.), The Oxford Handbook of Political Methodology (pp. 779-795), Oxford University Press, New York.

Comte, A. (1848/1999). Discours sur l'Ensemble du Positivisme, GF-Flammarion, Paris.

Copenhaver, P.B., \& Schmitt, B.C. (2002). Renaissance Philosophy (Foreword by P.O. Kristeller), Oxford University Press, New York.

Coppedge, M. (1999). Thickening Thin Concepts and Theories: Combining Large-N and Small in Comparative Politics. Comparative Politics, Vol.31, No.4, pp. 465-476.

Coppedge, M. (2007). Thickening Thin Concepts: Issues in Large-N Data Generation. In G.L. Munck (Ed.), Regimes and Democracy in Latin America: Theories and Methods (pp. 105122), Oxford University Press, New York.

Corcoran, J. (1974). Aristotelian Syllogisms: Valid Arguments or True Generalized Conditionals? Mind, Vol.83, pp. 278-281.

Corcoran, J. (2003). Aristotle's Prior Analytics and Boole's Laws of Thought. History and Philosophy of Logic, Vol.24, No.4, pp. 261-288. 
Corcoran, J. (2009). Aristotle's Demonstrative Logic. History and Philosophy of Logic, Vol.30, No.1, pp. 1-20.

Creswell, W.J. (2008). Educational Research: Planning, Conducting, and Evaluating Quantitative and Qualitative Research (3rd ed.), Pearson Education, Upper Saddle River, NJ.

Creswell, W.J. (2009). Research Design: Qualitative, Quantitative, and Mixed Methods Approaches (3rd ed.), Sage, Thousand Oaks, CA.

Crowther, D., \& Lancaster, G. (2008). Research Methods: A Concise Introduction to Research in Management and Business Consultancy (2nd ed.), Elsevier, Oxford, UK.

Descartes, R. (1987). Discours de la Méthode: Pour bien Conduire sa Raison et Chercher de la Vérité dans les Sciences (Texte et commentaire par Etienne Gilson), Vrin, Paris. (Original work published 1637)

Denzin, K.N. (1997). Interpretive Ethnography: Ethnographic Practices for the 21st century, Sage, Thousand Oaks, CA.

Denzin, K.N. (2009). Qualitative Inquiry under Fire: Toward a New Paradigm Dialogue, Left Coast Press, Walnut Creek, CA.

Denzin, K.N. (2010). The Qualitative Manifesto: A Call to Arms, Left Coast Press, Walnut Creek, CA.

Denzin, K.N., \& Lincoln, S.Y. (1998). Introduction: Entering the Field of Qualitative Research. In N.K. Denzin \& Y.S. Lincoln (Eds.), The Landscape of Qualitative Research: Theories and Issues (pp. 1-34), Sage, Thousand Oaks, CA.

Denzin, K.N., \& Lincoln, S.Y. (2003a). Introduction: The Discipline and Practice of Qualitative Research. In N.K. Denzin \& Y.S. Lincoln (Eds.), Collecting and Interpreting Qualitative Research Materials (2nd ed., pp. 1-45), Sage, Thousand Oaks, CA.

Denzin, K.N., \& Lincoln, S.Y. (2003b). Preface. In N.K. Denzin \& Y.S. Lincoln (Eds.), Collecting and Interpreting Qualitative Research Materials (2nd ed., pp. ix-xiii), Sage, Thousand Oaks, CA.

Denzin, K.N., \& Lincoln, S.Y. (2005). Preface. In N.K. Denzin \& Y.S. Lincoln (Eds.), The Sage Handbook of Qualitative Research (3rd ed., pp. ix-xix), Sage, Thousand Oaks, CA.

Denzin, K.N., \& Lincoln, S.Y. (2008a). Preface. In N.K. Denzin \& Y.S. Lincoln (Eds.), The Landscape of Qualitative Research (3rd ed., pp. vii-x), Sage, Thousand Oaks, CA.

Denzin, K.N., \& Lincoln, S.Y. (2008b). Introduction: The Discipline and Practice of Qualitative Research. In N.K. Denzin \& Y.S. Lincoln (Eds.), The Landscape of Qualitative Research (3rd ed., pp. 1-43), Sage, Thousand Oaks, CA.

Denzin, K.N., \& Lincoln, S.Y. (2008c). Preface. In N.K. Denzin, Y.S. Lincoln, \& L.T. Smith (Eds.), Handbook of Critical and Indigenous Methodologies (pp. ix-xv), Sage, Thousand Oaks, CA.

Denzin, K.N., \& Lincoln, S.Y. (2008d). Introduction: The Discipline and Practice of Qualitative Research. In N.K. Denzin \& Y.S. Lincoln (Eds.), Strategies of Qualitative Inquiry (3rd ed., pp. 1-43), Sage, Thousand Oaks, CA.

Denzin, K.N., \& Lincoln, S.Y. (2008e). Preface. In N.K. Denzin \& Y.S. Lincoln (Eds.), Strategies of Qualitative Inquiry (3rd ed., pp. vii-x), Sage, Thousand Oaks, CA.

Denzin, K.N., \& Lincoln, S.Y. (2011). Preface. In N.K. Denzin \& Y.S. Lincoln (Eds.), The Sage Handbook of Qualitative Research (4th ed., pp. ix-xvi), Sage, Thousand Oaks, CA. 
de Jong, L.H. (2010). From Theory Construction to Deconstruction: The Many Modalities of Theorizing in Psychology. Theory Psychology, Vol.20, No.6, pp. 745-763.

De Tarde, J.-G. (1993). Les Lois de l'Imitation, Kimé, Paris. (Original work published 1890)

De Tocqueville, A. (2004). Democracy in America (Vols. 1-2, A. Goldhammer, Trans.), Library of America, New York. (Original work published 1840)

de Vaus, A.D. (2001). Research Design in Social Research. Thousand Oaks, CA: Sage.

Dilthey, W. (1988). Introduction to the Human Sciences: An Attempt to Lay a Foundation for the Study of Society and History (R.J. Betanzos, Trans.), Wayne State University Press, Detroit, MI.

Dilthey, W. (2002). The Formation of the Historical World in the Human Sciences (R. A. Makkreel, \& F. Rodi, Trans.), Princeton University Press, Princeton, NJ.

Duden: Deutsches Universalwörterbuch 3. (1996), Duden, Mannheim, Germany.

Duignan, B. (Ed.). (2011). Ancient Philosophy: From 600Bce to 500ce, Encyclopaedia Britannica, New York.

Durkheim, E. (1893/2007). De la Division du Travail Social, PUF, Paris.

Durkheim, E. (1894/1988). Les Règles de la Méthode Sociologique, Flammarion, Paris.

Durkheim, E. (1912/2008). Les Formes Elémentaires de la Vie Religieuse: Le Système Totémique en Australie, Quadrige, Paris.

Eisenhardt, M.K. (1989). Building Theories from Case Study Research. Academy of Management Review, Vol.14, No. 4, pp. 532-550.

Flick, U. (2002). Qualitative Research: State of the Art. Social Science Information, Vol.41, No.1, pp. 5-24.

Flick, U. (2009). An Introduction to Qualitative Research (4th ed.), Sage, London. (Original work published 1995)

Flyvbjerg, B. (2001). Making Social Science Matter: Why Social Inquiry Fails and how it Can Succeed again (S. Sampson, Trans.), Cambridge University Press, New York.

Flyvbjerg, B. (2006). Five Misunderstandings about Case-study Research. Qualitative Inquiry, Vol.12, No.2, pp. 219-245.

Freud, S. (1913). The Interpretation of Dreams (A.A. Brill, Trans.), Macmillan, New York. (Original work published 1900)

Gaddis, L.J. (2002). The Landscape of History: How Historians Map the Past, Oxford University Press, New York.

Garfinkel, H. (1967). Studies in Ethnomethodology, Prentice-Hall, Englewood Cliffs, NJ.

Geertz, C. (2000). The Interpretation of Cultures: Selected Essays, Basic Books, New York. (Original work published 1973)

George, L.A., \& Bennett, A. (2005). Case Studies and Theory Development in the Social Sciences, MIT Press, Cambridge, MA.

Gilderhus, T.M. (2009). History and Historians: A Historiographical Introduction (7th ed.), Prentice Hall, Upper Saddle River, NJ.

Gill, L.M., \& Pellegrin, P. (Eds.). (2006). A Companion to Ancient Philosophy, Blackwell, Malden, MA.

Glaser, B., \& Strauss, A. (1967). The Discovery of Grounded Theory: Strategies for Qualitative Research, Aldine, Chicago. 
Gobo, G. (2005). The renaissance of qualitative research. Forum Qualitative Research, Vol.6, No.3, Art. 42.

Gomm, R., Hammersley, M., \& Foster, P. (Eds.). (2000). Case Study Method: Key Texts, Key issues. Sage, London.

Gottlieb, A. (2002). The Dream of Reason: A History of Western Philosophy from the Greeks to the Renaissance, W.W. Norton, New York.

Grabowski, A.F, III. (2008). Plato, Metaphysics, and the Forms, Continuum, New York.

Graziano, M.A., \& Raulin, L.M. (2010). Research Methods: A Process of Inquiry (7th ed.), Allyn \& Bacon, Boston, MA.

Gschwend, T., \& Schimmelfennig, F. (Eds.). (2007). Research Design in Political Science: How to Practice What they Preach, Palgrave Macmillan, New York.

Habermas, J. (1971). Knowledge and Human Interests (J. J. Shapiro, Trans.), Beacon Press, Boston, MA. (Original work published 1968)

Habermas, J. (1993). Modernity an Incomplete Project. In T. Docherty (Ed.), Postmodernism: A Reader (pp. 98-109), Harvester Wheatsheaf, New York.

Hakim, C. (2000). Research Design: Successful Designs for Social and Economic Research, Routledge, New York.

Haynes, R.S., \& Carroll, M.J. (2010). The Range and Role of Theory in Information Systems Design Research: From Concepts to Construction. ICIS 2010 Proceedings. Paper 136, April 15, 2011, Available from: <http:/ / aisel.aisnet.org/icis/>

Heckman, J.J. (2005). The Scientific Model of Causality. Sociological Methodology, Vol.35, No.1, pp.1-98.

Hellström, T. (2008). Transferability and Naturalistic Generalization: New Generalizability Concepts for Social Science or Old Wine in New Bottles? Quality \& Quantity, Vol.42, No.3, pp. 321-337.

Hendrickson, N. (2010, October 19). Counterfactual Reasoning and the Problem of Selecting Antecedent Scenarios. Synthese Online First.

Herodotus. (5th c. BC, 1957). Histories (Vol. 7, A.D. Godley, Trans.), Harvard University Press, Cambridge, MA.

Herodotus. (5th c. BC, 1960). Histories (Vol. 2, A.D. Godley, Trans.), Harvard University Press, Cambridge, MA.

Hiley, R.D., Bohman, F.J., \& Shusterman, R. (Eds.). (1991). The Interpretive Turn: Philosophy, Science, Culture, Cornell University Press, Ithaca, NY.

Holland, K., \& Herstad, J. (2000). The "Critical Case" in Information Systems Research. Working Paper Department of Informatics University of Oslo, October 23, 2010, Available from: <http://www.ifi.uio.no/in166/h00/criticalcase.pdf>

Honneth, A. (2007). Pathologien der Vernunft: Geschichte und Gegenwart der Kritischen Theorie. Frankfurt, Germany: Suhrkamp.

Horkheimer, M., \& Adorno, W.T. (1989). Dialectic of Enlightenment, Continuum, New York. (Original work published 1947)

Howell, M., \& Prevenier, W. (2001). From Reliable Sources: An Introduction to Historical Methods, Cornell University Press, Ithaca, NY.

Husserl, W. (1962). Ideas: General Introduction to Pure Phenomenology (W.R.B. Gibson, Trans.), Collier, New York. (Original work published 1913) 
Husserl, W. (1980). Phenomenology and the Foundations of the Sciences: Third Book Ideas Pertaining to a Pure Phenomenology and to a Phenomenological Philosophy (T.E. Klein \& W.E. Pohl, Trans.), Martinus Nijhoff, Boston, MA. (Original work published 1952)

Jaccard, J., \& Jacoby, J. (2010). Theory Construction and Model-building Skills: A Practical Guide for Social Scientists. New York: The Guilford Press.

Jonsen, R.A., \& Toulmin, S. (1988). The Abuse of Casuistry: A History of Moral Reasoning, University of California Press, Berkeley, CA.

Kincaid, H. (1996). Philosophical Foundations of the Social Sciences: Analyzing Controversies in Social Research, Cambridge University Press, New York.

King, L.M. (2003). The Renaissance in Europe, Laurence King, London.

Knoblauch, H., Flick, U., \& Maeder, C. (2005). Qualitative Methods in Europe: The Variety of Social Research. Forum Qualitative Research, Vol.6, No.3, Art. 34.

Korte, H. (1999). Einführung in die Geschichte der Soziologie. 5. Auflage, Leske \& Budrich, Opladen, Germany.

Koyré, A. (1939). Les Etudes Galiléennes, Hermann, Paris.

Kristeller, O.P., \& Mooney, M. (1979). Renaissance Thought and its Sources, Columbia University Press, New York.

Kukla, A. (2000). Social Constructivism and the Philosophy of Science, Routledge, New York.

Liddell, G.H., \& Scott, R. (1996). A Greek-English Lexicon (9th ed.), Oxford University Press, New York. (Original work published 1843)

Lincoln, S.Y., \& Denzin, K.N. (2003). The Seventh Moment: Out of the Past. In N.K. Denzin \& Y.S. Lincoln (Eds.), Collecting and Interpreting Qualitative Research Materials (2nd ed., pp. 1047-1065), Sage, Thousand Oaks, CA.

Lincoln, S.Y., \& Denzin, K.N. (2003). (2008a). Introduction: Critical Methodologies and Indigenous Inquiry. In N.K. Denzin, Y.S. Lincoln, \& L.T. Smith (Eds.), Handbook of Critical and Indigenous Methodologies (pp. 1-20), Sage, Thousand Oaks, CA.

Lincoln, S.Y., \& Denzin, K.N. (2003). (2008b). Epilogue: The Eight and Ninth moments -Qualitative Research in/and the Fractured Future. In N.K. Denzin \& Y.S. Lincoln (Eds.), The Landscape of Qualitative Research (3rd ed., pp. 539-554), Sage, Thousand Oaks, CA.

Little, D. (1998). Microfoundations, Method, and Causation: On the Philosophy of the Social Sciences, Transactions, Brunswick, NJ.

Lofland, J., Snow, D.A., Anderson, L., \& Lofland, L.H. (2006). Analyzing Social Settings: A Guide to Qualitative Observation and Analysis (4th ed.), Wadsworth Belmont, CA.

Lucas, W.J. (2003). Theory-testing, Generalization, and the Problem of External Validity. Sociological Theory, Vol.21, No.3, pp. 236-253.

Marshall, C., \& Rossman, B.G. (2011). Designing Qualitative Research (5th ed.), Sage, Thousand Oaks, CA.

Martin, J. (1996). The Dialectical Imagination: A History of the Frankfurt School and the Institute of Social Research, 1923-1950, University of California Press, Berkeley, CA.

Marx, K. (1977). Capital: A Critique of Political Economy (B. Fowkes, Trans.), Vintage Books New York. (Original work published 1867)

Mason, J. (2002). Qualitative Researching (2nd ed.), Sage, Thousand Oaks, CA. 
Maxwell, A.J. (2005). Qualitative Research Design: An Interactive Approach (2nd ed.), Sage, Thousand Oaks, CA.

McCullagh, B.C. (1984). Justifying Historical Descriptions, Cambridge University Press, New York.

Mill, S.J. (1872). System of Logic (8th ed.), Longmans, Green, London. (Original work published 1843)

Moran, D., \& Mooney, T. (Eds.). (2002). The Phenomenology Reader, Routledge, New York.

Morgan, L.D. (1996). Focus Groups. Annual Review of Sociology, Vol.22, pp. 129-152.

Morgan, L.D. (1997). Focus Groups as Qualitative Research (2nd ed.), Sage, Thousand Oaks, CA.

Morgan, L.D. (2007). Paradigms Lost and Pragmatism Regained: Methodological Implications of Combining Qualitative and Quantitative Methods. Journal of Mixed Methods Research, Vol.1, No.1, pp. 48-76.

Morgan, L.S., \& Winship, C. (2007). Counterfactuals and Causal Inference: Methods and Principles for Social Research, Cambridge University Press, New York.

Mueller-Vollmer, K. (Ed.). (2006). The Hermeneutics Reader, Continuum, New York.

Muirhead, H.J. (1900). Chapters from Aristotle's Ethics, John Murray, London.

Myers, D.M. (2009). Qualitative Research in Business \& Management, Sage, Thousand Oaks, CA.

Niaz, M. (2007). Can Findings of Qualitative Research in Education Be Generalized? Quality $\mathcal{E}$ Quantity, Vol.41, No.3, pp. 429-445

Noble, I., \& Bestley, R. (2005). Visual Research: An Introduction to Research Methodologies in Graphic Design, Sterling, New York.

Nussbaum, C.M. (2011). Creating Capabilities: The Human Development Approach, Harvard University Press, Cambridge, MA.

Onwuegbuzie, J.A., \& Leech, L.N. (2010). Generalization Practices in Qualitative Research: A Mixed Methods Case Study. Quality \& Quantity, Vol.44, No.5, pp. 881-892.

Parkinson, B.R. (1991). Voices from Ancient Egypt: An Anthology of Middle Kingdom Writers, University of Oklahoma Press, Norman, OK.

Parsons, T. (1938). The Role of Theory in Social Research. American Sociological Review, Vol.3, No.1, pp.13-20.

Patton, Q.M. (2002). Qualitative Research and Evaluation Methods (3rd ed.), Sage, Thousand Oaks, CA.

Peshkin, A. (1988). Understanding Complexity: A Gift of Qualitative Inquiry. Anthropology $\mathcal{E}$ Education Quarterly, Vol.19, No.4, pp. 416-424.

Piaget, W.F.J. (1926). La Représentation du Monde chez l'Enfant, PUF, Paris.

Pickard, A. (2007). Research Methods in Information, Facet, London.

Plato. (5-4th c. BC, 1921). Sophist (H.N. Fowler, Trans.), Harvard University Press, Cambridge, MA.

Plato. (5-4th c. BC, 1925). Phaedrus (H.N. Fowler, Trans.), Harvard University Press, Cambridge, MA.

Plato. (5-4th c. BC, 1966). Euthyphro (H.N. Fowler, Trans.), Harvard University Press, Cambridge, MA. 
Plato. (5-4th c. BC, 1997). Parmenides (A. Reginald, Trans.), Yale University Press, New Haven, CT.

Ragin, C.C. (1987). The Comparative Method: Moving beyond Qualitative and Quantitative Strategies, University of California Press, Berkeley, CA.

Ragin, C.C. (1998). Case-oriented Research and the Study of Social Action. In H.-P. Blossfeld \& G. Prein (Eds.), Rational Choice Theory and Large-scale Data Analysis, (pp. 158-168), Westview, Boulder, CO.

Ragin, C.C. (1999). The Distinctiveness of Case-oriented Research. Health Services Research, Vol.34, No.5, pp. 1137-1151.

Ragin, C.C. (2004). Turning the Tables: How Case-oriented Research Challenges Variableoriented Research. In H.E. Brady \& D. Collier (Eds.), Rethinking Social Inquiry: Diverse Tools, Shared Standards (pp. 123-138), Rowman \& Littlefield, Lanham, MD.

Ragin, C.C. (2009). Reflections on Casing and Case-oriented Research. In D. Byrne \& C.C. Ragin (Eds.), The Sage Handbook of Case-based Methods (pp. 522-534). Thousand Oaks, CA: Sage.

Ratner, C. (2006). Epistemological, Social, and Political Conundrums in Social Constructionism. Forum Qualitative Social Research, Vol.7, No.1, Art 4.

Rawls, A.W. (1996). Durkheim's Epistemology: The Neglected Argument. American Journal of Sociology, Vol.102, No.2, pp. 430-482.

Rickless, C.R. (2007). Plato's Forms in Transition: A Reading of the Parmenides, Cambridge University Press, New York.

Ricoeur, P. (1950). Philosophie de la Volonté. Tome 1: Le Volontaire et l'Involontaire, Aubier, Paris.

Ricoeur, P. (1969). Le Conflit des Interprétations: Essais d'Herméneutique I., Seuil, Paris.

Ricoeur, P. (1971). Sémantique de l'Action, Cercle de Philosophie, Louvain, Belgium.

Ricoeur, P. (1972). Herméneutique, Editions du SIC, Louvain-la-Neuve, Belgium.

Ricoeur, P. (1986a). A l'Ecole de la Phénoménologie, Vrin, Paris.

Ricoeur, P. (1986b). Du Texte à l'Action : Essais d'Herméneutique II., Seuil, Paris.

Ricoeur, P. (1995). L'Herméneutique à l'Ecole de la Phenomenology, Beauchesne, Paris.

Rosenau, M.P. (1995). Post-modernism and the Social Sciences: Insights, Inroads, and Intrusions, Princeton University Press, Princeton, NJ.

Rosenberg, A. (2008). Philosophy of Social Science (3rd ed.), Westview Press, Philadelphia, PA.

Ruddin, P.L. (2006). You Can Generalize Stupid! Social Scientists, Bent Flyvbjerg, and Case Study Methodology. Qualitative Inquiry, Vol.12, No.4, pp. 797-812.

Ryle, G. (1996). The Thinking of Thoughts: What is "Le Penseur" Doing? Studies in Anthropology, 11. (Original work published 1968), September 29, 2009, Available from: <http://lucy.kent.ac.uk/CSACSIA/Vol14/Papers/ryle_1.html>

Searle, R.J. (1995). The Construction of Social Reality, Free Press, New York.

Seawright, J., \& Collier, D. (2010). Glossary. In H.E. Brady \& D. Collier (Eds.), Rethinking Social Inquiry: Diverse Tools, Shared Standards (2nd ed., pp. 313-359), Rowman \& Littlefield, Lanham, MD.

Shoemaker, J.P., Tankard, W.J, Jr, \& Lasorsa, L.D. (2004). How to Build Social Science Theories, Sage, Thousand Oaks, CA. 
Silverman, D. (2010). Doing Qualitative Research: A Practical Handbook (3rd ed.), Sage, Thousand Oaks, CA.

Simmel, G. (1908). Soziologie: Untersuchungen über die Formen derVergesellschaftung, Duncker \& Humblot, Leipzig, Germany.

Smith, J.M. (2003). Social Science in Question: Towards a Postdisciplinary Framework, Sage, London.

Stake, E.R. (2008). Qualitative Case Studies. In N.K. Denzin \& Y.S. Lincoln (Eds.), Strategies of Qualitative Inquiry (3rd ed., pp. 119-149), Sage, Thousand Oaks, CA.

Sutton, B. (1998). Qualitative Research Methods in Library and Information Science. In A. Kent (Ed.), Encyclopedia of Library and Information Sciences (Vol. 62, pp. 263- 284), Marcel Dekker, New York.

Tashakkori, A., \& Teddlie, C. (2003). The Past and the Future of Mixed Methods Research: from Data Triangulation to Mixed Model Designs. In A. Tashakkori \& C. Teddlie (Eds.), Handbook of Mixed Methods in Social and Behavioral Research (pp. 671-702), Sage, Thousand Oaks, CA.

Tashakkori, A., \& Teddlie, C. (2010). SAGE Handbook of Mixed Methods in Social and Behavioral Research (2nd ed.), Sage, Thousand Oaks, CA.

The Oxford American Dictionary and Language Guide. (1999), Oxford University Press, New York.

Teddlie, C., \& Tashakkori, A. (2009). Foundations of Mixed Methods Research: Integrating Quantitative and Qualitative Approaches in the Social and Behavioral Sciences, Sage, Thousand Oaks, CA.

Thomas, R.J., Nelson, K.J., \& Silverman, J.S. (2005). Research Methods in Physical Activity (7th ed.), Human Kinetics, Champaign, IL.

Treibel, A. (1999). Einführung in soziologische Theorien der Gegenwart. 5. Auflage, Leske \& Budrich, Opladen, Germany.

Venable, R.J. (2006). The Role of Theory and Theorising in Design Science Research. DESRIST 2006 Proceedings, October 18, 2011, Available from: <http://desrist.org/conference/>

Vidich, J.A., \& Lyman, M.S. (1998). Qualitative Methods: Their History in Sociology and Anthropology. In N.K. Denzin \& Y.S. Lincoln (Eds.), The Landscape of Qualitative Research: Theories and Issues (pp. 41-110), Sage, Thousand Oaks, CA.

Wallerstein, I., Juma, C., Keller, F.E., Kocka, J., Lecourt, D., Mudimbe, Y.V., et al. (1996). Open the Social Sciences: Report of the Gulbenkian Commission on the Restructuring of the Social Sciences, Stanford University Press, Stanford, CA.

Ward, G. (2003). Postmodernism, Contemporary Books, Chicago.

Weber, M. (1921/1972). Wirtschaft und Gesellschaft: Grundriß der verstehenden Soziologie 5. (Revidierte Auflage, Besorgt von J. Winckelmann), J.C.B. Mohr (Paul Siebeck), Tübingen, Germany.

Whetten, A.D. (1989). What Constitutes a Theoretical Contribution? Academy of Management Review, Vol.14, No.4, pp. 490-495.

Wiggershaus, R. (1998). The Frankfurt School: Its History, Theories, and Political Significance (M. Robertson, Trans.), MIT Press, Cambridge, MA. (Original work published 1986)

Williams, M. (2000). Interpretivism and Generalization, Sociology, Vol.34, No.2, pp. 209-224. 
Windelband, W. (1980). Rectorial address, Strasbourg, 1894. History and Theory, Vol.19, No.2, pp. 169-185. (Original work published 1894)

Yeboah, A.D. (2008). Research Methodologies in Criminology, Nova Science, New York.

Yin, K.R. (2003). Applications of Case Study Research (2nd ed.), Sage, Thousand Oaks, CA.

Yin, K.R. (2009). Case Study Research: Design and Methods (4th ed.), Sage, Thousand Oaks, CA. 


\section{Section 3}

Coding and Cartographic Models 



\title{
A Simulation Approach to Validate Models Derived from Observational Studies
}

\author{
Pierre N. Robillard and Simon Labelle \\ Ecole Polytechnique de Montréal \\ Canada
}

\section{Introduction}

Protocol analysis is a suitable approach for studying cognitive behaviors. It is a psychological research method that elicits verbal reports from research participants, and is used to study the thinking process in the cognitive sciences (cognitive psychology, behavior analysis). Consequently, much of what we know in most domains and particularly in software engineering about practitioners' cognitive processes is based on researchers' use of protocol analysis (Robillard et al, 1998). Trickett \& Trafton (2002) provide a comprehensive summary of practical how-to primers on protocol analysis. Hughes \& Parkes (2003) provide an excellent survey of trends in the use of verbal protocol analysis in software engineering research. They found that some difficulties associated with the technique are: (i) the effort of devising a valid and reliable encoding scheme; (ii) the time-consuming nature of the encoding process; and (iii) the problem of comparing the results of researchers who have applied different encoding schemes.

Coding is a strategy used in protocol analysis to extract values for quantitative variables from qualitative data collected from observations. Human behavior is characterized by coding schemes, and statistical tools are used to derive models of the behavioral patterns emerging from the tasks under study (Ericsson \& Simon, 1993).

The coding scheme defines the formalism for encoding the protocol, and should be capable of encoding the moves adequately and yet be formal enough to support quantitative analysis. A transcript entry is called a (verbal) move. The protocol transcript or verbatim account is an accurate written representation of all the moves performed by the participants during the observed session. The coding of a verbatim account is a subjective task that is very time-consuming. Consequently, the coding activities are the weak link in any study based on protocol analysis, in that two coders who perform the same move characterizations may have a different interpretation of the moves, and may not replicate exactly the same coding pattern. This behavior is natural, and it is unrealistic to expect exactly the same coding patterns from two coders, or even from the same coder.

In protocol analysis, reliability is associated with stability between coders (Baer, 1977), such stability being obtained by measuring the degree of similarity between the resulting codes. Coding is a subjective activity based on the assignment of qualitative codes to natural moves, and many approaches have been developed to evaluate the reliability of 
coding activities (Carletta et al., 1997, Seaman, 1999, Foster et al, 2008). Stability could also mean that a coder codes in the same way during the entire experiment. One must verify that the coder will not introduce bias in one way or another during the various coding sessions (Medley \& Mitzel, 1963). For example, in software process assessments, studies have been conducted to benchmark Cohen's Kappa index, which is a measure of interrater reliability (Khaled, 1999).

The traditional scientific approach to validating models derived from experimental studies is to replicate the experiments. Experimental replication validates the appropriateness and reliability of the observed data, the correctness of the statistical analysis, and the significance of the results. Difficulties in replicating observational empirical studies arise from the impossibility of using the same people carrying out the same project in the same environment. However, a qualitative replication has a looser meaning, which is that it must only preserve the conditions set forth in the theory being tested (Seeman, 1999).

What is often recommended is to analyze the reliability of an empirical study before deriving a model based on these studies. Cronbach et al. (1972) suggest the theory of generalizability, where the factors that could affect reliability are identified, controlled experiments are repeated many times with one of the factors being modified each time, and the results are statistically compared. The idea is to define the environment for supporting the generalization of the results. This approach is considered the best for estimating reliability (Berk, 1979; Mitchell, 1979). However, it is very difficult to implement, since it requires a limited environmental setup and a relatively large amount of resources (Johnson \& Bolstad, 1973). Some authors also argue about the necessity for generalizability, finding this approach unduly complex (Nelson et al., 1977) and in some cases inappropriate for experiments where qualitative measurements are involved (Cooil \& Rust, 1994).

The term validity is potentially confusing when used in protocol analysis. The distinction is between the validity of the coding scheme, or variables derived from it, and the validity of the interpretation relating content variables to their causes or consequences. To assert that a category or variable is valid is to state that there is a correspondence between the category and the abstract concept that it represents. To assert that a research result based on protocol analysis is valid is to state that the finding does not depend upon the specific scheme or coder (Weber, 1990)

In the case of the validity of the coding scheme, measurement validity is defined by its representativeness and adequacy (Kerlinger, 1973; Curtis, 1980), validity often being confirmed by human experts (Herbert \& Attridge, 1975). Indeed, a detailed analysis by experts of the experimental setup may be necessary to judge the validity of the experiment. Another approach would be to measure the same phenomenon in various ways and then compare the results (Campbell \& Fiske, 1959). Many studies explore means to improve interrater reliability and use Monte Carlo investigation to measure the effect of the number of rating scale categories on levels of inter-rater reliability (Cicchetti et al., 1985).

Most studies are looking to improve the validity of the coding scheme and the reliability of the coders who are using it. The validity of the interpretation is crucially dependent on the validity of the model that is derived from the protocol analysis, and yet there is no single established definition of model validity and validation in the modeling literature. 
Building confidence in the usefulness of a model is a gradual process, dispersed throughout the methodology, starting with problem identification, the coding scheme, the reliability of the coders, and the structure of the resulting model. The ultimate objective is to establish the validity of the structure of the model. Accuracy of the model behavior's reproduction of real behavior is also evaluated, but this is meaningful only if we already have sufficient confidence in the structure of the model (Barnas, 1996).

Nowadays, model structures derived from protocol analysis are built from the activity of one coder, whose reliability has been tested against that of another coder on a part of the protocol. The major assumption in protocol analysis is that a reliable model structure is built by a reliable coder using a valid coding scheme. It is assumed that, if another coder had done the coding, the same model structure would emerge.

Our research work leads us to consider the validity of interpretation of the model structure resulting from protocol analysis. We found no approach in the literature to evaluate the impact of subjective coding on the model structure.

The idea behind Monte Carlo Coding Replication (MCCR) is to simulate replication of the coding in the protocol analysis. The simulation generates codes with random variation of the qualitative interpretation within an observed range of variability. This enables the researcher to determine in which qualitative parameter ranges the resulting model structure remains invariable, or to evaluate the sensitivity of the model structure to these parameters. This paper presents an original approach to measure the impact of coding variability between reliable coders on the structure of the resulting models.

The simulation procedure consists of three steps that have been added to a standard protocol analysis procedure. The steps of the simulation procedure are described in the context of a real protocol analysis project. The next section introduces the components needed for the simulation in the context of an overview of protocol analysis, which leads to the problem to be solved by MCCR. The third section, which constitutes the core of this paper, presents in detail the three steps of the simulation procedure, which are the definition of the variability matrix, the performance of the simulation procedure and the reconstruction of the model from the simulated moves. The last section illustrates the impact of coder variability on the structure of the model from a real model built by the human coder.

\section{Overview of protocol analysis and problem statement heading}

The objective of the research project used to illustrate MCCR was to observe the activities of software team members during technical review meetings and to use protocol analysis to derive a model of the team's behavior during the meetings. The analysis approach used to derive the model is based on Exploratory Sequential Data Analysis (ESDA) (Sanderson \& Fisher, 1994), which is applicable to the analysis of systems, environments, or behavioral data, the sequential integrity of which has been preserved. More details of this study and its approach can be found in the publications of the review meeting model ( $\mathrm{D}^{\prime}$ Astous \& Robillard 2002, D'Astous et al., 2004). The model at the time it was published did not take into account the results obtained subsequently from the MCCR approach presented in this chapter. 


\section{Traditional Approach}
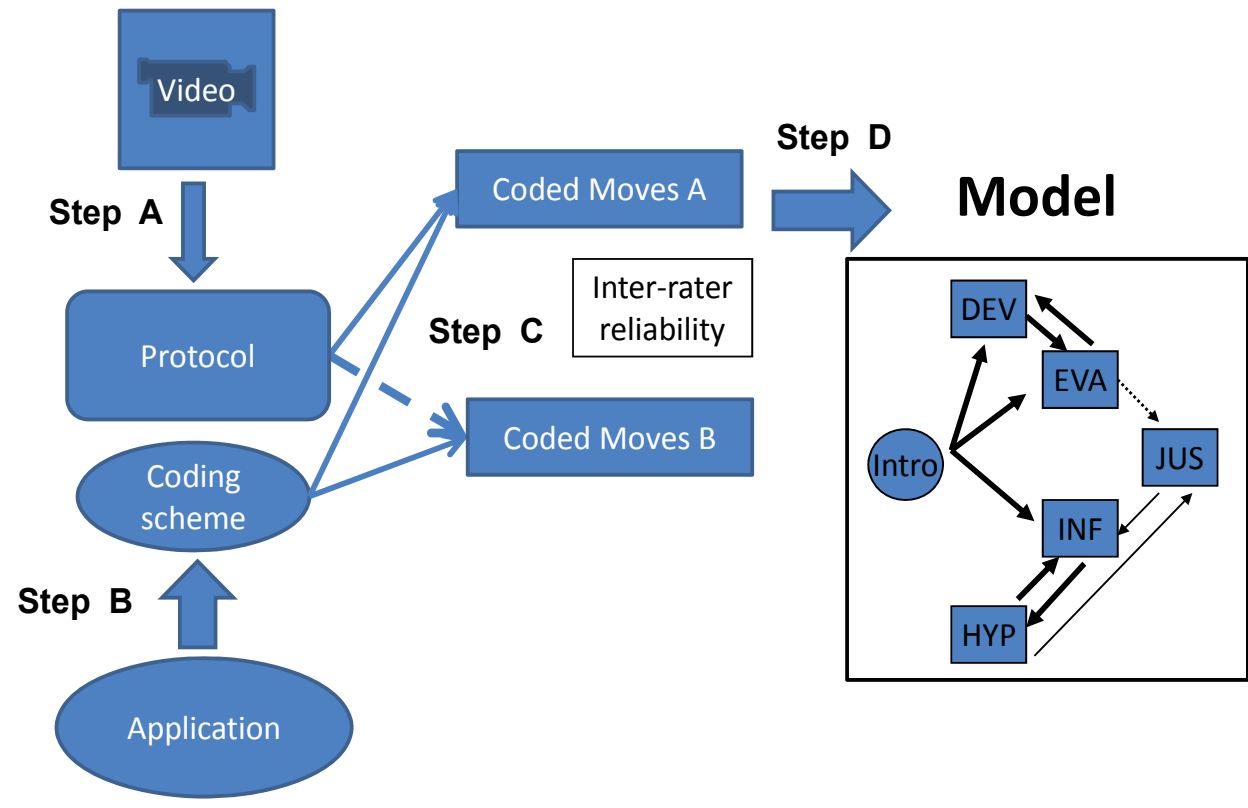

Fig. 1. The four steps of the traditional approach to protocol analysis

Figure 1 illustrates the four steps (A to D) of the traditional protocol approach to model building derived from qualitative analysis, where the data collection approach is to videotape participants. For more details on traditional approach to protocol analysis see Trickett \& Trafton (2002).

\subsection{STEP A: Protocol}

A specially trained typist transcribes the videos to produce a document called a protocol. The transcription is a technical activity that requires considerable skill. The main difficulties arise from the naturalness of the discourse, the technical vocabulary, and verbal familiarity. The transcripts form the basic data for the analysis, although it is sometimes useful to refer back to the video to validate the meaning of a statement. A transcript entry is called a (verbal) move (Sinclair \& Coulthard, 1975; Edmonson, 1981), a move being a statement made by a single speaker. A series of related moves is called a sequence and a series of sequences is called a verbal interaction. During a conversation, a move is the contribution of a single speaker to a given sequence. It is, therefore, the basic element to be identified from the transcript. The protocol (transcription) is an accurate written representation of all the moves made by participants. Moves may be very short and monosyllabic, like No or Yes, or extended and last for a few minutes. 


\subsection{STEP B: Coding scheme}

A coding scheme is derived from the application domain. The coding scheme defines the characteristics that are needed to describe a specific move, facilitate its identification, and provide the basis for coherent analysis. The definition of these characteristics is obtained through an iterative process of experimentation and validation of the coding scheme on one typical recording session. The coding scheme must be flexible, while at the same time maintaining the accuracy required by the researchers. The scheme should make it possible to focus on aspects judged to be relevant by the researchers. It should meet the theoretical attributes of interest and be objective (Olson et al., 1994). Objectivity deals with the reliability and validity of the coding scheme, while theoretical interest depends mainly on the domain and goals of the research. A coding scheme should be able to encode the moves adequately, and yet be formal enough to support quantitative analysis. Researchers in software engineering are even proposing a coding scheme to reduce the effort required to perform protocol analysis (Mayrhauser \& Lang, 1999). Cognitive ergonomists and software engineers are often involved in coding scheme definition.

The following illustrates the coding scheme developed for the model presented in this example (D'astous et al, 2004). Many characteristics are needed to describe a specific move, facilitate its identification, and provide the basis for coherent analysis. Table 1 defines the move characteristics.

An elementary syntax, including the separator symbol (/) between each characteristic, is used to code a move in such a way that it will facilitate automatic analysis. The generic code is then:

\section{ID/ACTIVITY/SUBJECT/DURATION/ATTRIBUTE}

For example, in the 60th move by teammate $M$ (ID), $M$ makes an assertion consisting in the evaluation (ACTIVITY) of the design solution SOLx (SUBJECT), lasting 5 seconds and referring to the format used to describe SOLx, which is criteria F, CRIT:F (ATTRIBUTE). Its code is:

M60/EVA/ SOLx/05/CRIT:F

Table 2 defines the limited set of activities for the review sequence moves.

\begin{tabular}{|l|l|}
\hline Characteristics & \multicolumn{1}{|c|}{ Definition } \\
\hline ID & Identifies the move through the speaker and the rank in the polylog \\
\hline ACTIVITY & Identifies the speaker's action \\
\hline SUBJECT & Identifies the informational entity on which the activity is performed \\
\hline DURATION & Identifies the duration of the move in seconds \\
\hline ATTRIBUTE & $\begin{array}{l}\text { Complements the subject, with respect to a criterion, fact, role, } \\
\text { content, project, sequence, etc. }\end{array}$ \\
\hline
\end{tabular}

Table 1. Move characteristics 


\begin{tabular}{|l|l|l|}
\hline \multicolumn{1}{|c|}{ Activity } & Abr. & \multicolumn{1}{c|}{ Definition } \\
\hline Evaluation & EVA & $\begin{array}{l}\text { Judging the value of a subject. This evaluation can be } \\
\text { negative, positive, or neutral. }\end{array}$ \\
\hline Justification & JUS & $\begin{array}{l}\text { Arguing or explaining the rationale for a certain choice. It is } \\
\text { often necessary to follow up an evaluation with a } \\
\text { justification of the approach taken. }\end{array}$ \\
\hline Information & INF & $\begin{array}{l}\text { Providing new knowledge with respect to the nature of a } \\
\text { subject. }\end{array}$ \\
\hline Hypothesis & HYP & $\begin{array}{l}\text { Expressing a personal representation of a subject. This } \\
\text { representation is made through the use of expressions such } \\
\text { as “I believe that..., “I think ...," or “...maybe..." }\end{array}$ \\
\hline Development & DEV & $\begin{array}{l}\text { Presenting a new idea in detail. This is considered a creative } \\
\text { activity. }\end{array}$ \\
\hline Introduction & Intro & Introducing the subject. \\
\hline
\end{tabular}

Table 2. Types of activity found in review sequence moves

\subsection{STEP C: Coder reliability}

The coding scheme is applied on the protocol by coders. More than one coder is needed on a least part of the protocol to measure the inter-rater reliability. Coder A, who collaborated in defining the coding scheme, performed the first coding, called Version A. Coder B, trained by coder A, performed coding in many sessions, and is called Version B. This approach provides two coded versions of the same session with different coder characteristics. The codes were shown to be reliable, according to accepted practices (Cone, 1977; Cooil \& Rust, 1994). Coding scheme must be validated such that inter-rater dissents are regarded as a source of variance, but not as a possible bias.

Table 3 presents the Perreault and Leigh (1989) reliability index and Cohen's Kappa (1960) for the two versions. These indices, obtained from two different statistical approaches, are above a threshold estimated to signify good agreement between coders.

\begin{tabular}{|c|c|c|c|}
\hline Versions & Reliability Index & Std dev & Kappa \\
\hline Version A and B & 0.83 & 0.020 & 0.68 \\
\hline
\end{tabular}

Table 3. Perreault and Leigh reliability index and Cohen's Kappa

\subsection{STEP D: Model building}

The last step is to build a model or a theory from the analysis of the coded moves. The purpose of this research was to identify typical configurations of exchanges (i.e. configurations occurring with a significant frequency) in review meetings. The model presented is based on the Latent Semantic Analysis (LSA) statistical methods (Sackett, 1979; Allison \& Liker, 1982). The analysis of sequential structures using LSA is grounded in information theory. It enables the identification of units (moves, exchanges) that follow each other, with or without other units in between. The analysis consists of determining whether 
or not the occurrence frequency of a given unit is independent of the occurrence frequency of another unit. These methods can be used in an iterative way, in several cycles, as long as LSA allows significant configurations to be detected.

This qualitative data analysis method is aimed at generating a model structure, and there are a number of approaches to strengthen, or confirm a proposition after it has been generated from the data. One method for helping to confirm findings, which is particularly well suited to most software engineering studies, is obtaining feedback on the findings from the subjects who provided the data in the first place. This strategy is sometimes called member checking (Seaman, 1999), and was the method used in this review meeting study. Participants, when presented with this model (see the box on the right-hand side of Figure 1 ), argue that it is only partially representative of the exchange patterns typical of a technical review meeting.

Confirmation of a qualitative model is a major endeavor. The whole qualitative approach is rigorous and objective, but the coding activity, which relies on the categorization of the protocol based on the coding scheme, may not be? What is the impact of coding variability on the derived model structure resulting from coder subjectivity? Would another coder deliver a different model structure?

Theoretically, each model could be different, depending on the intrinsic variability of sequences of coded moves. How can we know which model is the best? Assuming that it is feasible from a cost perspective to perform many codings and derive the corresponding model for each of them, it will be possible, for example, to consider only the model structure that is common to all models, or the features that are encountered most frequently.

This paper presents a novel approach based on Monte Carlo Coding Replication (MCCR), which enables the validation of the models by simulating numerous codings based on the inter-rater reliability measure. The approach consists of measuring the variability of the coding on a representative sample of the data, and then simulating that coding with the same variability for all the transcripts. The impact of the coding variability obtained from various simulated coders is then analyzed on the resulting model structures.

\section{Simulation procedure}

MCCR is an original simulation approach that has been developed to validate the model structure derived from protocol analysis. The main challenge is to characterize the variability of the coders in such a way as to be able to simulate sequences of coded moves that are within the inter-rater reliability level.

We present below a new approach to replicating coding activities based on Monte Carlo simulation. Underlying MCCR are the following two assumptions:

1. Reference sessions are representative of associated sessions, and consequently the variations between coders observed for one reference session are representative of the variations for any of the sessions associated with this reference session.

2. The simulated data will not generate hidden dependencies between the data, and the generated data will be similar to real data. 
The first assumption is necessary in order to reduce the effort required to measure variability. In theory, all the sessions are unique and variability could be measured on each of them. Pragmatic considerations and the homogeneity of the sessions, in terms of reviewing activities, make this assumption reasonable. In case of doubt, or given enough resources, it could be validated on many sessions. The example used in this chapter to illustrate the MCCR is based on a single representative session. Many sessions were coded by two coders, and it was estimated by the coders that session 2 versions $A$ and $B$ were representative of all the sessions coded. The second assumption is guaranteed by the parameters of the normal distributions used for the simulations, which are specific to, and derived from, each session.

MCCR is based on two parameters. The first takes into account the qualitative and subjective differences observed between coders in the reference session to simulate the variability. The second takes into account the profile of moves within the real session to reconstruct the simulated sessions.

Figure 2 shows the three steps added ( $E$ to $G$ ) in the MCCR approach from the traditional approach. In Step E, the variability between coders, which is based on the reference coding sessions used to compute the inter-rater reliability index, is characterized. In Step F, these variability characteristics are fed to the simulator. In Step G, models are reconstructed from the simulated moves. Finally, a new model structure is derived based on the invariant structures, or the structures that have the greatest probability of occurrence.

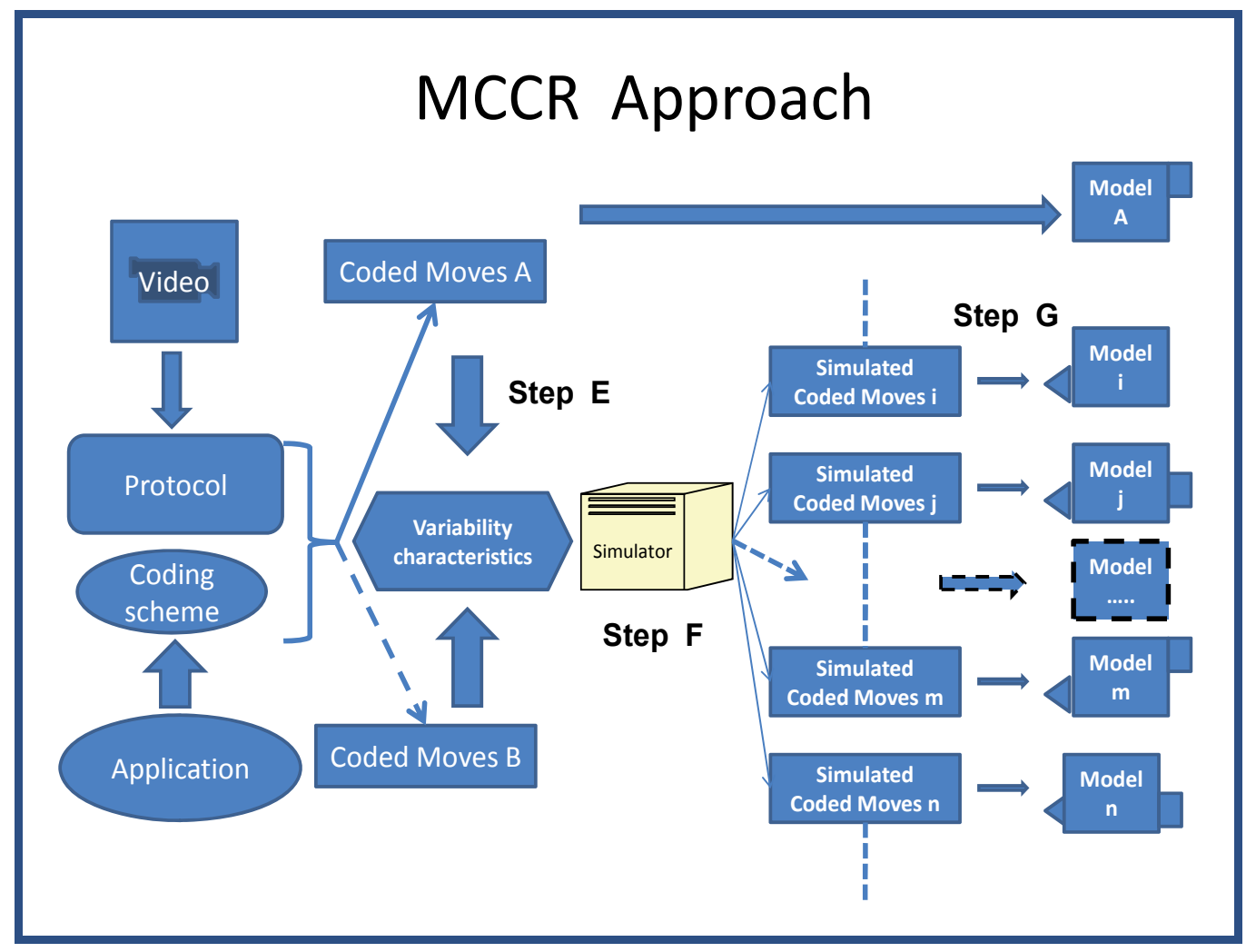

Fig. 2. Three added steps for the MCCR approach 


\subsection{Step E: Coded move variability characteristics}

Coded move variability is characterized according to two dimensions: coders (E1) and moves (E2). Step E1 characterizes the variability of code occurrence originating from the coders. Step E2 characterizes the variability of move duration originating from the protocol. In this step, simulated codes are assigned to appropriate moves, since the session duration is the same regardless of the coders (human or simulated).

The reliability and appropriateness of the coding scheme constitute major issues and are the subject of much research from a variety of viewpoints (Carletta et al., 1997) (Craggs \& Wood, 2005). The aim of the MCCR approach is to validate the resulting model structure by taking into account the variations in coder practices, assuming that the coding scheme is appropriate and the coders are reliable.

In Step E1, the variability for each code is characterized. For example, variation occurs when one coder evaluates a move as a DEV, while another considers it to be an EVA (See Table 2 for abbreviation meaning). The agreement matrix between two coders provides the probabilities of code occurrence.

Table 4 presents the number of occurrences of each code for Versions A and B for the reference session 2. For example, the element in the first line (DEV) and the first column (DEV) indicates that 11 moves were coded as DEV in both versions. The element in the second column (EVA) indicates that three moves coded DEV in Version A were coded EVA in Version B. Column JUS shows that no DEV move in Version A was coded JUS in Version B. The last column gives the total number of moves with a particular code in Version A. The last line gives the total number of moves with a particular code in Version B. For example, moves were coded DEV 20 times in Version A and 16 times in Version B, with 11 moves coded DEV in both versions. The values on the diagonal are the exact coded agreements for the two versions, which sums up to 106. An introductory move (INT), not shown in this table, is always the first move, the one that introduces the topic to be reviewed, and cannot be misjudged.

\begin{tabular}{|c|c|c|c|c|c|c|}
\hline \multirow{2}{*}{ Version $A$} & \multicolumn{7}{c|}{ Version B } \\
& DEV & \multicolumn{7}{c|}{ EVA } & JUS & HYP & INF & TOTAL \\
\hline DEV & $\mathbf{1 1}$ & 3 & & 2 & 4 & 20 \\
EVA & & $\mathbf{1 5}$ & & 2 & 4 & 21 \\
JUS & & 2 & $\mathbf{1 1}$ & 1 & 5 & 19 \\
\hline HYP & 5 & 1 & & $\mathbf{1 9}$ & 3 & 28 \\
\hline INF & & & 2 & 1 & 50 & 53 \\
\hline TOTAL & 16 & 21 & 13 & 25 & 66 & 141 \\
\hline
\end{tabular}

Table 4. Code occurrences from Version A and Version B of session 2

Table 3, presented previously, shows that the two coded versions A and B of the session 2 are reliable for discourse analysis. With the current state of the art, it is not possible to claim that one version is better than another. The main challenge is to understand and quantify the impact of this variability on the model structure derived from this protocol analysis, where only one coder coded all the sessions. It is very time-consuming, and almost 
impossible, to code all seven sessions many times in order to measure the variability of the resulting codes, as shown in this case for session 2.

In Step E2, the move duration, which is an intrinsic property of moves within a session, is characterized. Table 5 and Table 6 show the average duration of a move and its standard deviation respectively for the moves of Versions A and B. For example, the 11 DEV moves, which were coded DEV in both versions, have an average duration of 21.3 seconds with a standard deviation of 11.8 seconds. The 3 DEV moves of Version A that were coded EVA in Version B have an average duration of 8 seconds, with a standard deviation of 1.7 seconds. These tables provide the duration characteristics of code variability.

\begin{tabular}{|c|c|c|c|c|c|}
\hline \multirow{2}{*}{ Version $A$} & \multicolumn{5}{|c|}{ Version B } \\
& DEV & EVA & JUS & HYP & INF \\
\hline DEV & 21.3 & 8 & & 19 & 10.3 \\
\hline EVA & & 10.4 & & 9.5 & 5 \\
\hline JUS & & 20 & 20.6 & 5 & 8.5 \\
\hline HYP & 8 & 4 & & 15.7 & 19.7 \\
\hline INF & & & 9.5 & 3 & 15.1 \\
\hline
\end{tabular}

Table 5. Average move duration in Versions A and B

\begin{tabular}{|c|c|c|c|c|c|}
\hline \multirow{2}{*}{ Version A } & \multicolumn{5}{|c|}{ Version B } \\
& DEV & EVA & JUS & HYP & INF \\
\hline DEV & 11.8 & 1.7 & & 5.7 & 6.1 \\
\hline EVA & & 6.7 & & 3.5 & 2.9 \\
\hline JUS & & 5 & 13.3 & 3 & 4.4 \\
\hline HYP & 3.2 & 3 & & 10,7 & 16.2 \\
\hline INF & & & 4.9 & 2 & 12.9 \\
\hline
\end{tabular}

Table 6. Standard deviation for move duration in Versions A and B

The three tables needed for Step E can be easily and automatically computed from the coded move sessions. This approach requires that all the moves in a session be defined before coding. All coders code the same number of moves in a given session. All the moves are identified in the protocol with a unique ID. For example, coders will not have to decide whether an utterance accounts for one or two moves.

\subsection{Step F: Simulation procedure}

The Monte Carlo simulation of a session uses the coder and move duration characteristics obtained in Step E to produce a simulated session. The simulation procedure is performed following five steps. Step F1 and F2 simulates a new sequence of codes according to the coder variability characteristics. Step F3, F4 and F5 associates each simulated code to a corresponding move, according to the duration characteristics of each type of code. The number of moves per session is not modified by the simulation. First, all simulated sessions have to be coded by one human coder, which is Version A (see Figure 2). The 
reliability index and the Kappa coefficient are calculated for each simulated session based on Version A, and should be near or above the values computed in Table 3 to be considered reliable.

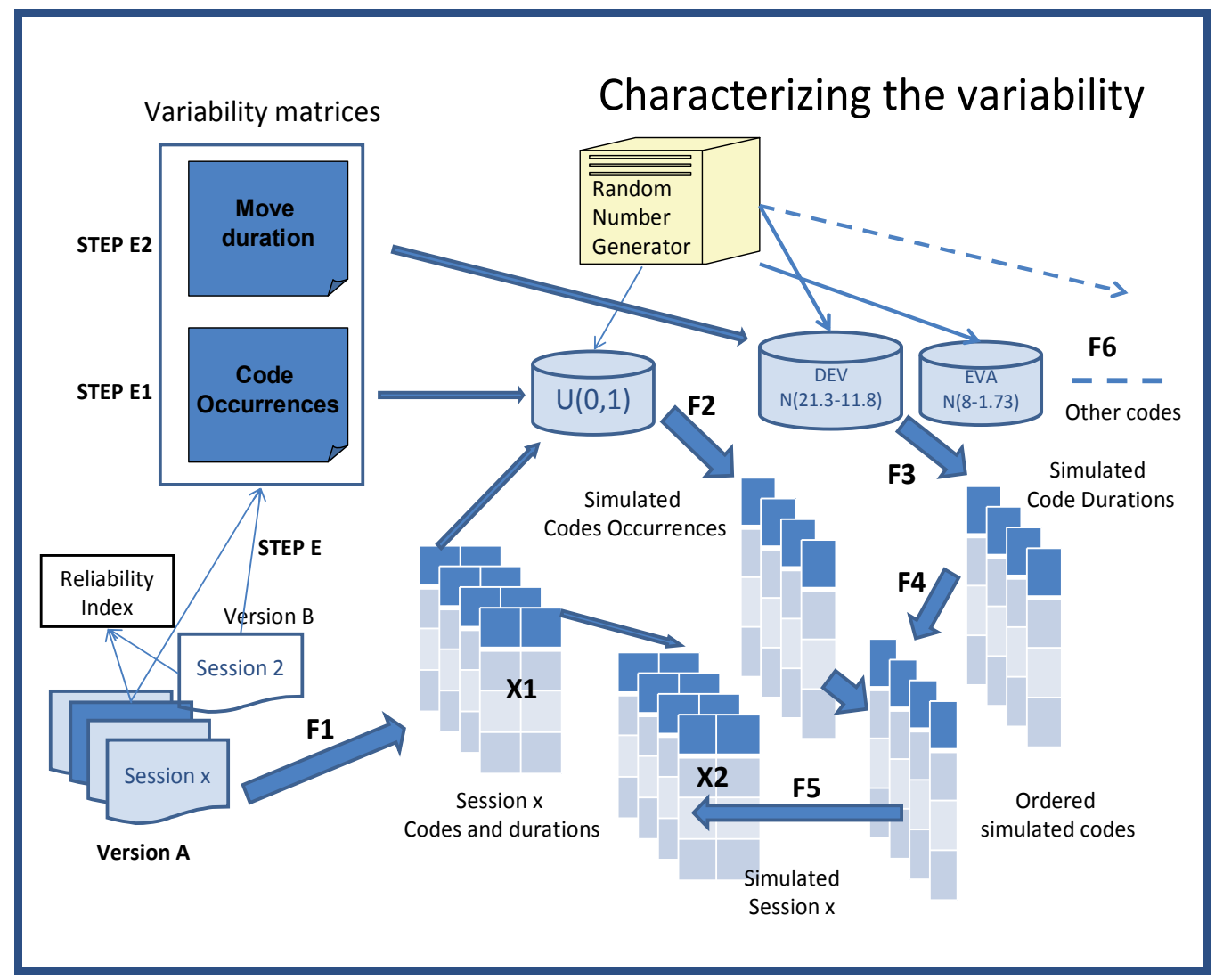

Fig. 3. Steps for the simulation of a session

Figure 3 illustrates the five steps of the simulation procedure, based on the simulation of session $\mathrm{x}$. In the bottom-left corner is Version A of all the sessions that were hand-coded. Session 2 is one of the sessions of Version B that was selected by another coder as the reference session from which are derived the variability matrices produced in Step E1 and Step E2, described in the previous section. We recall that there can be more than one reference session. For example, sessions A1 to A4 of Version A can have session B2 of Version $\mathrm{B}$ as the reference session, and sessions $\mathrm{A} 5$ to $\mathrm{A} 7$ can have $\mathrm{B} 5$ as the reference session, etc.

The simulation procedure is composed of five steps, labelled F1 to F5 in Figure 3. The table dimensions shown in the figure are for illustration purposes only and do not correspond to the real example. The first step (F1) is to extract all the moves with the same type of activity, and their duration, from a given session $x$ of Version A. For example, Table 7 (Table $\mathrm{X} 1$ in Fig. 3) presents the six DEV codes and their duration ordered according to move occurrence (ID). In the ID field, the letter representing the author is omitted, since it is irrelevant for this analysis. 


\begin{tabular}{|c|c|c|}
\hline ID & Code & Duration \\
\hline 4 & DEV & 15 \\
\hline 12 & DEV & 4 \\
\hline 13 & DEV & 37 \\
\hline 25 & DEV & 12 \\
\hline 32 & DEV & 20 \\
\hline 46 & DEV & 8 \\
\hline
\end{tabular}

Table 7. Duration of DEV codes to be simulated

The next step (F2) is to simulate the new codes that will correspond to the DEV codes assigned by the human coder. This new simulated code depends on the variability probability profile between coders.

The variability between coders is such that there is a probability that some of these DEV codes will be coded differently by another coder. This probability is obtained from the multi-coding performed on the reference session with different coders. The probabilities of code occurrence are presented in Table 8, which is derived from Table 4.

For example, the number in the first row and the first column (.55) represents $55 \%$ of the 20 total occurrences of DEV for Version A. The three occurrences of EVA account for $15 \%$ of the total occurrences. The probability of occurrence is expressed cumulatively, so EVA is at $70 \%$.

\begin{tabular}{|c|c|c|c|c|c|}
\hline Version $A$ & \multicolumn{5}{|c|}{ Version B } \\
& DEV & EVA & JUS & HYP & INF \\
\hline DEV & 0.55 & 0.7 & 0.7 & 0.8 & 1 \\
EVA & 0 & 0.71 & 0.71 & 0.81 & 1 \\
JUS & 0 & 0.1 & 0.69 & 0.74 & 1 \\
HYP & 0.18 & 0.21 & 0.21 & 0.89 & 1 \\
INF & 0 & 0 & 0.04 & 0.06 & 1 \\
\hline
\end{tabular}

Table 8. Probability of occurrence of codes

Six random numbers generated from a uniform distribution $U(0,1)$ are used to determine how many DEV codes, if any, will be coded differently. The ordered random numbers in the first column of Table 9 are compared with the probabilities on the DEV line of Table 8. In Table 9, there are four numbers lower than 0.55 (in bold) and two larger than 0.55 , but less than 0.7 , which means that the simulated session will have four DEV codes and two EVA codes, as shown in the second column of Table 9.

\begin{tabular}{|c|c|}
\hline $\begin{array}{c}\text { Ordered } \\
\text { Random number }\end{array}$ & $\begin{array}{c}\text { Simulated } \\
\text { Assigned Code }\end{array}$ \\
\hline $\mathbf{0 . 1 3}$ & DEV \\
\hline $\mathbf{0 . 2 2}$ & DEV \\
\hline $\mathbf{0 . 4 0}$ & DEV \\
\hline $\mathbf{0 . 4 9}$ & DEV \\
\hline 0.57 & EVA \\
\hline 0.68 & EVA \\
\hline
\end{tabular}

Table 9. Simulated code occurrences 
The next step (F3) is to determine which of the two DEV codes in the real session will be interpreted as an EVA code in the simulated session. Ordering and sequencing the codes is important, since many analyses like LSA rely on the code sequences. The assignment of the simulated codes to corresponding moves is based on the duration patterns of the moves, as characterized in Table 5 and Table 6 . This information is used to define the characteristics of the virtual duration of each of the new DEV and EVA codes. It is called virtual duration, because it is used only to find the appropriate moves, since the real duration of a move is fixed, regardless of how it is coded (human or simulated). The simulation is based on normal distributions with the parameters given in Table 5 and Table 6 for the corresponding codes. Normal distributions $\mathrm{N}(21.3-11.8)$ and $\mathrm{N}(8-1.73)$, which have been found to be appropriate, are used for DEV and EVA respectively. Analyses of the moves are performed to determine the most appropriate distributions for code duration simulations. Table 10 presents the results for the four DEV codes (in bold) and the two EVA codes in increasing order of simulated duration.

The next step (F4) is to match the simulated codes with the real moves. Table 11 shows the resulting association of simulated codes with the real codes. The shorter simulated code as given by Table 10 is associated with the shorter real code, and so on. The simulated duration is only used to find the code association.

\begin{tabular}{|c|c|}
\hline CODE & $\begin{array}{c}\text { SIMULATED } \\
\text { DURATION }\end{array}$ \\
\hline EVA & 7 \\
\hline DEV & $\mathbf{9}$ \\
\hline EVA & 10 \\
\hline DEV & $\mathbf{1 7}$ \\
\hline DEV & $\mathbf{2 5}$ \\
\hline DEV & $\mathbf{2 8}$ \\
\hline
\end{tabular}

Table 10. Simulated code durations

\begin{tabular}{|ccccc|}
\hline ID & CODE & Duration & Sim CODE & Virtual Duration \\
\hline 12 & DEV & 4 & EVA & 7 \\
\hline 46 & DEV & 8 & DEV & $\mathbf{9}$ \\
\hline 25 & DEV & 12 & EVA & 10 \\
\hline 4 & DEV & 15 & DEV & $\mathbf{1 7}$ \\
\hline 32 & DEV & 20 & DEV & $\mathbf{2 5}$ \\
\hline 13 & DEV & 37 & DEV & $\mathbf{2 8}$ \\
\hline
\end{tabular}

Table 11. Matching simulated codes on the basis of duration ordering

The last step (F5) is to build a new simulated version according to the simulated codes assigned to the real moves. Table 12 shows the ordered DEV codes, according to their ID, in the real and simulated versions of session $\mathrm{x}$. The process is performed for all the codes of Version A to build a full, simulated version that has the same duration and where qualitative variations of coding are performed according to the coder's characteristics provided by the reference session. 
MCCR provides a new version of the human-coded version that is likely to have the same characteristics as a version obtained by another coder. It will have the same number of codes with a variability that will be within the characteristics measured in the reference session. For example, Table 12 shows that a simulated coder will have coded moves 4, 13, 32 and 46 with the same code DEV as the human coder but will have coded moves 12 and 25, which are lasting 4 and 12 second respectively, as EVA codes. Kappa index shows that such coding is within the inter-reliability level found between the two human coders.

\begin{tabular}{|c|c|c|c|}
\hline ID & $\begin{array}{c}\text { Human-coded } \\
\text { Version }\end{array}$ & $\begin{array}{c}\text { Simulated } \\
\text { Version }\end{array}$ & $\begin{array}{c}\text { Real } \\
\text { Duration }\end{array}$ \\
\hline 4 & DEV & DEV & 15 \\
\hline 12 & DEV & EVA & 4 \\
\hline 13 & DEV & DEV & 37 \\
\hline 25 & DEV & EVA & 12 \\
\hline 32 & DEV & DEV & 20 \\
\hline 46 & DEV & DEV & 8 \\
\hline
\end{tabular}

Table 12. DEV code sequence in coded and simulated versions

The whole process of simulating coded sessions is summarized by the two matrices, labelled $\mathrm{X} 1$ and $\mathrm{X} 2$ at the bottom center of Figure 3. The left-most column containing the coded type of activity (DEV, EVA, etc.) is used for the simulation process, since this is the source of the variability, while the right-most column containing the move duration is transferred 'as is' (arrow with no label) in the resulting simulated sessions (X2). The same simulation process (F6) is repeated for each type of activity, as shown in Table 8.

MCCR is efficient and could be performed hundreds of times in any given session, which means that the whole spectrum of coding variability can be explored. The Kappa coefficient is used to evaluate the level of agreement between the simulated versions and the real version.

\section{Reconstructing the models (Step D)}

Reconstruction of the models (Figure 1, Step D) is the last step of protocol analysis. This section shows the impact of the MCCR approach on validating the exchange pattern model structure derived from protocol analysis performed by a single coder and on the model derived from multi-simulated coding.

The question MCCR has answered is: How do we know that the model structure would have been the same if another coder had performed the protocol analysis?

The analysis of model structure variability is not part of the MCCR approach. It is presented only to illustrate the usefulness of MCCR in a real case. Readers interested in the detail of model building and measurement of the link significance levels for the model structures are referred to the papers presenting the models (D'Astous et al, 2002, 2004).

The purpose of this protocol analysis was to build a model of the communication exchanges occurring in technical review meetings, in order to optimize their efficiency and to better integrate these activities into the software engineering process. 
Figure 4 shows the model structure resulting from the single-coder model on the left-hand side and model structure from the simulated model on the right-hand side. The single-coder model is based on the analysis of seven coded meetings. The patterns observed in each meeting are added together to create the left-hand cumulative pattern of the figure. The right-hand cumulative pattern is made-up of a combination of the simulated meetings, where each of the seven meetings is simulated 10 times. More simulation did not add any new information to the model structure. LSA is applied to each meeting independently.

The following briefly explains the model. The exchanges identified (Table 2) introduce the subject (INTRO), provide information (INF), make a hypothesis (HYP), generate a development (DEV), evaluate (EVAL), and justify (JUS) viewpoints. Each meeting is analyzed and the number of link occurrences is indicated by the size of the link. Links in large bold type indicate that they are statistically significant for all meetings, while solid narrow type indicates that the links are statistically significant for some of the meetings.

The single-coder model provides the following exchange patterns during a peer review meeting. Any sequence of exchanges starts with the introduction (INTRO) of the topic to be reviewed. Following the INTRO, there are three significant exchanges that could occur during a session: a development exchange DEV, an evaluation exchange EVA, or an information exchange INF. Peers are likely to continue on into DEV and EVA exchange sequences or into INF and HYP exchange sequences. Sometimes, a justification exchange JUS will follow an evaluation EVA or a hypothesis HYP exchange, and a justification JUS exchange may be followed by an information INF exchange. The single-coder model also indicates that an introduction INTRO may be followed by a hypothesis HYP exchange.

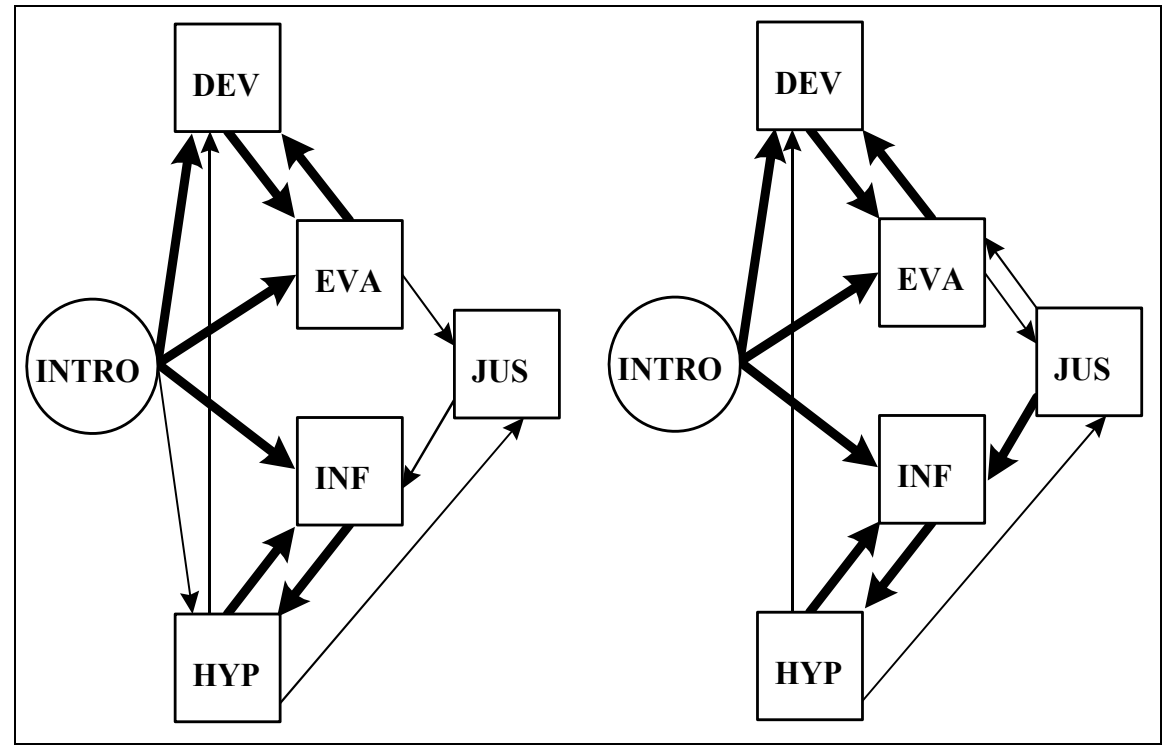

Fig. 4. Single-coder model (left-hand side) and simulated model (right-hand side).

At first glance, the single-coder and simulated patterns are similar, which means that the variability in coding is not dramatic. Nevertheless, there are some differences that emerge from the simulated model. For example, all but one of the large bold links is common to 
both models, except for the JUS-INF link, which appears only in the simulated model on the right-hand side of the figure. Secondary links, represented by solid lines, are also almost the same in the two models, except for the INTRO-HYP links, which appear only in the singlecoder model on the left-hand side of the figure, and the JUST-EVA link, which appears only in the simulated model.

The simulated model confirmed the salient features of the single-coder model. MCCR may resolve ambiguities that arise from the qualitative components of coding. For example, the exchange patterns INTRO and HYP observed in the single-coder model are clearly not significant based on the simulated model. In contrast, the weak link between JUS and INF observed in the single-coder model is, in fact, a significant one in the simulated model. The simulated model shows the occurrence of an evaluation-justification loop.

\section{Conclusion}

MCCR enables analysis of the impact of individual qualitative variability on coding transcripts from protocol analysis. It is much easier to perform than real replication of the protocol analysis by different coders, and requires far fewer resources. Moreover, it provides information on the components of the resulting models, which are sensitive to the qualitative components of coding. This Monte Carlo validation approach is generic, and not limited or specific to the case study presented. The simulation is essentially based on the number of categories, which is limited by the capacity of human beings to handle numerous categories. This number is always very small compared to the power of computer simulation.

To summarize, MCCR is performed on complete protocol analysis performed by a welltrained coder. The coding is redone in one or many representative sessions, called reference sessions, by another well-trained coder. The reliability of the two coded protocols is measured with known tools, such as agreement indices or Kappa coefficients. Qualitative differences between the coders, which are interpreted as the qualitative variations in the coding pattern, are characterized by a probability matrix derived from the two codings of the reference sessions. Profiles of coded moves are characterized by the average and standard deviation of their durations. These parameters are used to specify the parameters of the normal distributions of the random generator. All codes are simulated according to the distribution specifications provided by the reference sessions. Sequences of simulated codes are built according to the specific code duration within a given session. A simulated session of exactly the same duration is obtained with a coding variability that is within the range shown for the reference session. The Kappa coefficient is computed on the simulated sessions to validate their reliability. As many simulations as needed could be performed to obtain a statistically significant and stable model.

Observational studies are difficult to conduct in an industrial environment, and analysis of the data is very time-consuming. However, such studies constitute one of the few approaches that enable the modeling of real activities. MCCR is a new method developed to extract from the observed model structure the components related to the subjective and qualitative components of the observations. This method has been illustrated with an example based on modeling the technical review meeting, but it is of general interest and can be applied to any observational study. 


\section{Acknowledgment}

This research would not have been possible without the generous participation and patience of the software development team members from whom the data were collected. To all these people, we extend our grateful thanks. This research was supported in part by NSERC grant A-0141.

\section{References}

Allison, P.D. \& Liker, J.K. (1982). Analyzing sequential categorical data on dyadic interaction: a comment on Gottman, Psychology Bulletin, 91, 393-403.

Baer, D.M. (1977). Reviewer's Comment: Just because it's reliable doesn't mean that youcan use it, Journal of Applied Behavior Analysis, 10 (1), 117-119.

Barnas Y. (1996). Formal aspects of model validity and validation in system dynamics, System Dynamics Review, 12 (3), 183-211.

Berk, R.A. (1979). Generalizability of behavioral observations: A clarification of interobserver agreement and interobserver reliability. American Journal of Mental Deficiency, 83 (5), 460-472.

Campbell, D.T. \& Fiske, D. (1959), Convergent and discriminant validation by the multitrait, multi-method matrix. Psychological Bulletin, 56, 81-105.

Carletta, J., Isard, S., Doherty-Sneddon, G., Isard, A., Kowtko, J.C., \& Anderson, A.H. (1997). The reliability of a dialogue structure coding scheme, Computational Linguistics, 23(1), 13-31.

Cicchetti, V.D., Shoinralter, D., \& Tyrer, P.J. (1985). The effect of number of rating scale categories on levels of interrater reliability: A Monte Carlo investigation, Applied Psychological Measurement, 9 (1), 31-36.

Cohen, J. (1960). A coefficient of agreement for nominal scales, Educational and Psychological Measurement, 20(1), 37-46.

Cone, J.D. (1977). The relevance of reliability and validity for behavioral assessment, Behavior Therapy, 8, 411-426.

Cooil, B. \& Rust, R.T. (1994). Reliability and expected loss: A unifying principle. Psychometrika, 59(2), 203-216.

Craggs, R., \& Mc Gee Wood, M. (2005). Evaluating discourse and dialogue coding schemes, Computational Linguistics, 31(3), 289-296.

Cronbach, L.J., Gleser, G.C., Nanda, H., \& Rajaratnam, N. (1972). The dependability of behavioral measurements: Theory of generalizability for scores and profiles. John Wiley \& Sons, New York.

Curtis, B. (1980). Measurement and experimentation in software engineering. Proceedings of the IEEE, 68(9), 1144-1157.

D'Astous, P., \& Robillard, P.N. (2002). Empirical study of exchange patterns during software peer review meetings, Information and Software Technology, 44, 639-648.

D’Astous, P., Détienne F., Visser W., \& Robillard, P.N. (2004). Changing our view on design evaluation meetings methodology: A study of software technical review meetings, Design Studies, 25(6), 625-655.

Edmonson, W. (1981). Spoken discourse: A model for analysis. London: Longman,.

Ericsson, K.A., \& Simon, H.A. (1993). Protocol analysis: Verbal reports as data, Revised Edition. MIT Press, Cambridge. 
Foster, A., Urquhart, C. \& Turner, J. (2008). "Validating coding for a theoretical model of information behaviour". Information Research, 13(4) paper 358.

Herbert, J., \& Attridge, C. (1975). A guide for developers and users of observation systems and manuals. American Educational Research Journal, 12(1), 1-20.

Hughes, J., \& Parkes, S. (2003). Trends in the use of verbal protocol analysis in software engineering research, Behaviour \& Information Technology, 22(2), 127-140.

Johnson, S. M., \& Bolstad, O.D. (1973). Methodological issues in naturalistic observation: Some problems and solutions for field research. In Hamerlynck, L. A., Handy, L. C., Mash, E. J. (eds.) Behavior Change: Methodology, Concepts and Practice. Research Press, Champaign, Illinois, 7-67.

Khaled, E.E. (1999). Benchmarking Kappa: Interrater agreement in software process assessments, Empirical Software Engineering, 4, 113-133.

Kerlinger, F.N. (1973), Foundations of behavioralrResearch, 2nd edition. Holt, Rinehart and Winston, NY,

Mayrhauser, von A., \& Lang, S. (1999). A coding scheme to support systematic analysis of software comprehension, IEEE Transaction on Software Engineering, 25(4), 526-540.

Medley, D.M. \& Mitzel, H.E. (1963). Measuring classroom behavior by systematic observation. In Gage, N. L. (ed.) Handbook of Research on Teaching. Rand McNally, Chicago, 247328.

Mitchell, S.K. (1979). Interobserver Agreement, Reliability, and Generalizability of Data Collected in Observational Studies. Psychological Bulletin, 86(2), 376-390.

Nelson, R.O., Hay, L.R., \& Hay, W.M. (1977), Comments on cone's "The relevance of reliability and validity for behavioral assessment". Behavior Therapy, 8, 427-430.

Olson, G.M., Herbsleb, J.D., \& Rueter, H.H. (1994), Characterizing the sequential structure of interactive behaviors through statistical and grammatical techniques. HumanComputer Interaction, 9, 427-472.

Perreault, W.D.J., \& Leigh, L.E. (1989), Reliability of nominal data based on qualitative judgments, Journal of Marketing Research, 26, 135-148.

Robillard, P.N., d'Astous, P., Détienne, F., \& Visser, W. (1998), Measuring cognitive activities in software Engineering. Proceedings of the 20th International Conference on Software Engineering, 292-300.

Sackett, G.P. (1979), The lag sequential analysis of contingency and cyclicity in behavioral interaction research. In Osofsky, J. D. (ed.) Handbook of Infant Development. WileyInterscience Publication, 623-649.

Sanderson, P.M.,\& Fisher, C. (1994). Exploratory sequential data analysis: Foundations. Human-Computer Interaction, 9, 251-317.

Seaman, C.B. (1999), Qualitative methods in empirical sudies of software engineering, IEEE Transaction on Software Engineering, 25(4), 557-572.

Sinclair, A., \& Coulthard, R.M. (1975). Towards an analysis of discourse. The English used by Teachers and Pupils. Oxford University Press.

Trickett, S., \& Trafton, J.G. (2002), A primer on verbal protocol analysis, in Handbook of Virtual Environments: Design, Implementation, and Applications (Human Factors and Ergonomics), Kay Stanney (Editor), 332-346.

Weber, R.P. (1990). Basic content analysis, Series: Quantitative Applications in the Social Sciences, Sage University Paper 


\title{
Cartographic Generalization Applied to Social Networks Maps in the City of Curitiba in Brazil
}

\author{
Renan M. Pombo, Luciene S. Delazari and Claudia R. Sluter \\ Federal University of Parana, \\ Brazil
}

\section{Introduction}

Social network is a form of representation of affective or professional relationships between themselves or between groups of mutual interests. Generally, social networks are understood as a set of social actors (individuals, groups and institutions), called nodes, which are interconnected when these are relations between them, incorporating a social system (Delazari, Sluter and Kauchakje, 2007).

However, the representations used by analysts in social network, ie graphs and matrices of relationships are not georeferenced, and it does not allow to perform analysis about the network structure in relation to their spatial distribution and other phenomena that may affect this distribution.

In this research we intend to produce maps of social network in small-scale representations and digital media in order to allow user interaction with the map and to make handling easier and to be effective. Thus we used the cartographic generalization automatically of the elements that form the network, which was performed using the aggregation and displacement operators. Therefore we gained readable representations of all social actors together with their respective links in a map on a smaller scale.

The automation of the cartographic generalization process aims to represent the maps at smaller scales and to transmit efficiently the information. Today, maps are viewed even on mobile phone screens. Therefore several decisions should be modeled and implemented by algorithms. This research discusses, specifically, the problem of automation of aggregation and displacement operators applied to point features.

Maps are important to explore the influence of the geographic localization in the social networks. We can see where there is more actors, places with no organizations acting. And then the analysts can make your decisions about the position of a next actor or relocate them. The cartographic generalization allows users to use maps on digital screens in any scale through your operators.

\section{Social network}

The network analysis emphasizes the relationships between actors, which means that they usually are not sampled independently, as in other types of conventional data focusing on 
the actors and attributes. The study of complex networks was initiated by mathematicians and then used in sociology from the perspective of structural analysis of social networks ( Tomael and Marteleto, 2006).

Social networks associated with cartography gained support to expand the knowledge of their analysts through a spatial component originated from the georeferencing of social actors and their relationships (Delazari et al, 2007). In the graph without reference we can move an actor to avoid overlaps without any compromise with its geographical location. Network analysis can still show their degree of dependence between nodes and how this can be complex or not.

\section{Procedures}

This research aims to automate two existing cartographic generalization operators. Here we will focus on procedures needed to automate the aggregation and displacement operators applied on the reduction of scale of the representations of social networks.

In this study we used the same data survey of Marchis (2008) and Pombo (2009) that refer to the social actors of the social networks that guarantee the right to social assistance in the city of Curitiba. The data were collected through research on Internet search sites to issues concerning health, labor, dwelling, nutrition, education and social assistence.

The maps were produced using the software ArcGIS, which allows variation of the scale of representation and hiding of certain types of features on the map. In addition to allowing the variation of the symbology. For the organization of data in tables are used Microsoft Excel.

The main goal was to achieve that automated process of generalization results similar to manual work performed in Pombo (2009). In both studies was applied the aggregation to the overlaps involving point symbols with the same classification - with the same symbology. Otherwise, if there is conflict between different symbols will be used the displacement operator.

The work began with a file with the coordinates of the points (network nodes), the developed software calculates and stores the distances between all points of the network. To perform the aggregation procedure, the program determines which is the minimum distance to avoid the conflict in the symbology. This distance is defined as the product between the radius of the symbol in the representation and the value of the scale. Then the algorithm checks which the distances are shorter than the minimum allowed value and stores the involved points.

Then there is a classification of points. If the points have the same classification, are assembled in one whose coordinates are obtained by averaging the coordinates of the points in which it originated. The radius of this new symbol is determined by the aggregate amount of symbols.

If the symbols were different, the software would randomly selects one of the points involved and their coordinates were added or subtracted from the value of the radius multiplied by the scale until there is no more overlap between the symbols. If this 
displacement cause new conflicts, the procedure is repeated, but with the point that had not been drafted first. If there is still conflict, moves the third point that does not allow the separation of the symbols and so on until there are no more congestion. Finally, a new file is organized with the generalized points.

\section{Results}

With the developed software, several tests were performed by varying representation of the input parameters. An analysis with respect to the results obtained by changing the size of the minimum point symbol and the variation of the scale. The figures presented in this section refer to tests based on network social health data of Curitiba in the scale of 1:75,000. The goal is to produce representations on the scale of 1:250,000, which allows visualization of the whole base map of the city of Curitiba on computer screen of 15 ". But tests were made with intermediate stops to realize that if and when problems occur as in the representation. FIGURE 1 shows the scale of the original part of the social network of health, more specifically, in the central region (Downtown Regional), which has the largest concentration of social actors with a minimum radius of $1 \mathrm{~mm}$.

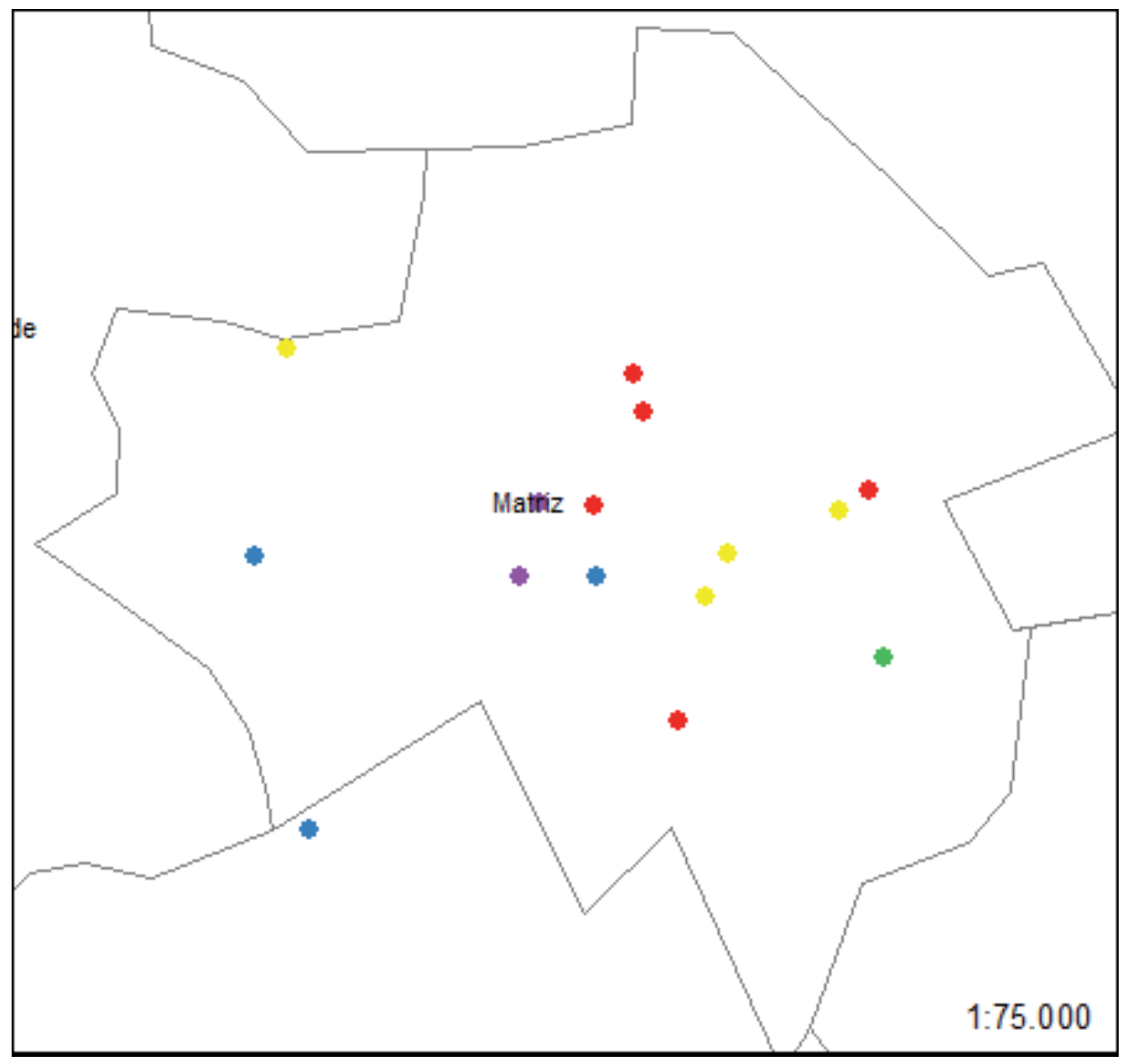

Fig. 1. Original data with 1:75.000 scale for regional division 
The three intermediate scales adopted in the tests were 1:100,000, 1:150,000, 1:200,000. On a scale of 1:100,000 (Figure 2) there were no changes in the positions of the actors, because there is no problems in the representation.

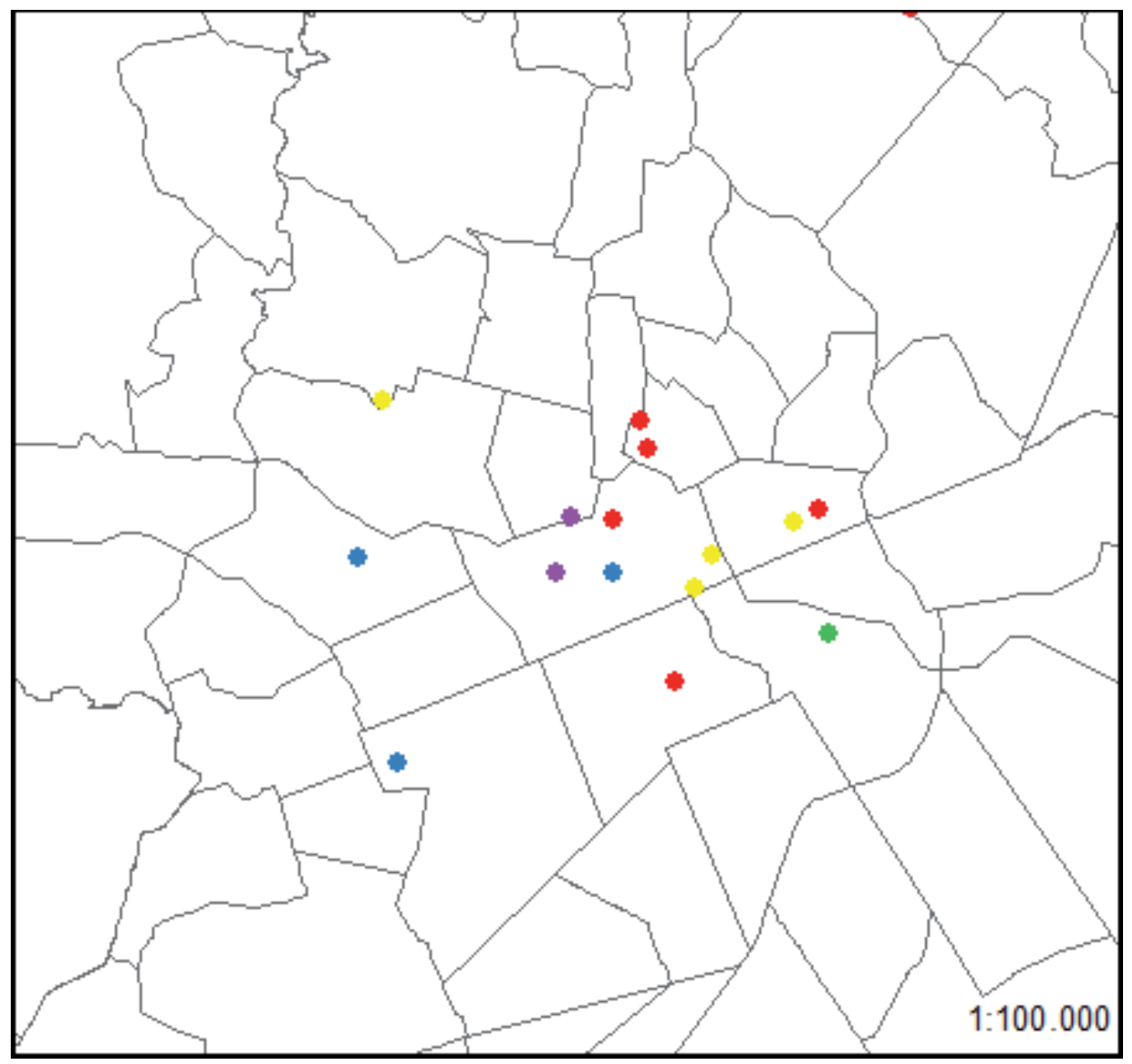

Fig. 2. Generalized data for 1:100.000 scale 
On a scale of 1:150,000 (Figure 3) we have the first generalization, in which the actors are separated by the displacement operator. It appears that the symbol red circle was moved north to not collapse as in Figure 4 - highlighted - the operator was applied so that, even after the displacement, the actors keep in their positions of origin.

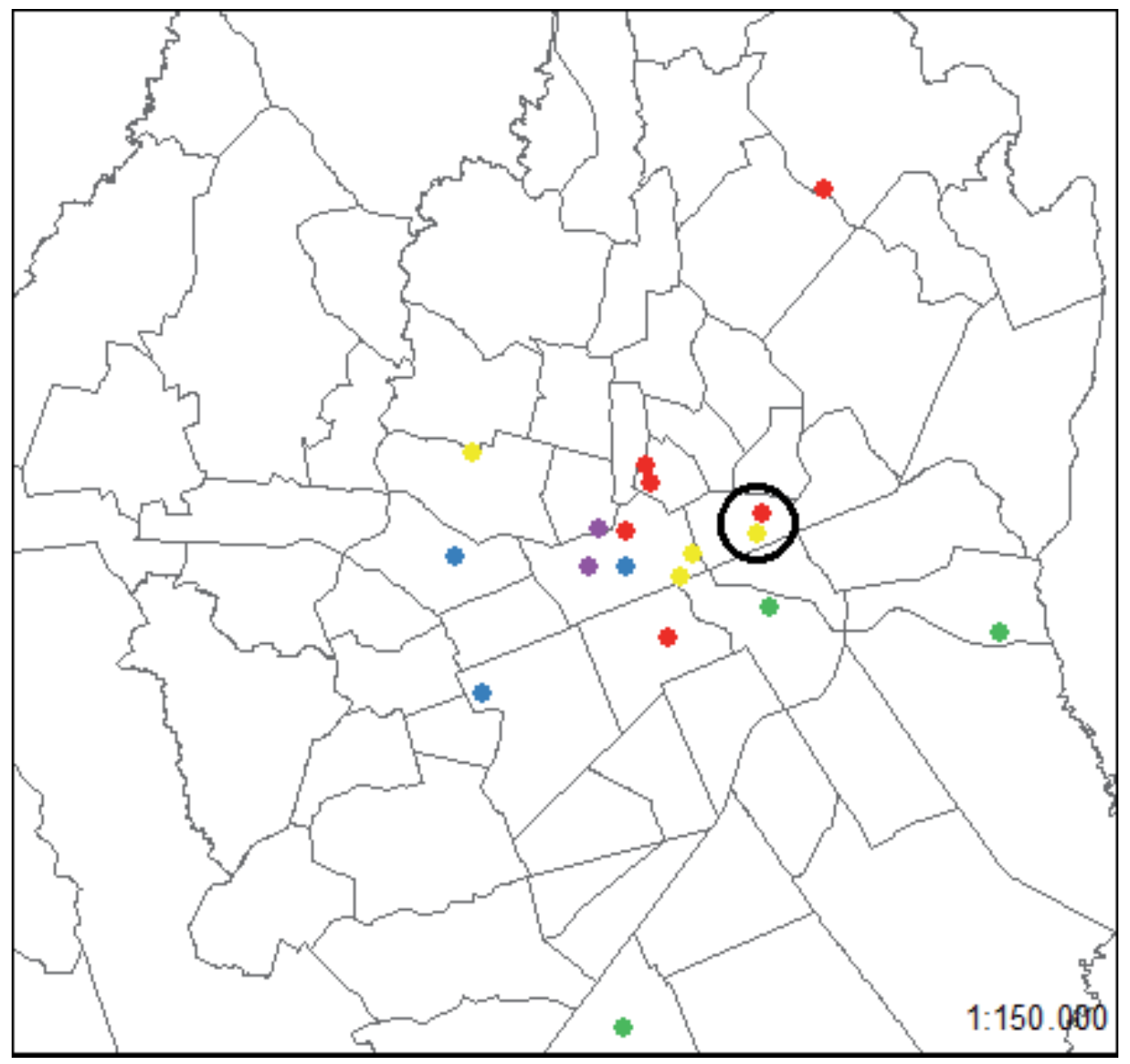

Fig. 3. Generalized data for 1:150.000 scale 


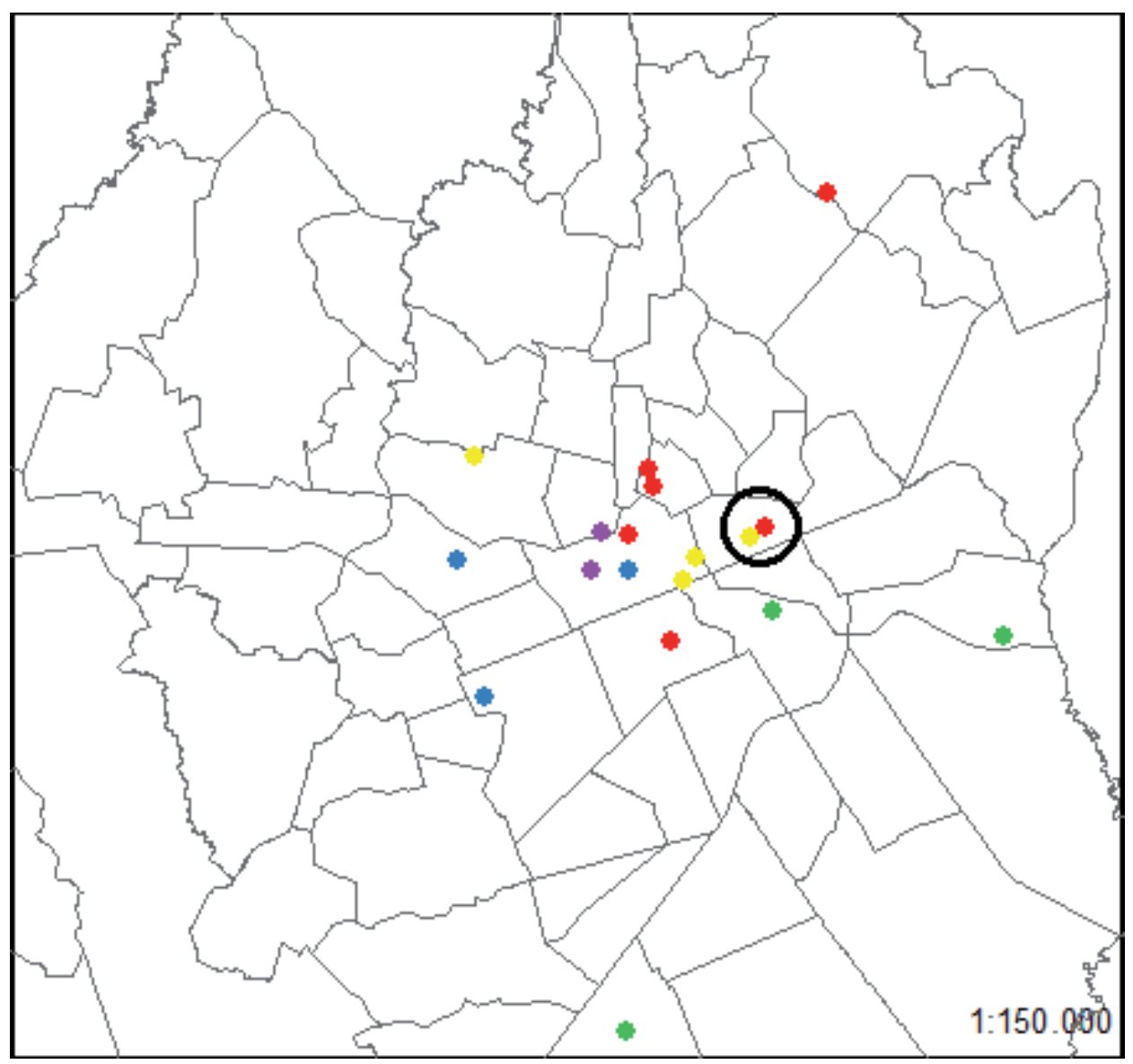

Fig. 4. No generalized data for 1:150.000 scale 
Although the new symbols are represented with circles of radius bigger than $1 \mathrm{~mm}$ it causes problems at the division representation by districts, because they could be represented in two city district on the same time. But with the regional division (Figure 5), this type of conflict does not exist.

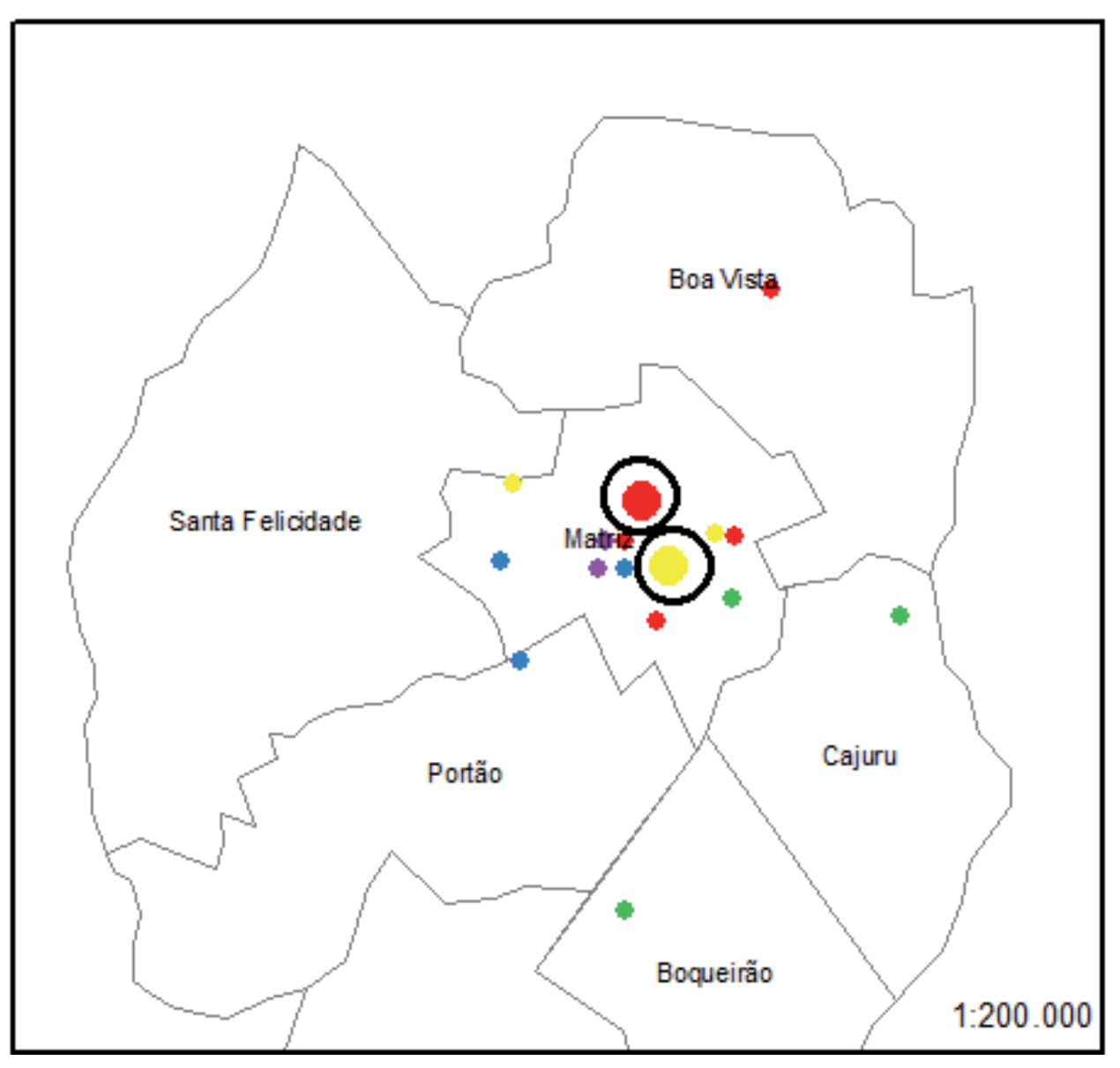

Fig. 5. Generalized data for the regional division for 1:200.000 scale 
The reduction of scale gives an increase in quantity of symbols, conflicts that can be seen in Figure 6, that is in the scale of 1:250,000.

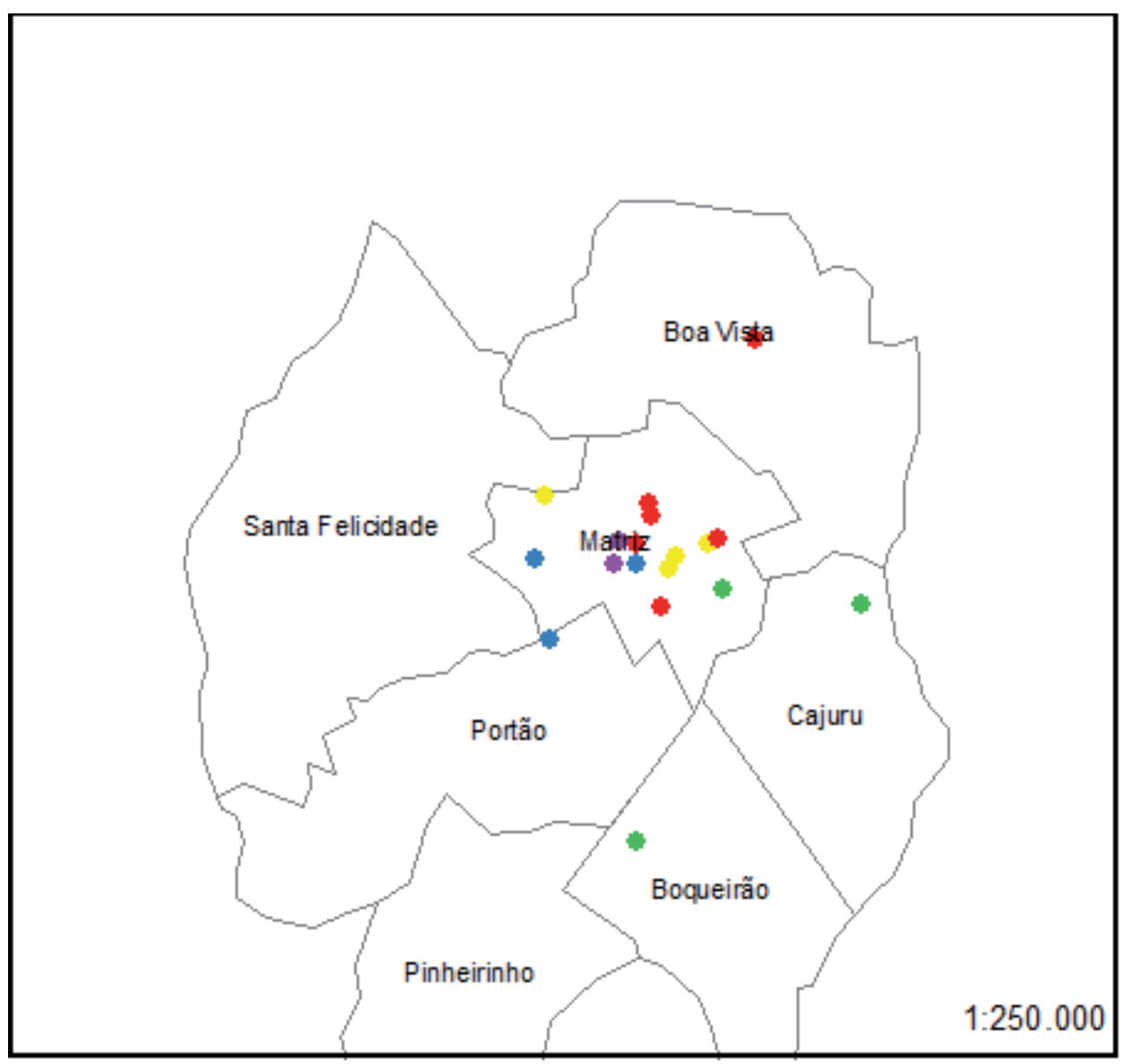

Fig. 6. No generalized data for 1:250.000 scale 
The result of generalization of the program shows that new displacements were implemented and applied to the circles ( 2 purple, 1 red and 1 blue) in the center of the illustration. The generalized representation can be seen in Figure 7. The actors who once seemed to represent the four points of a rectangle, were reconfigured to form a diagonal line.

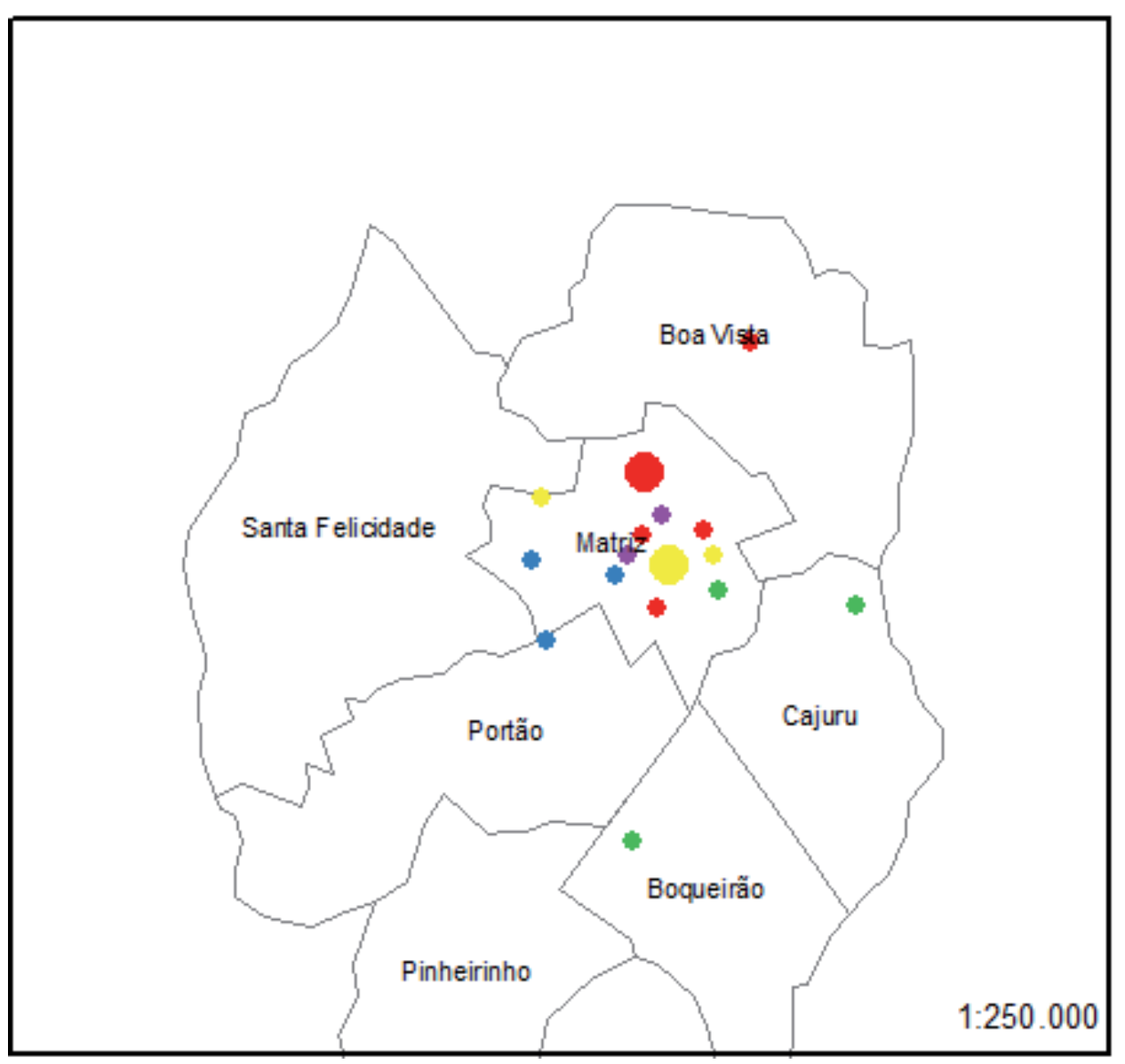

Fig. 7. Generalized data for 1:250.000 scale 
In Figure 8 presents the results obtained by the generalization made in the manual work of Pombo(2009). This process also ran avoiding overlaps with place names and sought a greater spread between the symbols for the addition of place names to social actors. The author was concerned with the relative orientation of the features specific to each other and it can be seen in Figure 7 that this was maintained in most situations. The biggest difference is between the two generalizations involving circles in purple. In automatic generalization between them there was a displacement because there were other symbols that preserved the position of the features. In the manual there was aggregation of these symbols in order to maintain the relative orientation between them.

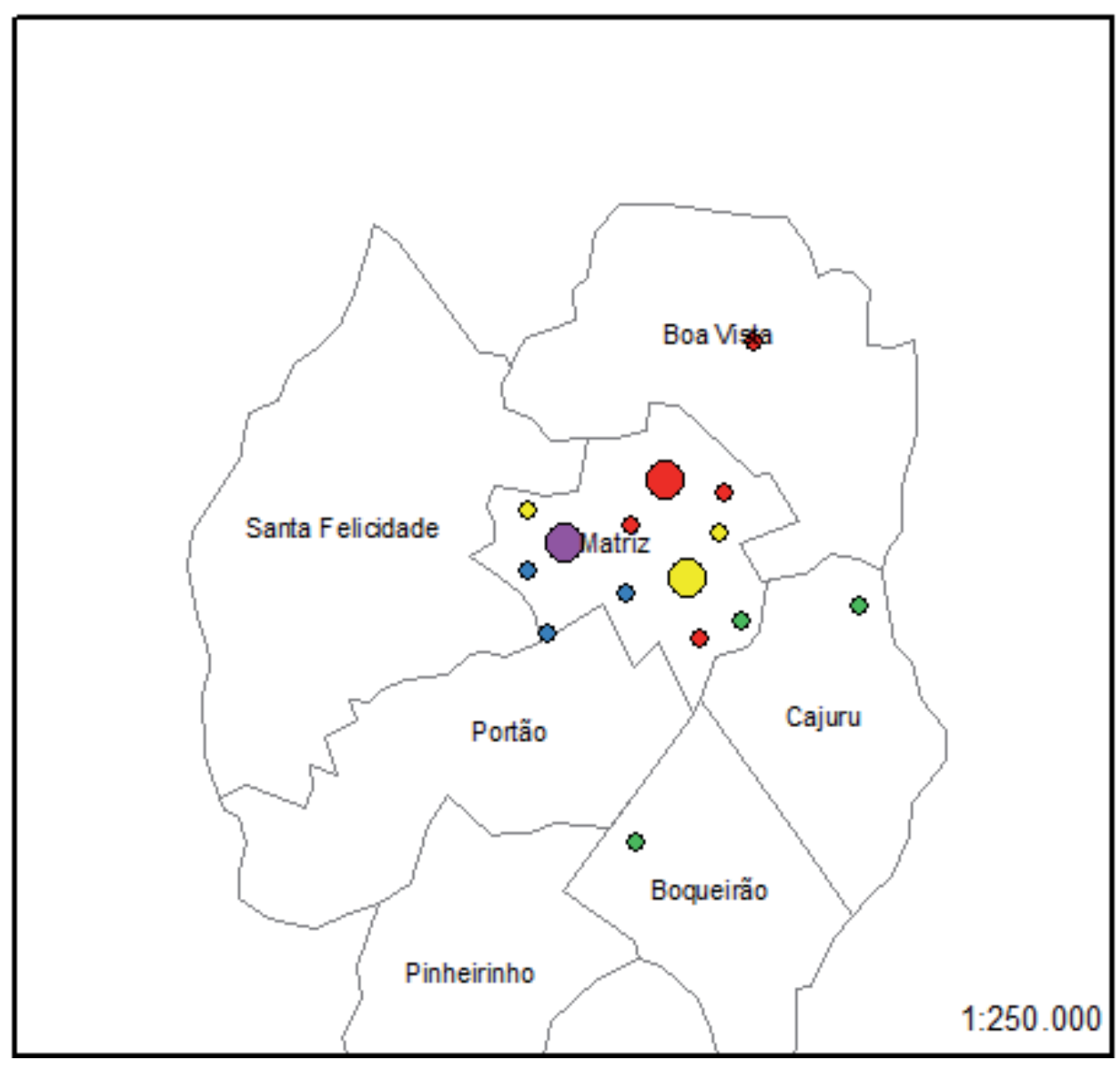

Fig. 8. Manual generalized data for 1:250.000 scale 
In the next figures 9, 10 11, 12,13 and 14, after the generalization, we can see how the generalized maps are applied to the city of Curitiba. On the left is the map with scale reduction without generalization and on the right the results of this research. In Figure 15 we have the result for the Parana state. And, finally, in the illustration in the Figure 16, we have the result for Brazil. These last two results are about the social network for social assistance, because is the only network in Curitiba that has relationship with institutions, that are not located in the same city.

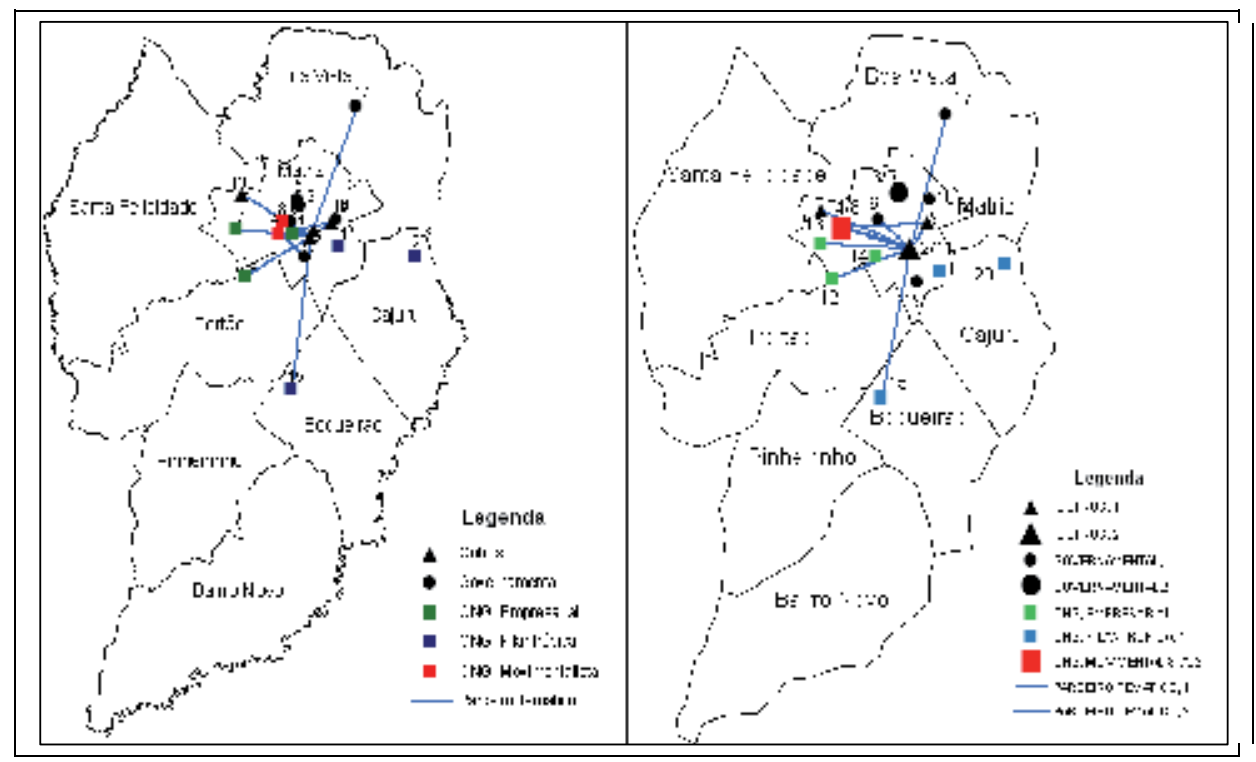

Fig. 9. Social Network for health

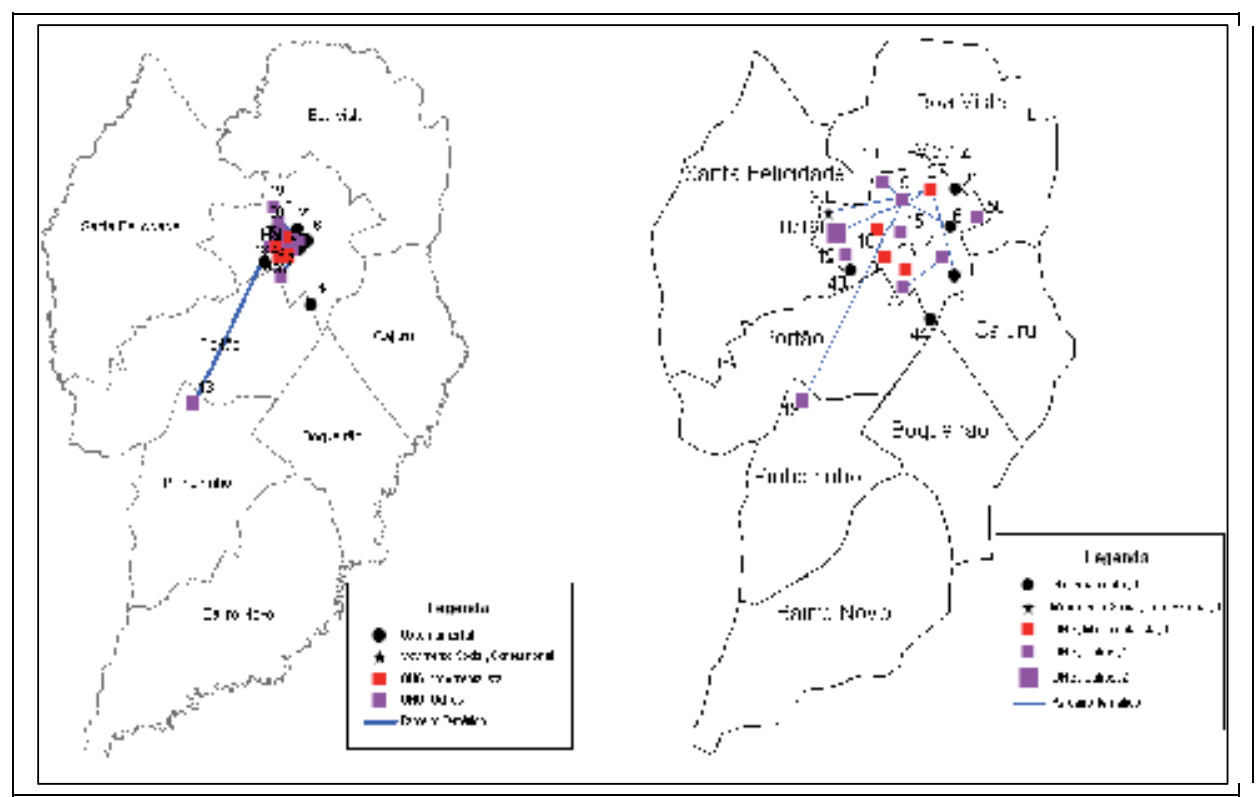

Fig. 10. Social Network for labor 


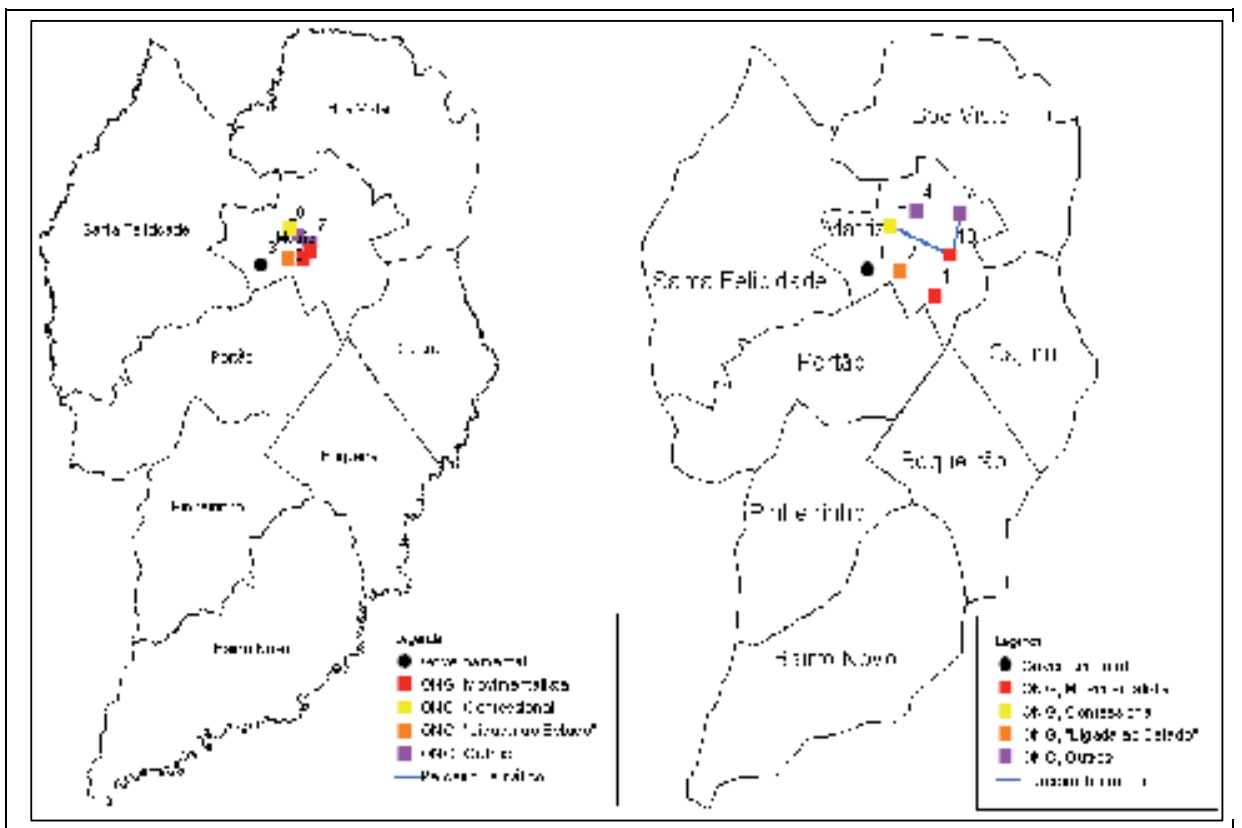

Fig. 11. Social Network for dwellling

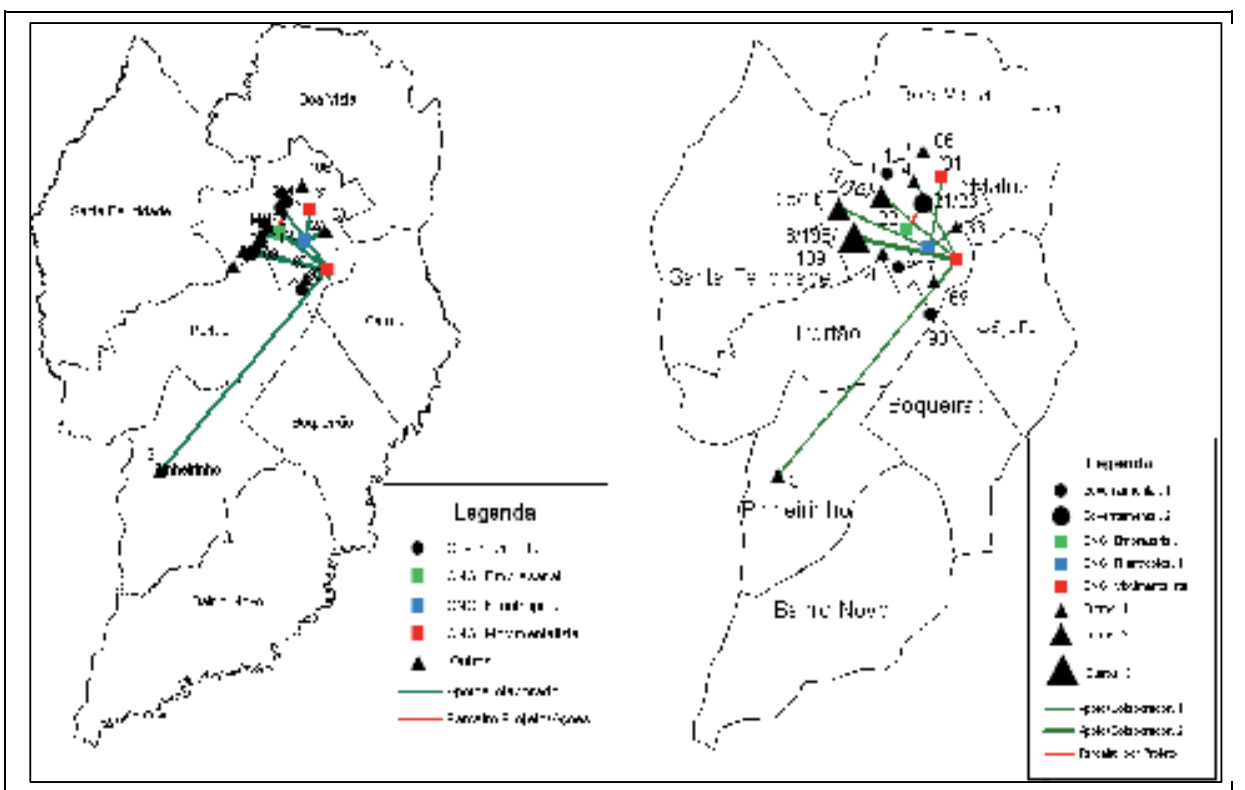

Fig. 12. Social Network for education 


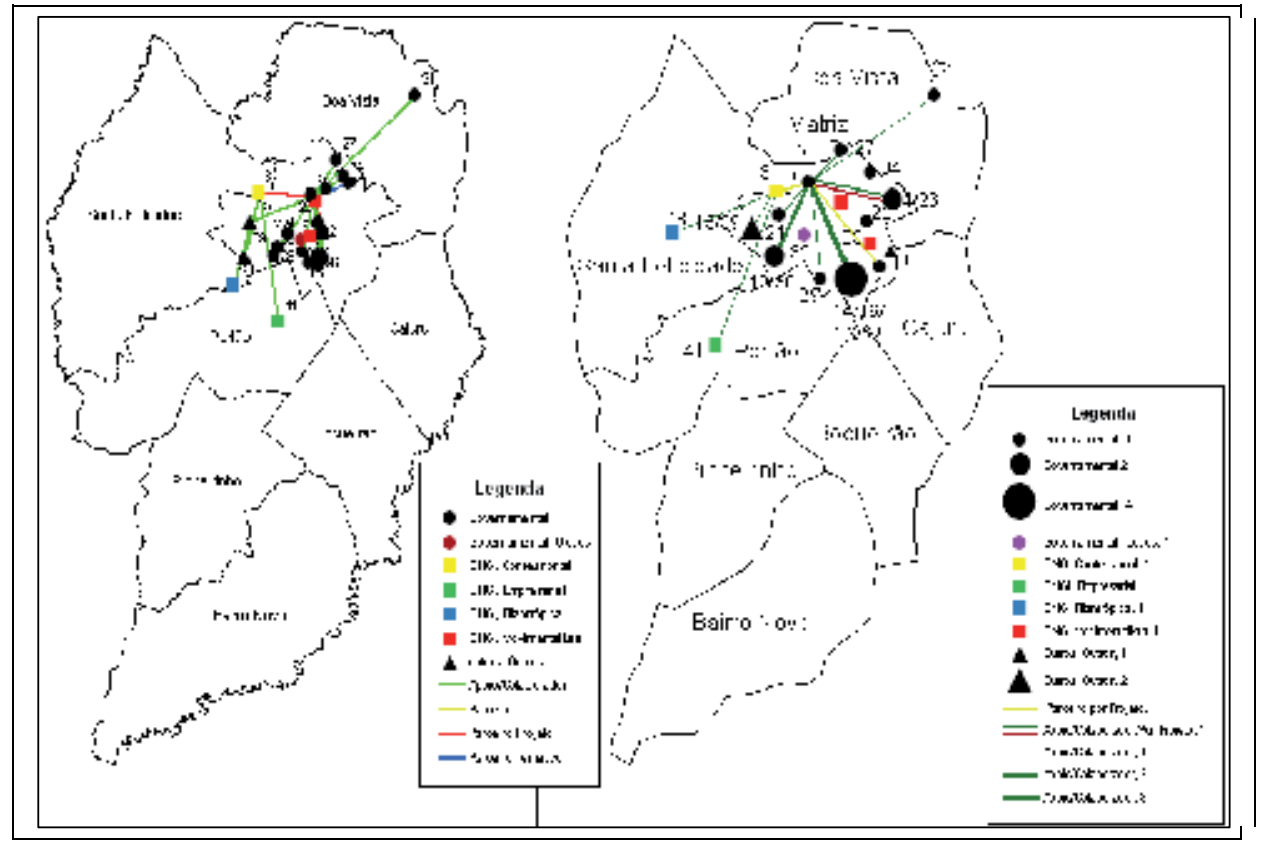

Fig. 13. Social Network for nutrition

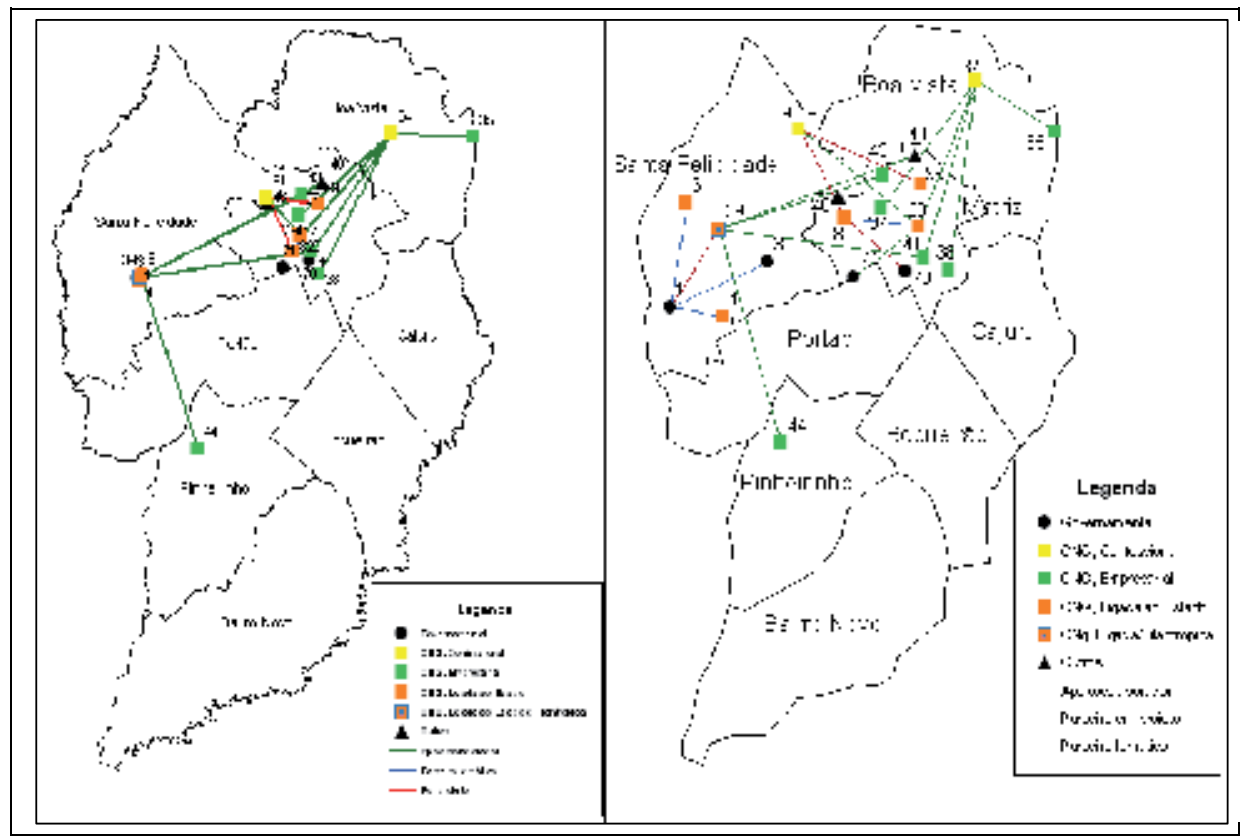

Fig. 14. Social Network for social assistence 


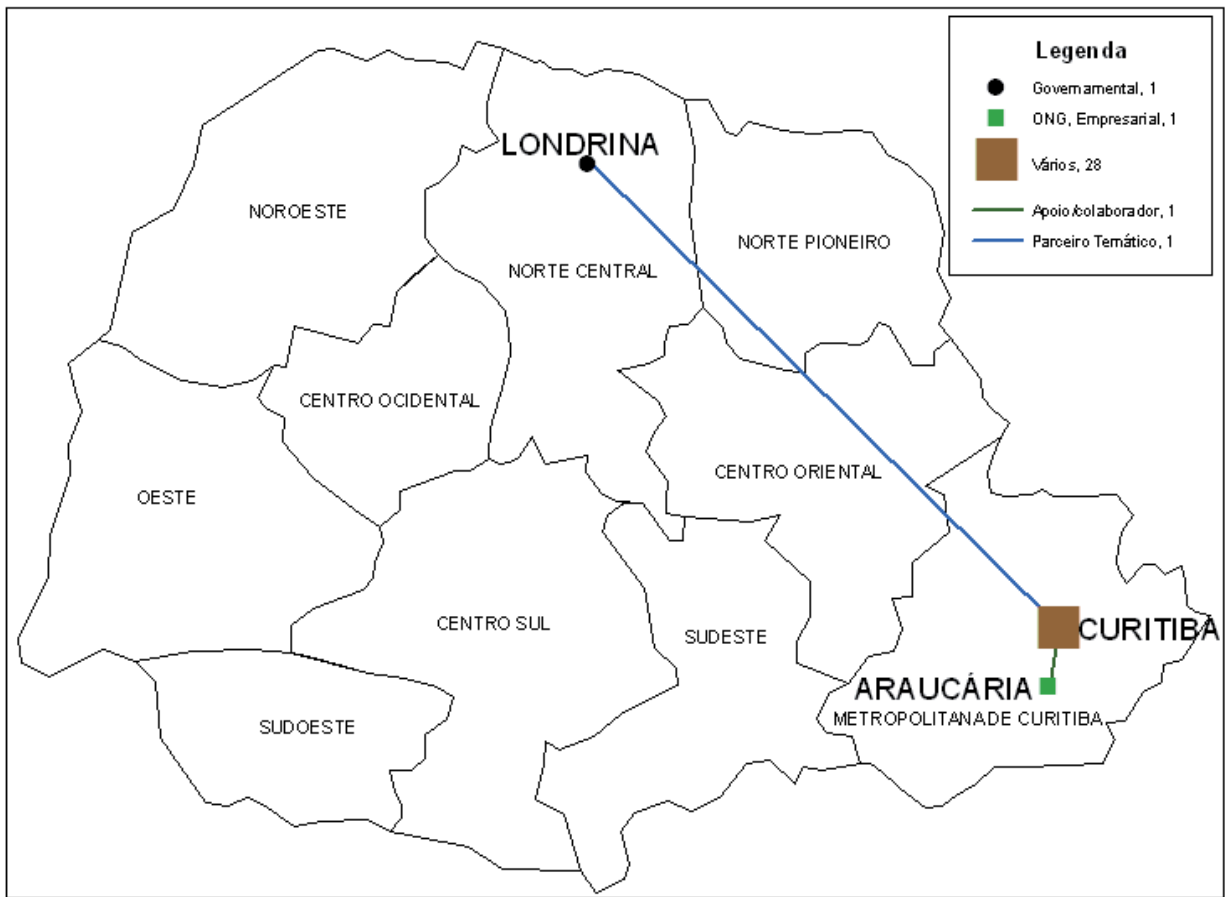

Fig. 15. Social Network for social assistence in Parana

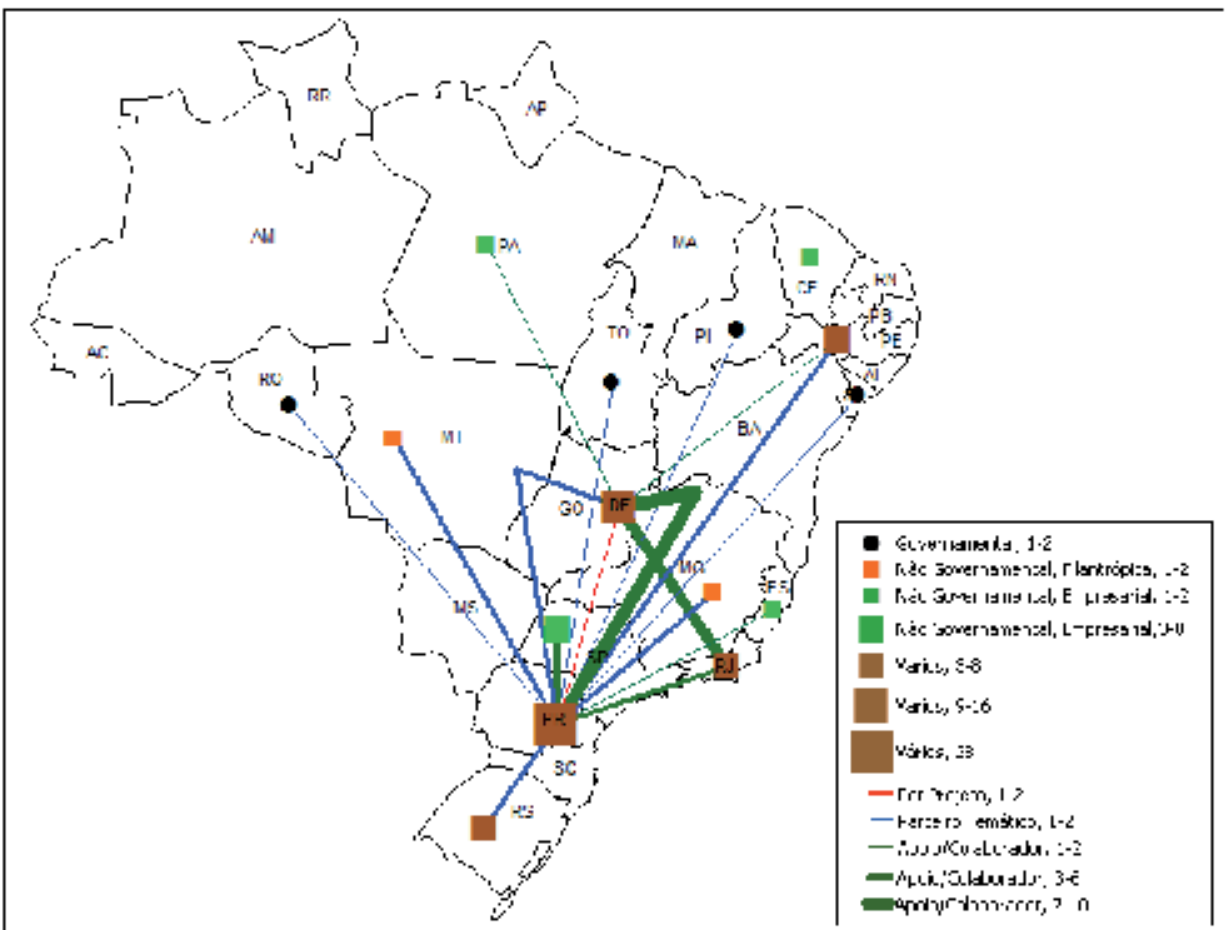

Fig. 16. Social Network for social assistence in Brazil 


\section{Conclusion}

This work aimed to contribute to the stdy of the process of cartographic generalization by automating the aggregation and displacement operators applied to specific features. This is the continuation of research that has as main objective to evaluate the contributions of cartography for the representation of social networks.

When symbols were used with radii of $1 \mathrm{~mm}$, the generalized representations were effective, because even after geometric transformations, the nodes have not lost their spatial reference, or even when displaced, remained in their original neighborhoods. This result exceeded original expectations, because the level of analysis takes place by regional (group of districts) would not be considered a problem if the actors change their position in the new neighborhoods, while still belonging to the same region.

The symbols aggregates, formed by two or more social actors, were represented by a variation in size. Therefore, we have to aplly displacement and make aggregations with the symbols because of the new conflicts caused by the bigger size of circles.

There is no single solution when the displacement operator is applied due to its random nature not always resulting generalizations have the same results. This is because in each use of the software, the algorithm picks which actor will be moved and its direction. However for the purposes of analysis, the conclusions are independent of small movements, provided there are no sudden changes in spatial reference. Compared to the manual generalization, results were quite similar.

To improve the software, it is recommended to implement restrictions on the location of the symbols, for example, create barriers for the displaced points do not exceed certain limits in order to preserve the spatial reference.

This work contribute to make easier the geografic analysis about social networks and the position of their actors. The cartographic generalization supports many scales of visualization of the social phenomena. Finally, when we have this processes in a automatic way, the analysts can make your own maps without a cartographer.

\section{References}

Delazari, L., Sluter, C., Kauchakje, S. Visualização Cartográfica Aplicada à Análise de Redes Sociais. V Colóquio Brasileiro de Ciências Geodésicas, p 538-542, 2007.Li, B.; Xu, Y. \& Choi, J. (1996). Applying

Firkowski, H. Generalização Cartográfica de Grades Retangulares Regulares Baseada na Teoria Matemática da Comunicação. Curitiba, 2002.Tese (Doutorado em Ciências Geodésicas) - Universidade Federal do Paraná.

Granovetter, M. The strength of weak ties: a network theory revisited. In: MARSDEN, Peter V.; LIN, Nan (Eds.). Social structure and network analysis. Beverly Hills: Sage, 1982. Cap.5, p.105-130.

Hanneman, R. Riddle M. Introduction to social network methods. Sociology Departament. 2001. $<$ http://faculty.ucr.edu/ hanneman/ >. 
Hardy, P. Map Production From An Active Object Database, Using Dynamic Representation and Automated Generalization. Laser-Scan Ltd, Science Park, Milton Road, Cambridge, CB4 4FY, UK, 1999.

Marchis, C. Proposição de projeto cartográfico para representação de redes sociais. Curitiba, 2008. Dissertação de Mestrado - Universidade Federal do Paraná.

Matheus, R. F.; Silva, A. B. O. Análise de rede social como método para a ciência da informação. Revista de Ciência da Informação, vol. 7, n. 2, 2006.

<http://www.netic.com.br/redeci/images/stories/docs/ars.pdf>

Pombo, R. M. Generalização Cartográfica Aplicada à Representação e Análise das Redes Sociais. Curitiba, 2009. Dissertação de Mestrado - Universidade Federal do Paraná.

Tomaél, M. I.; Marteleto, R. M. Redes Sociais: posições dos atores no fluxo da informação. Encontros Bibli., Florianópolis, n.esp., 1. sem. 2006.UCINET. Disponível em $<$ http://www.analytictech.com/downloaduc6.htm>. 


\title{
Open-Source Tools for Data Mining in Social Science
}

\author{
Paško Konjevoda and Nikola Štambuk \\ Ruđer Bošković Institute \\ Croatia
}

\section{Introduction}

Data mining can be defined as the application of machine learning algorithms (Mitchell, 1997) for semiautomatic or automatic extraction of information from data stored in databases (Chakrabarti et al., 2009; Witten et al., 2011). The goal of data mining is to extract knowledge from the data set in human-understandable structures. In recent years data mining has been used widely in the areas of science and engineering, such as bioinformatics, genetics, medicine, education and engineering. Textbooks on data analysis in social sciences largely deal with classical statistical methods, while data mining is usually mentioned only briefly (Moore, 2010). For example, in most of those books, the methods for solving the classification problem are separated from the analysis of data structure. Discriminant analysis and logistic regression are the most common classification methods found in social science textbooks, with PCA and factor analysis as representatives of the methods for analysis of data structures (Foster et al., 2006; Harlow, 2005; Tabachnick \& Fidell, 2007). Some data mining techniques, like classification trees, allow simultaneous analysis of classification and data structure, and are far easier to interpret than discriminant, PCA or factor types of analyses (Rokach \& Malmon, 2008; Zhang \& Singer, 2010). We believe that it is not appropriate to separate statistics and data mining, because those methodologies are complementary to each other. Fortunately, several good textbooks that successfully combine statistics and data mining have been published in recent years (Larose, 2005, 2006; Myatt, 2007, 2009). Commercial applications for data mining are very expensive, and as such inaccessible to many institutions and students. One of the solutions is to use open-source programs that allow high-quality statistical and data mining software to be available to all students and researchers (Janert, 2011). The reason for using open-source software is not just commercial. Many open-source programs have such a quick development cycle that commercial software can not compete with. A classic example is $\mathrm{R}$ ( $\mathrm{R}$ Development Core Team, 2011) which has more than 50,000 procedures for analysis and visualisation of data. Unable to follow the development of $\mathrm{R}$, many commercial vendors of statistical software have added the option of calling $\mathrm{R}$ from their products. Some examples are SAS JMP, SPSS, STATISTICA, Genstat, etc. Therefore, we can say that R has become the gold standard for data analysis and visualization. Furthermore, the use of open-source software enables standardization and reproducibility of studies. This problem is particularly pronounced in numerical analysis, when the coefficients of the options within the program can significantly 
affect the results and conclusions (Štambuk \& Konjevoda, 2011). The purpose of this paper is to describe the open-source data mining programs that the authors have found useful in their work (Štambuk et al., 2007a, 2007b). Advantages and disadvantages of those programs are described, the web addresses where they can be found are listed, and the most relevant textbooks and manuals that describe how to work with these programs are cited.

\section{2. $\mathbf{R}$}

$\mathrm{R}$ is an open-source programming language and software environment for statistical computing and data visualization ( $R$ Development Core Team, 2011). The $R$ project was started in 1995 by a group of statisticians at the University of Auckland, and has continued to grow ever since. It is named after the first initials of the first two $\mathrm{R}$ authors (Robert Gentleman and Ross Ihaka). Academic researchers in various fields of applied statistics have adopted $\mathrm{R}$ for statistical software development and data analysis. It has become a de facto standard among statisticians for developing statistical software. There are a lot of niches in terms of $\mathrm{R}$ users, including: environmental statistics, econometrics, medical and public health applications, bioinformatics, and social sciences (Hilbe, 2010; Vinod, 2010) among others. Pre-compiled binary versions of $\mathrm{R}$ are provided for various operating systems on the address: http://cran.r-project.org/ ( $R, 2011)$. $R$ is well documented, and free $R$ Journal (2-3 issues per year) is also available on the same web address. Two main reasons for the worldwide success of $R$ are its extensibility and superb data visualization (Fig. 1, Fig. 2). There are more than 2500 packages which enormously extend functionality of R. However, it is not easy for beginners (and even advanced users) to manage a huge number of procedures (over $50,000)$ that packages contain. A significant problem presents a command-line orientation of R. There are some graphical user interfaces under development. However, with the exception of Rattle (Fig. 3) none of them currently have the maturity and reachiness of menu-driven functionality associated with commercial statistical and data mining software.

\subsection{Recommended R books}

There are many books on R. Unfortunately, the vast majority of those books are too difficult for beginners, and it often happens that students completely withdraw from $\mathrm{R}$ after reading too advanced textbooks. Therefore, we cite the manuals which are simple and useful for the beginners.

\subsubsection{Introductory books}

1. $R$ in action: data analysis and graphics with $R$ (Kabacoff, 2011). If you were to read only one book on R, it should be this one. It is a little masterpiece of pedagogy and clarity of writing, and deserves a detailed study.

2. A beginner's guide to $R$ (Zuur et al., 2009) explains technical details of working with $R$. Therefore, it is oriented on users who already know statistics, but want to learn $R$.

3. Using $R$ for introductory statistics (Verzani, 2005) teaches introductory statistics and R. It is well written and examples are nice. However, some students find it difficult to master both subjects at the same time.

4. Statistics: an introduction using $R$ (Crowley, 2005) is similar to the previous book (3), but slightly more advanced. 
5. Business analytics for managers (Jank, 2011) is a user-friendly introduction to regression analysis with R. It also explains some of advanced techniques, like multivariate visualization, regression trees, and nonparametric regression.

\subsubsection{R Graphical User Interfaces (R GUI)}

1. $R$ through Excel: A spreadsheet interface for statistics, data analysis, and graphics (Heiberger \& Neuwirth, 2009) describes RExcel (Microsoft Excel add-in). It allows access to the $R$ from within Excel. Detailed information about RExcel available at the web page: http:/ / rcom.univie.ac.at/ (RExcel, 2011).

2. Getting started with Rstudio (Verzani, 2011). This short book (75 pages) explains how to use RStudio, an integrated development environment (IDE) for R (Fig. 4). It includes a variety of features intended to make working with $\mathrm{R}$ more productive and straightforward. RStudio is available at the page: http:/ / rstudio.org/ (RStudio, 2011).

\subsubsection{Reference books about $R$}

Reference books are must-have for any serious user of R. The $R$ book (Crowley, 2007), A handbook of statistical analysis using $R$ (Everitt, 2010) and $R$ cookbook (Teetor, 2011) are recommended manuals of beginner-intermediate level.

\subsubsection{Data mining books}

1. Data mining with $R$ and Rattle (Williams, 2011). This book describes Rattle (Fig. 3), a tabbased graphical user interface for data mining using $R$ (Williams, 2011). The book is readable and easily written. Rattle runs under GNU/Linux, Macintosh OS X, and MS Windows operating systems. Rattle is freely available at: http://rattle.togaware.com/ (Rattle, 2011). Rattle is probably the most mature R GUI; simple, but powerful.

2. Data mining with $R$ : learning with case studies (Torgo, 2011). The book is more advanced than Data mining with $R$ and Rattle. It is based on examples from ecology, economy and bioinformatics.

\subsubsection{Graphics with $\mathbf{R}$}

1. $R$ Graphics (Murrell, 2006) is a detailed description of using $\mathrm{R}$ for production of publication quality graphs. It is clear and straightforward, but it is not recommended as the first book for beginners.

2. Lattice: Multivariate data visualization with $R$ (Sarkar, 2008) describes the package lattice for multivariate data visualization (Fig. 1.).

3. ggplot2: elegant graphics for data analysis (Wickham, 2009) describes ggplot2 package that simplifies many of the details of creating statistical graphics. Graphs produced with ggplot 2 are both beautiful and meaningful.

\subsubsection{R programming}

A first course in statistical programming with $R$ (Brown \& Murdoch, 2007) is entry-level introduction to programming. No previous knowledge of $R$ is required, but the reader must know statistics and some calculus. 


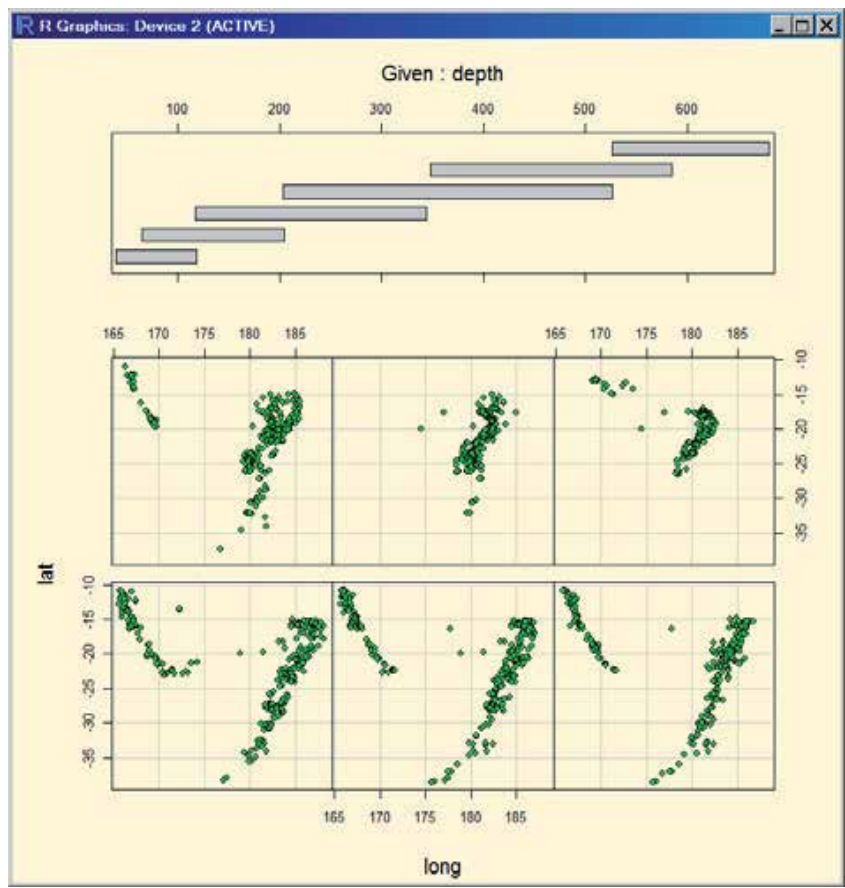

Fig. 1. An example of multivariate data visualization with $\mathrm{R}$. The plot was made using lattice package (Sarkar, 2008).

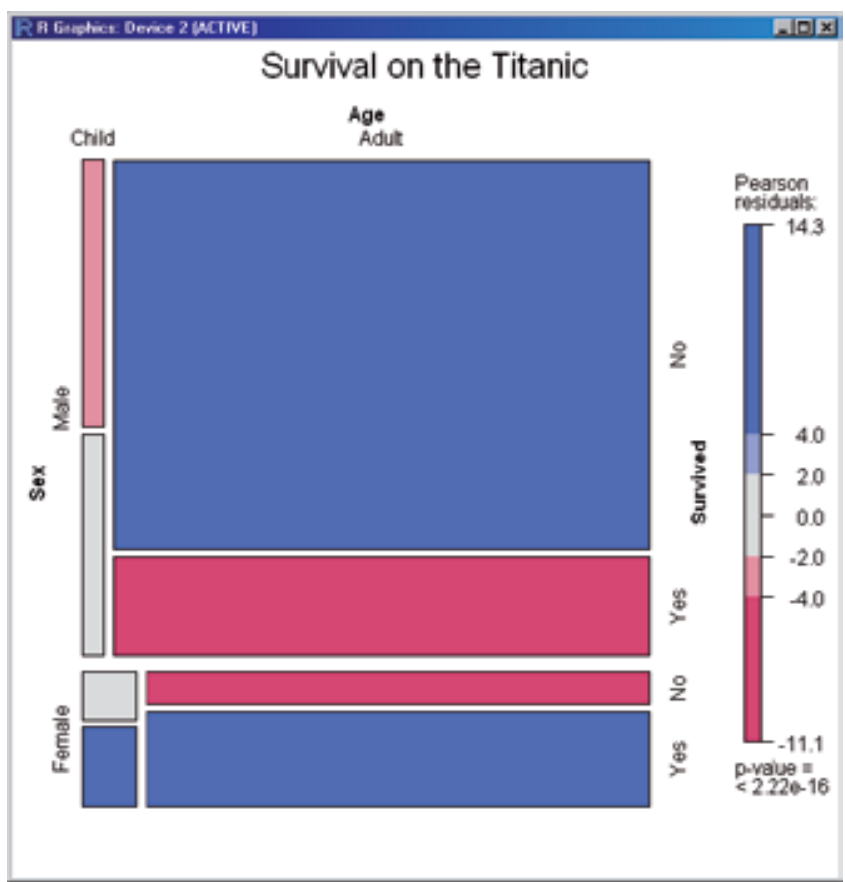

Fig. 2. An example of multivariate visualization of categorical data with $\mathrm{R}$. The plot was made using $v c d$ package. 


\section{WEKA}

WEKA is a collection of machine learning algorithms for data mining tasks, freely available at: http://www.cs.waikato.ac.nz/ml/weka/ (WEKA, 2011). WEKA is Java based software, and works well under Windows, GNU/Linux and Mac Os X operating systems. It contains tools for data preprocessing, classification, regression, clustering, association rules, and visualization (Fig. 5, Fig. 6, Fig. 7). The algorithms can either be applied directly to a dataset, or called from a user's Java code. It is also well-suited for developing new machine learning schemes.

A number of books use WEKA to illustrate principles of data mining (Larose, 2005, 2006; Myatt, 2007, 2009). Two books are especially useful for potential users:

1. Data mining: practical machine learning tools and techniques (Witten et al., 2010). This book, written by creators of WEKA, is now in its third edition, and is a standard reference on WEKA.

2. Data mining techniques and applications: an introduction $(\mathrm{Du}, 2010)$ is a short (about 300 pages) and readable introduction to data mining. All examples are explained using WEKA software.

WEKA is probably the most successful open source data mining software. It has inspired development of other programs with more elaborated graphical user interface and better visualization methods. Two of them are KNIME (Konstanz Information Miner) available from: http://www.knime.org/ (KNIME, 2011), and RapidMiner, available from: http:/ / rapid-i.com/ (RapidMiner, 2011).

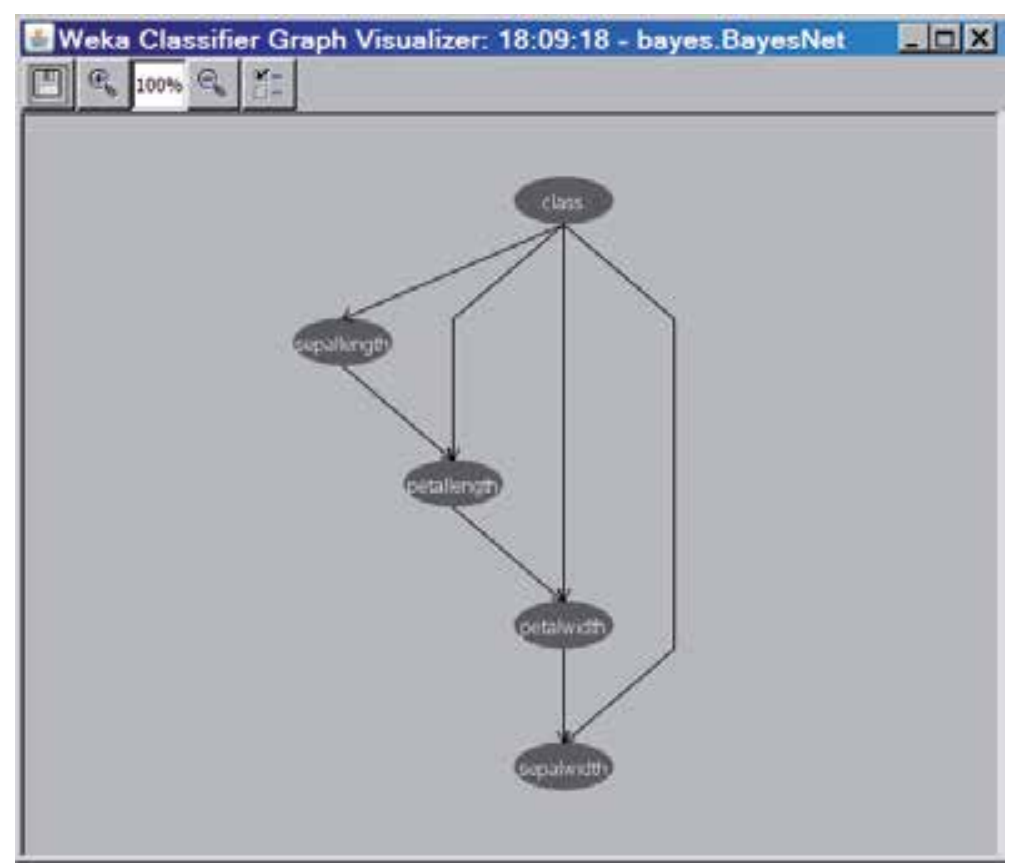

Fig. 5. WEKA implements various Bayesian network classifier learning algorithms. 


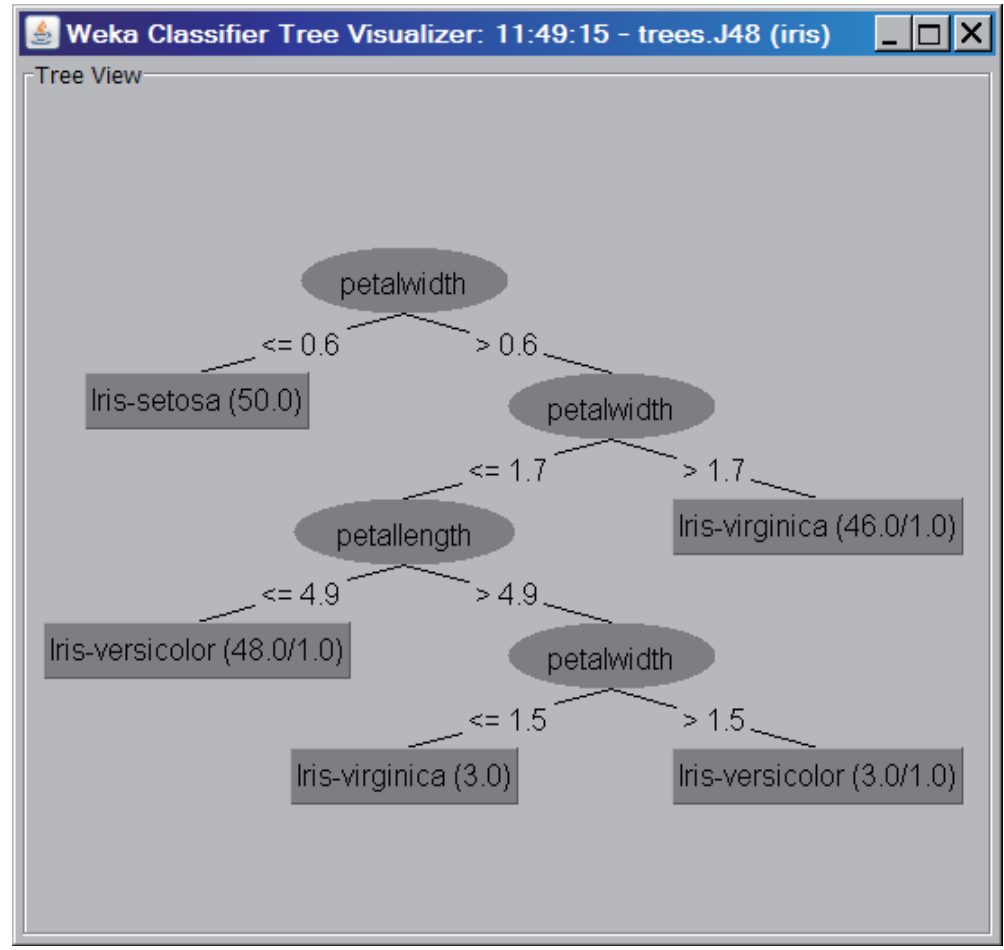

Fig. 6. An example of classification tree constructed with J48 classifier inside WEKA software.

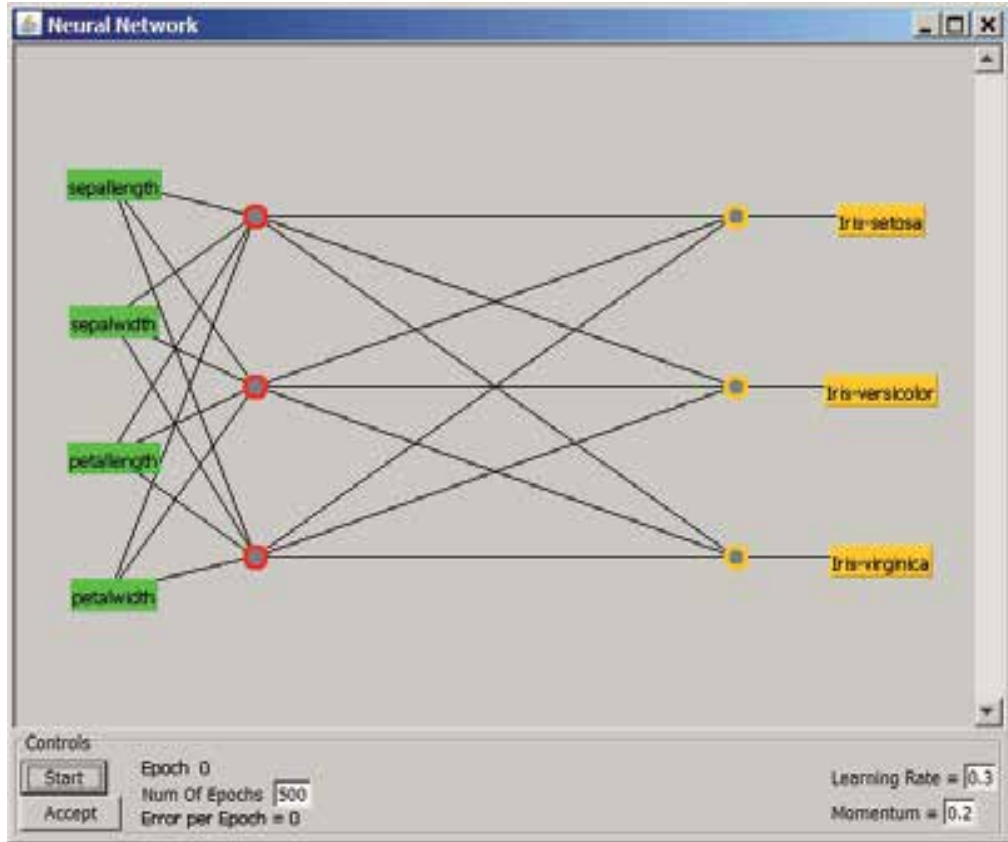

Fig. 7. A graphical representation of an artificial neural network (multilayer perceptron) implemented in WEKA. 


\section{TANAGRA}

TANAGRA (Rakotomalala, 2005) is open-source data analysis software for academic and research purposes which combines data mining techniques with statistical learning (Hastie et al., 2009; Mitchell, 1993; Witten et al., 2010). The program and detailed tutorials are available at: http:/ / eric.univ-lyon2.fr/ ricco/tanagra/en/tanagra.html (TANAGRA, 2011).

TANAGRA is a successor of SIPINA project (SIPINA, 2011) which implements various supervised learning algorithms, especially an interactive and visual construction of decision trees. TANAGRA is more powerful, it contains many supervised learning techniques, but also other paradigms such as clustering, factorial analysis, parametric and nonparametric statistics, association rule, feature selection, etc (Fig. 8). TANAGRA works under Windows operating systems and GNU/Linux if Wine is used.

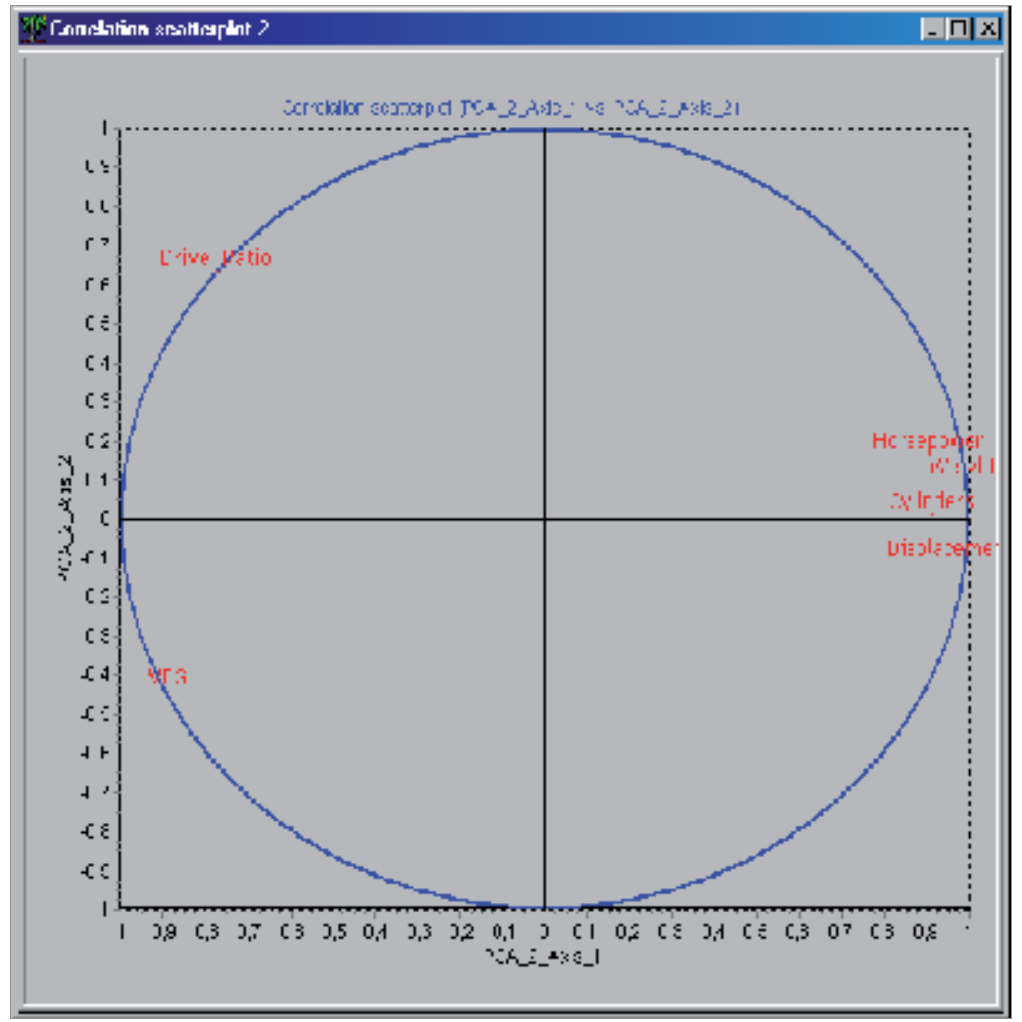

Fig. 8. Knowledge extraction with TANAGRA using combination of PCA and HAC 


\section{ORANGE}

ORANGE is open-source data mining and visualization software. It is freely available from: http://orange.biolab.si/ (ORANGE, 2011). It enables design of data analysis process through user friendly visual programming (Fig. 9). ORANGE contains different visualizations, from scatter plots, bar charts, trees, to dendrograms, networks, and heatmaps (Fig. 10). Most major algorithms for data mining are represented. It works under Window, Mac Os X and GNU/Linux operating systems. Orange is integrated within Python, so this programming language can be used as a scripting language for repetitive tasks.

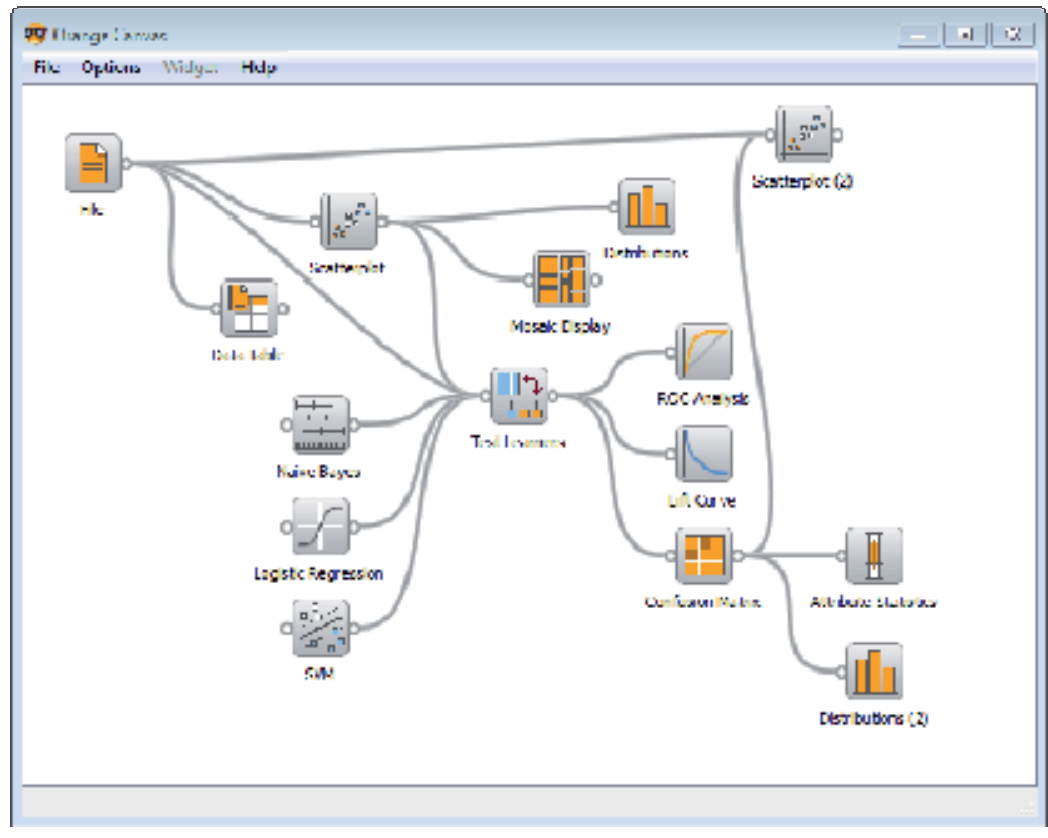

Fig. 9. ORANGE enables visual programming of data analysis process. It remembers user's choices, suggests most frequently used combinations, and intelligently chooses which communication channels to use.

\section{PAST}

The final application in our selection of data mining software is PAST (Hammer et al., 2001). PAST is freeware (not open-source), but we think it's a good replacement for R, especially for users who dislike a command line. Originally, it was aimed at paleontology, but now is also popular in many other fields. It includes common statistical, plotting and modeling functions. 


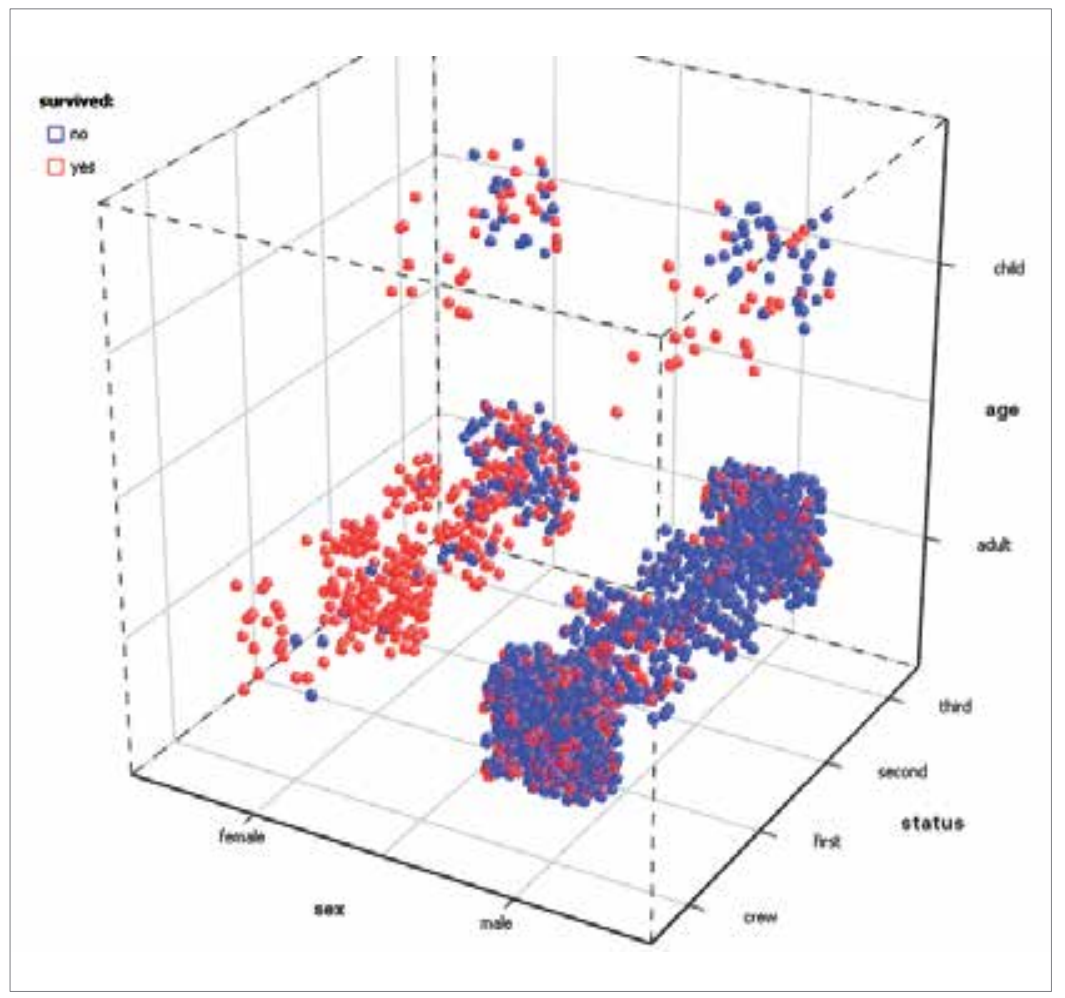

Fig. 10. ORANGE contains different visualization methods: from scatter plots, bar charts, trees, to dendrograms, networks, and heatmaps.

PAST is available on the following web address: http:// folk.uio.no/ohammer/past/ (PAST, 2011). The manual accompanying PAST is detailed, and examples are well explained. The theoretical foundations of algorithms implemented in the PAST are described in the textbook Paleontological data analysis (Hammer \& Harper, 2006).

PAST is a very user-friendly and feature-rich software (Fig. 11), especially remarkable for its large collection of cluster analysis algorithms (Everitt et al., 2011). It also implements a twogroup permutation test, an excellent replacement for Hotelling's T2 test in situations where data do not follow a normal distribution (Good, 2011).

It works under Windows operating systems and GNU/Linux if Wine is used. PAST includes a simple, Pascal-like, scripting (programming) language that allows user to make his own algorithms within the PAST framework. The language has a full matrix support and a library of mathematical, statistical and user-interface functions. 


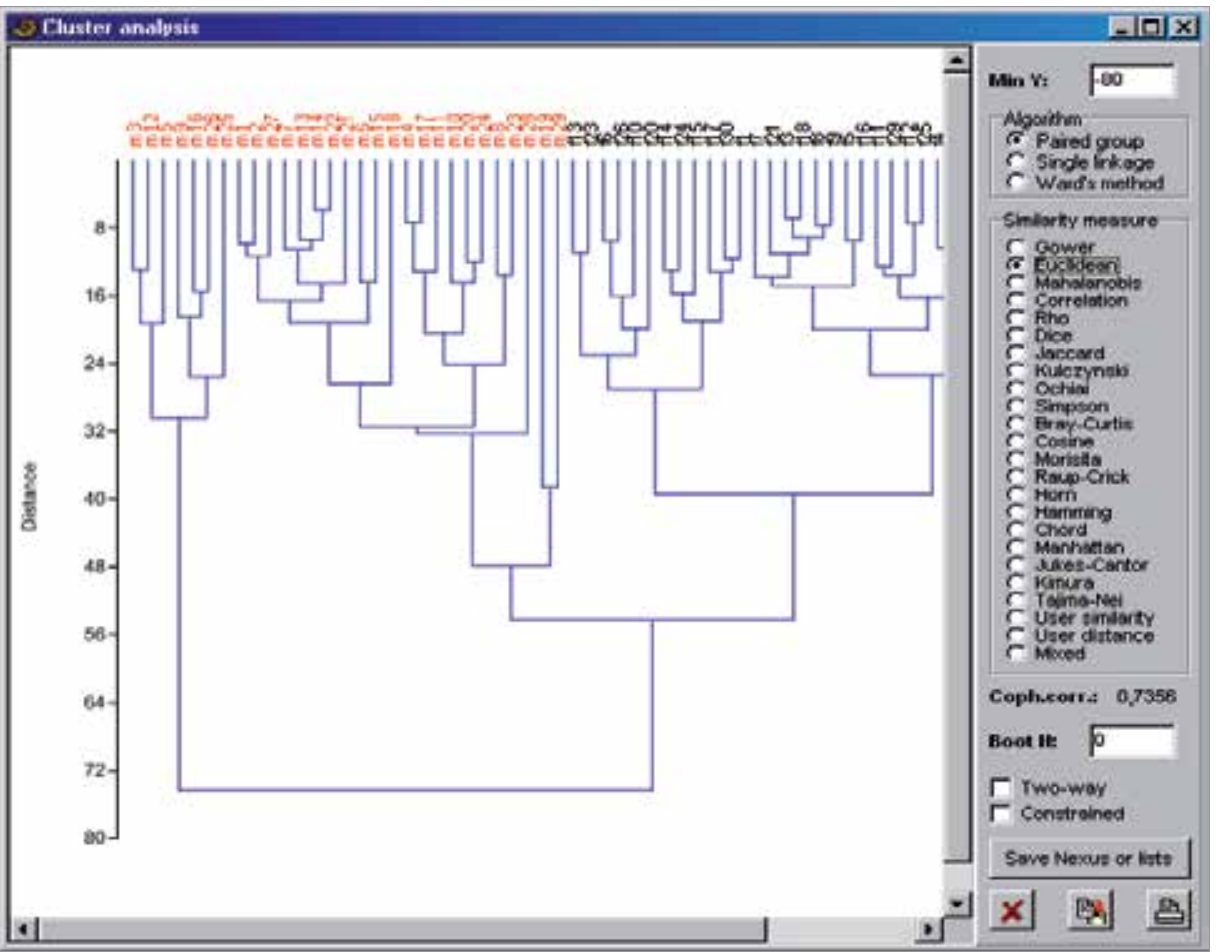

Fig. 11. PAST implements different unsupervised learning methods, including cluster analysis, neighbour joining, and $k$-means clustering.

\section{Conclusion}

Open-source data mining software is completely comparable to commercial programs according to the criteria of functionality and reliability. Moreover, some of them, such as $\mathrm{R}$ and Weka, have become the gold standard for industrial applications. According to Rexer's Annual Data Miner Survey in 2010, R has become the data mining tool used by more data miners (43\%) than any other (Rexer Analytics, 2010). Therefore, it can be concluded that the use of open source software for educational purposes is completely justified, because students will be able to continue using the same programs after they graduate.

\section{Acknowledgments}

The support of the Croatian Ministry of Science, Education and Sports is gratefully acknowledged (grant No. 098-0982929-2524).

\section{References}

Brown, W.J. \& Murdoch, D.J. (2007). A first course in statistical programming with $R$, Cambridge University Press, ISBN 978-0-521-69424-7, Cambridge

Chakrabarti, S.; Cox, E.; Frank, E.; Güting, R.H.; Han, J.; Jiang, X.; Kamber, M.; Lightstone, S.S.; Nadeau, T.P.; Neapolitan, R.E.; Pyle, D.; Refaat, M.; Schneider, M.; Teorey, T.J. 
\& Witten, I.H. (2009) Data mining: know it all, Elsevier, ISBN 978-0-12-374629-0, Amsterdam

Crowley, M.J. (2005) Statistics: an introduction using R, Wiley, ISBN 0-470-02297-3, Chichester

Crowley, M.J. (2007) The R book, Wiley, ISBN-13 978-0-470-51024-7, Chichester

$\mathrm{Du}, \mathrm{H}$. (2010). Data mining techniques and applications: an introduction, Cengage Learning EMEA, ISBN 978-1-84480-891-5, Andover

Everitt, B.S.; Landau, S.; Leese, M. \& Stahl, D. (2011). Cluster analysis, Wiley, ISBN 978-0-47074991-3, Hoboken

Everitt, B.S. \& Horthorn, T. (2010). A handbook of statistical analysis using R, CRC Press, ISBN 978-1-4200-7933-3, Boca Raton

Foster, J; Barkus, E. \& Yavorsky, C. (2006). Understanding and using advanced statistics, Sage, ISBN 1-4129-0013-1, London

Good, P.I. (2011). Analyzing the large number of variables in biomedical and satellite imagery, Wiley, ISBN 978-0-470-92714-4, Hoboken

Hammer, Ø., Harper, D.A.T., \& Ryan, P.D. (2001). PAST: Paleontological statistics software package for education and data analysis. Palaeontologia Electronica, 4, 1, June 2001, pp. 1-9, ISSN 1094-8074.

Hammer, Ø., Harper, D.A.T. (2006). Paleontological dana analysis, Blackwell, ISBN $978-$ 14051-1544-5, Malden

Harlow, L.L. (2005). The essence of multivariate thinking: basic themes and methods, Lawrence Erlbaum, ISBN 0-8058-3730-2, Mahwah

Hastie, T.; Tibshirani, R. \& Friedman, J. (2009). The elements of statistical learning: data mining, inference, and prediction, Springer, ISBN 0387848576, Dordrecht

Heiberger, R.M. \& Neuwirth, E. (2009). R through Excel: A spreadsheet interface for statistics, data analysis, and graphics, Springer, ISBN 978-1-4419-0051-7, Dordrecht

Hilbe, J. (2010). Statistical software for social sciences, In: Statistics in the social sciences: current methodological developments, Kolenikov, S., Steinley, D. \& Thombs, L. (ed.), pp. 175-190, Wiley, ISBN 978-0-470-14874-7, Hoboken

Janert, P.K. (2011). Data analysis with open source tools, O'Reilly, ISBN 978-0-596-80235-6, Beijing

Jank, W. (2011) Business analytics for managers, Springer, ISBN 978-1-4614-0405-7, New York

Kabacoff, R.I. (2011) $R$ in action: data analysis and graphics with $R$, Manning, ISBN: 9781935182399, Shelter Island

KNIME (Date of access 19 October, 2011), Available from: <http://www.knime.org/>

Larose, D.T. (2005). Discovering knowledge in data: an introduction to data mining, Wiley, ISBN 0-471-66657-2, Hoboken

Larose, D.T. (2006). Data mining methods and models, Wiley, ISBN 978-0-471-66656-1, Hoboken

Mitchell, T.M. (1997). Machine learning, McGraw-Hill, ISBN 0070428077, New York

Moore, D.S. (2010). The basic practice of statistics, W. H. Freeman and Company, ISBN 978-14292-0121-6, New York

Murrell, P. (2006) R Graphics, CRC Press, ISBN 978-1-58488-486-6, Boca Raton

Myatt, G.J. (2007). Making sense of data: a practical guide to exploratory data analysis and data mining, Wiley, ISBN 978-0-470-07471-8, Hoboken

Myatt, G.J. (2009). Making sense of data II: A practical guide to data visualization, advanced data mining methods, and applications, Wiley, ISBN 978-0-470-22280-5, Hoboken 
ORANGE (Date of access 19 October, 2011), Available from: <http://orange.biolab.si/> PAST (Date of access 19 October, 2011), Available at: <http://folk.uio.no/ohammer/past/> R Development Core Team (2011). R: A language and environment for statistical computing. R Foundation for Statistical Computing, Vienna, Austria. ISBN 3-900051-07-0, URL http://www.R-project.org/.

R (Date of access 19 October, 2011), Available at: <: http://cran.r-project.org/>

Rakotomalala, R. (2005). TANAGRA: a free software for research and academic purposes, Proceedings of European Grid Conference 2005, RNTI-E-3, vol. 2, pp. 697-702, Amsterdam, February 2005

RapidMiner (Date of access October 19, 2011), Available from: <http://rapid-i.com/> Rattle (Date of access October 19, 2011), Available from: <http:// rattle.togaware.com/> RExcel (Date of access October 19, 2011), Available from: <http://rcom.univie.ac.at/> Rexer Analytics (Date of access 19 October, 2011), Available at: http:/ / www.rexeranalytics.com/Data-Miner-Survey-Results-2010.html

Rokach, J. \& Malmon, O. (2008). Data mining with decision trees: theory and applications, World scientific, ISBN 978-981-277-171-1, Singapore

RStudio (Date of access October 19, 2011), Available from: <http:/ / rstudio.org/>

Sarkar, D. (2008) Lattice: Multivariate data visualization with $R$, Springer, ISBN 978-0-38775968-5, New York

SIPINA (Date of access October 19, 2011), Available from: http://eric.univlyon2.fr/ ricco/sipina.html

Štambuk, N. \& Konjevoda P. (2011). The role of independent test set in modeling of protein folding kinetics, In: Software tools and algorithms for biological systems (Book Series: Advances in Experimental Medicine and Biology, Volume: 696), Arabnia, H.R. \& Tran, Q.N. (ed.), pp. 279-284, Springer, ISBN 978-1-4419-7045-9, New York

Štambuk, A; Štambuk, N. \& Konjevoda, P. (2007) Application of Kohonen Self-Organizing Maps (SOM) based clustering for the assessment of religious motivation, Proceedings of the ITI 2007, 29th Int. Conf. on Information Technology Interfaces, Cavtat, Croatia, June 25-28, 2007, pp. 87-91

Štambuk, A.; Štambuk, N. \& Konjevoda, P. (2007) Analysis of Hoge Religious Motivation Scale by Means of Combined HAC and PCA Methods, Proceedings of the ITI 2007, 29th Int. Conf. on Information Technology Interfaces, Cavtat, Croatia, June 25-28, 2007, pp. 197-201

Tabachnick, B.G. \& Fidell, F.S. (2007). Using multivariate statistics, Pearson, ISBN 0-205-459382, Boston

TANAGRA (Date of access October 19, 2011), Available from: <http://eric.univlyon2.fr/ ricco/tanagra/en/tanagra.html>

Teetor, P. (2011). R cookbook, O'Reilly, ISBN 978-0-596-80915-7, Beijing

Torgo, L. (2011). Data mining with R: learning with case studies, CRC Press, ISBN 978-1-43981018-7, Boca Raton.

Verzani, J. (2005). Using $R$ for introductory statistics, CRC press, ISBN 1-58488-4509, Boca Raton

Verzani, J. (2011). Getting started with RStudio, O'Reilly, ISBN 978-1-449-30903-9, Beijing

Vinod, H.D. (Ed.) (2010). Advances in social science research using $R$, Springer, ISBN 978-14419-1763-8, New York 
WEKA (Date of access October 19, 2011), Available from: <http:/ / www.cs.waikato.ac.nz/ml/weka/ (Weka, 2011)>

Wickham, H. (2009). ggplot2: elegant graphics for data analysis, Springer, ISBN 978-0-387-981406, Dordrecht

Williams, G. (2011). Data mining with Rattle and R: the art of excavating data for knowledge discovery, Springer, ISBN 978-1-4419-9889-7, New York

Witten, I.H.; Frank, E. \& Hall, M.A. (2010). Data mining: practical machine learning tools and techniques, Morgan Kaufmann, ISBN 978-0-12-374856-0, Amsterdam

Zhang, H \& Singer, B.H. (2010). Recursive partitioning and applications, Springer, ISBN 978-14419-6823-4, Dordrecht

Zuur, A.F.; Ieno, E.N. \& Meesters E.H.W.G. (2009). A beginner's guide to R, Springer, ISBN 978-0-387-93836-3, Dordrecht 


\section{Section 4}

\section{Knowledge Management}





\title{
Applying Social Sciences Research for Public Benefit Using Knowledge Mobilization and Social Media
}

\author{
David J. Phipps ${ }^{1}$, Krista E. Jensen ${ }^{1}$ and J. Gary Myers ${ }^{2}$ \\ ${ }^{1}$ York University \\ ${ }^{2}$ KMbeing.com \\ Canada
}

\section{Introduction}

The social sciences and humanities (SSH) matter. They matter because they help us understand and address wicked problems. Wicked problems are persistent problems about which there is little agreement on solutions. Not all the stakeholders are known, end points are equivocal and when interventions are introduced the problems themselves might change. We can address wicked problems but we have a tough time eradicating them. Wicked problems "occur in a social context; the greater the disagreement among stakeholders, the more wicked the problem. In fact, it's the social complexity of wicked problems as much as their technical difficulties that make them tough to manage" (Camillus $2008,100)$. Wicked problems are therefore social problems. Wicked problems are problems of the social sciences.

All of the "wicked" problems such as poverty, housing, immigration, climate change, security, at risk kids, Aboriginal issues, social determinants of health - to name a few embrace issues related to the social sciences. New solutions that help address these wicked (i.e. social) problems are referred to as social innovations. Definitions of social innovation abound (Cahill 2010), but one of the most elegant is from Louise Pulford formerly of the Young Foundation in the United Kingdom who describes social innovations as "devising new and better ways to tackle social problems" (Policy Horizons Canada 2011). Social innovations can be developed by for-profit or not-for-profit organizations, by individuals (called social innovators, or social entrepreneurs) or by consortia of public, private and notfor-profit organizations. As Geoff Mulgan of the Young Foundation has written, "social change depends...on alliances between what could be called the 'bees' and the 'trees'. The bees are the small organizations, individuals and groups who have the new ideas, and are mobile, quick and able to cross-pollinate. The trees are the big organizations, governments, companies or big NGO s, which are poor at creativity but generally good at implementation, and which have the resilience, roots and scale to make things happen" (Mulgan 2008, 22).

SSH research deepens our understanding of these wicked problems and provides evidence and new knowledge of the actors, drivers, policies, barriers and enablers of wicked problems. But new SSH knowledge that isn't shared cannot contribute to decisions about 
social innovations. This chapter examines how universities can maximize the impact of SSH research for the benefit of society. This chapter examines knowledge mobilization.

Knowledge mobilization is the process of connecting academic SSH research (and other knowledge) to non-academic decision makers so that SSH research informs decisions about public policy and professional practice. As we have written (Phipps and Shapson 2009) knowledge mobilization (the process) enables social innovation (the outcome).

Universities are the main producers of new SSH research knowledge and graduate level talent. Both knowledge and talent have the potential to contribute to decisions about social innovations but neither of them can benefit society if SSH scholars limit themselves to traditional academic paradigms of scholarly communication and dissemination. Digital technologies are creating new opportunities for scholars to disseminate and engage their research beyond traditional academic audiences. Lowry and colleagues (Lowry et al. 2011) recently reviewed digital technologies, scholarly communication and knowledge mobilization. They observed that "it is evident, however, that in university engagement efforts-at York University, University of Victoria, and Memorial University of Newfoundland, among others-digital information sources can augment direct social interaction, although these initiatives are only now starting to emerge out of their pilot phases" (Lowry 2011, 58). Recognizing the need to go beyond traditional scholarship and the creation of new knowledge, Tim Brodhead wrote when he was CEO of the McConnell Family Foundation "the greater challenge now is not to generate new ideas per se but to create systemic supports for a continual process of social innovation in Canada: identifying promising initiatives [and] rigorously testing their efficacy" (Brodhead 2010, 109).

Invigorated engagement of SSH research and connections between the university and society will enable SSH research to have an impact on social innovation by creating a sustainable institutional platform that connects research and expertise beyond the academy for social benefit. Writing in Canadian Government Executive, the President of the Social Sciences and Humanities Research Council of Canada, Chad Gaffield, re-imagined campuscommunity collaborations for social innovation. "An international consensus is now emerging around a people-centered model of innovation for successful change in businesses, government, and communities. ... The new model calls upon us to re-kindle the relationship between knowledge and society; to re-imagine and renew the historic covenant between campuses and the public; and to exploit all the ways of knowing about the past and present to tackle the world's toughest challenges" (Gaffield 2011).

Knowledge mobilization is an emerging institutional research infrastructure that supports these multi-directional connections between academic research and society.

A note on terminology: Many organizations use a diversity of terms to describe what we call knowledge mobilization. There are subtle distinctions between knowledge transfer (KT), knowledge translation (also KT), knowledge exchange (KE), knowledge transfer \& exchange $(\mathrm{KTE})$, knowledge translation and transfer (KTT), knowledge mobilization $(\mathrm{KMb})$, and knowledge integration (KI); however, they are all terms to describe essentially the same process of connecting research to practice/policy. Recently an effort to move away from the terminology recommends the term $\mathrm{K}^{*}$ (K-star) as a solution to those entrenched in their own identities and resistant to other terms. We prefer to use Knowledge Mobilization $(\mathrm{KMb})$. Rather than use this chapter to further the debate, our focus instead will be on our $\mathrm{KMb}$ practice. It is this practice that we describe below. 
One more definition: decision maker. A decision maker, sometimes referred to as a research user or policy-maker, is a person who uses research to make decisions, frequently about public policy or professional practice. Some models of $\mathrm{KMb}$ imagine that researchers and decision makers sit in "two communities" with researchers producing research and decision makers being the receptors for research; however, this model has been shown to be too simplistic as many decision makers also produce research knowledge and all researchers use research to inform decisions about the research process (Davies, Nutley and Walter 2008).

This chapter will review the $\mathrm{KMb}$ literature by reviewing three books that provide a more detailed review of the literature than can ever be done in this chapter. Reflecting on our five years of $\mathrm{KMb}$ practice, our review of these books draws important observations from each body of work and moves to the conclusion that relationships between researchers and decision makers are key elements to the social process of $\mathrm{KMb}$. This chapter will then review Canadian examples of $\mathrm{KMb}$ and will describe efforts underway at universities that are developing an institutional capacity to support $\mathrm{KMb}$. We shall provide detail about the $\mathrm{KMb}$ Unit at our institution, York University (Toronto, Canada) and describe how York and KMbing.com: are experimenting with social media tools to support the social process of knowledge mobilization that connects SSH research to social innovations. We shall conclude with thoughts about future directions for both the study and practice of knowledge mobilization.

\section{Literature review}

Rather than attempting a whole scale review of the literature on diverse terms, structures and efforts underway to connect research to decision makers, we shall review three books published on different aspects of this emerging yet diffuse field:

1. Knowledge Translation in Health Care: moving from evidence to practice (Strauss, Tetroe and Graham 2009) presents the Knowledge to Action Cycle that describes how health evidence moves into health practice.

2. Knowledge Mobilization in the Social Sciences and Humanities: moving from research to action (Bennet and Bennet 2008) reviews different aspects of how social sciences research can engage with decision makers.

3. Using Evidence: how research can inform public services (Nutley, Walter and Davies, 2007) is a detailed and exhaustive review of the literature on research utilization, how research moves into use by practitioners and decision makers for social services and public policies.

These three books were chosen because they provide in depth reviews of the literature in different dimensions of the $\mathrm{KMb}$ process in health, social sciences and research utilization respectively.

In their book, Knowledge Translation in Health Care: moving from evidence to practice, Strauss, Tetroe and Graham examine the Knowledge to Action (KTA) Cycle in detail. The KTA Cycle is derived from planned action theories. The KTA Cycle provides a framework linking knowledge creation to outcomes and sustained knowledge use. The KTA Cycle, first published by Graham and colleagues in 2006, and reproduced with permission, is shown in Figure 1. 


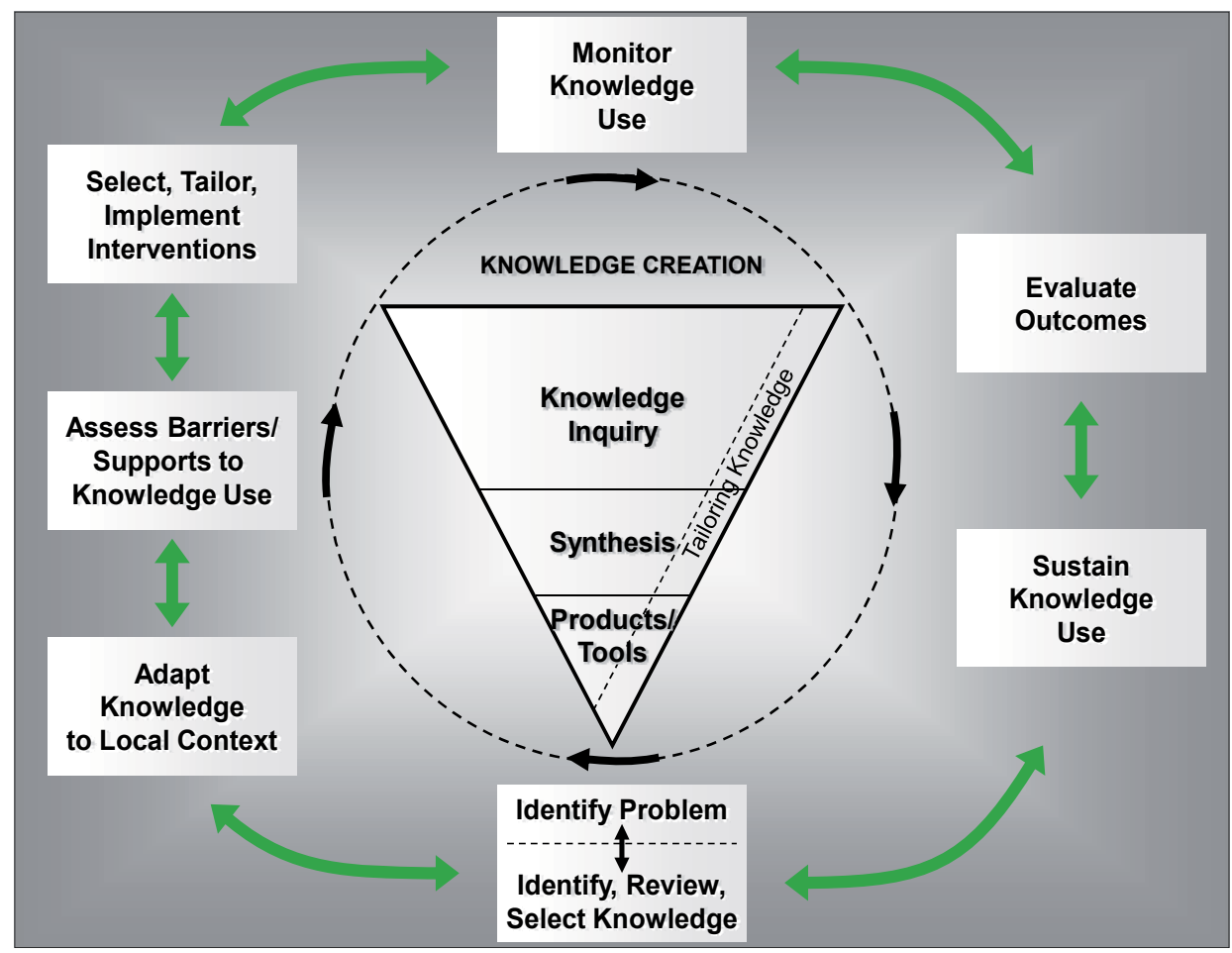

Fig. 1. Knowledge to Action Cycle

The KTA Cycle follows research from knowledge creation to knowledge synthesis (such as systematic reviews) to the production of knowledge products and tools that can be exported out of the knowledge creation space (the funnel at the centre of the model) and into the knowledge utilization space. In the knowledge utilization space (on the outside of the funnel) evidence is adapted, implemented, monitored, evaluated and then its use is sustained. Although presented in a stepwise fashion, the double headed arrows and the cycle around the knowledge "funnel" indicate that this is not intended to imply a linear process. The book dedicates a chapter to each of these steps investigating each in depth. The book provides an excellent review of how evidence derived from health research can be synthesized, packaged and moved into implementation for improved health practices and health policies. Each chapter examining each step in the KTA Cycle considers the barriers and enablers of success. The book is focused on evidence which is its main strength - but also its main limitation. Evidence that has gone through a systematic review resulting in actionable messages is the best evidence to move into implementation (Lavis et al, 2003). Yet by focusing solely on moving existing evidence into practice/policy, Strauss, Tetroe and Graham do not consider the literature that focuses on the relationships by which that evidence is implemented and by which policy makers and practitioners inform knowledge creation. In this book, Michelle Gagnon points out that "involving knowledge users as partners in the research process is a strong predictor that research findings will be used and that the research endeavour will achieve a greater impact" (Gagnon 2009, 240). Clearly in each of the stages described in the KTA Cycle, strong relationships between researchers, practitioners, policy makers and patient advocates will maximize the impact of evidence on policy/practice. 
Looking explicitly at knowledge mobilization in the social sciences and humanities, Alex and David Bennet's book, Knowledge Mobilization in the Social Sciences and Humanities: moving from research to action, focuses more on the relationships that underpin $\mathrm{KMb}$ rather than the evidence that flows as a result of those relationships. They indicate this is especially important for tacit knowledge (Bennet and Bennet 2007, 95). They describe $\mathrm{KMb}$ as "collaborative entanglement." "Collaborative entanglement means to purposely and consistently develop and support approaches and processes that combine the sources of knowledge and the beneficiaries of that knowledge to interactively move toward a common direction such as meeting an identified community need" (Bennet and Bennet 2007, 48). This description evokes the messy ("entanglement") yet social ("collaborative") nature of the relationships that are inherent in $\mathrm{KMb}$ processes. Bennet and Bennet also consider $\mathrm{KMb}$ as a program which is a distinction from much of the literature that looks at individual $\mathrm{KMb}$ interventions. When considering systematic supports for social innovation a systems or programmatic approach is required (Brodhead 2010).

The authors present a complex model of $\mathrm{KMb}$, shown in Figure 2, reproduced with permission.

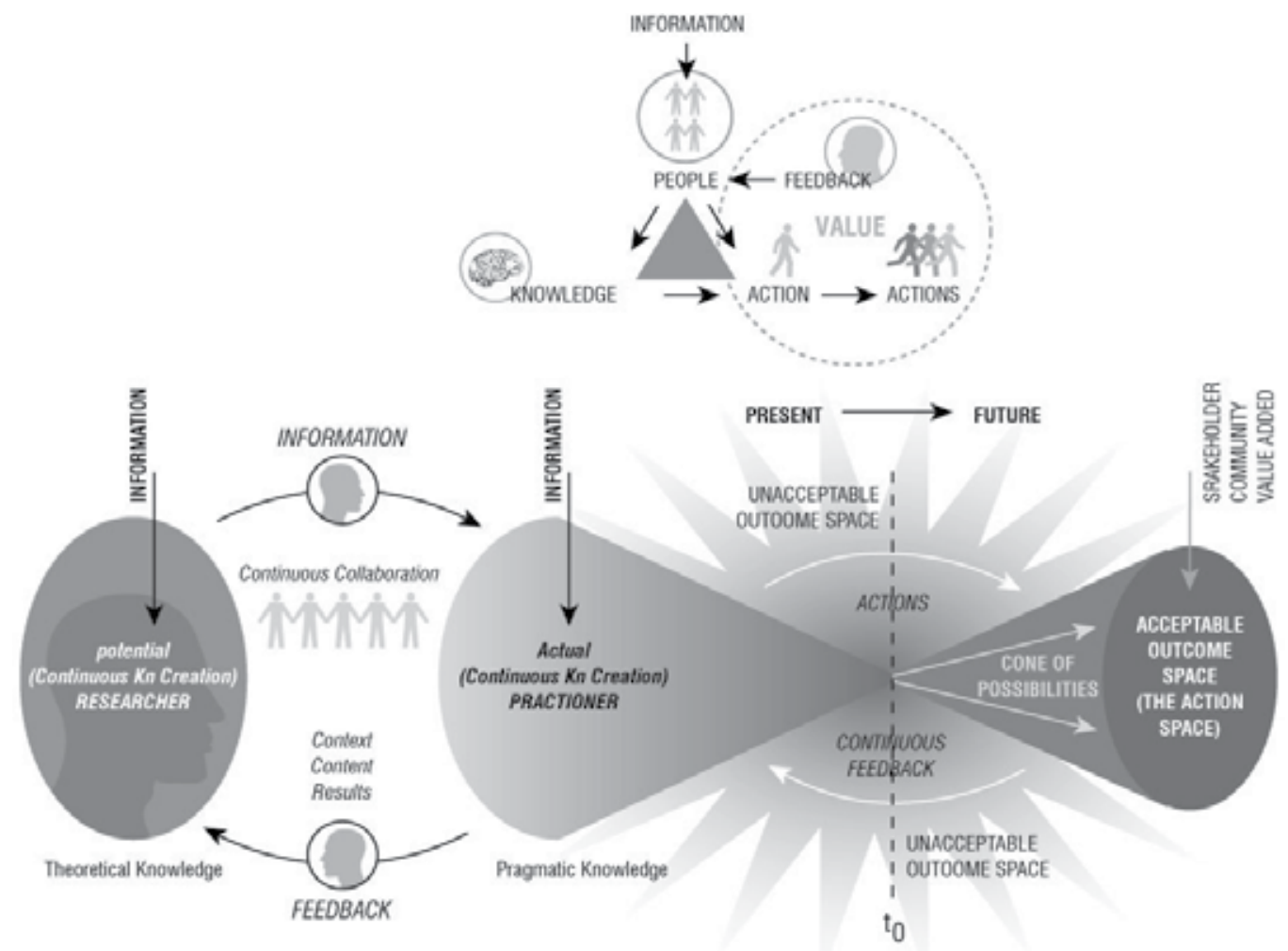

Fig. 2. Knowledge Mobilization - assimilating and applying the right knowledge to solve problems, make decisions and take effective actions.

The main challenge with this model is its complexity and the fact that the arrows are unidirectional, although the diagram's intent as described in the text of the book is to 
illustrate a multidirectional flow of knowledge. That is not to say that $\mathrm{KMb}$ is not complex and multidirectional - it is. But complex models do not aid in understanding.

The main strength of this model is that it moves beyond the transmission/translation of evidence in the Knowledge to Action cycle. Bennet and Bennet describe a process of knowledge flows, and their diagrams support this with pictures of flows between researchers and decision makers. The flows are sometimes mediated by social media. Social media can "enable information and knowledge sharing among stakeholders. These tools can contribute to the development of trust and respect among stakeholders by supporting the creation or expansion of communities of interest, learning, and practice." (Bennet and Bennet 2008, 85)

Knowledge brokers (Lomas 2007; Ward, House and Hamer 2009) also play a role in "both connecting and translating knowledge to end users (Bennet and Bennet 2008, 123). A flow of knowledge, whether supported by knowledge brokers, social media or both, builds on the Knowledge to Action model by focusing on relationships between researchers and decision makers. It is this focus that differentiates Bennet \& Bennet from the Knowledge to Action model. As Bennet and Bennet indicate (Bennet and Bennet 2008, 124), "it is through the interaction of all these relationships and activities and the sharing of understanding that desired change emerges."

Sandra Nutley and her colleagues (Nutley, Walter and Davies 2007), from the Research Unit for Research Utilization (http://www.ruru.ac.uk/) at the University of Edinburgh, published Using Evidence: how research can inform public services - a seminal review of the literature on research utilization. Nutley and colleagues review research utilization studies that show how research is used to inform public policy and professional practice. There is no "Nutley model" of research utilization. Rather the book focuses, in part, on the social nature of research utilization.

Nutley agrees with Bennet and Bennet by recognizing that research utilization is socially and organizationally situated and is an interactive process. Because it is a social process, interactive forms of engagement between researchers and decision makers should be encouraged. "Interpersonal and social interactions are often key to the use of research, whether among policy and practice colleagues, or more directly with researchers themselves" (Nutley, Walter and Davies 2007, 302). Nutley and colleagues identify that "the need for better links between researchers and potential research users is addressed through interactive strategies, by developing informal networks or more formal partnership arrangements" (Nutley, Walter and Davies 2007, 151). They conclude that "interactive approaches currently seem to show most promise in improving the use of research. Interactive strategies may range from simply enabling greater discussion of findings by policy makers and practitioners at presentations, through local collaborations between researchers and research users to test out the findings from research, to formal, ongoing, large-scale partnerships that support better connections between research, policy and practice over the longer term" (Nutley, Walter and Davies 2007, 152).

Much scholarship of research use has focused on relationships between individuals; however, those individuals sit within organizations. Creating a culture of research use within organizations (both research and policy/practice organizations) can help support the collective and embedded nature of the links between research and decision making. Although this might be true, Nutley and colleagues recognize that "the evidence that we 
have about developing effective organisational - and system - level research use strategies, while emerging, is still fairly thin on the ground" (Nutley, Walter and Davies 2007, 307). In 2012, Nutley and her colleague Sarah Morton state that systems models of research use are the future (Morton and Nutley 2011).

Sandra Nutley's work builds on that of Bennet and Bennet by considering not only relationships between researchers and decision makers but also the specific focus on the social nature of these relationships and the interactive approaches that can best enhance research use. Key also to current efforts to enhance research use and $\mathrm{KMb}$ is the role played by organizations.

Finally, whether working at the level of the individual or the organization, whether working to enhance the uptake of evidence or create interactive strategies to support enhanced research use, these activities happen within and between different sectors. As shown in Figure $3, \mathrm{KMb}$ occurs within and between sectors resulting in social benefit. Viewed holistically, $\mathrm{KMb}$ can be considered an ecosystem. "Knowledge Mobilization is the overall flow and ongoing and constant input and development of knowledge. It is the open process of putting available knowledge into active service to benefit not just one particular corporate or organizational structure, but for the greater benefit of all in society" (Myers, 2010).

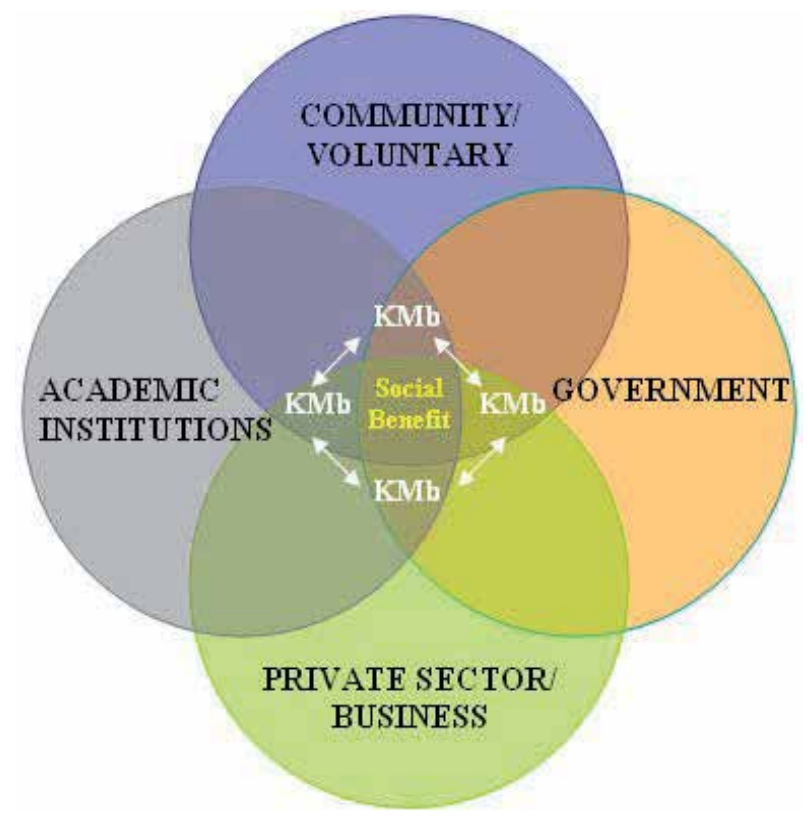

Fig. 3. KMb happens between and among sectors for social benefit.

All the $\mathrm{KMb}$ associated activities in the Knowledge to Action cycle, those reviewed by Bennet and Bennet and by Sandra Nutley and her colleagues occur in the arrows in figure 3 . Most flow diagrams focus on the activities that occur in the boxes at the beginning and the end of the arrows. It is these boxes that are the focus of Knowledge Translation in Health Care: moving from evidence to practice (Strauss, Tetroe and Graham, 2009). This book deepens our understanding of each step in the Knowledge to Action cycle, but Andrew Campbell and Nick Schofield (Campbell and Schofield 2008) remind us to also focus on the flows between 
the boxes. Recognizing that "diagrams of organizations and other systems usually attach the dollars to the boxes and not to the arrows between them", the authors recommend that funders also "fund the arrows" (Campbell and Schofield 2008).

Key Messages:

- $\mathrm{KMb}$ is a social process

- Efforts to enhance $\mathrm{KMb}$ need to be interactive and focus on the relationships between researchers and decision makers

- $\mathrm{KMb}$ happens at the level of the individual and is only beginning to emerge at the organization and the system/sectoral level

\section{3. $\mathrm{KMb}$ as an institutional capacity}

Having reviewed the $\mathrm{KMb}$ literature in the previous section we now illustrate the key messages using live case studies of Canadian organizations that have developed a capacity for $\mathrm{KMb}$. Canada is seen as an international leader in $\mathrm{KMb}$; however, there is an evidence gap since this international perception has never been empirically proven (Linder, Sudsawad and Phipps 2011). Nonetheless, Canada has created many public organizations that maximize the public benefit from public investments in research. Canada has created national institutions with a $\mathrm{KMb}$ mandate including the Canadian Institutes of Health Research (http://www.cihrirsc.gc.ca/), Canadian Health Services Research Foundation (http://www.chsrf.ca/Home.aspx), Canadian Partnerships Against Cancer (http://www.partnershipagainstcancer.ca/), Mental Health Commission of Canada (http://www.mentalhealthcommission.ca/english/pages/ default.aspx), and the Canadian Council on Learning (http://www.ccl-cca.ca/CCL/Home/ index.html). While not an organization of the federal government, Canada also has ResearchImpact-RéseauImpactRecherche (http://www.researchimpact.ca/ home/), a national network of university knowledge mobilization units. As a result of this effort to embed $\mathrm{KMb}$ within the Canadian research fabric a number of organizations have developed a $\mathrm{KMb}$ mandate to complement their research mandate.

The Institute for Work and Health (IWH) is an independent not-for-profit research organization in Toronto, Canada. IWH is one of the top five occupational health and safety research centres in the world. It has a mandate to undertake research in the prevention of work-related injury and illness and on treatment, return to work, disability prevention and management, and compensation policies for injured workers. IWH also has an active knowledge transfer and exchange unit that brokers relationships while making research accessible to policy makers and other work and health stakeholders. According to the IHW website (http://www.iwh.on.ca/knowledge-transfer-exchange) which uses KTE synonymously for $\mathrm{KMb}$ - "KTE is the process of sharing timely, useful evidence-based research findings with decision makers and others who use research. KTE also involves actively engaging external audiences in research to make studies more relevant" (Institute for Work and Health 2011). IWH produces a number of products including research articles, reports and working papers, but they also produce policy briefs and research summaries such as Sharing Best Evidence (Institute for Work and Health 2011) and Research Highlights (Institute for Work and Health 2011).

More importantly, because the literature above illustrates that $\mathrm{KMb}$ is a social process based on interactive relationships, the KTE team at IWH supports a variety of networks and 
events. Guided by a Health \& Safety Association Liaison Committee, IWH has established a variety of clinical networks and relationships with different occupational health and safety stakeholders - including labour, advocates and policy makers. "John Frank, director of the Scottish Collaboration for Public Health Research and Policy and founding director of research at IWH, sums up Gibson's contribution: 'Jane [Gibson, Director, Knowledge Transfer \& Exchange, IWH] has created at IWH perhaps the most effective and polished KTE unit in any research facility in North America. She has been able to forge exceptional links to IWH's diverse and dispersed stakeholder communities, and provide them with a steady flow of easily digestible research findings. Indeed, her IWH unit's novel conceptualization and actualization of KTE has led the field internationally (Institute for Work and Health 2011)."' $\mathrm{IWH}$ has embedded $\mathrm{KMb}$ at the organizational level, creating a system of $\mathrm{KMb}$ that supports institutional relationships between research and decision makers.

Another Canadian example of $\mathrm{KMb}$ embedded within a research-based organization is PREVNet: Promoting Relationships and Eliminating Violence Network, funded by the Networks of Centres of Excellence (NCE). According to the PREVNet website (http://prevnet.ca/) "PREVNet is a national network of Canadian researchers, national non-governmental organizations (NGOs), corporations, and governments committed to stop bullying and promoting healthy relationships for children and youth. Bullying is wrong and hurtful. Every child and youth has the right to be safe and free from involvement in bullying. It affects children and youth who are bullied, those who bully others, and those who know it is going on". PREVNet produces a number of knowledge products including videos and fact sheets (presented online and in downloadable formats) while also supporting the social side of knowledge mobilization by hosting a number of interactive exchange events.

PREVNet has recently been awarded an NCE Knowledge Mobilization (NCE-KM) New Initiative award to co-create 10 sustainable knowledge mobilization (shown here as KM) projects. The goal for this NCE-KM is to provide adults and youth with evidence-based knowledge, strategies, tools, and programs to prevent violence and promote healthy relationships. For adults to be effective in preventing violence, they require evidence-based knowledge about: healthy and unhealthy development and relationships, how development varies by age and gender, how to identify risks for violence, and what to do to support children who are developing behaviours that may lead to violence. Peers also require this knowledge for their own healthy development and to support friends.

PREVNet joins 58 researchers and 56 graduate students with 50 national NGOs, corporations, and government agencies. Through working groups, the expertise of NGOs, government ministries and diverse fields of research expertise are integrated and linked in comprehensive knowledge mobilization. Researchers collaborate with partners to exchange and translate knowledge to develop education, assessment, intervention and policy related to bullying. PREVNet is working for socio-cultural change in Canada regarding power and aggression in relationships by providing NGOs and governments with the capacity to adapt and disseminate specific scientific knowledge and technology to build awareness, change attitudes, assess the extent of bullying and victimization problems, implement evidence based strategies and develop policies that support these activities. Through these partnerships, the outcomes of improved understanding and practice will enhance social capital, promote mental and physical health, promote healthy relationships, school engagement and prevent crime. 
Although PREVNet is producing a wide range of knowledge products - including training manuals, reference guides, public service announcements and research communiqués - the focus of PREVNet is on relationships, collaborations and networking. The products produced arise from the research and knowledge exchange activities of the individuals and organizations in the network. PREVNet illustrates the social nature of $\mathrm{KMb}$ by using interactive methods to produce a system of knowledge mobilization to enhance the practices of all those involved with children and youth to promote their healthy development through healthy relationships.

Both IWH and PREVNet are organizations designed around a KMb mandate for a specific topic, workplace health \& safety and healthy relationships respectively. Some universities are beginning to invest in an institutional $\mathrm{KMb}$ capacity similar to the ubiquitous technology transfer and industry liaison services (Agrawal 2001) - although this is an emerging expertise and there are relatively few examples of universities that have invested in $\mathrm{KMb}$ and related support services. Three examples (from England, Scotland and USA) illustrate the varied forms in which universities have invested in an institutional capacity to connect research to policy and/or practice.

Starting with a grant from the Atlantic Philanthropies in 2003, the Community University Partnership Program (Cupp) now receives core funding from the University of Brighton. Cupp fosters relationships that allow academic expertise (faculty and students) to work with community opportunities. Cupp operates $a$ research helpdesk (Rodriguez and Millican 2008) that community organisations can call to get connected to academic researchers, communityuniversity research forums and "speed dating" all designed to make community-university connections around issues of importance to local communities (Hart and Wolff 2006). Cupp is one way that the University of Brighton is delivering on its aim of Corporate and Civic Engagement as articulated in its Corporate Plan (http://staffcentral.brighton.ac.uk/ xpedio/groups/Public/documents/staffcentral/doc007325.pdf).

Built on a community of practice model (Hart and Wolff 2006), Cupp embeds knowledge exchange within all of its activities. Cupp does not prioritize the production of knowledge products to transfer the results of university research but mainly seeks to create opportunities for researchers and community partners to develop relationships that will meet the needs of both community and university partners. Knowledge exchange tools used by Cupp include The Brighton and Sussex Community Knowledge Exchange (Hart, Maddison and Wolff 2007) and On Our Doorstep programs. Both of these programs create spaces where university faculty and students can meet potential partners from local community organizations and explore relationships for mutual benefit. Cupp brokers these relationships and supports community-university collaborations that emerge from conversations started through knowledge exchange. Cupp embodies the three key lessons learned from the literature showing that knowledge mobilization is a social process supported by interactive relationship building strategies and practiced at an institutional level.

The Centre for Research on Families and Relationships (CRFR)( http://www.crfr.ac.uk/) is based at the University of Edinburgh and has partners across other Scottish universities. "The mission of CRFR from its inception has been to make research accessible to a range of audiences and to work in partnership with policy-makers and practitioners to ensure that 
research is relevant and useful to policy and practice communities in Scotland, the UK and beyond" (Morton and Nutley, 2011). CRFR has strong collaborations with the Research Unit for Research Utilisation (http://www.ruru.ac.uk/) at the University of Edinburgh and grounds much of its effort in the foundational work of Sandra Nutley and colleagues (Nutley, Walter and Davies 2007). Since 2001, CRFR has sought to achieve its mission by undertaking project or contract work on behalf of decision makers, making research products available in formats that are accessible to a non-academic audience and brokering relationships between university researchers and decision makers.

As Morton and Nutley (2011) describe, "the provision of support for research use is important both in academic and in policy and practice settings. One of the key differences between working in research centres with a commitment to knowledge exchange and working in standard academic departments is often the level of knowledge exchange support available. If knowledge exchange is not costed into research funding applications, and is not supported by university practices it will be hard for individual researchers to carry out the activities necessary to increase the impact of their work.". CRFR is a university-based $\mathrm{KMb}$ service that provides the support necessary to maximize the impact of research on non-academic audiences. Like Cupp, CRFR supports the social aspects of knowledge mobilization by fostering relationships between researchers and decision makers. Although the centre is discipline specific, CRFR serves the needs of a number of academic institutions.

The Institute for Health Policy at the University of Texas School of Public Health has also started a project designed to connect research to policy. The first incarnation of Research Into Action is focused on connecting public health researchers and policy makers/practitioners, but there is also a desire to grow to serve needs more broadly at the university. According to their website (https://ktexchange.sph.uth.tmc.edu/ research_into_action.aspx), "Research Into Action (RIA) is a knowledge translation initiative that was founded in 2007 with a generous award from the ExxonMobil Foundation, with the aim of developing new methods of assisting researchers throughout the University of Texas Health Science Center in translating their technical findings into usable advice and practical recommendations".

The RIA team approaches knowledge translation (their term for $\mathrm{KMb}$ ) in three ways: 1) through translation activities undertaking contracted research in collaboration with public health agencies; 2) through a web portal and social media strategy and 3) disseminating key findings to public health stakeholders. RIA produces knowledge products such as briefing packages and FAQ sheets for alcohol and substance abuse screening and referral, as well as a research synthesis on noise pollution - among other projects. Their online portal boasts over 600 members, has a variety of blog posts and pod casts, including an online discussion board. It is this latter feature of social media that allows RIA to complement its knowledge products with interactive engagement of stakeholders.

Cupp, CRFR and RIA use different strategies to support different levels of engagement between researchers and decision makers. All three have professional staff supporting $\mathrm{KMb}$ activities of their institution. Cupp is almost exclusively based on an exchange model creating spaces and opportunities for the community and university to interact. CRFR produces products for policy makers and practitioners but they also rely on events and networks where their work can inform policy and practice. RIA relies more heavily on 
contracted collaborative research and the production of knowledge products to translate research into policy and practice settings, but RIA complements these with a strong online and social media engagement strategy. Cupp, CRFR and RIA illustrate how KMb services are social processes supported by interactive (in person or online) relationships between researchers and decision makers.

All three are working beyond the level of a single project and embedding the activity within institutional structures and systems. As mentioned above, Nutley, Walter and Davies recognize that "the evidence that we have about developing effective organisational - and system - level research use strategies, while emerging, is still fairly thin on the ground" (Nutley, Walter and Davies 2007, 307). Only Cupp serves the need of the entire university. In order to better understand the potential for university wide $\mathrm{KMb}$ we examine in detail York University's KMb Unit.

Key Messages:

- Institutional $\mathrm{KMb}$ support services require a variety of strategies to support the diverse $\mathrm{KMb}$ needs of stakeholders

- $\mathrm{KMb}$ often occurs at the level of a research project or research unit; however, some institutions are beginning to invest in organizational and systems level $\mathrm{KMb}$ services

- $\mathrm{KMb}$ creates value for the institution as well as for researchers and their decision maker partners.

\section{KMb at York University}

York University was the first university in Canada to develop a KMb Unit that is fully integrated into the university's research enterprise. York's KMb Unit delivers KMb services to faculty and students and to decision makers in local community organizations and (mainly municipal, regional and provincial) government agencies. Most of York's KMb services are social in nature and depend on interactive strategies to broker relationships between researchers and research users. York's KMb strategy and processes have recently been explained in detail (Phipps 2011).

As shown in Table 1, York's KMb Unit practices elements of producer push, user pull, knowledge exchange (Lavis, Ross, McLeod and Gildner 2003) and co-production (Hart, Maddison and Wolff 2008). Arising from each of these methods is a KMb service. While each service might be employed to address a specific opportunity or need, they all work in concert to support the development of collaborations between researchers and decision makers.

ResearchSnapshot clear language research summaries (KMb service \#1) are a form of producer push knowledge transfer in which the $\mathrm{KMb}$ Unit writes a two page summary of peer reviewed journals and edited book chapters according to clear language writing and design principles. The $\mathrm{KMb}$ Unit uses these as calling cards to facilitate introductions between researchers and decision makers - making these a tool to support the social and interactive nature of $\mathrm{KMb}$; however, they are also used in a non-iterative fashion to transfer the results of research to decision makers. Every other $\mathrm{KMb}$ service above is based on interactive methods that support personal relationships between researchers and decision makers. 
All of York's KMb services are detailed elsewhere (Phipps 2011). We shall focus some detail here on the core of the $\mathrm{KMb}$ Unit - the knowledge broker. The knowledge broker operates a research translation help desk (Rodriguez and Millican 2008). The knowledge broker is the first point of contact for researchers and decision makers seeking to connect with one another. The knowledge broker meets (in person or on the telephone) with the requester, clarifies the inquiry, translates the request into either a research or a policy/practice opportunity and then seeks candidates who might be interested in the collaboration. The broker makes an introduction between the researcher (or decision maker) and the potential decision maker (or researcher) collaborator. The knowledge broker also supports the emerging conversations as the parties explore collaboration. The broker does not become directly involved in the research.

\begin{tabular}{|l|l|l|}
\hline KMb Method & \multicolumn{1}{|c|}{ KMb Service } & \multicolumn{1}{|c|}{ Notes } \\
\hline $\begin{array}{l}\text { Producer } \\
\text { Push }\end{array}$ & $\begin{array}{l}\text { \#1 Clear } \\
\text { language research } \\
\text { summaries }\end{array}$ & $\begin{array}{l}\text { Develop clear language research summaries from } \\
\text { completed faculty research. York has over 170 } \\
\text { ResearchSnapshot clear language research summaries } \\
\text { posted in a searchable online database } \\
\text { www.researchimpact.ca/researchsearch }\end{array}$ \\
\cline { 2 - 4 } & $\begin{array}{l}\text { \#2 Lunch and } \\
\text { Learn }\end{array}$ & Seminar series at decision-maker sites \\
\hline User Pull & $\begin{array}{l}\text { \#3 Research } \\
\text { translation help } \\
\text { desk }\end{array}$ & $\begin{array}{l}\text { Use knowledge broker model to help decision-maker } \\
\text { partners identify, develop, and sustain collaborations } \\
\text { with researchers. }\end{array}$ \\
\hline $\begin{array}{l}\text { Knowledge } \\
\text { Exchange }\end{array}$ & $\begin{array}{l}\text { \#4 Research } \\
\text { forums }\end{array}$ & $\begin{array}{l}\text { KM in the AM: Monthly thematic knowledge } \\
\text { mobilization breakfasts. }\end{array}$ \\
\hline $\begin{array}{l}\text { Co- } \\
\text { production }\end{array}$ & $\begin{array}{l}\text { \#5 Social media } \\
\text { to support } \\
\text { collaboration }\end{array}$ & $\begin{array}{l}\text { Provide support for full suite of social media tools } \\
\text { including blogging, delicious bookmarks, Twitter, and } \\
\text { social collaboration tools. }\end{array}$ \\
\cline { 2 - 3 } \#6 KMb interns & $\begin{array}{l}\text { Graduate student KMb interns work in research } \\
\text { collaborations with decision-maker partners. }\end{array}$ \\
\hline
\end{tabular}

Table 1. York University's Suite of KMb Services

This last point differentiates York's KMb service from RIA and CRFR. The knowledge brokers at RIA and CRFR are themselves researchers and undertake research projects on behalf of decision makers. The knowledge brokers at York's KMb Unit do not get involved in the research project. As a project arises from a brokered collaboration the knowledge broker will monitor and be available to support any concerns arising. The knowledge broker will also follow up during and after the project to identify any outputs or outcomes of the collaboration - but the knowledge broker is not part of the research team.

A research translation help desk staffed by a knowledge broker makes research and research expertise accessible to non-academic research stakeholders by supporting interactive, social relationships between decision makers and researchers. As the foundation of the KMb Unit, the research translation help desk is the first service that should be established as part of an institutional capacity to support $\mathrm{KMb}$. 
It is also the job of the knowledge broker to match the $\mathrm{KMb}$ services provided with the $\mathrm{KMb}$ opportunity at hand. Phipps explains that "developing a KMb strategy for any particular research collaboration may employ one or more of these $\mathrm{KMb}$ services. The knowledge broker chooses the right $\mathrm{KMb}$ service(s) according to the available research, the researcher(s), the decision-maker(s), and the context of the potential collaboration (available resources, regulatory environment, political context, time pressure, etc.)" (Phipps 2011). In some cases multiple $\mathrm{KMb}$ services might need to be applied to individual opportunities. This is supported by a recent systematic review that demonstrated that multifaceted approaches to the implementation of research into practice were superior to those employing individual methods (Boaz, Baeza and Fraser 2011).

After more than five years of operating an institutional $\mathrm{KMb}$ service, what does success look like? As described by Phipps, "developing and using these six KMb services has created value for faculty, graduate students, and decision-maker partners as well as the university itself. In addition to the $\mathrm{KMb}$ services identified above, the $\mathrm{KMb}$ Unit has been part of the grant application team that supports all large-scale policy and practice-relevant research grant applications. Engaging the $\mathrm{KMb}$ Unit as part of the grant application team ensures that grant applications are submitted with a well-crafted and justified knowledge mobilization strategy, something that is becoming increasingly required by granting agencies. These large-scale grants have attracted over \$16 million in external research support for York University researchers. In addition, the $\mathrm{KMb}$ Unit has attracted over \$1 million in research funding from decision-maker partners to support research collaborations. York's KMb activities have also helped partner-organizations raise over $\$ 800,000$ in support of their services" (Phipps 2011). The activities of the KMb Unit are summarized in Table 2.

\begin{tabular}{|l|l|}
\hline \# Faculty Involved & 228 \\
\hline \# Graduate Involved & 141 \\
\hline \# Information sessions for faculty and students & 165 \\
\hline \# Information sessions for community & 184 \\
\hline \# collaborations brokered & 227 \\
\hline \# agencies involved in KMb partnerships & 205 \\
\hline Community Partner funding raised & $\$ 810 \mathrm{~K}$ \\
\hline Research Contract funding raised & $\$ 1.2 \mathrm{M}$ \\
\hline Total KMb associated grant funding raised & $\$ 16 \mathrm{M}$ \\
\hline \# web hits & $+4 \mathrm{M}$ \\
\hline \# Research Summaries & 158 \\
\hline \# tweets & 4253 \\
\hline \#Twitter followers & 1529 \\
\hline \# delicious bookmarks & 219 \\
\hline \# blog postings (+71,000 views) & 266 \\
\hline
\end{tabular}

Table 2. York's KMb Activities February 2006-August 2011

These numbers tell only part of the story. Most of these numbers are input metrics for $\mathrm{KMb}$ and do not present the outcomes or impact of this work. Some outcomes are seen in the short 
term. Of the +35 graduate student interns who have worked with community or government partners in projects brokered by the $\mathrm{KMb}$ Unit, eight have been hired by their internship partner. This is an immediate outcome with a significant impact on the graduate student who is now working in a chosen field of study. It also has an impact on the internship partner who now has increased their capacity to engage in research and also has an appreciation of the role that university research can play in their organization (Hynie, Jensen, Johnny, Wedlock and Phipps 2011). In contrast to the short term impact of a KMb intern hired by a partner, most impacts occur over the span of years. It is these stories that tell the impact that numbers do not. We illustrate this impact with four stories from our work.

\subsection{Evaluation of the Inclusivity Action Plan (public sector partner)}

York Region is home to the largest newcomer population in Canada. In January 2005 York Region held an Inclusivity Summit that resulted in York Region Counsel adopting an Inclusivity Action Plan that included opening a Welcome Centre where newcomers could get access to culturally sensitive health and human services. In 2007, York's KMb Unit brokered a collaboration between the Regional Municipality of York and York University researchers from the Faculty of Health to undertake an evaluation of the Welcome Centre. The evidence co-produced in that evaluation informed a decision by the Regional government to expand the program. York Region now has five Welcome Centres and newcomers across this large region have greater access to many settlement services including the culturally sensitive health and employment services they require. In this expansion 86 jobs were created and over 48,000 instances of service delivery have been performed between 2007 and 2011. The researchers published their results in an international journal (Singh and Hynie 2008) and you can read the ResearchSnapshot clear language research summary (http://researchimpact.ca/_app/impact/files/tables/files.path.22.pdf) about this research and its policy implications.

\subsection{Green Economy Centre (private sector partner)}

Nottawasaga Futures is a non-profit community futures agency in rural South Simcoe that supports rural businesses. Nottawasaga Futures approached York's KMb Unit in 2009 to assist them with the challenge of helping rural businesses make greener business decisions. York's KMb Unit held a meeting of faculty, funders and Nottawasaga Futures to imagine how green business services could be delivered in South Simcoe. Nottawasaga Futures engaged the services of two graduate student $\mathrm{KMb}$ interns who were supervised by faculty members from York University's Schulich School of Business and the Faculty of Environmental Studies. The interns undertook research that informed the development of a business case that subsequently was funded and launched the Green Economy Centre. The Green Economy Centre is an innovative research and education program that initiates, supports and facilitates green economic best practices, ensuring that the rural South Simcoe economy is healthy and sustainable. The Centre was launched in April 2010 and received funding in summer 2010. Since then, the Centre has contacted 604 local businesses and made 507 individual visits resulting in 39\% of companies expressing interest in the Centre's programs. With the support of the Centre, four companies have received loans for green projects totaling more than $\$ 1$ million, and seven other loans are pending. The services of the Green Economy Centre have already created 18 jobs while 
maintaining 221 jobs. This project is illustrated in a short $\mathrm{KMb}$ video (http://www.youtube.com/researchimpact\#p/a/u/0/J15_lBz5mYU).

\subsection{Heat Registry (community partner)}

In North America heat kills more people every year than all severe weather related event combined. In 2008, Tanya Gulliver was a graduate student in York's Faculty of Environmental Studies. She was awarded a York University KMb Internship to work for the summer with her partner, Parkdale Activity and Recreation Centre (PARC), a community centre providing support to a large and diverse group of psychiatric survivors, low income and marginalized persons. PARC and Tanya developed Canada's first heat registry that tracked vulnerable community members at risk of heat exposure. During heat alert days the heat registry was used by Tanya and a group of street engaged volunteers to track vulnerable community members and ensure that they received the services they needed to resist the heat. In 2010, Tanya developed a Heat Registry Manual. “This manual offers a way to track (through regularly updated documentation) and actively monitor people who might be at risk from suffering ill effects caused by extreme heat. It provides a system of checking in on, and checking up on (through outreach or some other effort), people who have voluntarily self-identified as being at risk and want to be on the Registry" (Toronto Disaster Relief Committee 2011). The Heat Registry Manual is now before the Toronto Board of Public Health and is being evaluated by Toronto's Medical Officer of Health to determine how it might be able to inform Toronto's cooling policies and services.

\subsection{Strength Investments (community sector partner)}

For five years The United Way of York Region (UWYR) and York University have been deepening their knowledge mobilization and social innovation partnership. Recently York and UWYR co-invested in three graduate student interns who undertook social asset mapping research in Markham, a suburban municipality in York Region. Their research developed the evidence needed to launch a wholly new form of United Way investment called Strength Investments that recently invested $\$ 150 \mathrm{~K}$ in six projects. As an example, UWYR invested in a project from the Markham African Caribbean Association titled Building Sustainable Capacities amongst Afro-Canadian Caribbean Youth in York Region. This initiative involves and listens to local youth as they identify their realities in York Region inclusion, education, family life - and then helps them identify and build their own solutions. Strength Investments and therefore this project would not have been possible without the collaboration between a UWYR opportunity and York research.

Three lessons can be learned from these stories: 1) KMb initiatives take time to develop. Impact is rarely measured in the short term. As you plan your institutional $\mathrm{KMb}$ services, be prepared to invest for the long haul (4-5 years) before expecting to see impacts of your work; 2) $\mathrm{KMb}$ is a process that results in social innovations. Strength Investments, Heat Registry, Welcome Centres and the Green Economy Centre are social innovations that were enabled by $\mathrm{KMb} . \mathrm{KMb}$ is not an end in itself. $\mathrm{KMb}$ is the process of brokering relationships between researchers and decision makers. The outcomes of those collaborations are social innovations; 3) Students are more frequently the source of research leading to social innovations than are faculty members. 
Key to the success of York's KMb Unit is its partnership with the United Way of York Region (Phipps, Zanotti and Johnny 2009; Phipps and Zanotti 2011). Established in 1976, United Way of York Region (UWYR) is a registered charity uniting people and resources to improve our communities. In its 2010 fundraising campaign, UWYR raised $\$ 8.1 \mathrm{M}$ to support human services provided by its 39 member agencies delivering 100 programs. UWYR also funds coalitions of residents and organizations. United Way identifies community priorities and works with partners to take action, supporting a network of 100 critical programs across the region's nine municipalities. More than just a funder of agencies and coalitions, UWYR is a community champion and strong advocate for the community sector. UWYR allows York's $\mathrm{KMb}$ services to be grounded in the perspectives of the community. This collaboration is a result of and reinforces the trust between the community and the University and creates an institutional relationship that transcends individuals.

Key Messages for those seeking to develop an institutional capacity for $\mathrm{KMb}$ :

- Collect data: Count everything (see Table 2) and track initiatives longitudinally to identify those social innovations that will become your success stories.

- The first action you should take is to hire the right knowledge broker as the core of your KMb Unit.

- Develop a suite of $\mathrm{KMb}$ tools and select the right tool(s) for the right opportunity.

- Develop institutional relationships between the university and non-academic partners.

\section{Social media as an emerging tool for knowledge mobilization}

Social media support is one of the $\mathrm{KMb}$ services offered by York's KMb Unit (Table 1). Social media are increasingly being used in the academy both as a teaching tool and as a research dissemination tool. Although formal research on social media is only beginning to emerge there is evidence that social media enhances transparency and trust, while supporting a variety of relationships. As a result, social media has the potential to support knowledge mobilization and research-based relationships. York is very active in social media to support an emerging $\mathrm{KMb}$ community of practice and we are using social media tools to support co-creation of research and knowledge; however, this experience is only now beginning to be formally evaluated - so this section will be a visioning section.

\subsection{Literature review of social media and $\mathrm{KMb}$}

Much of the literature exploring the use of social media comes from the business sector and is primarily concerned with the use of social media for marketing and sales purposes. Research of this type looks mainly at social media as a way to reach consumers (Kaplan and Haenlein 2010), or else focuses on issues of Return on Investment (ROI) and justifications for using social media (Fisher 2009). Due to the low or no cost of social media tools, the not-forprofit and charity sectors have also been quick to use these tools to engage potential volunteers and donors (Mattson and Barnes 2010). Researchers in computer science and computer mediated communication areas are also interested in social media; however, this research is mainly interested in examining these tools from an analytic, measurement or operational perspective (Finin, et al. 2007; Weng, et al. 2010).

Boyd and Ellison's survey of scholarship on social media found that "research has focused on impression management and friendship performance, networks and network structure, 
online/offline connections, and privacy issues" (Boyd and Ellison 2007, 9). However, research and the literature illustrating the utility of social media in the social sciences for knowledge mobilization was lacking from this list.

Research on the social media tool Twitter is also emerging and studies have been conducted to understand who is using the tool and how they are using it (Java et al. 2007; Honeycutt and Herring 2009), as well as how networks are formed through Twitter friends and followers (Huberman, Romero and $\mathrm{Wu}$ 2008). However, these studies are more focused on how people are using Twitter from an operational point of view and have not begun to provide insights into how people are using Twitter to establish relationships and/or communities of practice. They also do not look specifically at research communities.

More useful to our discussion of social media for knowledge mobilization is research that explores the establishment of virtual communities of practice (Sangwan, Guan and Siguaw 2009; Urquhart, et al. 2010; Michaelides, Tickle and Morton 2010) and more general discussions of knowledge transfer and sharing (Tortoriello, Reagans and McEvily 2011). These articles discuss the elements necessary for successful online community development and knowledge mobilization.

As the use of social media for knowledge mobilization is an emerging field of study, there are many opportunities for researchers to examine how these tools are currently being used to disseminate social science research, engage between researchers and decision makers and how the use of these tools might evolve in the future.

\subsection{How York's KMb Unit is using social media}

York's $\mathrm{KMb}$ Unit has been using social media tools since May 2008 when we launched our Wordpress blog Mobilize This! (http://researchimpact.wordpress.com). In 2009, we started micro-blogging using Twitter (http://twitter.com/researchimpact; @researchimpact) and using an online collaborative platform called O3 (http:// researchimpact.othree.ca), which is available free of charge to all researchers in the province of Ontario through the Ontario Research and Innovation Optical Network (ORION).

We now have a suite of social media tools that we use for various purposes, as shown below in Table 3:

\begin{tabular}{|l|c|c|c|c|}
\hline & Storytelling & Resource Sharing & Disseminating Research & Networking \\
\hline Blogging & $\square$ & $\square$ & $\square$ & \\
\hline Twitter & $\square$ & $\square$ & $\square$ & $\square$ \\
\hline O3 & $\square$ & $\square$ & $\square$ & $\square$ \\
\hline Delicious & & $\square$ & $\square$ & $\square$ \\
\hline LinkedIn & & & & $\square$ \\
\hline Flickr & $\square$ & & $\square$ & \\
\hline YouTube & $\square$ & & $\square$ & $\square$ \\
\hline Facebook & $\square$ & $\square$ & $\square$ & $\square$ \\
\hline
\end{tabular}

Table 3. Using Social Media Tools for Knowledge Mobilization 
We do not currently use facebook as our community is more active on Twitter but Facebook has the potential to be a useful tool, especially for engaging students.

Blogging: York's KMb blog, Mobilize This!, provides an opportunity for us to share news about our activities; promote upcoming events; highlight past events; allow readers to get to know our team through Meet a Mobilizer posts; share reviews of books and articles we are reading; and offer editorial comments on issues important to knowledge mobilization. Readers can follow our blog posts through the use of an RSS aggregator or by signing up to receive new posts via email, and they can comment on our posts which enables community engagement.

Twitter: Twitter allows us to send 140 character long updates called tweets to our followers, who are people who have voluntarily elected to receive our tweets. As of October 17, 2011, we have 1,631 followers and we follow 326 other people's tweets. We use Twitter to update our followers on our activities, share resources we find useful, connect to people who share our interest in knowledge mobilization, and in 2010, we also started tweeting daily about our ResearchSnapshot series of clear language research summaries. Every day, we send out a tweet with the title and link to one of our over 170 ResearchSnapshot summaries; for example, on October 17, 2011 we tweeted, "Today's ResearchSnapshot: Research summaries improve access and utility of academic \#research." The use of Twitter in this way increased web traffic to the searchable online database of ResearchSnapshot (www.researchimpact.ca/researchsearch) 270\% (York University, $\mathrm{KMb}$ Unit, unpublished data).

O3: This online collaborative platform contains a suite of social media tools, including a document repository, wikis, forum discussion lists, blogging features, an event calendar, member profiles and picture galleries. It also supports the importing of RSS feeds and other social media applications for further customization.

The York $\mathrm{KMb}$ Unit uses $\mathrm{O} 3$ for our own activities but also supports the use of $\mathrm{O} 3$ for other knowledge mobilization research projects conducted by York researchers and their community partners. One example of this is the use of O3 in Project Teen Mom, a collaborative research project focused on teen mothering that involved researchers and graduate students from York University's LaMarsh Centre for Research on Violence and Conflict Resolution, along with practitioners from two service agencies in York Region Kinark Child and Family Services and the York Region Children's Aid Society. Using O3 gave practitioners access to research relevant to serving teen mothers and provided insights into what some of the pressing research needs were in the community to the researchers and students. You can watch a video featuring some of the participants in the project talking about their experiences using O3 to network and share knowledge (http://www.youtube.com/researchimpact\#p/u/3/qwldIEBaPWY).

\subsection{Twitter profiles keyword study}

The KMb Unit's use of Twitter provides an open network (while you can block a follower you don't need to approve a follower) with the resulting increased availability of user profiles. In order to gain more understanding of how knowledge brokers can use Twitter, a pilot project was undertaken by York's $\mathrm{KMb}$ Unit to advance our understanding of how individuals might use Twitter to connect and form relationships. Analysis of what 
information Twitter users chose to include in their online profile information enhances our understanding of how individuals are connected within a social media network as a community of practice (Michaelides, Tickle and Morton 2010).

This study was based on the hypothesis that the words used in profiles are important as they describe the closeness of fit between the interests of Twitter account holders. The closer the fit of profiles will indicate greater group cohesiveness based on similar interest in a particular subject matter. Twitter users with similar interests can follow each other and develop as a community of practice.

\subsubsection{Methodology}

This study used two frameworks for analyzing Twitter profiles based on words found in two groups of Twitter users: Followers of ResearchImpact (FRI) $(\mathrm{n}=345)$ and those that ResearchImpact Follows (RIF) ( $\mathrm{n}=99)$. A randomly generated number of Twitter profiles was also included for analysis as a Control Group $(\mathrm{CG})(\mathrm{n}=145)$. The dataset used in this study was compiled by extracting the public profiles of all FRI and all RIF on March 09, 2010.

As an initial visual indicator for comparison, the words used in Twitter profiles from FRI, RIF and CG groups were generated into word clouds using the online tool found at www.wordle.net. Word clouds provide a visual representation of the frequency of words by representing the more frequently used words as greater graphic size within the word cloud. In the study of Twitter profiles, word clouds of profiles indicated a greater number of larger and similar words (social, knowledge, research, innovation) in both FRI and RIF (see Figure 4). The greater number of larger random and non-matching words in CG indicated a lack of common keywords compared to FRI and RIF - except for the word social and media used the most frequently. This could be explained given the popularity and ubiquitous use of the term social media on the Internet.

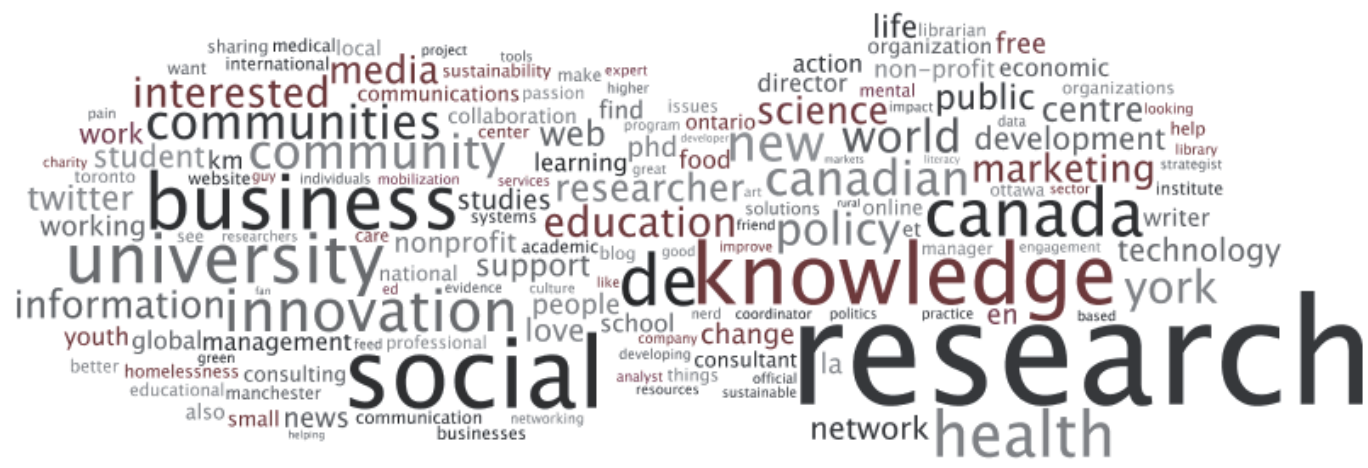

Fig. 4A. Word cloud of words used in Twitter profiles of Followers of ResearchImpact $(\mathrm{n}=345)$

Although the KMb Unit's hypothesis appeared to be supported by the word clouds, because FRI and RIF appear different than CG, statistical analysis beyond a visual representation was required to determine if there were any differences between the groups FRI and RIF. We explored the scoring of keywords used in Twitter profiles as a way of indicating closeness of fit between followers and @researchimpact. 


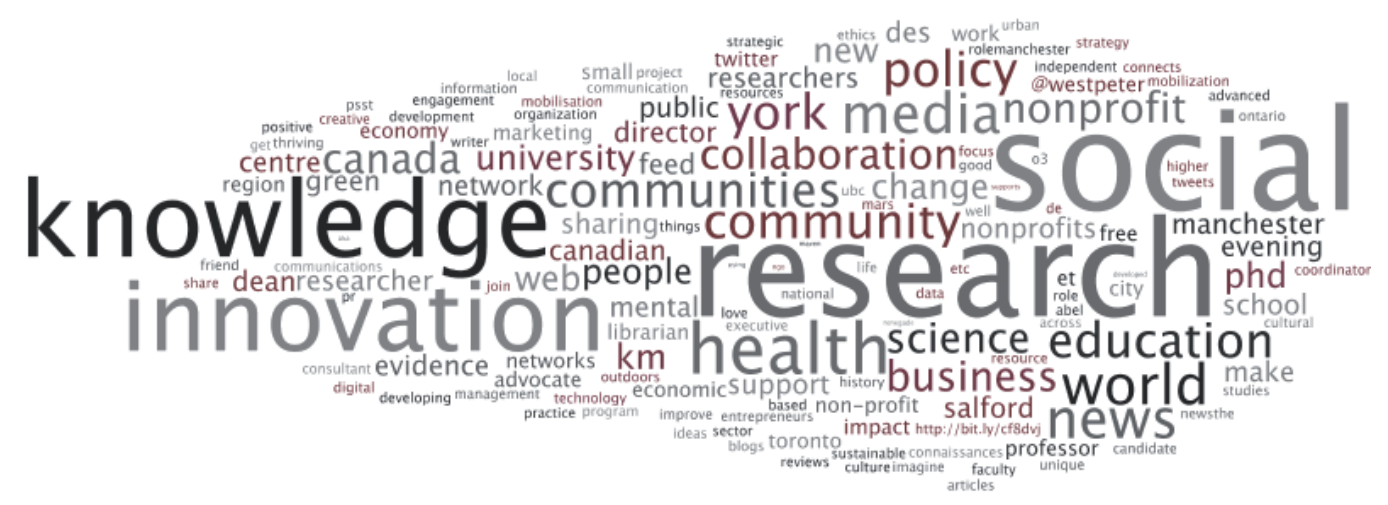

Fig. 4B. Word cloud of words used in Twitter profiles of those followed by ResearchImpact $(\mathrm{n}=99)$

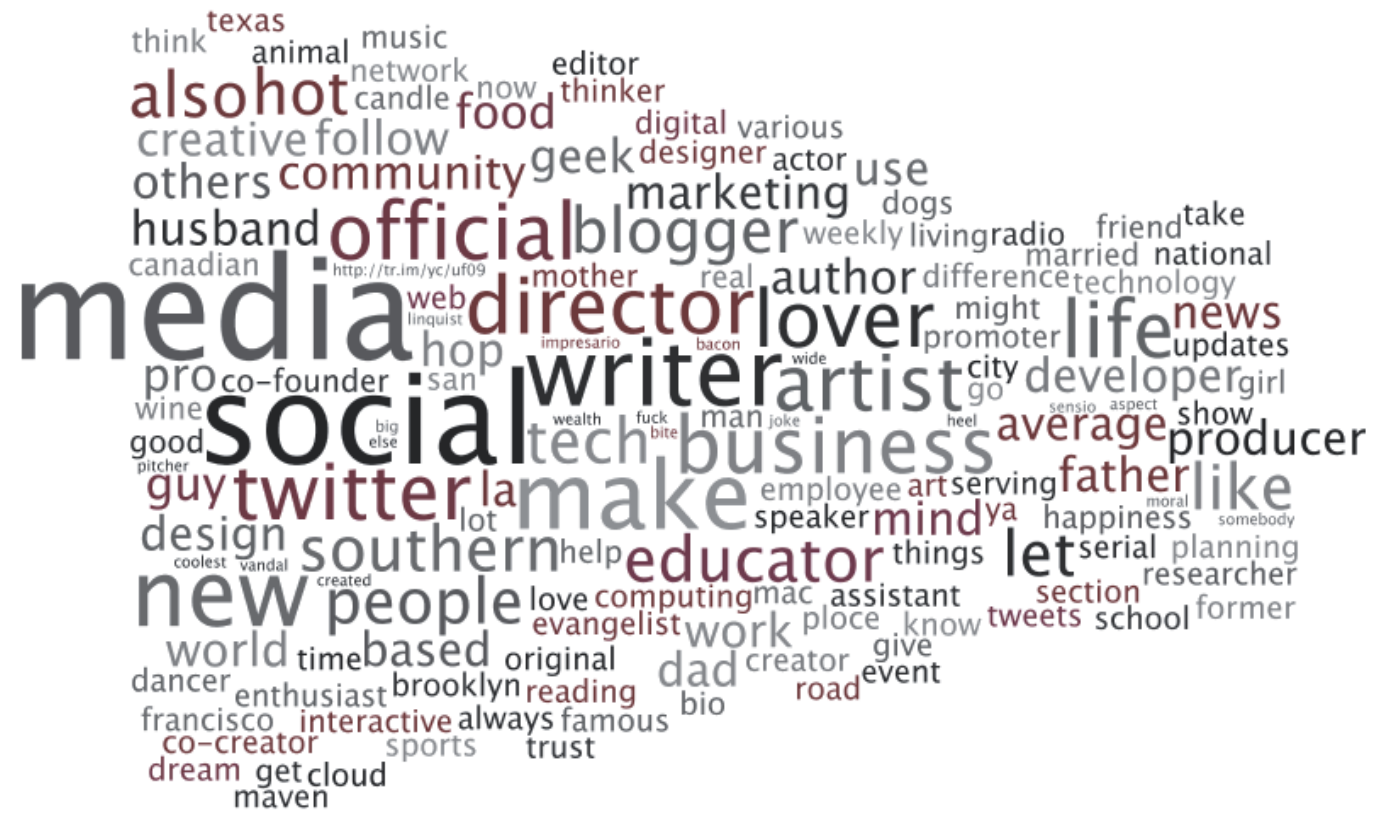

Fig. 4C. Word cloud of words used in Twitter profiles of the Control Group ( $n=145)$

Keywords were selected from ResearchImpact's website (http://www.researchimpact.ca) by considering relevant words connected to the work of our $\mathrm{KMb}$ practice. A brainstorming session with staff and students $(n=7)$ of the $\mathrm{KMb}$ Unit created a list of 16 keywords encompassing those words considered most relevant to our $\mathrm{KMb}$ practice. Each member of the $\mathrm{KMb}$ Unit then ranked their top ten keywords scoring each from 10 (top ranked) to 1 (bottom ranked). The ranked scores of each word were summed to develop a total weighted score for each word. As examples, the word "knowledge" received the highest score total of 60. The word "community" scored a total of 31 and web (using the words website, Internet 
as synonyms) received the lowest score total of 1 . The ResearchImpact profile with keyword scores is "Research (score 60) Impact (score 10) is Canada's knowledge (score 60) mobilization (score 49) network, connecting university (score 11), community (score 31) and government researchers (score 43) for social (score 26) innovation (score 29)" for a total score of 259. The scores in the RIF and FRI groups range from 0 to 194.

The keyword scores of the profiles of all 99 RIF and 345 FRI and 145 CG were averaged and means plus Standard Deviation (S.D.) compared using a two tailed $t$ test for independent variables to test for significant differences. The results are presented in Table 4.

\begin{tabular}{|c|r|r|r|r|r|r|}
\cline { 2 - 6 } \multicolumn{1}{c|}{} & \multicolumn{1}{c|}{$\mathrm{n}$} & \multicolumn{1}{c|}{ range } & \multicolumn{1}{c|}{ Mean } & \multicolumn{1}{c|}{ S.D. } & \multicolumn{2}{c|}{$\mathrm{p}$ value } \\
\hline FRI & 345 & $0-194$ & 17.8 & 33.084 & FRI & \multirow{2}{*}{ RIF } \\
\hline RIF & 99 & $0-194$ & 38.69 & 45.601 & $6.29 \mathrm{E}-07$ & $1.7 \mathrm{E}-15$ \\
\hline CG & 145 & $0-74$ & 4.31 & 13.92 & $2.89 \mathrm{E}-06$ & . \\
\hline
\end{tabular}

Table 4. Comparison of mean Twitter keyword scores

It is clear that all three groups were significantly different $(p<0.05)$ from each other and also that the average Twitter profile keyword score of the group that ResearchImpact follows $(\mathrm{RIF}=38.69)$ is more than double the keyword score of the group that is following ResearchImpact $(F R I=17.8)$ and both are much greater than that of the Control Group (CG $=4.31$ ). It appears that ResearchImpact chooses to follow a group that is not only smaller in number but that is more tightly aligned with ResearchImpact's own interests than those who follow ResearchImpact as judged by the higher keyword score. This supports our hypothesis that keywords are an important means for Twitter users to identify each other and identify membership in an online community of practice based on their self-identified interest in knowledge mobilization.

Keywords in Twitter profiles are thus important as they allow Twitter users to identify other users with self identified like interests. Like interest is the foundation of a community of practice. But then what can these members of this community accomplish? How can we evolve a Twitter-based $\mathrm{KMb}$ community of practice into a group that supports $\mathrm{KMb}$ and collaborations? We have yet to use Twitter to broker relationships between researchers and decision makers; however, we are developing closer ties with some Twitter followers as illustrated by the following example.

@KTExchange (the above mentioned RIA project at University of Texas School of Public Health) has been tweeting since July 7, 2009. They have 152 followers and follow 55 as of October 14, 2011. York's KMb Unit has been tweeting as @ResearchImpact since May 1, 2009. We have 1632 followers and follow 325 as of October 14, 2011. @ResearchImpact and $@$ KTExchange follow each other. We began a more in depth relationship by commenting on each others' blogs and exchanging on those comments using Twitter. Our comments were mostly about the Canadian and US KMb experience. We were invited by @KTExchange to participate on a panel at the CDC National Conference on Health Communication, Marketing, and Media (Austin, Susawad and Phipps 2011) and only weeks before the conference did we connect by phone and then in person at the conference. This presentation 
has led to co-publishing blogs and a formal collaboration is under development. In this case, Twitter identified a common interest that grew into an online collaboration that resulted in collaborating on a conference presentation and now is a collaboration supporting $\mathrm{KMb}$ practice in Toronto and Houston.

\subsection{Establishing communities of practice and mobilizing knowledge through social media}

Pick (2009) uses the concept of the four C's of social media to describe their use. Users develop Content within a given Context, i.e. specific applications and tools such as blogs, wikis or Twitter, to make Connections with others who share a similar interest; leading to Conversations; and when using social media for knowledge mobilization, we suggest that this model could be extended to include a fifth C-Collaboration as shown in Figure 5.

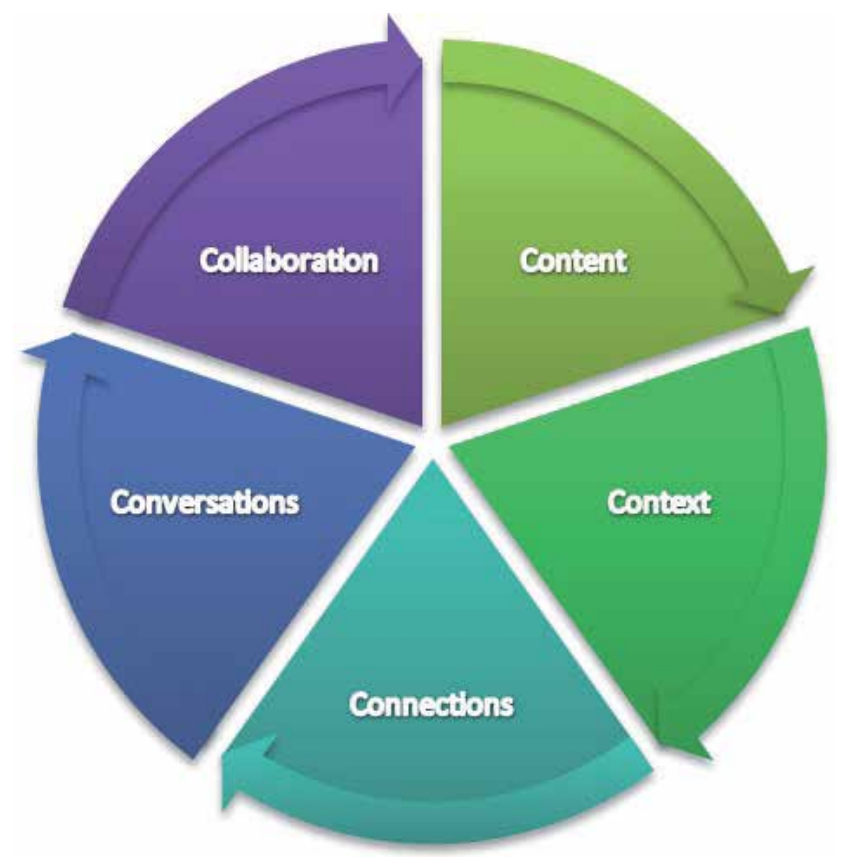

Fig. 5. The Five C's of Social Media: Adapted from Pick (Pick 2009)

The connections, conversations and collaboration aspects of social media work together to help establish Communities of Practice ( $\mathrm{CoP}$ ) online and support the social nature of $\mathrm{KMb}$. Wenger defines $\mathrm{CoP}$ as "groups of people who share a concern or a passion for something they do and learn how to do it better as they interact regularly" (Wenger 2006). Social media tools, such as Twitter, provide CoP with the ability to connect, share and interact on a regular basis, even if they can't meet in person. The concept of CoP is important to $\mathrm{KMb}$ as demonstrated by Cupp that operates on a CoP model (Hart and Wolff 2006).

In order to successfully share, mobilize and co-create knowledge within CoP, it is necessary to establish trust, reciprocity and relational ties, along with a shared vision, norms ( $\mathrm{Li}$ and $\mathrm{Li}$ 2010) and sense of community (Sangwan, Guan and Siguaw 2009) between members. These 
are all important aspects required for successful $\mathrm{KMb}$ and these elements can be seen in Twitter communities:

- $\quad$ Trust is gained by being a reliable and respected source of information;

- Reciprocity comes in the form of retweeting others' tweets;

- Relational ties are established by following others tweets and by being followed. Both strong and weak ties are present- some community members will be people you know in real life or others will only be known to you online;

- Shared vision, norms and sense of community come from using the tool itself. Those who are active on Twitter know how it works and agree to the rules simply by using it.

Also important to $\mathrm{KMb}$ is the presence of knowledge intermediaries or boundary spanners (Tortoriello, Reagans and McEvily 2011). These are individuals who are involved in multiple communities and who can transfer knowledge and help develop relationships across groups. In institutional knowledge mobilization units, like York's KMb Unit, knowledge brokers act as boundary spanners both in real life and online.

York's KMb Unit uses social media and specifically Twitter to disseminate and exchange information. We connect to others who share similar interests and form ties with individuals and organizations because of a mutual participation in these social media. Social media are tools for knowledge brokers and can help span boundaries. We hypothesize that we are better brokers because we use social media tools, but these tools will always supplement not replace -knowledge brokers who are central to $\mathrm{KMb}$.

Key messages:

- More research is needed to understand how social media can be used to mobilize social science research

- Social media can be used to disseminate and exchange research and knowledge

- Social media, especially Twitter, can help to connect individuals sharing a common interest and thus support a community of practice.

\section{Conclusion}

This chapter has drawn from the literature to show how $\mathrm{KMb}$ and related activities are social processes. These processes include how the social sciences and humanities along with other organizations and institutions attempt to address some of the ongoing wicked problems and more general problems in society to create greater social benefit. The chapter has shown how these processes benefit from interactive methods of $\mathrm{KMb}$ that focus on the relationships between researchers and decision makers, as well as how $\mathrm{KMb}$ is emerging as an institutional capacity that includes the use of knowledge brokers supported by social media. We illustrated these concepts with examples from Canada, the US and the UK and provided details about York University's institutional KMb support services. The stories from York's $\mathrm{KMb}$ practice illustrate that $\mathrm{KMb}$ is a process that results in social innovations. We then presented the case and some preliminary evidence to show that social media, especially with the use of Twitter, are tools that have the potential to support the social processes of $\mathrm{KMb}$. 
We will close with three predictions for the future development of KMb.

1. $\mathrm{KMb}$ will continue to develop at the institutional level:

Sandra Nutley observed four years ago that "the evidence that we have about developing effective organisational - and system - level research use strategies, while emerging, is still fairly thin on the ground (Nutley, Walter and Davies, 2007). York University, University of Edinburgh, University of Brighton and University of Texas School of Public Health have all made investments in institutional knowledge brokering. In the same way that almost all universities have developed a capacity to support technology transfer and commercialization we predict that universities will invest in $\mathrm{KMb}$ services that will support the co-production of knowledge by university researchers and their research partners from the community, government and private sectors.

York University leads ResearchImpact-RéseauImpactRecherche (RIR), Canada's KMb network. RIR is a pan-Canadian network of six universities ${ }^{1}$ who have all invested in knowledge brokers and an institutional capacity to support $\mathrm{KMb}$ for faculty, students and their local research partners. Such networked $\mathrm{KMb}$ has the potential to grow institutional $\mathrm{KMb}$ into a system of $\mathrm{KMb}$. System's level $\mathrm{KMb}$ will allow sharing of best practices, professional mentoring and has the potential to make research from one jurisdiction in the system accessible to decision makers in another jurisdiction.

2. Social media will evolve as a tool to broker relationships between researchers and decision makers:

Many organizations are moving from experimenting with social media to embracing social media as part of their business. We illustrated above that Twitter can support a community of practice and $\mathrm{KMb}$ collaboration. As more research and decision maker organizations adopt social media we anticipate that these tools will evolve from tools that disseminate information to tools that support collaboration and engagement. Despite this predicted adoption of social media, we anticipate that social media will become tools that will complement but not replace knowledge brokers.

3. Knowledge brokering will evolve as a profession:

Many organizations, some of them described in this chapter, have created professional knowledge broker positions to link research to policy and practice. Where there is critical mass, these local knowledge brokers are forming communities of practice. One example is the Ontario Knowledge Transfer and Exchange Community of Practice (www.ktecop.ca). The Toronto-based KTE CoP is funded by a grant from the Canadian Institutes of Health Research, has more than 300 members, has a social media presence through its blog and holds regular professional development meetings. Another example is the Knowledge Brokers' Forum (http://www.knowledgebrokersforum.org) which is an online community of practice for knowledge brokers in the field of international development.

As the profession of knowledge brokering grows there will increasingly be opportunities for professional exchange among knowledge brokers. On December 7,

\footnotetext{
${ }^{1}$ Memorial University of Newfoundland and Labrador, Université du Québec à Montréal, York University, University of Guelph, University of Saskatchewan, University of Victoria
} 
2011 the London School of Economics is hosted a knowledge brokers and knowledge intermediary workshop titled Bridging The Gap Between Research, Policy and Practice: The importance of intermediaries (knowledge brokers) in producing research impact (http://www.genomicsnetwork.ac.uk/cesagen/events/title,24718,en.html).

Knowledge Mobilization Works (www.knowledgemobilization.net) is an Ottawabased consulting firm that is organizing the Canadian Knowledge Mobilization Forum in June 2012. These conferences and workshops will help to build the profession of knowledge brokering.

In 2012, we see knowledge mobilization embedded within research projects, research units and research institutions. $\mathrm{KMb}$ is developing as a profession in many disciplines. Knowledge brokers are networking locally and nationally and national systems of $\mathrm{KMb}$ are forming. In 2007, Sandra Nutley concluded that evidence for organizational and systems level $\mathrm{KMb}$ was only beginning to emerge. What a difference five years makes. As our world is now part of a social network of knowledge and innovation - we look forward to being part of the next five years of knowledge mobilization.

\section{References}

Agrawal, Ajay K. 2001. “University-to-industry knowledge transfer: literature review and unanswered questions." International Journal of Management Review 3(4): 285302.

Bennet, Alex and David Bennet. 2008. Knowledge mobilization in the social sciences and humanities: moving from research to action. Frost, West Virginia, USA: MQI Press.

Boaz, Annette, Juan Baeza, Alec Fraser and the European Implementation Score Collaborative Group. 2011. Effective implementation of research into practice: an overview of systematic reviews of the health literature. BMC Research Notes 4:212 doi:10.1186/1756-0500-4-212.

Boyd, Danah M. and Nicole B. Ellison. 2007. "Social Network Sites: Definition, History and Scholarship." Journal of Computer-Mediated Communication 13(1): 1-20.

Brodhead, Tim. 2010. “On Not Letting A Crisis Go to Waste: An Innovation for Canada's Community Sector." The Philanthropist 23(1): 93-117. Accessed August 14, 2011. http://www.thephilanthropist.ca/index.php/phil/article/view/813.

Boyd, Danah M. and Ellison, Nicole B. 2007. "Social Network Sites: Definition, History and Scholarship." Journal of Computer-Mediated Communication 13(1):1-20.

Cahill, Geraldine. 2010. "Primer on Social Innovation: A Compendium of Definitions Developed by Organizations Around the World." The Philanthropist. 23(3): 259272. Accessed August 14, 2011. http://www.thephilanthropist.ca/index.php/phil/article/view/846 .

Camillus, John C. 2008 “Strategy as a Wicked Problem." Harvard Business Review 86(5): 99-106.

Campbell, Andrew and Nick Schofield. 2008. "The Getting of Knowledge: a guide to funding and managing applied research." 2nd edition. Land \& Water Australia. 
Accessed August 14, 2011. http://lwa.gov.au/files/products/land-and-wateraustralia-corporate/pr061240/pr061240.pdf

Davies, Huw, Huw, Nutley, Sandra and Walter, Isabel. 2008. "Why 'knowledge transfer' is misconceived for applied social research." Journal of Health Services Research \& Policy 13(3): 188-190.

Dede, Chris. 1999. "The Role of Emerging Technologies for Knowledge Mobilization, Dissemination, and Use in Education" Accessed October 22, 2011. http:/ / www.virtual.gmu.edu/edit895/knowlmob.html.

Finin, Tim, Anupam Joshi, Pranam Kolari, Akshay Java, Anubav Kale, and Amit Krandikar. 2007. "The Information Ecology of Social Media and Online Communities." AI Magazine 28, 3: 1-12.

Fisher, Tia. 2009. "ROI in Social Media: A Look at the Arguments." Database Marketing \& Customer Strategy Management 16(3): 189-195.

Gaffield, Chad. 2011. "In the new model of innovation people matter." Canadian Government Executive. Accessed August 14, 2011. http:/ / cge.itincanada.ca/index.php?cid=312\&id=13982\&np=1

Gagnon, Michelle. 2009. "Knowledge dissemination and exchange of knowledge." Knowledge Translation in Health Care: moving form evidence to practice Eds. Sharon Strauss, Jacqueline Tetroe and Ian D. Graham. Chichester, UK: WileyBlackwell.

Graham Ian D. et al. 2006. “Lost in knowledge translation: time for a map?" J Contin Educ Health Prof 26(1):13-24

Hamid, Saad. 2007. "Web 1.0 vs Web 2.0, the Difference." Sizlopedia. Assessed October 22, 2011. http://www.sizlopedia.com/2007/08/18/web-10-vs-web-20-the-visualdifference/.

Hart, Angie, Elizabeth Maddison and David Wolff. 2007. Community-university partnerships in practice. Leicester: National Institute of Continuing Education.

Hart, Angie and David Wolff. 2006 "Developing local 'communities of practice through local community-university partnerships'." Planning, Practice and Research 21(1): 121-38.

Honeycutt, Courtenay, and Susan C. Herring. 2009. "Beyond Microblogging: Conversation and Collaboration via Twitter." Proceedings of the 42nd Hawaii International Conference on System Sciences. Big Island, HI. IEEE: 1-10.

Huberman, Bernardo A., Daniel M. Romero, and Fang Wu. 2008. "Social Networks that Matter: Twitter Under the Microscope." Accessed November 17, 2011. http://ssrn.com/abstract=1313405

Hynie, Michaela, Krista E. Jensen, Michael Johnny, Jane Wedlock and David J. Phipps. 2011. "Student learning from community engaged graduate internships." Education and Training 53(1): 45-56.

Institute for Work and Health. 2011. "Tapping the tree of knowledge: How Jane Gibson bridged the research-to-action gap." Accessed September 10, 2011. http://www.iwh.on.ca/at-work/65/tapping-the-tree-of-knowledge. 
Java, Akshay, Song Xiaodan, Tim Finin and Belle Tseng. 2007. "Why we twitter: Understanding microblogging usage and communities." Accessed October 22, 2011. http://ebiquity.umbc.edu/_file_directory_/papers/369.pdf

Kaplan, Andreas. M. and Michael Haenlein. 2010. "Users of the world, unite! The challenges and opportunities of social media." Business Horizons 53: 59-68.

Lavis John N. et al. 2003. "How can research organizations more effectively transfer research knowledge to decision makers?" The Milbank Quarterly 81: 221-248.

Lavis, John et al. 2003. "Measuring the impact of health research:

Assessment and accountability in the health sector." Journal of Health Services Research Policy, 8(3):165-70.

Li, G. X, and Y. J. Li. 2010. "Knowledge Sharing Behaviour in Learning Online Communities: A Social Capital Perspective." IEEE ICMIT. IEEE: 910-915.

Linder, Stephen, Pimjai Sudsawad and David J. Phipps. 2011. "The Knowledge Translation Divide: What's the Canadian Secret?" National Conference on Health Communication, Marketing, and Media. Atlanta, GA. Accessed September 10, 2011. http:/ / www.cdc.gov/NCHCMM/pdf/ConfProgram2011.pdf

Lomas, John. 2007. "The in-between world of knowledge brokering." British Medical Journal, 334(7585):129-32.

Lorimer, Rowland et al. 2011. “Digital Technology Innovation in Scholarly Communication and University Engagement." Accessed August 14, 2011.

http:/ / tkbr.ccsp.sfu.ca/files/2011/02/Lorimer-DigInnovationScholCmn.pdf .

Mattson, Eric and Nora G. Barnes. 2010. "Blogging for the hearts of donors: Largest US charities use social media." Accessed October 22, 2011.

http:// www.umassd.edu/cmr/studiesandresearch/bloggingfortheheartsofdono rs/.

Michaelides, Roula, Matthew Tickle and Susan C. Morton. 2010. "Online Communities of Practice for innovation and knowledge transfer: A case study in the U.K. Management of Innovation and Technology (ICMIT). IEEE International Conference. Accessed October 14, 2011.

http:/ / ieeexplore.ieee.org/xpl/freeabs_all.jsp?arnumber=5492861.

Mina Singh and Michaela Hynie. 2008. "An evaluation of an inclusivity action plan." The International Journal of Diversity in Organisations, Communities and Nations 8(4):117124.

Morton, Sarah and Sandra Nutley. 2011. "What happens next? Getting Research into Policy and Practice" in Jamieson, L. Simpson, R. and Lewis, R.(eds) Researching Families and Relationships, Palgrave MacMillan, UK.

Mulgan, Geoff. 2008. "Social innovation: what it is, why it matters and how it can be accelerated." Accessed August 14, 2011.

http://www.sbs.ox.ac.uk/centres/skoll/research/Documents/Social\%20Innova tion.pdf

Myers, J. Gary 2010. “The Difference Between KM (Knowledge Management) \& KMb (Knowledge Mobilization)" Accessed August 27, 2011.

http:/ / kmbeing.com/2011/01/05/the-difference-between-km-knowledgemanagement-kmb-knowledge-mobilization/. 
Nutley, Sandra M., Isobel Walter and Huw T. O. Davies. 2007. Using evidence: How research can inform public services. Bristol: The Policy Press.

Phipps, David J. 2011. "A report detailing the development of a university-based knowledge mobilization unit that enhances research outreach and engagement." Scholarly Research and Communications. 2(2): 020502, 13 pp. Accessed February 5, 2012 http://src-online.ca/index.php/src/article/viewFile/31/57

Phipps, David J. and Danielle Zanotti. 2011. "It's the basement stories not the belt." Gateways Int'l J. Community Research \& Engagement. In press.

Phipps, David J., Danielle Zanotti and Michael Johnny. 2009. "Lessons learned from knowledge mobilization: turning research into action." Research Global 22: 2023.

Phipps, David J. and Stan Shapson 2009. “Knowledge mobilization builds local research collaborations for social innovation." Evidence \& Policy, 5(3):211-227.

Pick, Tom. 2009. "The Four C's of Social Media Marketing." Webbiquity, B2B Marketing Blog. Accessed October 22, 2011. http://webbiquity.com/social-mediamarketing/the-four-cs-of-social-media-marketing/

Policy Horizons Canada. 2011. The Social Innovation Exchange: A conversation with Louise Pulford of the Young Foundation, United Kingdom. Accessed August 14, 2011.

http:/ / www.horizons.gc.ca/page.asp?pagenm=2011_0061_Pulford

Rodriguez, Polly and Juliet Millican. 2008. “Community-university research engagement: The Cup research helpdesk." Community-university partnerships in practice: $32-$ 40. Leicester, U.K.: National Institute of Continuing Education.

Sangwan, Sunanda, Chong Guan and Judy A. Siguaw. 2009. "Virtual social networks: Toward a research agenda." International Journal of Virtual Communities and Social Networking 1(1): 1-13.

Strauss, Sharon, Jacqueline Tetroe and Ian D. Graham. 2009. Knowledge Translation in Health Care: moving from evidence to practice. Chichester, UK: WileyBlackwell.

Toronto Disaster Relief Committee. 2011. Protecting Vulnerable People From Health Impacts of Extreme Heat. Accessed October 14, 2011. http://www.tdrc.net/uploads/report_to_boh.pdf.

Tortoriello, Marco, Ray Reagans and Bill McEvily. 2011. "Bridging the knowledge gap: The influence of strong ties, network cohesion, and network range on the transfer of knowledge between organizational units." Organization Science 1-16.

Urquhart, Christine, Anne Brice, Janet Cooper, Sian Spink and Rhian Thomas. 2010. "Evaluating the development of virtual communities of practice that support evidence based practice." Evidence Based Library and Information Practice 4863.

Ward, Vicky, Allan House and Susan Hamer. 2009. “Knowledge brokering: The missing link in the evidence to action chain." Evidence \& Policy 5(3): 267-279.

Weng, Jianshu, Ee-Peng Lim, Jing Jiang, and Qi He. 2010. "TwitterRank: Finding Topicsensitive Influential Twitterers." WSDM'10. New York, NY. ACM: 261-270. 
Wenger, Etienne. "Communities of Practice: A Brief Introduction." Accessed October 13, 2011. http://www.ewenger.com/index.htm 


\title{
Re-Visiting Ethnographic and Orthodox Research Methodologies: Field Research Experiences from an African Perspective
}

\author{
Oliver Mtapuri \\ University of Limpopo \\ South Africa
}

\section{Introduction}

When undertaking research, there are many things that we take for granted. This concerns research being done either in an African, Asian, Western or any other context. This Chapter shall pay special attention to research carried out in an African context and draws out nuances that pertain to such a setting on the basis that other developing countries in Asia and Latin America might have their distinctive characteristics. Thus, this Chapter is informed by our own experiences as African researchers. A case study based on data from Mashonaland West in Zimbabwe is used to illustrate some of the nuances. Using Western tools of research in an African context has always been a challenge. For instance, English is the main tool of communication in the Western world whereas in Africa, there are different languages or dialects. Researchers have to find some ways to accommodate such specificities as languages or dialects. Women are not allowed to wear pants in certain African settings. Therefore, respondents may refuse to provide information to a woman researcher wearing pants. Yet, doing research while wearing pants, for a woman in a different context may well be acceptable. Casley \& Lury (1989) observe that:

The special difficulties of conducting surveys in developing countries derive from their socio-economic structure. Agriculture is still the main occupation of most of the population and it presents particular problems. Climatic and soil conditions may vary within one country. Some areas may be inhabited by nomadic pastoralists and others by small farmers. Thus there will often be wide regional disparities particularly since the differences in physical conditions will usually be accompanied by cultural differences (Casley et al., 1989).

There are no traditional barriers when negotiating entry for research in Western countries. In most cases the major barriers are the Ethical Committees at local level and satisfaction of the legal requirements such as the Data Protection Act and the Human Rights Act among others in the case of the UK. In Africa, a researcher has to negotiate with local Chiefs and some very limited extent with the government through ministries or departments of health if the research involves human subjects. This explains why most drug tests are done in Africa and this suggests some exploitable and slipshod laxity on the part of African governments on that score. At Universities, researchers have to obtain permission from the 
relevant Ethical Committees of that University who may approve or not approve the projects based on its relevance and quality and the treatment of human subjects in a humane way and guarantees of the observation of its ethical codes and standards. Furthermore, the poor state of infrastructure in rural areas of most African countries makes access to respondents very difficult. However, the major challenges faced by researchers when doing research in an African context relate primarily to data collection and sampling. This Chapter will attempt to unpack those challenges and dilemmas when doing research in such a context using essentially an interpretivist paradigm. The key components of the paradigm are 'subjective perceptions and understanding; which arise from experience; objective actions or behavior; and context' Ulin et al., (2002:22). The Chapter will also posit a few alternative methods with respect to data collection.

\section{Questionnaire dilemmas}

Western research methodologies do not take into account African development stages/dilemmas. Africa is a continent clamouring for education, technology, research capacity and skills. It is on these pre-requisites of development, among others, that she is lagging behind. In the rural areas of Africa, illiteracy is still rampant. The challenges experienced when using a questionnaire in an African context may relate to misinterpretation of the questions due to low levels of literacy. This implies that content errors will be committed as a result of the misinterpretations. Questions may also not be answered leading to non-responses. Whole questionnaires may also not be completed. Furthermore, one has to take into account the challenges of language. The multiplicity of languages in one country may present a challenge to researchers. South Africa has eleven official languages. According to the Ethnologue - Languages of the World, the number of individual languages listed for Zimbabwe is 20. Of those, 19 are living languages and one (1) is a second language without mother-tongue speakers (Lewis, 2009). However, Zimbabwe has two dominant languages, namely Shona and Ndebele with a multiplicity of dialects. This means that the research teams inevitably have to be multi-lingual in composition. Language use in the design of the questionnaire itself is also a problem. Care has to be taken with regard to wording and general phraseology. Different words mean different things to different people. For instance, the "you" would be very disrespectful in a research context which in Shona, a dominant indigenous language in Zimbabwe, would be, "iwe". A researcher cannot use such a word in research questions as that may offend the respondent if s/he is a household leader who would prefer to be called "imi", its "you" but used in its plural form as if addressing many people.

When the questionnaire is researcher administered, there is always the danger of the researcher bringing in his/her biases into the research. Most qualitative research is pragmatic from the point of view of the researcher and subjective from the point of view of the subjects. However, the researcher must strive to desist from leading the respondents and bringing in his/her bias into the research. Seasoned researchers have the capacity to do so which comes through (self-) training and experience. Structured questionnaires tend to channel people in a particular direction, directive approach, which are researcher-defined, and usurp discretion from the respondents who will withhold his/her "views', if not covered in the questionnaire, due to these 'structured responses' created by the researcher. It 
must be admitted that structured questionnaires are easy to analyse as compared to the unstructured ones. The trade off would be between the unquestionable ease of analysis provided by structured questionnaires or depth and variety of responses provided in unstructured questionnaires. These decisions rest squarely with the researcher.

Because of poor literacy levels in a rural African context, there is need to dedicate more time for holding the interviews or for questionnaire administration. Unstructured questionnaires and interviews give more discretion to the respondent to delve into issues based on their own volition, non-directive approach. This has often been found to be the case. Alternatively, respondents because of being "over-researched" tend to provide the researcher with the answers that the researcher expects in order to get rid of him/her as soon as is possible. To gain the people's confidence the research has to ensure the confidentiality and anonymity of respondents and the researcher must remain genuine/authentic and honest and also perhaps allow respondents to choose their own pseudonyms for the purposes of the study. Creating research partnerships, between the respondents, who purvey the information, and the researcher/learner/co-interpreter, carries with it serious ethical obligations anchored on mutual trust and understanding of common interests (Ulin et al., 2002). Hammersley \& Atkinson (cited in Silverman, 2001) posit that respondents are more concerned with the researcher as a person than the research itself - whether 'he or she can be trusted, what he or she might be able to offer as an acquaintance or a friend, and perhaps also how easily he or she could be manipulated or exploited'. The advantage of an 'outsider' is that respondents may be more inclined to discuss issues of a personal nature such as love and lust with them than with an 'insider' to avoid unnecessary mortification and humiliation. The bottom-line is that the questionnaire should better be administered by the researcher in cases where illiteracy is commonplace.

\section{Interviewing conundrum}

Interviewing is the process of directing a conversation to collect information (Angrosino, 2007). In an African setting we observe that interviews have their own advantages and disadvantages. One of the disadvantages is that the interviewer may push for his agenda that is, introduce interviewer bias as mentioned earlier. The advantage is that interviews cater for all people irrespective of their background, education, gender and other considerations. Face to face interviews are ideal for matters of a personal nature such as sexuality which should be understood based on personal experience as well as what people and society say about it - personal experience and what people say inform behavior. Telephonic interviews are hampered by the paucity of technology because in the rural areas, homes do not have telephones in many an African context.

\section{Focus group enigma}

Some topics are not ideal for Focus Group Discussions (FGDs). People cannot freely air their views. Some people may not feel free to answer questions in front of others and thus may not fully participate in the discussions in spite of the noble objectives the discussions/interviews may have. People may not be comfortable either in the company of others or the researcher herself. Some do not speak out because of lack of self confidence or plain respectfulness, timidity, and even reticence. Those who have the confidence, 
enthusiasm and buoyancy, dominate discussions with potential to hijack it. Some moderate their answers in the presence of peers. Group interviews tend to exert influence on respondents and in turn influence the outcomes of the research. Some tend to toe the line in the presence of others. Mixed gender groups may be considered appropriate in some contexts but not mixed age groups because of the sensitivity of certain customs. (By way of caveat, even mixed gender groups should be carefully considered because in some settings, African men prefer to have their early morning chats in the kraal with the exclusion of women. Their inclusion relies on the topic to be discussed). Even then, focus group discussions in mixed groups are characterized by male domination. Moreover, for cultural reasons, some people may not want to be isolated and be put into a group of people whose values they do not share. For example, Muslim women in Northern Nigeria would not want to be put in the same focus group with Christian women from the South and by extension, Muslim men may not want to be grouped with Muslim women. These create problems. Thus FGDs are important and relevant for matters of mutual interest such as community, social events but judiciously constructed.

\section{Participant observation riddle}

According to Angrosino (2007), observation is the act of perceiving the activities and interrelationships of people in the field setting. Participant observation is unique in that it combines the researcher's participation in the lives of the people under study while also maintaining a professional distance (Fetterman, 1998). In an African setting, those who are being observed may not feel free to be in the company of a stranger. To go about this, it may be meritable to employ researchers who are locals because they can literally 'connect' with their people. This could be particularly true for studies that seek to understand cultural aspects of a people. The disadvantage of using the services of local people is that the obvious things to them may be unique to a stranger and thus interesting to research. This may result in rich grassroot level data not being captured by using their services. Casley et al., (1989) claim that 'insiders' may 'over-identify with the subjects' such that their lines of inquiry are restricted. People tend not to trust strangers in their midst leading to deception of researchers. Furthermore, people usually want to be compensated for their time. Some of this could be attributable to economic disadvantage and some of it as due payment/recognition for effort.

\section{More on the divide}

\subsection{Cultural divergence}

There are differences in how issues can be conceived in an African as opposed to a Western perspective. The differences could be cultural/traditional, literacy and moral differences. A culture (Western) that allows both males and females to wear pants will not have a problem with a woman researcher to wear pants when doing research. As earlier mentioned, a culture that does not allow women wearing pants in an African context may not allow enumeration to be done by a woman wearing them. The appropriate dress code has to be observed. Makoni (n.d) observes that euthanasia, homosexuality and sex change are embraced in the Netherlands but are considered taboo in Zimbabwe. This is a reflection of the legal and moral schism between the two countries. 
Elderly people do not want to be "cross-examined" by the young ones on moral and cultural grounds, for example, on issues of family, governance and sex-related matters. They do not take to it lightly. Questions related to those matters should not be assigned to young researchers lest they be rebuffed and snubbed. The elders are the custodians of cultural heritage and indigenous knowledge of their communities which they pass on from generation to generation. Thus, secrecy is commonplace regarding ages-old tradition and taboos such that it becomes difficult to obtain credible information on such traditions and taboos. There are topics which cannot be discussed publicly or shared in public because they are thought to be sacred and sacrosanct. For example the initiation ceremonies among the Venda people in South Africa (most ethnic groups in Africa actually try by all means possible to keep their ceremonies sacred and guarded). Participants to those ceremonies do not easily share that information. This is different from Western respondents. One would find that some teenager girls do video tape their debut sex encounter to share with their friends. Issues related to sex and sexuality are neither discussed between fathers and sons, nor can that conversation transpire between mothers and sons (and fathers and daughters) in an African context. It may be slightly different in urban areas where western culture has been adopted. However, this shows that the level of openness differs in each of these two contexts. Cultural prohibitions on divulging issues related to initiation schools, mean that accessibility to facts becomes problematic. Even when respondents agree to be interviewed, the reliability of the information provided may still remain questionable given the 'sacredness' of the practice which is guarded and preserved jealously. Information will not be provided to a non-initiate because of the thorough screening process which involves elaborate test questions. It is unethical to use hidden cameras. Therefore there are those things that are considered to be sacred and therefore are held in secrecy. Those things become very difficult to observe overtly. Covert access, which is access secured without the respondent's knowledge, raises ethical issues and may well endanger the researcher's life if the researcher is found out and his/her disguise is publicly unmasked. Because Africans secretly guard these practices, sexuality among blacks is portrayed as uncultivated, unfettered and insatiable against a western self-portrait of 'restraint, purity, calculation and rationality' (Marais, 2005:15). Such ostentatious and conceited inferences are unwarranted and misplaced to the extent that such generalizations inspire wrong conclusions.

Diversity is not always embraced in certain settings as people tend to look inward to their own cultural and traditional practices to the exclusion of other perspectives. In that sense inclusivity is wantonly and shamelessly disregarded. Furthermore, many things are not properly documented in an African context, this makes researching them very difficult in cases of secondary data search.

\subsection{Seasonality}

Most African contexts are affected by seasons as in other contexts. There are times when people are too busy that they can hardly have time to take questions for example during rainy season and harvesting periods. Researchers have to accommodate these periods. This is particularly true when the researcher has to undertake research using participatory methods such as Seasonal Calendars and Gender Daily Calendar. Seasonality calendars can cover themes such as water availability, disease occurrence, patterns of household food security (e.g maximum food availability and food stress periods), labour demand and so 
forth. Daily activity charts show people's routines and chores from morning to evening during both dry and rainy seasons while seasonality in terms of disease occurrences may cover both animals and humans (bilharzias, cholera, malaria and measles). These are key ingredients in, for instance, preparing a community's action plan. What is important is to be aware of the moments when it is opportune to do research without causing severe disruptions to daily chores in their lives.

\subsection{Communication tools}

Another challenge that a researcher has to take into account is the issue of "communication tools" that one uses. Whereas in western countries, they can easily make use of the internet, mobile phones and so forth. This can be totally different in developing African countries as most successful interviews have to be done face to face. Financial resources and equipment are a huge problem for African researchers. They sometimes cannot afford to buy the photographic, Personal Data Assistant (PDA), audio and video equipment to undertake their research. Thus, the approach of using technology as a means of doing research is definitely different in an African context as opposed to a western one. In the West, researchers have a wider access to the internet which can be used to dispatch questionnaires and the PDA, particularly in the Health sector, has been found to be useful with potential for quick turnaround times between data entry and processing and reporting. Overall, their use has enhanced the quality of the data.

\subsection{Power dynamics}

African and western cultures show differences in the manner in which they open up to strangers/researcher. In an African setting, there are issues which one cannot discuss with a stranger/researcher which implies that respondents will withhold information making it difficult to collect data. In an African setting, it is extremely difficult to have access to respondents, wife and children, without the explicit permission of the head of the household who is usually male (in a patriarchal society). Therefore, culture is another challenge that one would have to contend with when using western methodologies. As an example, the head of a household in most African traditional set ups is a male and it is a male who normally speaks to a stranger - in this case a researcher. This means that gender dynamics are also another challenge which implies that a researcher cannot talk to or interview a woman alone in the absence of the husband or a male relative. All these situations tend to influence the type of responses that a researcher gets. In other words, these challenges have a bearing on the outcomes of the research in an African context. In essence, it seems there is enjoyment of privilege by males that power and authority bequeaths upon them because of their gender in an African context. This would be interpreted from a western perspective as a reproduction of inequities when the purveyors of this custom view it as normal. Gender stereotyping is also commonplace. In a place like Gwanda in Zimbabwe, one cannot talk to the wife without the approval of the husband. (In some places, men and women do not use the same toilets). This implies that researchers ought to be culturally, politically and contextually sensitive as they do their research in any given setting. Samples at the individual level are not always easy to build in African context - for example a woman is less likely to take questions in the absence of their husbands if they are married due, again, to the power dynamics at play. This is different from the West, for example, in UK or the 
Netherlands, women are free to take questions and in most cases they do not need the approval of a man to speak their mind or to answer questions. The power dynamics between males and females, between genders - between males and males; and females and females - are at the core of the melee for equity in the distribution of power, opportunity, authority, self-esteem and resources between traditionalists/cultural conservatives and feminists.

\subsection{Accessing respondents}

Access can also be limited because one has to consult the traditional authorities who use their discretion to grant the researcher the permission to canvas information from his/her subjects. Entry has to be negotiated. In some rural areas of Zimbabwe, for example, a family may not easily entertain "strangers" to come into their midst without the knowledge and sometimes approval of the Chief/gatekeeper. This means that the Chief needs to be informed of the research and its purpose before it is undertaken. He can either refuse entry or approve it. He can also disapprove the subject to be discussed, for example if it threatens their cultural values. Traditional authorities can thus make or break a survey or study. It is no wonder that people can boycott the study if the chief does not approve of the study. Traditional protocol has to be observed. It is advisable to know the ethics and norms of the people one is going to research and know how to talk and address them without offence. Participation is not always easy to secure in some contexts. The challenge is also that how do you assemble people without paying them, if the intention is not to compensate. Concomitant to this is the challenge of assembling people at the same time where they have to abandon their usual chores for this data collection event.

The following example shows the span of authority of the Chief. The third national census of South Africa since the attainment of democracy in 1994, Census 2011, took place on 10th 31st October, 2011. In terms of the Statistics Act of South Africa, it is obligatory for citizens to take part in the census. It was reported on National TV that a Chief, in the KwazuluNatal province, had refused entry to census enumerators because his subjects had not been offered the first opportunity to work as enumerators. Given the importance of the exercise to the country and after elaborate negotiations and concessions with the chief, his subjects were eventually enumerated. This illustrates the power and authority that chiefs/gatekeepers command in an African context.

Physical access to respondents can be impaired by the impassable roads, the forests and mountainous terrain that the researcher has to navigate. A researcher would need to establish a sample frame in order to draw a sample in such situations. There are a number of problems that a researcher would face in an African setting. For example, house numbers are found mainly in urban set ups and not in rural areas. It would be difficult to draw up a sample of houses in rural areas because the homes are not serially numbered or sequentially ordered and usually not located in a linear fashion (and often cyclical). It becomes a challenge to employ probability sampling methods such as systematic sampling in an African context. This is because the enumerator must cover all units in some sequence either even or odd until the last number in the sequence. The homeless also present their own challenges because of their homelessness and lack of fixed place of abode. The informal housing settlements in urban areas also pose their own problems as numbering is not sequential if at all it exists. 


\subsection{A case study based on my own experiences}

I did a study which focused on the perceptions of poverty in Mhondoro communal area which is located in Mashonaland West province of Zimbabwe.

While the abiding theme of my work was poverty alleviation and targeting in poor African countries such as Zimbabwe, the hypothesis central to my study was stated as follows:

Hypothesis: While income may be an aspect of poverty in Africa, access to assets is important in a self-definition of poverty that uses local norms as the measure of well-being.

Accordingly, the other questions that were subjected to interrogation were:

- What factors affect perceptions of poverty and thus should be taken into account in a consensual approach to poverty?

- What is the minimally adequate asset level (MAAL) that a person must have for him or her not to be considered poor?

- Is there a measurable threshold of assets that constitutes poverty?

- How many and what kind of assets do people need not to be poor?

- Does location matter?

\subsubsection{Aims of my study}

The study had one overarching objective: To establish an asset-threshold using the consensual approach through the eyes and experiences of the people of Mashonaland West in a self-definition of poverty which uses local norms as the measure of well-being.

\subsubsection{The research setting}

Mhondoro communal area is situated 40 kilometres from Harare, the Capital City of Zimbabwe. Villagers in the area grow plants and keep animals for day-to-day living. This communal area was chosen, firstly, because the area reflects a social landscape that is rural - this was one of the fundamental conditions underlying the quest for an assetthreshold steeped in a rural setting. Secondly, the researcher's familiarity with the location as well as knowledge of the local language, Shona, which is critical in understanding the perceptions of poverty and how these relate to assets. Shona is the dominant language spoken in this province. Familiarity with the location brings in some element of purposiveness in terms of site selection. Mashonaland West is not the only rural area representing a typical African setting. There could be other areas in Zimbabwe with similar conditions as elsewhere in Africa. This paper does not lay a claim to the generalisability of the results to the whole of Mashonaland West or Zimbabwe or Africa per se. Generalising results from case studies requires a judgment about the typicality of findings in the population about which a generalisation is made (Hammersley, 1992) cited in Shaffer (2002:48). According to Shaffer (2002) the crux of the issues lies in determining 'typicality' which in practice is very hard to do, especially given that the results [may be] highly contextual and may defy generalisation. According to the 2002 Census carried out by the Government of Zimbabwe, the total population of Mashonaland West province was 1,224,670 comprising 609,778 males and 614,892 females. About 72 per cent of the population of the province lived in rural areas while 28 per cent resided in the urban 
areas. The average household size was four persons. In terms of the type of dwelling units, 32 per cent of the households live in traditional dwelling units, while 40 per cent occupied modern dwelling units and the rest occupied a mixed type of dwelling units. About 66 per cent of the population had no electricity while 80 per cent of the population had access to safe water, either from piped water or from boreholes/protected wells. The remaining 20 per cent obtained water from unsafe sources such as unprotected wells, rivers and streams.

To gain entry I also had to obtain the permission of the kraal head despite the fact my father was born in that village. It was not a given that I would be allowed to collect data for my study without his permission. Since I was staying in the capital city and had last gone there many years back, my brother had to consult the kraal herd before I could do my study there. The kraal head knew my brother because my brother has built a house in the village and he commutes there every two weeks. He also has a small plot adjacent to his house on which he grows maize and vegetables. After negotiations on my behalf, permission was granted. In a way I felt like an outsider/stranger. However, after the permission was granted, work commenced. It was easy for the kraal head to assemble his subjects. He would hit with a heavy metal rod for about six to seven times onto a thick metal sheet shaped like a satellite dish which hung from a tree, which sounded to me like a Church bell, to call his subjects. In a few minutes, the villagers would start streaming from their roundavels and houses to the kraal head's compound. He announced the object of my visit and granted permission to his subjects to assist me with the information I needed for my study. With the kraal head's blessing, I had more households from whom I could canvass for information than I had anticipated.

I selected a sample of 100 households in the three villages of Zimucha, Chakavanda and Washayanyika These villages, collectively constituted my rural area for the purposes of my study. And a furthermore 25 households from the urban area of Chikonohono township in Chinhoyi for reasons of comparison. Chinhoyi, which is located approximately 120 kilometres north-west of Harare, is the provincial capital of Mashonaland West. My sample sizes of 100 households for the rural area and 25 households for the urban area were informed by the limitations of the budget as well as time constraints. I collected my data from 10 December 2005 to 10 January 2006 at both rural and urban sites.

In terms of research design, I used the ballot (or poll or voting) in which people were given ballot papers as is done during parliamentary and presidential elections. Instead of voting for their member of parliament or president, they voted for the assets they deemed to be necessary to achieve an acceptable standard of living. All participants in the poll were given a unique number which was used to link their ballot papers to their demographic data. Each ballot paper had the name and picture of each asset. Three categories of ballot boxes were used for this purpose: Most Important, Important and Less Important. Accordingly, in the process of voting, participants were asked to place their ballot papers in the appropriate box. After the votes were cast, the ballots were counted before the assembly of villagers for validation.

Six focus groups in the rural areas and 3 in the urban area as well as interviews complemented the vote in this multi-method approach which collected both quantitative 
and qualitative data. Interviews were carried out covering demographic information as well as definitions of poor and rich persons. The first choice participants for Focus Group Discussions (FGDs) were heads of household. In their absence any member of the community participated because assets are 'neutral'. If the concern was income, the researcher would have involved primarily only income earners in the FGDs. During the main survey, six FGDs were held in the rural area: one adult male-only group; one adult female-only group; one adult mixed group; one youth-male group; and one youth-female group and one mixed youth group. In the urban area, only three FGDs were conducted as follows: one adult male-only group; one adult female-only group; one mixed youth group. Assets concerned those who had them as well as those who did not. On average, between 8 and 15 participants took part in the FGDs.

There were separate FGDs for males and females. The purpose of separating the males from the females was to allow the different genders to speak their minds freely. It was also of interest to delve into the different perspectives that men and women had on a subject and separation of genders allowed this. Men and women may have different perspectives on say a house. Men may need a house, but women may look beyond a mere house to its quality as well. Males and females are not homogenous social categories in terms of needs and aspirations. Mixed groups of both males and females were also used to affirm views, to engage men and women in debate (if there was such a debate) and to establish dominant views in constructive engagement. The mixed group FGD dealt with the issues of how many of the items they voted for were required to lead a minimally acceptable way of life as well as to determine the cost of each item.

Accordingly, FGDs were useful in the clarification and elaboration of social norms, experiences and practices; and providing a broad overview of perceptions of what constituted assets that were necessities of life for the people in the area. In the rural area, the list consisted of 32 items, while in the urban area it contained 37 items. These items were chosen by participants in FGDs after starting off with a short-list of 14 items. The preliminary list used in the urban and rural areas had the following 14 items: cattle, hut, land, plough, wheel-barrow, scotch cart, hoe, goats, spade, axe, bed, chickens, radio and donkey. In the urban area, the list excluded a cultivator and harrow mentioned in the rural area and also separated a mobile phone from a telephone as distinct assets. The people selected these items as a broad yardstick by which the poor could assess their lifestyle.

The consensual approach is where public opinion is used via a ballot to determine socially perceived necessities of life. (See Mack and Lansley (1985) who are the proponents of the methodology used in this study). Middleton (2000) notes that consensus can also be reached through discussion, negotiation and eventual agreement. Thus, the lack of certain goods and services which are believed by the majority of the population to be essential falls under consensual measures (ibid).

Because of the success of this first leg of my study, following the rapport that I had established with the kraal head and his subjects, I followed it up with another study in 2009. I have turned my study into longitudinal study. This case study was sourced from Mtapuri, $(2008,2010)$. 


\section{Alternatives}

\subsection{Workshops and seminars}

Workshops and seminars are a form of data collection. Facilitators will solicit ideas, opinions and views from different and disparate stakeholders on a matter. Brainstorming can be used to gather information. The flow of information should be encouraged back and forth, unfettered. A trained facilitator can make that possible. Rich and useful information can be obtained for further processing from these open discussions. The advantages are many. For example, stakeholders will have an open opportunity to air their views. Workshops and seminars usually come at a great cost. These costs include hiring a venue and payment to participants and facilitators. Because of paucity of resources in an African context, workshops and seminars can be held under a tree, in a marquee, tent, a classroom, and so forth.

\subsection{Open mic (microphone) sessions}

Open mic sessions are ideal for the youth (especially in urban areas). These are sessions in which everyone has a chance to air his/her views in front of everyone in an open and transparent manner. It allows people to vent their anger, emotions, joy and happiness at the issues that matter most to them.

\subsection{Storytelling}

There variants to storytelling. One of them involves sensitizing respondents on the theme for discussion so that the researcher and the respondent are on the same wave length. The researcher then asks the respondent what they know about the issue. He/she further probes as the conversation continues. Alternatively, an imaginary story is presented and a respondent is asked to relate it to their own experience. Second variant is for people to form a group. And then let each person tell their own story which is recorded on tape with their permission but recorded anonymously. By giving them the tape recorder, the researcher will be giving them control over what they say. The tape recorder is passed on like a baton stick to the next person who also tells his/her story. The third variant is to engage with the respondents one-on-one and the researcher then asks a specific question to kick start the conversation. From then on the respondent will tell his/her story. The fourth variant is done without giving any direct prompt. The researcher captures stories spoken when people are busy with their chores for instance baking or stories spoken as people gossip. It assumes an open discussion. No topics is pre-determined. It is conversation between people who know each other. It may be about their individual aspirations, community aspirations, politics, sexuality, infidelity and so forth.

\subsection{Event search}

This approach to data collection which I call event search is a way to understand how people deal with life experiences. It seeks to gather information on their ways of life at key events in people's lives. These events include weddings, marriages, ritual ceremonies, funerals and so forth. It is these events that speak to the real life experiences as they reminisce about themselves, friends, workmates, their rulers and leaders and their 
communities in general. These are the stories that shape and point to the way people live their lives. One will not be interested in the wedding or funeral per se but about what people say and talk about in relation to socio-political issues such as housing, electricity, sanitation, life in general and so forth. It is at funerals that people talk about HIV/AIDS and sexuality and even laugh at some of the issues.

\section{The etiquette challenges}

What would a researcher do if he/she is offered water to drink when he/she can see that the source of the water is dirty or the water does not meet his/her hygiene standards? In rural areas of Zimbabwe, people drink traditional beer from the same gourd - a gourd is a hardskinned fruit which is dried and hollowed out to make a bowl or cup - which rotates from one drinker to another without being washed. Skipping is grimaced and frowned upon. What would a researcher do if offered a gourd of beer/water that is in rotation? Some may consider it unhygienic while beer has been traditionally drunk that way from generation to generation. In Rushinga in Zimbabwe, the people smoke marijuana openly. Even children are allowed to smoke marijuana in this region. However, in the rest of the country it is illegal to smoke marijuana. What would a researcher do if he/she observes an illegal activity during his/her research and children are partaking in it? This poses an ethical challenge. In the same region, beer is brewed in a rotational fashion from one household to the next on a weekly basis as an income generation venture and a practice. Anecdotal evidence shows that women in the region even drink more than men because it is the responsibility of the women to brew the beer. This has been the case since time immemorial. In such circumstances the researcher must not judge the subject and colour or taint his/her research with his/her own biases.

\section{The challenges and the search for alternatives}

Table 1 below shows some challenges and possible alternatives. Judgment on the way to execute the research solely rests with the researcher. Interviews from a western perspective can fall within a continuum from being open (approachable) to closed (privacy is treasured/precious). Alternative methods are shown in the table.

\begin{tabular}{|l|l|l|l|}
\hline Method & Western way & African way & Alternatives \\
\hline Interviews & $\begin{array}{l}\text { Easily approachable } \\
\text { Some consider their } \\
\text { privacy as precious. }\end{array}$ & $\begin{array}{l}\text { You need to have } \\
\text { consent of chief } \\
\text { People expect some sort } \\
\text { of compensation for } \\
\text { participation like food } \\
\text { parcels } \\
\text { Not easily approachable, } \\
\text { secretive. }\end{array}$ & $\begin{array}{l}\text { Ensure confidentiality } \\
\text { and anonymity. } \\
\text { Researcher must } \\
\text { remain authentic and } \\
\text { honest. } \\
\text { Story-telling. } \\
\text { Photo narrative where } \\
\text { permissible }\end{array}$ \\
\hline $\begin{array}{l}\text { Questionnaire } \\
\text { Self } \\
\text { administered }\end{array}$ & $\begin{array}{l}\text { There are poor } \\
\text { response rates. }\end{array}$ & $\begin{array}{l}\text { Literacy rate - some } \\
\text { cannot write. The } \\
\text { researcher has to be sure } \\
\text { that people understand. }\end{array}$ & $\begin{array}{l}\text { Face-to-face } \\
\text { administration to } \\
\text { explain or to write for } \\
\text { them. }\end{array}$ \\
\hline
\end{tabular}




\begin{tabular}{|c|c|c|c|}
\hline Method & Western way & African way & Alternatives \\
\hline $\begin{array}{l}\text { Questionnaire: } \\
\text { Researcher } \\
\text { administered }\end{array}$ & $\begin{array}{l}\text { A westerner can } \\
\text { encounter language } \\
\text { and cultural barriers }\end{array}$ & $\begin{array}{l}\text { Translation from African } \\
\text { to English, and vice } \\
\text { versa is problematic } \\
\text { because of possible loss } \\
\text { of meaning. You may } \\
\text { lose meaning during } \\
\text { data collection, because } \\
\text { of the inability to get } \\
\text { some information } \\
\text { verbatim and still retain } \\
\text { original meaning. Thus } \\
\text { misinterpretation may } \\
\text { lead to wrong } \\
\text { conclusions. } \\
\text { Intrusive at times; } \\
\text { Could face language } \\
\text { barriers }\end{array}$ & $\begin{array}{l}\text { Face-to-face. } \\
\text { Ensure confidentiality } \\
\text { and anonymity }\end{array}$ \\
\hline $\begin{array}{l}\text { Focus Group } \\
\text { Discussions }\end{array}$ & High response rate. & $\begin{array}{l}\text { Unlikely to get } \\
\text { responses in groups } \\
\text { because people are shy } \\
\text { to speak about certain } \\
\text { subjects in public, e.g } \\
\text { HIV, sex etc. } \\
\text { Cultural norms may } \\
\text { dictate who participates } \\
\text { in the focus groups } \\
\text { People tend not to want } \\
\text { to talk about health and } \\
\text { politics in focus groups }\end{array}$ & $\begin{array}{l}\text { Identify a chairperson } \\
\text { from the group. } \\
\text { Researcher must stay } \\
\text { outside. Use tape } \\
\text { recorder with their } \\
\text { permission or } \\
\text { Use smaller groups for } \\
\text { discussions and couple } \\
\text { it with sport or any } \\
\text { other activity such as } \\
\text { baking for women } \\
\text { while discussing issues }\end{array}$ \\
\hline $\begin{array}{l}\text { Participant } \\
\text { observation }\end{array}$ & $\begin{array}{l}\text { Misunderstanding of } \\
\text { the culture. } \\
\text { Getting permission is a } \\
\text { challenge }\end{array}$ & $\begin{array}{l}\text { Misunderstanding of } \\
\text { people's way of life, } \\
\text { language, culture and } \\
\text { cultural codes. Sacred } \\
\text { traditions are difficult to } \\
\text { observe. } \\
\text { Getting permission is a } \\
\text { challenge }\end{array}$ & $\begin{array}{l}\text { A researcher would } \\
\text { need an interpreter to } \\
\text { decode the cultural } \\
\text { codes, language and } \\
\text { explain way of life. }\end{array}$ \\
\hline
\end{tabular}

Table 1. Challenges faced by researchers when collecting data in an African context

Table 2 shows the opportunities and challenges that a researcher can anticipate in an African context. Opportunities are galore in the realm of poverty, unemployment, rituals and customs, gender as well as a myriad of other social and economic issues, including the environment. 


\begin{tabular}{|l|l|}
\hline Opportunities & Challenges \\
\hline $\begin{array}{l}\text { Learn African rituals and customs e.g } \\
\text { initiation ceremonies }\end{array}$ & $\begin{array}{l}\text { The customs are considered sacred and } \\
\text { opportunities are lost to study them. Using } \\
\text { hidden cameras becomes unethical. }\end{array}$ \\
\hline Learn about gender and gender imbalances & $\begin{array}{l}\text { Values and beliefs embedded in cultures } \\
\text { and passed on from generation to } \\
\text { generation }\end{array}$ \\
\hline $\begin{array}{l}\text { To use photo narrative - in which you use } \\
\text { photos to prompt discussion. People say } \\
\text { what they see in the photo and asked to } \\
\text { draw parallels with their own experiences. }\end{array}$ & $\begin{array}{l}\text { Technology is not readily available and not } \\
\text { affordable to many people in the rural } \\
\text { areas }\end{array}$ \\
\hline To use overt observation & $\begin{array}{l}\text { Access is regulated by the chief, kraal head } \\
\text { or male members of the family }\end{array}$ \\
\hline To appreciate and celebrate diversity & $\begin{array}{l}\text { The existence of many languages means } \\
\text { that meaning may be lost during } \\
\text { translation from vernacular to English and } \\
\text { vice versa. Diverse groups keep their } \\
\text { customs, beliefs and traditions secret }\end{array}$ \\
\hline
\end{tabular}

Table 2. Opportunities and challenges

\section{Conclusion}

In this Chapter I presented challenges of doing research in an African context. There are differences in how issues can be conceived in an African as opposed to a Western perspective. The differences could be cultural/traditional, literacy levels or of a moral nature. This does not imply that doing research in Africa is fruitless or impossible but rather the Chapter warns the researcher of what to heed and pay attention to. It was also about presenting a worldview that thrives in an African context so that the researcher is better prepared to navigate his/her way in that space. People do successful research in an African context by findings ways around those apparent and fundamentally technical ambiguities presented by Western and African perspectives. To be a successful researcher in Africa and perhaps elsewhere, it requires that a researcher is culturally, politically and socially sensitive in the environment in which one is doing his/her investigations. This is helpful in appreciating what permissions need to be obtained to gain entry into communities, what methods are ideal for data collection in the circumstances, who should be the subject of the investigation, how would one draw a sample and what sampling method should one choose and so forth.

In this chapter we exposed the challenges found in an African context such as:

- The chief and the headman becoming important role players in surveys because without whose consent, access to subjects may be denied. The chief may approve or not approve the topic for the research;

- In some patriarchal communities in Zimbabwe for example, a woman may not respond to questions in the presence of the husband. It is the husband first as his prerogative, which he can delegate to her.

- Certain rituals like initiation are done in secrecy to preserve their "sacredness", which poses a challenge when doing research; 
- In places where respondents are illiterate, self administered questionnaires become redundant.

- A team of multi-lingual research researchers and assistants may be required due to the availability of a wide array of languages thriving in African communities. Translation from vernacular to English and vice versa may not capture the essence of the discussion and hence meaning may be lost in the process.

- When sampling, literature talks about numbering the houses, street by street in some sequence, for example, for systematic random sampling. But in some rural areas of Africa, such streets do not exist as roundavels/huts in a village are clustered in a manner that may defy sequential numbering.

The challenges highlighted above do present opportunities. It is for the avid and passionate researcher to navigate in this less understood but interesting and exciting space for research - the African terrain. The African terrain, while providing a seemingly contradictory and ambiguous tapestry when juxtaposed with a western one, it presents a diverse and rich terrain of unlimited possibilities for research. As my experience has shown, my snowball was growing as more and more people wanted to be involved in my study through word of mouth. This happened because I observed traditional protocol to the letter as well as all relevant research ethics governing my work. The sky is no longer the limit. I conclude with these perceptive words by Khotsa and Sithole:

Africa has the potential to become a world leader in almost all spheres of life, but potential remains potential as long as it is not explored and exploited (Khosa, 2009:11).

Knowledge production, scientific knowledge production in particular, should continually be informed by discourse battles and debates, openness to innovation and discourses from elsewhere (Sithole, 2009:98).

I choose to take the opportunities and still imagine a rich and flourishing terrain for knowledge production in Africa.

\section{Acknowledgement}

I would like to thank Jana Sertic who managed the publication of this edition, my colleagues Pudurai Justin Mazengwa, Judy Mohale, Nompumelelo Thabethe, Mamoloko Rangongo and Osden Jokonya for their counsel and thoughtful suggestions during the preparation of this piece. I am grateful to Prudence Lebese and Maritjie Du Toit for their support during the data collection and analysis process and my colleagues at the Turfloop Graduate School of Leadership of the University of Limpopo for their support and guidance throughout this project.

\section{References}

Angrosino, M. (2007). Doing ethnographic and observational research, Sage Publications, Thousand Oaks, CA

Casley, D.J \& Lury, D.A. (1989). Data Collection in Developing Countries, Claredon Press, Oxford 
Lewis, M. P.2009. Ethnologue: Languages of the World, Sixteenth edition. SIL International. Dallas, Online version:Accessed 20 October, 2011, Available on http://www.ethnologue.com/

Fetterman, D. M. (1998). Ethnography: Step by step (2nd ed.), Sage Publications, Thousand Oaks, CA.

Khotsa, LE. 2009. The Sunnyside of the Dark Continent, Reach Publishers, ISBN 97819920261566, Wandsbeck, South Africa

Makoni, M. (n.d). Another-mans-meat-another-mans-poison. Mazwi - Zimbabwean Literary Journal, Available at http://www.mazwi.net/editorials/another-mans-meatanother-mans-poison Accessed on 29 October 2011. ISSN 2042-9126 [Online

Marais, H. (2005). Buckling: The Impact of AIDS in South Africa, University of Pretoria, Pretoria

Mtapuri, O. (2008). Exploring local conceptions of poverty, wealth and well - being: Field evidence from Mashonaland West Province of Zimbabwe, Development Africa, Codesria, Vol. XXXIII, No. 3, pp. 35-44

Mtapuri, O. (2010). Developing a Poverty Index for African Economies using the Consensual Approach: The Case of Mashonaland West, Zimbabwe, Lambert Academic Publishing, ISBN 978-3-8383-4514-7,Saarbrucken, Germany

Silverman, D. (2001). Doing Qualitative Research: A Practical Handbook, Sage Publications, London

Sithole, MP. (2009). Unequal Peers: The politics of discourse management in the social sciences, Africa Institute of South Africa, Pretoria

Ulin, P.R. Robinson, E.T, Tolley, E.E, \& McNeill, E.T. (2002). Qualitative Methods, A Field Guide for Applied Research in Sexual and Reproductive Health, Family Health International, North Carolina 


\section{Section 5}

\section{Public Opinion and Citizenship}





\title{
Social Physics: An Interdisciplinary Way to Explore the Mechanism of Public Opinion
}

\author{
Yijun Liu and Wenyuan Niu \\ Institute of Policy and Management, \\ Chinese Academy of Sciences, Beijing \\ P.R. China
}

\section{Introduction}

After Conde put forward the idea of social physics nearly 200 years ago, the discipline has experienced such three development phases as classical social physics, modern social physics and contemporary social physics. As an interdisciplinary field, contemporary social physics uses the concepts, principles and methods of natural science to explore, simulate, export, explain and find out social behavior rules and economic operation orders with efficiently extending, properly integrating and rationally modifying (Fan et al., 2006). During the last 50 years, great progress has been achieved in this field.

Public opinion reflects the public on certain social reality and phenomenon in different historical stages, the integration of mass consciousness, ideas and emotion. The subject of opinion is the general public, the object is a particular focus of the community, and the ontology is the tendentious comments or remarks of this focus. "Public opinion comes before the unrest" has become consensus. Before any major social changes happen, there is always an aura from public opinion. During the changes, some oscillations will be caused on public opinion. After the changes, some public opinions will be persisted to guide new social changes as experience, preparation and reference. Public opinion can be viewed as a social behavior of the public and presentation of forming legal or moral restriction. It's helpful to build harmonious society. In contrast, it can also induce social trouble. Therefore, it's very significant to find out rule of opinion formation and evolvement and then guide opinion infection.

Social physics insisted that mechanism of opinion formation and evolution, same as process of common incident, involves latent period, active period and close period. Three main theories, social combustion theory, social shock wave theory and social behavior entropy theory are involved in social physics. When large-scale individuals or group discuss some incident together, it enters active period from latent period of opinion. That indicates that opinion is built step by step and formed at last by integration of local viewpoints with key points from opinion leader. The level of opinion formation during different stages can be quantitatively decided by number, scale and intensity. In the following parts, social physics with three main theories will be applied to study the mechanism of public opinion. Part 2 will briefly review the state of art by focusing on a wide list of disciplines ranging from 
sociology, psychology, politics, journalism communication, mathematics, physics and system science. Three theories of social physics will be introduced in part 3. In part4, social combustion theory will be used to study mechanism of opinion formation, social shock wave theory is used for exploring process of opinion evolution, and social behavior entropy theory is used to analyze behavior of participants, mainly opinion leader, in opinion ' $\mathrm{Ba}^{\prime}$ which means a virtual or reality environment.

\section{Review of the public opinion under different disciplines}

This part will firstly introduce the existing research on public opinion under Social Sciences \& Natural Sciences. Social science puts more emphasis on straightforward analysis of social observation and investigation with qualitative description methods. In contrast, natural science focuses more on the mathematics or physics mechanism of opinion with quantitative methods. The latter ways produce more abstract results and may detach research from the actual media events.

\subsection{Research on public opinion in the field of social sciences}

\subsubsection{Research on public opinion from sociological perspective}

Sociology is a comprehensive course for studying the social structure, function, occurrence and development through social relations and social behavior (Chen, 1999). From the sociological perspective, research on opinion relates to the effect on the opinion formation from the process of socialization, the impact on the individual opinions from social organizations and social economic structures, and so on. Sociology concerns about the phenomenon of social life (the object of opinion) directly related to people (the subject of opinion), advocates to scan these phenomenon through the integrated and comprehensive perspective (the ontology of opinion). Therefore, there is an essential relationship between opinion research and sociology.

\subsubsection{Research on public opinion from social psychology perspective}

Social psychology is a branch of psychology to study the social and psychological phenomena of individuals and groups (Sha, 2002). Some scholars directly interpret opinion as the people's social and political attitudes. This definition limits opinion in the scope of research on the society or social activities. Only the subject and ontology of opinion are involved. Research on opinion also concerned about the various social phenomena and problems (the object of opinion) associated with the social and political attitudes besides the above two concerns. That is, different scopes are respectively owned by opinion research and social psychology which focus mainly on psychological development of the social groups.

\subsubsection{Research on opinion from politics perspective}

Politics concentrates on countries, political parties, classes, the political system, political democracy, political power distribution etc. and explores some regular essence (Wang, 2005). Opinion is defined as the social political attitude, which means that politics can guide social attitude of the civil society (ontology of opinion) and object of opinion is viewed as 
the social phenomena and problems of politics. It can be more properly described as that opinion is people's direct or indirect attitude towards government. Opinion reflects the support or opposition of people and affects people's actions and the development of the situation. Politicians usually build a situation in favor of themselves and against the competitors with different opinions. Progressive opinion is often the precursor of the revolution.

\subsubsection{Research on public opinion from journalism communication perspective}

Communication is a science to study all human communication, development rules and the relationship between communication and society. In short, communication is majoring in how people exchange information with some symbols. Journalism media is very important for reflecting, forming and guiding opinion. To different subject of opinion, media can provide respective help. It accelerates the process of formation and evolution of opinion for the public, and helps to supervise, guide and control opinion for government. "Public opinion", as a classic in communication field, presents a panoramic view for opinion first time and lets people realize the various internal and external relationships among all kinds of opinion phenomena (Lippman, 2006).

\subsection{Research on public opinion in the field of natural sciences}

\subsubsection{Research on public opinion from mathematics perspective}

Usually, researchers build some mathematical models and then try to describe, explain, forecast or find out some rules based on analysis of these models. An early formulation of such a mathematical model was given by J.R.P. French in 1956 in order to understand the complexity of group's activity (French, 1956). In 1974, De Groot M. applied Delphi method to build consensus (De Groot, 1974). Lehrer \& Wagner regarded rational theory as fundamental condition of modeling for social opinion from justice level to epistemology level in 1981 (Lehrer \& Wagner, 1981). Especially, Abelson R., Friedkin N. \& Johnsen E. investigated how to achieve consensus or form social opinion from divergent thinking (Friedkin \& Johnsen, 1999). This solution can be described concretely as following.

Let $\mathrm{n}$ be the number of agents in the group under consideration. Each agent $i$ will not blindly accept or reject another opinion but consider other opinions totally and then form owned viewpoint. That is, opinion of each agent can be modeled by regarding other opinions with different weight value $w$.

French Model: with discrete conditions, weight value $w$ is constant.

Given the same hypothesis, opinion vector can be denoted with continues condition at time $t$ as

$$
x(t)=\left(x_{1}(t), x_{2}(t), \cdots, x_{n}(t)\right)
$$

Where, $x_{i}(t)$, opinion of agent $i$, is a real number. Let $w_{i j}$ be weight of effect on agent $i$ from agent $j$, then

$$
x_{i}(t+1)=w_{i 1} x_{1}(t)+w_{i 2} x_{2}(t)+\cdots+w_{i n} x_{n}(t)
$$


$w_{i j}$ is a variable of time. It relates to $x(t)$ at $t$.

$$
W(t, x(t))=\left(w_{i j}(t, x(t))\right)
$$

That is:

$$
x(t+1)=W(t, x(t)) x(t)
$$

This model can be simplified. Assume $W$ is a constant random matrix, then:

$$
x(t+1)=W x(t)
$$

The above procedure is the kernel process of social opinion modeling by De Groot and Lehrer.

Based on the model by De Groot and Lehrer, Friedkin and Johnsen consider opinion of agent $i$ lies on two factors. The first one is insistence of owned opinion, described as $g_{i}$. The second one is effect from other opinions, described as $1-g_{i}$. Formula (2) can be changed as

$$
x_{i}(t+1)=g_{i} x_{i}(0)+\left(1-g_{i}\right)\left(w_{i 1} x_{1}(t)+w_{i 2} x_{2}(t)+\cdots+w_{i n} x_{n}(t)\right)
$$

With matrix mode, it can be expressed as,

$$
x(t+1)=G x(0)+(1-G) W x(t)
$$

This is Friedkin \& Johnsen Model. The difference between this model and Abelson model is that differential equation instead of difference equation is involved in the latter on. These models often involve matrix theory, Markov chain and graph theory, etc.

\subsubsection{Research on public opinion from physics perspective}

The Ising model has been well-known to be a simple model providing profound physical significances, which is helpful for discovering principles in our physical world (Zhang, 2007). It has been not only conceived as a description of magnetism in crystalline materials, but also applied to various phenomena as diverse as the order-disorder transformation in alloys, the transition of liquid helium to its suprafluid state, the freezing and evaporation of liquids, the behavior of glassy substances, and even the folding of protein molecules into their biologically active forms.

We consider an Ising spins chain $\left(S_{i} ; i=1,2,3, \ldots N\right)$ with the following dynamic rules:

if $S_{i} S_{i+1}=1$, then $S_{i-1}$ and $S_{i+2}$ take the direction of the pair $(i, i+1)$;

if $S_{i} S_{i+1}=-1$, then $S_{i-1}$ takes the direction of $S_{i+1}$, and $S_{i+2}$ takes the direction of $S_{i}$.

These rules describe the influence of a given pair on the decision of its nearest neighbors. When members of a pair have the same opinion, then their nearest neighbors agree with them. On the contrary, when members of a pair have different opinions, then the nearest neighbor of each member disagrees with him (her). 
Deriving from the Ising model, Sznajd model (Stauffer, 2002a, 2002b), Krause-Hegselmann model, Deffuant model (Stauffer, 2005) and Galam model (Galam, 1990, 2003) have also been proposed for opinion dynamics.

\subsubsection{Research on public opinion under system perspective}

Systems Science focuses on the structure, function (including evolution, coordination and control) and general rules. China's famous scientist Qian Xuesen gathers up and unifies achievements from the different disciplines with a systemic perspective, reveals the general rules and nature of system, and then builds the theoretical basis for systems science. Objective of system science is various types of systems. According to amount of the elements and their different types, as well as degree of complexity of the relationships between different elements, systems are divided into simple system and complex system.

Some scientists have tried to study opinion from the perspective of systems science. For example, Haken H., a famous physicist, proposed viewpoints as order parameter of opinion formation and considered that change in the number of viewpoints $\left(n_{+}, n_{-}\right)$is a cooperative effect. Also, he insists that the formation of viewpoint will be affected by the same or the opposite viewpoint. Haken simply divides opinion into two contrary, positive and negative. That means, opinion is viewed as a simple system here.

\section{Three theories of social physics}

Social physics presents three main theories, social combustion theory, social shock wave theory and social behavior entropy theory (Niu, 2001). Social combustion theory focuses on mechanism of society stability. Social shock wave theory explores spatio-temporal distribution of society stability. Social behavior entropy theory is for essential research on society stability.

\subsection{Social combustion theory}

Social combustion theory, which carries a reasonable analogy between the natural burning phenomenon and social disorder, instability and turmoil, was proposed in 2001(Niu, 2001). In nature, burning involves not only physics process but also chemistry process. Physics process indicates physical balance conversation of energy, and chemistry process mainly indicates physical change and the related conditions. Burning occurred only if all three basic conditions, namely burning material, catalysis and the ignition temperature or the last straw, exist. That is, any of the three ones is indispensable. The mechanisms of combustion process in nature can also be used for reference during studying on social stability. In detail, the basic cause of social disorder, such as conflict between people and nature and the disharmony between persons, can be viewed as the burning material. The non-rational judgments, malicious attacks by hostile forces and deliberately one-sided interests of the chase will work as catalysis. When both of the above exist, even a small emergency become the ignition temperature or the last straw, thus result in mass incidents with a certain scale and some impact and then cause social instability and discord at last.

This research studies on the mechanism of opinion formation based on social combustion theory. There are wide ranges of attitudes, discussions and demands as a collection of 
burning material. The hierarchical structure in ba of the public opinion will create more opportunities to move closer to consensus, which can be viewed as social "catalysis". What ultimately triggers the formation of public opinion is usually an unexpected incident or an authoritative source of speech. That is "the last straw".

\subsection{Social shock wave theory}

The shock wave is one of the most important phenomena in the high speed of gas movement process. It is the strong compression wave produced by strongly compressed gas, also known as strong inter-section. The thin interruption is called as shock wave(Zhi, 2003). In this thin layer, speed, temperature, pressure, and other physical quantities changes quickly from the wave front value to wave behind value. Also, the gradient of speed, pressure and temperature are great. Therefore, the shock wave theory is not very concerned about the flow in wave, but just explores changes of physical quantities after going through the shock wave.

At present, some ideas are absorbed from the shock wave theory to solve complex social problems (Niu, 2001), especially for those problems with wave phenomena, such as traffic, the flow of people, etc. The crowded can be viewed as a continual medium because any crowd disturbance is spread in the crowd with the form of waves. Besides, due to individual differences, non-linear distortion occurs on waves, which may result in the shock wave, crowded accident.

\subsection{Social behavior entropy theory}

Social behavior entropy is the essence of social unrest. The entropy theory in physics field is used for preference to explain the composing of group from individuals. There are six principles of social behavior entropy theory (Niu, 2001), namely 1) Universal 'minimal effort' principle, 2) Pursue 'Minimum entropy' principle, 3) Keep 'psychological balance' principle, 4) Sustain 'EQ resonance' principle, 5) social orientation 'was the trend-U' Principle, and 6) Long for social convention that limits any other people except himself.

During the process of public opinion formation and evolution, we may mainly concern with 'psychological balance' principle and sustain 'EQ resonance' principle.

'Psychological balance' principle. If the individual could calm down through persuasion and self-reflection after suffering some unfairness, great help can be provided for the building of a harmonious society. In other words, by persuading the participants can achieve selfacceptance, self-awareness, self-experience and self-control.

Sustain 'EQ resonance' principle. 'EQ resonance' means that only the people who own most respect, reputation or approbate is allowed to play a role for persuading. Whether to the public or to government leaders, an example is very significant.

Individuals always unconsciously follow some of the rules from social behavior entropy theory. Moreover, the above two principles indicate that the individuals prefer to seek the emotional support and the dependence on attitude from opinion leaders. This explained the indispensability of opinion leaders. 


\section{Studying on the mechanism of public opinion based on three theories of social physics}

\subsection{To determine formation of public opinion based on social combustion theory}

Public opinion during the process of collection and formation is presented with two forms (Liu, 2002), view flow and action flow. With view flow, the public continually express their opinions on some of the social reality and problems to vent their unhappiness. If a high degree of consensus is achieved among the view flows and the demands of the people can not be met, view flow will upgrade to action flow. That is, the individual and unprompted actions become organized and purposeful campaign, to promote the mass outbreak of emergencies. The critical point of opinion formation is the moment when view flow upgrades to action flow.

Some of the social injustice invoked a psychological dissatisfaction of people, which plays an important role in preparation for the opinion formation. Those voices of appeal and cumulative negative effects, and so on, can be viewed as burn the material from the people's suffering. Besides, some sensitive words, such as 'the rich', 'money', 'official' and 'corruption' etc. will work as catalysis of pricking up public discontent (Liu et al, 2008). With the above to preconditions, even a small event can play a role of the last straw. At this moment, the three conditions of opinion formation are in place and a consensus of opinion has been reached. Without active response, the mass incidents leading to crimes against property and social stability will eventually happen.

As a result, research on opinion formation should focus more on the mass incidents caused by the day-to-day events, analyze the opinions against social order and stability derived from public debate or views through continual friction and integration, and then give a correct guidance or even destroy this destructive force in a timely manner to avoid unexpected incidents and protect the security of the people and society property. As an important part of early warning system, public opinion research will take a prediction role through the grasp of opinion formation mechanism.

\subsection{To simulation evolution of public opinion based on social shock wave theory}

\subsubsection{Concept modeling for qualitative analysis}

Qualitative analysis produces scenarios or hypotheses for the complex problems, i.e. to expose some qualitative relations or structures of the concerned problems. Opinion represents diffusion of the explicit awareness and presents ups and downs state. According to the different ability of people, there are different reactions. Wave ups and downs are exhibited because of the gap produced from different strength of evolution. One conceptual model of opinion diffusion is proposed as below.

Hypothesis: there are $N$ opinion subjects, each of them owns viewpoint $o_{i}$ where $i=1,2, \cdots N$. The three basic elements of opinion infection involves $\{\sigma$ change of public behavior Eenvironment of opinion infection $t$ time of opinion infection\}, as following,

$$
Y=F(\sigma, E ; t)
$$


where, $Y$ is speed of opinion infection on some social phenomenon or event.

Opinion diffusion is the process of choosing or being persuaded of each individual. Participants (or part of them) get agree on behavior finally. Therefore, the law of gravity can be referred to reflect change of individual behavior between moment $t$ and $t+1$ due to interaction. That can be expressed as,

$$
\sigma_{i}=\sum_{j=1}^{N} k \frac{o_{i} \bullet o_{j}}{d_{i j}^{\alpha}}
$$

where $k$ is the constant coefficient, $d_{i j}^{\alpha}$ represents the distance between the individual $i$ and individual $j, \alpha$ is the parameter of power, $o_{i} \bullet o_{j}$ describes the consistency between the individual $i$ and individual $j$.

If $o_{i} \bullet o_{j}>0$, individual $i$ has the same viewpoint as individual $j$, then individual $i$ will hold the original viewpoint.

If $o_{i} \bullet o_{j}<0$, individual $i$ has the opposite viewpoint to individual $j$, then, we can take following two conditions:

when $\sigma>0$, individual $i$ will hold the original viewpoint.

when $\sigma<0$, individual $i$ will change its proposition.

\subsubsection{Multi-agent modeling for quantitative simulation}

Of course, having an explicit concept model with mathematical expression does not mean at all that one has explicit mathematical answers. With the development of complex adaptive systems theory, artificial life and distributed artificial intelligence technology, MAS (Multi Agent Systems) provides a good approach to address these issues. Agent (intelligent subject) is abstracted from the study of specific entities, which has their own initiative behavior, and is a 'live' individual. Through establishing different decision-making rules for agents, a simulation model can be set up. In this paper, we use multi-based modeling to simulate the agents' behaviors with different interactive strategies to understand the dynamics of public opinion.

During opinion diffusion, the behaviors of the participants can be classified as 'conformity', 'power' and 'egoism'(Liu \& Gu, 2008; Liu et al, 2009). In detail, 'conformity' involves more psychological factors. Participants are fear of loneliness and obey to majority. 'Power' mainly involves the moral values. Whether power or prestige is decisive factor, which is especially important in China. 'Egoism' is driven by people's values. For some benefit, people may even change their words and deeds. Therefore, the 'conformity', 'power' and 'egoism' are fundamental for the establishment of opinion infection simulation rules. Three transfer rules of opinion can be defined as following:

- The 'conformity' rule: ask all their neighbors for their opinion, and then follow the arithmetic average of them.

- The 'power' rule: convince all neighbors particularly if two neighboring agents have the same opinion. 
- The 'egoism' rule: each agent select one discussion partner at one time step. If their opinions differ by less than the confidence bound, their two opinions mutually get closer without necessarily agreeing completely.

Simulation 1: Hypothesis, when $t=0$, the number of opinion subject (agent) is $N=10000$, there are $n=5$ types of viewpoints, and the transfer probability is $p_{O_{i j}}=p_{O_{i i}}=0.1, a_{p i}$ is a random real number between 0 and 1 . We require that only people with similar opinions talk to each other, namely, agents with viewpoint 1 can be talk with viewpoint 2 , but can not be contact with viewpoint $3,4,5$, if we defined the bounded is 1 . The initial random spatial status can be described as figure 1 .

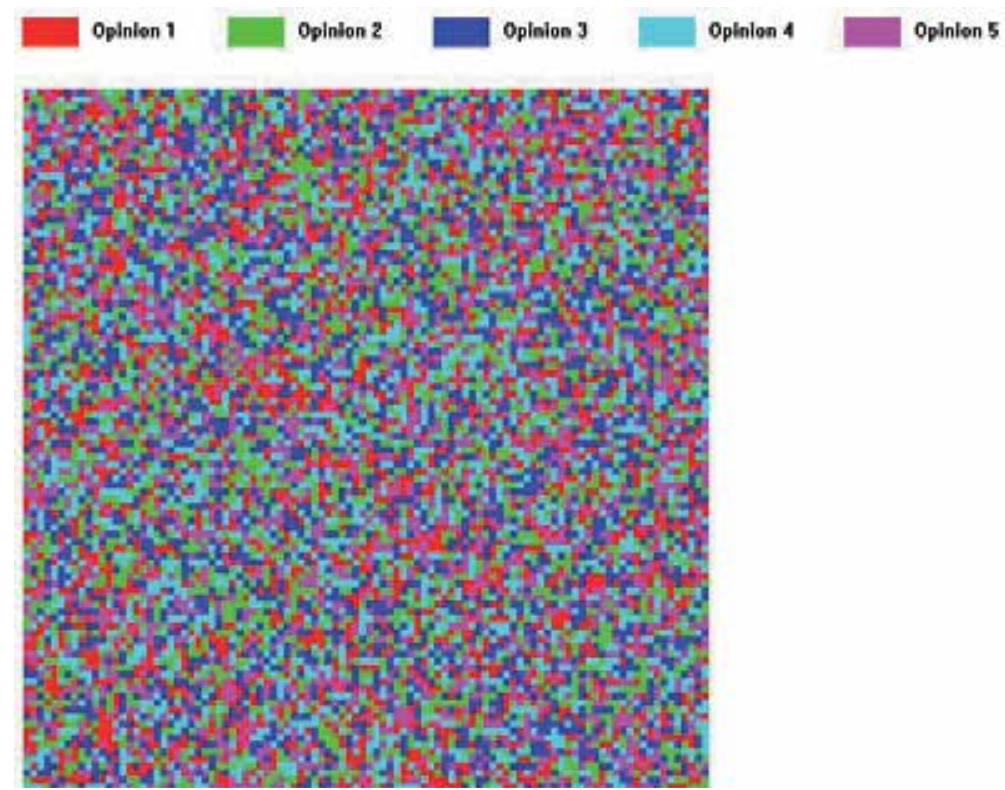

Fig. 1. Initial random spatial status of agents' distribution

According to the above conditions, with different rules of opinion transferring, respective results can be captured at $t=5$ as shown in figure $2(\mathrm{a}),(\mathrm{b})$ and (c).

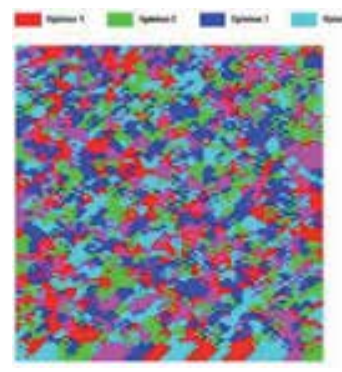

(a)

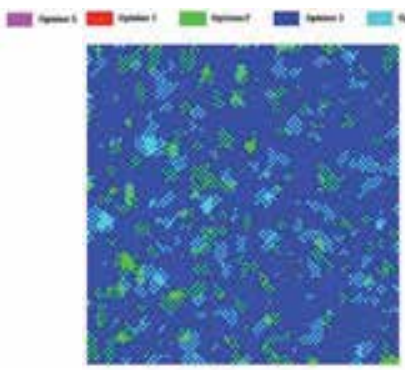

(b)

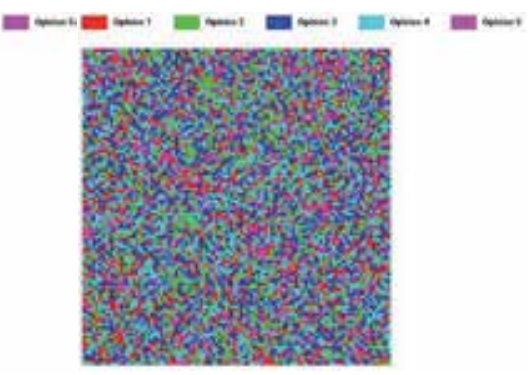

(c)

Fig. 2. when $t=5$, (a) is the agents' distribution with "power" rule, (b) is with "conformity" rule and (c) is with "egoism" rule. 
When $t=10$, the corresponding status is described as figure $3(\mathrm{a}),(\mathrm{b})$ and (c).

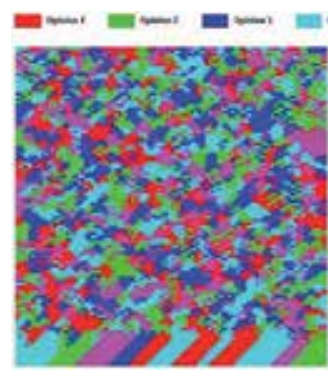

(a)

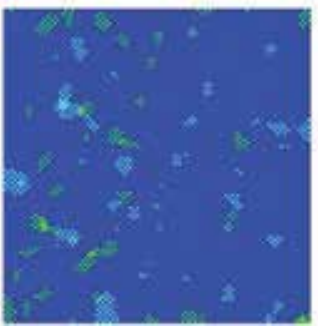

(b)

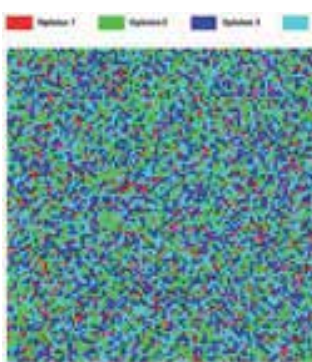

(c)

Fig. 3. When $t=10$, (a) is the agents' distribution with "power" rule, (b) is with "conformity" rule and (c) is with "egoism" rule.

When $t=20$, the corresponding result is shown as figure $4(\mathrm{a})$, (b) and (c).

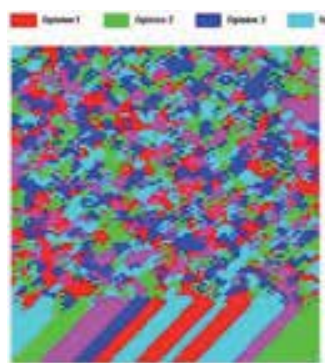

(a)

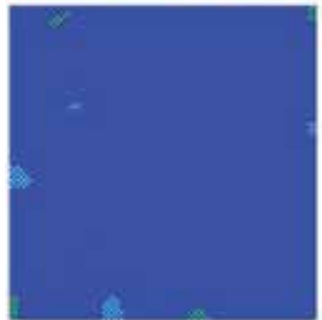

(b)

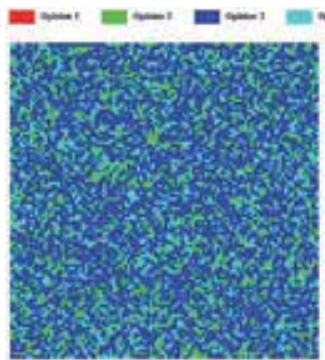

(c)

Fig. 4. When $t=20$, (a) is the agents' distribution with "power" rule, (b) is with "conformity" rule and (c) is with "egoism" rule.

Above are the opinions' spatial scenarios, figure 5 (a), (b) and (c) can be found whose temporal evolution process.

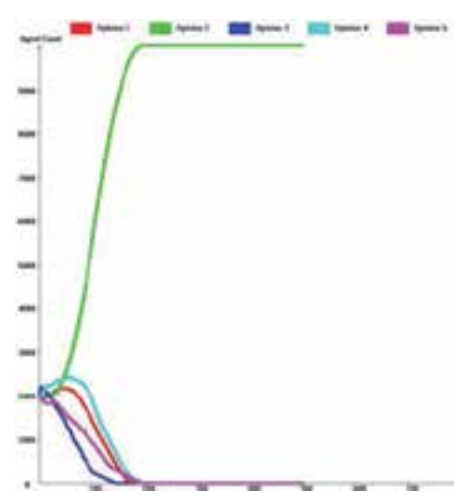

(a)

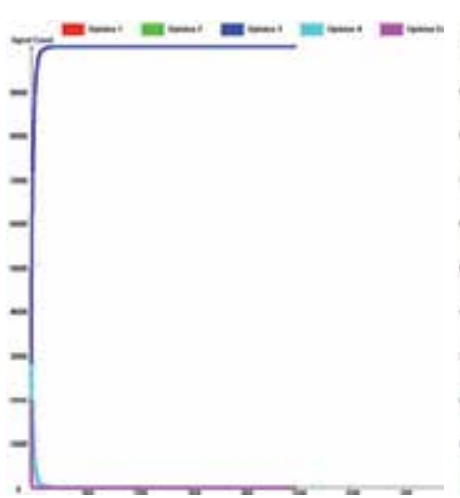

(b)

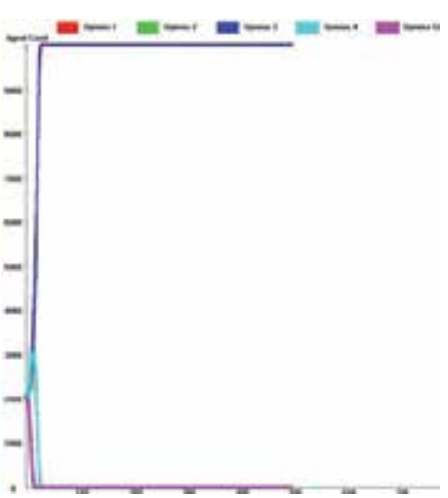

(c)

Fig. 5. From $t=5$ to $t=20$, five opinions temporal evolution process, (a) is the agents' distribution with "power" rule , (b) is with "conformity" rule and (c) is with "egoism" rule. 
Simulation 2: Hypothesis, most conditions are same with the simulation 1, only in $N=10000$ agents, triplicate individuals, namely $N_{1}=3333$, are prefer to "power" rule, $N_{2}=3333$ stand to "conformity" rule, rest of the $N$ adhere to "egoism" rule. The initial random spatial status also be described as figure 1. Figure 6 (a) and (b) respectively show the spatial status when $t=50$ and $t=100$, consensus formation can be captured at $t=500$ as shown in figure 7 . Temporal evolution process can be seen in figure 8.

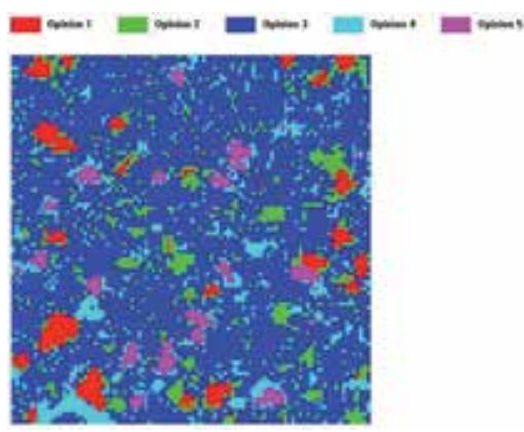

(a)

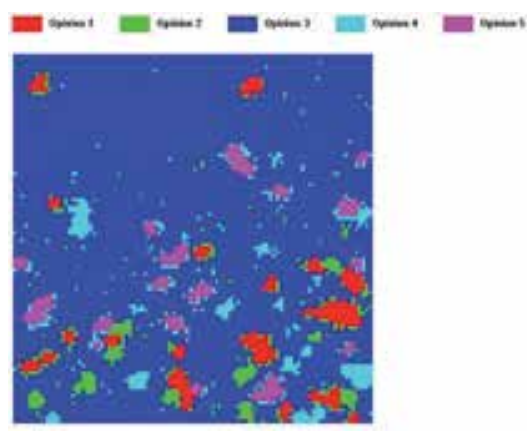

(b)

Fig. 6. (a) is the agents' distribution when $t=50$, (b) is the agents' distribution when $t=100$.

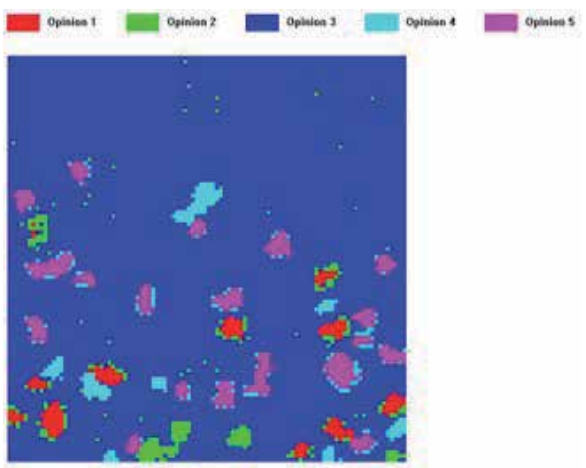

Fig. 7. Agents' distribution when $t=500$

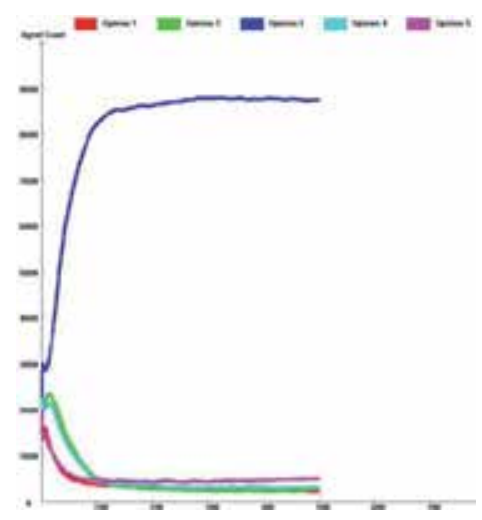

Fig. 8. From $t=50$ to $t=500$, five opinions temporal evolution process 
Based on the above simulations and analysis, some conclusions can be drawn as following.

For the simulation 1:

With the 'power' rule, given opinion subjects (agents) holding five viewpoints, the viewpoint supported initially by more agents will get agree among more and more participants during opinion dynamics. In the scenarios of this paper, the viewpoint 2 with green color dominates the process of opinion diffusion.

With the 'conformity' rule, an opinion subject (agent) will continuously get average value between the five viewpoints. Trend of opinion diffusion is able to be determined by viewpoint 3.

With the 'egoism' rule, viewpoint of each agent will be transferred depending on corresponding probability when meeting agents with same or different viewpoint. The process of evolution is slower than "conformity" rule in the same simulation steps.

For the simulation 2:

During the simulation steps, though viewpoint 3 is the consensus tendency in five viewpoints, but evolution speed is slowly than single preference by all the agents.

\subsection{To recognize opinion leaders based on social behavior entropy theory}

Hegselmann etc al. (Hegselmann \& Krause, 2002) figured out that opinion can be formed in a group as small as a few experts or as large as in the whole society. Based on this viewpoint, 4.3.1 of this paper will use meta-synthetic approach (MSA) and expert mining (EM) to identify and judge "expert leaders" during the process of experts argumentation. In 4.3.2, social network analysis (SNA) will be involved to find out the "opinion leaders" during the opinion formation and evolution over network.

\subsubsection{Recognizing opinion leaders in a group}

\subsubsection{Meta-synthetic approach and expert mining}

Meta-synthetic Approach(MSA), proposed by a Chinese system scientist Qian Xuesen (Tsien HsueShen), is one of the system methodologies to tackle with open complex giant system (OCGS) problems from the view of systems in the early 1990s (Qian et al, 1990). Here, we regarded OCGS problems such as social public opinion as ill-structured or wicked problems. This approach expects to unite organically the expert group, data, all sorts of information, the computer technology, and even scientific theory of various disciplines and human experience and knowledge for proposing hypothesis and quantitative validating. Later it is evolved into Hall of Workshop for Meta-Synthetic Engineering (HWMSE) which emphasizes to make full use of breaking advances in information technologies (Gu \& Tang, 2003, 2005).

Expert mining (EM), as a new mining method, is put forward based on the meta-synthetic approach ( $\mathrm{Gu}$ et al, 2008). This method emphasizes expert experience, ideas and wisdom mining. It is not built on the basis of mass data but in a smaller group of samples based on the thinking of experts to conduct in-depth experience in mining. This method is also different from those based on artificial intelligence-based expert system because it focuses 
more on people - machine, human-oriented to people's wisdom and the wisdom of the main groups. Mining expert system methodology, which combines science, scientific thinking and knowledge of scientific theories and makes full use of modern computer technology, is the development of the former theory and technology.

This section tries to identify and judge expert leaders by expert leader judgment module with guidance of MSA and EM.

\subsubsection{Hall for workshop of expert argumentation and expert leader judgement module}

Based on MSA, expert mining method and knowledge creation model, the Hall for Workshop of Expert Argumentation is to provide a distributed computer platform. On which, participants bring out new ideas and knowledge through communication and collaboration (Tang \& Liu, 2004; Liu \& Tang, 2005). The Hall integrates proposals and views from experts to build solution and compute quantitatively degree of centralization and consensus.

Aiming to the discussion topic, the Hall for Workshop of Expert Argumentation expresses the registered ID (shown in rectangular box) and keywords (shown in ellipse box) as a visualized two-dimensional map, as shown in Figure 9, The experts owning high degree of concerns will be centralized. This provides a new way to share knowledge and solve unstructured problems.

Discussion space is a joint thinking space for the participants. Via the 2-dimensional space, the idea association process to stimulate participants' thinking, idea generation, tacit knowledge surfacing and even wisdom emergence is exhibited based on the utterances and keywords from participants. The global structure and relationships between participants and their utterances are shared by all participants in the session. It helps the user acquire a general impression about each participant's contributions toward the discussing topic, and understand the relationships of each thinking structure about the topic between participants.

The expert leader judgement module of the Hall for Workshop of Expert Argumentation constructs the consistent matrix based on the sameness and difference of keywords from all participants. The largest eigenvector will be computed to achieve sort of speaker. The sort can also be used to exhibit contribution of each participant. The matrix A can be expressed as,

$$
a_{i i}=\left|U_{i}\right| \text { and } a_{i j}=\left|U_{i} \cap U_{j}\right|, i \neq j .
$$

Where, $U_{i}$ represents the set of keywords from No. $i$ participant.

After discussion, participants will be evaluated to help analyze quantitatively discussion result and try to find out key speaker based on effects on group from each participant. Those key speakers are "opinion leader".

Example: The Xiangshan Science Conference (XSSC, website: www.xssc.ac.cn), which is initiated in 1993 in similar to Gordon Research Conferences and denotes as the general designation of a series of small-scale academic meetings which bring together a group of scientists working at the frontier of research of a particular area and enable them to discuss 
in depth all aspects of the most recent advances in the field and to stimulate new directions for research, is a top-level science forum for interdisciplinary and cutting-edge studies and can be viewed as a platform for knowledge sharing and creation in China. Next we apply our tool to analyze Xiangshan Science Conference.

Figure 9 shows the process and result map of analyzing 'the brain, consciousness and intelligence' topic with experts meeting system. Detail of design and development of the system will not be explained here. Figure 9(b) is different from Figure 9(a) because one new expert ('Pan Yunhe') is added into the discussion. But the two maps own the same character that the expert 'Wang Yunjiu' locates at the center of the discussion. That indicates that he actively involved in the 'brain' research field and relative meeting. This result can be verified by the record in text mode from Xiangshan Conference.

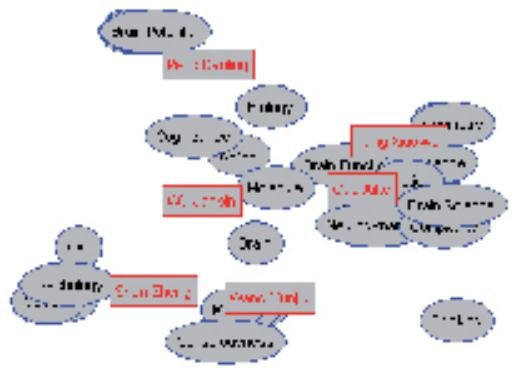

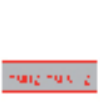

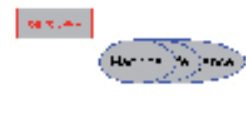

nuti: (a)
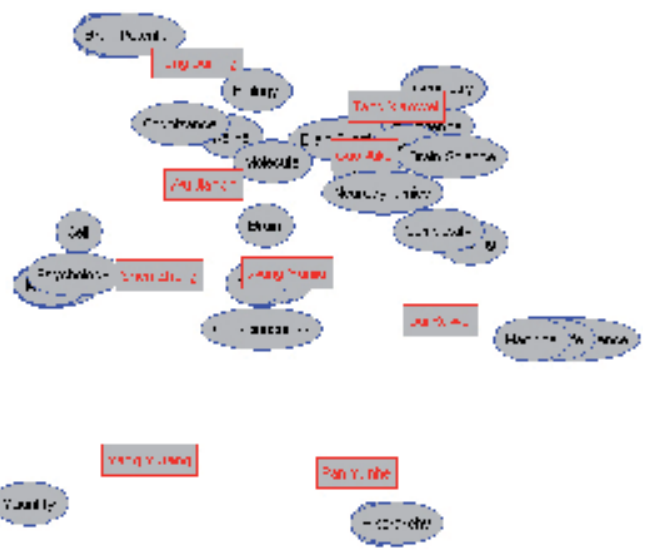

(b)

Fig. 9. Two-dimensional Distribution of Participants and Keywords

Table 1 lists the evaluation of participation based on agreement and discrepancy matrixes. It is shown that user 'Guo Aike' holds highest rank based on both eigenvectors, which may be justified by his active role as one of chairpersons or plenary speech contributors among those conferences, which furthermore exposes his big influence in neuroscience field in China.

\begin{tabular}{ll}
\hline Maximum eigenvector of agreement & $(0.3761,1.0914,0.3082,0.6179,0.2522,0.3618$, \\
matrix: & $0.3125,0.1937,0.1092)$ \\
\hline \multirow{2}{*}{ Rank of the top five participants: } & Guo Aike > Wang Yunjiu > Tang Xiaowei \\
& $>$ Peng Danling > Dai Ruwei
\end{tabular}

Table 1. Evaluction of 9 Participantions

Due to less staff and simple content, Prof. Guo Aike can not be defined as 'opinion leader'. Instead, 'leader expert' is better. However, such a new idea builds an important basis for research of identifying "opinion leader". 
The social network analysis proposed in the following section of this article can be used to identify "opinion leaders" from a large scale of participants.

\subsubsection{Detecting opinion leader in the society}

\subsubsection{Social network analysis}

Social network analysis (SNA), as a new paradigm for sociological research (Scott, 2007), is proposed in 1930s and enhanced in 1970s. This article intends to detect the 'opinion leaders' by this method. In fact, the opinion leaders are those special individuals who appear during the formation of opinion from microcosmic individual actions to macroscopical group behavior.

'Social network' refers to the social actors and the collection of the relationship between different actors. That is, a social network is a collection of a number of points (social actors) and the connection between the points (the relationship between actors). "Social network" emphasizes that each actor has a certain extent relationship with other actors. Social network analysis build models for these relationships, try to describe the structure of relations between group members and study the effect on group and individual from this structure.

Social network analysis can be used to identify quantitatively the 'opinion leaders' because this approach has exactly described the relationship between the subjects of opinion in a very good way. In which, the social network position refers to a series of individual actors who have the similar characters in social activities, relationship and interaction located in the same relationship network, network factor refers to combination of relations to link the social positions and mode of the relation between the actors or positions.

Some other concepts such as point, edge, degree, betweenness, cutpoint, component, subgroup and centralization and so on are involved in SNA. During the formation and evolution of opinion, this article particularly concerns the 'cutpoint'.

\subsubsection{Cutpoint in the SNA as the opinion leader}

In graph theory, the only one point connecting two sub-graphs is called as cutpoint. The cutpoint is very important because its absence will divide network into independent segments named after block. Such a point is important to not only network but also the other point, that is, cutpoint plays the "opinion leaders" role among the subjects of opinion.

Example: A series of serious terrorist attacks occurred in the in the eastern part of United States at September 11, 2001. With this incident, World Trade Center in New York, the Pentagon where U.S. Department of Defense locates in Washington and some other important buildings had been attacked and heavy casualties were caused. By the later survey, this is an organized and purposeful terrorist activity against the interests of the people, the U.S. security and even world peace. After that, not only the United States governments but also experts around the world analyze this incident in-depth for getting more meaningful and valuable information and forecasting such terrorism. Figure 10 (website: http:/ / www.orgnet.com/tnet.html) shows the social network analysis of key man of $9 \cdot 11$ terrorist events. 


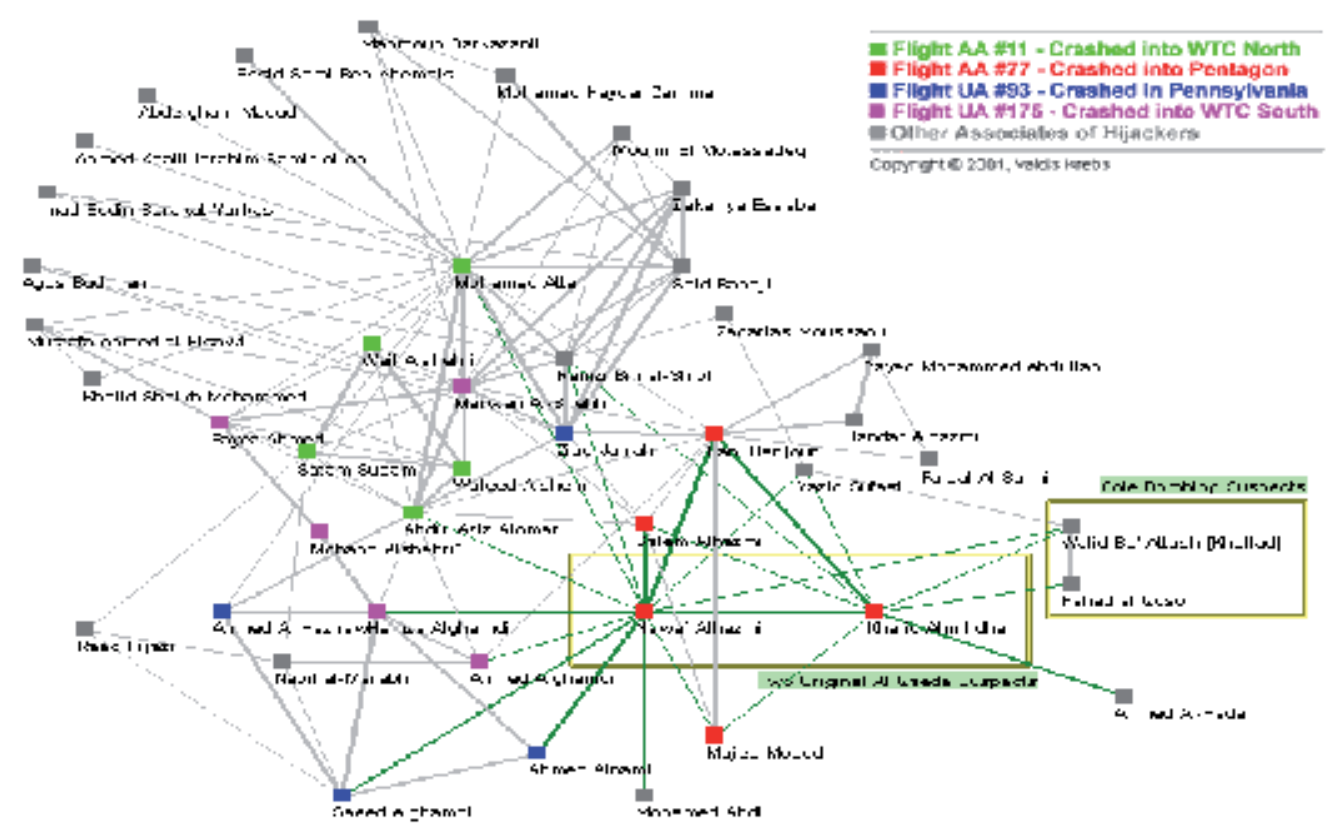

Fig. 10. Social network analysis of participants of $9 \bullet 11$ terrorist events

This case is involved here to indicate that social network analysis is a good method and technique to identify the "key persons". Analogously, opinion leader can be easily identified in a war of opinion through the "cut point" algorithm if the network topology of opinion subjects had been built out.

\section{Conclusion}

This paper tries to explore the mechanism of public opinion with perspective of social physics. Firstly, the research points out that public opinion are arisen by the herd behavior under the paroxysmal events, and then formed by the mass incidents based on social combustion theory. The development process is an important opportunity to catch essence of public opinion formation, which is useful for building a quantitative method to predict the herd behavior under the paroxysmal events and mass incidents. Then, opinion dynamics is explored and one kind of concept model and some simulate demonstrations based on social shock wave theory is produced. Such a kind of work aims to provide different perspectives for some systemic solutions instead of traditional ways toward social issues (topics about opinion). Forming of qualitative scenarios or hypotheses through concept modeling is the foundation for understanding the opinion's complex structure, simultaneously, multi-agent modeling as a core quantitative activity is also used to describe and analyze opinion's simulations based on assumptions. Finally, the public continually look for the emotional support and depend on opinion leaders due to the 'psychological balance' and 'emotional resonance' principles based on social behavior entropy theory. That is the root cause of generation of opinion leaders. MSA and SNA are studied to identify the 
opinion leaders and master their behaviors and traces for further exploring the nature of opinion and then effectively controlling and guiding opinion.

According to result presented in this paper and previous research, it can be summarized that the key value of opinion is from its prediction and alert function as a way to guide opinion infection, to get full, harmony and sustainable development of society. Of course, lots of further works are still under exploration. This paper only proposed two scenarios, more detailed simulation will be strengthened, three rules will be further explored in-depth, and the simulation platform for opinion diffusion will be gradually improved, etc. All which aim to explore more new perspectives, methods and ideas on public opinion, and provide theoretical and methodological support for building harmonious opinion society.

\section{Acknowledgment}

The authors gratefully acknowledge the support of National Natural Science Foundation of China (Grant No. 91024010).

\section{References}

Fan, Z.; Liu, Y.; Li, D.; Wang, Y.\& Fu, Y. (2007). Social Physics: The Forefront of International Research Perspective, Science press, ISBN978-7-03-019397-1, Beijing, China (in Chinese)

Niu, W. (2001). Social Physics: Significance of the Discipline's Value and its Application, Science, Forum. , Vol.54, No.3, pp.32-35 (in Chinese)

Chen, L. (1999). Public Opinion, China radio and television press, ISBN7-5043-3327-1/G, Beijing, China (in Chinese)

Sha, L. (2002). Social psychology, China renmin university press, ISBN7-300-07581-9, Beijing, China (in Chinese)

Wang, L. (2005). Study on Pubilc Opinion: Theory, Method and Foci of Society, Tianjin social sciences press, ISBN7-80688-051-8, Tianjin, China (in Chinese)

Lippman, W. (Translated by Yan, K. \& Jiang, H.). (2006). Public Opinion, Shanghai renmin press, ISBN978-7-208-06080-7, Shanghai, China (in Chinese)

French, R. (1956). A formal theory of social power, Psychological Review, Vol.63, pp181-194

De Groot, M. (1974). Reaching a consensus, Journal of American Statistics Association, Vol.69, pp118-121

Lehrer, K. \& Wagner, C. (1981). Rational Consensus in Science and Society, D. Reidel Publication Company , Dordrecht, Holland

Friedkin, N. \& Johnsen, E. (1999). Social influence networks and opinion change, Advances in Group Processes, Vol.16, pp1-29

Zhang, Z. (2007). Conjectures on exact solution of three-dimensional (3D) simple orthorhombic Ising lattices, In: arXiv, 0705.1045, Available from http://arxiv.org/abs/0705.1045

Stauffer, D. (2002a). Sociophysics: the Sznajd model and its applications, Computer Physics Communications, Vol. 146, pp93-98

Stauffer, D. (2002b). Sociophysics simulations, In : Arxiv, cond-mat, 0210213, Available from http://arxiv.org/abs/cond-mat/0210213v1 
Stauffer, D.(2005). Sociophysics Simulations II: Opinion Dynamics, In: Arxiv. Physics, 2F0503115, Available from http://arxiv.org/abs/ Physics/2F0503115

Galam, S. (1990). Social Paradoxes of Majority Rule Voting Renormalization Group, Journal of Statistical Physics, Vol. 61, pp943-951

Galam, S. (2003). Modeling Rumors: The No Plane Pentagon French Hoax Case, Physica A, Vol. 320, pp571-580

Zhi, Q. (2003). A discussion on shock wave, Journal of Guizhou Normal University (Natural Sciences), Vol.21, No.1, pp.25-27 (in Chinese)

Liu, J. (2002). Principle of Public Opinion, Huaxia Publishing Co., Ltd, ISBN7-5080-27965/G, Beijing, China (in Chinese)

Liu, Y.; Gu, J. \& Niu, W. (2008). Study on the Mechanism of Public Opinion Formation, Proceedings of the 15th Annual Conference of Systems Engineering Society of China, pp595-600, Nanchang, China, , Oct 10-12, 2008

Liu, Y. \& Gu, J. (2008). Systems Analysis and Modeling of Opinion Infection, IEEE International Conference on Systems, Man and Cybernetics, pp484-488, Singapore, Sep 4-6, 2008

Liu, Y.; Niu, W. \& Gu, J. (2009). Study on Public Opinion Based on Social Physics, Proceedings of the 20th International Conference on Multiple Criteria Decision Making, pp318-324, Chengdu, China, Jun 20-22, 2009

Hegselmann, R. \& Krause, U. (2002). Opinion Dynamics and Bounded Confidence Models, Analysis, and Simulation, Journal of Artifical Societies and Social Simulation, Vol.5, No.3, pp.1-33

Qian, X.; Yu, J. \& Dai, R. (1993). A new Discipline of Science - the Study of Open Complex Giant Systems and its Methodology, Chinese Journal of Systems Engineering \& Electronics, Vol.4, No.2, pp.2-12 (in Chinese)

$\mathrm{Gu}$, J. \& Tang,X. (2003). Some Developments in the Studies of Meta-Synthesis System Approach, Journal of Systems Science and Systems Engineering, Vol.12, No.2, pp.171-189

Gu, J. \& Tang,X. (2005). Meta-synthesis approach to Complex System Modeling, European Journal of Operational Research, Vol.166, No.33, pp. 597-614

Gu, J.; Song, W. \& Zhu, Z. (2008). Expert Mining and TCM knowledge, Proceedings of 9th Knowledge and System Sciences, Guangzhou, China, Dec11-13, 2008

Tang, X. \& Liu, Y. (2004). Computerized Support for Idea Generation During Knowledge Creating Process, Proceedings of Second International Conference on KEST, pp. 8188, Tsinghua University Press, Beijing, China, Apr28-30, 2004

Liu, Y. \& Tang, X. (2005). The Introduction of Some Mental Models and Tools for Creativity Support, Systems Engineering -Theory \& Practice, Vol. 5, No.2, pp. 56-61 (in Chinese)

Scott, J. (Translated by Liu J.). (2007). An Introduction to Social Network Analysis, Chongqing university press, ISBN978-7-5624-2147-4, Chongqing, China (in Chinese) 


\section{Section 6}

Enterpreneurship, Employment and Industrial Relations 



\title{
The Methodology of Formulating Iranian National Policy of Entrepreneurship: A Conceptual Framework
}

\author{
Hassan Danaeefard and Mohammad Reza Noruzi \\ Tarbiat Modarres University, (TMU), Tehran, \\ Iran
}

\section{Introduction}

Theorists believed that the "entrepreneurship improvement" can be the intervention matter of governments on the basis of market failure. This intervention becomes practical in the form of a pack named "policy". The process of policy making includes problem identification, instruction compiling, formulation, performance, evaluation, changing and finishing policy, but policy formulation (i.e. counting alternatives related to solving a public problem) is of high significance because of its efficacy on other stages. The methodology of policies' formulation can be advantageous in compiling efficient and effective policies for country's policy makers. But there is no clear methodology over entrepreneurship policy making. Iran like other countries pays much more attention to the entrepreneurship and its related issues and tries to be developed in this era. The researchers of this study are going to represent a conceptual methodology for compiling national policy of entrepreneurship in Iran. This methodology is represented in an innovative-analysis way through reviewing the knowledge and participation of author in planning the process of forming policy alternatives. Therefore, the process of counting policy alternatives and the manner of compiling national policy of entrepreneurship as its performing practical content will be performed after representing conceptual framework.

\section{Entrepreneurship, government and public policy}

There was no clear entrepreneurship till 1990. There were some scattered scientific works all over the world until the $1{ }^{\text {st }}$ decade of that year but no especial focus can be observed among these works (Hart, 2003, p.3). Then, the governments started a kind of change in their policy course on the basis of public management in order to encourage and facilitate entrepreneurship. In fact, this course change can be supposed as a reaction to quick and developing economic and social environments of countries which are represented in increasing technology, improvement evolutions, global competition growth, appearance of knowledge-based economic, economic and industrial reformation, increase of democratic values acceptance (significance) and development of private sector. The governments have detected entrepreneurship as a managing solution to these quick changes. In other words, 
the governments have entered the entrepreneurship into policy making system as a solution to a wide range of problems such as occupation necessity, integrating labor force, social integration, efficient and competition improvement, economical innovation and wealth production. Therefore, the government should be aware of accepting entrepreneurship policies according to economic, social, cultural and political circumstances and should emphasize various methods and measures of entrepreneurship policies. "Policy refers to a complex of government fixed and intended actions in order to solve a public problem (Alvani \& Sharifzade, 2008)." There are not many studies on entrepreneurship policies because it entered into public systems just in the last few decades. So, there are some discussions over main material and elements of entrepreneurship policy, territory and attributions. The main problem with forming new desirable offices environments and removing business obstacles is entrepreneurship policy but there is still limited knowledge over entrepreneurship identification as policy territory. The aim of this study is to represent some kind of methodology for compiling the national policy of entrepreneurship in Iran. The following questions should be responded in order to reach this aim: 1) what does public policy mean? 2) What is the wisdom source of government entrance into entrepreneurship? 3) What is the national policy of entrepreneurship? 4) What are the elements of entrepreneurship elements? 5) What are the performance evaluation measures of entrepreneurship policy? 6) What are the elements of the methodology of compiling national policy of entrepreneurship?

\section{The nature of public policy}

In general, the term policy refers to the treatment of a simple or more complex set of actors like a public officer, institution or policy maker in a public environment of activities (public transportation, supporting consumers etc.). Although it can refer to what the governments select in order to act or not to act. These kinds of definitions will be sufficient for public discourses but an exact definition is needed in order to systematizing thoughts of public policy theorists and facilitating affective relations.

Literature of politics is full of various definitions of public policy. Everyone who has written something on policy has represented a definition of policy too. In this part, it is going to have critical review on public, performing and understandable definitions of policy.

Eayston defines policy as the relation of public unit to its environment. This kind of definition is general and cannot clarify the limitations of this relation (Eyston, 1971, p.18). Tomas Dye declares that policy refers to whatever the government chooses to do or not to do. This kind of definition does not clarify that whether the selection of government refers to policy or not. Furthermore, definition of government major and minor activities is placed in public policies limitation which is not true (Dye, 1984, p.1). Richard Rose defines public policy in this way: Public policy includes a complex of activities related to each other which are performed in a long period of time and affect individuals and is not a definite decision. However this definition is not clear, it clarifies the idea that policy is a complex of activities not a definite decision (Rose, 1969, P.X). Finally, Friedrich states that policy is a proposed performance of a group, individual or government in a definite environment which 
prepares some occasions and obstacles in order to achieve, eliminate obstacle or benefit a chance.

An important point of this definition is that the policy is connected to an ultimate aim. The ultimatum of public actions may be hard to identify but the thoughtful aim is the necessary part of this definition. A disadvantage of this definition is that policy refers to what the governments do and not what is decided or proposed.

Anderson's definition seems to be the most comprehensible one. He believes that policy clarifies constant and aimed actions which are followed by an individual or some actors in order to pay attention to a problem. This proposition refers to what the governments do and not what is proposed or decided and it separates policy from a decision that is especial option among others. So, public policy refers to a complex of public actions which are taken in order to solve a public problem in a constant way (Anderson, 2000,p.4).

\section{The rationale of government intervention in entrepreneurship}

There are 3 main theories over public intervention or lack of intervention about economic, social and cultural affairs. In other words; there are 3 theories over the reasons of government entrance in different nation stages: 1) The theory of market failure (Brown; Lane, 1991, Wolf, 1988) 2) The theory of government failure (Hausman, 2008;Nelson, 1987). 3) The theory of public value failure (Bozeman, 2002). The theory of market failure can be the basis of government entrance into entrepreneurship from among other theories. Market failure will theorize goods and services in allocating resources and producing producer. Economists believe that the market will be failed through following 4 conditions i.e. it cannot produce goods and services in a suitable manner and allocate the resources in an efficient way.

- $\quad$ Market Power: The companies will decrease the production in order to increase prices and their benefits when they occupied market power. This will produce limited number of goods in non-competition markets and when price monopoly of some goods are in the power of one or several companies, the competition is ruined and numerous goods will be produced in non-competition markets. This means that incomes will be gathered by the persons who have the power of market in the cost of individuals who do not have this power.

- Externalities: Externalities mean that a third party, who is not competed in a trade, has received benefits or is imposed costs voluntary. For example, residential regions near Mehrabad airports suffer landing and flying noises (negative externalities) or in a positive condition, the rural around South Pars benefit road facilities of refinery although they bear the pollutions too. The costs and benefits are not calculated in bills of companies in both conditions. If polluting companies try to take actions they will pay more costs and as a result the price of good will be increased. The same result applies to positive externalities too.

- Public Goods: Public goods benefit people but people cannot be excluded from their utilization because of goods' nature whether they have paid the price or not, i.e. a productive company cannot benefit production of these goods itself because it cannot control efficiently produced goods. Therefore, free market is not capable of profit 
production. National defense is a sample of public good which benefits all the people when producing and no one can be excluded. All the individuals, who pay or do not pay taxes, utilize it. This is called free rider in economic matter. Free market would not produce such goods because it is going to gain benefit always.

- $\quad$ Equity: There is a kind of tend toward inequity distribution of income in a market system which is not fair. Everyone who a bargain negotiates and buys others power can quickly reach financial resources in a free market system. The people will receive bank resources whose repaying is proved. Some are always preferred to others in various realms. How this inequity can be decreased? The governments should intervene.

- Stability of Macroeconomics: Market system rises and falls. Sometimes it booms (economic growth is high and joblessness is low) and other times slack and depression rules over the market (economic growth is low and negative and joblessness is high). Resource allocation and goods production will not be efficient and productive therefore the government should intervene and balance the market.

\section{The emergence of entrepreneurship policy}

Until two last decades, the focus of industrial and economic develop was on a proposition that the main resources of economic growth is several big companies in countries which act as economic lever. The governments tried to make efficient or affective main parts of national economic through passing especial rules for companies, tax encouragements and performing supporting laws in order to decrease prices and making healthy competition. This economic paradigm ignored the role of new companies as main resources of entrepreneurship, job making and economic developers. In fact, governments' method for global competition improvement of big companies did not encourage or affect the appearance of new companies, economic entrepreneurship and national competition (Aundretch \& Thurik, 2001). As theorists declare encourage of innovative actions is the minor production of industrial policies during industrial ages.

In 1979, Brich (Brich, 1987) showed that the major portion of new occupations in U.S.A. was created by small enterprises. Public policy makers realized the significance of small companies after this representation. Governments started emphasis on small and medium sized institutes' policy compiling in 1980 and they emphasized small companies' establishment in 1990. The main axis this policy is focusing on measures for improving performing environments of small companies which is controversial topic of establishing new company.

On the basis of economic and development cooperation organization's document (OECD, 1995, 1997 and 1998) and European commission (1998), a tend to entrepreneurship policies growth related to joblessness growth in most of the member countries of this organization and European countries was appeared in the middle of 1990. As the findings of different researches on the basis of the connection of new companies, innovation and economic growth was publicized, the attention of governments to entrepreneurship improvement and encourage of entrepreneur responsible was intensified in the second half of 1990.

Researchers (Audretsch, 1995, 1999; Reynolds et al, 2004) assert that almost all governments have accepted that establishing small and medium companies is a significant factor in 
economic growth and this can be achieved by making job, innovation and efficient improvements in the structure of the company and government. However, governments in developed countries emphasize big companies, the emphasis focus is on environment improvement for new and developing countries. In other words, researchers (Audretsch \& Thurik, 2001) believe that entrepreneurship policy appearance is a reaction to changing from managed economic to entrepreneur economic whose attribution is transition from "industrial economic" to "knowledge-based economic", "structure and production part" to "service part", "big companies" to "small companies" and from "small companies" to "new companies".

\section{A holistic entrepreneurship policy}

This policy is the most comprehensive policy in the typology of entrepreneurship policies including measures of three other types. National policy of the state aims to reduce blocks on the road to entry and exit, ease access to resources for better businesses (finance, information and technical and managerial aids) and meeting the needs for establishing target groups. However, promotion of entrepreneurship culture necessitates a positive atmosphere for entrepreneurship and inserting it into the educational system.

The main motive for the holistic view is reaching high levels of dynamism, innovation, efficiency, and growth through entrepreneurial activities or creating a more entrepreneur community. This view helps states have a more comprehensive perspective on the domain of their failures (systematic failures, social failures, educational failures, and market failures) and focus on positive side effects of entrepreneurship in order to affect short and long-term development of an entrepreneur community. The results of a study (Stevenson and Lundstrum, 2001) revealed that only four of the thirteen countries under study had taken this view. The following features distinguished these countries from others:

1. There are clear statements on the importance of business dynamism (rate of entry and exit activities) over innovation and economic growth and the share of new firms in efficiency and general competitiveness of the economy in upstream documents.

2. The state has plans to accelerate entrepreneurial activities in its policies' documents with the wisdom, long-run targets, operational objectives of the policy and set of measures and priorities.

3. Policy includes quantitative operational objectives aiming to increase the number of people conducting entrepreneurial activities, rate of founding companies, number of entrepreneurs or level of entrepreneurial dynamism.

4. The budget funds innovative entrepreneurial plans (not just for few of the measures that may be in accordance with the framework).

5. They perform the entrepreneurship policies with the help of all ministries (or levels of the state) under supervision of one single ministry that acts in a large context of entrepreneurial activities.

6. There are indexes evaluating measures of improving entrepreneurship atmosphere and cultural conditions to found real firms in the framework of the national entrepreneurship policy.

The above-discussed typologies are demonstrated in fig. 1 . 


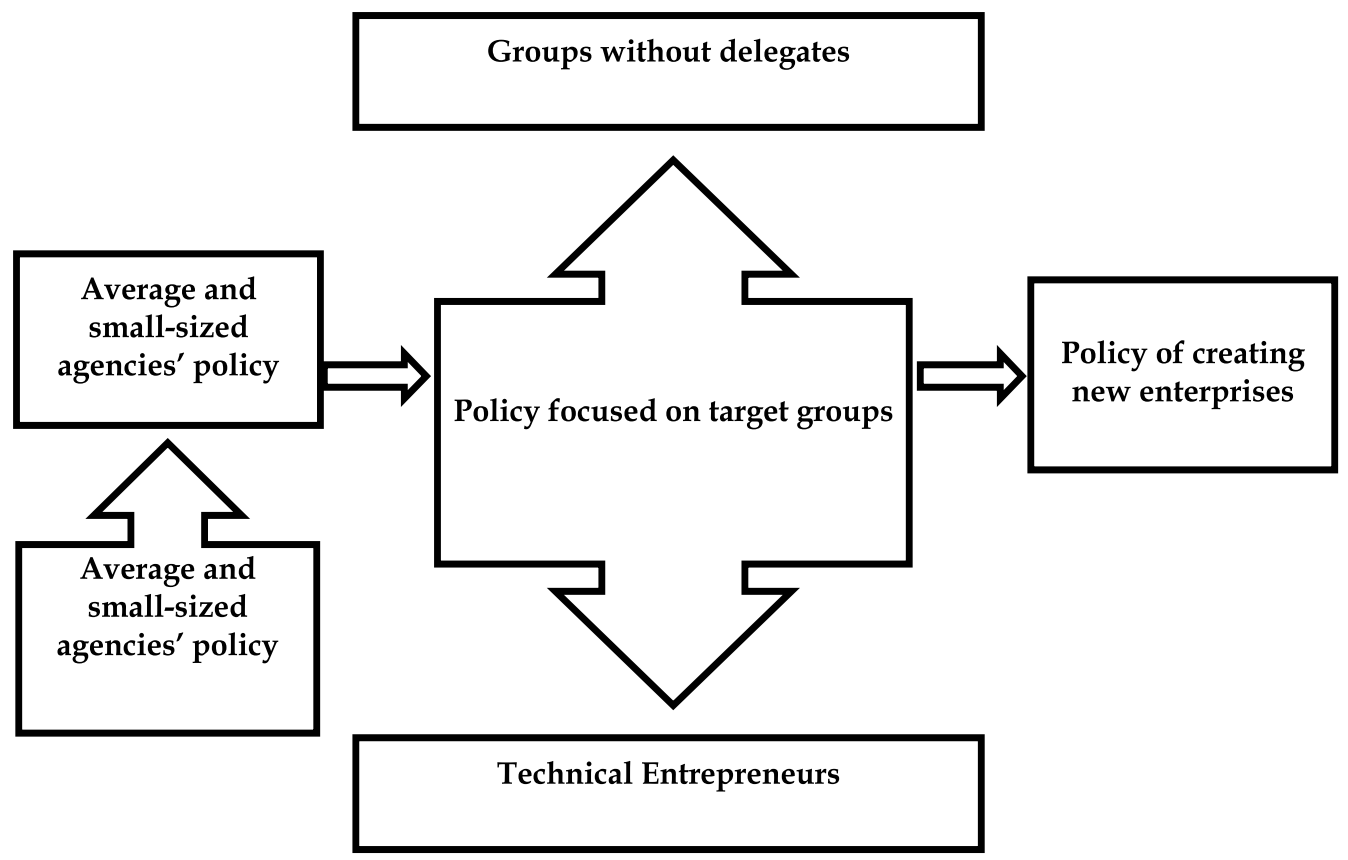

Fig. 1. Typology of entrepreneurial policy

\section{The cycle of public policymaking in Iran}

There are four management levels in Iran managing the country in a close relationship with each other: 1- level of supreme policies (The leader and his special departments); 2- level of policies (government as legislative, executive and judiciary systems or the political management); 3- level of policies strategies (public management); and 4- level of agencies strategies (private sector management). In other words, the first level makes macro policies and the second level designs public policies considering them. The third level participates in the process of making policies and helps operate them. Helping the public sector, the fourth level compiles agency strategies in order to reach the states macro targets. The cycle of policymaking is shown in fig. 2.

As it can be seen in the cycle, the problems (like unemployment) are diagnosed firstly and then they are given to the decision-making department of the executive system (cabinet) through different canals (special departments of the government, press, beneficiary groups etc.). The bill is given to the parliament after being passed in the cabinet. Then it goes into the parliament's agenda and becomes a policy. The act passed in the parliament (under the supervision of the Guardian Council) is then given to the president for execution. Finally, the president orders public managers to enforce the laws with the help of the private sector. Passage of time lets the laws be evaluated and they may experience some changes or even they may be terminated. These laws' side effects may result in the repetitions of this cycle over. Formulation of the policy is of a great importance in the cycle. Although this step is taken in the cabinet and the parliament members have to make the selection, the parliament 
sometimes enters in formulation of some policies. According to the department of the cabinet offering the policy, the bill contains it or the parliament members give a plan that includes it. The question here is that how this formulation (as a part of the cycle of policymaking) is conducted. The present study attempts to compare the methodology of the formulation of the policy with the content of entrepreneurship policy.

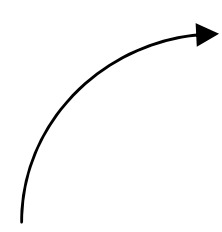

Changing or ending the policy

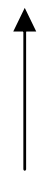

Evaluation of the policy

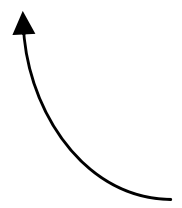

\section{Diagnosing the problem}

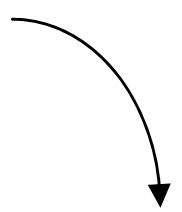

Compiling the agenda

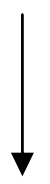

Formulation of the policy

\section{Performing the policy}

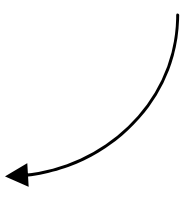

Fig. 2. the Cycle of Public Policymaking in Iran

\subsection{Formulation of public policies}

Public policymaking is exclusively conducted by the government and since its formulation is highly dynamic and complex, no pre-defined theoretical pattern has been offered for it. According to Dror (1997: 12), public policy making is dynamic and complex process in which several elements play various roles and complement it. Different factors directly or indirectly affect formulation of policymaking. Public organizations play the direct role and non-governmental ones influence it indirectly. Islamic Counseling Assembly, the cabinet, public organizations, city councils and committees, courts; mass media, political parties, pressure groups, and public opinion are other actors on the formulation scene.

Thinkers in the field of policymaking (e.g. Dye, 2000; Anderson, 1971; and Eyeston, 1992) dedicate a chapter of their books to formulation of policymaking but they all have failed to offer a comprehensive theory to help policymakers. For instance, Yoorace (1993) suggested the cooperative model for the formulation of the government's policymaking. In this model, several committees of citizens are formed in different fields and various alternatives of dealing with a public issue are designed and one is selected by these committees. 


\section{Methodology suggested for the formulation of entrepreneurial policymaking}

The issue of national policy of entrepreneurship has become one of the major discussions of the scientific bodies of entrepreneurship. The author of the present study has attempted to clarify the ambiguities in the process of the formation of the national policy of entrepreneurship through reading the key literature in the field (see Lundstrum et.al., 2007; Stevenson, 2005). The methodology proposed by the author has some roots in the abovementioned literature but the author has attempted to offer a comprehensive framework to help the nation's policymakers. Here, the process of the formulation is defined expressively (Fig. 6 and 7).

\section{Diagnosing the public problem and identifying Its roots}

The beginning point for the formation of a policy or a Macro policy is the existence of a public problem. Policy problem may be defined as "a condition creating need or dissatisfaction among people and inducing the government to take actions to alleviate or solve it" (Anderson, 2000, p. 81). Therefore, the distressful situation causing concern among people can be considered to be a public problem. Thus, identification of the public problem is an extremely important point in the beginning of the compilation of a policy. The main part of the definition of a public problem is finding its roots. A condition may be defined as a public problem, but the reasons for creation of such condition should also be defined. Many problems may have different reasons. For example, "inflation" may be the result of "the inequality of supply and demand", "large amounts of liquidity in the country", or "social psychology". "Unemployment" may as well be caused by "national laziness", or "friendship in governmental positions" and the solution to all these problems is the government's policies. For instance, the medicine curing "national laziness" is entrepreneurship. When the reason for unemployment is diagnosed to be "national laziness", a solution under the title of "national entrepreneurship" may become the agenda in cabinet meetings. Therefore, it can be said that policies are the cures for public sufferings.

\section{Finding the solution for a public problem}

After the public problem is identified, the solutions must be considered. These suggestions may be the outcome of expert talks, understanding discourses in the community or referring to scientific texts. Experts may believe that the solution for national laziness rising at the hope of being employed in a governmental department is entrepreneurship in the country. The second phase discusses this point.

\section{A study on theoretical framework of the solutions}

Mere identification of the problem does not necessarily end in a good result. Policymakers ought to offer their suggestion. The references should be introduced. There is a scientific branch in the field of entrepreneurship abundant with theories defining entrepreneurship from different aspects. Some have attempted to promote it from psychological point of 
view and others have tried to elevate it from sociological, political, managerial etc. points of view. Understanding these views helps reach a better understanding of strategies for promotion of entrepreneurship. The next part discusses the benefits of these theories for policymakers.

\section{Basic principles of entrepreneurship policies}

When the solution of the public problem of "national laziness" through national entrepreneurship policy is certain, the components of the policy ought to be found in order to be modified and elevate the spirit of "national over-activity" and get rid of "national laziness". This step requires a reference to entrepreneurship theories. Entrepreneurship theories of behavior clarify the entrepreneurship phenomenon. Therefore, major components of the theories could be supposed as the main ingredients of the public policy of entrepreneurship. There are three bases for entrepreneurship policies: opportunity, motivation, and proficiency. According to these three, when an opportunity is provided, and the necessary motivation and proficiency exists, one becomes entrepreneur. In order to create an opportunity, entry barriers should be reduced and availability of resources ought to be increased. Motivation is created through increasing the possibility of business, offering technical skills and teaching entrepreneurial gimmicks. However, it should be noted that all these points are discussed under various theories in the literature of entrepreneurship.

\section{Identifying factors currently affecting the bases of the policy}

The government can indeed modify three bases of entrepreneurial policies by taking different criteria. The author believes that considering Lundstrum and Stevinsons' view (Lundstrum and Stevinsons, 2005, P. 208) one can find what parameters influence entrepreneurial activities and promote entrepreneurship through modifying those triple bases. Identification of these factors helps the government reach the key points in the triple bases of entrepreneurship. (Figure 3)

In short, it is worth mentioning that the level of entrepreneurial activity is influenced by some factors which are affected by some other parameters. For instance, profit influences level of entrepreneurship but in order to increase it, the structure of cost and price and taxing system go under some modifications. Studying these points is suggested for all those interested in studies in the field.

\section{Determining domains of the policy}

If definition of policy as a set of criteria with relative stability and purposefulness compiled to solve a public problem is accepted, another point will rise and it is that each policy contains several other fields of policies inside. According to the material and factors affecting level of entrepreneurship, these fields of policy (better-said modification fields) are divided into six subcategories. (Figure 4).

In other words, macro policy of the government in the field of entrepreneurship is based on identification of subsidiary parts of the entrepreneurship system. Systematizing the plans in the sextet identified domains; the national macro policy of entrepreneurship comes up. 


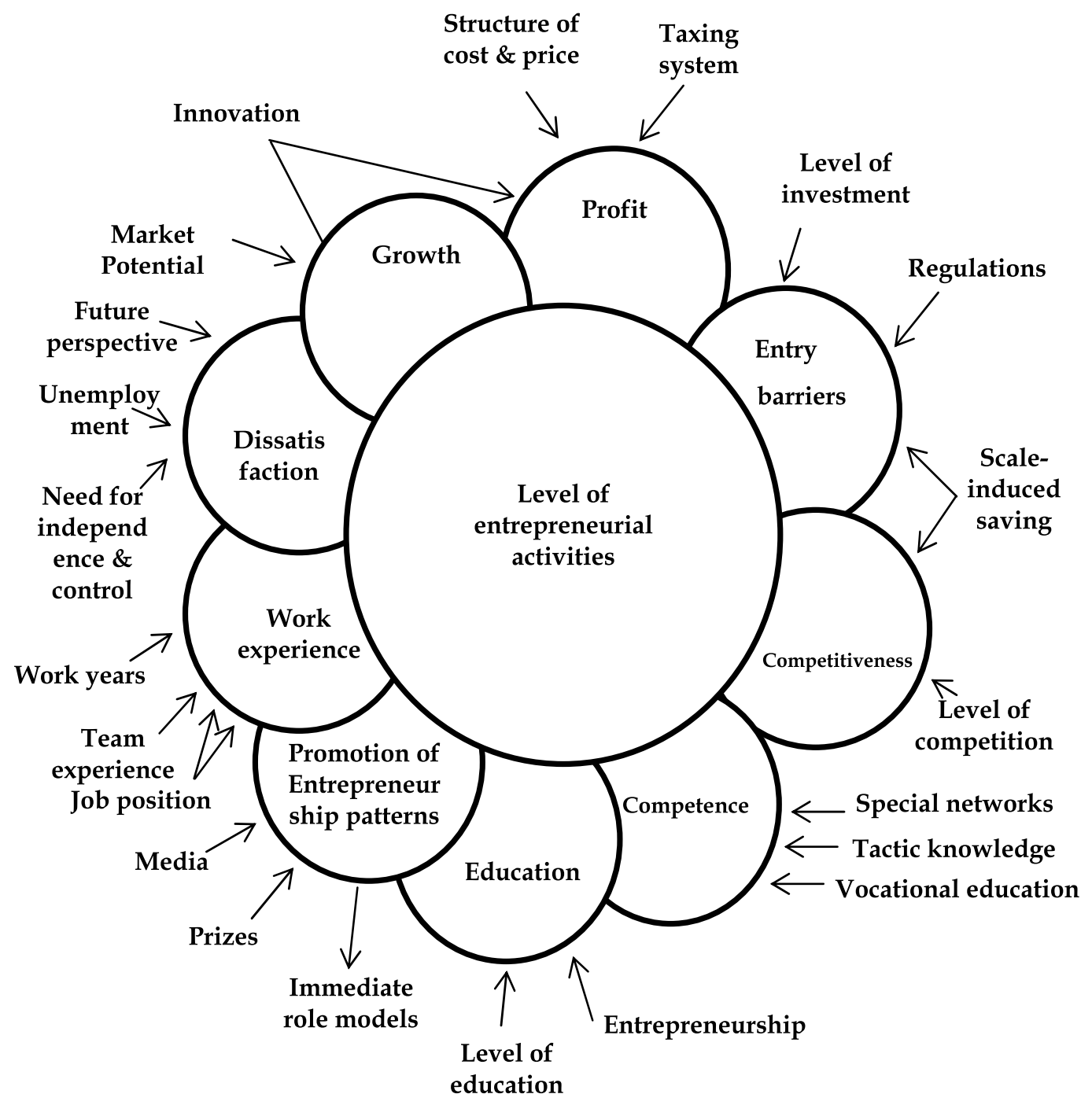

Fig. 3. Factors affecting level of entrepreneur ship

\begin{tabular}{|l|l|}
\hline \multirow{5}{*}{ Entrepreneurship policy } & Promotion of entrepreneurship \\
\cline { 2 - 2 } & Education of entrepreneurship \\
\cline { 2 - 2 } & Entry and exit barriers \\
\cline { 2 - 2 } & Supporting founders of businesses \\
\cline { 2 - 2 } & Providing the initial investment \\
\cline { 2 - 2 } & Special target groups \\
\hline
\end{tabular}

Fig. 4. Domains of the entrepreneurship policy 


\section{Setting the goals of the policy}

In modern communities, any policy designed by the government has to have a goal. The government strengthens the agriculture sector by policy of paying subsidy to farmers. Sometimes the goal is not stated explicitly in the statements of the policies for they may hamper contradiction in the country but all policies sure have goals. In the forth step the goals of a policy have to be defined. (See table 1 for examples of goals for different domains).

In the next phase, the criteria have to be assigned to let policymaker monitor performance of the policies. The conceptual methodology suggested is presented in fig.6 and its compatibility with entrepreneurial policies is illustrated in fig.7.

Figure 5 demonstrates the links between the bases of the policies, their goals, and their domains and the criteria. (Stevenson and Lundstrum, 2005)

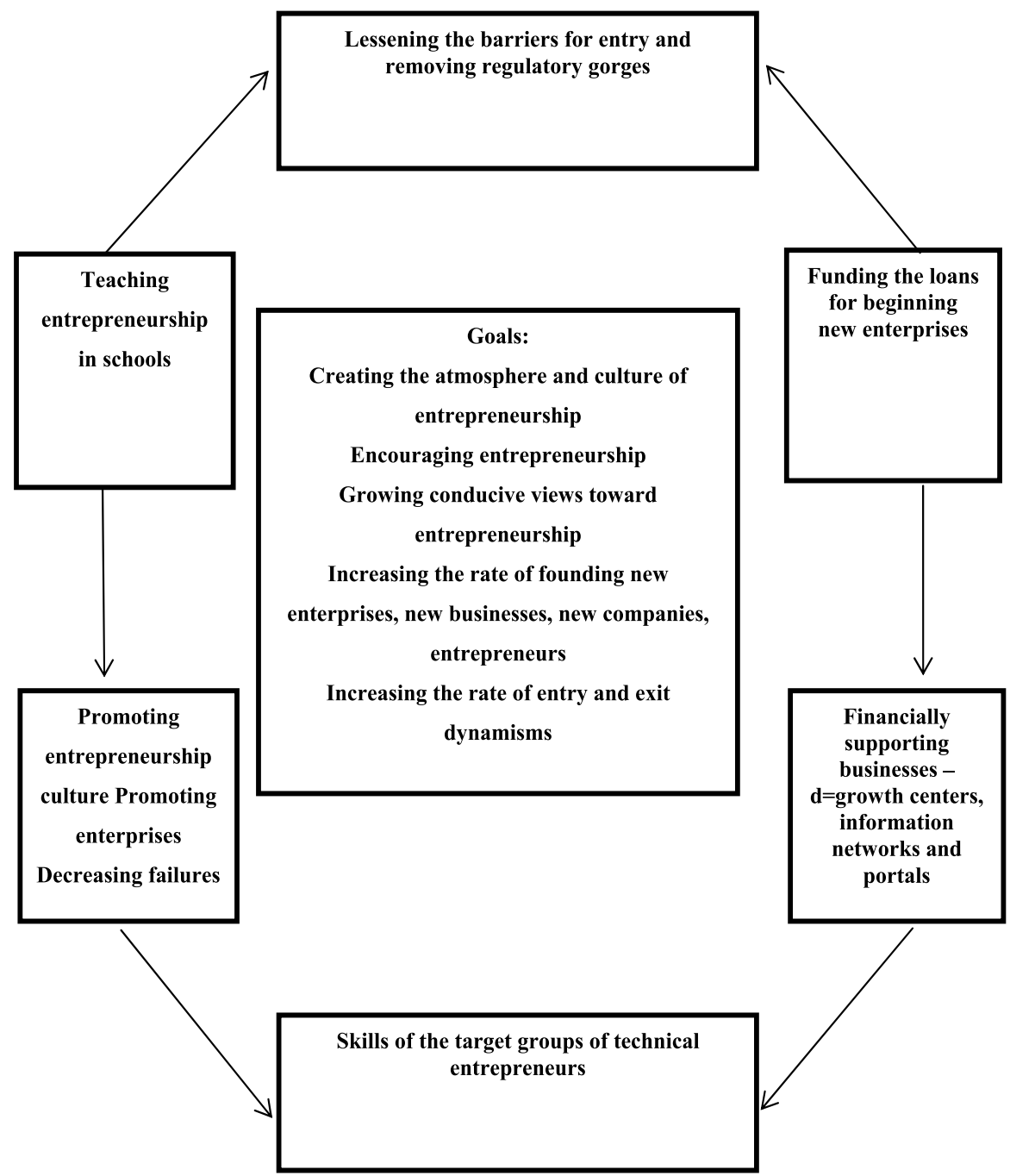

Fig. 5. The outline of the criteria for entrepreneurship policies 


\begin{tabular}{|l|l|}
\hline Domain of the Policy & Goals of the policy \\
\hline $\begin{array}{l}\text { Promotion of } \\
\text { entrepreneurship }\end{array}$ & $\begin{array}{l}\text { Increasing social value of entrepreneurship } \\
\text { Promoting a better knowledge of entrepreneurship in } \\
\text { society } \\
\text { Promoting creditable role models }\end{array}$ \\
\hline $\begin{array}{l}\text { Education of } \\
\text { entrepreneurship }\end{array}$ & $\begin{array}{l}\text { Improving individuals' opportunities to reach better } \\
\text { technical knowledge } \\
\text { Inserting entrepreneurship into different levels of official } \\
\text { education }\end{array}$ \\
\hline
\end{tabular}

Table 1. Sample goals of different domains of entrepreneurship

\begin{tabular}{|c|c|c|c|c|c|c|c|}
\hline $\begin{array}{l}\text { Identifying } \\
\text { public } \\
\text { problem } \\
\text { for a policy } \\
\text { and } \\
\text { finding its } \\
\text { roots }\end{array}$ & $\begin{array}{l}\text { Finding } \\
\text { solutions } \\
\text { for the } \\
\text { public } \\
\text { problem- } \\
\text { alternative } \\
\text { strategies }\end{array}$ & $\begin{array}{l}\text { Under- } \\
\text { standing the } \\
\text { theoretical } \\
\text { framework of } \\
\text { the solutions- } \\
\text { policies }\end{array}$ & $\begin{array}{l}\text { Defining } \\
\text { the main } \\
\text { ingredient } \\
\mathrm{s} \text { of the } \\
\text { policy for } \\
\text { practicing } \\
\text { the } \\
\text { solution }\end{array}$ & $\begin{array}{l}\text { Identifying } \\
\text { the factors } \\
\text { affecting the } \\
\text { bases of the } \\
\text { policy }\end{array}$ & $\begin{array}{l}\text { Deter- } \\
\text { mining the } \\
\text { domains of } \\
\text { the policy }\end{array}$ & $\begin{array}{l}\text { Deter- } \\
\text { mining the } \\
\text { goals of the } \\
\text { domains of } \\
\text { the policy }\end{array}$ & $\begin{array}{l}\text { Deter- } \\
\text { mining } \\
\text { the } \\
\text { criteria } \\
\text { for } \\
\text { the } \\
\text { policy }\end{array}$ \\
\hline $\begin{array}{l}\text { Presenting } \\
\text { scientific } \\
\text { justification } \\
\text { in the } \\
\text { country } \\
\text { Deciding } \\
\text { on the } \\
\text { public } \\
\text { problem } \\
\text { Finding } \\
\text { the reasons } \\
\text { behind the } \\
\text { public } \\
\text { problem }\end{array}$ & $\begin{array}{l}\text { Defining } \\
\text { the } \\
\text { solution } \\
\text { for each } \\
\text { of the } \\
\text { problems }\end{array}$ & $\begin{array}{l}\text { What } \\
\text { suggestions } \\
\text { do theories } \\
\text { have for } \\
\text { practicing the } \\
\text { solutions }\end{array}$ & $\begin{array}{l}\text { Defining } \\
\text { the keys of } \\
\text { the } \\
\text { theories } \\
\text { and } \\
\text { modifying } \\
\text { them to } \\
\text { practice } \\
\text { solutions }\end{array}$ & $\begin{array}{l}\text { Economic } \\
\text { factors } \\
\text { Political } \\
\text { factors } \\
\text { Social factors } \\
\text { Cultural } \\
\text { factors } \\
\text { Psychological } \\
\text { factors } \\
\text { Concordance } \\
\text { of the } \\
\text { theoretical } \\
\text { bases and } \\
\text { the condition }\end{array}$ & $\begin{array}{l}\text { Identifying } \\
\text { the modifi- } \\
\text { cation fields } \\
\text { to reach } \\
\text { prescriptive } \\
\text { solutions }\end{array}$ & $\begin{array}{l}\text { Deter- } \\
\text { mining the } \\
\text { goal of } \\
\text { every } \\
\text { domain of } \\
\text { the policy } \\
\text { expected to } \\
\text { be reached } \\
\text { after } \\
\text { practicing }\end{array}$ & $\begin{array}{l}\text { Deter- } \\
\text { mining the } \\
\text { criteria to } \\
\text { measure the } \\
\text { success of } \\
\text { the } \\
\text { domains of } \\
\text { the policy }\end{array}$ \\
\hline
\end{tabular}

Fig. 6. Conceptual model of the methodology of formulating national policies 


\begin{tabular}{|c|c|c|c|c|c|c|c|}
\hline \begin{tabular}{|l|} 
Ident- \\
ifying \\
public \\
problem \\
for a \\
policy and \\
finding its \\
roots \\
\end{tabular} & $\begin{array}{l}\text { Finding } \\
\text { solutions for } \\
\text { the public } \\
\text { problem- } \\
\text { alternative } \\
\text { strategies }\end{array}$ & $\begin{array}{l}\text { Understan } \\
\text { ding the } \\
\text { theoretical } \\
\text { framework } \\
\text { of the } \\
\text { solutions- } \\
\text { policies }\end{array}$ & $\begin{array}{l}\text { Defining the } \\
\text { main } \\
\text { ingredients } \\
\text { of the policy } \\
\text { for } \\
\text { practicing } \\
\text { the solution }\end{array}$ & $\begin{array}{l}\text { Identi- } \\
\text { fying } \\
\text { the } \\
\text { factors } \\
\text { affecting } \\
\text { the } \\
\text { bases of } \\
\text { the } \\
\text { policy }\end{array}$ & $\begin{array}{l}\text { Determining } \\
\text { the domains } \\
\text { of the policy }\end{array}$ & $\begin{array}{l}\text { Determining } \\
\text { the goals of } \\
\text { the domains } \\
\text { of the policy }\end{array}$ & $\begin{array}{l}\text { Determining } \\
\text { the criteria } \\
\text { for the } \\
\text { success of } \\
\text { entrepreneur } \\
\text { ship }\end{array}$ \\
\hline \begin{tabular}{|l|} 
Practical \\
laws: the \\
youth \\
invasion \\
to \\
governme \\
ntal offices \\
for \\
employ- \\
ment \\
Public \\
problem: \\
national \\
laziness \\
Social \\
reason: \\
Assisting \\
govern- \\
ment
\end{tabular} & $\begin{array}{l}\text { Improving } \\
\text { the spirit of } \\
\text { entrepreneur } \\
\text { ship in the } \\
\text { country }\end{array}$ & $\begin{array}{l}\text { Psychologi } \\
\text { cal theories } \\
\text { Sociologica } \\
1 \text { theories } \\
\text { Political } \\
\text { theories } \\
\text { Manageme } \\
\text { nt theories }\end{array}$ & \begin{tabular}{|l|} 
Lack of \\
entrepreneur \\
ship \\
Opportunity \\
Motivation \\
Proficiency
\end{tabular} & $\begin{array}{l}\text { Profit } \\
\text { Entry } \\
\text { barriers } \\
\text { Growth } \\
\text { Work } \\
\text { ex- } \\
\text { perience } \\
\text { Edu- } \\
\text { cation } \\
\ldots \\
\ldots\end{array}$ & $\begin{array}{l}\text { Domain of } \\
\text { promoting } \\
\text { entrepreneur } \\
\text { ship } \\
\text { Domain of } \\
\text { teaching } \\
\text { entrepre- } \\
\text { neurship } \\
\text { Domain of } \\
\text { entry } \\
\text { barriers } \\
\text { Domain of } \\
\text { supporting } \\
\text { founders of } \\
\text { enterprises } \\
\text { Domain of } \\
\text { supplying } \\
\text { the } \\
\text { company's } \\
\text { capital } \\
\text { Domain of } \\
\text { target } \\
\text { groups }\end{array}$ & $\begin{array}{l}\text { For example: } \\
\text { Domain of } \\
\text { promoting } \\
\text { entrepreneur } \\
\text { ship } \\
\text { Increasing } \\
\text { social value } \\
\text { of } \\
\text { entrepreneur } \\
\text { ship } \\
\text { Increasing } \\
\text { the } \\
\text { knowledge } \\
\text { on } \\
\text { entrepreneur } \\
\text { ship } \\
\text { Making } \\
\text { entrepreneur } \\
\text { ship role } \\
\text { models }\end{array}$ & $\begin{array}{l}\text { For example: } \\
\text { Prize-giving } \\
\text { plans } \\
\text { Occurrence } \\
\text { of entrepre- } \\
\text { neurship } \\
\text { Number of } \\
\text { entrepre- } \\
\text { neurship } \\
\text { events }\end{array}$ \\
\hline
\end{tabular}

Fig. 7. Compatibility of the suggested methodology and the way national policy of entrepreneurship is compiled

\section{Compiling the criteria of the policy}

Evaluation of the performance is impossible without the criteria that help measure the realization of each policy. In this stage, the criteria for different domains of the policy have to be defined. (See table 4.- Stevenson and Lundstrum, 2005)

\section{Discussions and results}

The science of policymaking in Iran does not have a long history compared to the practice of policymaking. In other words, the nation suffers a great loss of experts in the field. Lack of a proper connection between political sciences and public management has contributed to this loss. This means policymakers of the country are in urgent need for guidelines from experts both scientifically and practically to compile policies. Very few books and papers have been published in this area. In addition, since the science of policymaking is a novel approach in Iran, the real life condition may be much worse. 


\begin{tabular}{|c|c|c|}
\hline $\begin{array}{l}\text { Domain of } \\
\text { entrepreneurship }\end{array}$ & Goals of the policy & Criteria of the policy \\
\hline $\begin{array}{l}\text { Promotion of } \\
\text { entrepreneurship }\end{array}$ & $\begin{array}{l}\text { Increasing social value of } \\
\text { entrepreneurship } \\
\text { Increasing society's } \\
\text { knowledge on } \\
\text { entrepreneurship } \\
\text { Promotion of role models of } \\
\text { entrepreneurship } \\
\end{array}$ & $\begin{array}{l}\text { Prize giving plans } \\
\text { Activities of mass media } \\
\text { Entrepreneurial events }\end{array}$ \\
\hline $\begin{array}{l}\text { Education of } \\
\text { entrepreneurship }\end{array}$ & $\begin{array}{l}\text { Increasing the opportunities } \\
\text { for accessing technical } \\
\text { knowledge of } \\
\text { entrepreneurship } \\
\text { Inserting entrepreneurship } \\
\text { into different levels of } \\
\text { official education }\end{array}$ & $\begin{array}{l}\text { Inserting entrepreneurship into } \\
\text { different levels of official education } \\
\text { Education of teachers to teach } \\
\text { entrepreneurship } \\
\text { Supporting young entrepreneurs and } \\
\text { common business activities of students } \\
\text { Supporting entrepreneurial prizes and } \\
\text { competitions }\end{array}$ \\
\hline $\begin{array}{l}\text { Entry and exit } \\
\text { barriers }\end{array}$ & $\begin{array}{l}\text { Reducing the time and cost } \\
\text { of beginning a new business } \\
\text { Reducing barriers and } \\
\text { improving the opportunities } \\
\text { for founding a company and } \\
\text { growth } \\
\text { Removing discouraging } \\
\text { barriers to entrepreneurial } \\
\text { decisions }\end{array}$ & $\begin{array}{l}\text { Increasing the efficiency of business } \\
\text { registration } \\
\text { Integrating public departments related } \\
\text { to entrepreneurial affairs } \\
\text { Removing discouraging barriers in the } \\
\text { market } \\
\text { Social security and taxing systems } \\
\text { Investigating competition policy, } \\
\text { companies act, bankruptcy acts, and } \\
\text { regulations affecting transferring the } \\
\text { ownership of businesses } \\
\text { Reducing the tax loads and office loads } \\
\text { on new enterprises } \\
\text { Tax exemptions and discounts in order } \\
\text { to encourage investments and offering } \\
\text { capital to new companies } \\
\text { Creating new compilation departments } \\
\text { inside the cabinet for better regulations }\end{array}$ \\
\hline $\begin{array}{l}\text { Supporting new } \\
\text { enterprises }\end{array}$ & $\begin{array}{l}\text { Simplifying access to } \\
\text { information, counseling and } \\
\text { other forms of institutional } \\
\text { supports for founding new } \\
\text { companies and easing } \\
\text { transfer of technical } \\
\text { knowledge }\end{array}$ & $\begin{array}{l}\text { Networks of centers offering services to } \\
\text { founders of companies, } \\
\text { Integrated public departments related } \\
\text { to entrepreneurship of new } \\
\text { entrepreneurs } \\
\text { Information portals for new enterprises } \\
\text { Technical educational programs for } \\
\text { new entrepreneurs } \\
\text { National strategies for growth centers } \\
\text { and supporting entrepreneurship } \\
\text { networks }\end{array}$ \\
\hline
\end{tabular}




\begin{tabular}{|l|l|l|}
\hline $\begin{array}{l}\text { Domain of } \\
\text { entrepreneurship }\end{array}$ & Goals of the policy & Criteria of the policy \\
\hline $\begin{array}{l}\text { Financially } \\
\text { supporting new } \\
\text { enterprises or } \\
\text { supplying their } \\
\text { initial capital }\end{array}$ & $\begin{array}{l}\text { Studying the failures in the } \\
\text { market and gaps in } \\
\text { financially supporting new } \\
\text { enterprises or companies in } \\
\text { their primary levels } \\
\text { Reducing information gaps }\end{array}$ & $\begin{array}{l}\text { Small loans, initial or pre-investment } \\
\text { finance for new entrepreneurs } \\
\text { Loan guarantee plans } \\
\text { Investment capital for new technical } \\
\text { entrepreneurs } \\
\text { Incentives for common investments } \\
\text { Investments in new enterprises and } \\
\text { companies in their primary levels }\end{array}$ \\
\hline Target groups & $\begin{array}{l}\text { Reducing systematic } \\
\text { barriers to increasing the } \\
\text { number of new companies } \\
\text { by special groups of the } \\
\text { community; reducing the } \\
\text { risk of companies with } \\
\text { growth technology in order } \\
\text { to increase the wealth }\end{array}$ & $\begin{array}{l}\text { Accessing information about available } \\
\text { financial supports } \\
\text { Establishing partnership with banks } \\
\text { and other financial agencies } \\
\text { Special agencies for target groups, } \\
\text { prizes, counseling, education and } \\
\text { following the services, colleagues } \\
\text { networks, information portals and loan } \\
\text { plans }\end{array}$ \\
\hline
\end{tabular}

Table 4. Goals and criteria of each domain of national entrepreneurship policy

The present paper attempted to offer a conceptual methodology at first and then evaluate its compatibility with the national policymaking in the domain of entrepreneurship. The author does not claim this methodology is flawless and perfect but a lot has been done to offer practical and scientific hints for policymakers.

One of the main concerns of policymakers in national level is determining alternatives of the policy in the cycle of policymaking in the formulation level (fig. 6). In other words, what steps should be taken for the alternatives of the policy to be determined. As a new field of studies on entrepreneurship, the governments' interference brings up such a gap. The author has attempted to offer a new analytical and innovative methodology for compiling fresh alternatives for the policy.

Formulation of the policy is an important step in the process of policymaking and there is a theory named the formulation theory in the literature of entrepreneurship. General tips from experts in the field fail to make a big contribution for the policymakers. The author believes that formulation as a key stage plays an important role in designing the road map for national policy of entrepreneurship. Compatibility of the elements of this map depends largely on the correct selection of individualistic policies and plans forming the meta-policy. Having a logical framework may help policymakers make a logical arrangement of the map of the policy.

\section{References}

Alvani, Mahdi, Fattah Sharifzade, (2008), The process of policy making," Payam e Noor University press. 
Anderson, James E. (2000). Public policy making, New York: Houghton Mifflin.

Audretsch, David B.; Grilo, Isabel and Thurik, A. Roy (2007). Handbook of research on entrepreneurship policy, Northampton, MA: Edward Elgar.

Bozeman, Barry (2002). Public value failure and market failure, lead article, Public Administration Review, 62/2), 145-161.

Brich, D. (1979). The Job creation in America, New York: Free Press

Brown, Peter (1992). "The failure of market failures" Journal of Socio- Economics 21 (1): 1-24.

Dror, Y. (1968). Policy making re-examined.Sanfransisco: Chandler Publishing company.

Dye, Thomas R. (1992). Understanding public policy, Englewood cliffs, NJ: Prentice-Hall.

European Commission (1998). Fostering entrepreneurship in Europe: priorities for future, communication from the commission to the council, Brussels: commission of European communities, 07.04.

European Commission (2004). Action plan: The European agenda for entrepreneurship, COM (2004) 70 final communication from the commission to the council, The European parliament, the European economic and social committee and the committee of the regions, Brussels: Commission of the European communities, 11. 02.

Eyeston, Robert (1971). The threads of public policy: A study in policy leadership, Indianapolis: Bobbs-Merrill.

Hart, D.M. (ed.) (2003b). The emergence of Entrepreneurship policy, Cambridge, MA: Combridge University Press.

Housman, Daniel M. (2008). "Market failure, government failure, and the hard problems of cooperation", Ethiqueet economique/Ethic and Economics, 6(1), 1-6.

Lane, Robert (1991). The market experience, Cambridge, MA: Cambridge university Press.

Lundström, A., Stevenson, L. (2005). Entrepreneurship policy: Theory and practice, New York: Springer science Business Media Inc.

Nelson, Richard (1987). Roles of government in a mixed economy, Journal of policy Analysis and Management 6(4): 541-557.

OECD (1995). Thematic overview of entrepreneurship and job creation policies, Paris: OECD.

OECD (1997). Small businesses, job creation and growth: faets, obstacles and best practices, Paris: OECD.

OECD (1998). Fostering entrepreneurship, paris: OECD.

Reynolds, P.D.M., M. Hay and S.M. Camp (1999). Global entrepreneurship Monitor, 1999 executive report, Wellesley, MA, Kansas City and London: Babson college, Kauffman center for entrepreneurship leadership and the London Business school.

Rose, Richard (ed.) (1969). Policy making in Great Britain, London: Macmillan. 


\title{
Theoretical Approaches to Employment and Industrial Relations: A Comparison of Subsisting Orthodoxies
}

\author{
Christopher Odogwu Chidi ${ }^{1}$ and Okwy Peter Okpala ${ }^{2}$ \\ ${ }^{1}$ Department of Industrial Relations \& Personnel Management, University of Lagos, \\ ${ }^{2}$ Department of Accounting, University of Lagos, \\ Nigeria
}

\section{Introduction}

Theory could be viewed as a coherent group of assumptions or propositions put forth to explain a phenomenon. A theory is an abstraction of reality and is synonymous with perception, viewpoint, assumption, frame of reference or a perspective. The relevance of theory in any field of endeavour cannot be over emphasised. Theory attempts to observe, understand, explain, predict and control events or phenomena. "It helps in our understanding of events and problems in the practical world" (Fajana, 2000, p.21). Without theory, there is no practice, thus, according to Luthans "it has often been said (usually by theoreticians) that there is nothing as practical as a good theory" (Luthans, 1998, p.13). Hyman (1975, p.12) argues that the whole point of an explicit theoretical perspective is to provide a framework within which the complex detail of the real world can be organised. Hyman (1975, p.2) further asserts that "those who glory in their pragmatism and insist that they are immune from theory are simply unaware of their own preconceptions and presuppositions". Without theory men cannot act, for a theory is a way of seeing, of understanding and of planning. Phoenix (1964) as cited in Asika (1995, p.53) opines that "a theory or model provides an abstract pattern whose structure in relevant respects is congruent with the structure of the physical (and social) world, as demonstrated by agreement between observations and predictions made from the theory or model".

We view theory as the substructure upon which practice or action which can be likened to the superstructure is based. A Theory is different from a model. Thus, a model or paradigm refers to the representation of reality. Models are simplified descriptions of real situations (Waters, 1998). According to Cooper and Schindler (2001), there is a distinction between a model and a theory. Thus, models differ from theories in that a theory's role is explanation whereas a model's role is representation and simulation. Model is a representation of a system that is constructed to study some aspects of the system or the system as a whole (Cooper \& Schindler, 2001). There are various typologies of models. Models could be iconic, analogue, statistical, mathematical, descriptive, graphical, as well as verbal. Theories and models abound in the field of industrial relations. There are multifarious theories of 
industrial relations as a result of its multidisciplinary nature; looking for a universal definition of industrial relations may be as stressful as looking for an ocean in the desert. This is so because, over the years, the concept has been subjected to different conceptual treatment (Ogunbameru, 2004). According to Farnham and Pimlott (1995), there are five theories by which industrial relations institutions, structures and processes are analysed. They opine that the theories which individuals develop about industrial relations are attempts to construct logically consistent ways of understanding and explaining social behaviour and real-life activities in this complex field of human interest. These are the unitary, systems, conflict, Marxist and social action theories. Salamon (2000) posits that industrial relations theories are unitary, pluralist or pluralistic, Marxist, systems and social action. Green (1994) classifies industrial relations theory as follows: Unitary perspective and a more recent variant, the neo-unitarist perspective; conflict theory, including the Marxist and pluralist perspectives; systems approach and the contrasting social action perspective. No one perspective gives a perfect view but each illuminates our understanding of the subject. There are a number of variations on all these themes. Green asserts that generally, a viewpoint, perspective or theory is put forward and this is then modified in the light of experience, criticism and changing circumstances. According to Otobo (2000, p.42), "theorising is a continuous exercise and no one model has satisfied everyone in terms of taking into account all variables at play; attempts to provide more comprehensive theoretical expositions on the industrial relations system have not stopped". There are paucity of studies or researches on theoretical approaches to employment and industrial relations in the Nigerian context. This study is an attempt to address this gap. In addition, it will add to the limited theoretical knowledge in this exciting area of study in a developing country like Nigeria.

The objective of this paper is to attempt a comparison of the five most influential theoretical frameworks of employment and industrial relations and to bring to the fore similarities and differences in the theoretical formulations. The paper also examines a critical review of the theories and also highlights the major themes inherent in the theories. To achieve this objective, the paper adopts a theoretical approach.

\section{Theoretical and conceptual framework}

Employment relations is the study of the regulation of the employment relationship between employer and employee, both collectively and individually, and the determination of substantive and procedural issues at industrial, organisational and workplace levels (Rose, 2008). According to Kaufman (2010), industrial relations is viewed as the process of rule making for the workplace (Dunlop, 1958); job regulation (Flanders, 1965); social regulation of production (Cox, 1971); the employment relationship as structured antagonism (Edwards, 2005); social regulation of market forces (Hyman, 1995); process of capitalist production and accumulation and the derived political and social class relations (Caire, 1996 as cited in Kaufman, 2010); conflict of interests and pluralist forms of workplace governance (Kochan, 1998); class mobilization and social justice (Kelly, 1998); the advancement of efficiency, equity, and voice in the employment relationship (Budd, 2004); collective representation and social dialogue (European Industrial Relations Observatory, 2002). According to Bain and Clegg (1974), a traditional approach to employment and industrial 
relations has been to regard it as the study of the rules governing employment, and the ways in which the rules are changed, interpreted and administered. We now turn to discussing the theoretical themes starting with the unitary theory.

\subsection{Unitary theory}

The unitary frame of reference is credited to Alan Fox (1966). The unitary perspective views the organisation as pointing towards a single or unified authority and loyalty structure. Emphasis under the unitary perspective is placed on common values, interest and objectives. Those subscribing to this view see all organisational participants as a team or family thereby implicitly emphasing shared values, shared goals and common destiny. Unitarism in essence implies the absence of factionalism within the enterprise (Fajana, 2000).Conflict is viewed as irrational and the sacking of striking workers is preferred to consultation or negotiation. Conflict is regarded as pathological or evil or bad. Trade unionism is outlawed and suppressed as it is viewed as an illegitimate intrusion or encroachment on management's right to manage. According to Rose (2008), under the unitary perspective, trade unions are regarded as an intrusion into the organisation from outside, competing with management for the loyalty of employees. The unitary theory tends towards authoritarianism and paternalism. It is pro- management biased and emphasises consensus and industrial peace. The underlying assumption of this view is that the organisation exists in perfect harmony and all conflict is unnecessary (Rose, 2008).

\subsection{Conflict theory}

Conflict theory is synonymous with the pluralist or the pluralistic frame of reference which is also credited to Alan Fox (1966). Conflict theory views the organisation as coalescence of sectional groups with different values, interests and objectives. Thus, employees have different values and aspirations from those of management, and these values and aspirations are always in conflict with those of management. Conflict theorists argue that conflict is inevitable, rational, functional and normal situation in organisations, which is resolved through compromise and agreement or collective bargaining. Conflict theorists view trade unions as legitimate challenges to managerial rule or prerogatives and emphasise competition and collaboration. This view recognises trade unions as legitimate representative organisations which enable groups of employees to influence management decisions (Rose, 2008). Rose further states that the pluralist perspective would seem to be much more relevant than the unitary perspective in the analysis of industrial relations in many large unionised organisations and congruent with developments in contemporary society

\subsection{Systems theory}

The concept of system derives from the structural/functionalist perspectives of social system (society). This also connotes the macro-sociological, order or social system view of society. There are several senses or meanings of the word 'function'. These are (i) teleological, where one asks about the goals or ends something serves (ii) mathematical, where one refers to the co-variation of a set of variables e.g. $y=f(x)$; (iii) configurational, where one speaks of the interdependence of a set of elements within a system, and asks 
what contribution each makes to the whole. The systems approach to industrial relations is configurational. Thus, Dunlop developed his theoretical approach of industrial relations on the basis of a systems concept and was heavily influenced by the prior work of Parsons (Fajana, 2000). According to Ogunbameru (2004), the American system approaches to the study of industrial relations were strongly influenced by structural/functionalist sociology. Dunlop based his model explicitly on Parsons social system, which assumed an inherent bias towards order and stability. Otobo (2000, p.17) posits that Dunlop began his explanatory model with a series of questions.

"What meaning, then, is to be given to an industrial relations system"? (Otobo, 2000, p.17). "In what sense is a 'system' involved? Can the term be given rigorous and analytical definition, or shall it remain a perceptive phrase corresponding to the insights of practical experience? Are there characteristics common to all industrial relations system? What factors distinguish one industrial relations situation from another? Can the same concept be used to facilitate analysis among sectors within a country and also among countries?" (Otobo, 2000, p.17). These questions posed by Dunlop (1958) were then followed by six general propositions.

- "An industrial- relations system is to be viewed as an analytical sub-system of an industrial society on the same logical plane as an economic system, regarded as another analytical sub-system. The industrial relations system is not coterminous with the economic system; in some respects the two overlap and in other respects both have different scopes. The procurement of a work force and the setting of compensation for labour services are common centers of interest. A systematic explanation of production, however, is within economics but outside the scope of industrial relations. The full range of rule-making governing the work place is outside the scope of an economic system but central to an industrial relations system.

- An industrial relations system is not a subsidiary part of an economic system but is rather a separate and distinctive subsystem of the society, on the same plane as an economic system. Thus, the theoretical tools designed to explain the economic system are not likely to be entirely suitable to another different analytical subsystem of society.

- Just as there are relationships and boundary lines between a society and an economy, so also are there between a society and an industrial relations system. All analysis of the economy makes some assumptions, explicitly or implicitly, about the remainder of the social system, so also must an analysis of an industrial relations system make some assumptions about the rest of the social system.

- An industrial relations system is logically an abstraction just as an economic system is an abstraction. Neither is concerned with behaviour as a whole. There are no actors whose whole activity is confined solely to the industrial relations or economic spheres, although some may approach this limit. Neither an economic system nor an industrial relations system is designed simply to describe in factual terms the real world of time and space. Both are abstractions designed to highlight relationship and to focus attention upon critical variables and to formulate propositions for historical inquiry and statistical testing.

- This view of an industrial relations system permits a distinctive analytical and theoretical subject matter. To date the study of industrial relations had little theoretical content. At its origin and frequently at its best, it has been largely historical and 
descriptive. A number of studies have used the analysis of economics particularly in treating wages and related questions and other studies, particularly of factory departments; have borrowed the apparatus of anthropology and sociology. Although, industrial relations aspires to be a discipline, and even though there exists separate professional societies, industrial relations has lacked any central analytical content. It has been a crossroads where a number of disciplines have met, history, economics, government, sociology, psychology and law. Industrial relations requires a theoretical core in order to relate isolated facts, to point to new types of inquiries and to make research more additive. The study of industrial relations systems provides a genuine discipline.

- Three separate analytical problem are to be distinguished in this framework (a) the relation of the industrial relations to the society as a whole (b) the relation of the industrial relations system to the subsystem known as the economic system and (c) the inner structure and characteristics of the industrial relations subsystem itself."(Otobo, p.17-19)

Otobo (2000, p.19) citing Dunlop (1958) argues that "An industrial-relations system at any one time in its development is regarded as comprised of certain actors, certain contexts, an ideology which binds the industrial-relations system together and a body of rules created to govern actors at the workplace and work community". According to Dunlop, systems theory provides the analytical tools and the theoretical basis to make industrial relations an academic discipline in its own right.

\subsubsection{Certain actors}

The actors that make up the industrial relations system are

- A hierarchy of managers and their representatives in supervision

- A hierarchy of workers (non-managerial) and their spokesmen

- Specialised governmental agencies and specialised private agencies created by the first two actors, concerned with workers, enterprises and their relationships.

\subsubsection{Contexts}

This refers to the setting which these actors operate, that is the larger environment which shapes the conduct of, and the rules established by workers, employers and the state. Dunlop highlights three aspects of the environment.

- Technological characteristics of the work place and work community: These influence the form of management and employee organisation and the problems posed for supervisors. Thus, the adopted technology will greatly determine the size and skills of work force as well as availability of labour. It also affects the health and safety at the workplace. The adopted technology has far-reaching consequences in determining IRs rule making.

- Market/budgetary constraints: The products market or budget is a decisive factor in shaping the rules established by an industrial relations system. More so, the market or budgetary constraints also indirectly influences the technology and other characteristics of the work place, including the scale and size of operations. In all, an industrial 
relations system created and administered by its actors is adaptive to its market and budgetary constraints (Otobo, 2000). More so, the profitability of the enterprise depends on its product market.

- The locus and distribution of power in the larger society: The relative distribution of power among the actors in the larger society tends to a degree to be reflected within the industrial relations system. Thus, the distribution of power within the industrial relations system is affected by the distribution of power in the wider society. Dunlop is not concerned about the distribution of power within the industrial relations system, nor with the relative bargaining powers among the actors, nor their controls over the processes of interaction or rule setting, rather the reference to the distribution of power outside the industrial relations system. Thus, the wider society is seen as providing certain external influences and constraints but not as completely dominating industrial relations system.

\subsubsection{A body of rules}

The actors in given contexts establish rules for the workplace and work community. Actors establish rules that govern their own interactions. Dunlop referred to this as the "web of rules" that governs the parties. There is no assumption by Dunlop that these rules are jointly made by the actors. These rules he referred to as the substantive and procedural rules. Thus, the creation of rules is seen to be the central aim of the industrial relations system. The substantive rules pertain to issues involving wages/salaries, hours of work and other terms and conditions of employment. On the other hand, the procedural rules relate to the rules governing discipline, redundancy, settlement of disputes, periodicity of meetings, renewal of collective agreements and the like. The rules of the industrial relations systems may be expressed in a variety of forms such as the regulations and policies of the management hierarchy, the laws of any worker hierarchy, the regulations, decrees, decisions, awards or orders of governmental agencies, collective bargaining agreements and the customs and traditions of the work place and work community. The rules may be written, an oral tradition or customary practice. Thus, the establishment and administration of these rules is the major concern or output of the industrial relations sub-system of industrial society.

\subsubsection{Ideology}

Ideology connotes a set of ideas and beliefs commonly held by the actors that helps to bind or integrate the system together as an entity. According to Otobo (2000, p.28) citing Dunlop "each of the actors in an industrial relations system may be said to have its own ideology. Dunlop insists rather strongly that all these ideologies must be sufficiently compatible or consistent to permit a common set of ideas which recognise an acceptable role for each actor". Dunlop assumes that the ideology of IRs system must be one or the same among the actors.

As could be deduced from the above model, there are three sets of independent variables in an industrial relations systems, the actors, the contexts and the ideology of the system, while the rules represent the dependent variable or the output of the industrial relations system. The dynamic model of the systemic paradigm, open system analysis and the oxford school are further elaboration of the Dunlopian model. 


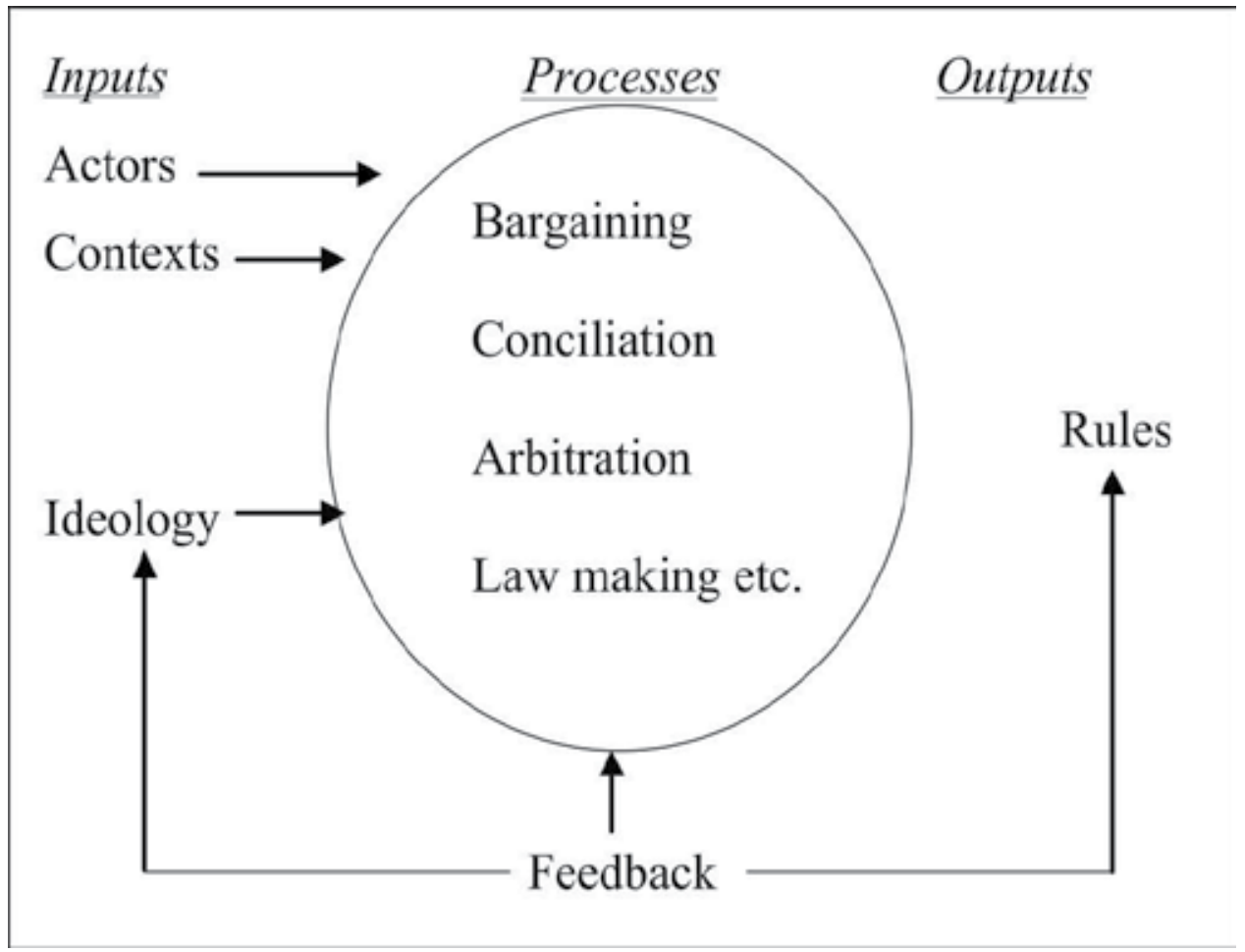

Source: Farnham, D. \& Pimlott, J. (1995). Understanding Industrial Relations. (5thed). London: Cassell

Fig. 1. A Simple Model of an Industrial Relations System

\subsection{The dynamic model of the systemic paradigm}

The dynamic model of the systemic paradigm of industrial relations is a refinement to Dunlop's analytical framework. This dynamic model is credited to Blain and Gennard (1970). The duo adopted Dunlop's proposition of an industrial relations system being on the same logical plane as the economic subsystem. Their work centred on classifying the variables in an industrial relations system into dependent and independent variables, a task the Dunlopian model made difficult to achieve. They expressed the industrial relations system algebraically as shown below:

$$
r=f(a, t, e, s, i)
$$

Where, $r=$ the rules of the industrial relations system

$\mathrm{a}=$ the actors

$\mathrm{t}=$ the technical context of the work place.

$\mathrm{e}=$ economic or the market/budgetary constraint 
$\mathrm{s}=$ the power context and the status of the parties

$\mathrm{i}=$ the ideology of the system.

From the above equation, the rules can be viewed as the dependent variables being determined by the interaction of the five independent variables. Thus, the function of the industrial relations system is to establish a set of rules for the workplace and work community. In dynamic society the rules will frequently alter as a result of changes in the contexts or environment. Thus, the dynamic model emanated as a response to the criticisms levelled against the Dunlopian system model. It has been criticised as having a static view of industrial relations.

\subsection{The open system analysis}

Dunlop's systems theory uses the term 'system' in a too loose and undefined manner. The open system analysis is concerned with looking at industrial relations system in terms of inputs and outputs and the interaction with the environment. According to Koontz, $\mathrm{O}^{\prime}$ Donnel and Weihrich (1980, p.19) "almost all life is a system. Our bodies certainly are. Our homes and universities are, as are our government agencies and our businesses." Systems have inter-related parts which work together to form a complex unity or whole. The features of a system are as follows:

- Whole: a system is more than the sum of its parts. It must be viewed as a whole.

- Closed or open: A system is regarded as open if it exchanges information, energy or material with its environment. A closed system is one that does not have interactions with its environment. All social systems are by nature open systems.

- Boundary: Every system has boundaries which separate it from its environment.

- Input and output: All systems which interact with the environment are amenable to receiving inputs from other systems and giving output to other systems.

- Feedback: An informational input that tells whether the system is indeed at least achieving a steady state and is not in danger of destruction.

- Homeostatic: This is referred to as dynamic homeostatic (steady state). Hence an organisation will not be able to survive if its inputs do not at least equal its outputs.

- Subsystems: With the exception of the Universe, all systems are subsystems. That is every system is a component of other larger systems.

- Equifinality: All open systems have common ends or objectives as everyone performs in a manner that will enhance the attainment of the broad objectives of the system

- Differentiation and Elaboration: As the system grows, it tends to become more specialised in its elements and to elaborate its structure. This is exemplified by the expansion of product lines or creation of new sales offices by an organisation.

Having stated some of the characteristics of a system, one would be apt to state that the Dunlopian model of an industrial relations system ought to have followed the open system concept in formulating an industrial relations system instead of seeing it as a system of rules, which appears too parochial. The systemic paradigm by Dunlop has attracted an avalanche of criticisms, some of which are as follows:

- The heroic assumption taken by Dunlop that an industrial relations system will necessarily be homeostatic has been criticised. This is because industrial conflicts are 
never truly resolved and one problem arises after another. So, the system is not completely stable as claimed by Dunlop

- The model provides no explanation of the causes of industrial action but laid more emphasis on conflict resolution.

- Dunlop's formulation of an industrial relations system largely omits such behavioural variables as human motivation, perception and attitudes, personality and small group interaction. He laid more emphasis on institutions (trade unions, employers associations).

- Dunlop identified three main actors in the industrial relations system but failed to make reference to the owners of industrial property. It has been argued, that this omission stems from the fact that decisions in the industrial relations system are made by managers and not owners. Some have argued that the number of actors has to be increased.

- Another flaw is that Dunlop's idea of a system is a deterministic mechanism. Dunlop's actors are not persons. The model suffers from reification. No provision for the role of individual personalities was advanced.

- Limited predictive value associated with the systems model makes it difficult to forecast whether the system will experience more or less conflicts as a result of a given change in one or more of the environmental contexts.

- It suffers a handicap in that it does not take into account the processes by which the rules of the system are determined or made.

- One of the criticisms of the system approach is the difficulty in defining a system. There was no clear definition of what was meant by the concept of a system itself. This failure may have caused some writers to misrepresent the theory of industrial relations system. What is the substance of a system of industrial relations? This was the question raised by Flanders. Not until recently has it been stated that a system of industrial relations is a system of rules. However, a system of industrial relations as propounded by Dunlop is not a system of rules but a conceptual framework in which one component element is the rules. The systems approach has been misrepresented by a Sociologist, Eldridge who conceptualised the model as being comprised of only three elements (the actors, rules and ideology).

- The claim by Dunlop that the industrial relations system is on the same logical plane as the economic system is not correct, as Wood, Wagner, Armstrong, Goodman and Davis (1975) have pointed out, once Dunlop accepted the Parsonian social system that the social system is comprised of four functional sub-systems (the economic, political, integrative and pattern- maintenance) the industrial relations system could not therefore be on the same logical plane as the economic system, but it should be construed as on a lower logical plane than the economic system.

- It is criticised that the framework is static, not dynamic in time as processes are ignored.

- The systems theory concentrates on formal rules as against informal rules and processes.

- The systems model does not entail an account of the ways in which inputs are converted into outputs.

- Power could not rightly be a property of the external context of industrial relations system only, instead, power is considered central internally to the conduct of the parties themselves for the establishment and defence of rules and their application. It is a fact 
that workers/union and management are involved in a power relationship within the enterprise and industry.

- Dunlop did not pay sufficient attention to all facets of conflict in the industrial relations system, his emphasis being on conflict resolution and not its generation. Why and how conflicts occur are likely to reveal more about industrial relations processes and institutions than how their manifestations are sorted out (Otobo,2000). The overall argument is that Dunlop misunderstood the Parsonian system analysis.

\subsection{Oxford school}

Since the oxford school does not necessarily have to constitute a self- contained approach, and has the elements of the systems theory, it should probably be viewed merely as a variant of the systems approach (Fajana, 2000). The oxford school emerged from the systems approach as both focus on institutions of industrial relations, although the point of difference is merely on emphasis. This approach is credited to Allan Flanders a British academic. According to Flanders as cited in Hyman (1975, p.11), "industrial relations is the study of the institutions of job regulation". He opines that the rules of any industrial relations system are seen as procedural and substantive. The procedural rules regulate the behaviour of parties to the collective agreements- trade unions and employers or their associations, whereas, the substantive rules regulate the behaviour of employees and employers as parties to individual contracts of employment. In fact, it is the substantive rules of collective bargaining that regulate jobs. Thus, the collective agreement is made up of both the procedural and substantive clauses. Some of the institutions of job regulation are internal as well as external. Internally, we have joint consultation, the grievance procedure, a code of disciplinary works' rules, a factory wage structure, and a host of others. Externally, there are other institutions which limit the freedom of the enterprise and its members in their rule-making activities, such as a protective labour legislation, the rules of trade unions and employers' association. The rules of the industrial relations system are viewed as being determined through the rule making process of collective bargaining which is regarded as a political institution involving a power relationship between employers and employees. The oxford approach can be expressed algebraically in the form of an equation. $r=f(c)$

Where, $r=$ the rules governing industrial relations system.

$$
c=\text { collective bargaining }
$$

When the equation is compared with the equation of the dynamic systems model which states that $r=f(a, t, e, s, i)$, it can be seen that the distinction between the dynamic systems model and the oxford approach lies in the right hand side of the equation. But both have the same output but different inputs. The oxford approach has stressed the process of rule making through collective bargaining while the dynamic system model emphasises the role of wider influence on rule determination. For the oxford approach, political variables are seen as of paramount importance but for the dynamic system model, economic, sociological and ideological variables are thought to be significant.

The criticisms of the oxford approach are as follows: (a) It is too narrow to provide a comprehensive framework for analysing industrial relations problems (b) It over- 
emphasised the importance of the political process of collective bargaining and gives insufficient weight to the role of the deeper influences in the determination of rules.

\subsection{Marxist theory}

Marxism is, more or less, a general theory of society and of social change with implications for the analysis of industrial relations within capitalist societies and does not strictly explain the theory of industrial relations. The application of Marxian theory as it relates to industrial relations today derives from later Marxist scholars rather than directly from the works of Karl Marx himself (Ogunbameru, 2004). According to Hyman (1975) the contribution of both Dunlop and Flanders are giant strides in the formulation of industrial relations theory, but argues rather strongly that to define industrial relations exclusively in terms of rules and institutions for job regulation is far too limited or restrictive. What this implies is that industrial relations is all about the maintenance of stability and regularity in industry. He argues that the issue of conflict was not given proper analysis by the duo, as they focused on how any conflict is contained and controlled, rather than on the process through which disagreements and disputes are generated. Hyman asserts that the perspectives of the duo however influential, is onesided and inadequate. Hyman (1975, p.12) defines industrial relations" as the study of the processes of control over work relations and among these processes, those involving collective worker organisation and action are of particular concern". Hyman further argues that unceasing power struggle for control is a central feature of industrial relations. To him, this struggle for control emanates from the nature and characteristics of capitalist society. He summarised the major characteristics of capitalism as (i) the ownership and or control of the means of production by a small minority (ii) the domination of profit as the fundamental determinant of economic activities (iii) the obligation on most of society to sell their productive abilities on the market as a commodity. Against this background, two major classes are located within capitalist industrial relations which are also a reflection of what obtains in society. Thus, capitalist industrialism bifurcate society into two classes. These are the owners of means of production which is the capitalist or bourgeoisie and the owners of labour, which are the workers or proletariat. This being so, the interests of employers and employees are diametrically opposed and conflictual. The capitalist endeavours to purchase labour at the lowest possible price whilst labour on the other hand tries to sell his only asset at the highest possible price in order to ensure his existence. The capitalists tend to maximise profit whilst the workers tend to maximise wages/salaries. Thus, in capitalist industrial society, the interests and aspirations of both labour and employers are divergent and in conflict. The Marxist perspectives typify workplace relations as a reflection of the incidence of societal inequalities and the inevitable expression of this at the work place. To sum it up, Hyman further states that industrial relations is all about power, interests and conflict and that the economic, technological and political dynamics of the broader society inevitably shape the character of relations among industrial relations actors which he described as the political economy of industrial relations. Conflict is viewed as a disorder precursor to change and to resolve conflict means to change the imbalance and inequalities in society in terms of power and wealth. Trade unions are viewed as 
employee response to capitalism. Marxist theory emphasises exploitation and alienation. This perspective is critical of capitalist society and its system of production, distribution and exchange and emphasises the importance of collective action including strike action and action short of strikes (Rose, 2008). Hyman (1975) argues that given the nature of capitalist society, industrial relations can be analysed from a more radical perspective. This theory is also known as the radical perspective.

\subsection{Social action theory}

According to Green (1994, p.4), "the social action theory views industrial relations from the individual's viewpoint and motivation". According to Rose (2008), the social action approach considers the organisation from the position of the individual members or actors who will each have their own goals. This perspective regards conflicts of interests as normal behaviour and part of organisational life (Rose, 2008). It is credited to Max Weber (18641920); a German Sociologist. Social action theory represents a contribution from sociologists to the study of organisations. It attempts to view the organisation from the standpoint of individual members or actors of industrial relations. The theory seeks to analyse why the actors take certain lines of action. This contrasts with the systems approach which states that behaviour is a result of the structure and processes of the system. Social action arises out of the expectations, norms, attitudes, values, experiences, situation and goals of the individuals working in the system. Thus, according to Green while the system approach is up-down, the social action theory is a bottom-up approach. Salamon (2000) opines that the importance of the social action theory of industrial relations is that it weakens the fatalism of structural determinism and stresses that the individual retains at least some freedom of action and ability to influence events in the direction that he/she believes to be right or desirable. Social action theorists emphasise the use of interview, survey and participant observation in determining the reality of both society and of organisations.

\section{Comparison of subsisting orthodoxies}

The central focus of comparative analysis of these theoretical formulations of employment and industrial relations is to examine the degree of differences and commonalities or similarities between and among them. The heroic assumption by Dunlop that the ideology of the industrial relations system must be one, or compatible in spite of the fact that each actor has its own ideology has been challenged by Hyman. According to Hyman (1975, p.12), "if the system of industrial relations is so well integrated, and if the goals and values of the actors are so much in agreement, how is it that industrial conflict occurs at all?" Thus, while this may be true for the unitary approach, it is not true for the social action theory, conflict and Marxist theory. This is so because, the unitary theory emphasises common values while the others emphasise differing values, interests and objectives among actors. Thus, this assumption is spurious in reality even within the unitary perspective. The systems theory views industrial relations system as being stable regulated and in a steady state or homeostatis; again, this position is favoured by the unitary approach which emphasises consensus and industrial peace and views conflict as irrational. Marxist and other conflict theorists do not subscribe to this idea. Hyman maintains that the definition by the systems and the oxford school should be broadened to accommodate the sources as well 
as the consequences or aftermaths of industrial conflict on the social partners. He views conflict as inevitable and rational in the industrial relations system. Conflict and disorder cannot be excluded from industrial relations system.

All five theories differ at the level of conceptualisation of an industrial relations theory, the Dunlopian model and its variants see the product or output of an industrial relations system as a network or web of rules (both substantive and procedural rules). To the system theorists, the central core or focus of an industrial relations system is the rule-making process to govern the actors and work community. Hyman and other Marxists see it as the study of the processes of control over work relations and among these processes; those involving collective worker organisation and action are of particular concern. Hyman's view is applicable to the unitary and conflict theorists. The unitary theory emphasises unified authority and loyalty structure whilst the conflict theory emphasises competitive authority and loyalty structures.

The social action theory contrasts with the systems approach. Whilst the systems approach opines that behaviour of actors is a function of the structure and processes of the system, social action arises out of the expectations and other attributes of the individuals working in the system. Salamon (2000) argues that the importance of the social action theory of industrial relations is that it weakens the fatalism of structural determinism and stresses that the individual retains at least some freedom of action and ability to influence events in the direction that he/she believes to be right or desirable. In reality, the actors in the system are influenced by the system and in turn they influence the system.

Dunlop's actors are institutions not persons. The systems theoretical formulation suffers from reification. This means that individuals involved at the workplace and whose activities are industrial relations have been relegated to the background, while institutions like trade unions, collective bargaining, employers' collectivities and various state organs have been employed in his analysis. This Hyman finds abnormal as it creates the tendency to conceive industrial relations solely in terms of relationships between agencies and organisations, rather than between people. To Hyman, this is a "mechanical and depersonalized approach to social analysis. In other words, treating abstract collective entities which are the creation of human activity, as the active agencies in social relations and in consequence devaluing the part played by human actors"(Hyman, 1975, p.13). Hyman argues that the analysis of industrial relations should not only focus on trade unions as organisations, but also on workers and their grievances and aspirations. Hyman's view is shared by social action theorists who stress that the individual retains at least some freedom of action and ability to influence events based on their aspirations, values and goals. Hyman states that the fact that labour is treated as a commodity is one of the causes of conflicts in industry. Thus, the subjugation, exploitation, and alienation of workers are the sources of workers grievances. To this effect, workers aspire to control their own work, rather than being subject to constant instructions and supervision. Hyman notes that the notion of regulation conceals the centrality of power, conflict and instability in the processes of industrial relations. With respect to the resolutions of conflict in work relations, the unitary, systems, conflict and Marxist approaches favour different methods. While the unitary favours coercion, the systems theory adopts the rule-making process, conflict theory favours compromise and 
agreement and Marxists favours changing the imbalance and inequalities in society in terms of wealth and power.

Trade unionism is accepted by the systems theory, conflict theory, Marxist theory as well as the social action theory. However, the unitary theory views trade unionism as illegitimate intrusion or encroachment on management prerogatives and is outlawed and suppressed. The theories have the following commonalities: All five theories recognise the importance of context. Industrial relations does not and cannot exist in a vacuum. It exists at the micro, macro and global levels. The significant aspects of context are economic, technological, political, social and legal dynamics of the broader society. Despite the deluge of criticisms levelled against these theoretical formulations, they have been referred to as monumental contributions to scholarship (Onabanjo, 2001). The five theories have sociological etymology, suggesting that they derive their fountain from sociology. All five theories recognise that three actors or participants or social partners are involved in industrial relations in line with the concept of tripartism in industrial relations as proposed by the International Labour Organisation (ILO). However, with the emergent transnational organisations and the influence of host communities emphasis is now shifting to the concept of "tripartism-plus." None of the theories can boast of having a comprehensive coverage of the subject area of industrial relations. However, we take solace in Hyman's comment that "in defining the scope of industrial relations, it is necessary to define the subject more narrowly than the total network of social relationships in industry" (Hyman, 1975, p.31).

\section{Conclusion}

The practice of employment/industrial relations has benefited immensely from theoretical frameworks of leading theorists in the field of industrial relations. It has been observed that despite the criticisms levelled against some of these theories they have stood the test of time and have contributed immensely to scholarship and practice. Among these theories, there are areas of commonalities and differences as could be deduced from the comparative analysis. Although, Dunlop in the preface to his Industrial Relations System gave his objective as the advancement of a general theory for the examination of industrial relations (Fajana, 2000) ; this objective is yet to be achieved. Fajana (2000, p.21) argues that "a large number of industrial relations theories have been accepted into the body of knowledge of industrial relations, although each valid theory emphasises only little aspects of the field. There is yet to emerge a general theory of industrial relations". While giving kudos to Dunlop for his pioneering efforts, one may ask; can there be a general/ unified theory of industrial relations? This is food for thought for industrial relations academics and practitioners alike.

\section{References}

Asika, N.M. (1995). "Theoretical Perspectives on the Issue of Administration".UNILAG. Journal of Business. Vol. 1 No. 1 pp 50-70.

Bain, G.S. \& Clegg, H.A.(1974). “Strategy for Industrial Relations Research in Great Britain”. British Journal of Industrial Relations. Vol.12 №.1, pp.91-113 
Blain, N. \& Gennard, J. (1970). "Industrial Relations Theory: A Critical Review" British Journal of Industrial Relations Vol. Viii №. 3 pp. 389-392.

Budd, J. (2004). Employment with a Human Face: Balancing Efficiency, Equity, and Voice. Ithaca: Cornell University Press.

Cooper,. D. R. \& Schindler, S.P.(2001). Business Research Methods (7th ed).New York: McGraw- Hill Companies.

Cox, R. (1971). "Approaches to the Futurology of Industrial Relations." Bulletin of the Institute of Labour Studies, Vol. 8, №. 8, pp. 139-64.

Dunlop, J.T. (1958). Industrial Relations Systems. New York: Holt (title now owned by Cengage Learning)

Edwards, P. (2005). 'The Employment Relationship and the Field of Industrial Relations." In. Edwards, P. (ed.) Industrial Relations: Theory \& Practice, (2nd ed.). (pp. 1-36).. London: Blackwell.

European Industrial Relations Observatory. (2002). Towards a Qualitative Dialogue in Industrial Relations. Dublin: EIRO.

Fajana, S. (2000). Industrial Relations in Nigeria: Theory and Features (2nd ed.). Lagos: Labofin and Company.

Farnham, D. and Pimlott, J. (1995). Understanding Industrial Relations (5 $5^{\text {th }}$ ed.).London: Cassell Educational Ltd.

Flanders, A. (1965). Industrial Relations: What is Wrong with the System? An Essay on Its Theory and Future. London: Farber \& Farber.

Fox, A. (1966). Industrial Sociology and Industrial Relations. Royal Commission Research Paper N0. 3. London: HMSO

Green, G.D. (1994). Industrial Relations Text and Case Studies (4th ed) U.K: Pitman Publishing.

Hyman, R. (1995). "Industrial Relations in Theory and Practice." European Journal of Industrial Relations, Vol. 1, No. 1, pp. 17-46.

Hyman, R. (1975). Industrial Relations: A Marxist Introduction. London: Macmillan.

Kaufman, B.E. (2010). "The Theoretical Foundation of Industrial Relations and its Implications." Industrial and Labour Relations Review.Vol.64, Issue1, pp.73-108.

Kelly, J. (1998). Rethinking Industrial Relations: Mobilization, Collectivism, and Long Waves. London: Routledge.

Kochan, T. (1998). "What is Distinctive about Industrial Relations Research?" In Whitfield, K. \& Strauss, G. (eds.). Researching the World of Work.(pp.31-50).Ithaca: Cornell University Press

Koontz, H; O' Donnell, C. \& Weihrich, H. (1980). Management (7th ed).Japan: McGraw-Hill Luthans, F. (1998). Organisational Behaviour (8thed.). New York: Irwin/McGraw-Hill.

Ogunbameru, A. O. (2004). Organisational Dynamics. Ibadan: Spectrum Books Ltd,

Onabanjo, I. (2001). "Globalization: Implications for Industrial Relations". Knowledge Review Vol. № 1 pp. 7-13

Otobo, D. (2000). Industrial Relations: Theory and Controversies. Lagos: Malthouse Press Ltd

Rose, E.D. (2008). Employment Relations. (3rd ed).London: Pearson Education Ltd.

Salamon, M. (2000). Industrial Relations Theory and Practice. (4th ed). London: Pearson Education Ltd. 
Waters, D. (1998). Essential Quantitative Methods: A Guide for Business. New York: Addison Wesley Longman Ltd.

Wood, S., Wagner, A., Armstrong, E., Goodman \& Davis, E. (1975). "Industrial Relations Systems as a Basis for Theory in Industrial Relations". British Journal of Industrial Relations Vol. 13 № 3, pp 291-308 


\title{
Human Capital Resourcing Practices and Organisational Performance: A Study of Selected Organisations in Lagos State, Nigeria
}

\author{
Christopher Odogwu Chidi ${ }^{1}$ and Okwy Peter Okpala ${ }^{2}$ \\ ${ }^{1}$ Department of Industrial Relations \& Personnel Management \\ University of Lagos, \\ ${ }^{2}$ Department of Accounting, University of Lagos, \\ Nigeria
}

\section{Introduction}

Organisations are microcosms of society. They are our source of niche as well as our source of scourge (Chidi \& Ogunyomi, 2010). What a paradox? It is a truism that organisations are the dominant feature of our society. According to Etzioni (1964, p.1), "our society is an organisational society. We are born in organisations, educated by organisations, and most of us spend much of our lives working for organisations. We spend much of our leisure time paying, playing, and praying in organisations. Most of us will die in an organisation, and when the time comes for burial, the largest organisation of all- the State must grant official permission". "Organisations are social units (or human groupings) deliberately constructed and reconstructed to seek specific ends or goals" (Etzioni, 1964, p.2). For organisations to meet their goals, they should be able to attract qualified and competent human capital. There is a popular maxim that the human capital is a source of competitive advantage. This is the view of the resource-based theory. The focus of this study is on recruitment and selection which are subsets of human capital resourcing practices; and to examine their relationship with organisational performance. Organisational performance has been the focus of intensive research efforts in recent times. How well an organisation implements its policies and programmes and accomplishes its strategic intent, in terms of its mission and vision is of paramount concern. Managers and administrators in both private and public organisations are becoming increasingly aware that a critical source of competitive advantage does not often come from product and services, best public relations strategy or state-of-the-art technology but from having an appropriate system of attracting and managing the organisation's human resources (Oladipo \& Abdulkadir, 2011).

Many scholars have viewed the concept of organisational performance from different perspectives, and this has been problematic since there are no universal or unified criteria for measuring the concept of organisational performance. Researchers have used financial 
and non-financial metrics to measure organisational performance. Financial measures include profit, sales, and market share. Non-financial measures include output or productivity, quality, efficiency, and the attitudinal and behavioural measures such as commitment, intention to quit, and satisfaction (Khan, 2010). Dimensions of organisational performance could therefore be financial and non-financial. Wang, Chich-Jen, and Mei-Ling (2010) citing Choi and Mueller (1992) argue that an enterprise should simultaneously consider financial and non-financial indices for performance evaluation. In other words, qualification and quantification factors should be considered at the same time, because such non-financial indices as employee morale and product quality are very important for its long-term operation.

The absence of good human capital resourcing practices could lead to dysfunctional or negative organisational outcomes and high rates of employees' separation (voluntary and involuntary). Organisations try to avoid the need for involuntary separation and to minimise voluntary separation especially among top performers as both kinds of turnover are costly (Noe, Hollenbeck, Gerhart \& Wright, 2004). Haphazard human capital resourcing and separation of valued employees could be insidious to the well-being of organisations the world over. In some organisations, be it unionised or non-unionised, public or private, manufacturing or service-oriented, the human capital resourcing function is conducted in a haphazard and informal fashion. More so, nepotism, favouritism and god-fatherism have been rife in the execution of human capital resourcing function in some organisations. According to Fashoyin (2005), recruitment and selection are heavily influenced by relations rather than by objective assessment of the suitability of the job applicant. Thus, family affiliation and friendship have substantial influence. These may lead to negligent hiring with adverse implications for employees' satisfaction, retention as well as organisational performance. In spite of such challenges, researches on effective human capital resourcing are few. According to Ploy hart (2006), in spite of the critical needs for effective staffing practice, many organisational decision makers overlook the need for staffing research necessary to identify research-practice gaps. Such studies are imperative to abridge the theory-practice gaps in human capital resourcing practices in Nigerian workplaces. An organisation reputable for incessant voluntary labour turnover will find it extremely difficult to attract talents or prospective applicants from the external labour market. Dreher and Dougherty (2002) argue that voluntary turnover is costly because replacements must be recruited, selected and trained. Thus, an excessive voluntary turnover rate can disrupt the social and communication patterns among stayers and increase stayers' workloads and stress levels. The escalation in the rate of labour turnover is a grave concern for organisations. Dess and Shaw (2001) opine that voluntary turnover represents significant direct costs (replacement, recruitment, selection, temporary staff and management time); indirect costs (staff morale, pressure on remaining staff, cost of training, product/ service quality and organisational reputation) and loss of social capital.

According to Peretomode and Peretomode (2001), the efficiency and effectiveness of any work place (whether the private or the public sector) largely depend on the calibre of the workforce. The availability of a competent and effective labour force does not just happen by chance but through an articulated recruitment exercise. Recruitment is the process of 
announcing job vacancies with a view to attracting a pool of qualified applicants to fill up vacancies in an organisation. It is regarded as a positive process. According to Etomi (2002), recruitment is concerned with the process of attracting a sufficient number of individuals with the right profile in terms of qualifications, experience, skills and other relevant attributes to indicate their interest in working for an organisation. Recruitment is the process of generating a pool of qualified applicants for organisational jobs (Mathias \& Jackson, 2004). Selection is the process of evaluating those who have been searched for and obtained through recruitment with a view to deciding whether they can be employed or not. It is also the process of choosing the individual or individuals who best meet the job-related criteria. "Selection is a decision-making activity: the psychological calculation of suitability" (Price, 2004, p.408). According to Yoder and Staudohar (1982, p.169) "selection is the process in which candidates for employment are divided into two classes - those who will be offered employment and those who will not"

The objective of this paper is to examine human capital resourcing practices and organisational performance in selected organisations in the food, beverage and tobacco industry in Lagos State, Nigeria.

\section{Literature review}

Attempt is made to review some theoretical and conceptual issues, as well as empirical studies germane to human capital resourcing practices. Human capital is the bedrock upon which all other organisational resources rest upon. Bontis, Dragonetti, Jacobsen and Roos (1999) view human capital as representing the human factor in the organisation; the combined intelligence, skills and expertise that gives the organisation its distinctive character. The human elements of the organisation are those that are capable of learning, changing, innovating and providing the creative thrust which if properly motivated can ensure the long-term survival of the organisation. According to Armstrong (1998), the word resourcing describes what managers need to do to ensure that they get the people they want in terms of numbers, skills and capabilities. This entails forecasting requirements, job analysis, recruitment and selection. Price (2004) views employee resourcing (human capital resourcing) as the process by which people are identified and allocated to perform necessary work. Thus, resourcing has two strategic imperatives: first is to minimise employee costs and to maximise employee value to the organisation; and secondly to obtain the correct behavioural mix of attitude and commitment in the workforce. Katou (2008) views human capital resourcing as comprised of recruitment, selection, separation and flexible work arrangements. This study leans on the following theories; the resource-based view or theory, contingency theory, universalistic theory and decision-making theory. Theoretical framework provides the explanations on which a study is based (Oyeniyi, 2006). What follows is an examination of the theoretical underpinnings of the study.

\section{- $\quad$ Resource-based view or theory}

Human resource has been regarded as an important factor for an organisation to gain competitive advantages and realise organisational goals or targets as advanced by the resource-based theory (Barney, 2001). This theory holds the view that the strategic 
capability of an organisation is dependent on its resource capability in the shape of people (Armstrong, 2009). The resource-based theory provides the rationale for human capital resourcing. It maintains that the long-term competitiveness of a company depends upon the resources that not only differentiate it from its competitors, but are also durable and difficult to imitate and substitute. The resource-based view, advocates that an organisation can gain competitive advantage by attracting and retaining best human resources.

\section{- Contingency theory}

Contingency theory states that the relationship between the relevant independent variables (e.g HRM policies and practices) and the dependent variable (performance) will vary according to the influences such as company size, age, technology, capital intensity, degree of unionisation, industry/sector, ownership and location (Paauwe, 2004). This theory holds the view that human resource management is influenced by the organisation's environment and circumstances (Legge, 1978; as cited in Armstrong, 2009). This is also true for human capital resourcing practices and organisational performance since they cannot exist in a vacuum but within an operating milieu or environment. Organisational performance is influenced by variables viewed as contingencies (Katou, 2009). This supports the argument of Kuchinke (2003) that organisations do not operate in a vacuum.

\section{- Decision-making theory}

The basis of the decision-making theory is the fact that decision- making is at the very heart of business success of any organisation (Gberevbie, 2006). For this study, the decisionmaking theory is adopted because human capital resourcing which promotes the achievement of organisational goals and objectives entails management decision. Thus, the more quality a decision taken by an organisation is, particularly as regards who to hire and from what source, the higher their profitability and productivity (Gberevbie, 2006). Noe, Hollenbeck, Gerhart, and Wright (2004) observed that through personnel selection, organisations make decisions about who will or will not be allowed to join the organisation. This theory assumes that decision-making is the essence of all human resource management actions and processes. It also assumes that decisions are rational and that managers examine the pros and cons of taking a particular decision.

\section{- Universalistic theory}

The universalistic theory is associated with terms such as best practices and high performance work practices. It assumes that there is a linear relationship between HR practices or systems and organisational performance and that best practices are universally applicable and successful. It posits that organisational success is best measured in terms of financial performance indicators like profits, or by market share and sales levels. Universalistic theorists are Osterman (1994), Pfeffer (1994) and Huselid (1995).

What follows are empirical studies which have established connections between human capital resourcing practices and organisational performance. Delany and Huselid (1996) 
established that practising an effective recruitment and selection process has positive relationship with organisational performance. Researchers have found a positive and statistically significant association between use of recruitment and selection procedure and profits (Terpstra \& Rozell, 1993). A good recruitment exercise enhances productivity. It also reduces cost as it reduces labour turnover rate and employee dissatisfaction (Nwachukwu, 2000). In a study in Greece, Katou and Bedhwar (2006) found that staffing and other HRM practices such as training and promotion, involvement of employees, incentives, safety and health have positive relationship with firms' performance. Gberevbie (2010), in a study on strategies for employee recruitment, retention and performance in the Nigerian civil service, argued that for an organisation to realise its goals, appropriate strategies for employee recruitment and retention are sine-qua-non for enhanced performance. In a study on recruitment, and quality of academic staff selection, the case of Covenant University, Nigeria; Gberevbie (2006) declared that the success and failure of any organisation to a greater extent depend on the quality of its work-force arising from the level of importance attached to the recruitment of its work-force. Gberevbie (2006) further observed that there is a linkage between labour sourcing decision and organisational performance. The linkage is contingent on the fact that for organisation to achieve its goals, labour sourcing decisions on where to get quality staff and when to hire them become very crucial to the performance and survival of the organisation. In a similar vein, Armstrong (2001) observed that employee resourcing strategy is a vital part of both the formulation and implementation of business strategies needed to achieve organisational performance/effectiveness.

Selection has been found to be positively related to firm performance (Terpstra \& Rozell, 1993). Researchers found a positive relationship between effective recruitment and selection practices and top-class performance (Harel \& Tzafrir, 1996; Delany \& Huselid, 1996). Selective selection is negatively related to employee turnover and is positively related with perceived profit, market share and investments in the near future (Verburg, 1998). Fajana (1995) noted that in the selection process of small and medium enterprises (SMEs) and some large enterprises (LEs), selection was often based on subjective criteria as interviews, psychological tests and medical examinations were ignored or not formally or seriously executed. References were sometimes asked for but not given proper consideration or scrutiny. From empirical research, firm size is very important for the formalisation of HR policies and practices. Hornsby and Kurato (1990) analysed personnel policies and activities on five important aspects namely; job analysis and job description, recruitment and selection, remuneration, training and performance assessment. They found that sophistication of personnel activities were directly linked with firm size. The bigger the company the more sophisticated and extensive were the policies and activities in use. Thus, with increasing size, HR policies and activities become more complex, more formalised and sophisticated. If an organisation hires an applicant without checking his/her references and background and the applicant later commits a crime while in the employ of the organisation, the organisation may be found guilty of negligent hiring (Aamodt, 1999). From experience, reference checks is one of the selection tools usually taken for granted by many organisations. The danger of this attitude towards reference checks could be negligent hiring. Dessler (2008) defined negligent hiring as hiring 
workers with questionable backgrounds without proper safeguards. Negligent hiring is injurious to the organisation, customers and co-workers. Aamodt (1999) suggested that employers via the HRM department should obtain information about the quality of previous performance by relying on an applicant's references from previous employers. Ahmed and Schroeder (2003) investigated the effect of selective hiring on organisational performance (quality, cost, flexibility, delivery and commitment). The study confirmed a positive and significant relationship with firms' operational performance. Delaney and Huselid (1996) reported that selectivity in staffing is positively related to firm performance. Employees could be hired via sophisticated selection procedures designed to screen out all but the very best potential employees that would impact on the bottom line.

\subsection{Research hypotheses}

Two research hypotheses were formulated for the purpose of this study and both tested at $5 \%$ level of significance. The authors hypothesised:

i. That there is a positive relationship between recruitment practices and organisational performance.

ii. That there is a positive relationship between selection practices and organisational performance.

\section{Methods}

The study adopted the descriptive or survey research design. There are other research designs such as observational and experimental designs. The justification for choosing the survey method is based on the fact that the study is interested in gaining an understanding of the problem being investigated without any attempt to manipulate or control the sample subjects (Asika, 1991). More so, the focus of the study is to examine the attitude or opinion of respondents as opposed to their behaviour. The survey method is ideal since large sample of respondents are required and the researchers are interested in subjecting the collected data to rigorous statistical analysis and hypotheses testing. Primary, secondary and tertiary sources of data were utilised. With a view to eliciting information from respondents, a self-developed questionnaire was designed using the 4point Likert scale ranging from strongly agree (4) to strongly disagree (1). Also, the 3point scale ranging from always (3) to never (1) was deployed. An in-depth interview was carried out to corroborate some of the responses to the items in the instrument. The instrument has 24 items. Section A sought the views of respondents on adopted human capital resourcing practices. This section has nineteen (19) items. Section B x-rayed the demographic profile of respondents with five (5) items. Cronbach's Alpha or Coefficient Alpha is .89 or $89 \%$. This implies that the instrument is reliable. A total of 105 samples were drawn from the target population using the simple random sampling technique. However 78 questionnaires were returned upon which the data analysis was based. This represents $74 \%$ response rate. Data analysis was carried out with the aid of SPSS (Statistical Package for Social Sciences) version 17. Correlation statistical technique was used to test the hypotheses at $5 \%$ level of significance. 


\section{Results}

\begin{tabular}{|c|c|c|c|c|c|c|c|c|c|}
\hline S/N & STATEMENTS & $\mathbf{N}$ & $\mathbf{S}$ & A & $\mathbf{A}$ & D & SD & MIS & STD \\
\hline & & & & 4 & 3 & 2 & 1 & & \\
\hline 1 & $\begin{array}{l}\text { Organisational performance is } \\
\text { positively related to recruitment } \\
\text { practices. }\end{array}$ & 78 & & \begin{tabular}{l|l}
$35)$ & \\
$9 \%$ & 5
\end{tabular} & \begin{tabular}{l|l}
$(40)$ \\
$51.3 \%$
\end{tabular} & $\begin{array}{c}(3) \\
3.8 \%\end{array}$ & ----- & 3.41 & .57 \\
\hline 2 & $\begin{array}{l}\text { Job analysis exercise (systematic } \\
\text { investigation in to the nature and } \\
\text { requirements of a job) is carried out } \\
\text { prior to recruitment of staff in my } \\
\text { organisation. }\end{array}$ & 78 & & $\begin{array}{l}\text { 2) } \\
4 \%\end{array}$ & $\begin{array}{l}(56) \\
1.8 \%\end{array}$ & $\begin{array}{l}(7) \\
9 \%\end{array}$ & $\begin{array}{c}(3) \\
3.8 \%\end{array}$ & 2.99 & .63 \\
\hline 3 & $\begin{array}{l}\text { Job vacancies are often advertised } \\
\text { externally in my organisation to fill } \\
\text { job openings }\end{array}$ & 78 & $\begin{array}{r}(12 \\
15.4\end{array}$ & $\begin{array}{l}\text { 2) } \\
4 \% \\
\end{array}$ & \begin{tabular}{l|}
$(29)$ \\
$37.2 \%$
\end{tabular} & $\begin{array}{c}(24) \\
30.8 \%\end{array}$ & $\begin{array}{c}(13) \\
16.7 \%\end{array}$ & 2.51 & .95 \\
\hline 4 & $\begin{array}{l}\text { Job vacancies are advertised } \\
\text { internally in my organisation to fill } \\
\text { job openings }\end{array}$ & 78 & $\begin{aligned}(20 \\
25.6\end{aligned}$ & $\begin{array}{ll}0 \% \\
6 \% & 5\end{array}$ & $\begin{array}{l}(43) \\
55.1 \%\end{array}$ & $\begin{array}{c}(13) \\
16.7 \%\end{array}$ & $\begin{array}{c}(2) \\
2.6 \%\end{array}$ & 3.04 & .73 \\
\hline 5 & $\begin{array}{l}\text { Both internal and external } \\
\text { recruitment sources are used in } \\
\text { filling job vacancies whenever they } \\
\text { arise. }\end{array}$ & 78 & & \begin{tabular}{l|}
$00)$ \\
$.6 \%$ \\
5
\end{tabular} & \begin{tabular}{|l|l|}
$(41)$ & \\
$52.6 \%$ &
\end{tabular} & \begin{tabular}{|c|}
$(15)$ \\
$19.2 \%$
\end{tabular} & $\begin{array}{c}(2) \\
2.6 \%\end{array}$ & 3.01 & .75 \\
\hline 6 & $\begin{array}{l}\text { In the recruitment of staff, my } \\
\text { organisation adopts realistic and } \\
\text { honest recruitment messages. }\end{array}$ & 78 & $\begin{aligned}(14 \\
17.9\end{aligned}$ & \begin{tabular}{l|l}
$4)$ & 6 \\
$9 \%$ & 6
\end{tabular} & $\begin{array}{l}(53) \\
57.9 \%\end{array}$ & $\begin{array}{l}(5) \\
6.4 \%\end{array}$ & $\begin{array}{l}(6) \\
7.7 \%\end{array}$ & 2.96 & .75 \\
\hline 7 & $\begin{array}{l}\text { The use of outsourcing recruitment } \\
\text { strategy is practised by my } \\
\text { organisation. }\end{array}$ & 78 & & \begin{tabular}{l|l}
$8 \%$ & \\
$1 \%$ & 4
\end{tabular} & \begin{tabular}{l|l}
$(34)$ \\
$43.6 \%$
\end{tabular} & $\begin{array}{l}(22) \\
28.2 \%\end{array}$ & $\begin{array}{l}(4) \\
5.1 \%\end{array}$ & 2.85 & .84 \\
\hline 8 & $\begin{array}{l}\text { The use of contract staff is } \\
\text { commonplace in my organisation }\end{array}$ & 78 & & & $\begin{array}{l}(32) \\
41 \%\end{array}$ & $\begin{array}{c}(26) \\
33.3 \%\end{array}$ & $\begin{array}{l}(1) \\
1.3 \%\end{array}$ & 2.88 & .79 \\
\hline 9 & $\begin{array}{l}\text { E-recruitment or online or internet } \\
\text { recruitment is practised by my } \\
\text { organisation to attract prospective } \\
\text { applicants from external labour } \\
\text { market }\end{array}$ & 78 & & & $\begin{array}{l}(10) \\
22.8 \%\end{array}$ & $\begin{array}{c}(23) \\
29.5 \%\end{array}$ & $\begin{array}{c}(26) \\
33.3 \%\end{array}$ & 2.28 & 1.17 \\
\hline 10 & $\begin{array}{l}\text { Recruitment practices are related to } \\
\text { organisational size }\end{array}$ & 78 & $\begin{array}{r}(2 \\
26 .\end{array}$ & \begin{tabular}{l|l}
$9 \%$ & 5 \\
\end{tabular} & $\begin{array}{l}(44) \\
66.4 \%\end{array}$ & $\begin{array}{c}(13) \\
16.7 \%\end{array}$ & ---- & 3.10 & .66 \\
\hline 11 & $\begin{array}{l}\text { How often does your organisation } \\
\text { deploy the following internal metho } \\
\text { of recruitment? }\end{array}$ & & $\mathbf{N} \mid A$ & $\begin{array}{c}\text { Alway } \\
3\end{array}$ & & $\begin{array}{l}\text { eetimes } \\
2\end{array}$ & $\begin{array}{c}\text { Never } \\
1\end{array}$ & MIS & STD \\
\hline i & Promotion & & 78 & $\begin{array}{c}(31) \\
39.7 \%\end{array}$ & & $0.3 \%$ & --- & 2.40 & .49 \\
\hline ii & Transfer & & 78 & $\begin{array}{c}(31) \\
39.7 \%\end{array}$ & & $\begin{array}{l}(44) \\
6.4 \%\end{array}$ & $\begin{array}{c}(3) \\
3.8 \%\end{array}$ & 2.36 & .56 \\
\hline iii & Job p & & 78 & $\begin{array}{c}(23) \\
29.5 \%\end{array}$ & & $\begin{array}{l}(50) \\
4.1 \%\end{array}$ & $\begin{array}{c}(5) \\
6.4 \%\end{array}$ & 2.23 & .56 \\
\hline
\end{tabular}




\begin{tabular}{|c|c|c|c|c|c|c|c|}
\hline $\mathbf{S} / \mathbf{N}$ & STATEMENTS & $\mathbf{N}$ & SA & A & D & SD & MIS \\
\hline & & & 4 & 3 & 2 & 1 & \\
\hline iv & Employee referral & 78 & $\begin{array}{c}(12) \\
15.4 \%\end{array}$ & $\begin{array}{c}(55) \\
70.5 \%\end{array}$ & $\begin{array}{c}(11) \\
14.1 \%\end{array}$ & 2.01 & .55 \\
\hline 12 & $\begin{array}{l}\text { How often does your organisation } \\
\text { deploy the following external } \\
\text { method(s) of recruitment? }\end{array}$ & $\mathbf{N}$ & $\begin{array}{c}\text { Always } \\
3\end{array}$ & $\begin{array}{c}\text { Sometimes } \\
2\end{array}$ & $\begin{array}{c}\text { Never } \\
1\end{array}$ & MIS & STD \\
\hline $\mathbf{i}$ & Advertisement & 78 & $\begin{array}{c}(37) \\
47.4 \%\end{array}$ & $\begin{array}{c}(41) \\
52.4 \%\end{array}$ & 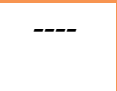 & 2.47 & .50 \\
\hline ii & Executive search agency(head hunters) & 78 & $\begin{array}{l}(7) \\
9 \%\end{array}$ & $\begin{array}{c}(50) \\
64.1 \%\end{array}$ & $\begin{array}{c}(21) \\
26.9 \%\end{array}$ & 1.82 & .58 \\
\hline iii & Employee referral & 78 & $\begin{array}{c}(15) \\
19.2 \%\end{array}$ & $\begin{array}{c}(53) \\
67.9 \%\end{array}$ & $\begin{array}{c}(10) \\
12.8 \%\end{array}$ & 2.06 & .57 \\
\hline iv & Through Unions & 78 & $\begin{array}{c}(2) \\
2.6 \%\end{array}$ & $\begin{array}{c}(25) \\
32.1 \%\end{array}$ & $\begin{array}{c}(51) \\
65.4 \%\end{array}$ & 1.37 & .54 \\
\hline $\mathbf{v}$ & Educational Institutions(scouting) & 78 & $\begin{array}{c}(8) \\
10.3 \%\end{array}$ & $\begin{array}{c}(31) \\
39.7 \%\end{array}$ & $\begin{array}{l}(39) \\
50 \%\end{array}$ & 1.60 & .67 \\
\hline vi & Private employment agency & 78 & $\begin{array}{c}(9) \\
11.5 \%\end{array}$ & $\begin{array}{l}(46) \\
59 \%\end{array}$ & $\begin{array}{c}(23) \\
29.5 \%\end{array}$ & 1.82 & .62 \\
\hline vii & Public employment agency & 78 & $\begin{array}{c}(5) \\
6.4 \%\end{array}$ & $\begin{array}{c}(25) \\
32.1 \%\end{array}$ & $\begin{array}{c}(48) \\
61.5 \%\end{array}$ & 1.45 & .62 \\
\hline viii & Professional bodies & 78 & $\begin{array}{c}(6) \\
7.7 \%\end{array}$ & $\begin{array}{c}(35) \\
44.9 \%\end{array}$ & $\begin{array}{c}(37) \\
47.4 \%\end{array}$ & 1.60 & .63 \\
\hline ix & Unsolicited applications & 78 & $\begin{array}{c}(4) \\
5.1 \% \\
\end{array}$ & $\begin{array}{c}(53) \\
67.9 \%\end{array}$ & $\begin{array}{c}(21) \\
26.9 \% \\
\end{array}$ & 1.78 & .53 \\
\hline $\mathbf{x}$ & Walk-in & 78 & $\begin{array}{c}(3) \\
3.8 \% \\
\end{array}$ & $\begin{array}{c}(59) \\
75.6 \%\end{array}$ & $\begin{array}{c}(16) \\
20.5 \%\end{array}$ & 1.83 & .47 \\
\hline 13 & $\begin{array}{l}\text { How often does your organisation use } \\
\text { the following recruitment metrics to } \\
\text { determine the effectiveness and } \\
\text { efficiency of recruitment } \\
\text { sources/methods in your organisation? }\end{array}$ & $\mathbf{N}$ & $\begin{array}{c}\text { Always } \\
3\end{array}$ & $\begin{array}{c}\text { Sometimes } \\
2\end{array}$ & $\begin{array}{c}\text { Never } \\
1\end{array}$ & MIS & STD \\
\hline $\mathbf{i}$ & Yield ratio & 78 & $\begin{array}{c}(19) \\
24.4 \%\end{array}$ & $\begin{array}{l}(39) \\
50 \%\end{array}$ & $\begin{array}{c}(20) \\
25.6 \%\end{array}$ & 1.99 & .71 \\
\hline ii & Cost per hire & 78 & $\begin{array}{c}(13) \\
16.7 \%\end{array}$ & $\begin{array}{l}(39) \\
50 \%\end{array}$ & $\begin{array}{c}(26) \\
33.7 \%\end{array}$ & 1.83 & .69 \\
\hline iii & Survey & 78 & $\begin{array}{c}(3) \\
3.8 \% \\
\end{array}$ & $\begin{array}{c}(28) \\
35.9 \%\end{array}$ & $\begin{array}{c}(47) \\
60.3 \% \\
\end{array}$ & 1.44 & .57 \\
\hline iv & Data on turnover & 78 & $\begin{array}{c}(13) \\
16.7 \% \\
\end{array}$ & $\begin{array}{c}(45) \\
57.7 \%\end{array}$ & $\begin{array}{c}(20) \\
25.6 \% \\
\end{array}$ & 1.91 & .65 \\
\hline $\mathbf{v}$ & Leads or applicant generated & 78 & $\begin{array}{l}(7) \\
9 \%\end{array}$ & $\begin{array}{c}(31) \\
39.7 \%\end{array}$ & $\begin{array}{c}(40) \\
51.3 \%\end{array}$ & 1.58 & .66 \\
\hline
\end{tabular}




\begin{tabular}{|c|c|c|c|c|c|c|c|c|}
\hline & & $\mathbf{N}$ & SA & $\mathbf{A}$ & D & SD & MIS & STD \\
\hline & & & 4 & 3 & 2 & 1 & & \\
\hline 14 & $\begin{array}{l}\text { There is positive relationship } \\
\text { between selection practices and } \\
\text { organisational performance }\end{array}$ & 78 & $\begin{array}{c}(28) \\
35.9 \%\end{array}$ & $\begin{array}{c}(49) \\
62.8 \%\end{array}$ & $\begin{array}{c}(1) \\
1.3 \%\end{array}$ & ---- & 3.35 & .51 \\
\hline 15 & $\begin{array}{l}\text { Selection practices are related to } \\
\text { organisational size }\end{array}$ & 78 & $\begin{array}{c}(12) \\
15.4 \%\end{array}$ & $\begin{array}{l}(51) \\
65.4 \%\end{array}$ & $\begin{array}{c}(15) \\
19.2 \%\end{array}$ & ---- & 2.96 & .59 \\
\hline 16 & $\begin{array}{l}\text { Employees' tenure in an } \\
\text { organisation is related to adopted } \\
\text { recruitment and selection } \\
\text { practices }\end{array}$ & 78 & $\begin{array}{l}(5) \\
6.4 \%\end{array}$ & $\begin{array}{c}(35) \\
44.9 \%\end{array}$ & $\begin{array}{c}(35) \\
44.9 \%\end{array}$ & $\begin{array}{c}(3) \\
3.8 \%\end{array}$ & 2.54 & .68 \\
\hline 17 & $\begin{array}{l}\text { Orientation/induction of new } \\
\text { staff is done by my organisation }\end{array}$ & 78 & $\begin{array}{c}(34) \\
43.6 \%\end{array}$ & $\begin{array}{c}(40) \\
51.3 \%\end{array}$ & $\begin{array}{c}(3) \\
3.8 \%\end{array}$ & $\begin{array}{c}(1) \\
1.3 \%\end{array}$ & 3.37 & .63 \\
\hline 18 & $\begin{array}{l}\text { Staff are properly placed after } \\
\text { selection in jobs for which they } \\
\text { are economically most useful }\end{array}$ & 78 & $\begin{array}{c}(20) \\
25.6 \%\end{array}$ & $\begin{array}{c}(50) \\
64.1 \%\end{array}$ & $\begin{array}{c}(8) \\
10.3 \%\end{array}$ & ---- & 3.15 & .58 \\
\hline 19 & $\begin{array}{c}\text { How often does your organisation } \\
\text { use the following selection } \\
\text { method (s) in making hiring } \\
\text { decisions? }\end{array}$ & $\mathbf{N}$ & \multicolumn{3}{|c|}{\begin{tabular}{|c|c|} 
Always & Sometimes \\
3 & 2
\end{tabular}} & $\begin{array}{l}\text { Never } \\
1\end{array}$ & MIS & STD \\
\hline $\mathbf{i}$ & & 78 & $\begin{array}{r}(69) \\
88.5\end{array}$ & & & ----- & 2.88 & .32 \\
\hline ii & & 78 & $\begin{array}{r}(56) \\
71.8\end{array}$ & & & $\begin{array}{c}(1) \\
1.3 \%\end{array}$ & 2.71 & .49 \\
\hline iii & $\begin{array}{c}\text { Assessment centre (case analysis, } \\
\text { in-basket, leaderless group } \\
\text { discussions) }\end{array}$ & 78 & $\begin{array}{r}(20) \\
25.6\end{array}$ & & $\begin{array}{l}8) \\
7 \%\end{array}$ & $\begin{array}{c}(20) \\
25.6 \%\end{array}$ & 2.00 & .72 \\
\hline iv & Work sample(practical test) & 78 & $\begin{array}{r}(19) \\
24.4\end{array}$ & & & $\begin{array}{c}(15) \\
19.2 \%\end{array}$ & 2.05 & .66 \\
\hline $\mathbf{v}$ & $\begin{array}{l}\text { Graphology(hand writing } \\
\text { analysis) }\end{array}$ & 78 & $\begin{array}{r}(10) \\
12.8\end{array}$ & & & $\begin{array}{l}(39) \\
50 \%\end{array}$ & 1.63 & .71 \\
\hline vi & $\begin{array}{c}\text { Polygraph( honesty or integrity } \\
\text { test) }\end{array}$ & 78 & $\begin{array}{r}(15) \\
19.2\end{array}$ & & & $\begin{array}{c}(38) \\
48.7 \%\end{array}$ & 1.71 & .78 \\
\hline vii & Medical examination & 78 & $\begin{array}{r}(50) \\
64.1 \\
\end{array}$ & & $\begin{array}{l}0) \\
6 \% \\
\end{array}$ & $\begin{array}{c}(8) \\
10.3 \%\end{array}$ & 2.54 & .68 \\
\hline viii & Reference report & 78 & $\begin{array}{r}(33) \\
42.3\end{array}$ & & $\begin{array}{l}6) \\
2 \%\end{array}$ & $\begin{array}{c}(9) \\
11.5 \%\end{array}$ & 2.31 & .67 \\
\hline ix & $\begin{array}{c}\text { Investigation from previous } \\
\text { employers }\end{array}$ & 78 & $\begin{array}{r}(27) \\
34.6\end{array}$ & & $\begin{array}{l}0) \\
3 \%\end{array}$ & $\begin{array}{c}(11) \\
14.1 \%\end{array}$ & 2.21 & .67 \\
\hline
\end{tabular}

Source: Field Survey, 2011.

Legend: $N=$ Respondents: $\mathrm{SA}=$ strongly agree: $\mathrm{A}=$ Agree: $\mathrm{D}=$ disagree: $\mathrm{SD}=$ strongly disagree:

MIS= mean Item Score: $\mathrm{STD}=$ standard deviation/ variation ratio

Table 1. Descriptive Statistics of Primary Data 


\begin{tabular}{|c|c|c|c|}
\hline $\mathrm{S} / \mathrm{N}$ & Variables & $\begin{array}{l}\text { Absolute } \\
\text { Frequency }\end{array}$ & $\begin{array}{l}\text { Relative } \\
\text { Frequency }\end{array}$ \\
\hline \multirow{3}{*}{1.} & Sex of Respondents & & \\
\hline & Male & 69 & $88.5 \%$ \\
\hline & Female & 9 & $11.5 \%$ \\
\hline \multirow{6}{*}{2.} & Age of Respondents & & \\
\hline & Less than 20years & ----- & --- \\
\hline & $20-29$ & 4 & $5.1 \%$ \\
\hline & $30-39$ & 48 & $61.5 \%$ \\
\hline & $40-49$ & 25 & $32.1 \%$ \\
\hline & 50 and above & 1 & $1.3 \%$ \\
\hline \multirow{5}{*}{3.} & $\underline{\text { Marital Status }}$ & & \\
\hline & Married & 49 & $62.8 \%$ \\
\hline & Single & 29 & $37.2 \%$ \\
\hline & Separated & -- & --- \\
\hline & Divorced & -- & ---- \\
\hline \multirow{6}{*}{4.} & Qualifications & & \\
\hline & OND & 1 & $1.3 \%$ \\
\hline & HND & 30 & $38.5 \%$ \\
\hline & First degree & 27 & $34.6 \%$ \\
\hline & Masters & 20 & $25.6 \%$ \\
\hline & Ph.D & --- & ---- \\
\hline \multirow[t]{4}{*}{5.} & Job Category & & \\
\hline & Junior & ----- & ---- \\
\hline & Senior & 62 & $79.5 \%$ \\
\hline & Management Staff & 16 & $20.5 \%$ \\
\hline
\end{tabular}

Source: Field Survey, 2011.

Table 2. Demographic Profile of Respondents 
In testing the first hypothesis that there is a positive relationship between recruitment practices and organisational performance, it was found that there is a positive relationship between recruitment practices and organisational performance $(r=0.45 ; \mathrm{p}<.05)$. This result is statistically significant. In testing the second hypothesis that there is a positive relationship between selection practices and organisational performance, it was found that there is a positive relationship between selection practices and organisational performance $(r=0.49$; $\mathrm{p}<.05)$.This is also statistically significant. This means that as recruitment and selection practices are properly executed or improve, so does organisational performance. It should be noted that this interpretation in no way implies causality. The significant relationship merely indicates that the variables covary.

\section{Discussion of findings}

From the test of hypotheses, it was found that there is a positive relationship between recruitment practices and organisational performance $(r=0.45 ; \mathrm{p}<.05)$. It was also found that there is a positive relationship between selection practices and organisational performance $(\mathrm{r}=0.49 ; \mathrm{p}<.05)$. These results are statistically significant. These findings are consistent with the findings of Delany and Huselid (1996) who found that practising an effective recruitment and selection process has positive relationship with organisational performance. The findings also corroborate the views of Terpstra and Rozell (1993) who found an association between use of recruitment and selection procedure and profits. The findings are also consistent with those of Ahmed and Schroeder (2003) who investigated the effect of selective hiring on organisational performance (quality, cost, flexibility, delivery and commitment) and found a positive and significant relationship with firms' operational performance.

\section{Conclusion}

This study set out to investigate human capital resourcing practices and organisational performance. From the findings of this study, it could be concluded that there is an association between human capital resourcing practices and organisational performance. From the findings of this study, the authors hereby recommend that organisations should pay adequate attention to recruitment and selection practices to gain competitive advantage.

\section{References}

Aamodt, M.G. (1999). Applied Industrial/ Organisational Psychology. (3rd ed). U.K: Wadsworth Publishing Company.

Ahmed, O. \& Schroeder, R.G. (2003). “The Impact of Human Resource Management Practices on Operational Performance: Recognising Country and Industry Differences". Journal of Operations Management. 21, pp. 19-43.

Armstrong, M (2009). Armstrong's Handbook of Human Resource Management Practice. (11 ${ }^{\text {thed.). }}$. U.K: Kogan Page Ltd.

Armstrong, M. (2001). A Hand Book of Human Resources Management Practice (8th ed). U.K: Kogan Page Ltd. 
Armstrong, M. (1998). Managing People: A Practical Guide for Line Managers. U.K: Kogan Page Ltd.

Asika, N. (1991). Research Methodology in the Behavioural Sciences. Lagos: Longman Nigeria Plc.

Barney, J.B. (2001). “Resource-based Theories of Competitive Advantage: A Ten-Year Retrospective on the Resource-based View". Journal of Management. (27), pp. 643650.

Bontis, N., Dragonetti, N.C., Jacobsen, K. \& Roos, G. (1999). “The Knowledge Toolbox: A Review of the Tools Available to Measure and Manage Intangible Resources." European Management Journal. 17(14), pp. 391-402

Chidi, O.C \& Ogunyomi, P.O. (2010). "Research and Organisational Development as Pivots of Corporate Growth and Effectiveness in Nigerian Organisations". European Journal of Scientific Research.Vol.44, No.1, pp.152-158.

Delaney, J.T. \& Huselid, M.A. (1996). “The Impact of Human Resource Management Practices on Perceptions of Organisational Performance". Academy of Management Journal. Vol.39, № 4. pp. 949-969.

Dess, G.G., \& Shaw, J.D. (2001) "Voluntary Turnover, Social Capital and Organisational Performance." Academy of Management Review. 26(3), pp.446-456

Dessler, G. (2008). Human Resource Management. (11 th ed.). New Delhi: Prentice-Hall of India Private Ltd.

Dreher, F.G. \& Dougherty, T.W. (2002). Human Resource Strategies: A Behavioural Perspective for the General Manager. New York: McGraw-Hill/Irwin.

Etomi, E. (2002). "The Changing Face of Recruitment and Selection". Human Resource Management, Journal of the Institute of Personnel Management of Nigeria.Vol.11, No.1, pp.26-33.

Etzioni, A. (1964). Modern organisations. Englewood Cliffs NJ: Prentice Hall.

Fajana, S. (1995). "Managing Human Resource for Productivity in Small and Medium Sized Enterprises."In Ojo. A.T (ed) Management of Small and Medium Scale Enterprises in Nigeria (pp.121-134). Lagos: Pumark Nig. Ltd (Educational Publishers)

Fashoyin, T. (2005). "Management in Africa”. Lagos Organisation Review.Vol.1, N0.1, pp.43-45

Gberevbie, D.E. (2010). “Strategies for Employee Recruitment, Retention and Performance: Dimension of the Federal Civil Service of Nigeria". African Journal of Business Management.Vol.4 (8).pp.1447-1456.

Gberevbie, D.E. (2006). “Recruitment and Quality Academic Staff Selection: The Case Study of Covenant University. Ife Psychologia. Vol.14, Nㅁ. 2, pp.117-141.

Harel, G.H. and Tzafrir, S.S. (1996). “The Effects of Human Resource Management Practices on the Perceptions of Organisational and Market Performance of the Firm". Human Resource Management. 38, pp.185-200.

Hornsby, J.S., \& Kurato, D.F. (1990). “Human Resource Management in Small Business: Critical Issues for the 1990s".Journal of Small Business Management.28 (3), pp.9-18.

Huselid, M.A. (1995). "The Impact of Human Resource Management Practices on Turnover, Productivity, and Corporate Financial Performance" Academy of Management Journal, 3(38): 635-672. 
Katou, A. A. (2009). “The Impact of Human Resource Development on Organisational Performance: Test of a Causal Model." Intitute of Behavioural and Applied Management, pp. 335-356.

Katou, A. A. (2008). "Innovation and HRM: The Greek Experience".Organizacija. Vol.41, №.3, pp.81-89

Katou, A. A. \& Budhwar, P. S. (2006). “The Effect of Human Resource Management Systems on Organisational Performance: Test of a Mediating Model", International Journal of Human Resource Management, 17(7), pp. 1223-1253.

Khan, M.A. (2010). "Effects of Human Resource Management Practices on Organisational Performance -An Empirical Study of Oil and Gas Industry in Pakistan". European Journal of Economics, Finance and Administrative Sciences. Issue 24, pp.157-175

Kuchinke, K.P. (2003). "Contingent HRD: Towards a Theory of Validation and Differentiation in Formal Human Resource Development". Human Resource Development Review. 2(3), pp.294-309

Mathias, R.L \& Jackson, J.H. (2004). Human Resource Management. (10th ed). Cincinnati, Ohio: South-Western Publishing.

Noe, R. A., Hollenbeck, J.R., Gerhart, B. \& Wright, P.M. (2004). Fundamentals of Human Resource Management. New York: McGraw - Hill/ Irwin.

Nwachukwu, C. C. (2000), Human Resources Management. (2nd ed). Nigeria: Davidstones Publishers

Oladipo, J.A \& Abdulkadir, D.S. (2011). “An Evaluation of Strategic Human Resource Management in Nigerian Universities: The Impact of Ownership Type and Age". European Journal of Economics, Finance and Administrative Sciences. Issue 32, pp.7-25.

Osterman, P. (1994). "How Common is Workplace Transformation and Who Adopts It?" Industrial and Labour Relations Review. 47: 173-88.

Oyeniyi, A.A. (2006). “The Influence of Students' Industrial Work Experience Scheme (SIWES) on the Development and Utilisation in Oyo and Osun States, Nigeria." Unpublished Ph.D Thesis, University of Ibadan.

Paauwe, J. (2004). HRM and Performance: Achieving Long-term Viability. Oxford: Oxford University Press.

Peretomode, V.F \& Peretomode, O. (2001). Human Resources Management: Principles, Policies and Practice, Lagos: Onosomegbowho Ogbinaka Publishers.

Pfeffer, J. (1994). Competitive Advantage Through People. Boston: Harvard Business School Press.

Ployhart, R.E. (2006). "Staffing in the 21st Century: New Challenges and Strategic Opportunities." Journal of Management. Vol. 32, № .6, pp.868-897.

Price, A. (2004). Human Resource Management in a Business Context. (2 ${ }^{\text {nd }}$ ed). London: Thomson Learning.

Terpstra, D.E. \& Rozell, E.J. (1993). “The Relationship of Staffing Practices to Organisational Level Measures of Performance." Personnel Psychology. 46, pp. 27-48.

Verburg, R.M. (1998). Human Resource Management: Optimale HRM-praktijken en Configuraties. Dissertation. Amsterdam: Vrije Universiteit. 
Wang, F., Chich-Jen, S., \& Mei-Ling, T. (2010). “Effect of Leadership Style on Organisational Performance as viewed from Human Resource Management Strategy". African Journal of Business Management .Vol. 4(18), pp. 3924-3936

Yoder, D. \& Staudohar, P.D. (1982). Personnel Management E Industrial Relations. (7th ed.). Englewood Cliffs, N.J: Prentice-Hall Inc. 


\section{Section 7}

Lean Behaviour and Sustainability 



\title{
Enhancing Productivity Through Lean Behavior
}

\author{
A. Perumal Puvanasvaran \\ Technical University of Malaysia Malacca \\ Malaysia
}

\section{Introduction}

Productivity is a measure of output from a production process. It reflects the efficiency of production. A change in technology today, however, might increase production output with a given quantity of inputs, such an increase in productivity would be more technically efficient, but might not reflect any change in allocative efficiency. Lean initiatives that focuses on tools and techniques, often stumble as excitement from initial achievements diminishes and the lean way becomes more difficult. Behavioral change is the key to making lean initiatives sustainable and successful. Lean behavior on the other hand, improves the problem solving capabilities of people in the development system while eliminating wastages, reducing cost, increasing efficiency and enhancing productivity.

Furthermore, lean is one of the popular concepts that have been implemented in many companies. According to Bhasin and Burcher (2006), very few companies succeed in implementing lean manufacturing practices. The number of lean tools, techniques and technologies available to improve operational performance is growing rapidly, however a few companies that put effort to use them failed to produce significant results. One of the major reasons for unsuccessful implementation of lean manufacturing is the typical behaviors exhibited by people at the workplace, which are known to be deficient in trust and gain commitment too. Orr (2005) stated that the term "lean" manufacturing seems to have forgotten the debate on human motivation, and has focused on techniques, where the emphasis has been on deploying new methods, rather than understanding how work is organized and lead. The practice of lean behavior is shown to be an essential element for producing healthy work environments that can lead to economic improvement. At the same time, lean produces as stated by Emiliani (1998). Emiliani and Stec (2004) that lean behavior practices must apply all the lean principles where most companies failed to apply all the lean principles together in order to get significant result. It is essential that the right behavior among organization employees is maintained, so that the full benefits of lean can be enjoyed as stated by Sanjay and Peter (2006). Implementation lean is a long journey process and not easily implemented. To fully benefit the company for lean implementation, both the concept and techniques should be considered. Lean behaviors typically are essential factor and should be assessed for a successful and complete implementation.

Thus, it is important to enhance productivity through lean behavior and inspect lean result after implementation of lean. This study will presents the results of the comparison being made between lean behaviors after implementing lean for one year through lean behavior of 
the people in the development system. The importance of the problem solving capabilities of people in implementing lean process management will be also discussed. The survey was to conduct in an aerospace composite manufacturer in their kitting department. Selfadministered questionnaire has been selected to be the survey instrument in enhancing productivity. These questionnaires were distributed to 45 employees working in the kitting department. Results of the findings and feedback are collected and analyzed by using the Statistical Package for Social Science (SPSS) software. The outputs of the analysis were in the form of index values, percentages and hypothesis testing. The result shows the improvement on lean behavior with the help of people development system implementation which enhance the people capabilities in eliminating wastages, reducing cost and increasing productivity. These are supported by comparing the results of surveys on lean behavior for the beginning and end of the year with the monitoring of real life data on the case study.

\section{Lean behavior}

Lean behavior is defined as behaviors that add or create value to waste. It is the minimization of waste associated with arbitrary or contradictory thought and actions that leads to defensive behavior, ineffective relationship, poor co-operation, and negative attitudes stated by M.L. Emiliani (1998). According to case study on Motorola, behavior is important to change culture to sustain implementing of lean concept. Many efforts failed due to the behavior of the management. Employees will follow the management's behavior if they are ordered to do new things. Anonymous, Worley and Doolen (2006) investigated two specific variables impact on lean implementation which is management support and communication. For management support, top management should not only demonstrate commitment and leadership, it must also work to create interest in the implementation and communicate the change to everyone within the organization. Comm (2005) states, that five best practiced components must present in order to apply lean. The five best practiced components are environment change, leadership, culture, employee empowerment, and communication. The management is required to have these lean behaviors which will influence the employees to practice the five components. Meanwhile, Orr (2005) stated that leadership is the fundamental aspect in engaging this different approach in thinking. Leaders are not necessarily top and senior management. Leaders are employees with influence on the work, at whatever level of seniority and responsibility. A leadership must have nine lean behaviors. The nine lean behaviors are teaches and engages workgroups, Respect For people, Process Focus, Support and recognition, Lead by example, Deploy policy and objectives, Commitment to standards, Understand lean vision and principles and Support the change process. Meanwhile, there are other findings on factors that act as barrier for implementing lean concept. Emiliani (1998) stated four primary causes that management lack influence over employees; the four components are the barrier for the commitment of whole employees to implement lean concept. The four components are Trust, Communication, Processes and Environment. Even a case study was conducted by O'hEocha (2000) on Cooke Brother Ltd manufacturing company about the influence of employee's attitudes on the use of $5 S$ which is one of the lean tools for improvement environment management. After the company applies the lean tool in their company, employees were asked to identify the potential issues that may act as barriers to effective implementation. 
A survey was conducted by interviewing the top management, middle management and the shop floor. The top management barrier to implement the tool related to issues of communication and power. There were concerns that middle managers and supervisors may feel threatened by the perceived loss of control as shop floor staffs gain more power to use initiatives and make certain changes without consultation with line managers. Even they felt that there were difficulties when it came to making decisions regarding throwing away certain pieces of equipment and machinery that are very old, do not work and take up valuable space. Middle management representatives commented that the 5Ss started off well but dwindled in certain areas. It was because they lost interest and it fell down on custom and practice/self-discipline. They also felt that they should have more power to make decisions relating to their positions. Shop floor felt that some employees had attitude problems, and put minimum effort into their jobs and were not bothered to use or implement the 5Ss, while others were actively involved. Besides, they also commented that their initiative was sometimes held back by their line managers. It was felt that certain line managers were fearful of their subordinates shining and potentially threatening their position. As a result, they did minimum and took no interest in the initiatives of $5 S$ that were likely to be protected by their line managers. From the survey, it was clear to show that the management behavior is the important barrier to implement the lean tool. In Table 1, lean behaviors practices of impact lean manufacturing are highlighted.

\begin{tabular}{|c|c|c|}
\hline Authors & Component & Lean Behaviors Practice \\
\hline Worley J.M. and Doolen T.L & $\begin{array}{l}\text { i. Management support } \\
\text { ii. Communication }\end{array}$ & $\begin{array}{l}\text { - Commitmest withoat fear, respeet to people, recognition to people } \\
\text { - Clear communication }\end{array}$ \\
\hline Clare L. Comm & $\begin{array}{l}\text { i. Environment for change } \\
\text { ii. Leadership } \\
\text { iii. Culture } \\
\text { iv Employee empowerment } \\
\text { v. Commanication }\end{array}$ & $\begin{array}{l}\text { - Understanding and proactive } \\
\text { - Assist and coact employees, respossible } \\
\text { - Honest and respect to people } \\
\text { - Given recognition for employees } \\
\text { - Share information, understand the goal }\end{array}$ \\
\hline Emiliani & $\begin{array}{l}\text { i. Trust } \\
\text { ii. Communication } \\
\text { iii. Processes } \\
\text { iv. Envircenment }\end{array}$ & $\begin{array}{l}\text { - justice without favors some people, meet the promise } \\
\text { - Clear message, quick feedback, } \\
\text { - Clear about their responsibilities, follow procedures } \\
\text { - Given recognition to people, understanding people problem }\end{array}$ \\
\hline Cameron & i. Leadership & $\begin{array}{l}\text { Teaches and engages workgroups, respeet for people, process focus, support } \\
\text { and recognition, lead by example, deploy policy and objectives, commitment } \\
\text { to standards, understand lean vision and principles, support the change process }\end{array}$ \\
\hline
\end{tabular}

Table 1. Component and Lean Behaviors Practice Of Impact Lean Manufacturing

\section{Tips to build lean behavior}

It is a present-day instance of the recurring theme in human history toward increasing efficiency, decreasing waste, reducing cost and using empirical methods to decide what matters, rather than uncritically accepting pre-existing ideas. In order to enhance productivity, we therefore acquire a broader set of skills, creative and innovative approaches to analysis, using up-to-date tools and design through the implementation of lean production methodology and management. As an example, manufacturers must be able to use various of methodologies, ideas and many others of present-day high technology tools to be able to enhance problem productivity and do lean production effectively. Meanwhile, both manufacturers and consumers that comprise the different status levels in the community will have easy and convenience access to the real-time oriented systems for problem solving capabilities and productivity enhancement. For this to occur, tips to build lean behavior are proposed in this chapter for enhancing productivity. 
The organizational vertical integration has parallels input values with other lean disciplines such as lean behaviors are therefore important. Positive organizational behavior which is linked to human resource strengths and psychological capabilities for performance improvement and productivity enhancement in today's workplace. Worker inappropriate behaviors are difficult to change because they are functional: they serve a purpose as an employee. The practice of lean behaviors is shown to be an essential element for producing healthy work environments that can lead to economic growth, as well as help businesses sustain efforts to become lean producers. The principal focus is on how individuals can consistently behave in ways that create value, with the goal of eliminating waste in both intra and interpersonal relationships M.L. Emiliani (1998).

Several tips that leaders can use to improve their team lean behavior. Such as:

1. Defining lean behavior

Most people like to see the correct or the right way of handling work but the correct or right way is not always clear. They might think of doing a right way without looking into lean principals. Lean wants problems and abnormal conditions to be exposed and no wastage nor costliness. Leaders have to make sure that doing the right way doesn't have a high cost to team members.

2. Rewarding who has lean behavior

When the demand for goods is decreasing, workers stop to do overtime. The leader needs to thank to workers for their action. If the workers continue doing their overtime, they are doing it for the wrong reason and that is to increase the overhead cost of the company. Some people like to bypass a process to make things happen quickly. Leader should stop rewarding them of heroism. This might be sending a wrong message.

3. Making system support lean behavior

Leaders must make the workplace look like $5 S$ environment that support lean behavior. Workers will do and follow what the leader normally says. If the workers keep collecting data without analyzing it or no change is being made. Do you think this system support lean behavior? Definitely not! This will lead to wasting of time and expenses.

4. Building up a right lean behavior team

When people see others exhibiting good behavior, they are more likely to continue or follow it. Leaders need to set up a good example of team that can perform good lean behaviors so that others will follow the right steps and procedures.

5. Eliminating problem members in the team

If a member doesn't meet productivity and quality standards after repeated attempts to work with in order to improve, then get a new member. If a customer has expectations that are unreasonable and unprofitable to company, find more customers and nothing to say that you need to deal with them. If an employee chooses not to show lean behavior, they may not be a good match for the team.

\section{Comparison of behavior attributes}

The organizational vertical integration reflects the level of business community that exhibits the most consistent generative behaviors, as well as helpful tendencies, will be the partner of choice for employees, suppliers, customers, and investors. Lean behaviors exhibited by the organization culture should be a strong source of competitive advantage. Table 1a below 
compares common fat behaviors that result in waste and selected lean behaviors that promote flow between people as stated by Cleary (1989) and (1996). It is important to realize that interpersonal skills and organizational effectiveness are developed by practicing and improving upon weaknesses (i.e. lean behaviors), not strengths (i.e. fat behaviors) as mentioned by M.L. Emiliani (1998).

\begin{tabular}{ll}
\hline Fat behaviors & Lean behaviors \\
\hline Confusion & Self-awareness \\
Unnecessary commentary & Humility \\
Irrelevant observations & Compassion \\
Random thoughts & Suspension \\
Self-imposed barriers & Deference \\
Ego & Calmness \\
Irrationality & Quietude \\
Revenge & Reflection \\
Inaction & Honesty \\
Positions & Benevolence \\
Interpretations & Consistency \\
Uncertainty & Generosity \\
Negativity & Patience \\
Excess & Humor \\
Gossip & Understanding \\
Sarcasm & Respect \\
Preoccupation & Listening \\
Ambiguity & Observation \\
Extreme flattery & Trust \\
Cynicism & Sincerity \\
Subjectivity & Equanimity \\
Bias/prejudice & Objectivity \\
Deception & Discipline \\
Selfishness & Rectitude \\
Pride & Wisdom \\
Criticism & Balance \\
\hline
\end{tabular}

Table 1.a Comparison Of Behavior Attributes by Cleary (1989) and (1996).

\section{Do lean behaviors correlate with productivity?}

The answer is probably yes, when we consider the practice of lean behaviors as shown to be an essential element for producing healthy work environments that can lead to productivity growth, as well as help production sustain efforts to become lean producer. The principal focus is on how individuals can consistently behave in ways that create value (lean behavior), with the goal of eliminating waste in interpersonal relationships and development of people that possess basic capabilities for problem solving in their thoughts and actions. If the performance of lean behaviors can be practiced by individuals and then integrated into the organization, the productivity will definitely be enhanced by lean behavior.

Liker (2004) stated about how organization should implement the lean process management, there is no exact definition for a fully lean organization. Even though the goal of becoming a 
fully lean organization can only be reached if the employees are well aligned with new philosophy. In that case, it is important for an organization to understand and apply all lean behavior practices and principles, in addition to a comprehensive lean thinking which affects the whole business model as a key and not solely learner production. Figure 1 shows the key participants in a business, each having a relationship governed by processes and behaviors governing generative relationships. Each of these relationships should be carefully managed in order to minimize waste and maximize the benefits to productivity enhancing.

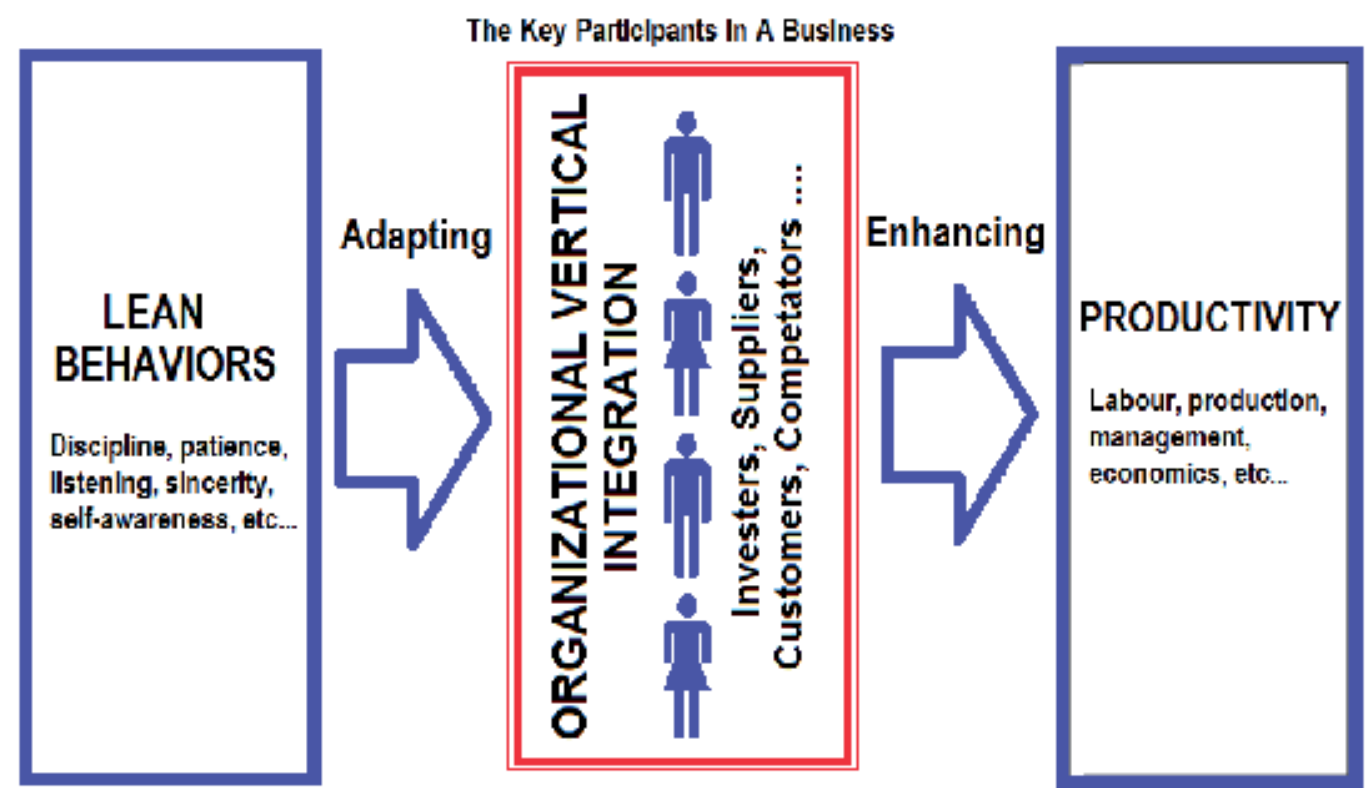

Fig. 1. Enhancing Productivity Through Lean Behaviors In A Business

\section{Background of case study}

The Company where the case study was conducted was incorporated on 16th August 1994. Currently, numbers of employees are 1155 person. The nature of business for this company is to manufacture composites components for aero and non aero structures. The name of this company is changed to $A B C$ in terms of confidential issues. $A B C$ was given a mandate by the government to spearhead Malaysia's foray into the high technology industry of aerospace and composites manufacturing.

The objective of the establishment of $\mathrm{ABC}$ is to become the manufacturing arm for $A B C^{\prime} \mathrm{s}$ work cluster. $A B C$ created the work cluster with the aim to provide design, manufacturing and aircraft production services to relevant industries. This company fits into the business plan by participating in manufacturing activities for sub-contract work. To date, $A B C$ has succeeded in securing major wing manufacturing programmed with leading aerospace companies, BAE systems, specifically for the manufacture of Airbus A300, A320 and A380 range of aircrafts. The company has also secured non-aerospace composites component manufacturing of the Alvis Bridging Launch Rail in Advance Composites. Within a short span of time since its formation, $\mathrm{ABC}$ has emerged as a leading aerospace company in the 
region and a known industry player in the world. Though $\mathrm{ABC}$ having start to implement Lean Manufacturing System since 2004, but there are some mistakes and frailness due to the lack of implementation which is observed as in production system, where the knowledge and understanding of lean manufacturing system as common and primary root cause problem. The whole of problems occurred throughout from the top level to the bottom. Due to this, the top management commitment, teamwork, and people capabilities in eliminating wastages are also lacking. Furthermore, the problems occurred because the lean implementation was not linking to the individual, department, and company's key performance indicator which was unmotivated the total employees of the company to practice the real of lean concepts. Therefore, based on this reality the lean behavior among the employees never rooted. Effectively in the end of 2006, the company overcomes the past problems with new perspective of lean implementation by developing the integration and heuristic approach of lean concepts as a new strategy that involve all aspects of the company in their operation that correlated to the KPI. The scope of study for this project is conducted only at kitting department.

\section{Developing a new system to productivity enhancing}

In today's competitive world, no company can afford to waste resources. The most underutilized resource of most manufacturing company is their people assets. The number one asset of any organization is also its people. In fact, people are one of the few appreciating asset an organization has. The real advantages of employee's involvement are to focus a group of employees with different perspective on a single objective that support the organization's strategic focus. The companies that develop and leverage the capabilities of all their employees will achieve better performance than those that do not. The companies that fail to unlock the potential of their workforce will be forced to carry more overhead, have more layers of management, will be slower to react to market change and opportunities. Therefore, since we implement lean as a system in which the people functions need to be developed into a system which called "People Management Systems" to provide the capability for rapid improvement and adoption to change. Each of the three systems in framework has its own objective. The objective of the lean process management system is to identify and eliminate wastages by removing non value added activities. People management systems need to provide the capability for rapid improvement and adoption to change. Here, again, we must accept the fact that change is inevitable and that the speed with which the necessary modification are made is the deciding factor in our survival. The objective of the business management system is to apply carefully the organization's limited resources, including capital and hard assets as well as time and human assets. Three integration elements with total employee involvement from top to bottom play an important role for sustaining problem solving among employees in practicing lean concept. It is important to create people development system which consists of all these three elements with total involvement of people to increase problem solving capability. People management system, Business management system and lean process management system are integrated by principles that, in a sense, hold them together. These principles are meant to provide a framework (Figure 2) to focus the direction in enhancing productivity and problem solving capability among employees by forming as people development system in lean process management. They are: 
- Key performance indicator - KPI for every level such as company, department, section and individual levels which is linked towards the organization goal.

- $\quad$ Respect for people - Respect for people which mainly focuses on the lean behaviors that each employee in organization should build in their mind.

- Skill and Knowledge - Skill and Knowledge for employees will support them in practicing lean concept effectively and efficiently by utilizing the lean tool and techniques.

Another important element incorporated with this people development system framework is teamwork of top, middle and bottom management. The total commitment of all these three levels will enhance the productivity and the problem solving capability in lean process management among employees.

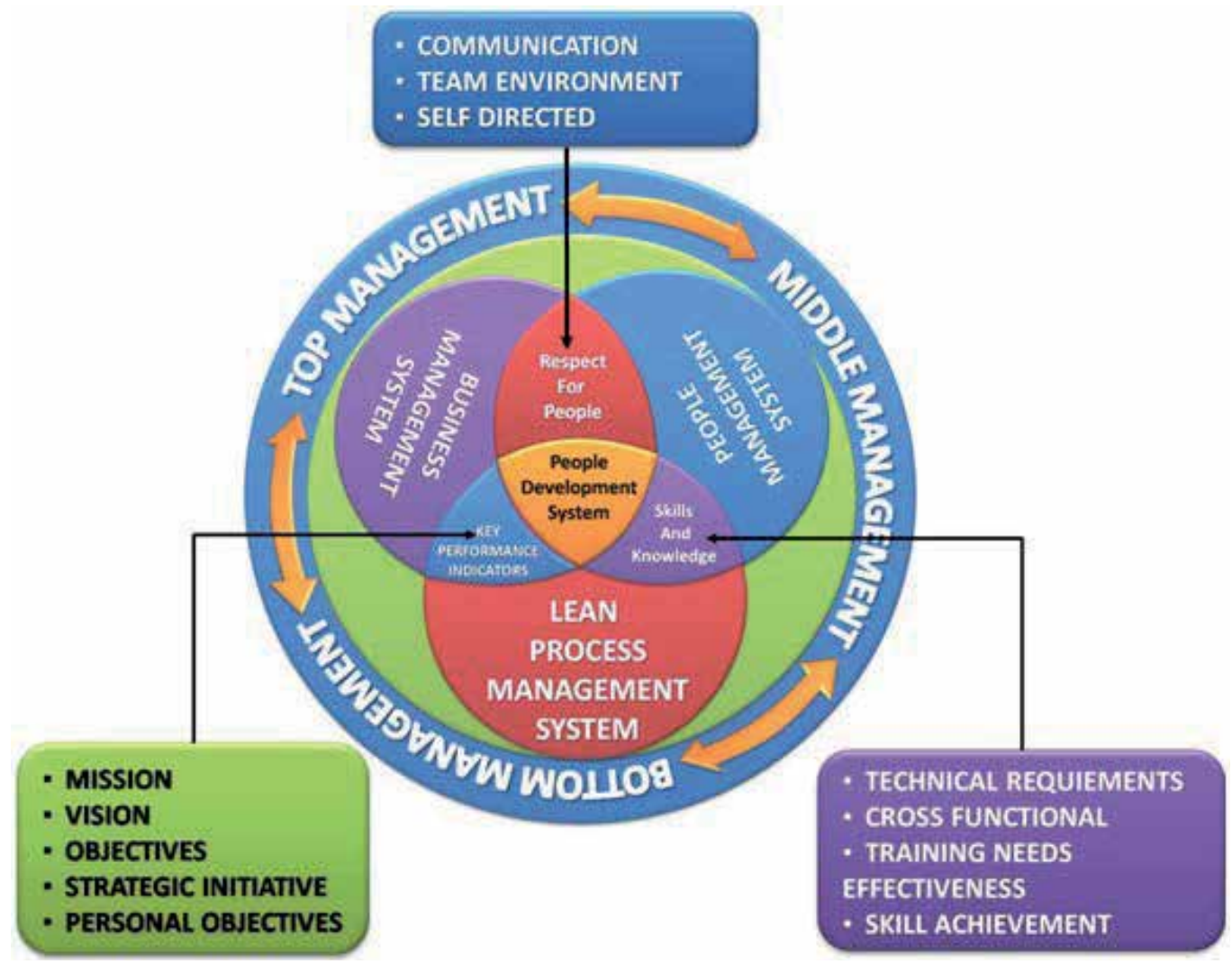

Fig. 2. PDS Framework for Enhancing Productivity and Problem Solving Capabilities Among Employees.

\section{Key characteristic, critical success factors (CSF) and related performance matrix}

The following key characteristics, CSFs and related performance metrics are identified A.P. Puvanasvaran et al (2008), as crucial in people development system of lean process management and are highlighted in Table 2. 
- KPI in lean process management determination through Mission, Core Value, Vision, Objective, Strategy, Strategy Initiative and Personal Objective for people development system is crucial. This will align overall workforce of the company to follow one common goal. Each level has its own portion of contribution towards the target. The results are compared with the target or goal used to measure the success of KPI. The accumulation of success from each portion will reflect the overall achievement of the company goal.

- $\quad$ Respect for people in lean process management is another crucial factor in developing the lean culture throughout organization. In order to measure the lean behaviors, top management commitment, leanness level of the company and perception of team member's capability, Liker (2004) type scale is used to get the responses from respondent. For example, one can ask managers to rate the degree of support by top management on five-point scale from no support (1) to total support (5). Beside this, the problem solving capability also can be measured by counting the number of ideas generated, Level of people involved and the total cost of the project.

- Skill and Knowledge in lean process management is the fundamental requirement for employees to equip themselves. Without this they can't perform well in solving problem to identify and eliminate wastages. Lean tools and assessment techniques by using assessment criteria to determine the level of implementation using spider web chart with rating of 1 (beginning to introduce) to 5 (practice with excellent). Another measurement on employee skill metric will emphasize on employees skill and their cross functionality.

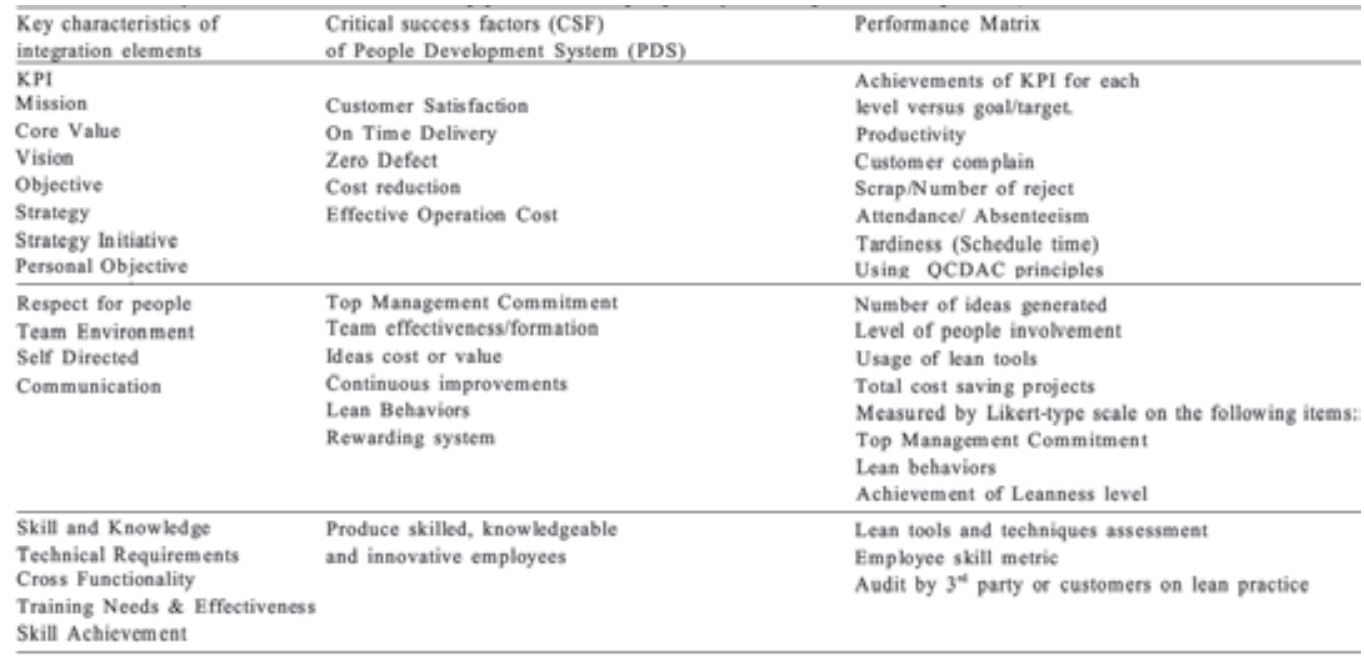

Table 2. An Analytical Framework For Measuring Problem Solving Capability In Lean Process Management

\section{Methodology}

To conduct the case study survey, the questionnaire was used which was developed by the Ford Motor company. The questionnaires contain the criteria of lean behavior practices based on the literature review Orr (2005). The lean behavior practices are divided into three 
categories which are; respect for people (RFP), continuous learning and improvement (CL\&I) and process and result driven (P\&RD). This is exactly to fulfill the Toyota "4 P model" Orr. For the RFP and CL\&I, there are nine variables asked, and for P\&RD, there are twelve variables to answer. The answers of questionnaire were using the four-point scale and circle the appropriate number. The response scale ranges from 1 to 4 representing the range of strongly agree, agree, disagree, and strongly disagree.

The index value is used to determine the strength and weakness of lean behavior practices. The index value is calculated by formula provided by Nesan and Holt (2002).

$$
\text { Index }=\left[\left(n_{1}\right)+2\left(n_{2}\right)+3\left(n_{3}\right)+4\left(n_{4}\right)\right] /\left[4\left(n_{1}+n_{2}+n_{3}+n_{4}\right)\right]
$$

Where $n_{1}, \ldots, n_{4}$ represent the number of respondents that indicated the respective practices on the scale 1 to 4 . The formula yields indices ranging from 0 to 1 , where below 0.2 represent minimum strength and above 0.8 represents maximum strength

Nesan and Holt (2002). The second analysis is to determine the relationship or mean score of each level of management for each practice. Analysis of variance (ANOVA) is used to analyses situations in which there are several independent variables and how these independent variables interact with each other Field. Before calculating the ANOVA, one assumption must be considered is the score of variable is normally distributed. For the third analysis, correlation is used to measure the difference between each principle variables and how are they related. Before calculating a correlation coefficient, there are a few assumptions for correlation analysis which are normality and linearity Coakes (2005). Pearson's correlation coefficient is a measure of linear association with the score for each variable is normal distributed. If the relationship is not linear and normal distributed, Spearman's rho will be used to measure the correlation between the variables Coakes (2005).

\subsection{Pilot test}

Pilot test is conducted to ensure the finding from the questionnaire is valid and meet the objective of this project. This is done by sending the questionnaire to two lean expertise of the company. Discussion on the questionnaire was held when the company was visited. Opinion was given which help researcher to modify the questionnaire. Besides that, from the pre-test, the total time spend to answer the questionnaire also can be identified.

\subsection{Sending and receiving questionnaire}

The questionnaires send to a composite manufacturing company in Malaysia. The questionnaire is directed to three levels of the company, which are, top management, engineers and operators, and shop floor workers. For the top management level respondent, the questionnaires were answered by all department of the company. Meanwhile, the questionnaire only rated by kitting area department for the last two level respondents. The feedback is received within two weeks from the company. The total feedbacks are 53 . The questionnaires send to one of composite manufacturing company in Malaysia. The questionnaire is directed to three levels of the company, which are, top management, engineers and operators, and shop floor workers. For the top management level respondent, the questionnaires were answered by all department of the company. Meanwhile, the 
questionnaire only rated by kitting area department for the last two level respondents. The feedback is received within two weeks from the company.

\subsection{Analysis using SPSS}

After getting the result from company, authors will use the software SPSS version 13 to make the analysis. In the 2 part of the question (about lean behavior), the outputs of the analysis were in the form of index values, percentages and hypothesis testing. In the literature review state that Emiliani and Stec (2004) explain lean behavior is applying lean principles and tools to improve leadership behaviors and eliminate behavioral waste.

\section{Results and discussion}

In order to assess the lean behavior before and after the lean implementation, a questionnaire was distributed and then an internal consistency analysis was used to evaluate the reliability of questionnaire.

\subsection{Respondent rate}

The questionnaire distributed directly to the employees to do the survey. The beginning of the year (January) questionnaire was distributed to 45 employees of the kitting department. However, 3 employees already resign. Thus, the questionnaire only assigned by 42 people and the feedback collected exactly 42 respondent results. At the end of the year, questionnaire was distributed to 44 people and collected back exactly 44 responds.

\subsection{Reliability test}

Internal Consistency Analysis: An internal consistency analysis was used to assess the reliability of questionnaire. It is an indicator of how well the different items measure the same issue. The measurement of internal consistency involve for calculation of Cronbanch's coefficient alpha. The values of alpha range from 0 to 1 where the value close to 1 indicate higher reliability. Alpha value should be positive and usually greater than 0.7 which are considered acceptable for testing the reliability of factors. As shown in table 3, the alpha value for the January 2007 in the three categories range is from 0.721 to 0.821 . For respect for People, the scale of reliability can be increased by eliminating Q02 which show 0.815 . For Continuous Learning and Improvement, the scale of reliability it is better to include all of the nine questions and if any questions were eliminated, will reduce the scale reliability. Last but not least for Process and Result Drive, the scale of reliability can be improved by eliminating Q30 which show 0.829. For the December 2007 the alpha value in three categories ranges from 0.718 to 0.758 . For respect for People, the scale reliability can be increased by eliminating Q03 which show 0.739. For Continuous Learning and Improvement \& Process and Result Driven the scale of reliability were reduced Q12 and Q23,which show 0.768 and 0.801 .

Although, the alpha value for the 3 categories for December 2007 is decreased when compare with January 2007, but the range is greater than 0.7 , so the instrument are consider acceptable. Furthermore, the elimination questions are not necessary as the alpha value increase slightly after eliminating. Since the alpha value are greater than 0.7 , it can conclude that this instrument is reliable. 


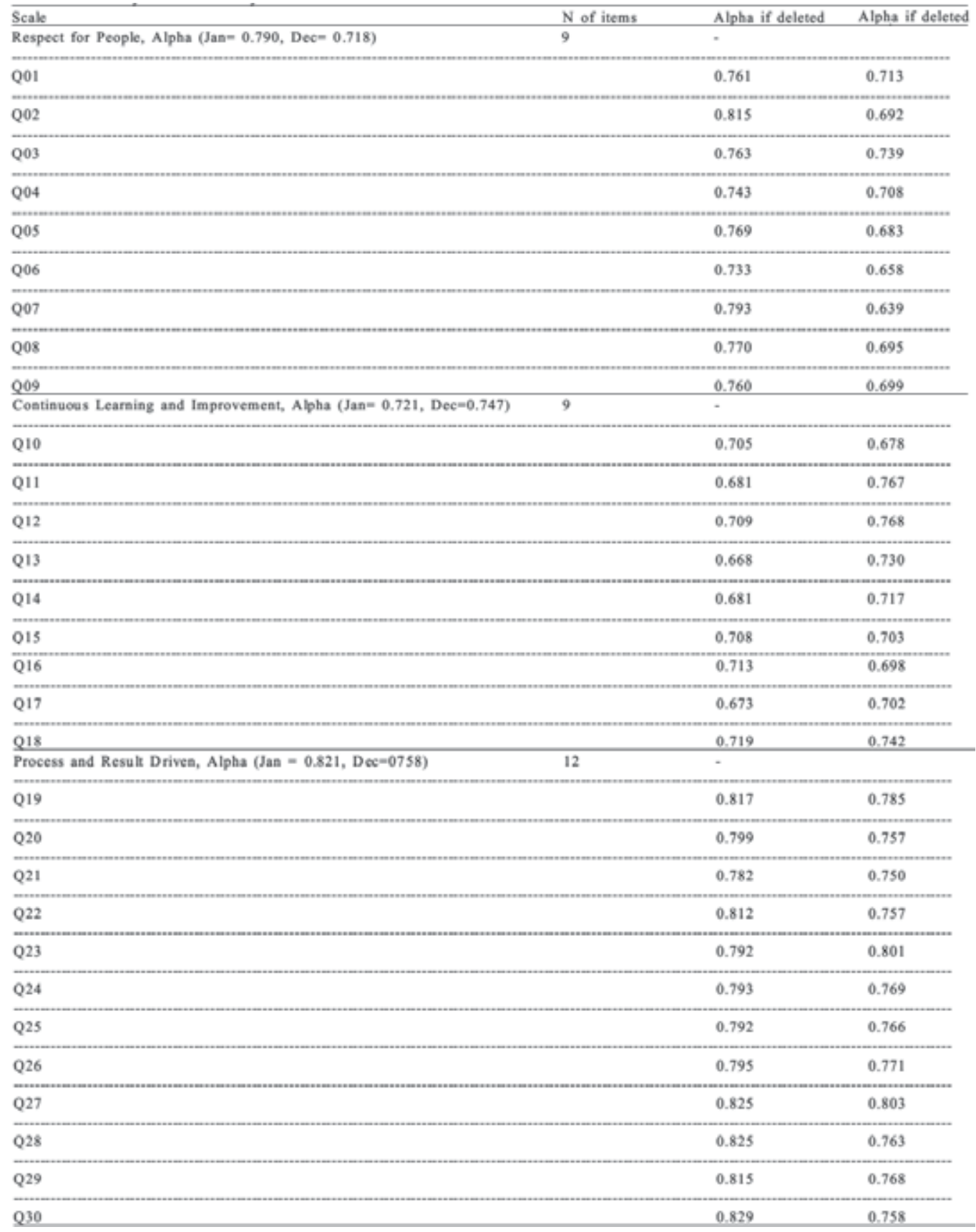

Table 3. Reliability Statistic January 2007 and December 2007

\subsection{Analysis and results}

The structured postal questionnaire survey was designed to assess initial literature search finding concerning the 30 practices, in two different dimensions. Dimension 1 investigated 
the strength and weakness of lean behavior practices within the organization. Dimension 2 explored the relationship between the lean principles. For each dimension, four Likert (2004) scales ranging from 1 to 4 were provided and the scale was used is the agreement scale. Agreement scale is used to determine agreement on of the 30 lean behaviors practices, the scale ranged from 1 (strongly disagree) to 4 (strongly agree).

\subsection{Analysis of strength and weakness of the lean behaviors practice}

Data obtained from the survey were subjected to relative index calculations for agreement factor. The relative index was calculated by using the formula:

$$
\text { Index }=\left[\left(n_{1}\right)+2\left(n_{2}\right)+3\left(n_{3}\right)+4\left(n_{4}\right)\right] /\left[4\left(n_{1}+n_{2}+n_{3}+n_{4}\right)\right],
$$

Where $\mathrm{n}_{1}, \mathrm{n}_{2}, \mathrm{n}_{3}, \mathrm{n}_{4}$ represent the number of respondents that indicated the respective practices on the scale 1 to 4 . The formula yields indices ranging from 0 to 1 , where below 0.2 represent minimum strength and above 0.8 represents maximum strength Nesan and Holt (2002). From the table 4, the indices calculated for all of the lean practices showed a similar pattern, with indices ranging between 0.464 and 0.78 for January 2007. For the December 2007, the range is between 0.597 and 0.818 . In addition, the min index for 30 practices also increased from 0.691 to 0.7614 , total increments is about $10 \%$. This indicates that lean behavior practices in aerospace composite manufacturer are improved, and it is near to the lean behavior standard. After comparing the 2 group of index, we found that the index for the practices is increased a lot. Such are:

- $\quad$ Meetings start on time. +0.215

- $\quad$ People from outside areas help to solve problems. +0.207

- People share ideas and knowledge.+0.151

However, there is also some practices need to be improved where the indices show dropped. Such practices are:

- People contribute openly and honestly in the meetings I attend.-0.029

- People are coached and trained by their leaders/Supervisors.-0.017

- People deliver what was promised.-0.013

Basically, for the new result, many performances were practiced very well. It can be seen clearly In Figure 3 and the sum of index of practices is increased a lot and meets the lean behavior standard (0.800) already. The results are better if compared with the January 2007 which is totally negative and only one practice meet the lean behaviors standard. Below are the practices that meet the standard:

- $\quad$ Before making decisions, people gather the information -from 0.708 to 0.818

- People share ideas and knowledge-from 0.667 to 0.818

- People are encouraged to improve their knowledge and skills at work -0.738 to 0.813

- People look for ways to improve their work -from 0.750 to 0.801

- People focus on the customer and the customer need (inside and outside the plant) from $0.750-0.818$.

However, one of the practices that needs improvement and need to be given more attention is the indices showed very low. The practice is: 


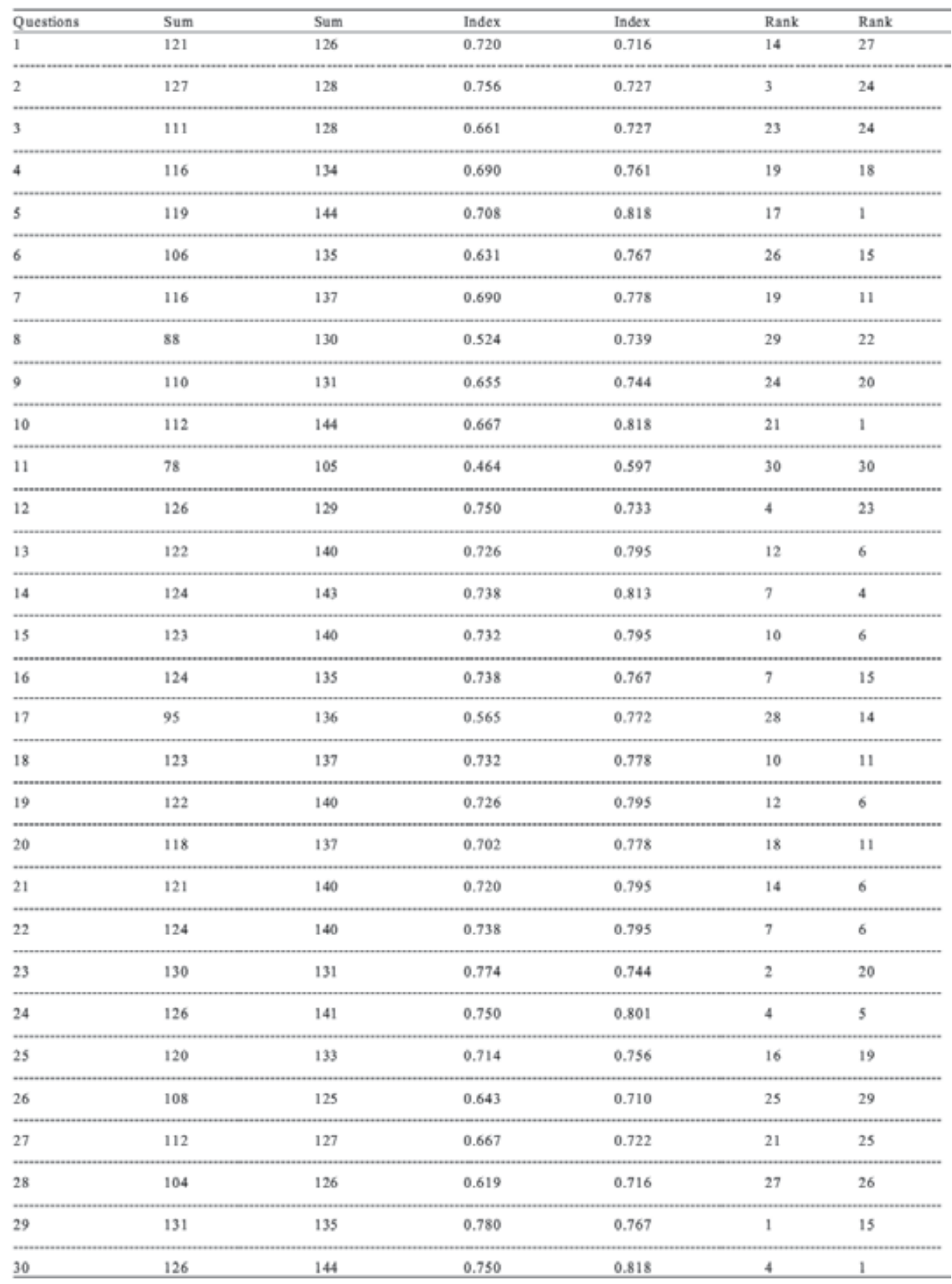

Table 4. Strength Values For January 2007 and December 2007 
- Plant leadership is on the plant floor daily to provide assistance and improve the business is 0.597 . However; the index also improved already, for the Jan 2007 just 0.464

Beside the index, after comparing the 2 group of ranking the authors found that the ranking for the practices also change drastically. These are:

- People share ideas and knowledge, it is raise 20 rank, from ranking 21 raise to 1 . It is the biggest lift practices:

- Before making decisions, people gather information. It is raise to 16 rank, from ranking 17 raise to 1 .

- People from outside help to solve the problem. It is raised 14 rank, from ranking 28 raise to 14 .

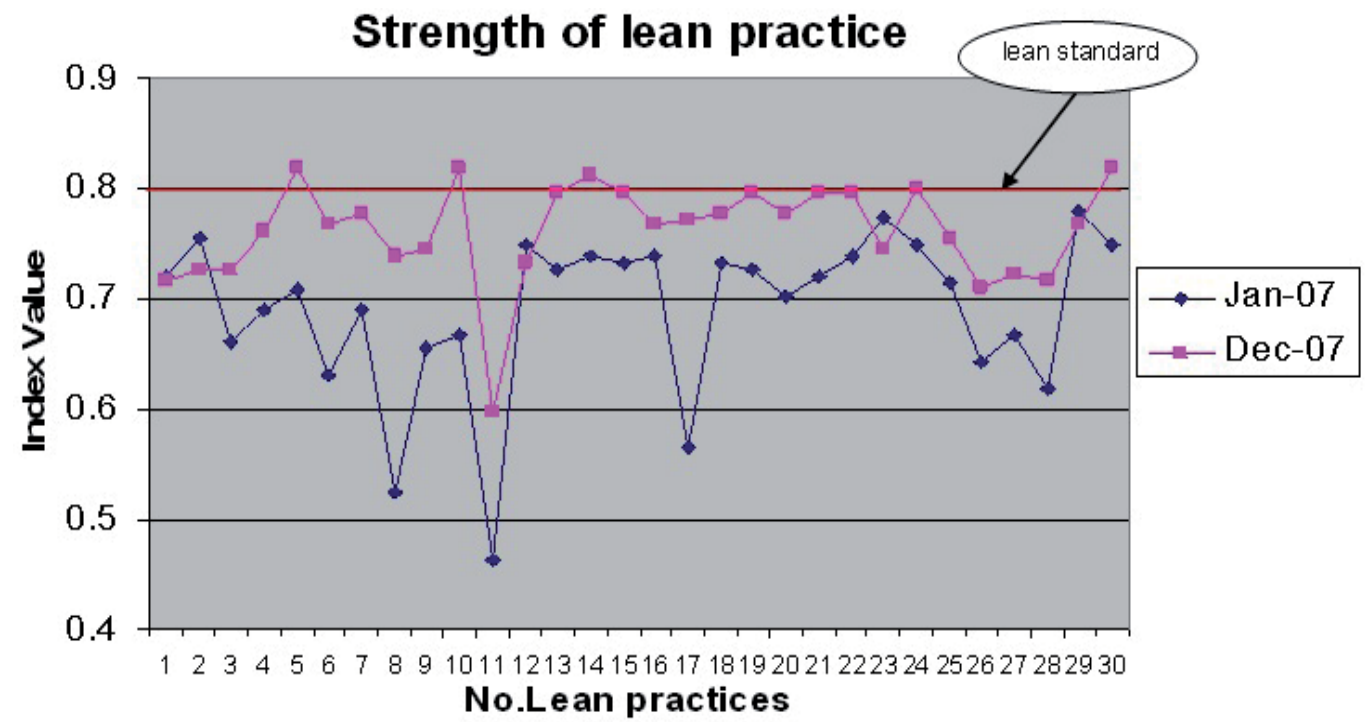

Fig. 3. Strength of Lean Practices.

The result at Table 5 shows that the increment respects for people was the highest. The practices for RFP such as, people contribute openly and honestly in the meeting will give employees operational autonomy encouraged an innovative culture and let employee contribute more ideas to solving problems. Furthermore, the practices "people can participate in decision relevant to their job and focus on the problem" in RFP also got strong relationship with PSC. In a study among the employees of a manufacturing plant, it found a positive relationship between participation and employees' innovative behavior, measured using self-ratings of employees' suggestions and implementation efforts will contribute to the idea of solving problem.

\begin{tabular}{lll}
\hline Construct & Jan 2007 & Dee 2007 \\
\hline Respect for people ( RFP) & 0.67 & 0.773 \\
Contiauces learniag asd improvement (CLAI) & 0.679 & 0.763 \\
Process and feselt Driven (PARD) & 0.653 & 0.698 \\
\hline
\end{tabular}

Table 5. Analysis Mean Value Index RFP, CLAI, PARD. 
The Figure 4 shows that index value of three main categories which all have significant increase. Especially, respect for people, which is increased from 0.67 to 0.773 . This is followed by continuous learning and improvement raised from 0.679 to 0.763 and the process and results driven shows improvement from 0.653 to 0.698 in each. Overall the results shows the company improved in all 3 construct, Thus, we can say, the company really put a lot of effort in practices lean behavior as shown in the comparison of lean behaviors in Table 8 and Figure 5. In conclusion, authors found that most practices of the lean behavior will improve the PSC of the employee. Thus, after implementing lean process after one year, problem solving capability of employee had been increased and make the lean result of company increase.

\section{Lean Behavior}

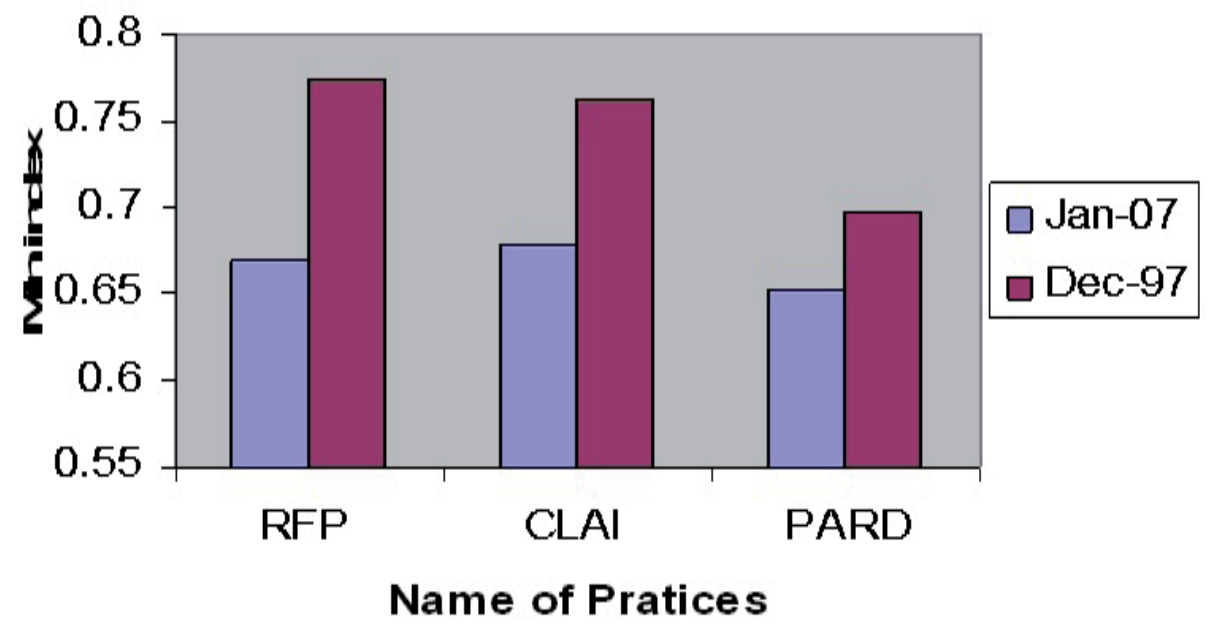

Fig. 4. Lean Behaviors Index Value

\subsection{Analysis of the relationship between the lean principles}

Correlation between Respect for People, Continuous Learning and improvement and Process and Result Driven are shown in Table 6 and Table 7. The data obtained was analyzed by using Statistical Package for Social Science (SPSS) software version 13. Correlation method was used where correlation is a measure of relationship involving variables Field. Table 6 and Table 7 show a matrix that is displayed giving the correlation between the three variables. For the January 2007correlation coefficient 0.587 between

\begin{tabular}{|c|c|c|c|c|}
\hline & & $\begin{array}{l}\text { Respect for } \\
\text { People }\end{array}$ & $\begin{array}{l}\text { Contiauous Learning } \\
\text { and improvement }\end{array}$ & $\begin{array}{l}\text { Proeess a ad } \\
\text { Result Drivea }\end{array}$ \\
\hline Respect for People & Correlation coefficient & 1.000 & $0.456 * *$ & $0.562 * \bullet$ \\
\hline \multirow[t]{2}{*}{ Continucus Learning and improvement } & Correlation coefficient & $0.456 *$ & 1.000 & 0.193 \\
\hline & Significast value & 0.001 & - & 0.111 \\
\hline Process and Resulk Driven & Significant value & 0.000 & 0.111 & . \\
\hline
\end{tabular}

Table 6. Correlation between Factors In January 2007. 
Respect for People and Continuous Learning and Improvement is 0.456, and the significance value of this coefficient is 0.001 . But for December 2007, the values become 0.129 , and significance value is 0.202 while the correlation coefficient between Respect for People and Process and Result Driven is 0.526 with the significance value is 0 . But for new result it is 0.101 and the significance value is 0.258 .last but not least, correlation coefficient between Continuous Learning and improvement and Process and Result Driven is 0.193 with the significance value is 0.111 . For new result is 0.310 and 0.020 .

\begin{tabular}{|c|c|c|c|c|}
\hline & & $\begin{array}{l}\text { Respect for } \\
\text { People }\end{array}$ & $\begin{array}{l}\text { Continuous Learning } \\
\text { asd improvemeat }\end{array}$ & $\begin{array}{l}\text { Process and } \\
\text { Result Drivea }\end{array}$ \\
\hline Respect for People & Correlation coefficient & 1.000 & $0.587 * 0$ & 0.101 \\
\hline \multirow[t]{2}{*}{ Contineous Learniag and improvement } & Correlation coefficient & $0.587^{* *}$ & 1.000 & 0.310 \\
\hline & Significant value & 0.001 & . & 0.020 \\
\hline Process and Resulh Driven & Significant valec & $0.258^{*}$ & 0.020 & . \\
\hline
\end{tabular}

Table 7. Correlation between Factors In December 2007

\begin{tabular}{lll}
\hline Variable & Index (Jan) & Index (Dec) \\
DOB & 0.691 & 0.7164. \\
\hline
\end{tabular}

Table 8. Index Value of Lean Behavior In January 2007 and December 2007

Comparison for Lean behaviors

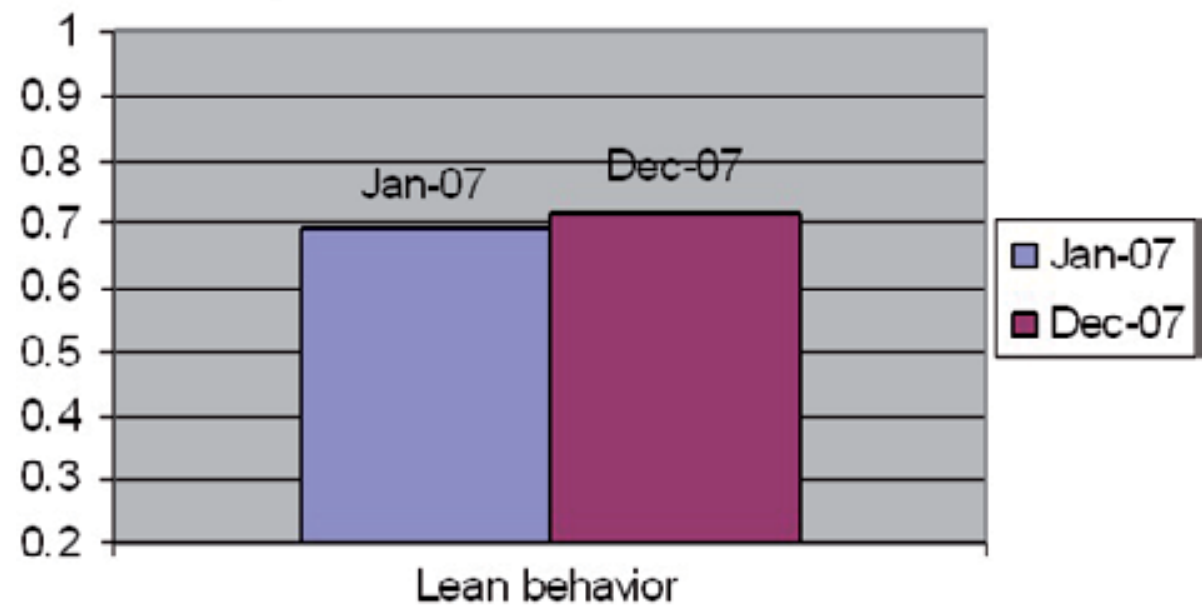

Fig. 5. Comparison for Lean Behaviors.

\section{Success of people development system in case study company}

The importance of problem solving capabilities of every employee in implementing lean process management to make the improvement in lean behaviors is evident as depicted by the real life data of kitting department as the company case study. 


\subsection{Idea generated and level of involvement}

Many studies focus mainly on the creative or idea generation stage of problem solving. In this context, employees can help to improve business performance through solving problem, such as generating ideas and use them as building blocks for new and better products, services and work processes Joreon. P.j.de.long (2007). From the graph shown below, every week at least one idea had been generated in kitting department, and the highest is 5 ideas generated per week. In past one year, a total of 139 ideas have been generated .It is proved that kitting department proactively and continually sought ideas to solve problems, indicates that employees have the capability to solve problem to become a central tenet of lean manufacturing best practice Kerrin (1999).

The employee involvement is categorized according to three main levels which are top, middle and bottom management. The Figure 6 below shows the level of involvement of employees by generating ideas for the year 2007. The highest contribution is coming from bottom level which is 38 and followed by middle level with 12 to top level which is 2 . Besides this, there is also a combination level involvement in generating ideas. Bottommiddle level is 52, middle-top is 35 and bottom-top is 1 . Furthermore, total idea generated for group combination level is 87 and single group level is 52 . The percentage for combination level is $63 \%$ for total ideas generated and 3 type levels is $37 \%$. However if we compare 2 groups, the result shows that total idea generated by group combination level is 35 more than single group level. Thus, the result indicates the teamwork of bottom; middle and top management in both sharing and applying knowledge for generating ideas in solving problems that are important Delbridge et al (1998).

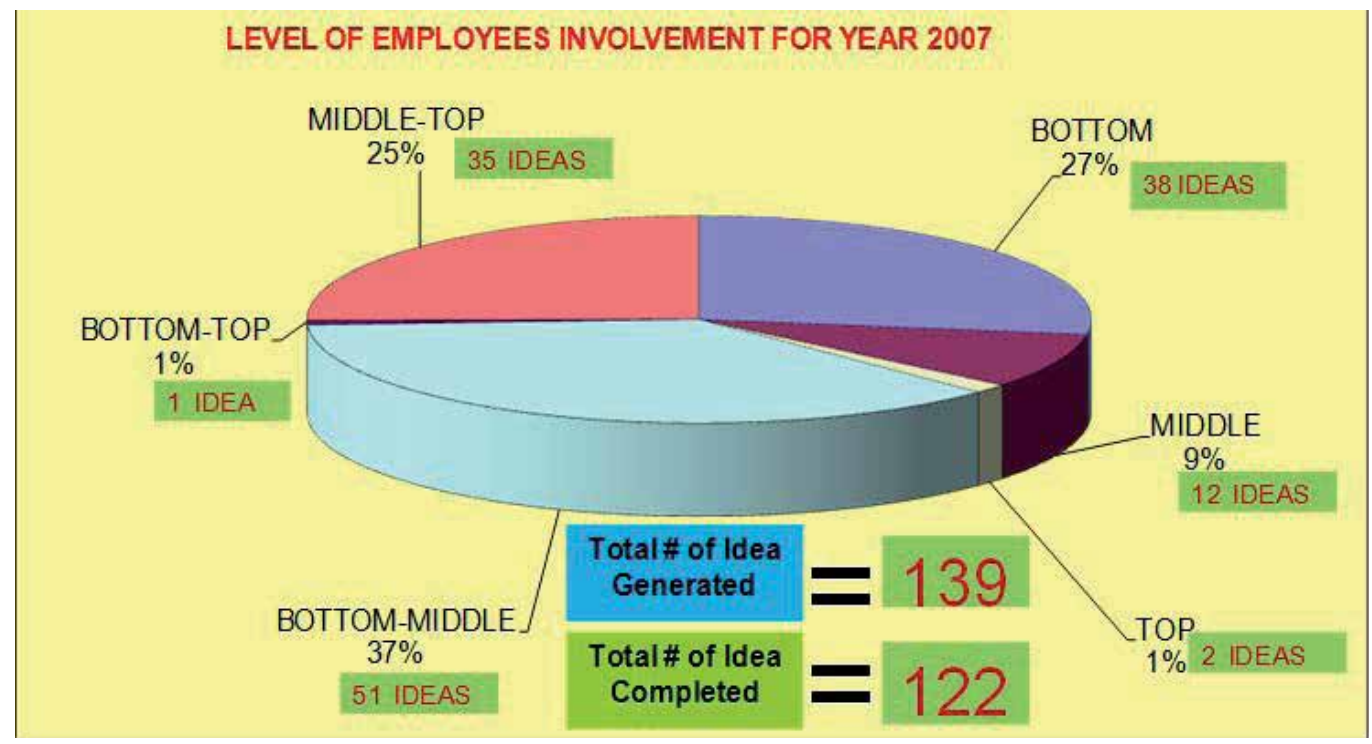

Fig. 6. Level of Employee's Involvement for Year 2007

\subsection{Total of wastages}

The graph at Figure 7 gives us an idea about type of wastages identified at the kitting department, where it is classified into 9 categories. It is obviously noted that the highest 
waste for company is the waste of space which is 39 , the second one is time waste at 21 , and the lowest waste is transportation which is at 5 . Without classification of any wastage into performance measurement, no monitoring can be made and no problem solving can be done to reduce the waste, where the impact is the failure of lean process management implementation A.P. Puvanasvaran et al (2008). It indicates that the employees of the company have capabilities to solve waste problem using the performance measurement.

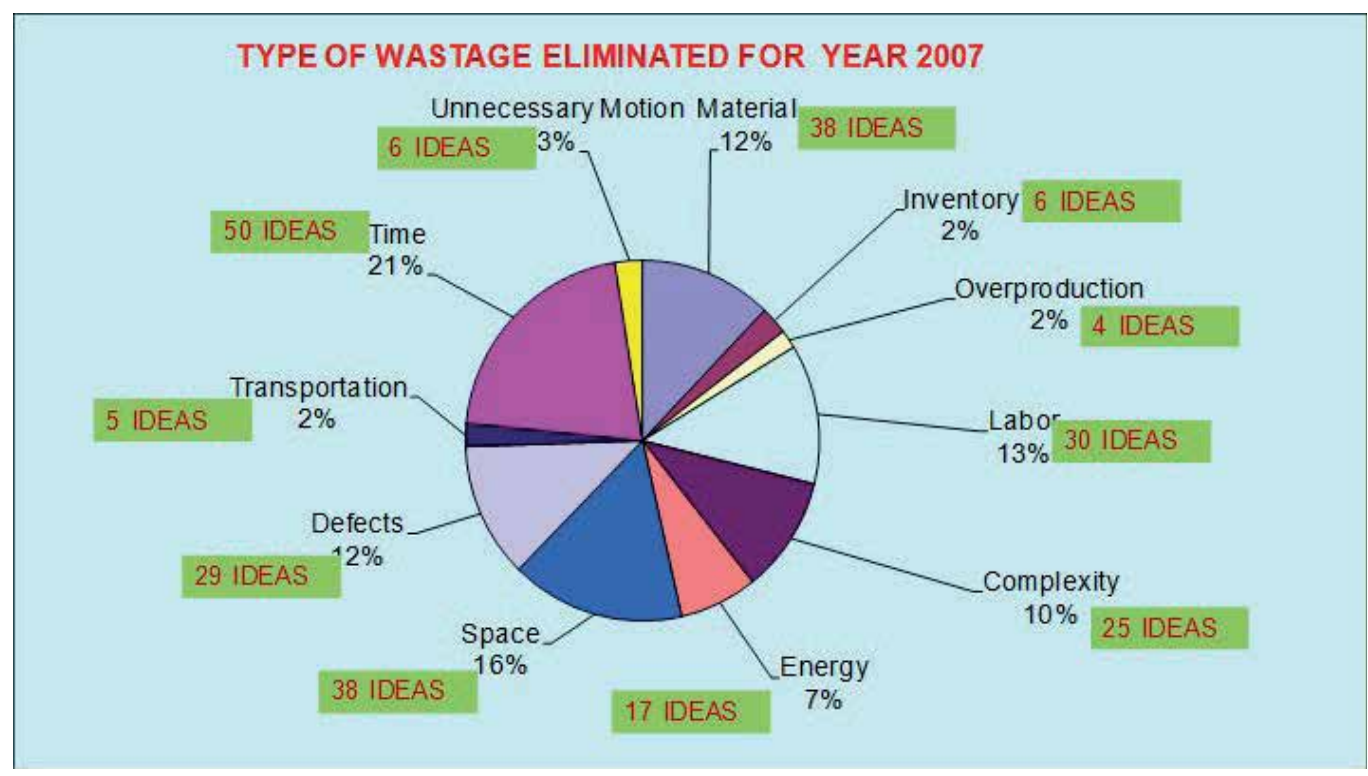

Fig. 7. Type of Wastage Eliminated for Year 2007.

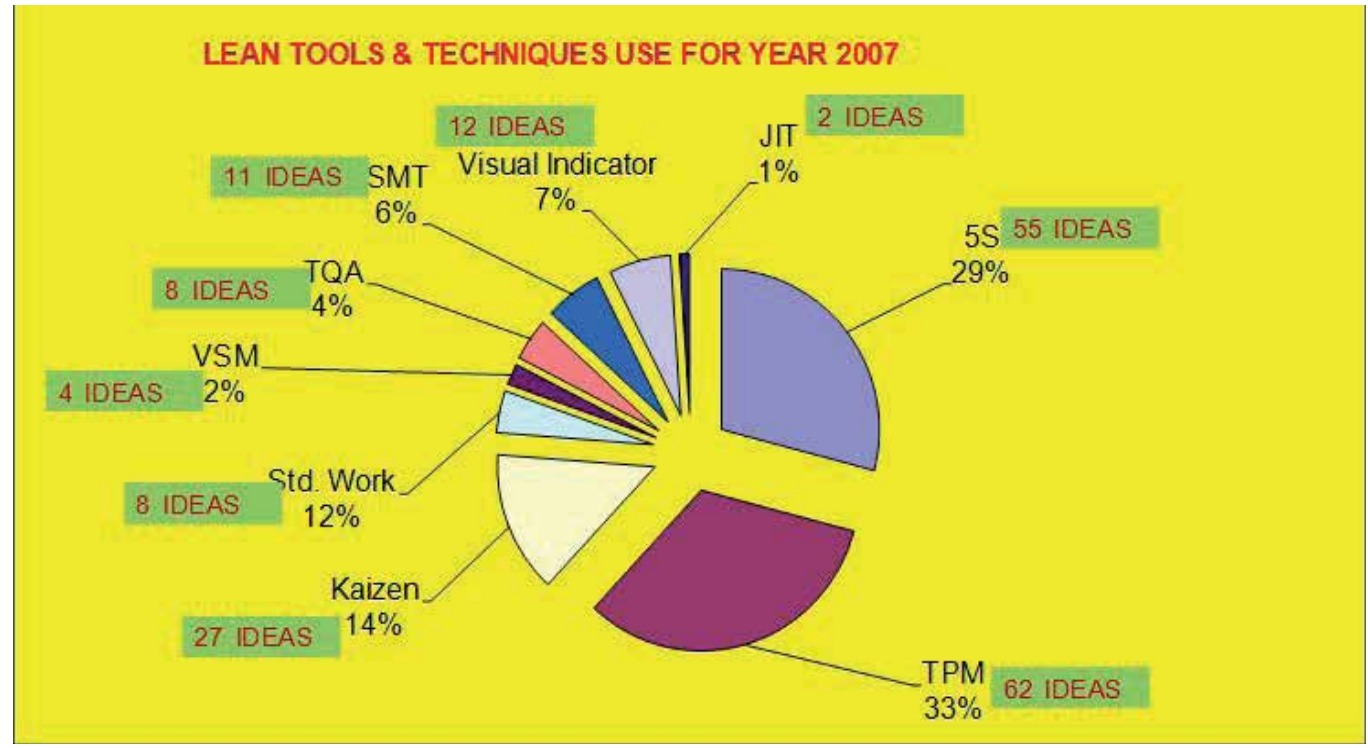

Fig. 8. Lean Tool and Techniques Use for Year 2007. 


\subsection{Cost saving}

Cost saving is an important standard to indicate the problem solving capability of the organization. The aim of lean manufacturing is elimination of waste in every area of production and includes customer relations, product design, supplier networks, and factory management. To meet the objectives of saving cost, Womack and Jones (1996). Kitting department ran a Kaizen Project in 2007, and the total amount of saving for reduces wastages in past one year is RM1,952,617.98. Thus, achievement of cost saving for company indicates employee had contributed most of the solutions to solve the problem of waste. So, it can be construed that problem solving capability of employees actually improved significantly. The Table 9 indicates total cost savings of the kitting department with the reference to their kaizen project generated form the problem solving activities.

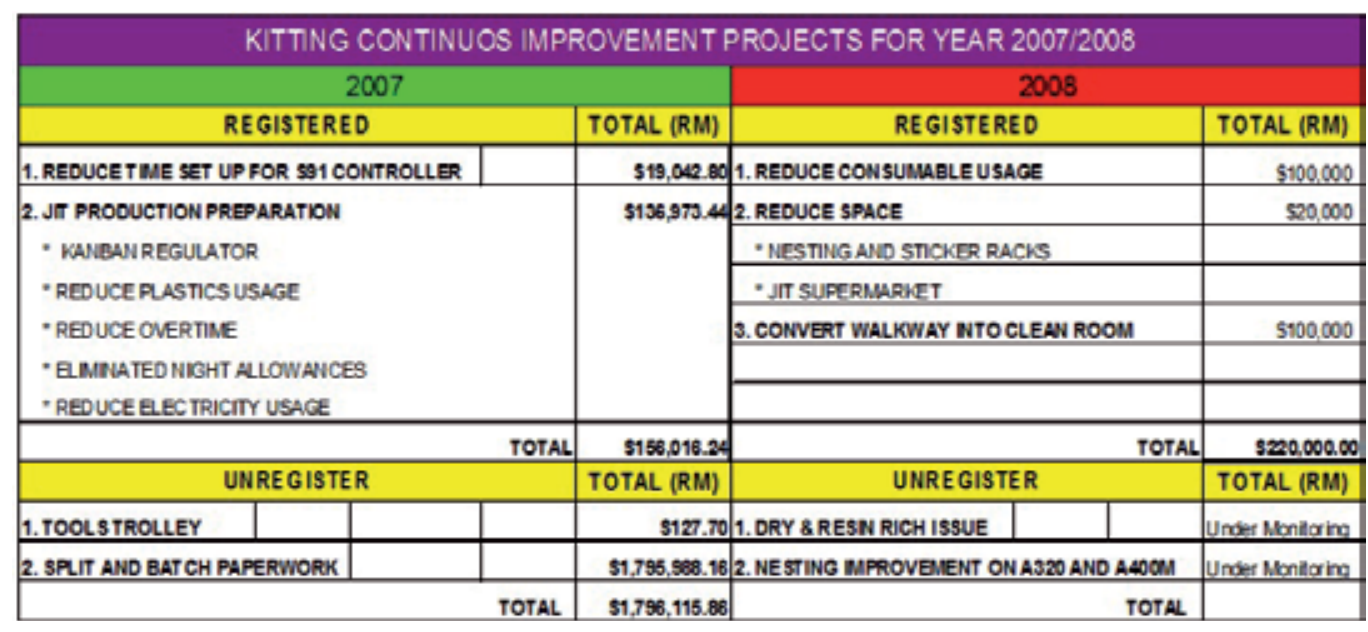

Table 9. Continuous Improvement Projects for the Year 2007 and 2008. 


\section{Lean tools used in problem solving and productivity enhancing}

In general, lean tool for kitting department can be categorize to 9 types as shown in Figure 8, such as five $S$, total productive maintain, Kaizen, visual stream map, visual indicator, just in time, and standard work chart. The most common tool used by kitting department is TPM, which is 62 times, followed by $5 \mathrm{~s}, 55$ time and mean values of using lean tool is 21 times. In the past one year, the kitting department total used 189 times of lean tool. In order to introduce lean thinking within manufacturing environment, the philosophy relies on the identification and elimination of the waste problem, which have effectively targeted and applied the various lean tools B.J. Hicks (2007). Thus, the frequency of employee using the lean tool indicates employee understanding identification and elimination of waste problem. In deduction, the employees have capability of problem solving.

\section{KPI achievement}

KPI is an important element that enables the achievement of vision, mission, core value, strategy, and the personnel objective for people development is crucial. Achievement of KPI shows the evidence of people involvement to drive high performance to gain stakeholder and customer satisfaction. Monitoring on each performance, measurement and countermeasurement taken to solve any problem occurring have contributed to the achievement of KPI.

The Table 10 shows total monthly man hours percentage of Overtime at kitting department has set the limit to be below $12 \%$ for the year 07. During PDS implementation, overtime was controllable all the time; not even a month exceeded the limit of overtime, which eventually gave a value of $11.5 \%$ for the whole year. It indicates that the company has saved considerably on labors cost in the past one year. The cost saving is due to employee success in lowering the stop time for the machine DCS 1, DCS 2, DCS 3.

\begin{tabular}{|c|c|c|c|c|}
\hline Principles & Matrix & Unit & Goal/Limit & $\begin{array}{c}2007 \\
\text { Achievement }\end{array}$ \\
\hline \multirow{4}{*}{ Quality } & Scrap & $\mathrm{MQ} \%$ & $2.60 \%$ & $1.97 \%$ \\
\hline & NCR & $\%$ & $7.80 \%$ & 0 \\
\hline & Snag Sheet & Control Limit $\%$ & $20 \%$ & 0 \\
\hline & Audit & \# of CAR & Zero & 1 \\
\hline \multirow{6}{*}{ Cost } & Overtime & $\begin{array}{c}\text { Total Monthly man } \\
\text { hours } \%\end{array}$ & $12 \%$ & $10.50 \%$ \\
\hline & Downtime & $\%$ & $10 \%$ & \\
\hline & DCS 1 & $\%$ & $10 \%$ & $9.14 \%$ \\
\hline & DCS 2 & $\%$ & $10 \%$ & $8.80 \%$ \\
\hline & DCS 3 & $\%$ & $10 \%$ & $7.65 \%$ \\
\hline & S91 & $\%$ & $10 \%$ & $10.34 \%$ \\
\hline Delivery & Output & $\%$ & $97 \%$ & $100 \%$ \\
\hline \multirow{5}{*}{ Accountability } & Attendance & $\%$ & $92 \%$ & $90.7 \%$ \\
\hline & Training & Hours & 18shrs & 2314hrs \\
\hline & Staff/rg hours & $\%$ & 47 staff & $100 \%$ \\
\hline & Major Accidents & Qty accidents & Zero & 0 \\
\hline & Accident Free Days & \# of days & 90days & 365 \\
\hline \multirow{3}{*}{$\begin{array}{l}\text { Continuous } \\
\text { Improvement }\end{array}$} & Kaizen & $\mathrm{RM}$ & $150 \mathrm{~K}$ & $156 \mathrm{~K}$ \\
\hline & SMT & Level & Level 4 & L4 \\
\hline & $5 S$ & Level & Level 4 & L4 \\
\hline
\end{tabular}

Table 10. KPI Achievement. 
The table also shows the achievement for the value scrap is $1.97 \%$ which achieved the goal of $2.6 \%$. The reason why the value is achieved is because the employees use the PDS methods to solve many scrap problems such as material dry and ply damage for the whole year. Complaints on product produce from internal and external customer shows null. It shows that quality of kitting department undergone significant upgrading due to problem solving capability of employees.

The Kitting department of the Company has gained benefits from many elements that have not been monitored before, the implementation of PDS. Wastages have reduced dramatically. Thus, the achievement KPI proves that productivity and problem solving capability has increased.

\section{Conclusion}

The purpose of this project has been to evaluate the improvement for the lean behavior possessed by the company in past one year. It reflects the enhancing productivity through lean behavior. The result show lean practices had been making improvement of the company in lean direction and important of problem solving capabilities in eliminating waste and saving cost thus, enhancing productivity. The results have provided support to the two proposed hypotheses. Besides that, evidence was found to support the relation between improvements of kitting department with problem solving capability on enhancing productivity through lean behaviors of employees in the organization.

The main findings show that the company is improving in past one year. Initial result of the kitting department was in moderate level stage to become lean, but result at the end of the year showed that they had nearly meet the high level stage of lean they need to keep their efforts in order to have productivity success in lean manufacturing implementation. It is also helpful to the company to recognize the importance of increased problem solving capability and productivity enhancing for employee to eliminate waste by adapting lean behaviors. In short, we are all looking toward "Better Productivity Through Better Lean Behavior".

\section{References}

[1] Puvanasvaran, A.P., B.H. Tan, M.H.M.A. Megat, Tang S.H, Muhamad, M.R, A.M.S. Hamouda, 2008c. "Degree Of Leanness And Managerial Commitment In An Aerospace Company" Journal of Statistics and Management System, Vol. 11, No. 4, pp. 653-673, ISSN 0972-0510, Taru Publications, India.

[2] Puvanasvaran, A.P., M.H.M.A. Megat, Tang S.H, Muhamad, M.R, A.M.S. Hamouda, 2008a. "A Review of Problem Solving Capabilities in Lean Process Management" American Journal of Applied Sciences Vol.5 No. 5, 2008,ISSN 1546-9239 (C) 2008 Science Publications, pp: 504-511.

[3] Puvanasvaran, A.P., M.H.M.A. Ooi, Megat, S.H. Tang, M.R. Muhamad, A.M.S. Hamouda, 2008b.“Lean Behavior Among Employees In Aerospace Company" 
Journal of Productivity, National Productivity Centre, Petaling Jaya, Malaysia, 25 pp: $29-44$.

[4] Anon, 1997. "Case study: Guiding cultural change at Motorola", Management Development Review, 10(4/5): 185-187.

[5] Bhasin, S. and P. Burcher, 2006. "Lean viewed as a philosophy", Journal of Manufacturing Technology Management, 17(1): 56-72.

[6] Cleary, T. (1989), Zen Lessons - The Art of Leadership, Shambhala, Boston, MA.

[7] Cleary, T. (1996), The Human Element, Shambhala, Boston, MA.

[8] Coakes, S.J., 2005. SPSS: Analysis without Anguish-version 12.0 for Windows, John Wiley \& Sons Australia. Ltd, Queensland.

[9] Comm, C.L., 2005. "A case study in applying lean sustainability concepts to universities", International Journal of Sustainability in Higher Education, 6(2): 134146.

[10] Delbridge, R., 1998. “Life on the Line in Contemporary Manufacturing," International Journal of operation \& Production Management, Oxford University Press, Oxford.

[11] Emiliani, M.L. and D.J. Stec, 2004. "Leaders lost in transformation", Leadership and Organization Development Journal, 26(5): 370-387.

[12] Emiliani, M.L., 1998. “Lean behaviors”, Management Decision, 36/9, pp: 615- 631.

[13] Field, A., 2005. “Discovering Statistics Using SPSS”, Sage Publications, pp: 107.

[14] Hicks, B.J., 2007. "Understanding and eliminating waste" international Journal of information Management.

[15] Jeroen, P.J. and N.D.H. Deanne, 2007. "How leaders influence employees' innovative behavior. Emerald Group Publishing Limited, pp: 761-771.

[16] Kerrin, N., 1999. Managing Innovation and Change: A Critical Guide for Organizations, Thomson, London.

[17] Liker, J.K., 2004. The Toyota Way: 14 Management Principles from the World's Greatest Manufacturer. McGraw Hill: New York.

[18] Nesan, L., Jawahar and D. Holt Gary, 2002.“ Assessment of organizational involvement in implementing empowerment ", Integrated Manufacturing Systems, 13/4, pp: 201-211.

[19] O'hEocha, C., 2000. "Case studies: A study of the influence of company culture, communications and employee attitudes on the use of 5Ss for environmental management at Cooke Brothers Ltd", The TQM Magazine, 12.(5): $321 \pm 330$.

[20] Orr, C., 2005. "Lean Leadership in Construction”, Management of People and Team, Proceedings IGLC-13.

[21] Ronald, M. Becker, 2001. "Automotive Manufacturing and Production ", http://www.findarticles.com/p/articles/mi_m0FWH/is_6_113/ai_76445159 (accessed on June 2001).

[22] Sanjay Bhasin \& Peter Burcher, 2006. "Lean viewed as a philosophy", Journal of Manufacturing Technology Management, 17 (1): 56-72.

[23] Womack, J.P., D.T. Jones, 1996. "Lean Thinking", Emerald Group Publishing Studies, 30(5): 739-58. 
[24] Worley, J.M. and T.L. Doolen, 2006. "The role of communication and management support in a lean manufacturing implementation", Management Decision, 44 (2): 228-245.943 


\title{
Organizational Sustainability: The Case of Handcrafts Micro-Business in Southern San Sebastian, Jalisco, México
}

\author{
José G. Vargas-Hernández \\ National System of Researchers of Mexico, \\ University Centre for Economic and Managerial Sciences, University of Guadalajara, \\ Núcleo Universitario Los Belenes CUCEA, Zapopan, Jalisco, \\ México
}

\section{Introduction}

The new conditions of globalization underlie life conditions and the importance of a generational future as a component of competitiveness. What constitutes globalization is the interaction that changes the scenarios for the individuals, organizations and society, who are constantly hounded by contradictory forces and uncertainties. The appropriate use of natural resources can meet present and future interests, having a change in current practices. In comparison to the economic rationality's logic that drives the functioning of organizations and has as a lead obtaining maximum present return, before that if natural resources yield greater benefit under their exploitation than taking care of them, they are sacrificed. Thus, the immediate economic profit is the current enemy of the environment. The ecological proposal in organizations, widen its model of interactions which can integrate the environmental paradigm to the organizational system. An approaching to sustainability of organizations is affected by the combination of ambiguous environmental economic policies, the abrupt adoption of production technologies and market practices. Sustainability in business organizations as an implementation strategy of process reengineering and the adoption of production technologies are oriented toward avoiding waste materials, recycling trash and eliminating toxics.

Lacking acceptance of the role that business organizations play in sustainability, it influences the global debate questioning real causes of pollution which poses safeguards to organizations and justify poverty as the main cause generating environmental degradation. It also suggests as a consequence of deterioration the inadequate economic policies that allow for business actions less friendly with the environment. This paper sets as aims, firstly to determine the level of organizational sustainability for the environmental and economic development of cutting treatment and exploitation activities of the grass called tule thypa spp from Zapotlán Lake. Similarly, the paper pretends to analyze potentialities and economic benefits derived from a marketing orientation of international business in the making of art craft out of tule and palmilla (a kind of palm) that growth spontaneously in Zapotlán Lake. To achieve these objectives, it is drawn a conceptual, theoretical, methodological and 
contextual reference framework, out of which departs to analyze the case of art craft micro enterprises processing tule in the locality of Southern San Sebastian following the design of a traditional research beginning with the hypothesis formulation. Finally, the results are analyzed and recommendations are formulated.

\section{Organizational sustainability and organizational social capital}

A sustainable organization integrates the ecological vision and the institutional theories in organizational systemic values. The acquisition of a common sense in the production of goods and services is utilized as a stronghold to promote the eco-efficiency as a friendly culture of organizations with their environments to achieve emission reductions and rational exploitation of natural resources. The environmental variable in organizational culture is inserted as a rational interpretation of the functioning for the environmental protection, reducing insecurity and context's social pressure. Environmental protection is a technical variable composed by other environmental values such as the promotion of environmental caring, environmental risk control, adequate relationship between organizations, and integration of working groups, orientation and permanent staffing on sustainability. In addition, economic instruments tie sustainable development to micro and small business enterprises. Instruments such as governmental policies, low market tariffs to diminish costs, promotion and incentive of employment opportunities, detection of opportunity areas, etc. are required. Also, it is convenient to have the diagnostic of adaptable enterprises to sustainability because not all of the micro and small business enterprises are capable to form an environmental internal culture. From the point of view of general theory of organizations, according to studies on social capital by Baker (2000) and Burt (1997) (cited by Portes, 1999:247) it is necessary to gain a greater insight, comprehension and understanding of market competitiveness mechanisms. Similarly, Joyce (1998) focuses his analysis of social capital in the leadership phenomena. Either the organization or each one of its members can be incorporated as public and private issues to social capital. Thus, from social capital emerge two patterns, the emphasis on public goods and the emphasis on private goods.

Leana y Van Buren III (1999) define organizational social capital as a resource which reflects the character of social relations within the organization, achieved through the levels of members' orientation by collective objectives and shared trust. Social capital is a collective attribute more than aggregation of individual social connections. It is a byproduct of other organizational activities and thus, it constitutes and indispensable component for collective action. Social organizational capital is an asset whose joint possession between members and the organization benefits both. A new organization has the advantage to create its own organizational social capital in such a way that can maintain optimum equilibrium between stakeholders, individual and other organizational interests in spite of their contingent nature. This is to say, different situations and persons in their relation to organizational performance. A community accounts on social organizational capital when their organizations are characterized by relationships of trust that develop and make predictable their behavior. The capacity of a community is reflected in its level of endogenous development.

The organizations are concrete reality with resources' rules and ordinances for the pursuit of objectives. Members of an organization have as expectations to solve problems of collective action to get supply of some goods and services. Organizations that follow general strategies 
and the ones that follow strategies of niches occupy different environmental resources to produce innovative responses that form inter-dependent connections with other specialized organizations in other industries such as structures of community support. Processes of economic structural change in a local economy require profound changes in trust levels and inter-relationships of cooperation fomented by arrangements of institutions and organizations. Thus, local government must define action lines which function as a catalytic converter of the community efforts.

Finally, population ecologists suggest that the environment selects the organizations which structural features provide the highest values for adjustment, emphasizing the competitive process as a driver of institutional change. Besides, population ecology suggests that organizations founded in a specific organizational form, combined with differences on the surviving rates between organizations with different organizational forms, produce institutional change.

\section{The case of micro and small business enterprises involved in the exploitation of Tule Typha spp. from the lake Zapotlán}

\subsection{Background of the problem}

Similarly to the great majority of aquatic bodies localized in closed basins of Mexican national territory, Zapotlán Lake is the natural receptacle, dump and outlet where converge the sewage, trash and black waters from the human settlements of Cd. Guzmán and san Sebastián del Sur (Southern San Sebastián)

The presence of these natural elements form a nutrients blend that facilitates the growth and development of an abundant aquatic mix which has covered almost the totality of the mirror's surface of the lake and it extends further beyond the shores while penetrating humidity. The transformations of the environment's lake of Zapotlán have a strong impact on the population's socio-economic issues, mainly in San Sebastián del Sur in the municipality of Gomez Farías, and to a lesser extent to the settlers of Cd. Guzmán in the municipality of Zapotlán el Grande, despite the higher levels of pollution in which in is actually found

Deterioration of this lake-body has achieved alarming levels as a consequence of the increasing population and its corresponding enlarging urbanization processes, industrial, farming and agricultural activities. Specifically, one of the natural resources offered by the lake of Zapotlán, the aquatic tule typha spp has been benefiting the settlers and inhabitants of San Sebastián del Sur mainly for its exploitation through the crafting of several products and handicrafts. Thus, the exploitation of tule and the elaboration of handicrafts create direct employments and constitute the income base for around 300 families and their members, a roughly estimation of one thousand and five hundred individuals making a living out of these activities in the municipality of Gomez Farías. However, more are the benefits obtained from tule chubby and plump those other kinds, followed by the one known as palmilla (palm). In its natural habitat, the palmilla tule harms tule chubby's growing and development. This problem, among others, added to the problems derived from environmental degradation which transforms nature of Zapotlán's lake, limit the economic activities derived from the extraction of tule, whose tendency, if it is going to continue in the 
future, and threatens the disappearance of an important employment's source for living sustainability of the inhabitants in San Sebastian del Sur. To aggravate this problem, The Pan-American Olympic Games has chosen Zapotlán's Lake as the location where the aquatic games will take place the year 2011. For that reason, the lake is having a profound transformation which implies the clearance and cleaning of the lake's mirror from any type of grass and bush, including the cutting off and taking out of tule. Other factors contributing to limit the environmental and economic sustainability of development and the scope of benefits from economic activities and exploitation of tule are the following:

a. Null orientation toward a sustainable exploitation of tule as a natural resource.

b. Lack of organization between the cutters of tule and the handcrafters'

c. Weak infrastructure for the development of a more advanced handcrafted production.

d. Excessive interest of hoarders and middlemen in the processes of commercialization and distribution of elaborated products.

e. Lack of mechanisms of governmental institutions to foster and develop economic activities, such as credits, training and technical assistance.

f. Null knowledge of techniques and systems to export their products to the international markets where more acceptances have.

Until now, handcrafted products derived from tule as the main raw material, are elaborated with a strong artistic content to attend local, regional, national and international markets, which traditionally consume because there is a strong historic presence in Mexican culture since pre-colonial times. In order to be organized to attend the regional market, a group of 42 craftsmen acting as partners integrating the Association of Craftsmen (Asociación de Artesanos) initiated the construction of the "House of Craftsmen" in the early nineties to operate as an outlet for selling their products. This business still operates until now having only six partners, although it can be inferred from simple observation that the partners are undergoing heavy conflicts, dividing the building and infrastructure in small areas to operate their own personal business.

Most of the craftsmen deliver their production to middlemen who always perform as hoarders, being a link in the distribution channel and contributing to the commercialization of the handcrafted products in the local, regional and national markets and incipiently in the international markets. These middlemen are the ones who hold the greater percentage of profits. Similarly, by the same token, production is oriented to meet the fundamental needs and wishes of our own local, regional and national markets. Because it is a captive and non competitive market, craftsmen do not perform product design and product development activities. The crafted products implicitly have a higher craftsmanship content in order to meet a more sophisticated demand of international markets which value and appreciate the artistic sensibility and good taste of skillful craftsmen. These craftsmen work tule as a raw material for the creation of handcrafts. The exploitation of tule from Zapotlán's lake has followed irrational patterns which affect not only the environmental sustainability and equilibrium, but also generate problems of low family income and in the running time lesser employment for manpower. The rehabilitation of the Lake requires a more rational exploitation of tule, in such a way that does not affect the environmental sustainability and the economic activity derived and the treatment of tule, while on the other hand, also it is required to improve the family income who work tule and inclusive to generate new employments. 


\subsection{Delimitation of the problem}

Zapotlán's Lake has been consciously subjugated to environmental degradation by the inhabitants of the municipalities of Zapotlán el Grande y Gómez Farías. This situation together with the over exploitation of tule are the two are the two main factors contributing to limit sustainability of the economic activity and derived benefits of cultivation, treatment and elaboration of tule handcrafts. There are other factors that limit the sustainable development. Thus, employment creation and increasing the income of around three hundred families highly depending of the economic activity are found seriously threatened given the current conditions of development. In the last few years, the selling of tule crafted goods has been favored. However, the industry still faces certain problems, among which we can mention:

- Increments in the cost of raw materials (Wood and tule)

- Considerable increasing of craftsmen in the region

- Bad reduction of handcrafted products

- Low and slow commercialization of handcrafted products

- Little support for the promotion of their goods.

This paper is the result of a research that initiated with the following questions: What is the sustainability of the economic efficiency and environmental development of the activities and expected benefits of tule's cultivation and exploitation in Zapotlán's Lake for almost three hundred families, whose income is depending on these activities, given the dysfunctional environmental conditions in which they are actually found? What are the potentials and economic benefits derived from introducing in the international markets the handcrafted products made based on tule from Zapotlán's Lake as the main raw material?

\subsection{Objectives}

- To determine the level of organizational sustainability taking into account the economic and environmental development out of tule's cutting, treatment and exploitation activities at Zapotlan's Lake.

- To analyze potentialities and economic benefits derived from a marketing orientation of international business in the making of handcrafted products made of tule and palmilla from Zapotlán’s Lake.

\subsection{Hypothesis}

One orientation of organizational sustainability in the craft making of handcraft goods made of tule from Zapotlan's Lake as the main raw material contribute to increase family income, the economic benefits of workers and the employment creation

\subsection{Handcrafts in San Sebastian Del Sur}

In the last decades, at San Sebastian del Sur located in the municipality of Gómez Farías, the main sources of income have been agriculture, livestock, fishery and handcrafts production. The handcrafts are made from different materials that are taken from natural resources abundant in the region Southern Jalisco. Inhabitants from San Sebastian del Sur take advantage of the benefits provided by what it is known as Zapotlán's Lake that offers means of sustenance from several economic activities that are done there. Among these economic 
activities, stand out commercial fishery and tule extraction. From this last economic activity, it is generated a large quantity of handcrafts that for several years, families and inhabitants of San Sebastian del Sur have as their main source of income.

\subsection{Main goods and handcrafts made out of tule}

Among the handcrafts and goods handcrafted and made out of tule in the locality of San Sebastian del Sur, can be mentioned chairs, curtains, armchairs, easy chairs, different types of containers for different purposes, such as the tortillas' containers, tables, blowers, bedrolls and matting (petates), baskets, etc. Tule as a raw material is extracted from Zapotlán's Lake where it growths spontaneously and wildly. This natural resource is recollected by the inhabitants of the municipality of Gómez Farías, who do not depend or are required to hold a permission granted by municipal authorities to cut tule, to dry it and to sell it.

\section{Research methods}

A study was conducted to analyze the economic and social development of handcrafters who carry on productive activities using tule as a raw material in the making of handcrafts at the locality of San Sebastian del Sur, in the municipality of Gomez Farías, Jalisco. Data was obtained by means of a random survey based on the roster of handcrafters, which is a list of registered ones. This was done with the purpose of getting to know the different economic and social aspects of producers. The following data was obtained: Number of surveyed: 33; number of workshops visited: 33. Trades of handcrafters and producers were classified in 4 groups, out of which it was found that the 33 persons surveyed were dedicated to the following activities: Manufacturing of rustic furniture: 9. Hand-woven of furniture and handcrafts: 7. Manufacturing of matting and bedrolls: 15. Cutters of tule: 2 .

\section{Analysis of results}

The percentages of families who are dependent of related activities with tule according to the survey results in the locality of San Sebastián del Sur, showed in Fig. 1 are the following:

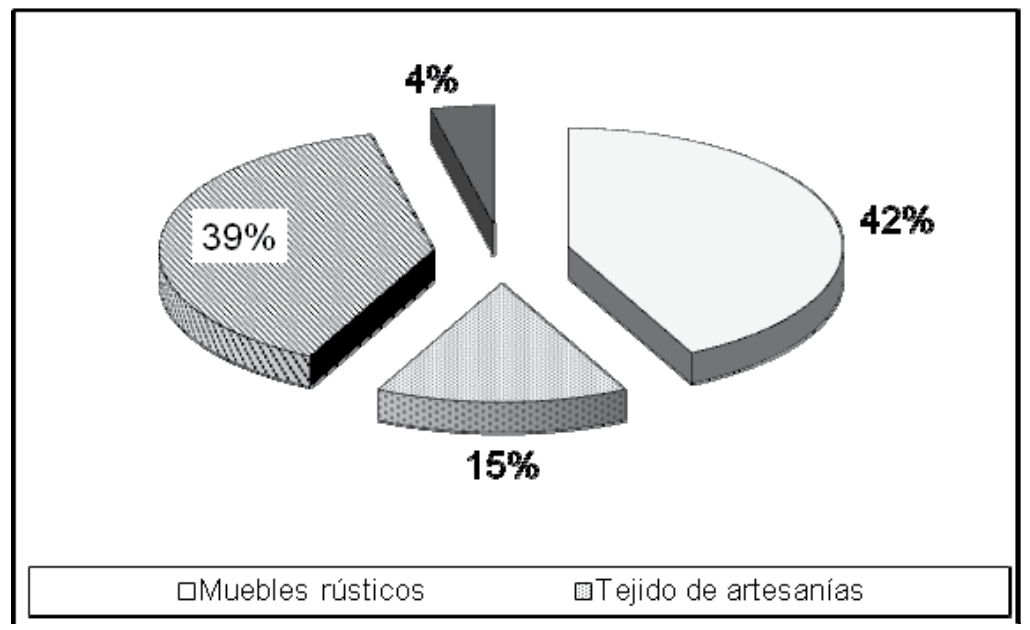

Fig. 1. Percentage of families depending of related activities with tule 
At the location of San Sebastián it was estimated the weekly average salary per worker in the different activities related to the processing of tule. This data was obtained according to the survey conducted by the craftsmen in the field, leading to the following results showed in Fig. 2:

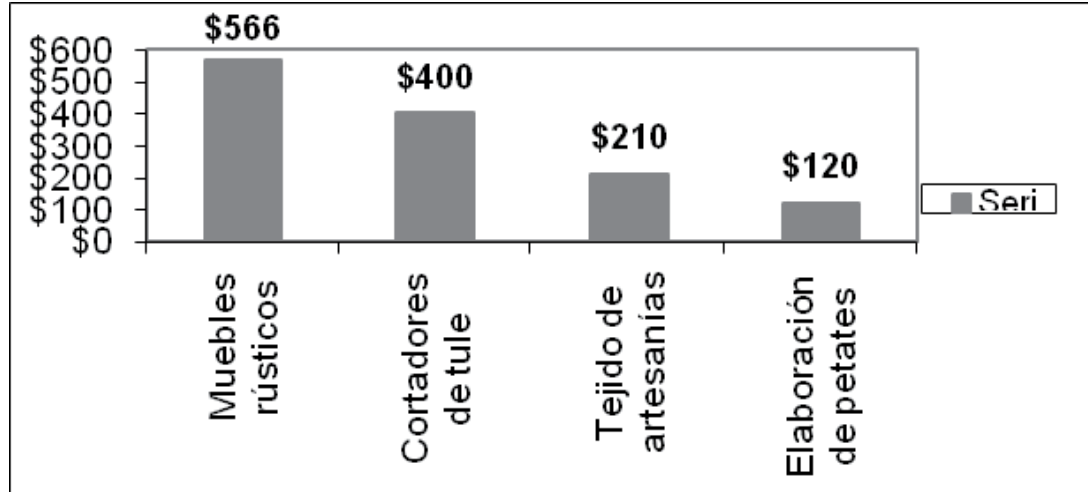

Fig. 2. Weekly average salary per worker.

According to the conducted study in the locality of San Sebastian, the percentage of craftsmen affiliated to associations and organizations was the following:

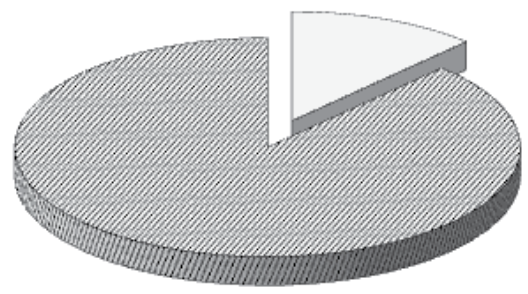

Fig. 3. Percentage of craftsmen affiliated to any organization or association

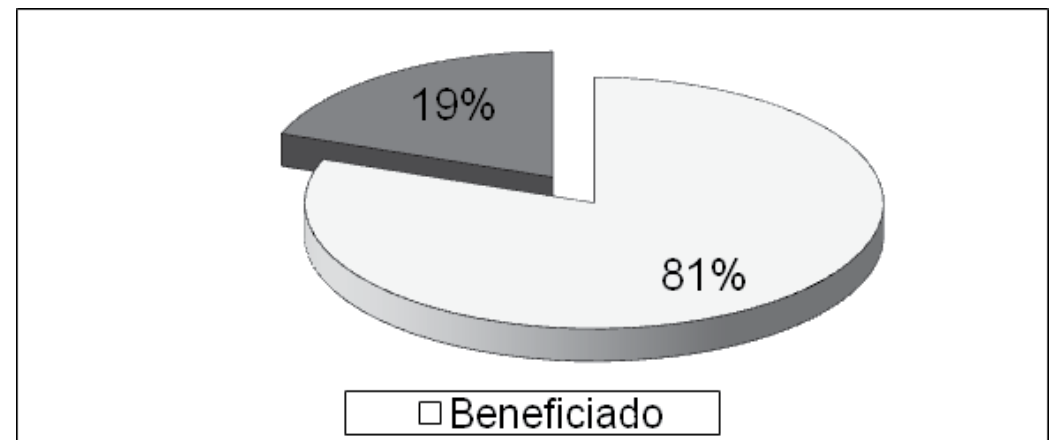

Fig. 4. Persons' opinion that have been benefited or affected the market for their products 
The majority of craftsmen (94\%) in the location of San Sebastian do not receive any type of support from any institution or dependence, while a small number of workers sustained not receiving economic support and promotion for their products and goods mainly from state and municipal institutions. $81 \%$ of the surveyed persons have the opinion that the market has been benefited in the last years due to there are more demand of their products and goods. Only $19 \%$ perceived that has been affected selling their products.

$45 \%$ of persons are sure that exists more pollution, $42 \%$ say to be in equal conditions tan before and only 13\% perceives less pollution in Zapotlan's Lake.

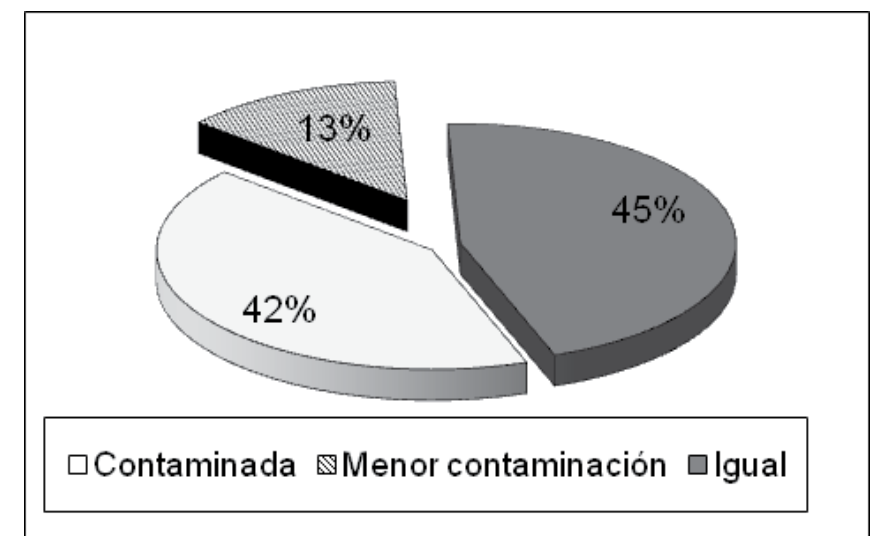

Fig. 5. Perceptions of surveyed persons on pollution at zapotlan's lake

\section{Discussion and comments on organizational sustainability based on the research results}

The presence of bundles of tule covering around one third of surface at Zapotlán's Lake (Universidad de Guadalajara, 1995), it is important for the nesting, refuge and protection of several species of fishes, tilapia, and carps and also for several species of birds. Tule's plant serves as the food for some species beginning from the organic material which becomes detached from the roots, regulates water's temperature thus the environmental temperature. In the following months after November, the plant is affected by groups of birds know as zanates who build their nest to sleep and to protect against the bundles of tule that the same birds break down. The biggest problem faced is that the proper bundles of tule palmilla (tule palm) used to weave chairs are flouting and invading the chubby tule used to manufacture matting and bedrolls and impede its normal growth and development. Thus, the bundles of tule need cleaning, or the tule palmilla damages the chubby tule. This problem has a solution. If there are extracted the bundles of tule palmilla that walk soils when the level of water goes up and cover the chubby tule.

The cutters claim that, among other problems, they do not count on the support and back up of any governmental dependence or institution through the granting of permissions to cut and exploit the tule. Also, the cutters claim that they do not receive any financial support in credits for the creation of facilities and infrastructure to carry on their activities and increase their productivity. One good example is the lacking acquisition of tools, cutters or motorboats to facilitate cutting of tule or conditioning of plots of land (patios or country 
yards) adjacent to Zapotlan's Lake. Similarly, there is lack of support to facilitate the drying of the cut tule. This situation always represents problems when tule is green. When tule is spread out and laid out in a country yard or patio for the processing of drying, it last from 3 to 4 days. There are some spells, mainly during the period of ebb tide in which tule is dried without being cuted, stating a little bit green. However, ecologically and environmentally these processes have some serious consequences which affect the local economics and environment.

Cultivation and exploitation of tule constitutes an important economic activity, mainly for the community of San Sebastian del Sur because it provides economic support to around three hundred families. About 80 families are economically benefited with the income received from the cutting, drying and transport of tule. Around 140 families are benefited from the handcrafted production of goods made of both varieties of tule, the palmilla tule and the chubby tule as the main raw material. Out of these 140 families, 120 are self-considered handcrafters of matting and bedrolls (petateros).

Around half of the 55 handcrafters registered in the Trade and Commercial Association have their own workshops, while the other half only work in assembling plant because they do not count on the instruments of a workshop properly equipped. Around 70 families receive income from intermediation, hoarding, stockpiling and commercialization activities of products derived from tule. The cutters of tule dry their raw material and make even up to two bunches per one day. The two bunches are sold at an average price of 75-80 pesos each one, making 150-160 pesos per day. Regularly, this sale represents the daily family income of around 13 USA Dollars. Tule palmilla used for the manufacturing of chairs is sold to intermediaries; most of them are owners of small stores who stockpile it. Tule chubby is sold to the craftsmen at a price a little bit less expensive to be used in the manufacturing of matting and bedrolls (petates).

Most part of manpower employed in the cutting of tule is permanent and their trade was inherited from antecedent generations. According to the conducted field research, and due to the ongoing changes taking place in Zapotlán's Lake, every year there are less cutters, thus, in the years before, the cutting of tule was an activity which generated more employment than nowadays. In the years before, the cutters formed a group and became a formal association of tule's cutters. Some of the actual cutters are temporarily workers in this activity, more specifically when they do not find a better remunerated employment.

The craftsmen produce different goods using as the main raw material tule, such as matting, bedrolls, hats, chiquihuites (containers in form of wide baskets), shadows, traditional chairs, high chairs, seamstresses, etc. From a roll to a bunch or bundle of tule handcraft up to seven matting which the craftsmen sell to a price of 30 pesos each one, an equivalent of two USA dollars and 40 cents, obtaining up to 210 pesos (almost 17 USA dollars) with an original investment averaging 80 pesos, the cost of one bunch of tule. A bunch of tule is formed with 5 or 6 big hands (manotadas) of tule. The cost of one big hand is approximately 20 pesos and it is the base to manufacture one chair which is sold at a price of 50 pesos to the intermediary o middleman. The cost of materials of one chair also includes besides the big hand, a wood branch of pine whose costs is up to 15 pesos, a cost that has been increasing when used to be 1.50 pesos several years ago. In one workshop where regularly work five craftsmen achieve to produce up to 70 chairs per week which are sold to the intermediaries and middlemen at a price of 70 pesos as an average. 
This price can improve depending on negotiations and trading with intermediaries and middlemen. Many cutters supply raw material to their own families who are in charge of the crafting of handcrafted products, widening with this situation the economic benefits and adding value. In other cases, there are conducted some practices of half by half ("medieros") between the cutters and the handcrafters. This is to say, after the selling of products; both cutter and handcrafter share the benefits in the same proportion, half and half.

Nowadays, the relationship among handcrafters of tule registered in one association at the location of San Sebastian del Sur is 55 . 16 years ago, the association was formed by 42 partners and set as the main aim the founding and building of the craftsman's house (Casa Del Artesano) as the market place in which their handcrafted production can be exhibited and sold. The three levels of government, Federal, State and Municipal contributed with donations of materials for the construction, while the partners made manpower contributions. However, most of the craftsmen members of the Association do not made any contributions of manpower and finally only six partners did it, and who nowadays exhibit and sell their handcrafted products in a separated way at the craftsman's house. This craftsman's house is located at one side of the Federal Highway Guadalajara- Cd. Guzman, in an adjacent location to the Municipal Cemetery. The person who was at the front and in charge of the Association, he has also the ownership of land and building. Originally the land was signaled by the Municipal Government of Gomez Farías for the construction of the craftsman's house. However, lately it was found that this land did not have any proprietorship according to the archives of the Public Register of Ownership.

Thus, nowadays, the ownership of the craftsman is the "apple of discord" and the cause of several conflicts due to the other craftsmen wants to participate of the benefits with the support of the municipal government. One of the former municipal officials who tried to benefit a group of craftsmen who do neither exhibit nor sell their products at the craftsman's house, had sent official letters to the Public Notary requesting a change in ownership regime and claiming that it is municipal's ownership. Despite of this, there is not one organization of craftsmen to whom it may classify among these who count on their own workshops, approximately half of the 55 registered members, and those who sell or "maquila" their own manpower for the former. Thus, those who sell their own manpower, do not have their own equipment, tools and facilities to establish their own workshop.

According to our own research, the interviewed assure that sales are declining. Today, they argue, sell less than 10 years ago. The middlemen and intermediaries are who more profits obtain from the commercialization of the handcrafted products. In order than a handcrafted product reaches the final consumer, it is required at least a three level's distribution channel, and this is to say that at least there are two intermediaries between producers and consumers. The intermediaries are the ones who attend national markets and to a lesser extent they export the handcrafted products to some parts of United States, Canada, Puerto Rico, England and Japan. The terrorist actions of September 11, 2001 had affected missing some orders of handcrafts. Some of the problems that the craftsmen face are those related to the lack of training programs to develop and preserve handcrafter techniques, financial support, and obtaining credits to extend basic production infrastructure, and of course, staffing and consultancy for direct exports of their products to the international markets. All these actions will benefit the economic income of more than 300 families in San Sebastián del Sur, Jalisco. 


\section{Conclusions}

The exploitation of tule represents an economic activity that provides income to approximately three hundred families living at the settlement of San Sebastian del Sur. Nevertheless, in the last few years the income has been decreasing due to the environmental changes and to the rehabilitation of Zapotlan's Lake to host the Pan-American Games in 2011. Both, the environmental changes and rehabilitation of the lake represent a serious threat to the economic efficiency and environmental sustainability. To achieve equilibrium between environmental sustainability and economic sustainability must be one of the main goals of the rehabilitation programs. Thus, the best indicators are required under a systematic study to determine the most adequate levels of environmental sustainability and economic efficiency. The limited social organizational capital and the lack of adequate forms of organization for the productivity, contribute to limit the scope and economic benefits that must provide an adequate exploitation of tule from Zapotlan's Lake. Disorganization of cutters and craftsmen of tule is the source of profound and increasing conflicts that not only block and limit the scope of better levels of productivity and family income, but also make difficult the pacific coexistence and living together and spoil the community's quality of life. Lacking a consultancy program to exports of handcrafted products has an impact on lower income to the families of cutters and craftsmen, because who actually obtain the greater part of profits are the intermediaries who take part on the commercialization processes and distribution channels.

Some characteristics of specificity and appropriateness of social and human capital involve economic, social and political relationships among individuals who are members of organizations, making complex their effects. Market is a social construction that makes operational social relations. Both capitals can be important resources of the competitive advantage, assuming that reside in the members or it is specific to the organizations as integral parts of resources that are unique and that are no observables. Organizations with higher levels of social and human capital generate more competitiveness than those with lower levels. A sustainable economy requires programs aimed to improve social and human capital.

\section{Recommendations}

Some recommendations after this research are formulated below:

- Design a program to promote handcrafted activities derived from the exploitation of tule that in a parallel form to the rehabilitation program of Zapotlán's Lake, establish the right indicators to achieve equilibrium between economic efficiency and environmental sustainability.

- To set a program of export consultancy with the support and technical staffing from students of international business at University of Guadalajara that provide the knowledge, skills and contacts in such a way that tule's craftsmen directly commercialize and market their own handcrafted products in the international markets.

- To propose a program for development of organizational and social capital and new forms of organization aimed to increase productivity and competitiveness of the craftsmen, thus increasing their family income and promoting employment creation and improve the quality of life of the whole community of San Sebastian del Sur. 


\section{References}

Baker, W. E. (2000). Achieving success trough social capital. San Francisco, CA: Jossey-Bass.

Burt, R. S. (1997). “The contingent value of social capital” Administrative science Quarterly, 42, 339-365

Joyce, Paul (1998). "Management and innovation in the public services". Strategy Change, 7. 54-63

Leana, R. Carrie; Van Buren III, Harry (1999). “Organizational social capital and employment practices" Academy of Management Review, Vol. 24, No. 3. Ohio.

Portes, Alejandro (1999). "Capital social: Sus orígenes y aplicaciones en la sociología moderna", Carpio, Jorge y Novaconvsky, Irene (comp.), De igual a igual. El desafí del Estado ante los nuevos problemas sociales, Fondo de Cultura económica-SiemproFlacso, Ciudad de México.

Universidad de Guadalajara (1995). Ordenamiento Ecológico de la Cuenca de la Laguna de Zapotlán el Grande Jalisco, Gobierno municipal, Cd. Guzmán, Jalisco. 


\title{
Strengthening Institutional Capacity for Science, Technology and Innovation in Uganda ${ }^{1}$
}

\author{
Muriisa R. Kabeba \\ Senior Lecturer/Dean, Faculty of Development Studies, \\ Mbarara University of Science and Technology (MUST), \\ Uganda
}

\section{Introduction}

The contribution of science and technology to development is well observed world over and has been repeatedly emphasised in Uganda as a development priority. This however, may not take place unless institutions that favour technological innovations and investments into science and technology knowledge systems are considered. Investing in science and technology is the global new catchword not only for individual countries but also donors, such as the World Bank. The new focus on science and technology in Uganda calls for right institutions if Uganda is to benefit from investing in S \& T. I hypothesise that promotion of science, technology and innovation cannot take place unless right institutions are put in place not only to allow generation of scientific knowledge but also to allow diffusion of this knowledge. This paper is about strengthening institutions for science and technology in Uganda. The paper discusses institutions and the institutional challenges for the promotion of science and technology and proposes the nature of institutions required for $S \& T$ to transform society.

In the recent years development initiatives have centred on the role of science and technology (S\&T) in promoting and sustaining development. The emphasis by Uganda's president on the need for promoting science and technology is a reminder that the world revolves around science and that scientific innovations are the ones making the world revolve. The call for developing and teaching science and technology in schools however should also put into consideration the institutional requirements for the technological development to occur. This paper discusses the different institutions that can positively contribute to the development of science and technology in Uganda.

The World Summit for Sustainable Development (WSSD) held in Johannesburg - South Africa in 2002 centred on key role of S\&T in sustainable development. The United Nations Millennium Project task force on science, technology, and innovation reiterates the need to harness science and technology sustainably to accelerate development. The development distance between different countries is now being attributed to the levels of science and technology in these countries. Thus,

\footnotetext{
${ }^{1}$ An earlier version of the paper was presented at Pre CHOGM Symposium "Science and technology for social and economic transformation in Uganda" 13 - 15 September 2007, Lake View Hotel, Mbarara
} 
The lessons of the last two centuries have shown the crucial difference between having and not having the science and technology capability, even for countries with vast natural resources. The difference lies in being able to exploit these resources themselves or be exploited by others. In this new century, the winner will be the one who can, with capability of science and technology and innovation, extract the most value out of them. www.logelproject.org 15/07/07

Apart from the expected or direct benefits for the promotion of science and technology, the new lending "conditionality" for developing countries is the promotion of science and technology or funding geared towards the promotion of science and technology. Therefore, recent funding requirements by donors such as World Bank is that recipient countries can only benefit from donor funds and aid if they put in place "right" institutions to promote science, technology and innovation. This requirement is based on the premise that promotion of science, technology and innovation cannot take place unless right institutions are put in place not only to allow generation of scientific knowledge but also to allow diffusion of this knowledge ${ }^{2}$. The new focus on science and technology in Uganda calls for right institutions if Uganda is to benefit from investing in S \& T. This paper is about strengthening institutions for science and technology in Uganda. The paper discusses institutions and the institutional challenges for the promotion of science and technology in Uganda, and proposes the nature of institutions required for $S \& \mathrm{~T}$ to transform society. The paper is guided by the following questions:

a. What are institutions?

b. What are the existing institutions in Uganda?

c. What institutions are required for science and technology to develop and to transform Uganda?

d. How can these institutions be strengthened for $S$ and $T$ to be effectively generated and utilised?

The paper proceeds as follows; section one conceptualises institutions of science and technology. Section two discusses the different institutional requirements for the promotion of science and technology, section three discusses the institutions for science and technology in Uganda. Section four discusses institutional challenges for the promotion of science and technology in Uganda, lastly section five draws conclusions from the discussions.

\section{What are institutions?}

Narrowly conceived institutions refer to structures such as research and technology institutes, universities and technical colleges set up for promotion of innovation. Broadly, institutions may be conceived as to include political habits, practices, norms and rules regulating relations and interactions among people.

Technological knowledge is crucial to development. However, designing the right institutions to absorb, retain, advance and sustain knowledge, has turned out to be much more challenging. The efficiency with which knowledge is created and diffused depend on a variety of institutions promoting innovation. It is worth mentioning that the failure to

\footnotetext{
${ }^{2}$ It is not the intention of this paper to discuss science and technology as a new lending conditionality for developing countries, but to appraise the institutional requirements for the success of technology as conditionality.
} 
innovate and promote benefits of science and technology is often due to failed institutions. This failure is further compounded by the gap between scientific knowledge generation systems and diffusion mechanisms. Scientific knowledge is erroneously seen as that generated in laboratories. Little attention is paid to the modes and mechanisms of diffusion and how this knowledge and skills could fit into existing systems. Thus, Chataway, Smith and Wield (2005: 3) argues that,

capacity building in science and technology enables innovation that can enable countries meet their development goals and improve people's lives. But innovation is not something that happens in sophisticated research laboratories, it can happen also by developing new and effective approaches and institutions.

The process of innovation is not only confined to formal arrangements, it involves significant elements of informal collaboration, learning and exchange of knowledge between individuals, organisations and across disciplines (Joanna Chataway, Smith, \& Wield, 2005b). Science and technology should be understood as far from the conventional sense of building stocks of research infrastructure and trained scientists. Science and technology designed to work as selfcontained activities are insulated from the very systems they seek to influence. This gap between science and its market is rooted in old thinking that equates science and technology generation through formal research with innovation. This conventional view promoted the mistaken notion that scientists and technology specialists, through their research organisations, were the innovators and producers of new knowledge. Research and emerging global challenges such as environmental, diseases, climate change and poverty however, show that scientific innovations cannot provide solutions if insulated from other disciplines. Thus, Nightingale and Scott (2007), point out that the justification for public funding of the biological sciences is "... largely at odds with the outcomes because major causes of illness, such as poverty, lack of education, and poor housing and healthcare are social and political issues that are poorly addressed by the current science-intensive research systems". Thus we need to draw on more inter and multidisciplinary research and take into account that science and scientists do not exist and may not work in isolation, they do so within a network of other actors and require a supportive environment. A look at the collaborative and coordinating institutions becomes crucial for successful science, technology and innovation.

For the promotion of science and technology, we need to create different institutions that facilitate innovation, diffusion of knowledge and change, in addition to formal institutions for science, technology and innovation. Thus we need to create coordination, reward systems, accountability and governance institutions. The discussion that follow, focuses on different institutions necessary for the promotion of science and technology in any given country or community.

\subsection{Institutions for coordination}

There is need to build institutions that allow proper exchange of knowledge between different systems and actors. Whereas innovation is crucial for development, it is noted above, that diffusion of knowledge is much more required for sustainable development. There is therefore, a need for the existence of strong institutions to coordinate different actors and to promote proper integration of scientific knowledge into development goals. It is in this light that the Uganda National Council for Science and Technology (UNCST) was established. Thus, one of the objectives of the UNCST is coordination of all scientific and technological activities 
in the country through national programme geared to the national socio economic development and one of the strategic goals of the institution is to "improve or streamline national science and technology policy environment to foster scientific and technological innovation" . http://www.uncst.go.ug/index.php/about-us.html accessed on 27/10/2011

\subsection{Governance and accountability}

For the promotion of science and technology, enormous funding for research and human capacity is required. However, funders are attracted to organisations whose governance structures are clear and solid enough to ensure monitoring of results and financial accountability. The strengthening of governance systems will allow organisations to redirect funders from funding specific projects to providing funding for a wide range of projects and programmes. In addition governance and accountability institutions will observe that funds and research grants are utilised in a transparent manner and according to set goals, standards and that researches conducted are in tandem with national development goals and priorities.

\subsection{Need for local systems that enhance the capacity to innovate}

One of the most pressing needs in Africa in general and Uganda in particular, is to build local systems that enhance the capacity to innovate. Local systems to generate and apply knowledge and information are necessary to build absorptive capacity, accelerate poverty reduction, manage natural resources, better boost productivity, compete in local and export markets and improve well being.

To make the above a reality, there is a need to take into account local needs and contexts. The understanding of local needs and local context includes taking account of local abilities to find solutions. Local citizens should be involved in providing solutions for problems and challenges they face. Consultations with local communities should be made to find out if there are alternative technologies at the local level which can be improved instead of imposing new technologies on them. In addition, science and technology should be demand driven so as to address local needs.

\subsection{Reward systems}

There should be continuous support of scientists and researchers in the field of technology by rewarding excellent scientists and innovative researches. In its objectives, UNAS aims at establishing "a regular award scheme for recognition of outstanding achievements in science that are pertinent to the prosperity of Uganda". The presidential award scheme for best scientists was put in place to recognise science and innovation.

\subsection{Ethical, legal and Quality Assurance (QA) institutions}

Ethical considerations consider the way research is conducted, protection of research subjects and use of other people's research. In some instances this may be influenced by the existing legal systems. In this regard, one of the challenges facing researchers is the assurance of protection and patent rights. Uganda still remains miles far from granting patents to its renowned researchers. Therefore, sharing of researches and innovative knowledge is still limited. Individual scientists are not free to pronounce/share the process of their researches to 
fellow scientists, because of lack of protection of their rights. Legal institutions are meant to create an enabling environment for innovation and technological diffusion.

In the agricultural sector, access and use of technology depends on an enabling environment, thus, "the ability of the agricultural innovation system to be able to access, use and diffuse knowledge embedded in agricultural technologies depends on the presence of an enabling framework that supports the emergence of technological capabilities by strengthening existing linkages, promoting new linkages and fostering inter-organisational learning that leads to capital accumulation and technical change. Such an enabling environment, by definition, is one that strengthens the absorptive capacity of local actors while protecting their interests through a policy framework that recognises their legal rights and privileges, linkages, socio-cultural norms and historical context" (Bartel 2010 at http://www.atdforum.org/journal/html/2009-34/11/ accessed on 26/10/11). An enabling environment for technology and innovation in agriculture is defined as one that provides the actors, skills, institutions and organizations required to promote the use, dissemination, diffusion and creation of knowledge into useful processes, products and services. This is one that promotes right laws, structures and systems; systems that allow innovation for example systems that allow patent rights and promotes individual initiatives by being recognised as sole claimants to their efforts and contribution to technological innovation.

Apart from legal and ethical institutions, the quality assurance institutions should be in place to promote science and technology. For example, the Uganda National Bureau of Standards (UNBS) not only challenges the quality of products, but also challenges the processes of production and the technology used in the production. Ministry of education is in charge of observing quality of teaching science and technology in schools. For example, it is a requirement that every secondary school to get registration as a teaching institution should have science laboratories. And to qualify this requirement, physical science subjects biology, physics and chemistry were made compulsory for all students.

\subsection{Policy institutions and sub-systems}

Developing an appropriate science and technology policy requires the involvement of multiple stake holders and to put into consideration the overall development programme. In addition, developing a sound $\mathrm{S}$ and $\mathrm{T}$ policy takes a number of stages ranging from policy agenda to policy evaluation. These stages require different institutions which must be well coordinated. A sound policy will depend on the extent stake holders are involved in the whole process of policy formulation. To what extent people who technology intends to benefit, participate in the design of the policy remains the key question? Often policy designs come as blue prints; it is a top-down process with limited participation of the people the policy is intended to benefit or the implementers of the policy.

Note: The description and categorisation made above is for analytical purposes and is not a blue print. Institutions are functional and may combine different functions; reward, coordination and control, and quality.

\section{Strengthening institutions for science and technology in Uganda}

Uganda has gone through stages of transformation; Pre-colonial, colonial, post colonial up to the late 1980s and present day. All these stages have their own characteristics for science and technology 
The pre-colonial Uganda was characterised by progressive technological innovations. Scientific knowledge and innovation can be exhibited in blacksmithing and food preservation. Setting granaries some meters above the ground protected food from dump and rodents. This is still practiced in villages. In traditional Ankole and Kigezi, some families/clans specialised and were known for their skill, innovation and technological advance in pottery making, basket weaving and black smith. Technological advance and innovations in these areas was a preserve of such families and clans. We well know of specific families called "Abahesi" literary meaning blacksmiths in Ankole and Kigezi. These families, made hoes, knives, spears and other tools, from smelt iron. Although such families continue to exist in societies in Uganda, they play a limited role in science and technology and innovation and are less recognised in the process of progress.

Their contribution to science, technology and innovation was affected by colonialism which killed family initiative through importation of the foreign hoe, china plates to replace locally made clay-pots and plates. During colonialism, institutional arrangements to foster development in Uganda did not in any way emphasise technology and science (Akampumuza, 2005). Colonial economic interests were the primary focus of institutions to foster "development".

The post-colonial Uganda faced many challenges in which teaching of science and was confined to laboratory teaching. Studying science and technology was left as an individual choice with limited carrier or professional guidance. Schools operated without laboratories and science equipments, and sometimes without science teachers.

The National Resistance Movement (NRM) government which came to power in 1986 was the first government to institutionalise science and technology, research and innovation and to integrate them in the overall development planning. To streamline teaching of science and technology, the Uganda National Council of Science and Technology (UNCST) was established.

\section{Uganda National Council of Science and Technology (UNCST)}

The Uganda National Council of Science and Technology (UNCST) was established by an act of parliament in 1990 with its line ministry of Finance Planning and Economic Development. The UNCST was established as a semi-autonomous institution with the mandate to develop and implement strategies for integration of science and technology in the national development process. And to advise government in formulation of policies that enhances and fosters the integration of science and technology in Uganda's social, economic and cultural development.

\subsection{Functions of UNCST}

- Provide advice to government and coordinate formulation and management of an explicit science and technology policy in all fields of science and technology.

- Promote the application and development of science and technology to serve the national socio economic development needs.

- Develop strategies and carry out activities that ensure sustainable integration of science and technology in the national development process. 
- Strengthen and promote the development of human resource capacity in science and technology.

- Coordinate all scientific and technological activities in the country through national programme geared to the national socio economic development.

- To coordinate and guide national research and development

The UNCST works hand in hand with different research organisations responsible for developing different technologies in various fields. The National Agricultural Research Organisation for example, is responsible for the coordination of research in agriculture and to develop appropriate technology and plant varieties.

\subsection{Research and professional bodies}

There are a variety of professional bodies aimed at not only protecting the rights of scientists but also to voice new needs and requirements for technological improvements and innovation. These bodies and institutions include; national medical research council, the Association of Uganda Engineers, National Agricultural Research Organisation (NARO), Uganda National Academy of Science (UNAS) and other autonomous bodies. Each of these bodies is geared towards promoting science and technology. For example NARO coordinates research in agriculture, food and science and technology, UNAS takes a multidisciplinary approach and brings together scientists from physical, biological and behavioural/social sciences. Objective number four of UNAS is to "encourage, stimulate, design and coordinate interdisciplinary and trans-disciplinary scientific research and development. Objective number five is to "get involved in planning, convening and coordination of scientific education programmes and help in the development and nurturing of high-level scientific and technological manpower in Uganda.

\subsection{Institutions of learning and innovation}

The role of learning is fundamental to the development of technology, merging knowledge bases, and to maximize benefits from science and technology innovations. There is a need therefore, to create institutions to promote learning, research and innovation. Creation of education systems for innovation is crucial for science and technology capacity building and technological diffusion. I contend that there cannot be a viable science and technology policy if it is not underpinned by well designed measures for addressing issues such as, learning, technology diffusion and transfer, research and development. The need therefore to make the teaching of science in schools compulsory and the setting up different institutes that could directly link with local communities is the beginning of addressing the above challenge.

The teaching of science in schools in Uganda is a long-time practice but since the 1990s, this has received a lot of emphasis. Science subjects are emphasised and made compulsory at secondary education level, while, state funding for higher education especially in public universities is more inclined towards supporting students who excel in science subjects than counterpart arts subjects.

Universities are teaching science and technology tailored to local needs and to address development challenges. Mbarara University of Science and Technology (MUST) for example, has a variety of community oriented programs in medicine, science and 
development studies. This is a move away from conventional way of teaching science and technology restricted to laboratories. Problem-based learning takes place in communities.

In the field of research, Mbarara University has had innovative researches addressing local needs. For example, in 2006, in the faculty of science, they found out that in semi-arid areas of Kashari County, the water dams without water run-offs get bacterial contamination during the dry season and are using "Mringa"-a local herb to purify water, making it safe for cattle consumption (discussion with a lecturer in faculty of science).

\subsection{The family/community and utilisation of Indigenous Knowledge (IK)}

As noted in the introduction, the family is an important institution in the development of science and technology in Uganda. Families utilise indigenous knowledge to process food such as making of ghee and pasteurised milk. They also use indigenous knowledge to control pests and disease; for example, the combination of cattle urine and ash to kill banana weevils, or the use of ash to protect seeds from weevils. Warren (1991) argues that development projects cannot offer sustainable solutions to local problems without using local knowledge. In fact to ignore local people's knowledge is almost to ensure failure in development projects (Brokensha et al. 1980). Global science recognises the relevance of indigenous knowledge, and the world conference on science recommended that scientific and traditional knowledge should be integrated in interdisciplinary projects dealing with links between culture, environment and development in areas as conservation of biodiversity, management of natural resources, understanding of natural hazards and mitigation of their impacts. "In Ethiopia, the absence of effective linkage between indigenous knowledge and conventional ones has been identified as one of the major problems that hinder the effectiveness of the development of agriculture in general and of agricultural research and extension systems in particular"(Fenta, 2006). In agriculture and climate change mitigation, IK is very important and may be a cheaper option than modern scientific knowledge. Indigenous knowledge is most effectively used in development projects as source of innovative solutions because of its perspective of being intensively local and long term (Huysamen, 2003). Affirming use of traditional approaches to improve agricultural production is effective and relatively cheap in addition, it preserves people's culture and tradition, and their dignity, and therefore they are easily embraced and owned by people.

It should be noted however that the traditional approaches have not received much attention at the national and international level. Most climatic change policy documents hardly recognise the use of traditional methods and approaches. In 2007 at the UN conference in Bali, indigenous people voiced concern about their exclusion from ongoing climate change debates, this was mostly done during the protests on 7 December 2007.3 In the Sahel region, the local populations in their indigenous knowledge systems have developed and implemented extensive mitigation and adaptation strategies that have enabled them to reduce their vulnerability to past climate variability and change which exceed those predicted by models of future climate change. However this knowledge is rarely taken into consideration in the design and implementation of modern mitigation and

\footnotetext{
${ }^{3} \mathrm{http}$ :// portal.unesco.org/science/en/ev.php-URL_ID=6550\&URL_DO=DO_TOPIC\&URL_SECTION=20 1.html
} 
adaptation strategies (Nyong F, Adesina, Elasha O. 2007). Speaking at the launch of the report of the minority rights group international in 2008, David Pulkol (a former Ugandan Minister) noted that, "There is no integration of indigenous knowledge into development planning, and so people are becoming more powerless." 4 He further noted that the luck of interaction between the state and informal leaders is a hindrance to dealing with climate change emergencies such as drought. IK therefore, can be crucial for debates on agricultural change and climate change adaptation strategies. This knowledge need to be utilised during the process of technology development and innovation, policy and change.

\subsection{Governance institutions}

As earlier mentioned, these are institutions responsible for observing that there is transparency in the way research and award of research grants are utilised. Further, these institutions are responsible for observing that researches are inline with development goals and national priorities. These institutions include the committee of parliament on science research and technology.

\section{Institutional challenges for science, technology and innovation in Uganda}

Coordination and collaboration is limited. There is often duplication of services and activities

Diffusion of technological knowledge is still a challenge. There are also budgetary constraints. Often there is little funding available to support science and technology institutions which act as centres for research, innovation and knowledge dissemination. For example the amount of funding going to universities is far small compared to that going to administration and primary education.

\section{Conclusion}

This paper has argued that science and technology are crucial for development. However, the paper has emphasised that scientific and technological knowledge is only effective if diffused. There is need to create institutions that can facilitate the diffusion of technology and enable its integration in the overall development goals. The paper has argued that for the promotion of science and technology in Uganda, there is a need for the existence of institutions that would ensure that research is done in a transparent manner, and that the teaching of science and technology are in tandem with national priorities and goals. Above all the paper has argued that scientists need to be motivated not only through availing them with research funds, guaranteeing the protection of their rights as researchers but also through acknowledgement and recognition of their distinct scientific works. Despite the long history of lack of institutionalised framework for the promotion of science and technology in Uganda, since the 1990s, different institutions to promote science, technology and innovation have been established. Finally, the paper discusses that the Uganda still faces a challenge of technological diffusion and coordination of different researches and research organisations in the country.

\footnotetext{
${ }_{4}^{4}$ Same as foot note 3
} 


\section{References}

Akampumuza, J. (2005). The Institutional Framework for Development Since Colonialism: Challenges of A Developmental State, In P. Mbabazi \& I. Taylor (Eds.), The Potentiality of 'Developmental States' in Africa: Botswana and Uganda Compared. Darkar: CODESRIA.

Bartel Constantine 2010, Enhancing food security in Africa through science, technology and innovation, Summary of the UNACTAD Technology and Innovation Report 2010, http://www.atdforum.org/journal/html/2009-34/11/ accessed on 26/10/11

Brokensha, D.; Warren, D. M and Werner, O. (1980) Indigenous knowledge systems and development. University Press of America, Lanham

Chataway, J., Smith, J., \& Wield, D. (2005). Partnerships for Building Science and Technology Capacity in Africa: Canadian and UK Experience. Paper presented at the conference, "Building Science and Technology Capacity with African Partners", London Jan 30th to Feb 2nd 2005.

Chataway, J., Smith, J., \& Wield, D. (2005b). Partnerships and Building Capabilities for Science, Technology, Innovation and development in Africa Working Paper No 2, November 2005, Open University Research Centre on Innovation, Knowledge and Development, Milton Keynes UK.

Fenta, Getahun (2006) “Farmers' Indigenous Knowledge - The Missing Link In The Development Of Ethiopian Agriculture: A Case Study of Dejen District, Amhara Region", Social Science Research Report No. 34, Addis Ababa, OSSREA,

Huysamen, R. (2003) Indigenous knowledge systems: relevance and future in SA organizations. HR Future, July: 46-48, Available at ,

http:/ / www.worldbank.org/afr/ik/iknt19.pdf

Mukandawile, Thandika (2007), WSSD. 2002. Plan of Implementation. World Summit of Sustainable Development, Johannesburg, South Africa (Advance Unpublished Text).

Nightingale, P., \& Scott, A. (2007). Peer Review and the Relevance gap: ten Suggestions for Policy makers. Science and Public Policy, 34(8), 543 - 553.

Nyong F, Adesina, Elasha O (2007), 'The value of indigenous knowledge in climate change mitigation and adaptation strategies in the African Sahel' "Challenges in Integrating Mitigation and Adaptation as Responses to Climate Change" Vol. 12 (5) 787 - 797

Uganda National Academy of Sciences (UNAS) news letter, issue number 2, January 2007June 2007.

www.ugandanationalacademy.org

www.logelproject.org 


\title{
A Didactic and Methodological Lesson of the Study of Economics and the Skill Development of Students
}

\author{
Maria Covadonga De la Iglesia Villasol \\ Complutense University of Madrid, \\ Spain \\ "... The complexity of models is elaborated merely for display, \\ far and away beyond the possibility of application to reality", \\ Joan Robinson (1979) ${ }^{1}$
}

\section{Introduction}

The consolidation of the European Higher Education Area (EHEA), into which many European Universities have thrown themselves, especially Spanish Universities, means for many senates the revision and the methodological adaptation of the contents to be taught and professors' work in the new Bachelor's degrees. The piece of work presented is a reflection on some methodological aspects of how to tackle the teaching/learning process in the study of Economics and, for this purpose, it rescues some lessons by important scholars, especially those by lecturer Joan Robinson. Works by Joan Robinson are innumerable; her legacy in the study of economics and social sciences does not limit to her great work The economics of imperfect competition, but it covers different topics as the diffusion, spread and explanation of master Keynes's contributions, since she was one of his young disciple and was part of "Circus", or, for many, her great contribution The Accumulation of Capital, written in 1956 (check Iglesia, 2007, for a summary).

The starting point to face the proposed aim is to reopen the famous box of tricks with which we, the economists of the latest generations have been trained, under the didactic approach, in order to assess once again its use in the current world; a world which is ever-changing, more and more open and dependent on new technologies and which points out the learning by doing concept as the guiding principle to follow; a world in which interdisciplinarity is becoming more important in the learning and in the work environment into which graduates must enter and in which cooperation between disciplines, which are close in the arch of knowledge, provides a greater degree of creativity when addressing the analysis and the search for solutions to the more and more complex economic problems, a search done by society and its institutions. In fact, any authors have stated that the solution to the current economic crisis should come from sociocultural fields, not only from economic ones.

${ }^{1}$ Cited in Walsh, V. y Gram, H. (1980), p XI 
New technologies have developed both new and changing teaching/learning spaces in which professors almost always lag behind their students. This is because students were born in the generation of both the incorporation and expansion of computing in learning, professional and leisure environments and the development of new ways of synchronous and asynchronous relations, of search and selection of the vast amount of freely available information, and even the establishment of rules of conduct and, in short, new models to which professors must adapt and, where appropriate, develop a range of abilities/ competencies/skills for which they have not been trained. In this sense, Gilbert (2001) states in an interesting text that professors need to assume the roles of information consultant, team collaborator, facilitator, lone worker, resource provider and academic supervisor in this technological age. These roles complement the mentor of writing (provider of feedback to the pieces of work done by students) and orchestrator (because he performs administrative, pedagogical, technological and link functions) pointed out by Wake, Dysthe and Mjelstad (2007).

Therefore, this article tries to address some of the aspects related to the didactic action of economics, following certain works by Professor Robinson where she showed concern, particularly in the last years of her academic life, for methodological aspects and the future of the study of the economic analysis and economics. Some of her statements prove it and are, at least, thought-provoking and often a source of controversy. Examples of this can be found, for instance, when she states that economic theorists of the last fifty years have been more concerned about the training and propagation of ideology than about the understanding of the world we live in, submerged in different arguments and moral values (statement referred to the controversy about the capital measurement The relevance of economic theory, published in Monthly Review, 1971). She even states that logic is corrupted by opinions, and she also writes in another piece of work that economists have abdicated to sociological and psychological views in explaining those issues that concern them (in Economics Today, lecture given in December, 1969 at the University of Basel accumulated in Collected, 1973). These and other fragments rescued from her innumerable writings are the guide of the methodological lesson tackled in this piece work, which explains how to review the teaching and learning process of economics in a context where the principle of learning by doing is vital. Therefore, this article will not question the specific contents of the studies in economics, nor is it to deal with some of the reviews which have generated interesting "controversies" in which leading academics have highlighted some theoretical inconsistencies in the patterns of economic thought in the modelling. On the contrary, it will collect and review some of the lessons that are either valid or adaptable to the current educational context.

Thus, it is important to state that the necessary methodological revision brought by the Bologna Process (Bologna Declaration in 1999 on the European space for Higher Education, EHEA, ratified by 32 countries in the Prague Communiqué, 2001), based on how to redefine the calculation of the ECTS (European Credit Transfer System) credits and the extra effort for professors and students of a teaching focused on the learning, has to strengthen the bases for a solid improvement in the quality of teaching. This revision has to be done through the updating of contents and the development of different activities and teaching resources that let students gain knowledge and skills that are specific in their degree during their training, while developing the transverse, systemic skills (as the Tuning Project refers to them) that the multidisciplinary, globalized work environment where they will practice their profession demands increasingly. 
In this respect, it is worth considering some empirical studies on the level of development of skills of graduates and students enrolled in either Economics or Business Management degrees (ECO and ADE are, respectively, the acronyms used in Spanish). These degrees show clear mismatches between the requirements of the labour market of professionals with high levels of competence and the degree of development acquired during their university training. Such mismatches vary depending on the previous professional experiences, expectations of the graduates or students and, therefore, on their degree of familiarity with the work environment in which they enter. It might be because they have usually done an internship or because they have had previous temporary jobs, or they have completed part of their degrees abroad by means of the Erasmus programme. Differences in the assessment of the importance of skill models between academic and labour field have been found in other studies with different analysis methodologies, objectives and samples. These results, which should be a reflection and a self-criticism, may be related to the way the didactic action has been addressed for a long time. It has focused on learning through the memorizing of mere concepts usually away from practice and simulated professional environments. The results show how to incorporate those activities to increase the skill development of our students in both the active learning process and its continuous evaluation.

This essay provides a symbiosis between some of the thoughts of various authors within the field of social sciences in general and Economics, dealing also with some of the trends and challenges faced by the European University nowadays, which are marked by internationalization and multidisciplinarity. It also alludes to the need to respond to the real, changing, global problems of society with fully qualified professionals both in content and specific competencies of their qualifications, and also, in a very special way, in generic, systemic, transverse and even emotional skills that favour a successful inclusion in the labour market. This approach to a quality teaching action, appropriate to the technological age and needs of today's society, is a real reform, desired by many, and it transcends the "Bologna Process" itself, which requires a commitment from academic authorities, business organizations and senates in which the actors and the primary beneficiaries are the students themselves, as they are the human capital of future generations.

Therefore, the chapter consists of the two marked parts: a guided methodological reflection on didactic action and a revision of empirical aspects related to the levels shown by graduates/students towards skill development within areas dealing with Economics.

\section{Some methodological considerations for the study of Economics}

Obviously the current University is a reflection of the society in which we live and there are remarkable differences with the one where some of the great masters of economics went to. This is mainly because of the use of technology in the teaching-learning processes, in the classroom and outside it. It can also be because of the way students mix themselves with professors, the way of searching the necessary information to enjoy themselves, etc. The technological revolution framed in a change of curricula adapted to the EHEA, has been the opportunity and/or the reason for professors to seriously reflect on how to address the specific contents of the degree.

That is, a reflection on both those specific contents that graduates should acquire and the methodological aspects of their study that otherwise would almost certainly be beyond our immediate interests. In this new methodological framework, the teaching work is increasingly 
done in a diverse educational context, where professors are not only a mere expert in content designed for oral presentation in master classes, but they also become designers of new learning environments. Professors combine and use a variety of educational resources and techniques and, at the same time, they become a generator and evaluator of useful resources for self-learning. Furthermore they also embody the necessary and fundamental aspect of being counsellors, mentors, motivators, and co-learners of their profession. They effectively teach the content and the subject to their students for their future professional development, that is, they are companions in the learning process as Iglesia (2006) stated.

But referring again to Cambridge and Professor Robinson, who makes us think about some methodological aspects of the study in today's economy, it is worth noting her unceasing interest in the study and the modelling of microeconomic problems when she said that "in my young days the trick was worked by concentrating on micro problems"... (in the essay "Marginal Productivity", published by The Indian Economic Review, 1976 and accumulated in the work Collected, 1973, cited by many authors). This ability has been, is and will be necessary to understand how the stylized economic models and the interrelationships among the relevant variables work. Both are necessary to make predictions about the behaviour of agents and future expectations and to specify explanatory equations in empirical analysis studies. This is then the main purpose of the article: to reflect on Joan Robinson's box of tricks. These tricks allow us to model the behaviour of agents from restrictive, simplifying assumptions, or skills, because their use makes it easier to deal with the learning of the interrelationships between the relevant economic variables. Thus, our students should definitely learn to use these tricks, which are a method of analysis and $a$ way to think like economists to solve problems of this increasingly complex, globalized economy. It has been 50 years since Joan Robinson showed their know-how through the classrooms. Although we have undergone a severe methodological change, some aspects should not, cannot change the study and the learning of Economic Analysis, such as the deep knowledge of the essentials that tighten up and support economics. But this statement does not preclude that such learning fits the current and unpredictable future development of the Information Technology (IT), as the UNESCO Universal Declaration on Cultural Diversity (Article 1) states. It refers to all disciplines and hence is applicable to economics, since it considers innovation and creativity to be a source of the change. Both are beneficial for present and future generations, and for whom IT has transformed the perception, the lifestyle, the way they communicate and the learning tools of an increasingly higher part of the population. Following this theory, when referring to the main method of thought and study of economic theory in the text Essays on Economic Analysis (1964, Exercices in Economics Analysis, in 1960), a pleasant to read book for any professor from this field, whose validity is timeless, Robinson describes it as "a model of construction", which "consists in a very simplified mental picture, which shows the behaviour of people in a social and physical environment, and eliminates what is not essential to the problem of the day, the way of focusing attention on what is essential" (1964, p. 10). In this regard, she points out some methodological rules to observe in the systematic study of economic theory, which, being basic, are often forgotten or simply not specified: 1) to consider the time variable in the analysis, 2) to specify the units used to measure the variables so that the different amounts have a meaning and 3) to distinguish the technical and physical relations between a human being and nature and between two human beings (1964, p. 7). Regarding to the time influence in the economy, as Carpintero says (1999, p. 137-38), Robinson states in 1953 in the essay Lecture in Oxford by an economist from Cambridge that "the distance between today and yesterday is 24 hours when we 
advance, but an eternity when we move back", but in 1975, in the article "The Time in Economics", in an homage to Georgescu-Roegen, John Hicks talks about the incoherence of representing the time in an economist's blackboard as it was a space, since, although ... "we can move in the space in any direction, the time only advances, it does not move back". Thus, Joan Robinson sets out the steps for students to "... learn something by doing it", and she shows to the readers "how to get to the propositions in economic theory, so they can continue by themselves from the point where the book ends" (1964, p. 7). She says it in the prologue of the quoted text, written in 1959 (published in 1960), and which, as she alleged, is "an uncommon book" (at prologue 1964, p. 7). In this book where she recommends "to use (referring to the suggested exercises) as a basis for group discussion", since she believes that "each student must work on an exercise and then compare the results with others and discuss their meaning and application" (p. 7): un approach distinguished from the common practice in our classrooms where the student resolves the exercise and checks the solution, taking the first of the steps suggested by Robinson, both for her comfort or, in many cases for the teacher's comfort. The fact that it is an unusual text has also been reflected in their methodological approach, in which she highlights the almost total absence of mathematical and graphic symbols (in a 299-page book there are exactly only 9 simple equations and no graphics), wanting to reflect, perhaps, what Joan Robinson once said ¿or was attributive at her? "I do not know mathematics, therefore I have to think" (if we check her more than 100 published works, it is easy to state that this statement is false). This phrase is often quoted by students of Economics when they criticize the difficulty of addressing the learning of abstract theoretical models. Although this phrase seems opposed to the common practice in standard texts of Economic Analysis and collects tradition expressed by Koopmans (1957) when he states that perhaps the numerical example and the diagram are the oldest mathematical tools in economics, it is perfectly compatible with it, since the mathematical formalisation allows styling and modelling the interactions of economic variables, providing the scheme of thought in which the reflection, the evaluation and the study are supported, without the need of being written.

Note that these suggestions recommend considering some basic, transversal skills that the student must develop in the learning process of economic theory, such as the ability to synthesize and to analyze, oral presentations, critical discussions or teamwork, etc.

Some of the tools of analysis are retrieved and analyzed. They are necessary to progress in the teaching-learning process of the behaviour of economic operators and the basic functioning of the models supporting economics, and they should be adapted around today's technology.

\subsection{On the subject of mathematical economics and multidisciplinary analysis}

These pages are not meant to reopen the discussion of the use/abuse of mathematics in the study of Economics (we take Frank Hahn's advice for the youth taking his baton: as it was a plague, to avoid discussions about Mathematics in Economics, quoted in Streeten, 2007, p. 49), they just collect some quotes for the critical reflection on some methodological aspects of the theoretical modelling and its abstract approaches. Whereas Samuelson (1947) stated that mathematics is more than a language, it is also a "powerful" tool for the solution to some central problems of Economics, Joan Robinson (see Walsh and Gram, 1979, in introduction pages) argued that "...the apparent precision of Mathematics has generated imprecision. The mathematical operations are performed on entities that cannot be defined, the calculations are made in terms of units that cannot be measured, correlations are confused with occasional laws, differences are identified with changes.... The complexity of models is made just for display, far away from the 
possibility of applying it to reality". Because of its abstraction, the virtue of mathematics is an accepted and not arguable fact in the economic analysis to state exactly and precisely the generic objectives of the economic operators and the axioms and decision criteria and restrictive assumptions and simplifications of reality that frame or define the context of the analysis, deductions and conclusions of models. However, the excessive use of formulation and mathematical symbols can have some risks if it confuses the student and it does not clarify the exposure and/or research. And this is out of the controversy, never close enough, of the realism of the assumptions of the economic analysis, since the results of the models are only valid in the compliance with the postulations made. Therefore, the use of mathematics in deductive method of the economic analysis makes explicit the assumptions of the models.

The current box of tricks combines classical analytical instruments, such as the numerical example, the mathematical symbols, the diagram, quantitative methods, with some more sophisticated and powerful utilities, expanded by the development of virtual technology, whose teaching virtues have widespread providing new media, ranging from the interactive whiteboard, tablet PC, virtual learning environments, simulation platforms, Web 2.0, etc.

We have gone from white chalks to laptops both in the teaching and in the learning of each of the pieces of the puzzle that the economic reality is and that at the same shapes the Economic Theory. Once they are analysed, these pieces must fit together perfectly to set up the studied reality and to give answers to the economic problems that society is facing. In this regard, Joan Robinson, when justifying the proposed method, points out in the already mentioned text, Essays in Economic Analysis (1960, in introduction pages), that "we must first divide the question into parts, and discuss each one separately, and piece them together again as best we can". Today the availability of a powerful mathematical programming software supports the analytical modelling of such pieces and the simulation of real environments and various scenarios of economic policy, which are increasingly used both to study the comparative statics and to determine the economic forecasts and its future projection. Different international public organizations and important study centres have been developing sophisticated simulation models for more than three decades in order to, for instance, predict the behaviour and development in different countries and to check possible imbalances in the same, or the effect on the saving, the private consumption or the investment of a change in the marginal tax rate. Although the term simulation may have several definitions, which could be summarized in exploring the behaviour of an experimental or virtual model similar to the real one in order to understand and/or predict its path, there is no doubt that it is a method that generates scientific knowledge and it is also a tool for learning how the economic reality works, which is studied through basic theoretical models. According to the paradigm of the study of Economic Analysis, we only understand an economic fact/phenomenon when we know the theoretical model that reproduces it, that is, when the interaction of behavioural assumptions that are set and the assumptions that define the scope of the problem/model, determine outcomes. Being this the case, simulation is hence a tool in the learning process of a changing world, plunged in a context of uncertainty.

This tool of simulation is not new and it has boosted its use with the technological development and algorithmic software, increasingly framed in virtual spaces, which also facilitates the integral view of teaching and learning in the economic analysis, materialized in the use of electronic didactic resources, which are available on Internet, and characterized by being: 1) radial, usable in different training levels, 2) multidisciplinary, since they address topics from different areas, approaches, methodologies and fields, 3) geared to the 
summary of the theoretical models with practical developments and uses in a professional environment and 4) updated, as Gracia and Iglesia (2007) point out. It thus builds a network of interrelated content that facilitates the student to approach Economics from different perspectives: theoretical models, empirical applications, case studies, actual experiences, the basic study of comparative statics of equilibrium and its implications in public policies, economic indicators, or historical references, etc. This integral, radial and multidisciplinary vision of the learning in economic analysis makes students learn to "think like economists" and develop certain skills that are needed in the economics' workplace. Students have rarely been developed them with the traditional teaching methodology.

Regarding this vision, in today's globalized world, there can be economic problems of other disciplines, as interdisciplinary boundaries are increasingly permeable so that they make intersections that go far beyond what our old masters could imagine half a century ago. Some examples are the Economics of education, environmental economics, economics of health, of natural resources or experimental economics whose development began in the investigation and later turned to teaching. Thus, Streeten (2007) identifies three reasons, with different methodological implications, that justify the disciplinary or interdisciplinary work, that can be moved to the learning process of students of economics: 1) the specialists of different disciplines, when working together on a single problem, in a cooperative way, contribute to the more complete and richer integrated solution, 2) certain assumptions, concepts, methods or techniques, when applied to other disciplines, may provide useful or satisfactory explanations and 3 ) it is possible that the approach of a separate discipline is not valid in a time or in a region, due to interactions between economic, social, political, historical variables, or the institutional knowledge (see the various examples he gives about interdisciplinary studies on Economics).

The fact that current economists work beyond the traditional borders in economics and with multidisciplinary approaches does not imply, as Streeten argued in the quoted text, the strengthening of other disciplines for the Economy against the opinion of Hicks (1979) (quoted by Carpintero, 1999, p. 129) who said that "the field of phenomena with which economics deals is so tight, that economists are continually banging their heads with their limits", generating new knowledge, the result of "riding cheerfully certain academic borders", the ones to which Dumont (1982) refers (quoted by Carpintero, 1999, p. 129). What it implies is that the new transdisciplinary knowledge searches intersections between different disciplines, absorbing background knowledge and taking advantage of permeable classic borders. In this point, it is necessary to refer to Georgescu-Roegen, who according to Zamagni (1986, p. 516), and although "his message has the peculiarity of not being identified with any school of economic thought", his intellectual aid in this century can be seen as "...the main contribution to cross the frontiers of economic theory and methodology". Later on, Carpintero (1999), and regarding this transdisciplinar focus, added that it is located at the base of the review performed by the Romanian economist of the mechanistic basis of the conventional economic approach, which not only affects the level of consumption, but also the level of production, and for this to be done, he was based upon the teaching of some subjects from the natural sciences such as thermodynamics and biology.

To strengthen the current relevance in the different areas of the science of inter/multi/transdisciplinarity mentioned (the semantic differences between the tree terms are clear, although they are usually used indiscriminately), there has been some clear examples, such as the Border Intramural Project initiated in 2004 by the Spanish National Research Council (CSIC) 
whose aim was to promote the incursion of scientists into new themes emerging from the interaction of several areas of knowledge (for instance, see the call for grants of the 10th of June of 2006), or the revision of the thematic lines of international conferences that show the relevance of multidisciplinarity in all areas of science. Another example is the final text of the Seventh Framework Programme of the European Community for research, technological development and demonstration (2007-13), which specifies that "it will strengthen the excellence ... through cooperation programmes, ideas, people and skills", and that includes some of the amendments proponed (documents: PE 360.033v010-00, amendment 312 presented by Rübi, P. -appendix I, paragraph 1- and PE 360.033v010-00, amendment 332 presented by Niebler, A. appendix I, chapter I "Cooperation", paragraph 6), in which reference was made to the relevance of interdisciplinary and multidisciplinary studies, quoting the European Union Research Advisory Group, which reports in its Indisciplinary in research (EURAB 04.009, April 2004 ) that the interdisciplinarity "is essential in keeping Europe at the forefront of scientific and technological research. Interdisciplinary research is a necessary feature of progress in scientific exploration". Moreover, it reports that in the field of "scientific cooperation", the promotion of such "joint calls" will provide new impulses and ideas, as "it is precisely in the boundaries between disciplines where knowledge and brand new products emerge."

In this sense, professors should not be away either from the economic, social and cultural development around us, or from the nearest scientific community, or from technological progress and its applications in learning, or from new and emphatic forms of media and entertainment which are suitable for learning, thus reformulating the teaching task, which is in a continuous process of adaptation to the environment, needs, perspectives and technology.

\subsection{About discussions, the creation of knowledge and independent learning}

It is worth returning to the discussion of those pieces of work to which Joan Robinson referred as a tool of learning. It is also worth pointing out that in the history of the economic thought there have been many episodes of scientific debate and controversy among the different positions or schools, which have promoted the progress in the development of theoretical models, without being alien to -the "unavoidable"?- ideological influence. If it was advisable not to enter the debate on the use/abuse of mathematics in the study of Economics, it is even more advisable not to enter the ideological presence in the approaches and orientations of economic problems.

Professor Robinson should be referred again, especially her book Economic Philosoph (1964), when dealing with the influence of ideology on economics. Robinson gives an answer to the question of determining the "criteria of an ethical proposition opposed to the criteria of a scientific proposition". She states that "if an ideological proposition is treated in a logical way, it may be dissolved in meaningless junk, or it may be a circular argument." These two metaphysical propositions still have their content and, "although they do not belong to the field of science, they are necessary for it", because they "have played a role, even an indispensable one," at least in the social sciences. She also adds that "although the ideology may or may not be removed from the world of thought in the social sciences, in the real world ... is certainly indispensable," ... and that "every economic system requires a set of rules, an ideology to justify them, and an awareness of the individual that makes him/her strive to fulfil them" (see pages 7-9, 18 and the following ones). It is very important to remember that debates in any science, especially in economics, have generated knowledge 
through logic considerations and conclusions. Therefore it is a good idea to expose students to a creative learning process from debates as a useful tool for searching a new knowledge. For this purpose, the debates need to be guided, thought, meditated and experimented by the professor. Regarding the use of debates, both in the generation of knowledge and in the progress of learning, Joan Robinson refers to them in the previously quoted text, Essays on Economic Analysis (1964), when she recommends "to use the exercises as a base of a team debate" (see introduction pages, p. 7). For this to be carried out, the professor should learn how to reformulate the standard exercises that make it easier to understand the models, and how to complement them with the incorporation of a set of open, well-defined questions. It is worth noting the difficulty of students when reviewing and answering coherently questions such as: what is the objective?, how is it interpreted?, what does it provide?, how is it related to?, etc. These questions should lead the student to create a new knowledge, to discover new concepts which have not previously been taught in the classroom and to go beyond what the professor has explained in class. Thus, Robinson believes that "the first step in the practice of the analysis is to learn how to know what a debate is about" (p. 13). This is why, it is very important to choose the questions so that students make progress in their knowledge and, as our mentor does throughout the above mentioned text, the professor can lead, advise and help each person to find which their own standard of judgement is.

It would be a mistake to stay in the lexicon and in the vehemence debated by several schools, which are not an end in themselves, but a way both for the process for creating knowledge among the academic ones of a discipline and for the active learning process of our students. For this purpose, some rules of the dialectic game should be set in motion; a game to which they (or even we) are not often used to and which is required as a transversal skill in any professional environment. It would be equally important to promote the search of important information to tackle any particular topic and to be critically assessed. In order to make the debate a useful way to learn, students should formulate clear questions to themselves for the search of answers. This is the only way to encourage the autonomous learning. In this sense, the quote by Isaac Rabí, Nobel Laureate for Physics in 1944, is very revealing. He affirms that when he came back from school, his mother would ask him the same question: "have you asked a good question today?" (In Szenberg, 1994, p. 5). It would be appropriate to add that Joan Robinson herself was a clear example of activism in her time in the construction of knowledge resulting from debates and discussions in every format possible. This activism went from written correspondence, meetings or seminars, to scientific essays. She was also part from, and sometimes she even provoked and provided feedback for, the most famous and scientific controversies in the economic field. As a historical note, it is worth pointing out that, from 1931, Joan Robinson was part of the Circus, together with her husband Austin, Sraffa, Harrod and Meade, whose aim was in to analyze Treatise on Money published by Keynes in 1930. She opened a deep debate when discussing with the master some elements of the General theory before its publication and she had with him a long, productive correspondence, more than 137 letters from April 1932 until April 1947, after the publication of the works Treatise on Money and A parable on saving and Investment. Furthermore, she was also in correspondence with authors such as Khan or Kalecki, since it was a very common practice among scholars from that time.

Regarding these disagreements, she stated that participants in these controversies are divided into schools -the conservative and the radical school- and the ideology seems to ooze in the logic. In economics, arguments are devoted, as in theology, to hold doctrines 
more than to test hypothesis (Robinson, 1977). Apart from that, she also reiterated that economists cannot resort to controlled experiments, as other scientists do, and the only way to have access to reality is by (means of) historic evidence; and most of the times that evidence could be read in both ways: the conservative and the radical one.

The written correspondence with other partners was a widespread way of discussing. This has nothing to do with today's society, where this activity has been relegated to the immediate email, which is very useful for the communication between students and professors so to solve doubts. In many cases, it is supplemented with thematic forums separated by categories, by topics within a subject and feasible with the methodology of e-learning platforms, virtual campus of the university, forums, personal blogs, wikis, etc. Everybody knows that the massive use of the different virtual resources is opening the catalogue of professions and there are, for example, bloggers that give a qualified opinion about several social, political and sports topics. These bloggers also comment on the new trends and what the latest news are, they open discussions, mobilize people and they are able to organise thousands of followers on a particular way of thinking or behaving so that they can set goals and vindicate them in a highly linked society that is developing very vague channels of communication.

Going back to the importance given by Joan Robinson to the political debate as a generator of knowledge, and referring to the controversies among academics, she states that debates should lead to an agreement, since the rules of logic and the available proof are the same for everybody. Moreover she points out the five reasons that, according to her view, set the starting point of a controversy (Introduction to the Theory of Employment, 1937): 1) The participant cannot make their opponents understand their point of view; 2) Any of the parts has made a logic mistake in the reasoning; 3) Participants assume different suppositions; 4) There is not enough evidence to clarify or to empirically contrast any aspect, and 5) The ideology of the participants is different.

These elements should be taken into account in the teaching/educational use of debates, since students should acquire knowledge and, therefore, they should justify their points of view, using logic and reasoning supported by known and, in many cases, mathematical material. They should specify the restrictive assumptions of the model supporting the presented arguments, giving different evidence. Therefore, they should be creative when searching and block out from the starting ideology. In short, this work is to make us think about the education that an economist, a good one, should obtain. In relation with this, Streeten (2007, p. 40) argues that the economist "will be better theorist if he knows philosophy and a better economist if he knows political science and history". He agrees on supplementing the use of the basic tools of economic analysis with the study of other adjoining disciplines such as history, the economic institutions, the elements of politics, etc. Furthermore, he points out that "education is more than acquiring skills and aptitudes, but also acquiring attitudes", and he also wonders about the limits: "what shall we do in order to make (students/graduates in Economics) properly use the toolbox with which we equip them so that they avoid being victims of the law of the hammer...? (the law of hammer is that law according to which a little boy, given a hammer, finds everything worth pounding, not only nails but also Ming vases) (Streeten 2007, p. 41). This is the question that leads us to the following section: to delimit how the learning and teaching processes should be reflected in the skill development of our students, taking their subjective perception to answer the required challenges in the working environment that they will enter, or they have already entered. Issues, those before mentioned, related to different indicators of educational quality. 


\section{From the theoretical training to the skill development}

Many readers may see a huge somersault between the two sections of this piece of work, but this happens only when the author cannot define clearly the conducting thread between them. In other words, the reader must be shown how important is to convert the methodological aspects mentioned above about the teaching/learning of the economic analysis, guided by some quotes from Joan Robinson's works, into a satisfactory skill development of the students. This development should meet the needs of the uncertain, changing, globalized, complex social and economic environment surrounding us.

The first test to be passed is the establishing of the generic concept of the word skill, a concept which is quite diverse, elastic and flexible because, as Climent (2010) states, though these several terms are often used without distinction, they are not always synonyms and some of them have important conceptual differences between them. This happens, for example, with the words abilities, dexterity, skills, qualities, aptitudes and/or talents, mainly when the Competence-Based Education school appears (the different definitions and typologies included in it are very instructive; they let laypeople delimit the concept and establish several dimensions of it). In order to delimit the point of view from which this work is based, in accordance with Holmes (2000), ability is a causative tool of what a person does, inside or outside the labour or social reality where this person lives, while skill is the ability that lets a person use a set of skills, knowledge and values in a suitable way in order to perform a task successfully, in certain labour or social circumstances. In line with this, Sladogna (2000) talks about complex abilities that have different degrees of integration and that appear in a wide range of situations in the different aspects of the personal and social human life, as these abilities are a synthesis of all the experiences the person has undergone in the frame of his past and present personal environment.

Thus, through the pragmatic way followed in this piece of work, and given that the author is not an expert on this subject, skill could be defined as "ability obtained through the combination of the knowledge with the social environment and personal characteristics, the aptitudes and experiences, which professionals, in this case university graduates, must have in order to perform their professional responsibilities in their jobs". Some of the elements of this definition are remarkable as it integrates the knowledge, gained in the process of the teaching-learning, with environmental, social and personal characteristics, but also from their own experiences. For more information about the theoretical development of the skill models, consider scholars such as McClelland (1973), Spenser and Spenser (1993), or LévyLeboyer (2001). However, the guide that has been used in the empirical piece of work that is presented below is more similar to the institutional models in which the REFLEX study (Aneca, 2004) and the Tuning project (see González and Wagenaar, 2003) were carried out.

\subsection{Empirical approach to the skill development of university graduates in the area of Economics}

In the following pages certain empirical evidence is presented in a descriptive way. It relates to the already proved phenomenon of the existence of clear imbalances between the perception university graduates have about the degree of their skill development and the assessment they make about how important it is in the working environment. This is 
obtained from the subjective points of view of students, from their own social, work, personal experience and from the learning environment in which they have been brought up. This fact confirms the positive effect of the work experience in the reduction of the gap observed.

This empirical approach here included shows how important is to improve the skill development among students during the university training process, which should go hand in hand with a specific technical training in each particular degree. This empirical approach gathers data collected from an opinion poll conducted on students in their fourth and fifth year of the Administration and Business Management [ADE] or Economics [ECO] degrees, which are taught in the Faculty of Economic and Business Sciences of the Complutense University of Madrid (UCM) and Alcalá de Henares (UAH), in January 2010. It should be explained that although the selection of the years, the fourth and the fifth, may bias the results obtained. This can be justified, however, since students in those years have already more precise criteria about the working environment. In fact, most of them were doing an internship or had already done one. They also had previous work experience and therefore they were able to determine if having previous work experience improved the skill development among university graduates, a fact that can justify certain reorientations of the training plans when designing the aim and the scope of the practicum.

The opinion poll used in the empirical analysis contains a comprehensive list of 40 standard skills, considered as independent variables. The questions related to different skills are presented in two symmetric groups, one refers to the skill degree developed during the university training (_UT) and the other one to the importance students think it has in the working environment in order to carry out their professional activities (_IW). Students assigned a numerical evaluation using the Likert scale, from 1 to $5(1=$ strongly disagree and $5=$ strongly agree).

As a summary,table 1 below describes the population who participated in the opinion poll and from the 444 of those polled who were attending classes on site, $49.55 \%$ of them were men, and $42.57 \%$ were in their fifth year, $34.45 \%$ of them studied ECO and $65.31 \%$ of them stated they had previous professional experience. There are not significant differences between the subsamples of the two universities (UCM and UAH). Furthermore, in ADE, $50.86 \%$ of the students were men and $47.06 \%$ were also men in ECO, $47.45 \%$ of the students enrolled in fourth year are men. This percentage is higher in the fifth year as $52.23 \%$ of the students were also men. Among the students that stated they did not have previous working experience, the percentage of men was $55.19 \%$ while the ones who had previous working experience dropped to $46.55 \%$. This fact shows that women are relatively getting closer to the working environment during their academic training.

It can be concluded from the descriptive analysis that, as the table A1 of the annex shows (for more details see Iglesia and Ionita, 2010), when students were asked about the degree of skill development during their university training (_UT), the more representative option was "average", with percentages between 27 and $40 \%$, and it turned to "very much" regarding to the degree of importance of the skills in the working environment in order to carry out their professional activities (_IW), with percentages that ranged from $32 \%$ to $50 \%$ with exceptions when students chose mainly the intermediate option (that is, a 3). This 


\begin{tabular}{|c|c|c|c|}
\hline \multicolumn{2}{|c|}{$\begin{array}{c}\text { Administration and Business Management degree (ADE): } \\
65.5 \% \text { to sample }\end{array}$} & \multicolumn{2}{c|}{ Economics degree (ECO) } \\
\hline Men & $50.86 \%$ & Men & $47,06 \%$ \\
\hline \multicolumn{2}{|c|}{$4^{\text {th }}$ year: $57,43 \%$ of the sample } & \multicolumn{2}{|c|}{$5^{\text {th }}$ year } \\
\hline Men & $47,45 \%$ & $\begin{array}{c}\text { Wen } \\
\text { experience }\left(\_C\right)\end{array}$ \\
\hline Without professional experience (_S): $34,68 \%$ of the sample & $\begin{array}{c}\text { Men } \\
\text { exsional }\end{array}$ \\
\hline Men & $55,19 \%$ & Men & $46,55 \%$ \\
\hline
\end{tabular}

Source: Personal Compilation. Opinion Poll about the Need for a Skill Development. Faculty of Economics.

Table 1. Distribution of sample by course, degrees and experience, by sex and total

asymmetry or imbalance in the assignment of answers between the options is proved in the estimated mean values and therefore in the differentials of those values for both questions (tables with the average values to facilitate the reading haven't been presented, although they are available in the quoted text). Thus, the average obtained is higher than 3.2 regarding the importance in the working environment, reaching even 4.3. The averages fluctuated between 2.1 and 3.4 regarding the degree of training as we can see in the Figure 1 attached, which clearly shows the difference in the average values between the two series.

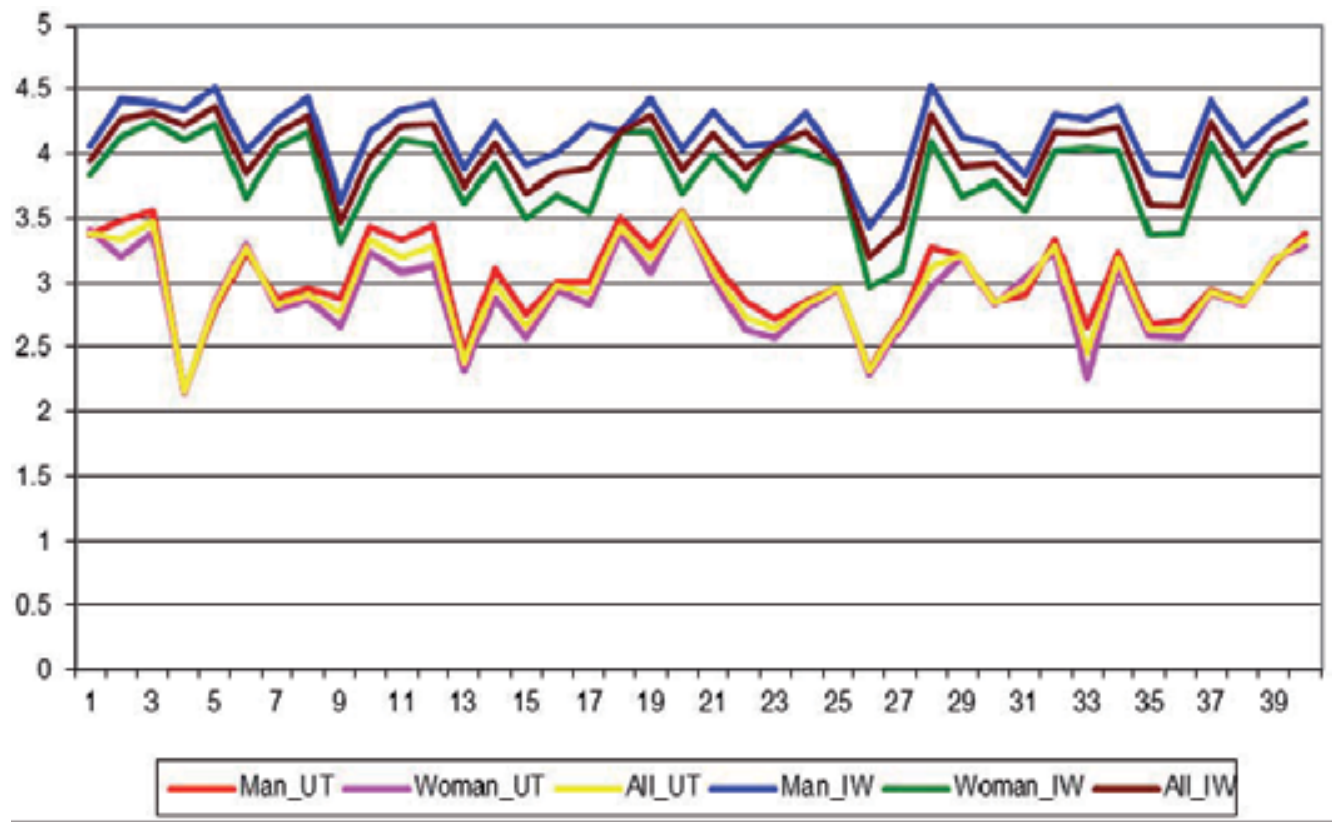

SOURCE: Personal Compilation. Opinion Poll about the Need for a Skill Development. Faculty of Economics and Business.

Fig. 1. Average values: Importance in the working environment/Training at University, total and by sex 
Furthermore, the different segmentations confirm the general rule already mentioned: the average rating that students assigned to the importance of the different skills in the working environment was greater than the degree of skill development they stated they have, regarding all students and segmented by sex, being in both cases the average values assigned by men higher than those assigned by women. Fluctuations were wider in the series related to the importance of skills in the working environment (_IW) than in the perception of the degree of formation gained during university training (_UT): in other words, students had a more uniform opinion about how important university training was for their skill development and they presented a higher variability in the assessment of the importance of the skill development in their working environment. Similarly, when the sample was segmented according to the year students were enrolled, the average assessment of the degree of skill development was higher among those who were in their fifth year than among those who were still in their fourth year. Nonetheless, the opposite occurred regarding the importance of the skill development in the working environment. In the segmentation, students with previous work experience conferred more importance to skill development than those who did not have it. Previous work experience also helped to change the perception they had, which helped to adjust their assessments and hence reduce the differential between the averages of both questions.

In brief, tables A2 and A3 appearing in the Annex show the results of the descriptive analysis of this piece of work, that is, the differential between the two questions in the average assessment of the students who were consulted, for all the skills, reflected in the figures 2 and 3, attached in the text. In general, the average assessment of men was slightly higher than the average assessment of women, nearly in all the skills, regardless of the fact they had or not professional experience or in which year, fourth or fifth, they were enrolled. Results were similar in both questions: the one about the importance they think skills have in their working environment and the one about to what extent they think university training contributes to their skill development. This fact determines a positive differential in the averages estimated, with a wide value range depending on skills, for the total population and by sex, and it was higher for men than for women, with few exceptions. Thus, for the total population, the differential estimated was lower when there was work experience. The reason could be that the student would have done or would be doing an internship or that maybe the student would have had a temporary employment, with a few exceptions not very significant.

In short, figure 2 (and table A2 in the Annex) shows the differential averages for the different skills and for the totals and subsamples by sex, according they had or not work experience. It could be observed that there was quite general pattern with a lower profile in the differential for the subsamples of women with work experience, a fact that meant a better adjustment and therefore a lower differential between the assessment they did about how important was skill development in their work environment and the degree of the skill development. There was a higher profile of men who stated that they did not have work experience, an aspect that suggested greater imbalances in the assessments. In fact, exceptions to this pattern were found in the subsamples of students with work experience, only in the skill "Negotiate efficiently" and among those who did not have work experience in "Organisation and Planning", "Oral and written communication in first language", "Ability to make yourself understood", "Work well under pressure", "Manage effectively your time", "Leadership" and "Trade efficiently". 


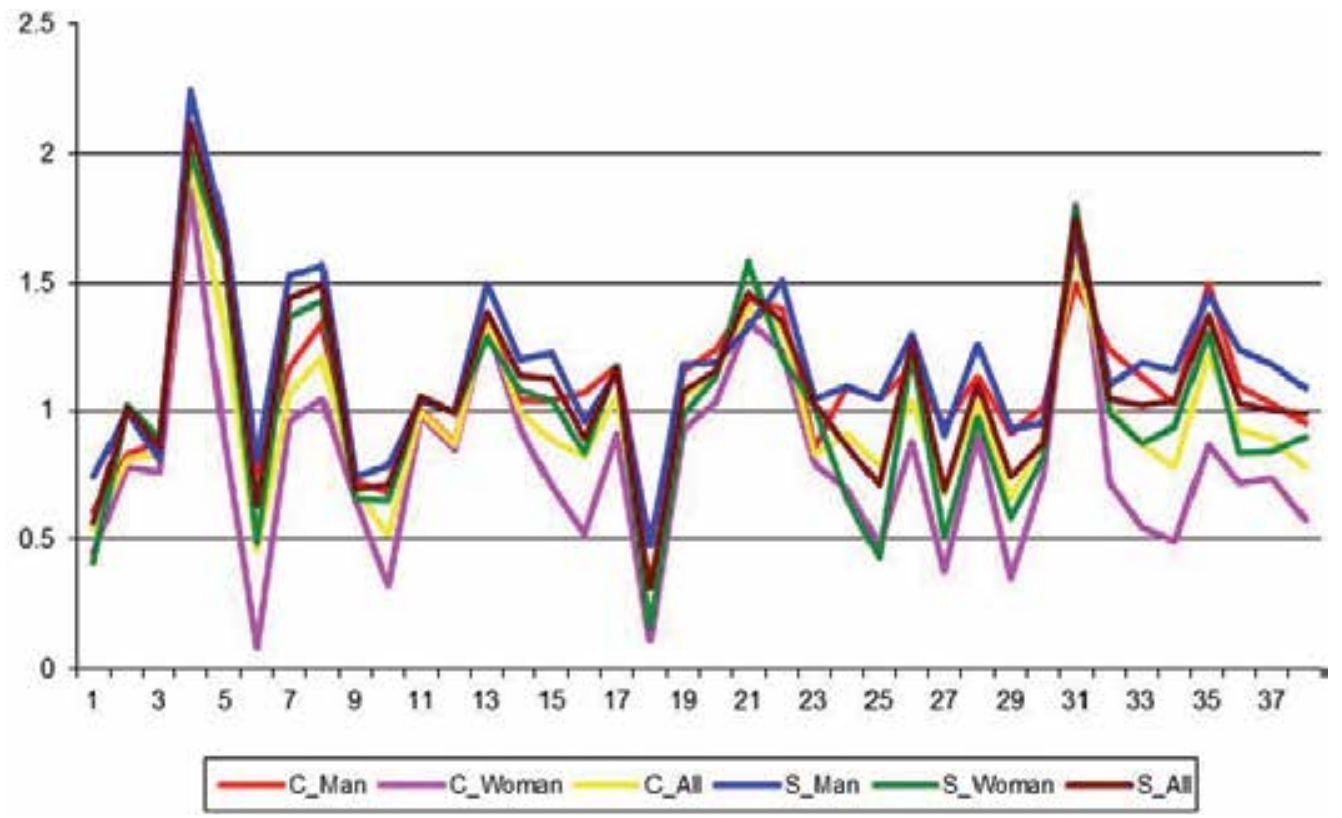

SOURCE: Personal Compilation. Opinion Poll about the Need for a Skill Development. Faculty of Economics and Business

Fig. 2. Differential importance in the work environment - Training at University, total and by sex, according the students have or not work experience

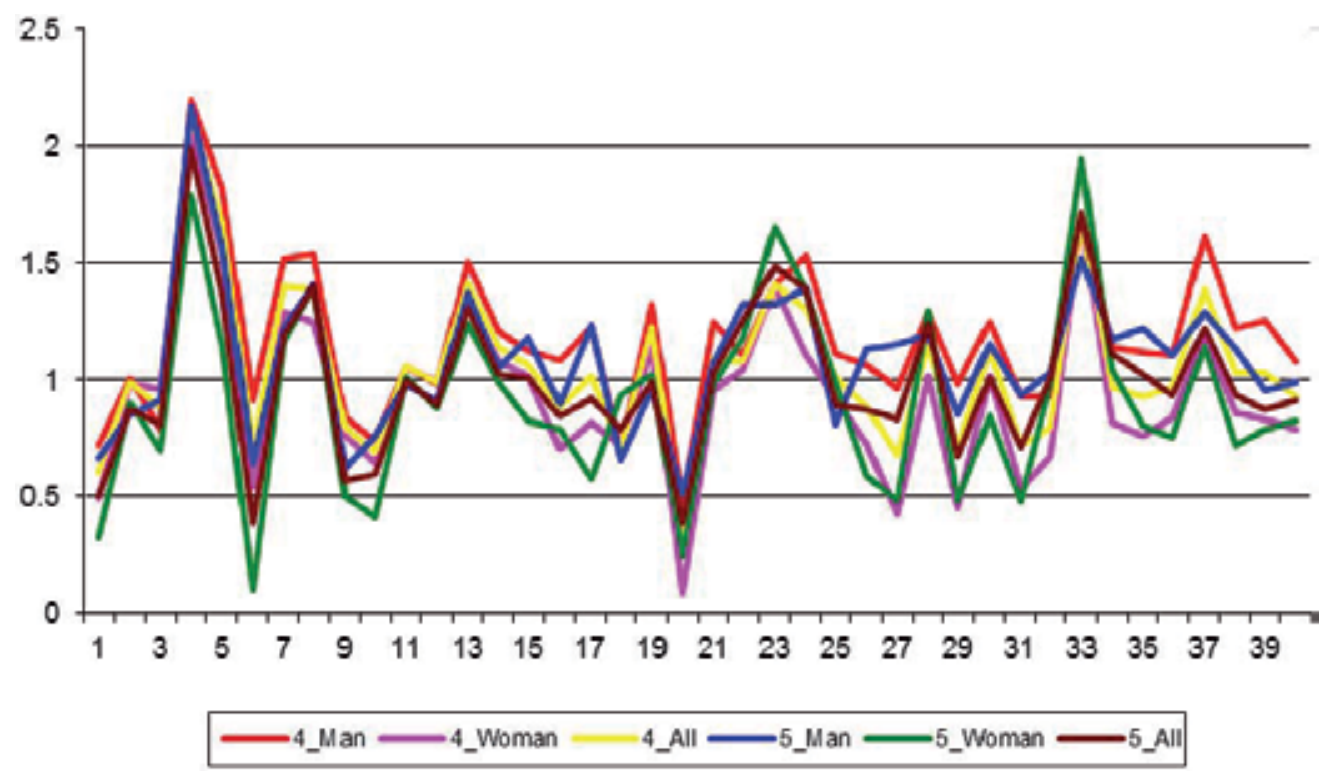

SOURCE: Personal Compilation. Opinion Poll about the Need for a Skill Development. Faculty of Economics and Business

Fig. 3. Differential Importance in the working place - Training at University, total and by sex, according they are in the fourth or fifth year of the degree 
Figure 3 (and table A3 of the Annex) above shows this differential for the different skills, by total and subsample by sex, according to the year in which the student was enrolled with a quite similar pattern to the one seen in the previous segmentation by work experience. This is because this experience increased every year, with a lower profile in the differential for the subsample of women who were enrolled in their fifth year and higher in men who were enrolled in their fourth year although this profile was not so clear, with some crossings in the assessments.

These descriptive results support the hypothesis already expressed previously: having previous work experience, either through temporary contracts or through internships, improves the perception of a higher degree of skill development, as they pass their fourth year and enrol in their fifth year and they are finishing their university training. Therefore, the studied differential reduces, and the adjustment between the assessments they did about the importance in the work environment and the degree of skill development achieved improves considerably. This information, considered to be quite important, should be interpreted as an indicator showing in which direction teaching should be addressed: the student, who has to learn specific knowledge of this subject, has to develop also some basic generic skills, including systemic, personal and instrumental ones. The added value that brings this information will result in a better teaching and research activity of the professor, with a greater understanding of the skill profile of students. This new situation will result in practical activities that will let students and future graduates be more trained for the work environment they are going to enter in a short period of time.

Some pieces of work which continue with this approach and which are being written in other universities should be pointed out in order to find the needs and the skill model of their students and graduates. An instance of this is the empirical study written by Ramírez and Velásquez (2010) that confirms the existence of differences between the skill profile for employment assessed by students from an academic point of view and professor and skill profile assessed by graduates and employers from a work point of view. In this way, the first ones consider that instrumental skills, such as languages and office automation, that is, the ones they cannot use correctly, are more important than the systemic skills, related to social, organizational and work management areas.

\section{Conclusion}

The aim of this piece of work was to reflect (or in this case make you think) about some of the methodological aspects of the study and teaching in Economic Analysis nowadays, recovering some lessons given by important scholars, mainly Professor Robinson, in an historic moment for the Spanish University which has completed the process of European convergence as a regenerating element of its university training and their necessary adaptation to an academic world which is a work, social, changing and globalized word.

The starting point for this piece of work is to use again the box of tricks with which all economists have graduated at our universities, and reopen it to assess one more time its usefulness in an uncertain, open and changing world that in some cases depends too much on new technologies, characterized by the inter/multi/transdisciplinarity analysis and the necessary scientific cooperation between the different disciplines in order to tackle and solve the social and economic problems that society and institutions have nowadays. This box of 
tricks refers to a set of basic concepts, categories, analytical instruments that all economists should know how to use, as well as their prospects, scopes and the adaptation to this period of time.

Nowadays, we work, teach/learn and do research collectively, connected through the Internet, with teaching tools that range from e-learning platforms to web-quest, where students download all kinds of teaching materials (text, audio or video) in order to make progress in their learning process. For many people, this is the learning economy (Lundvall and Johnson, 1994, Archibugi and Lundvall, 2001), whose key is the ability to learn from people, organisations, networks and regions, learn to present other criteria and scopes and to use the potential of new technologies to completely change new processes, ideas, products and so on.

Therefore, an economist should not merely know the proper use of tools as analytical instruments. Instead, those tools, as many important economists have stated, should be complemented with other aspects that are suitable to develop a dose of creativity that let us find the solution to the important economic problems that our society and institutions have to face up. In this context, Krueger et al. (1991, cited in Streeten, 2007, p. 57) state, in relation to Economics postgraduate studies in the U.S.A., that the main weakness of these studies was not the excessive use of Mathematics, but insufficient emphasis on links between theoretical and econometric tools and the problems of the real World. In words of Streeten (2007, p. 42), according to the Petition to Reform Undergraduate Education signed by more than 460 professors who are involved in hiring new professors who teach economy to undergraduate students in the U.S.A., the selected economists should have an understanding of the economic debates and the literature of the previous 20 years,... with a strong knowledge of the models that are taught to students, knowledge of the economic institutions and about the role those carry out in the economy, ability to communicate both verbally and in prose the main ideas of the Microeconomics and the Macroeconomics, knowledge of alternative approaches in economics and ability to contrast them and also knowledge of Econometrics and the limits of their proofs. All graduates in Economics in this country and in this economic system should therefore meet these requirements perfectly, which consist not only of technical knowledge but also include several skills.

As for the need for higher doses of creativity to face economic problems above mentioned, this creativity is institutionally considered as the driving force for innovation and a key factor for the development of personal, work, business and social skills of people, as well as the social welfare (text extracted of the Decision number 1350/2008/EC OF European Parliament and of the Council, of 16, December 2008, Official Journal of the European Union). Therefore, according to Perinat (2004), being a competent professional means not only knowing (having a theoretical or specific knowledge), but also knowing-how (practical knowledge) and knowing how to be (providing axiological professional attitudes).

Professors know that changes in the methodological didactic environment are aimed at only one objective: contributing to improve the quality of education. For this to be done, the activity of the professor-tutor-orchestrator-companion should be "redefined", and they must learn continuously the new technological applications which lead to "relearn" professional teaching practice "doing it". One important factor is to have a better and greater knowledge of the effectiveness of the classes given, through multiple indicators 
(assessment of educational attributes, causes of absenteeism, relationship between attendance and performance, incidence of tutoring in professor assessment, consideration of sex in these assessments, imbalances in the skill development of students and so on). For this purpose, professors can and should combine their teaching activities with research in order to find out strengths and weaknesses and generate with this a feedback process that improves our facet as professors as well as putting research about teaching at the disposal of the teaching activity itself, no matter which discipline nor which area of expertise, given the heterogeneity of students, subjects, disciplines, professors and degrees.

From this point of view, this piece of work has combined the methodological aspects of economic studies, the requirements of professional economists and a skill model balanced with their work environment. The empirical analysis let us highlight as a general rule for all students consulted that the average assessment given by students about the different segmentation proposals at work is higher than the degree of skill development they state they have. This fact determines always a positive differential between both values. Furthermore, in the segmentation by year in which the student is enrolled, the average assessment of the degree of development or preparation is generally higher among those students who are in their fifth year than those who are in their fourth year, although it is just the opposite when we talk about its importance in the work environment. It should be pointed out that, as a general rule and for the total of students, the average assessment of the degree of the development of skills at university and of its importance in the work environment is higher when students have previous work experience. In this way, the estimated differential for the different skills shows a quite general pattern with lower values in the differential for the subsamples of women with work experience, a fact that implies a better adjustment between the assessments they do of the importance in the work environment and of the degree of skill development, and the higher values for men who do not have previous work experience.

Professors should not only bear in mind what happens in a classroom but they also should think about the professional world students are going to enter, in order to facilitate the adaptation of these students to their changing, globalized and multidisciplinary work environments with creative vision which will let them solve problems, with greater specific knowledge and with a demonstrable background. Professors should have knowledge of other disciplines, how to deal with the different scientific areas, learn from other approaches because as Myrdal (1975; 2002 p. 70) states, in fact, problems are not economic, sociological, ecologic or psychological, but "they are all mixed and composite". We should open our classical box of tricks in order to achieve that those (many people) who think that the person "who is just an economist is a bad economist" (Streeten, 2007, p. 36) change their mind.

\section{Acknowledgment}

The data used come from the Project of Innovation and Improvement of the Education Quality: Educational plan aimed to improve the teaching of generic skills o students. From university to the work environment, financed by the Complutense University of Madrid (UCM). Thanks are due to C. Ionita, who tabulated the questions and did a first descriptive approach to the data.

The translation of the chapter was made by Guirado López, P. and Sánchez Moya, A. 


\section{Annex}

\begin{tabular}{|c|c|c|c|c|c|c|c|c|c|c|}
\hline \multirow{3}{*}{ Skills } & \multicolumn{5}{|c|}{ University Training (_UT) } & \multicolumn{5}{|c|}{ Importance at Work (_IW) } \\
\hline & \multicolumn{5}{|c|}{ Distribution of frequencies (\%) } & \multicolumn{5}{|c|}{ Distribution of frequencies (\%) } \\
\hline & 1 & 2 & 3 & 4 & 5 & 1 & 2 & 3 & 4 & 5 \\
\hline Analytical thinking and Ability to Synthesis & 2.25 & 12.16 & 38.74 & 38.29 & 8.56 & 0.90 & 4.05 & 20.50 & 48.65 & 25.90 \\
\hline Organization and Planning & 2.93 & 22.30 & 28.15 & 31.08 & 15.54 & 0.90 & 1.80 & 9.91 & 43.24 & 44.14 \\
\hline Oral and written communication in the mother tongue & 4.05 & 13.51 & 31.98 & 31.98 & 18.24 & 0.45 & 1.80 & 12.61 & 35.59 & 49.55 \\
\hline Oral and written communication in a foreign language & 38.29 & 28.60 & 17.79 & 9.91 & 5.41 & 2.25 & 3.15 & 14.86 & 28.83 & 50.90 \\
\hline Ability to use computer tools & 11.71 & 29.05 & 31.76 & 19.37 & 8.11 & 0.68 & 1.13 & 8.78 & 39.19 & 50.23 \\
\hline Ability to look for information in several resources & 4.05 & 19.59 & 31.76 & 34.46 & 10.14 & 0.68 & 4.50 & 26.58 & 46.17 & 22.07 \\
\hline Resolving conflicts or solving problems & 10.36 & 27.03 & 34.91 & 23.42 & 4.28 & 1.13 & 3.15 & 15.09 & 40.32 & 40.32 \\
\hline Making decisions & 10.81 & 21.40 & 39.41 & 22.52 & 5.86 & 0.45 & 2.93 & 10.36 & 38.29 & 47.97 \\
\hline Knowledge of other areas and disciplines & 13.29 & 28.60 & 30.41 & 22.97 & 4.73 & 2.03 & 11.49 & 36.49 & 37.39 & 12.61 \\
\hline Gaining new knowledge quickly & 3.60 & 15.99 & 36.26 & 32.43 & 11.71 & 0.23 & 3.83 & 22.30 & 45.50 & 28.15 \\
\hline Ability to make yourself understood/to communicate & 4.73 & 18.69 & 36.94 & 31.08 & 8.56 & 0.23 & 2.93 & 14.86 & 37.61 & 44.37 \\
\hline Working in a multidisciplinary team & 5.64 & 15.58 & 33.18 & 35.44 & 10.16 & 0.45 & 2.25 & 13.74 & 40.32 & 43.24 \\
\hline Working in an international context & 26.35 & 31.08 & 24.77 & 13.74 & 4.05 & 3.60 & 6.31 & 25.23 & 40.99 & 23.87 \\
\hline $\begin{array}{l}\text { Ability to manage personal relationships and } \\
\text { interpersonal communication }\end{array}$ & 7.88 & 22.07 & 38.74 & 24.77 & 6.53 & 0.90 & 2.25 & 17.12 & 46.62 & 33.11 \\
\hline Working in different and multicultural environments & 18.24 & 29.95 & 25.90 & 19.37 & 6.53 & 3.38 & 8.33 & 27.48 & 36.26 & 24.55 \\
\hline Critical and self-critical capacity & 6.98 & 25.00 & 37.39 & 24.32 & 6.31 & 1.13 & 5.41 & 25.68 & 43.02 & 24.77 \\
\hline Ethical commitment at work & 13.74 & 20.27 & 36.04 & 20.27 & 9.68 & 3.83 & 4.95 & 22.07 & 36.49 & 32.66 \\
\hline Working well under pressure & 7.66 & 11.71 & 29.73 & 30.86 & 20.05 & 1.58 & 3.15 & 14.64 & 36.94 & 43.69 \\
\hline Managing effectively your time & 9.91 & 17.79 & 33.11 & 23.20 & 15.99 & 0.90 & 2.25 & 9.68 & 40.09 & 47.07 \\
\hline Self-learning & 3.83 & 11.49 & 27.70 & 39.86 & 17.12 & 1.13 & 3.83 & 25.45 & 46.40 & 23.20 \\
\hline Adaptation to new situations & 6.08 & 20.05 & 39.41 & 26.80 & 7.66 & 0.23 & 2.70 & 15.09 & 44.37 & 37.61 \\
\hline Creativity & 14.64 & 25.68 & 35.59 & 19.14 & 4.95 & 1.80 & 5.63 & 20.72 & 45.27 & 26.58 \\
\hline Leadership & 15.77 & 31.31 & 31.76 & 14.86 & 6.31 & 1.13 & 3.60 & 18.24 & 39.86 & 37.16 \\
\hline $\begin{array}{l}\text { Initiative and entrepreneurial spirit : finding new } \\
\text { ideas and solutions }\end{array}$ & 10.36 & 28.38 & 33.78 & 2 & 4.50 & 0.90 & 2.70 & 15.32 & 40.77 & 40.32 \\
\hline Motivation by quality & 9.68 & 22.30 & 36.49 & 24.77 & 6.76 & 0.45 & 4.95 & 25.23 & 40.32 & 29.05 \\
\hline Environmental sensitivity & 31.53 & 28.60 & 21.40 & 13.29 & 5.18 & 7.66 & 20.27 & 30.18 & 28.15 & 13.74 \\
\hline Sensitivity to social issues & 18.02 & 27.03 & 30.18 & 18.47 & 6.31 & 4.50 & 15.32 & 30.63 & 32.43 & 17.12 \\
\hline Putting knowledge into practice & 9.68 & 18.02 & 33.56 & 28.15 & 10.59 & 0.68 & 1.58 & 14.41 & 33.11 & 50.23 \\
\hline Ability to look for information and research & 5.86 & 18.92 & 31.53 & 36.04 & 7.66 & 0.90 & 3.60 & 23.42 & 48.87 & 23.20 \\
\hline Design and management of projects & 11.71 & 23.20 & 38.29 & 22.07 & 4.73 & 1.35 & 4.73 & 20.50 & 47.07 & 26.35 \\
\hline Ability to popularize economic issues & 7.88 & 21.85 & 40.32 & 24.55 & 5.41 & 1.13 & 6.08 & 34.01 & 39.64 & 19.14 \\
\hline Present products, ideas or reports in public & 6.08 & 17.34 & 31.31 & 32.88 & 12.39 & 0.90 & 3.15 & 18.69 & 32.88 & 44.37 \\
\hline Trade efficiently & 26.13 & 26.13 & 27.48 & 16.22 & 4.05 & 1.35 & 3.38 & 12.84 & 42.57 & 39.86 \\
\hline Writing reports or documents & 9.01 & 18.02 & 30.63 & 31.08 & 11.26 & 1.13 & 3.60 & 11.71 & 40.77 & 42.79 \\
\hline Emotional self-awareness & 19.59 & 25.00 & 32.66 & 17.57 & 5.18 & 3.60 & 6.08 & 32.66 & 41.44 & 16.22 \\
\hline Emotional self-assessment & 19.14 & 26.35 & 29.73 & 20.72 & 4.05 & 3.83 & 6.98 & 34.23 & 35.81 & 19.14 \\
\hline Self-confidence & 15.54 & 22.07 & 27.25 & 24.10 & 11.04 & 1.35 & 4.05 & 12.61 & 32.43 & 49.55 \\
\hline Empathy & 13.96 & 22.52 & 33.78 & 24.10 & 5.63 & 2.03 & 6.08 & 26.80 & 36.04 & 29.05 \\
\hline Guidance to the achievement/success & 8.11 & 17.57 & 32.66 & 32.66 & 9.01 & 0.90 & 2.48 & 16.44 & 42.79 & 37.39 \\
\hline $\begin{array}{l}\text { Expressing ideas and fighting for rights without } \\
\text { attacking }\end{array}$ & 8.33 & 15.99 & 28.15 & 29.28 & 18.24 & 1.35 & 1.80 & 14.19 & 35.81 & 46.85 \\
\hline
\end{tabular}

SOURCE: Opinion Poll about the Need for a Skill Development. Faculty of Economics and Business. Personal Compilation.

Table A1. Distribution of skill frequencies, by degree of university training and importance in the work environment 


\begin{tabular}{|c|c|c|c|c|c|c|}
\hline \multirow{3}{*}{ Skills } & \multicolumn{6}{|c|}{$\begin{array}{c}\text { Differential average values } \\
\text { Importance at work - University Training }\end{array}$} \\
\hline & \multicolumn{3}{|c|}{$\begin{array}{c}\text { With professional } \\
\text { experience }\end{array}$} & \multicolumn{3}{|c|}{$\begin{array}{c}\text { Without professional } \\
\text { experience }\end{array}$} \\
\hline & Man & Woman & All & Man & Woman & All \\
\hline Analytical thinking and Ability to Synthesis & 0.612 & 0.449 & 0.539 & 0.748 & 0.413 & 0.569 \\
\hline Organization and Planning & 0.836 & 0.782 & 0.812 & 0.993 & 1.019 & 1.007 \\
\hline Oral and written communication in the mother tongue & 0.882 & 0.768 & 0.832 & 0.815 & 0.897 & 0.859 \\
\hline Oral and written communication in a foreign language & 2.106 & 1.855 & 1.994 & 2.237 & 2.013 & 2.117 \\
\hline Ability to use computer tools & 1.694 & 0.899 & 1.337 & 1.711 & 1.593 & 1.648 \\
\hline Ability to look for information in several resources & 0.765 & 0.087 & 0.461 & 0.808 & 0.484 & 0.634 \\
\hline Resolving conflicts or solving problems & 1.165 & 0.957 & 1.071 & 1.526 & 1.368 & 1.441 \\
\hline Making decisions & 1.341 & 1.044 & 1.208 & 1.563 & 1.426 & 1.489 \\
\hline Knowledge of other areas and disciplines & 0.729 & 0.652 & 0.695 & 0.748 & 0.658 & 0.700 \\
\hline Gaining new knowledge quickly & 0.682 & 0.319 & 0.52 & 0.793 & 0.651 & 0.718 \\
\hline Ability to make yourself understood/to communicate & 1 & 0.985 & 0.993 & 1.037 & 1.058 & 1.048 \\
\hline Working in a multidisciplinary team & 0.878 & 0.855 & 0.868 & 0.993 & 0.987 & 0.99 \\
\hline Working in an international context & 1.365 & 1.334 & 1.351 & 1.496 & 1.284 & 1.383 \\
\hline $\begin{array}{l}\text { Ability to manage personal relationships and interpersonal } \\
\text { communication }\end{array}$ & 1.035 & 0.942 & 0.993 & 1.2 & 1.084 & 1.138 \\
\hline Working in different and multicultural environments & 1.035 & 0.71 & 0.889 & 1.222 & 1.039 & 1.124 \\
\hline Critical and self-critical capacity & 1.07 & 0.522 & 0.825 & 0.956 & 0.839 & 0.893 \\
\hline Ethical commitment at work & 1.165 & 0.682 & 0.949 & 1.274 & 0.735 & 0.986 \\
\hline Working well under pressure & 0.611 & 0.55 & 0.585 & 0.718 & 0.916 & 0.824 \\
\hline Managing effectively your time & 1.177 & 0.913 & 1.059 & 1.149 & 1.174 & 1.162 \\
\hline Self-learning & 0.494 & 0.116 & 0.325 & 0.482 & 0.167 & 0.314 \\
\hline Adaptation to new situations & 1.153 & 0.928 & 1.052 & 1.178 & 0.981 & 1.073 \\
\hline Creativity & 1.235 & 1.029 & 1.143 & 1.185 & 1.129 & 1.155 \\
\hline Leadership & 1.435 & 1.348 & 1.396 & 1.326 & 1.574 & 1.459 \\
\hline $\begin{array}{l}\text { Initiative and entrepreneurial spirit : finding new ideas a } \\
\text { solutions }\end{array}$ & 1.4 & 1.232 & 1.324 & 1.511 & 1.206 & 1.348 \\
\hline Motivation by quality & 0.859 & 0.797 & 0.831 & 1.044 & 1.013 & 1.027 \\
\hline Environmental sensitivity & 1.094 & 0.695 & 0.915 & 1.096 & 0.659 & 0.862 \\
\hline Sensitivity to social issues & 1.047 & 0.478 & 0.792 & 1.044 & 0.432 & 0.717 \\
\hline Putting knowledge into practice & 1.165 & 0.884 & 1.039 & 1.296 & 1.239 & 1.266 \\
\hline Ability to look for information and research & 0.941 & 0.377 & 0.688 & 0.911 & 0.503 & 0.693 \\
\hline Design and management of projects & 1.129 & 0.899 & 1.026 & 1.252 & 0.968 & 1.1 \\
\hline Ability to popularize economic issues & 0.917 & 0.348 & 0.662 & 0.933 & 0.587 & 0.748 \\
\hline Present products, ideas or reports in public & 1.012 & 0.753 & 0.896 & 0.949 & 0.82 & 0.879 \\
\hline Trade efficiently & 1.494 & 1.797 & 1.63 & 1.689 & 1.787 & 1.742 \\
\hline Writing reports or documents & 1.236 & 0.725 & 1.006 & 1.103 & 0.987 & 1.042 \\
\hline Emotional self-awareness & 1.129 & 0.55 & 0.87 & 1.185 & 0.877 & 1.02 \\
\hline Emotional self-assessment & 1.023 & 0.493 & 0.785 & 1.155 & 0.942 & 1.041 \\
\hline Self-confidence & 1.494 & 0.869 & 1.215 & 1.452 & 1.303 & 1.373 \\
\hline Empathy & 1.094 & 0.725 & 0.928 & 1.237 & 0.839 & 1.024 \\
\hline Guidance to the achievement/success & 1.023 & 0.74 & 0.896 & 1.178 & 0.845 & \\
\hline Expressing ideas and fighting for rights without attacking & 0.953 & 0.579 & 0.786 & 1.089 & 0.903 & 0.990 \\
\hline AVERAGE INDICATOR & 1.097 & 0.801 & 0.965 & 1.166 & 0.993 & 1.074 \\
\hline
\end{tabular}

SOURCE: Opinion Poll about the Need for a Skill Development. Faculty of Economics and Business. Personal Compilation.

Table A2. Differential: Importance at Work - University Training, by experience, sex and total 


\begin{tabular}{|c|c|c|c|c|c|c|}
\hline \multirow{3}{*}{ Skills } & \multicolumn{6}{|c|}{$\begin{array}{c}\text { Differential average values } \\
\text { Importance at Work - University Training }\end{array}$} \\
\hline & \multicolumn{3}{|c|}{ 4th year } & \multicolumn{3}{|c|}{ 5th year } \\
\hline & Man & Woman & All & Man & Woman & All \\
\hline Analytical thinking and Ability to Synthesis & 0.719 & 0.492 & 0.6 & 0.667 & 0.322 & 0.503 \\
\hline Organization and Planning & 1 & 0.978 & 0.988 & 0.848 & 0.9 & 0.873 \\
\hline Oral and written communication in the mother tongue & 0.785 & 0.963 & 0.878 & 0.909 & 0.7 & 0.81 \\
\hline Oral and written communication in a foreign language & 2.198 & 2.082 & 2.138 & 2.172 & 1.789 & 1.989 \\
\hline Ability to use computer tools & 1.818 & 1.537 & 1.67 & 1.566 & 1.144 & 1.365 \\
\hline Ability to look for information in several resources & 0.909 & 0.538 & 0.713 & 0.647 & 0.1 & 0.386 \\
\hline Resolving conflicts or solving problems & 1.52 & 1.291 & 1.4 & 1.222 & 1.166 & 1.196 \\
\hline Making decisions & 1.537 & 1.246 & 1.385 & 1.404 & 1.4 & 1.402 \\
\hline Knowledge of other areas and disciplines & 0.834 & 0.761 & 0.796 & 0.626 & 0.5 & 0.566 \\
\hline Gaining new knowledge quickly & 0.743 & 0.642 & 0.69 & 0.758 & 0.411 & 0.593 \\
\hline Ability to make yourself understood/to communicate & 1.058 & 1.052 & 1.054 & 0.98 & 1.011 & 0.995 \\
\hline Working in a multidisciplinary team & 0.984 & 0.992 & 0.988 & 0.905 & 0.878 & 0.892 \\
\hline Working in an international context & 1.505 & 1.336 & 1.415 & 1.373 & 1.245 & 1.312 \\
\hline $\begin{array}{l}\text { Ability to manage personal relationships and interpersonal } \\
\text { communication }\end{array}$ & 1.207 & 1.075 & 1.137 & 1.051 & 0.988 & 1.022 \\
\hline Working in different and multicultural environments & 1.124 & 1.015 & 1.066 & 1.182 & 0.822 & 1.011 \\
\hline Critical and self-critical capacity & 1.082 & 0.709 & 0.886 & 0.899 & 0.789 & 0.846 \\
\hline Ethical commitment at work & 1.231 & 0.813 & 1.012 & 1.232 & 0.578 & 0.921 \\
\hline Working well under pressure & 0.694 & 0.717 & 0.706 & 0.656 & 0.933 & 0.789 \\
\hline Managing effectively your time & 1.323 & 1.142 & 1.227 & 0.959 & 1.022 & 0.989 \\
\hline Self-learning & 0.471 & 0.09 & 0.271 & 0.506 & 0.245 & 0.381 \\
\hline Adaptation to new situations & 1.248 & 0.955 & 1.094 & 1.071 & 0.978 & 1.026 \\
\hline Creativity & 1.107 & 1.045 & 1.074 & 1.323 & 1.178 & 1.254 \\
\hline Leadership & 1.405 & 1.403 & 1.404 & 1.323 & 1.656 & 1.482 \\
\hline $\begin{array}{l}\text { Initiative and entrepreneurial spirit : finding new ideas and } \\
\text { solutions }\end{array}$ & 1.529 & 1.105 & 1.306 & 1.394 & 1.378 & 1.387 \\
\hline Motivation by quality & 1.108 & 0.918 & 1.008 & 0.808 & 0.989 & 0.894 \\
\hline Environmental sensitivity & 1.066 & 0.724 & 0.887 & 1.131 & 0.589 & 0.873 \\
\hline Sensitivity to social issues & 0.959 & 0.425 & 0.678 & 1.152 & 0.478 & 0.831 \\
\hline Putting knowledge into practice & 1.29 & 1.015 & 1.146 & 1.192 & 1.3 & 1.243 \\
\hline Ability to look for information and research & 0.984 & 0.455 & 0.706 & 0.849 & 0.478 & 0.672 \\
\hline Design and management of projects & 1.248 & 1.015 & 1.126 & 1.152 & 0.844 & 1.006 \\
\hline Ability to popularize economic issues & 0.926 & 0.537 & 0.721 & 0.93 & 0.477 & 0.714 \\
\hline Present products, ideas or reports in public & 0.926 & 0.672 & 0.792 & 1.031 & 0.989 & 1.01 \\
\hline Trade efficiently & 1.694 & 1.686 & 1.69 & 1.516 & 1.945 & 1.719 \\
\hline Writing reports or documents & 1.141 & 0.813 & 0.968 & 1.172 & 1.044 & 1.112 \\
\hline Emotional self-awareness & 1.115 & 0.762 & 0.929 & 1.222 & 0.8 & 1.021 \\
\hline Emotional self-assessment & 1.107 & 0.836 & 0.965 & 1.101 & 0.756 & 0.936 \\
\hline Self-confidence & 1.612 & 1.187 & 1.388 & 1.293 & 1.145 & 1.222 \\
\hline Empathy & 1.223 & 0.858 & 1.031 & 1.132 & 0.722 & 0.936 \\
\hline Guidance to the achievement/success & 1.256 & 0.828 & 1.031 & 0.95 & 0.789 & 0.873 \\
\hline Expressing ideas and fighting for rights without attacking & 1.075 & 0.791 & 0.925 & 0.99 & 0.822 & 0.91 \\
\hline AVERAGE INDICATOR & 1.169 & 0.938 & 1.047 & 1.082 & 0.908 & 0.999 \\
\hline
\end{tabular}

SOURCE: Opinion Poll about the Need for a Skill Development. Faculty of Economics and Business. Personal Compilation.

Table A3. Differential: Importance at Work - University Training, by year, sex and total 


\section{References}

ANECA (Agencia Nacional de Evaluación de la Calidad y Acreditación), (2004). Encuesta de Inserción laboral. Madrid.

Archibugi, D. and Lundvall, B.-Å. (Eds) (2001). The Globalizing Learning Economy, Oxford University Press. ISBN 9780199241095

Carpintero, O. (1999). Economía y Ciencias de la Naturaleza: algunas consideraciones sobre el legado de Nicholas Georgescu-Roegen. ICE Revista de Economía n ${ }^{0} 779$, pps. 127142. ISSN 0019-977X. Ministerio de Industria Comercio y Turismo, Madrid.

Climent, J. B. (2010) Reflexiones sobre la Educación Basada en Competencias. Revista Complutense de Educación. Vol. 21 Núm. 1, pps. 91-106. ISSN 1130-2496. UCM, Madrid.

Gilbert, M. (2001). El profesor del siglo XXI: de transmisor de contenidos a guía del ciberespacio. I Encuentro internacional "La informática en las Universidades InfoUni'2001 (La Habana, Cuba). Reference: january, 2009. Available from http://tecnologiaedu.us.es/bibliovir/pdf/203.pdf

González, J. y Wagenaar, R. (2003). (Coord.) Tuning Educational Structures in Europe. Informe final. Fase I. ISBN: 84-7485-892-5. Universidad de Deusto. Bilbao Available from: http://www.relint.deusto.es/TUNINGProject/spanish/doc_fase1/Tuning $\% 20 \mathrm{Ed}$ ucational.pdf

Gracia, E. e Iglesia, M. C. (2007). La coordinación y diseño de materiales multidisciplinares para el campus virtual, en el área del Análisis Económico, Proceedings of Libro Actas III, Campus virtual, UCM. Madrid. Pps, 38-50. Available from: http:/ / eprints.ucm.es/5646/1/III_JORNADAS_CAMPUS_VIRTUAL.pdf

Holmes, L. (2000) Questioning the skills agenda, in S. Fallows y C. Steven (comps.) Integrating key skills in higher education: Employability, transferable skills and learning for life. pps. 201-214. ISBN 9780749432652. Londres: Kogan Page LDT

Iglesia, M. C. (2006). Varios retos y un único destino en la adaptación de los contenidos de la Teoría Económica al nuevo contexto de enseñanza aprendizaje. El campus virtual como herramienta de apoyo docente. Aplicaciones Prácticas de la Convergencia Europea. Edita: Servicio de Publicaciones y Oficina de Convergencia, UEX. Badajoz, 2006. ISBN: 84-7723-746-8

Iglesia, M. C. (2007). Joan Robinson, Keynesiana de izquierdas, capítulo 10, Mujeres economistas. Coord. Perdices, L. y Gallego, E. Ecobook, (2007). Pps 315-348. ISBN: 978-84-96877-02-3. Madrid.

Iglesia, M. C. y Ionita, C. (2010). Percepción de la necesidad de un desarrollo competencial en los estudios de economía y administración de empresas. Documento de trabajo, Enero 2010, Instituto de Análisis Industrial y Financiero. UCM. Available from: http:/ / eprints.ucm.es/10860/1/documento_de_trabajo_CIV.pdf

Koopmans, T. C. (1957). Three Essays on the State of Economic Analysis, Mc Graw-Hill. ISBN 70353352 New York

Krueger, A. et al. (1991). Report of the Commission on Graduate Education in Economics, Journal of Economic Literature, 29, pps. 1035-1053.

Levý-Leboyer, C. (2001). Gestión de las competencias: cómo analizarlas, cómo evaluarlas, cómo desarrollarlas. Ed. Gestión 2000. ISBN: 8480886382, Barcelona 
Lundvall, B. A. and Johnson, B. (1994). The learning economy, Journal of Industry Studies, Vol. 1,No. 2, December 1994, pps. 23-42.

McClelland, D. C. (1973). Testing for competente rather than intelligence. American Psycologist, $\mathrm{n}^{\mathrm{o}} 28$, pps. 1-14

Myrdal, G. (1975). The unity of social sciences. Human Organization, 34, 4 pps. 327-31 (See in The Dynamics of Applied Anthropology in the Twentieth Century: The Malinowski Award Papers Society for Applied Anthropology, Cap 5, pps. 70-76 Oklahoma City 2002. Reference: september, 2011, Available from http://www.sfaa.net/malinowski/monograph/malinowski.pdf\#page=70)

Perinat, A. (2004). Conocimiento y educación superior. Nuevos horizontes para la universidad del siglo XXI. Ediciones Paidós Ibérica S.A. ISBN 84-493-31-1664-3 Barcelona, España.

Ramírez, K. M. y Velásquez, A. (2010). Valoración de las competencias para el empleo en los titulados universitarios. Proceedings of I Congreso Internacional virtual de Formación del profesorado. Available from: congresos.um.es/cifop/cifop2010/paper/download/9831/9801 (3, 10, 2011)

Robinson, J. (1937) Introduction to the Theory of Employment. MacMillan (reedited in 1969), London, ISBN 0333043952,

(1953), "Disertación en Oxford por un economista de Cambridge", en Robinson, J, (1979), “Contribuciones a la Teoría Económica Moderna", , Siglo XXI, pps. 171-178, Madrid, ISBN 968-23-0527-6,

(1960), [1964] Essays in Economics Analysis. Macmillan, Londres, Ensayos sobre Análisis Económico. Ed. Fondo de Cultura Económica. México, ISBN 84-375-0021-4

(1964), [1966] Economic Philosophy., Londres; Filosofía Económica. Ed Gredos. Madrid. ISBN 84-249-2602-1

(1973) Collected Economic Papers, Vol IV. Ed. Basil Blackwell. Oxford ISBN: 0631144803

(1977) What Are the Questions?. Journal of Economic Literature, Vol. 15, Issue 4 (Dec.), pps.1618-1339

Samuelson, P. (1947). Foundations of Economic Analysis, Cambridge Mass: Harvard University Press, enlarged edition 1983. ISBN 0674313038

Sladogna, M. G. (2000), “Una mirada a la construcción de las competencias desde el sistema educativo. La experiencia Argentina." En: CINTERFOR-OIT. Competencias laborales en la formación profesional. Boletín Técnico Interamericano de Formación Profesional. No 149, ISSN: 02542439, pps. 109-134

Spenser, L. M. y Spenser, S. M. (1993). Competence at work, models for superior performance. New York. Ed. John Wilwy Sons Inc., ISBN 047154809X

Streeten, P. (2007). “QQué está mal en la Economía contemporánea?”, (initially published in Interdisciplinary Science Review 27,1, 2002 p13-24) Revista de Economía Institucional, vol 9, $\mathrm{n}^{\circ} 16$ pps. 35-62. Available from:

http:/ / www.economiainstitucional.com/pdf/No16/pstreeten16.pdf

Szenberg, M. (1994). Grandes economistas de hoy. Debate Pensamiento, Madrid. ISBN 84-7444$825-5$

Wake, J., Dysthe, O. y Mjelstad, S. (2007). New and Changing Teacher Roles in Higher Education in a Digital Age. Educational Technology and Society. Vol. 1, n. ${ }^{\circ} 10$, pps. 4051. 
Walsh, V y Gram, H. (1980). Classical and Neoclassical Theories of General Equilibrium, Oxford University Press, New York and Oxford. ISBN 0-19-502674-8, p. XI

Zamagni, S. (1986). Georgescu-Roegen, Nicholas, Eds. John Eatwell, Murray Milgate and Peter Newman. Palgrave Macmillan, 1987, The New Palgrave Dictionary, First Edition. vol. II, pps. 515-516. ISBN 0-333-52722-4 


\section{Section 8}

Reforms and Responses to the Global Crisis 



\title{
New Public Management and Reforms in Iran: Analysis of Government Downsizing
}

\author{
Hassan Danaee fard, Tayebeh Abbasi and Mohammad Reza Noruzi \\ Public Administration, Tarbiat Modarres University, (TMU), Tehran,
}

Iran

\section{Introduction}

The transformation of the public sector, generally termed 'new public management', is performed in most countries in association to economy, aiming at development of organizations along capitalist models of global economy, This has been particularly the case in Britain during the last two decades [1,2]. The representation of clear performance critieria, decentralization, government downsizing, cost decrease, competition, marketing, delegating non governance duties to half-independent, $[3,4]$.

This management method is introduced as a solution to insufficiency, weak service, unsuitable responding and reaction to previous failures of governments in context of economic growth as one eminent point of international evolution over public affair management [5, 6 and 7]. Undoubtedly, new theory is the main incentive of fundamental changes in public organizations and a basis for passing welfare state after the World War II.

The first part of this paper will study the bases and principals of new public management with a glimpse at streaks of this management in public sector of Iran, a method of performing new public management i.e. government downsizing In the last part, I shall show that downsizing has not significant value in Iran, and that predicted aims have been achieved in a combined way.

\section{Theoretic principals of new public management}

Different interpreters and claimers of new public management emphasize various aspects of this doctrine $[9,10,11,12,13$ and 14$]$

One of the authors summarizes principales of administration reformations related to new public management in five axes as following:

- Redefinition of administration duties, separating government from agencies, centralizing government on national economic management and representing public services.

- Restructuring public organizations through optimizing administration structure, downsizing public service and improving efficiency of affairs.

- Rationalizing public affairs' responsibility and decreasing duty interference in response framework. 
- Guiding reformation of public organizations on the basis of economic and social condition of each region.

- $\quad$ Establishing a legal and standard framework for affair performance [15].

1st Principle: Managerial orientation that Pollit introduces it as:

Efficiency increase, the use of complicated technologies, regular labor force in order to to ensure productivity and accurate performance of professional management role

$2^{\text {nd }}$ Principle: New public management on the basis of indirect control instead of direct one. Some characteristics of this principle are:

Continuous improvement of quality, emphasizing self-control, contractors, observation, suitable information systems, focusing on market and performance gauge

$3^{\text {rd }}$ Principle: Utilizing engineering techniques of industrial production in public sector.

$4^{\text {th }}$ Principle: Emphasizing cooperation preference based on market [18].

Steven Hood has declared seven indices constructing public management doctrine:

1. Placement of professional management in public sector: Hood says the first aspect of new public management emphasizes active control of public organizations by super managers having power. A sample of this aspect is "Let Managers Manage". This clarifies that there is a need to professional management at a high level [6].

2. Clear gauges and standards of performance: An important tool for the performance of the professional management model, is to have aims, achieve indces, emphasize aims, achievement indices, emphasizing qualitative results and justification on the basis of response. This focuses on results and outputs which are different from input focus (traditional public affairs).

3. More emphasize on controlling public organizations and innovation management: Controls are carried out via plan budgeting, performance, long-term planning and managing organization strategic. Strategic management focuses on aims to which the organization should reach in a very changeable environment. Focus on results instead of process means that the managers mostly pay attention to lower levels.

4. Separating units in public sector: Large Ministries are divided in order to separate policy maker units from performing units smaller ones.

5. Encouraging more competition in public services: The use of marketing principles can be applied to the public sector as jeans of separating performance and production from competition. This may have the advantage of decreasing costs and improving standards (technology transmission from input to output), competition between various producers in order to decrease costs and improving standards.

6. Emphasizing management methods of private sector: This idea means distancing from moral principles of public toward stretching in employment and awarding, performance evaluation and paying on the basis of qualification which emphasizes inserting successful management tools of private sector into public sector.

7. More emphasis on regulation and saving resources: Decreasing direct costs, increasing personnel discipline, resistance against desires of units, limiting other costs in the level of private sector costs and applying information and relation technology [16, 17]. 
Hays and Kearney have represented five principles of new public management through studying published cases:

1. Downsizing: Decreasing the size of government

2. Manageability: Applying principles of commercial management in government

3. Decentralization: Approaching decision making to service receivers

4. Removing bureaucracy: Restructuring government with emphasizing conclusion instead of processes

5. Privatization: Guiding good and service production toward outside of public institutions (private companies' production) [18].

It seems that Hays and Kearney's theoretic frameworks of new public management is the most complete framework. On the basis of this framework, this paper is going to focus on its first axis i.e. government downsizing, its meaning, bases, achievements and failures.

\section{Downsizing}

Governments have encountered economic and official problems during past two decades. Unnecessary group employment made public sector massive. On the basis of latest evaluations, at least $1 / 3-1 / 2$ of state owned personnel are working without bringing any profitability [21].

The governments have decided to achieve their constructions and compensating government capacity lose via granting some tenure activities to independent units or via privatization and contracting [22].

\section{Downsizing concept}

Different theories are represented over downsizing. Some theorists emphasize on decreasing human force in downsizing concept [25, 26]. On the basis of Casio theory (1993), downsizing is planned omission of occupations or posts which does not consist being fired or existing of personnel through retiring etc. [27].

Chan (1990) defines downsizing as decrease, diminution, omission, simplification of efficacy of government activities or public institutes and its internal plans which consists of consumption resource decrease such as budget and human force [24].

Cameron supposes downsizing as a complex of organizational activities which are formed into a management tool in both public and private sector and its aim is to decreases costs and increase of productivity and reengineering of processes [28, 29]. In this definition, downsizing process has the followings lowing characteristics: it is intentional, leads to a decrease in personnel number (via retirement, officer transmission to non-public sectors), facilitates organization or institute productivity via cost control and finally causes process performance via labor force and less resources.

Kettle declares that downsizing follows a simple aim i.e. downsizing the public sector size and contains a complex of clarified tactics of performing all acceptable political actions in order to decrease the number of organizations, costs and the number of officers. He believes in that downsizing would be carried out through malcontent citizen pressure on government who desire decrease of public costs. Main methods believe in that misappropriation is very 
high in public organizations and should be prevented. Therefore, semi-market activities, privatization, reorganizing of public institutes and limitation on the basis of government size are of significant methods [30].

Downsizing is a class of performance which lead to improvement and productivity of organization and decrease of human force cost. They expect some results related to downsizing such as: decrease of per capita costs, less bureaucracy, quick decision making, easier organizational relationships, more innovations and better productivity of organization [31].

There are various downsizing approaches:

\section{Downsizing approaches}

1. Downsizing as Reorientation: Governments or organizations downsizing whose aim is balancing internal environment to external environment should take reorientation approaches. In this approach, the organization tries not only regulate the problems according to the manner of performance but also pay attention to what is demanded in the first step. The aim of reorientation approach is to take an affective, productive and new structure via analyzing and redefinition of mission and the strategic orientation of an organization [31].

2. Downsizing as Convergence: Convergence approach does not refer to any main change in organization, it desires continuous improvement. In this approach, all people participate in downsizing.

Convergence is well performed in minute level of organization such as posts and processes and demands wide participation of people affected by downsizing. The whole organization is aroused in order to take a continuous improvement approach while management tries to cause downsizing aims.

One advantage of downsizing as convergence is that the whole organization participates in permanent improvement therefore an organization of reorientation should follow permanently the experience related to convergence approach.

The aim of reorientation is re-planning of the whole organization, while the aim of convergence approach is strengthening present structure and strategy through permanent effort in order to make a productive organization [31].

When an organization decides to downsize in every approach that it selects, it should utilize suitable strategy or strategies. Appelbaum et al. represented 3 common strategies via studying wide literature related to downsizing:

a. Strategy of decreasing human force: the first selection of downsizing organizations. This is a short term solution with quick effect and includes personnel transition, retirement, redemption, temporary fire and encouraging personnel to leave the organization. Also compulsory or voluntary transitions and firing with or without placement are of other methods utilized in decreasing human force performance. Each method follows a kind of decreasing support strategy. Cameron (1994) believed that this strategy leads to a competency enemia on the whole organization and without considering basic skills and vital human force [26]. Mentzer (1996) proposed that the most effective method of 
decreasing number of personnel is to select in an accurate and precise way instead of equal considering of them.

b. Replacing strategy: The aim of this strategy is to decrease work mass instead of reducing personnel and will be performed via gradual omission of duties, hierarchical levels, units and decrease of weekly work hours.

c. Systematized strategy: The main aim of this strategy is that it gives assure in order not to have human force decrease in a continuous and repeated form in the future. Systematized strategy will connect downsizing to simplification of all work contents and organization units such as supplying primary materials, planning process, marketing, production manners and the whole organization. This strategy emphasizes change of current position, culture, detecting suitable time of performance and longterm results.

These 3 strategies are not independent. Cameron believes that it is better for most organization of downsizing to utilize one strategy instead of various options.

Human force decrease is of several advantages among strategies. Some of these advantages are quick shrinkage of organization, drawing colleagues' attentions to undesirable position of organization, economizing in daily performance cost, attention of remaining personnel to changes and coming predictable decreases.

Some negative results are related to this downsizing strategy. When human force decrease strategy is considered, it is not clarified that who will be omitted from organization, which skills will be lost in organization and what will be the result of human force leaving it. Also downsizing strategy represents mis-performance and inefficacy of organization and contains faithfulness and commitment of personnel. Therefore individualism and "I" will rule the organization instead of "organization" and "we". At first, the organization benefit human force decrease in a short term but some theorists such as Bruton Ana et al. (1996) believe in that these benefits will be harmful to organization.

These organizations can follow other options in the framework of human force policies instead of human force decrease. Some of these actions are human force cost, performance facility, stopping new employments, decrease or omission of personnel benefits and shortening of weekly work hours.

When the organizations take re-planning strategy, they will be able to increase their productivity because of simpler structure. This short-term strategy will cause some changes and limitation in some parts of organization and will not have widespread efficacies.

Other scientists refer to downsizing methods especially in public sector which are mostly cost and human force decrease:

1. Human force balance via optional and before appointed retirements, stopping employment, service compensate plans, redemption policies, re-educating personnel in order to facilitate their transmission to private sector etc. [32, 33].

2. Reforming payment policies such as decrease of salary, advantages and limiting their payments [32]. On the whole, new public management and its main axis are spread in various countries and this point of view has become a public affair management. The following section will address this point 


\section{The experience of new public management in Iran}

The first streaks of new public management were observed in Iran in the form of reformation of Islamic Republic government as the first bill passed in 1982after Islamic revolution victory. The third bill of constitution emphasizes the necessity of suitable system existence and omitting unnecessary structures in order to reform official system. The investigations show that the government system reformations are carried out in five phases during past two decades:

The first bill in 1982

The second bill in 1983

The third bill in 1993

The fourth bill in 1997

The fifth bill in 1999

On the bases of documents, the first and the second ones were organized in order to perform the parliament passed acts on Islamic Republic Iran's administrational system plan. The third bill was established according to changes of management structure furthermore to the above reasons by employment organization in1989.

It can be concluded that the performance of government in bill 1-3 is ignored so the authority and duty of performing organizations should be ordered on the basis of following process.

The main axis of mentioned bills is deletion of repeated and parallel roles and uniting homogeneous roles into a system and the role of administration while its sovereignty and authority are not mentioned. The law of human force modification in public organizations was planned in 1986.

The methods of above mentioned bills were focused on administration duties on the basis of activity topics of government and the nature of duties was less considered. The first economic, social and cultural development plan of Iran was passed according to facilities, resources and limitations in 1989 and continued till 1993.

Some of this reformation plan's aims in budget and finance are making logical public system and decreasing government expenses through transmitting present duties to the private sector.

The plan of imposing some changes in the structure of ministries and specially planning and budget organization structures were passed in 1993. The bases of mentioned reformations are making homogeneous duties and activities of organizations stronger and preventing any repeated and intermixing duties. Therefore the units of common activities were merged and in the cases which duties could be transmitted to other parts, the units were omitted.

The second plan of economic, social and cultural development of Iran was begun in 1995 and it was predicted to be finished in 1999. The main aims of this plan were: the reformation of observing, performing and judiciary structure, balance in cooperative economic sectors of public and private. The second plan's 7th clause determines 12 clauses whose 4 cases are related to the administrational structure reformation: 
1. The administrational order and regulation and omitting parallel systems and imposing observation on the performance of public organizations, companies, banks and municipalities

2. The centralizing, administrational bureaucracy omission, utilizing the most simple performance methods and ceding affairs to the provinces

3. The reformation of administrational and organizational structures in order to give the affairs to private and cooperative sectors and strengthening the province centers

4. Gathering government duties in sovereignty actions in order to strengthen sovereignty activities and decrease of duties as much as possible

The public performance policies of government are defined as following:

1. Decreasing the density of public sector through omitting some structures, merging public ministries, institutions and companies and giving some actions to the private and cooperative sector through demanded regulations and law

2. Privatizing public and under public category companies

3. Giving some public services to municipalities

As the first action of the second plan and in order to reform administrational structural, organizational systems and methods and the management of human force the supreme administrational council composed of president, vice-president, the head of budget and planning organization, some of ministers, the head of employment organization, 2 members of parliament as observers and 3 administrational experts selected by a president are responsible for performing the second plan's policies in the context of administrational system reformation.

According to the results of the first and the second development plans it seemed that the government need one qualified and sufficient administrational system in order to develop plans, revise management affairs, administrational system problems, determining internal pro and cons and the international and internal opportunities and threats. Therefore, the administrational and employment organization gathered the first strategic evolutions plan through a wide study of administrational system conditions, problems, pro and cons. After merging planning and budget and employment organizations into management and planning organization in late of spring 2000, the administrational reformation movement (in the direct of aims and policies of "the 2nd step" at the time of presidency election in 2001 (Khatami's presidency)) changed into a form different from different from the previous ones.

The mentioned reformation movement changed into a lawful formed via gathering the third economic, social and cultural development plan. In most cases this plan is focused on government duties in order to perform reformation in administrational and management system. Its main duties are as following:

1. The decrease of governmental tenure and strength of government sovereignty and observation (articles 1, 2 and 64 of $3^{\text {rd }}$ plan)

2. Providing people participation, private sector and cooperation in management (article 1,2 and 64)

3. Downsizing of government in a logical way (article 1 and 64) 
4. The decrease of the number of governmental personnel to $5 \%$ through gathering human force plan of public sector (article 3)

5. Revision on the internal structure of ministries, organizations, companies and their subcategories in order to structure cohesion and omission of parallel, similar and repeated duties (article 1)

The council of ministers passed a comprehensive development plan of administrational system in 2000 on the basis of article 1 of $3^{\text {rd }}$ economic, social and cultural plan of Iran in order to achieve political aims of "2nd step" of Khatami presidency.

a. Making a logical size of government and its facilities in order to develop the activities of non-governmental sector through clarifying and separation of sovereignty and tenure affairs on the basis of article 64 of $3^{\text {rd }}$ development plan

b. Revolution in structure of government through investigation and identification of government problems, regulating public systems structure, investigation of performance offices and internal and international companies, representing suitable propositions in order to their resistance, omission, merge, etc.

c. Revolution plan in management systems: investigation of council and decision-making assemblies' duties, organization and reformation of public structures, establishing evaluation systems on performing systems, encouraging qualified managers, etc.

d. Revolution plan in employment systems through investigating and reforming employment regulations, occupation classification in order to match the studied fields to organization demands, strengthening experts, solving the problems of personnel income, etc.

e. Education and improvement plan of public human force through reforming evaluation system of public personnel, increasing motivations, supplying officers' salary, etc.

f. Reforming plan of processes, methods, official technology development through regulating wide reformation plan of all performing systems, gathering suitable software standards of representing public services to public sector, existing order and regulation plan, official space organization and preparation, etc.

g. The improvement plan of respecting people in official system through their priority rights over system and officer profits, widespread plan for respecting customers, etc.

As reviewing the $1^{\text {st }}-3^{\text {rd }}$ development plan in Iran and the government's performance in official reformation; it can be concluded that the $3^{\text {rd }}$ development plan is the first comprehensive plan of official reformation after Islamic revolution which has had significant achievements in some fields.

The significant streak of new public management in Iran is depended on government downsizing which is going to be explained in coming part.

\section{Downsizing in Iran}

A tendency of making public affairs existed in Iran after Islamic revolution victory which spread gradually. The lack of development in previous decades forced the government to take performing and comprehensive actions. Therefore, the number of performing systems increased upto 3 times as much the early revolution and the number of officers was 4 times as much. 


\begin{tabular}{|c|c|c|}
\hline Organization & $\begin{array}{l}\text { The number of } \\
\text { subcategory institutions } \\
\text { and companies }\end{array}$ & $\begin{array}{c}\text { The number of the } 2^{\text {nd }} \\
\text { and } 3^{\text {rd }} \text { generations } \\
\text { companies }\end{array}$ \\
\hline Leadership institution & 18 & 1 \\
\hline State expediency council & 5 & \\
\hline Islamic revolution institutions & 440 & 412 \\
\hline Economic ministry & 247 & 212 \\
\hline Education ministry & 279 & 12 \\
\hline State ministry & 1 & \\
\hline Intelligence ministry & 1 & \\
\hline Commercial ministry & 61 & 55 \\
\hline Health and medical ministry & 177 & 110 \\
\hline Agriculture ministry & 100 & 86 \\
\hline Cooperation ministry & 3 & 1 \\
\hline Transportation ministry & 29 & 8 \\
\hline Urbanization ministry & 35 & 35 \\
\hline Power ministry & 162 & 141 \\
\hline Oil ministry & 113 & 103 \\
\hline Science and research ministry & 164 & \\
\hline culture and Islamic guidance ministry & 24 & \\
\hline Defense ministry & 29 & 9 \\
\hline IT ministry & 41 & 32 \\
\hline Social affair ministry & 7 & 2 \\
\hline Industry ministry & 344 & 313 \\
\hline Interior ministry & 38 & 3 \\
\hline \multicolumn{3}{|l|}{ Judiciary ministry } \\
\hline Presidency organization & 31 & 17 \\
\hline Environmental protection organization & 1 & \\
\hline Physical education organization & 4 & 1 \\
\hline Atomic energy organization & 6 & \\
\hline $\begin{array}{c}\text { Cultural heritage and tourism } \\
\text { organization }\end{array}$ & 4 & 3 \\
\hline $\begin{array}{l}\text { Management and planning } \\
\text { organization }\end{array}$ & 28 & 21 \\
\hline Islamic council parliament & 4 & \\
\hline Judiciary branch & 8 & \\
\hline $\begin{array}{l}\text { Companies under municipality } \\
\text { observation }\end{array}$ & 9 & 9 \\
\hline $\begin{array}{l}\text { The number of independent ministries } \\
\text { and institutions }\end{array}$ & 30 & \\
\hline Total number & 2443 & 1574 \\
\hline
\end{tabular}

Table 1. The number of ministries, institutes, public companies, public institutions and public companies of the $2^{\text {nd }}$ and $3^{\text {rd }}$ generations in 2004 
The most important reasons of this action can be:

1. The lack of a clear definition of government role

2. High tendency of political and public managers toward service performing of government

3. The widening of social services because of population growth

4. Urbanism and service (quantity) development

5. Making public schools, hospitals, rehabilitation centers, etc. which were private

6. Changing private banks and insurances into public ones

7. Establishing new public institutions and organizations; the number of public ministries, institutions and companies was 345 in 1996 which is increased to 1130 (upto 4 times). Also, about 2000 companies have been established as second generation.

8. The number of organizational units shows a high digit in comparison to other countries. There are 15348 organizations and offices in national, provincial and urban levels. There are 20 ministries, 21 ministers and 17 performance units under the direct observation of president.

9. The increase of public personnel in comparison to private one is another index of government wide size. The public personnel ratio to the whole personnel was $19 \%$ in 1965 which has increased to $33.7 \%$ in 2001.

10. The conditions of employing as manager has been facilitated and the percentage of management post taking has increased upto $60 \%$.

11. The wrong interpretation of constitution which leads to the increase of public tenure.

12. The culture of parliament responses and members in order to receive more resources and public tenures and starting new constructive projects in related geographical region by government.

13. The lack of reliance in qualification and efficiency of private sector

The complexity of these ideas and actions led to a tinge role of non-public in authority which became worse through the lack of competition. In most of the developed countries, the logical size of government and people participation in taking authority actions were the main part of official reformation plans. Therefore, the authority of government in sovereignty is limited but the main authorities in Iran and other developing countries are the roles and responsible of government because of inefficient management power of nonpublic sector. Some of downsizing policies such as privatization and granting public companies can be seen in the $1^{\text {st }}$ and $2^{\text {nd }}$ plans. The aims and policies of the $2^{\text {nd }}$ plan are based on economic freedom and privatization. The basic shifts occurred in declared strategies but the public tenure and authorities were not followed. A cohesion planning is made in the $3^{\text {rd }}$ plan whose main regulations are:

1. Granting performing systems to non-public sector

2. Merging public ministries and institutions (article 2 of the $3^{\text {rd }}$ plan)

3. Limiting taking the public services (article 3)

4. Granting public companies management and shares to private and cooperative sectors (article 9)

5. The manner of granting public credits to various activities (article 64)

6. Gathering laws related to a part of public financial regulations and predicting a 4-year chance in order to perform it

7. The article 192 is related to the decrease of health and care ministry and article 152 emphasizes the activities of physical education organization 


\section{Government downsizing strategies in Iran}

According to the $3^{\text {rd }}$ plan and policies of political system, these strategies include:

1. Privatization strategy of public companies on the bases of:

- Preventing new capitalization in public companies

- Making logical privatization of companies in order to finish it till to the end of 5-year $4^{\text {th }}$ plan

- Preparing capitalization development context, omitting public monopolies, free and useful competition, supporting capitalism and creativity, reforming work regulation and laws and eliminating export obstacles

- Controlling public systems' activities and granting some social, cultural, service and production tenures through:

- Article 192 over decrease of health and cure ministry

- Article 152 over decrease of physical education organization

- Performing the laws of article 88 of regulating financial activities in order to buy services of non0public sector instead of government's service production and cooperation of nonpublic sector and granting management to this sector

- Granting some of public system activities to municipals

- Supporting non-public sector for developing cooperation in social, economic and cultural activities

2. Reengineering government structure via omitting or merging performance parallel, unnecessary or similar systems in order to reform processes, utilizing information technology and omitting extra organizational levels and posts

3. The number of officers should decrease upto $1.5 \%$ ( $2 / 3$ of present number)

It was predicted that the annual GDP in performance plan regulation of mentioned policies will decrease $2 \%$ to reach $30 \%$ but, it could not be achieved. Also, it was expected these policies do not encounter quality and quantity of public services, the force decreases not to be imposed equally in all sectors, its tendency to be in the direct of economic and social activities and above policies not to lead to joblessness and to be achieved by controlling services and human forces.

The first strategy: Granting public companies: The privatization and share granting of public companies were considered for the first time in the $1^{\text {st }}$ economic, social and cultural development plan and the demanded actions in order to identify the grantable companies were taken in 1990. This policy was followed during the 2nd plan too. The granting committee composed of a president, the head of central bank, economic and asset minister, the head of budget and plan organization (former) and the related organization and ministry boss started granting public companies. This committee decided to grant 538 and omission or merging of 48 companies but no affective action is taken for achieving this aims.

Some reasons of not achieving predicted results in the $1^{\text {st }}$ and $2^{\text {nd }}$ plans are:

1. The lack of management and observation organizations over granting plan

2. The lack of attention toward the expectations of public companies' buyers, focusing on its supply via identifying grantable companies, grant kind and pricing

3. Transmitting shares or activities from an organization to another public organization instead of transmitting it to a private organization 
4. Unclearness or unsuitability of country's economic policies and lack of suitable context of privatization and granting to non-public sector

5. The lack of sufficient past information over grantable companies' activities which show the ability of company in the mentioned activities

6. Some problems of public regulation and orders

The granting of public companies' shares started in 2001 in the frame of the $3^{\text {rd }}$ plan. Therefore, the gathering of demanded information over profitable and detrimental was possible via reforming public companies changeable budgets since 2002.

Some changes occurred along the $3^{\text {rd }}$ development plan which is of 3 main reasons:

1. The position of public companies in share granting and privatization process which leads to the decrease of public companies especially profitable ones.

2. Reactivity of companies which were inactive during the performance years of plan and have not been omitted by the ministry.

3. The change of country divisions and forming new provinces which lead to new companies in these provinces.

These changes of public companies, banks and profit-making institutions which were 500 companies in 2000, reached 507 companies in 2001 through existing 43 companies. The composition of profitable and detrimental companies has been 97 and 410 respectively, which shows the increase of detrimental companies.

\begin{tabular}{|c|c|c|c|c|c|c|}
\hline $\begin{array}{c}\text { Company } \\
\text { separation }\end{array}$ & 2000 & 2001 & 2002 & 2003 & 2004 \\
\hline $\begin{array}{c}\text { The } 1^{\text {st }} \text { and } \\
2^{\text {nd }} \text { generation }\end{array}$ & $1^{\text {st }}$ generation & 174 & 170 & 167 & 170 & 96 \\
\cline { 2 - 7 } & $2^{\text {nd }}$ generation & 376 & 337 & 343 & 348 & 437 \\
\hline $\begin{array}{c}\text { Benefit and } \\
\text { loss }\end{array}$ & Benefit & 457 & 410 & 401 & 408 & 414 \\
\cline { 2 - 7 } $\begin{array}{c}\text { Total number } \\
\text { of companies }\end{array}$ & loss & 93 & 97 & 104 & 110 & 119 \\
\hline
\end{tabular}

Table 2. The number of public companies along the $3^{\text {rd }}$ development plan (2000-2004) on the basis of profit and loss with a separation of $1^{\text {st }}, 2^{\text {nd }}, 3^{\text {rd }}$ etc. generations

The number of public companies increased to more than 510 companies in 2002. This process continued in 2003-2004 and reached 533 companies despite government policies for decreasing the number of companies.

On the basis of planning and management organization reports, the number of detrimental companies has increased from 110 to 11 in 2003-2004.

\begin{tabular}{|c|c|}
\hline Granting year & The number of public companies for granting \\
\hline 2001 & 395 \\
\hline 2002 & 126 \\
\hline 2003 & 108 \\
\hline 2004 & 7 \\
\hline total & 636 \\
\hline
\end{tabular}

Table 3. The number of companies for granting (2000-2004) 
The second strategy: Controlling public systems activity development and granting some of social, cultural, service and production tenures: Furthermore article 88 of regulation laws, other regulation and orders are predicted for granting organizations and ministries activities in order to control their activities and financial tenures.

\section{Health and medical education ministry}

a. Total number of health centers increased from 7089 in 2000 to 7633 in 2004 . The ratio of public centers decreased from $80.05 \%$ to $77.78 \%$ and the private ones increased from $6.38 \%$ to $8.91 \%$ and the ratio of other centers was decreased from $13.57 \%$ to $13.31 \%$ [57].

b. There were 112590 beds that public beds decreased from $69.78 \%$ to $69.67 \%$.

c. Total number of medical laboratories increase from 3701 units (2001) to 4126 units (2004). The public ratio decreased from $50.61 \%$ to $48.18 \%$, private ratio increased from $36.61 \%$ to $38.88 \%$ and other groups increased about $12.49 \%$.

d. Total number of rehabilitation centers increased from 1679 units (2001) to 2125 units (2004). The public ratio was decreased from $13.40 \%$ to $12.47 \%$, private ratio increased from $56.76 \%$ to $63.31 \%$ and other groups decreased from $29.84 \%$ to $25.22 \%$.

e. Total number of drugstores increased from 5918 units (2001) to 6380 units (2004). The public ratio decreased from $5.88 \%$ to $5.41 \%$ and private ratio increased from $90.57 \%$ to $91.27 \%$.

Despite above mentioned affairs and policies related to non-development of public sector, 13 hospitals were added to public sector 2000-2004 [58].

\section{Welfare organization}

On the basis of state economic regulations, welfare organization is obliged to activate in taking care of the handicapped, rehabilitation centers and kindergartens. The results of performance related to this organization concord to the reports from management and planning organization:

a. More activity of non-public sector in the framework of representing services to handicapped people in 2000. This index was 60\% which increased to $75 \%$ in 2004 (an increase of $15 \%)$.

b. The ratio of groups related to social welfare services in non-public sector was $30 \%$ in 2000 which increased to $71 \%$ in 2004 [57].

\section{Physical education organization}

On the basis of article 155 of the $3^{\text {rd }}$ plan, 7 exercising place and 1200 granted units that equaled 20 million dollars in the form of incomes were added to public fund. The government had an economy of 5 million dollars in 2004.

Other performance is giving certification to 3257 gyms along the $3^{\text {rd }}$ plan which is of predicated affairs in the context of representing demanded facilities in order to making the private sector more activate [57].

\section{Education ministry}

The number of non-public students is increased from 853000 in 2000 to 940000 in 2004 (a growth rate of $2.4 \%$ ). 
The non-public ratio in informal technical educations was increased from $38.3 \%$ in 2000 to $52 \%$ in 2004 (a growth rate of $8 \%$ ) [57].

\section{Job and social affair ministry}

The main affairs of this context were performed in 2 ways: decrease of public activities and more activity of non-public sector:

- Granting 50 educational and technical educational centers

- Issuing certificate 54 of internal private placement

- Issuing certificate 30 of international private placement [49]

\section{Agricultural ministry}

Some affairs are carrying out following activities with participation of public sector in order to decrease ministerial tenures:

Protecting national resource, vaccinating, artificial insemination, observing livestock productions, education, distributing chemical fertilizers, irrigational performance, transplanting and their granting, compiling technical criteria of agriculture and livestock sector and granting exercise and health centers depended to ministry.

\section{Transportation ministry}

Participating in non-public sector in representing services of keeping roads, supplying road safeties, road repairs and etc.

Also ministries of communication and IT, culture and Islamic guidance and defense have granted some parts of their activities to non-public sector [59].

The third strategy: Reengineering governmental structures through omitting or merging parallel, unnecessary or similar performing systems: The evolution of official system and lack of government structure development was done with emphasizing a logical downsizing of government size along the $2^{\text {nd }}$ plan. The supreme official council of country as a policy maker organization took actions in order to reform these structures by passing demanded acts (omission, merging or granting internal or abroad organizations and officers). Some of these reformations since 2003 are:

- Merging agricultural ministry and agriculture ministry, industry ministry and mine and metal ministry and the merge of planning and budget organization to employment organization in 2002

- The merge of merchant company to developing merchant service organization and the mission of cereal and rural-urban cooperation organization in 2003

- The merging of all education centers of agriculture research organization, public management education center and the supreme institution of research and development planning, national documents and national library, the omission of Iran carpet company and transforming its duties to merchant ministry, the establishment of Iran trade development organization, the merge of good supply and distribution center, the center of export development and the office of abroad merchant, the omission of road safety 
company and all the sub-companies of transportation ministry which are abroad and the division of finance and economy ministry and taxation organization in 2004.

- The necessary actions related to these affairs were taken in 2004 according to the acts of 2003. The most important of which are:

The assembling of all duties of the martyr, magnanimous and devotee in the office of Islamic revolution martyr affairs, 9 cases of omitting agriculture ministry's abroad offices, the merge of cultural heritage and tourism organizations into cultural heritage and tourism organization, the merge of scientific applied educational institution of Iran industries into mine and industries university, the merge of international law service offices of presidency, the merge of international center of civilization negotiation in foreign ministry, the merge of Islamic medical research center, the national center of medical science and technologies of health and medical educational research assistance, the merge of research centers depended on education ministry, the merge of education planning and innovations in education research and planning organization, the omission of agricultural machinery development company and a decrease in the number of abroad news agencies.

The fourth strategy: Decreasing the number of public offices up to $2 / 3$ of present number (0.5\% annually):

The mentioned strategy was taken into action by public organizations in order to decrease costs and optimizing human force composition. Therefore, the whole number of public officers should be decreased $0.5 \%$ till the end of the $3^{\text {rd }}$ pln.

The statistics of Iran management and planning show that the number of public human force has increased about $3.26 \%$ along the first 2-year of the 3 rd development plan however a decrease of $2 \%$ was predicted for the 3 rd plan. Graph.1 represents a descending process related to the number of officers in contrast to 2002 which has decreased to $2.89 \%$ in 2003 .

Investigations show that some ministries have decreased the number of their officers and some others have increased them along the $3^{\text {rd }}$ plan. Some ministries and organizations such asmerchant, power, urbanization, agriculture, Islamic culture and guidance, foreign, transportation, oil, cooperation, health cares and presidency ministries have decreased their officers number more than $4 \%$ along the $1^{\text {st }} 4$-year of plan. Other ministries have had increased the number of officers.

\section{Conclusion}

The political system of Iran is a unique kind of systems which should take native theoretical official reformations. New public management is one of the main strategies of official evolution. This strategy considers the separation of politic form office and replacing management sovereignty instead of office as its achievement demand. The serious approach of politic to offices has made the public management very problematic. This difficulty has caused much more failures of official reformations in Iran. Also this approach has affected the downsizing which is minor strategy of new public management. The second point is unclearness of official theory over country. Is the official theory especially for this system or is it a composition of capitalism and socialism? Therefore, there is always a kind of suspicion toward the increase or decrease of government role in society. This action has caused a less affective official system movement over downsizing of governments' changes. 
The government increases the budget of public companies coinciding with downsizing and the other government does the downsizing just in order to representing an achievement political symbol of its government. Various analyses show a complex of conclusions i.e. in some cases the downsizing plan is performed on the basis of regulations and in some others, the public officers has decreased and much more officers have been employed. It seems that the clearness of economy theory can affect the official reformations of country. Also the public management professionalization can show the downsizing necessary or unnecessary.

The downsizing decision is taken through decrease of public costs and establishing strong and productivegovernment in Iran. The performance of Islamic Republic of Iran can be taken into consideration in the form 4 strategies. Firstly, the number of public detrimental companies is increasingdespite granting some of companies and the continuous of 8 processes. Some reasons of the failure of government in granting public companies can be: transferring public companies' shares or activities from a public company to another public one instead of a private or non-governmental company, the lack of suitable context for privatization and granting to non-public sector, the contradiction of granting conditions of public companies in the $3^{\text {rd }}$ plan in contrast to public policies over the decrease of public tenures, policies and methods. Secondly, the granting of public services and activities in the form of article 192 of health affairs, article 152 of physical education decrease and article 88 of regulating public financial laws have predicted following activities and services in some ministries and organizations: the education ministry, transportation ministry, agriculture ministry, social affairs and job ministry, health ministry, post ministry, urbanization ministry, welfare organizations, tourism, technical education, document establishment and physical education.

The investigation of government performance shows that activities granting has not been performed in some ministries and organizations of health, social affairs, transportation, agriculture, education, physical education and welfare. Some of this action's reasons can be: the lack of managers' tendencies in granting activities in order to power resistance, disharmony of policies and decisions of legislative and executive branch over granting activities and the lack of powerful non-governmental sector. There needs to have affective non-governmental sectors' activities in order to strengthen government sovereignty and decrease of public actions.

Thirdly, the $3^{\text {rd }}$ plan has increasingly emphasized the composition of government structure and decrease of human force which has caused the merge, omission or gathering of ministries and their internal or abroad offices. These affairs haveonly decreased the number of ministries and their offices and have not affected the costs or productivity of government. The ascending number of employing human forces is in continuous and the aim of balancing $5 \%$ of this force has not been achieved.

It can be concluded that the main part of public resources is consuming in personnel cost sector (the size of government, public costs and the number of public personnel). Therefore, the role of government should be investigated, public costs should be controlled seriously, and some activities of low productivity and high energy consuming should be identified and revised. In that case the government can represent optimized services in the coming years.

The government should be able to resist its power and capacity along downsizing. The downsizing should be performed without any significant harm in welfare affairs. This 
should be taken into action on the basis of exact investigations over public organizations' capacity (human force and other resources).

Another challenge of downsizing is lack of sufficient information over human force productivity in public sector. The problem of brain drain causes significant disorders in this sector and demands productive human force employment in the service representation.

\section{References}

[1] Jackson Audrey And Japsley Irvine (2003) The Diffusion Of Accounting Practices In The New Management Public Sector, The International Journal Of Public Sector Management, Vol.16, No.5, pp.359-372.

[2] Hood Christopher (1990) De-Sir Humphrefying The Westminster Model Of Bureaucracy: A New Style Of Governance, Governance, Vol.3, No.2, , pp.205-214.

[3] Pedersen Christoffer Green (2002) New Public Management Reforms Of The Danish And Swedish Welfare States: The Role Of Different Social Democratic Responses, Governance: An International Journal Of Policy, Administration And Institution, Vol.15, No.2, pp.271-294.

[4] Boden Rebecca, Gummett Philip, Cox Deborah and Barker Kate (1998) Men In White Coats... Men In Grey Suits... New Public Management And The Funding Of Science And Technology Services To The UK Government, Accounting, Auditing And Accountability Journal, Vol.11, No.3, Pp.267-291.

[5] Manning Nick And Parison Neil (2004) Implications For The Russian Federation, International Public Administration Reforms, P.75

[6] Hood Christopher (1991) A Public Management for All Seasons, Public Administration, No69, pp.3-19.

[7] O,Flynn Janine (2004) Adding Public Value: A New Era Of Contractual Governance? Institute Of Public Administration Australia, Public Administration Challenges And Capabilities: Research And Practice, 10 November.

[8] Ocampo Romeo.B (1999) Modals Of Public Administration Reform: New Public Management (NPM), Asian Review Of Public Administration.

[9] Shaw Ian And Sozen Soleyman (2002) The International Applicability New Public Management: Lessons From Turkey, The International Journal Of Public Sector Management, Vol.15, No.6, PP.475-486.

[10] OECD (1996) Governance In Transition: Public Management Reforms In OECD Countries.

[11] Kim Shin Bok (2000) Reform Efforts Toward New Public Management In Korea, Llas/ Japan Joint Panel On Public Administration In Bologna, June 21.

[12] Olowu Dele (2002) Introduction New Public Management: An African Reform Paradigm?, Aferica Development, Vol.Xxvii, No.53, Pp.1-16.

[13] Oven Huse (1998) New public management: Strategic view on thoughts, concepts and theories, Tehran, Morvarid publication.

[14] Kolthoff Emile, Huberts Leo And Heavel Hans van Den (2003) The Ethics Of New Public Management: Is Integrity At State, Ethics \& Integrity Of Governance, Portugal, September.

[15] Asborn.P Stephen (2002) Public Management Critical Perspectives, London, Rotledge, P.185. 
[16] Ferlie.E, Ashburner.L, Fitzgerald.L And Pettigrew.A (1996) Characterizing The New Public Management, The New Public Management In Practice, 11-20.

[17] Economist (2000) Vol.346, No.8, 14 March.

[18] OECD (2004) Renewal of public sector: organizational structure in change, Mahmud Tavassoli, Mashhadi Ahmad, pp. 25-34.

[19] Wallis Joe.L and Dollery.E.Brian (2000) The Appropriateness Of The New Zealand Model Of Public Management Reform To South Aferica, Working Paper Series In Economics, No.8, August, http:/ /Www.Une.Edu.Au.

[20] Haun S Chan (2004) Downsizing federal government. Morteza Atufinia: Logicality of government size in 5 continents, vol.1, pp.227-270.

[21] Kivimaki Mika, Vahtera Jussi, Pentti Juana, Thomson Louise, Griffiths Amanda and Cox Tom (2001) Downsizing: Changes in Work and Self Related Health of Employees, Anxiety, Stress and Coping, Vol.14, Pp.59-73.

[22] ameron.K (1994) Strategies for Successful Organizational Downsizing, Human Resources Management, Vol.33, No.2, Pp.189-211.

[23] Manson.J Bonita (2000) Downsizing Issues, the Impact on Employee Moral and Productivity, Stuart Bruchey, New York.

[24] Lamsa Anna Maija (1999) Organizational Downsizing: An Ethical versus Managerial View Point, Leadership and Organization Development Journal, Vol.20, No.7, Pp.345-353.

[25] Burgin Robert (1997) Downsizing in Libraries: Experiences and Expectations, Library Management, Vol.18, Num.4, pp.187-182.

[26] Monavarian Abbas (2000) Downsizing of public sector, No.50, pp.54-73.

[27] Appelbaum.H Steven, Everard Andrea and Hung T.S Lorella (1999) Strategic Downsizing: Critical Success Factors, Management Decision, Vol.37, No.7, pp.535552.

[28] Mark Shakter (2005) Reformation in public sector of developing countries: coming problems, lessons and directions, Mahshid Foruhari, extract of public management, no.1, pp.16-24.

[29] Armstrong Jim and Jack Dafi (2004) government downsizing: problems and their solutions, Seyyed Hoseyn Mirhoseyni.

[30] Plan and budget organization (1997) The evolution circle of plan and budget organization: Tehran.

[31] Plan and budget organization (1997) The rule of first plan related to economic development, cultural and social of Islamic Republic of Iran, Tehran.

[32] Employment organization (1999) The appendix of official evolution plan, Tehran.

[33] Employment organization (1998) Strategic plan of official evolution system, Tehran.

[34] Planning and management organization (2004) The articles of government size logicality, Tehran

[35] Planning and management organization (2005) Annual economic report of 2004 and observing the 5-year third development plan, Tehran.

[36] Planning and management organization (2004) The articles of government structural reformation, Tehran.

[37] Planning and budget organization (1997) The rule of the second economic development plan, social and cultural of Islamic Republic of Iran, Tehran. 
[38] Planning and management organization (2004) A report on development plan in official system of country, Tehran.

[39] Planning and management organization (2004) The passed laws of management system plan, Tehran.

[40] Planning and management organization (2004) The passed laws of employment system plan, Tehran.

[41] Planning and management organization (2004) The passed laws of education and improvement system plan, Tehran.

[42] Planning and management organization (2004) The passed laws of process reformation plan, Tehran.

[43] Planning and management organization (2004) The passed laws of people improvement and respect plan, Tehran.

[44] Planning and management organization (2005) A report on articles of management organization.

[45] Planning and management organization (2004) A performance report on public organizations

[46] Planning and management organization (2000) Economic, social and cultural development plan of Islamic Republic of Iran, Tehran.

[47] Planning and management organization (2001) A regulation on government fiscal rules, Tehran.

[48] Planning and management organization (2005), An economic report of 2004 and observing 5-year third development plan, Tehran.

[49] Planning and management organization (2002), Economic report of 2001 and observing the second year of the third development plan, Tehran.

[50] Planning and budget organization (1999), A report on performance of the first 4 years of the second development economic, social and cultural plan of Iran, Tehran.

[51] Planning and management organization (2003), An economic report on 2002 and observing 3-year of the third development plan, Tehran.

[52] Planning and management organization (2004), The regulations of official supreme council and the performance of evolution plans in official system, Tehran.

[53] Planning and management organization (2005), An economic report of 2004 and observing 5-year performance of the third development plan, Tehran.

[54] Planning and management organization (2000), An economic report of 2004 and observing 4-year performance of the third development plan, Tehran.

[55] Planning and management organization (2005), An economic report on 2004 and observing 5-year performance of the third development plan, Tehran.

[56] Planning and management organization (2002), The regulations of official supreme council and evolution plan performance in official system, Tehran.

[57] Planning and management organization (2003), The regulations of official supreme council and evolution plan performance in official system, Tehran.

[58] Band.C David And Tustin.M Charles (1995) "Strategic Downsizing, Management Decision, Vol.33, No.8m, pp.36-45.

[59] Beirut A (1999) Public Sector Downsizing Early Retirement Schemes \& Voluntary Severance Pay, Surf For Arab States, Undp.Com.

[60] Vito Tanzi And Ludger Schaknecht (1996) Reforming Government Industrial Countries, Finance \& Development, PP.22-37. 
[61] Rama Martin (2001) Public Sector Downsizing: An Introduction, the World Bank, Economic Review, Vol.13, No.1, pp.1-22.

[62] Estache Antonio, Laffont Jean-Jacques And Zhang Xinzhu (2004) Downsizing With Labor Sharing And Collusion, Journal Of Development Economics, No73, PP. 519540 . 


\title{
Media of Exchange and Liquid Assets of Political and Market Enterprises: A New Monetary Perspective on Medieval French Monetary Mutations
}

\author{
Thomas Marmefelt \\ 1 University of Södertörn, Department of Social Sciences, \\ ${ }^{2}$ Åbo Akademi University, Åbo/Turku, \\ ${ }^{1}$ Sweden \\ 2Finland
}

\section{Introduction}

Liquidity constitutes a crucial link between the financial system and the real economy. Schumpeter (1911) argues that credit gives entrepreneurs the additional purchasing power required to realize innovations, which contribute to qualitative improvement of the economic process. In other words, bankers inject the liquidity required for innovation. However, following Austrian business cycle theory (see e.g. Mises, [1912] 1924, 1928; Hayek, 1929, 1931, Huerta de Soto, 2006), credit creation may cause economic cycles by creating an artificial boom that leads to a bust, that is harmful injections of liquidity cause business cycles.

In his formalization of Austrian business cycle theory within a capital-based macroeconomic model, Garrison (2001) finds that credit expansion yields unsustainable growth because it makes the market rate of interest lower than the natural rate of interest, which reflects consumers' intertemporal preferences. Huerta de Soto (2006) argues that credit creation unbacked by real saving gives malinvestment that distorts the production structure because the latter no longer corresponds to what consumers voluntarily would maintain.

This paper combines these two perspectives and argues that liquidity of enterprises in the real economy provided through credit creation by the financial system ex ante must be absorbed by innovation desired by consumers ex post. Hence, sustainable growth requires that the financial system, through a trial-and-error process, finds the correct liquidity which meets the financing needs of innovation desired by consumers.

What do we mean by liquidity? According to Yeager (1968), there are two concepts of liquidity; one is the amount of the medium of exchange and the other the total purchasing power of firms and individuals available from their asset holding and borrowing possibilities. He argues that the actual medium of exchange constrains the total purchasing power of firms and individuals, because attempts to sell liquid assets or borrow would 
mean declining prices of financial assets, higher interest rates, and tighter credit rationing. Following Gurley and Shaw (1955), Yeager stresses that asset preferences limit the expansion of near moneys if money supply is constant.

Similarly, Hicks ([1967] 1979) stresses that John Maynard Keynes used two different concepts of liquidity; one is money in the General Theory and the other is the relative concept of more or less liquid assets in the Treatise; the former Hicks calls fully liquid and the latter more or less liquid. He argues that, like real assets, financial assets may be categorized as running, reserve, or investment assets; money and trade credit are running assets, some level of liquidity is necessary - at least more or less liquid - for reserve assets, which represents the precautionary demand for money, while industrial assets may be interestbearing and possibly illiquid. Hicks argues that banks put strains on their reserve assets by giving credit, which can be contracted, thus forcing industrial clients to draw money from their running assets, while the monetary authorities may increase liquidity of banks by purchasing securities. In Hicks's (1977) view of the credit economy, representing Keynes's non-competitive banking system, the financial system consists of a monopolistic core and competitive mantle, while the rest of the economy is called industry, whose real investments require the core to increase its liability money.

Consequently, the amount of the medium of exchange constrains liquidity, since increased real investments when money supply is constant require that liquidity is reduced, either in the mantle, in case of borrowing, or in industry, in case of drawing on reserves. However, Greenfield and Yeager (1983) propose the BFH system, which lacks base money and separates the unit of account, defined by the government as a comprehensive commodity bundle, from privately issued media of exchange subject to competition. In such a system of monetary separation, the amount of the media of exchange is demand-driven, because there is no base money impeding them, while payments will be made by transfer of ownership shares in BFH funds, mutual fund banks, a blend of banks and mutual funds. The financing requirements of the real economy determine the amount of the media of exchange and thereby the liquidity. Liquidity as the required purchasing power of agents in the real economy, in terms of more or less liquid assets, would determine liquidity as the amount of media of exchange, the fully liquid assets.

While the BFH system represents what Cowen and Kroszner (1987) call a complete functional separation of unit of account from medium of exchange, in pre-modern times, there has been at least a partial separation in Europe from Charlemagne to the French Revolution (see Einaudi, 1936; Einzig, 1966), implying a distinction between imaginary money - the measure of value, the unit of account - and real money - medium of exchange, various metal coins. This paper analyzes monetary mutations of debasement and enhancement and the liquidity of political and market enterprises, considering medieval French monetary mutations from the perspective of new monetary economics. A theoretical interpretation by means of new monetary economics of medieval French monetary mutations will be made, using the literature on this historical phenomenon.

\section{Monetary separation and liquidity: An evolutionary perspective}

By liquidity this paper means the liquidity of political and market enterprises, which may be studied by means of fiscal sociology. According to Wagner (2007), the real economy consists 
of political and market enterprises that operate in the state and the market, which are two arenas of interaction, constituting the public squares and the market squares of society that are linked together by the economy-wide network of political and market enterprises. $\mathrm{He}$ considers both the state and the market as emergent orders with their own set of enterprises, connected through links between market enterprises and political enterprises. In his view, taxation provides the equity of political enterprises in the state, while fiscal phenomena emerge through human interaction, like market phenomena, thus implying that the state budget is an outcome of an evolutionary process. In that process, parliamentary assemblies are intermediaries connecting taxpayers with political entrepreneurs.

However, as Wagner argues, taxpayers, who sponsor a political enterprise, may leverage their own capital with capital provided by other taxpayers, who are forced investors. Accordingly, societies have different kinds of networks of political and market enterprises, which all need sufficient liquidity to conduct their real investments and deal with uncertainty. Both political and market enterprises look for additional purchasing power to fund their investments.

Monetary separation may influence the liquidity of political and market enterprises, as Greenfield and Yeager's (1983) proposed BFH system illustrates. In the BFH system, monetary separation means a functional separation between a physically defined unit of account and privately issued media of exchange; the unit of account being defined by the government as a nearly comprehensive commodity bundle and the privately issued media of exchange being subject to competition.

In the BFH system, the amount of the media of exchange is demand-driven, because there is no base money impeding them. As Yeager (2001) argues, money is an instrument of financial intermediation and the quantity of money changes with the demand for media of exchange in an economy without base money, using the BFH system. Consequently, with monetary separation the liquid assets of political and market enterprises giving them sufficient purchasing power determine the amount of the media of exchange.

Monetary separation of the medium of exchange from the unit of account with non-tangible means of payment is the basic feature of new monetary economics (Cesarano, 1995), to which the BFH system belongs. Cowen and Kroszner (1994) modifies the BFH system to develop an evolutionary account why traditional monetary functions, medium of account and medium of exchange can be separate assets rather than a single asset, money. They compare the viability of the laissez-faire BFH system with other commodity bundle media of account systems: directly convertible media with a limited bundle and a governmental compensated dollar. Cowen and Kroszner define five functions of money:

i. Store of value: a durable asset or commodity, which serves as an intertemporal abode of purchasing power;

ii. Medium of exchange: an asset or commodity which is held for purposes of indirect exchange and can be transferred to make purchases and retire debts;

iii. Medium of settlement: an asset or commodity which is delivered to extinguish the legal claims that the payee has upon the payer;

iv. Medium of account: a standard in which prices and debts are quoted;

v. Unit of account: a specific quantity of the medium of account. 
In the BFH system, the nearly comprehensive commodity bundle is the medium of account, in which the unit of account is physically defined. The medium of settlement is the redemption medium of Yeager and Greenfield's (1989) indirect convertibility, according to which banknotes and deposits would be redeemable in some redemption medium, say gold. In the BFH system, indirect convertibility pegs the price level, since it requires banks to redeem media of exchange of other banks with an amount of gold, or some other redemption medium, giving arbitrage that reverses any deviation of the price of the bundle from its defined price (Woolsey, 1992). Media of exchange are redeemed in some redemption medium, some commodity or security, in amounts equal to the medium of account of the commodity bundle defining the unit of account (Woolsey \& Yeager, 1994). In other words, $x$ units of a medium of exchange are redeemed indirectly in the quantity of some redemption medium having the same current market value as $x$ commodity bundles, defining the unit of account (Yeager, 2001).

While Greenfield and Yeager try to design a system with monetary separation, Cowen and Kroszner take an explicit evolutionary approach, combining Menger's $(1892 a, b)$ theory of the evolution of money, in which money evolves to overcome the inconvenience of barter with Wallace's (1983) legal restrictions theory, according to which without any legal restrictions interest-bearing securities would be preferred to money. While Menger argues that media of exchange evolve to overcome the inconvenience of barter, Cowen and Krozner (1994) argue that the development of media of account is historically and logically prior to the development of media of exchange. They examine how financial innovation separate media of account from media of exchange and settlement in historical, current, and hypothetical future economies, stressing that change come about as technological and institutional constraints change, while regarding the net return on media of exchange and settlement as a driving force behind their evolution. Considering legal restrictions, the Act of 1765 raised the impediments to interest-bearing notes in Scotland, as a consequence of the prohibition of the option clause, which gave interest to banknote holders during the postponement of redemption (Cowen and Kroszner, 1989).

According to Cowen and Kroszner (1994), increases in asset liquidity, financial innovation, and new transaction technologies decrease the importance of fixed nominal value demand deposits, and liquid securitized assets traded directly through mutual funds bypass traditional banks, while competition in financial markets brings about multiple media of account, exchange, and settlement. In particular, they stress that multiple media of account have been common throughout history. Their story of the evolution of media of account and exchange take place in the following stages:

1. Barter with media of account: Media of account to facilitate trade as language that communicates price information

2. Credit transactions as a substitute for the use of medium of exchange: Intertemporal barter

3. Separate media of exchange easy to store, divide and carry: Generally accepted exchange media arise as the most salable or liquid of assets

4. Unification of medium of account and medium of exchange in a single asset or commodity: media of exchange produced in homogeneous units and more liquid, eventually becoming a commonly understood value

5. New exchange media evolve: monetary evolution continues; assets with superior pecuniary returns displacing extant exchange media assets 
They put the Mengerian story, where marketable intermediary commodities to overcome the double coincidence of wants evolve and generally acceptable media of exchange arise as the most salable or liquid of assets through a market process, at the third and fourth stages. They argue that media of account evolve before media of exchange and that these two functions are eventually united in a single commodity or asset, as liquidity plays an increasing role in the choice of medium of account. The reason for liquidity being so important, they point out, is that the medium of exchange comes to represent a commonly understood value by being more liquid than the medium of account, but they also stress that the development of homogeneous units for media of exchange is a relatively recent historical development.

Nevertheless, they have a process orientation and regard monetary evolution as continuous, so new media of exchange will evolve, as the economy-wide adaptation of media of account and exchange leads to trading posts - spot and futures markets - for assets that bear pecuniary returns that begin to displace extant media of exchange, involving a preference for assets in form of electronic or bookkeeping entries rather than physical form. This is the introduction of intangible money that Cesarano (1998) considers as a key institutional change in the $21^{\text {st }}$ century.

According to Cowen and Kroszner (1994), technological progress increases the pecuniary benefits of using claims to productive assets as media of exchange and settlement and may eventually exceed the cost of separating the media of account from the media of exchange and of settlement, and the technologies that support monetary separation also lower the costs of multiple exchange media. Exchange would be based upon a medium of account value. However, multiple media of exchange and settlement would not be enough. Cowen and Kroszner point out that multiple media of account prevail in the early and the later stages of financial evolution, in advanced stages due to heterogeneous traders in terms of endowments and desired portfolio position. The media of account have a communicative function.

Yeager (1998) views money as a record-keeping device, a mechanism to facilitate trade, stressing that money prices convey information that gives coordination. This would be the medium of account function of money, but also the medium of exchange function has informational aspects. According to Cesarano (1999), the Mengerian selection process for commodities with the highest "salability" was guided by the search for informationally more efficient ways of settling transactions. Yeager (2000) argues that money is an example of institutional evolution from a commodity to a clearing device. Money becoming a clearing device opens up for monetary separation again. Dembinski and Perritaz' (2000) regard the weakening of money as a thing and increasing complexity of institutional arrangements as a break-up of money.

From a Searlean perspective, money prices possess language-like symbolism (Horwitz, 2007; Marmefelt, 2009). As Searle (1999) argues, all institutions but language require language or language-like symbolism. Searle's description of the symbolizing role of language as the foundation of the social universe is quite analogous to the symbolizing role played by money prices in the economic universe, and money prices communicate contextual and tacit knowledge, which is beyond the capacity of language (Horwitz, 2007). Market prices 
possess a language-like symbolizing function, but language is required to establish a shared meaning of money, property, exchange, and price, in order to give market prices that function (Marmefelt, 2009).

Stressing money's role as record-keeping device facilitating the clearing of multilateral exchanges, Yeager (1998) regards the unit of account as vocabulary, grammar, and idioms, while the media of exchange constitute particular documents and speech recordings. The latter may become electronic. Cowen and Kroszner (1994) points out that assets with superior pecuniary returns begins to displace the extant medium of exchange and market participants will prefer assets in the form of electronic or bookkeeping entries, while liquidity increases. However, they also point out that both the early and the late stages of financial evolution involve considerable multiplicity among the media of account. Hence, these are characterized by multiple vocabularies, grammars, and idioms and selection among them.

For communication it is essential that they remain clear, which requires a stable unit of account. Unless price signals are clear, communication by means of prices becomes less efficient and investments more inconsistent, thus forcing the financial system to constrain liquidity. In the BFH system that Greenfield and Yeager (1983) propose, a physically defined unit of account, as the total market value of definite quantities of specific commodities, is separated from the media of exchange subject to competition, and since the unit of account has its general purchasing power fixed by definition, it is a stable unit for pricing, invoicing, accounting, economic calculation, borrowing and lending, and writing contracts. However, it is based upon weights that represent the worth of the commodities at the time of definition. The commodity bundle is nearly comprehensive, including most commodities (Woolsey \& Yeager, 1994), thus giving stability for some time period, but as the economy evolves over time, the worth of the commodities in the commodity bundle may shift, both in absolute and relative terms.

A physically defined unit of account in terms of a bundle of commodities cannot maintain itself in the long run. Some commodities may become obsolete for industrial use and new may enter, while innovation will increase the services obtained from physical quantities of various commodities. For example, the refrigerator and the freezer decreased the worth of salt in food conservation, and more fuel efficient car engines decreased the quantity of crude oil required to drive a certain distance.

The economic value in terms of services we may obtain from the various commodities to be included in the bundle defining the unit of account is what really matters, not their physical quantities. Among classical economists, Steuart (1810) argues that the money of account is the ideal scale of equal parts that performs to, with regard to value what degrees do to angles, and when it becomes realized in gold and silver, it becomes the price and the measure of value, because only the money of account constantly preserves an equal value, the only permanent and equal scale. As examples he mentions the florin banco of the Bank of Amsterdam and the macoutes on the coast of Angola. The first he calls "an invention, of men instructed in the arts of commerce" that provides a standard on which the prices of all things are regulated, while precious metals, with their intrinsic value, vary with regard to this standard. He points out that bank money is unaffected by 
alterations in weight, fineness, and denomination of coins, while the macoute, which is divided in ten pieces, operates where there is no real money, but is used to estimate worth of things. According to Steuart, the money of account cannot be fixed to any material substance. This means that the use of a bundle of commodities, defined by weight, is problematic.

Meulen (1934) clarifies the importance of an invariable unit of value, referring to Steuart's money as arbitrary scale of equal parts, invented for measuring the value of things vendible and not fixable in perpetuity to any commodity. He also criticizes John Stuart Mill for describing the macoute, but failing to see that it constitutes an invariable unit. Meulen's historical examples of invariable units include:

i. the bank-notes in England during the Bank Restriction Act (1797-1819), where drainage of gold made commodities offered in bank-note prices only;

ii. the Assignats in France, which were a medium to pay government debt rather than a true credit medium, but still managed to function as unit of price before the real depreciation of the paper franc; and

iii. standardized paper as price unit in both the United States during the Civil War and in Russia with the practically inconvertible rouble notes.

Considering gold as unit of value, Meulen argues that a commodity unit of value has disadvantages, that a valuable metal used to measure value is a yard-stick made of guttapercha, and that stability in the unit of value requires that one fixes upon and standardizes the worth of some particular commodity at some particular time, stressing that value represents worth, not weight. He lets the banknote become the invariable unit of price, while the price of gold fluctuates with supply and demand. His reform is inspired by the French banquier system, a bill of exchange system where sellers draw drafts on their customers and their bankers discount these drafts for them that existed in France for some time and later in Belgium, Switzerland, Germany, and Austria. For commodity money systems, Sargent and Velde (2002) make a related distinction between coins exchanging by weight and coins exchanging by tale, and they point out that fiat money implies exchanges by tale. We may say that worth is reflected in tale.

However, also a banknote system may be problematic for the invariability of value. Wicksell ([1906] 1966) provides an example from $18^{\text {th }}$ century Sweden, the inconvertible notes issued by Sveriges Riksbank from 1745 that were too abundantly issued, especially during the Pomeranian War, causing the notes to depreciate relative to silver, which eventually led to the appreciation of the specieriksdaler - Sweden's international coin with virtually invariable weight and metal content - and the riksdaler Hamburger banco, ${ }^{1}$ used for most bills of exchange, relative to the bank notes in daler or mark. Consequently, the increase of liquid assets of political enterprises could be detrimental to the invariability of value of a banknote system. The determination of an adequate level of liquidity in a credit economy is therefore of great importance.

1 The Hamburger banco provided a unit of account. Wicksell points out how all great merchants required that all payments to them be calculated in the Hamburger banco and made settlements through giro payments. 


\section{Liquidity in the credit economy}

Cesarano (1999) refers to Wicksell's ([1906] 1966)pure credit economy as a main contribution to the understanding of a payment system devoid of currency, pointing to the accounting system of exchange possibly evolving today. The pure credit economy may be the direction to which the advanced economies today evolve. Information and communication technology plays an important role. Cesarano (2008) explicitly says that it moves actual economies towards a Wicksellian credit economy by making tangible money obsolete, while Dembinski and Perritaz (2000) argue that it drives a break-up of money, meaning that more specialized instruments and institutional arrangements take over the traditional functions of money. Hence, information and communication technology contributes to the evolution of an economy based upon intangible money that provides liquidity.

Based on Keynes, Hicks (1977) develops a credit economy model with a banking core, a financial mantle, and an outer industry as three concentric sectors, and he shows that an increase in real investment with constant money supply reduces liquidity somewhere, either in industry itself, if drawing on its reserves, or in the financial mantle, if borrowing. As Leijonhufvud (1984) argues, Hicks' credit economy model implies that bank financing of investment, which increases productivity, gives an endogenous increase in the money supply. Monetary separation may be helpful in this respect.

In Greenfield and Yeager's (1983) proposed BFH system, where monetary separation is complete, the amount of the media of exchange is demand-driven, because there is no base money impeding them, since mutual fund banks simply adjust fund shares depending on the portfolio selection of market participants between money and bonds. Provision of cheap credit to overcome under-consumption is the solution Meulen (1934) provides, arguing for the adequacy of the Scottish option clause, involving deferred redemption with interest being paid during the time the payment was deferred.

According to Meulen, credit is necessary in any advanced system of division of labor and legal restrictions cause capital to go only where the demand is extremely keen, and the combination of a limited exchange medium volume and high dividends to shareholders lead to an accumulation of the medium of exchange in the hands of the financial class not performing due labor. Having the gold standard as frame of reference, he continues that the banker, whom he calls the professional judge of commercial integrity and ability, is precluded from making long-term loans due the danger of being forced to redeem media of exchange into gold, forcing employers to turn to the unprofessional public. He mentions several disadvantages:

i. Employers obliged to submit themselves to a board of directors

ii. The banker as professional judge of commercial worth more likely to encourage true ability than the untrained public

iii. Fraudulent company promotion due to the limited liability company, an irresponsible organism without personal responsibility

iv. Credit extended at a fixed rate by a banker, in contrast to shareholders who provide equity on condition of sharing increased profits proportionally

This is the opposite of the more recent idea of capital markets as markets for corporate control. Not only does Meulen strongly advocate a credit economy rather than a loanable funds economy, but in his credit economy, the credit system will make sufficient injections of credit to monetize the powers of workers, thus preventing over-production. Meulen's 
ideal system of credit uses credit tokens, which are confidence-based, thus ruling out the need for redemption into gold; being issued by a reliable person who would redeem debt in due time is all a banknote requires. In other words, the media of exchange, the credit tokens, will be demand-driven to achieve full employment. The establishment of such a system implies that the economy moves from assignment of function in virtue of physical structure to pure status, like Searle (1999) characterizes the move from commodity money to fiat money. The media of exchange of credit economies have a pure status function and lack a function in virtue of physical structure.

Wicksell ([1906] 1966) argues that credit is a remedy to scarcity of money and explains how bills of exchange increase virtual velocity, before turning to the development of banking with certificates of deposit and banknotes, and the rise of the modern bank when deposit and giro banks started lending out deposits. In his pure credit system, credit is substituted for gold and the value of money is separated from gold. His essential issue is the exchange value of money and he considers an index based on the product of commodity price and quantity, but he rejects the possibility of such an index having an invariable value, because commodities mean different things to different individuals. An inconvertible paper coin would, according to Wicksell, imply the replacement of gold by credit media, and he stresses that the unit of value should be independent of material things. Hence, Wicksell's analysis implies an abstract medium of account, like Meulen's analysis. With the development of intangible money, Wicksell's paper coin may, in turn, be replaced by an intangible coin. However, liquidity is not limited to coins and banknotes.

Gurley and Shaw (1960) show that non-monetary indirect financial assets contribute to liquidity. They classify financial markets into submarkets for:

1. primary securities: bonds, equities, mortgages, and consumer debt;

2. indirect securities: (i) money: means of payment; (ii) non-monetary indirect securities: time and savings deposits, savings and loan shares

They describe how a short-term security, the Treasury bill, reduces the demand for money, but also how a Treasury issuing bills is a competitor to the monetary system, and that nonmonetary indirect financial assets grow. This can be seen as an injection of liquidity into political enterprises by market enterprises, but Gurley and Shaw point out that Treasury bills constitute a liquidity substitute and may be used by banks to regulate their own liquidity to obtain a desired level of reserves to primary securities. For example, banks could decrease their liquidity by selling Treasury bill and buying bonds. Hence, the existence of non-monetary indirect financial assets means that the form of liquidity between money and non-monetary indirect financial assets may vary.

Gurley and Shaw's non-monetary financial intermediation corresponds to intermediation in Wicksell's pure credit economy, or Meulen's ideal system of credit. Gurley and Shaw point out that non-monetary intermediaries may increase the supply of loanable funds by demanding primary securities, thus raising the total supply of loanable funds if not offset by a reduction if demand by spending units and the monetary system. ${ }^{2}$ Consequently, there are ways to expand liquidity of enterprises beyond money.

${ }^{2}$ Loanable funds have in their analysis a more general meaning, including credit. 
Monetary separation may play an important role in this process. The historical experience of liquidity with partial monetary separation in pre-modern economies, facing monetary mutations, such as the medieval French economy, may provide some valuable lessons that shed light on liquidity with the BFH system, as an alternative system of complete monetary separation.

\section{Liquidity with medieval imaginary and real monies subject to monetary mutations: The French experience in the light of new monetary economics}

The medieval French economy was subject to monetary mutations under a monetary régime of partial monetary separation. As such, it provides a case in point of monetary practices in pre-modern Europe. The monetary system in pre-modern economies in Europe from Charlemagne to the French Revolution was characterized by partial monetary separation, having two complementary monies: imaginary money and real money. Fantacci (2005) calls this period in European monetary history, a remarkably long period in which precious metals were used as means of payment without being defined as standard of value, during which ideal money or imaginary money was used as unit of account, the measure of value without having its metal equivalent legally defined, and real money as medium of exchange in the form of coins made of gold, silver, or copper.

Bloch (1954) describes how the Carolingians in France concentrated monetary power by controlling the mint, thus creating a "good money", ending the minting of gold, so rather than paying a sou (shilling) of gold, one paid twelve deniers (pennies) of silver, following Charlemagne's definition 1 livre (pound) $=20$ sous (shillings) $=12$ deniers (pennies); the sou and the livre being monies of account representing a real matter, a numerical expression of a certain value in deniers, a system lasting until the $18^{\text {th }}$ century. Einaudi (1936) considers the European monetary system between the $9^{\text {th }}$ and the $18^{\text {th }}$ centuries with a distinction between a monetary unit of contract and a unit of payment as rather common and durable, and he finds that the relation between imaginary money and effective money of payment varied inversively with the amount of the latter.

He shows that with a bimetallic standard, the imaginary money kept the monetary system in equilibrium, where the functional relationship reflected the relative market price between gold and silver, and that with imaginary money, three units had to be correlated: the imaginary monetary unit, the real monetary unit, and the unit of an economic good, so if the prices of both the real monetary unit and the unit of the economic good - the commodity - in terms of the imaginary monetary unit doubled, then the price of the unit of economic good in terms of the real monetary unit remains the same. In such a system, real money, having a market price in terms of imaginary money, provides liquidity. Coin shortages were a problem in medieval France that coincided with debasements and the gold écu replaced the livre as unit of account during the 1577-1602 period (Sargent \& Velde, 2002). The return of the livre suggests that the system with partial monetary separation, using an abstract unit of account, was resilient.

The stability of a bimetallic standard of gold and silver coins could be maintained by adjusting the legal ratio of gold to silver according to the market ratio, Einaudi (1936) argues. Referring to the case of Milan in $1762,{ }^{3}$ he finds that fifty-one gold and silver coins

\footnotetext{
${ }^{3}$ Einaudi's data is from Cesare Beccaria," Del disordine e de' rimedi delle monete nello stato de Milano nell' anno 1762," in Le opere, Vol. I, Florence: Le Monnier, 1854.
} 
were used, most of them foreign, each having its legal ratio expressed in the Milanese money of account, the lire di imperiali, an imaginary money holding the system together. However, he clarifies that it could happen that errors were made, attributing to coins with different metal content - weight and fineness - the same value in imaginary money, the money of account, but were easily adjusted when the coins were of the same metal, either gold or silver. It was more complex for coins, which were coined in two or more different metals.

Einaudi presents a simplifying example, with the initial ratio of gold to silver at 1:12, having only two coins:

i. gold fiorino (florin): weighing 120 grains of fine gold; valued at 24 imaginary lire; and

ii. silver scudo: weighing 120 grains of fine silver; valued at 2 imaginary lire, a twelfth of the gold coin according to the initial ratio.

Einaudi shows how the value of the silver coin varies. Letting the ratio of gold to silver change to $1: 11^{1 / 2}$ and 1:12 $\frac{1}{2}$, respectively, he finds how the value changes from 2 imaginary lire to 2.0869 and 1.92, respectively. From this he concludes that the function of the imaginary money in a bimetallic monetary system is to maintain the system in equilibrium by changing the current rate of real money in money of account.

As media of exchange, the two real monies in the example above constitute two liquid assets, while their respective value is expressed in imaginary money. Based on the relative legal rate of the two metals - the current ratio of gold to silver - that should adopt the relative market rate, the value of the liquid assets of enterprises, that is the liquidity of enterprises, in money of account, imaginary money, is maintained by compensating a relative rise or decline by adjusting the price of the real monies used in exchange, thus demanding a higher amount of real money, whose price in imaginary money has fallen.

However, in order to maintain liquidity, enterprises had to adjust their portfolios of liquid assets, shifting from real monies falling relative to the imaginary money into real monies stable relative to the imaginary money. Consequently, they would be able to improve their liquidity by shifting from real monies falling relative to the imaginary money into real monies rising relative to the imaginary money.

However, Bloch (1954) shows that the legal ratio between the two metals used in real money gold and silver - was fixed by the money of account, as general barometer of value, in France during the ancien régime: in 1660, a gold louis with gold content 6.81 gram, equal to 10 livres, and a silver écu with silver content 27.95 gram, equal to 3 livres. According to Bloch, this gives a ratio of approximately 1:13.6, although 1:13.7 would be more accurate $(932 / 68 \approx 13.7)$.

More important, Bloch argues that it may not be totally accurate to talk about a bimetallic standard, since gold was used essentially in international commerce, while most of domestic payments and all small payments were made in silver; and that the kings who paid in silver had an interest in valuing silver coins highly, while the discordance between the legal and commercial rate caused trouble, and the money of account provided the means to establish good order; the monetary system of germinal year XI established a variable ratio between gold and silver. The explanation, Bloch finds in the preceding monetary mutations that according to him followed a pattern of radical debasement, gradual reinforcements, and radical debasement again. They represent monetary mutations, but those are more involved. 
Monetary mutation, Fantacci (2005) calls any variation in the relationships between imaginary money, real money, and metal and he distinguishes two types:

i. Change in metal content at the mint: debasement (decrease), reinforcement (increase)

ii. Change in the tariff: enhancement (increase), abatement (decrease)

He shows that debasement and enhancement have identical effects on metal parity, debasement reducing metal content without affecting the legal value; enhancement by augmenting the legal value without affecting the metal content. For Savoy from 1500 to 1600, he finds exclusive use of debasement for small coins, but almost exclusive use of enhancement for large coins, representing the two exchange circuits: local trade, where extrinsic value matters, and long-distance trade, where intrinsic value matters.

For France in the early 1690s, Bloch (1954) shows how the legal value was changed in a way that looks as enhancements, increases in the tariff. Nevertheless, he calls them debasements, which he finds to be combined with gradual reinforcements. According to Bloch, in 1690 the old silver écu worth 3 livres was replaced by the new silver écu worth 3 livres 6 sous, thus debasing the silver écu by raising the silver écu from 3 livres to 3 livres 6 sous, followed in 1693 with a similar operation raising the silver écu to 3 livres 12 sous; while old coins were invalidated and redeemed at 3 livres 2 sous in 1690 and 3 livres 4 sous in 1693, thus giving the king a profit of 4 and 8 sous, respectively.

Why would the king engage in such activity? As Rolnick et al. (1996) show, seigniorage revenues were substantial in debasement years, but minting volumes increased both during debasement and reinforcement. In France during the ancien régime, the kings aimed at activating the minting of money, and the price of the gold mark increased from 60 livres to 63 livres 10 sous from December 1360 to January 1374, while the price of the silver mark increased from 5 livres to 5 livres 17 sous from April 1360 to October 1373, and debasement with increased tariff were very frequent, thus activating the minting, while enhancement of the coins preserved or increased the seigniorage, according to Landry ([1910] 1969).

Monetary mutation should, therefore, be considered as a means to increase the liquidity of political enterprises. A political enterprise that found sufficient liquidity was the defence by mercenaries. Bloch (1954) refers to transition from feudal military service to mercenaries as a new need created and that debasement gave increased means of payment available to the king. The Hundred Years War between France and England meant an increase in the demand for money. Military innovations in the fourteenth century induced the hire of foreign crossbowmen, but the French fleet also required the hire of foreign ships, according to Miskimin (1963).

In addition, foreign credit was also important. Miskimin (1963) argues that foreign credit mattered to the domestic economy only when included in the coinage, because a reminted foreign loan increased money supply, but still medieval credit relied more upon increasing velocity than the money supply. He mentions how forced loans brought hoardings into circulation, thus increasing velocity, but also that debt instruments, including bills of exchange, did not become means of payment. Nevertheless, as Wicksell ([1906] 1966) argues, bills of exchange increase virtual velocity, thus overcoming the scarcity of money through credit, while Gurley and Shaw (1960) point out that non-monetary indirect securities may increase the supply of loanable funds, thus contributing to liquidity. 
According to Miskimin (1963), the survival of France in the fourteenth century required elaborate fiscal expedients, such as credit, and France and England, both utilized substantial credit operations to finance the war, including loans from foreigners, while both French and English kings tried to prevent the circulation of foreign coins, which had to be carried to the mint. The French ordinance of June 30, 1306, stated that foreign gold and silver coins had no exchange rate in the Kingdom of France, but had to be carried to the mint (Saulcy, 1879), thus activating the minting of money and thereby creating seigniorage. This leads us back to the issue of monetary mutations, in particular their relationship with seigniorage.

Rolnick et al. (1996) define monetary mutation as any change in the mint equivalent, which is the ratio of a coin's legal tender value (the official rate in units of account) to its silver or gold content; mint price as the units of account paid per unit of weight for metal; and gross seigniorage as the difference between mint equivalent and mint price. Gross seigniorage increases with the mint equivalent, which in turn increases by raising the legal tender value (enhancement) or decreasing the gold or silver content (debasement).

Rolnick et al. compare the monetary history of England and France during the $14^{\text {th }}$ and $15^{\text {th }}$ centuries and find that in France between 1285 and 1490, there were 123 debasements of the silver currency, out of which 112 with more than 5 percent, but 64 debasements in gold currency, out of which 48 with more than 5 percent, while England enjoyed monetary stability that ended with the Great Debasement from 1542 to 1551.

The gross seigniorage rate - the ratio of gross seigniorage to mint equivalents - they find to be 7.5 percent for silver and 2.0 percent for gold in normal years, increasing to 21.7 for silver and 4.3 for gold during debasement years in France during the 1354-1490 period, but 4.6 percent for silver and 1.1 percent for gold in normal years, increasing to 16.2 percent for silver and 6.9 percent for gold during debasement years in England during the 1280-1600 period. Hence, debasements were important sources of liquidity of political enterprises. In addition, they find that minting volumes increased after both debasements and reinforcements. This implies that the media of exchange expanded. Consequently, monetary mutation increased the liquid assets of political enterprises and liquidity as media of exchange.

In late medieval France, the king determined the metallic value of the unit of account by specifying the number of livres to be struck from the mark of Paris, a metallic unit of weight, while the pied de monnaie, which expresses a relationship between the alloy, face value, and weight of the coin, was used to control the silver coinage, according to Miskimin (1963). He considers the pied de monnaie to be an index of debasement, since the number of livres to be struck from the mark is one fourth of the pied de monnaie. However, this may reflect enhancement.

According to Landry ([1910] 1969), monetary separation allowed the kings to augment and diminish the legal value of money, controlling minting through the pied de monnaie, while there was interaction between the legal value and the market value. In particular, he shows how the tariff chosen by the king influenced the proportion of silver that is minted relative to that used for other purposes. Through this kind of interaction between state and market, the kings were able to influence the minting of money and thus money supply. 
Landry stresses that in France during the ancien régime, the kings were always concerned about activating the minting of money, while the existence of a money of account facilitated monetary mutations. Landry's definition of debasement, which includes enforcement, and of reinforcement, which includes abatement, implies that his concern is metal parity, but still he mentions that enhancement was more frequent, because it was seen as seen as more honorable.

While Landry argues that debasement activated the minting of money to the benefit of the seigniorage, Miskimin states that debasement decreased the amount of metal that had to be brought to mint, but it also increased commodity prices, at least the price of wheat that he considers, although monetary mutation cannot always explain price changes. The price of the commodity increased with debasement, while holding the face value of debased money constant, according to Miskimin. In France, the ordinance of September 8, 1306 stated that weak money will be valued according to its intrinsic value, so that three deniers of it would correspond to one denier of good and strong money (Saulcy, 1879).

Miskimin (1963) stresses that the number of livres struck to the mark matters to money supply, unlike debasement. This means that the unit of account can be adjusted to control liquidity, while media of exchange are subject to market forces, like commodities. The geographic origin of the media of exchange is less important. Miskimin finds that wheat prices were rather insensitive to mint output and metal inflow, because there was alteration in coinage between France, Flanders, and England, reflecting foreign trade.

However, in order to give market enterprises sufficient liquidity, credit became necessary, including bills of exchange, along the lines of Wicksell ([1906] 1966), Meulen (1934), and Bloch (1954), the latter pointing out how the bill of exchange was established in the $13^{\text {th }}$ century to facilitate settlement between different locations in different monies. Hence, bills of exchange belonged to the long-distance exchange circuit and they were therefore very susceptible to debasements, thus explaining why enhancements were made for large coins used in the long-distance exchange circuit. However, the rise of bills of exchange as media of exchange is a matter for further research.

\section{Conclusion}

Liquidity is a crucial link between the financial system and the real economy. Sustainable growth requires that the financial system, through a trial-and-error process, finds the correct liquidity which meets the financing needs of innovation desired by consumers.

In the BFH system that separates a physically defined unit of account from privately issued media of exchange, the amount of the media of exchange is demand-driven, because there is no base money impeding them. Nevertheless, a commodity bundle is problematic in terms of stability of the medium of account, but so is banknote money. In a credit economy, an abstract medium of account with credit media of exchange may be appropriate, as media of exchange can expand beyond bank money.

Monetary separation in pre-modern Europe meant that imaginary money was used as unit of account and real money as medium of exchange. The former was an institution, while the latter to some extent can be seen as a commodity. In the BFH system, the unit of 
account is defined as a commodity bundle, in which each commodity is included in a specific quantity.

Under the gold standard, when the medium of account and medium of exchange functions were united, money was a pure commodity, a specific quantity of gold. In the pre-modern European monetary system, however, the medium of account was independent of any commodity, while coins as media of exchange had an intrinsic value, in that sense, being a commodity, but also an extrinsic value, in that sense, being an institution. Pre-modern money could be seen above all as a clearing device that later with the gold standard turned into a commodity.

Pre-modern money was subject to monetary mutations changing the metal parity of the unit of account, thus economizing on metal, as illustrated by medieval French monetary mutations. Debasements reduced metal content without affecting the legal value, thus decreasing the intrinsic value without changing the extrinsic value, while enhancements augmented the legal value without affecting the metal content, thus increasing the extrinsic value without changing the intrinsic value. This relative increase of extrinsic value to intrinsic value injected liquidity into the real economy, because seigniorage augmented liquid assets of political enterprises and the media of exchange expanded due to greater minting volumes, although enhancement, unlike debasement, seems to have influenced money supply.

The liquid assets of political enterprises improved, but the liquid assets of market enterprises should also have improved, but a credit medium, such as the bill of exchange was necessary. The unit of account can be adjusted to control liquidity, while media of exchange are subject to market forces, like commodities. Unlike the BFH system, where the unit of account is commodity-based, the units of account in pre-modern Europe were based on social convention and could therefore be adjusted to control liquidity.

\section{Acknowledgement}

An earlier version of this chapter with the title "Media of Exchange and Liquid Assets of Political and Market Enterprises: Some Lessons from Pre-Modern Monetary Practices" was presented at the European Association for Evolutionary Political Economy Conference 2010: The Economic Crisis and the Renewal of the European Model(s), Bordeaux, France, October 28-30, 2010.

\section{References}

Bloch, M. (1954). Esquisse d'une historie monétaire de l'Europe, Cahiers des Annales No. 9, Librairie Armand Colin, Paris

Cesarano, F. (1995). The New Monetary Economics and the Theory of Money. Journal of Economic Behavior \& Organization, Vol. 26, pp. 445-455, Reprinted in Cesarano (2008)

Cesarano, F. (1998). Providing for the Optimum Quantity of Money. Journal of Economic Studies, Vol. 25, pp. 441-449, Reprinted in Cesarano (2008)

Cesarano, F. (1999). Monetary Systems and Monetary Theory. Kredit und Kapital, Heft 2/1999, pp. 192-208, Reprinted in Cesarano (2008) 
Cesarano, F. (2008). Money and Monetary Systems: Selected Essays of Filippo Cesarano, Edward Elgar, ISBN 978-1-84720-519-3, Cheltenham \& Northampton, MA

Cowen, T., \& Kroszner, R. (1987). The Development of the New Monetary Economics. Journal of Political Economy, Vol. 95, pp. 567-590, ISSN 0022-3808

Cowen, T., \& Kroszner, R. (1989). Scottish Banking before 1845: A Model for Laissez-Faire? Journal of Money, Credit, and Banking, Vol. 21, pp. 221-231, ISSN 1538-4616

Cowen, T., \& Kroszner, R. (1994). Explorations in the New Monetary Economics, Basil Blackwell, ISBN 1-55786-071-8, Oxford and Cambridge, MA

Dembinski, P. H., \& Perritaz, C. (2000). Towards the Break-Up of Money, In: Georg Simmel's Philosophy of Money: A Centenary Appraisal, Backhaus, J. G., \& Stadermann, H.-J. (Eds.), pp.175-200, Metropolis-Verlag, ISBN 3-89518-297-4, Marburg

Einaudi, L. (1936). Teoria della moneta immaginaria nel tempo de Carlomagno alla revoluzione francese. Revista di storia economica, Vol. 1, pp. 1-35

Einzig, P. (1966). Primitive Money in Its Ethnological, Historical, and Economic Aspects (Second Edition, Revised and Enlarged), Pergamon, Oxford

Fantacci, L. (2005). Complementary Currencies: A Prospect on Money from a Retrospect on Premodern Practices. Financial History Review, Vol. 12, pp. 53-61, ISSN 1474-0052

Garrison, R. W. (2001). Time and Money: The Macroeconomics of Capital Structure, Routledge, ISBN 0-415-07982-9, London and New York

Greenfield, R. L., \& Yeager L. B. (1983). A Laissez-Faire Approach to Monetary Stability." Journal of Money, Credit, and Banking, Vol. 15, pp. 302-315, Reprinted in Yeager, L. B. (1997). The Fluttering Veil: Essays in Monetary Disequilibrium, Selgin, G. (Ed.), Liberty Fund, ISBN 0-865-97146-3, Indianapolis, IN

Gurley, J. G., \& Shaw, E. S. (1955). Financial Aspects of Economic Development. American Economic Review, Vol. 45, pp. 515-538, ISSN 0002-8282

Gurley, J. G., \& Shaw, E. S. (with a Mathematical Appendix by Einthoven, A. C.) (1960). Money in a Theory of Finance, The Brookings Institution, Washington, DC

Hayek, F. A. von (1929). Geldtheorie und Konjunkturtheorie, Beitrage zur Konjunkturforschung, Österriechischen Institut für Konjunkturforschung, no.1

Hayek, F. A. von (1931). Prices and Production (with a foreword of L. Robbins), Routledge, London

Hicks, J. R., ([1967] 1979). Critical Essays in Monetary Theory, Clarendon Press, ISBN 0-19828423-3, Oxford

Hicks, J. R., (1977). Economic Perspectives: Further Essays on Money and Growth, Clarendon Press, ISBN 0-19-828407-1, Oxford

Horwitz, S. (2007). Learning, Monetary Exchange, and the Structure of the Economic Universe: An Austrian-Searlean Synthesis. In: Economics and the Mind, White, M., \& Montero, B. (Eds.), pp. 75-88, Routledge, ISBN 978-0-415-77056-9, London and New York

Huerta de Soto, J. (2006). Money, Bank Credit, and Economic Cycles, Ludwig von Mises Institute, ISBN 978-0-945466-39-0, Auburn, AL

Landry, A. ([1910] 1969). Essai économique sur les mutations des monnaies dans l'ancienne France de Philippe le Bel à Charles VII, Bibliothèque de l'École des Hautes Études 185e fascicule, Librairie Honoré Champion, Paris 
Leijonhufvud, A. (1984). Hicks on Time and Money. In: Economic Theory and Hicksian Themes, Collard, D. A., Helm, D. R., Scott, M. F. G., \& Sen, A. K. (Eds.), Oxford University Press, Oxford, Reprinted in Leijonhufvud, A. (2000), Macroeconomic Instability and Coordination: Selected Essays of Axel Leijonhufoud, Edward Elgar, ISBN 1-85278-967-0, Cheltenham

Marmefelt, T. (2009). Human Knowledge, Rules, and the Spontaneous Evolution of Society in the Social Thought of Darwin, Hayek, and Boulding. Journal of Economic Behavior E Organization, Vol. 71, pp. 62-74, ISSN 0167-2681

Menger, C. (1892a). Geld. Handwörterbuch der Staatswissenschaften III vgl. No. 24 (Third Edition (1909) Handwörterbuch der Staatswissenschaften IV), Gustav Fischer, pp. 555610, Jena

Menger, C. (1892b). On the Origin of Money. Economic Journal, Vol. 2, pp. 238-255, ISSN 0013-0133

Meulen, H. (1934). Free Banking: An Outline of a Policy of Individualism, (Second Edition), Macmillan, London

Mises, L. von ([1912] 1924), Theorie des Geldes und der Umlaufsmittel, (Second Edition, Reprint 2005), Duncker \& Humblot, ISBN 3-428-11882-0, Berlin

Mises, L. von (1928). Geldwertstabilisierung und Konjunkturpolitik, Gustav Fischer, Jena

Miskimin, H. A. (1963). Money, Prices, and Foreign Exchange in Fourteenth Century France, Yale University Press, New Haven and London

Rolnick, A. J., Velde, F. R., \& Weber, W. E. (1996). The Debasement Puzzle: An Essay on Medieval Monetary History. Journal of Economic History, Vol. 56, pp. 789-808, ISSN 1471-6372

Sargent, T: J., \& Velde F. R. (2002), The Big Problem of Small Change, Princeton University Press, ISBN 0-691-11635-0, Princeton and London

Saulcy, F. de (1879). Recueil de documents relatifs à l'histoire des monnaies frappées par les rois de France. Depuis Phillipe II jusqu'à François Ier, Volume 1, Imprimerie nationale, Paris

Schumpeter, J. A. (1911). Theorie der wirtschaftlichen Entwicklung, Duncker \& Humblot, Leipzig

Searle, J. (1999). Mind, Language, and Society: Philosophy in the Real World, Weidenfeld \& Nicholson, ISBN 0-75380-921-4, London

Steuart, J. (1810). Principles of Banks and Banking of Money, as Coin and Paper, Davis, London

Wagner, R. E. (2007). Fiscal Sociology and the Theory of Public Finance, Edward Elgar, ISBN 978-1-84720-246-8, Cheltenham \& Northampton, MA

Wallace, N. (1983). A Legal Restrictions Theory of the Demand for 'Money' and the Role of Monetary Policy. Federal Reserve Bank of Minneapolis Quarterly Review, Vol. 7, pp. 1-7

Wicksell, K. ([1906] 1966). Föreläsningar i nationalekonomi, Häfte II: Om penningar och kredit, (Fifth Edition), Gleerups (first edition: Berlingska), Lund

Woolsey, W. W. (1992). A Model of the BFH Payments System. Southern Economic Journal, Vol. 59, pp. 260-272

Woolsey, W. W., \& Yeager, L. B. (1994), “Is There a Paradox of Indirect Convertibility?" Southern Economic Journal, Vol. 61, pp. 85-95

Yeager, L. B. (1968). Essential Properties of the Medium of Exchange. Kyklos, Vol. 21, pp. 4569, Reprinted in Yeager, L. B. (1997). The Fluttering Veil: Essays in Monetary Disequilibrium, Selgin, G. (Ed.), Liberty Fund, ISBN 0-865-97146-3, Indianapolis, IN 
Yeager, L. B. (1998). Are Markets Like Language? Quarterly Journal of Austrian Economics, Vol. 1, pp. 15-27

Yeager, L. B. (2000). Against Mistaken Moralizing. Quarterly Journal of Austrian Economics, Vol. 3, pp. 49-54

Yeager, L. B. (2001). The Perils of Base Money. Review of Austrian Economics, Vol.14, pp. 251266, ISSN 0889-3047

Yeager, L. B, \& Greenfield, R. L (1989). Can Monetary Disequilibrium Be Eliminated? Cato Journal, Vol. 9, pp. 405-419, Reprinted in Yeager, L. B. (1997). The Fluttering Veil: Essays in Monetary Disequilibrium, Selgin, G. (Ed.), Liberty Fund, ISBN 0-865-971463, Indianapolis, IN 



\section{Edited by Asunción López-Varela}

This is a unique and groundbreaking collection of questions and answers coming from higher education institutions on diverse fields and across a wide spectrum of countries and cultures. It creates routes for further innovation, collaboration amidst the Sciences

(both Natural and Social), the Humanities, and the private and public sectors of society. The chapters speak across sociocultural concerns, education, welfare and artistic sectors under the common desire for direct responses in more effective ways by means of interaction across societal structures. 